\title{
ASSESSMENT OF ELECTROSPINNING AS AN IN-HOUSE FABRICATION TECHNIQUE FOR BLOOD VESSEL MIMIC CELLULAR SCAFFOLDING
}

\author{
A Thesis \\ Presented to \\ The Faculty of California Polytechnic State University \\ San Luis Obispo
}

\author{
In Partial Fulfillment \\ of the Requirements for the Degree \\ Master of Science in Biomedical Engineering
}

by

Colby M. James

August 2009 
(C) 2009

Colby M. James

ALL RIGHTS RESERVED 


\section{COMMITTEE MEMBERSHIP}

TITLE:

Assessment of Electrospinning as an In-House Fabrication

Technique for Blood Vessel Mimic Cellular Scaffolding

AUTHOR: $\quad$ Colby M. James

DATE SUBMITTED: $\quad$ September 2009

COMMITTEE CHAIR: $\quad$ Dr. Kristen O’Halloran Cardinal, Assistant Professor

COMMITTEE MEMBER: Dr. Lanny Griffin, Department Chair

COMMITTEE MEMBER: Dr. Robert Crockett, Professor 


\begin{abstract}
Assessment of Electrospinning as an In-House Fabrication Technique for Blood Vessel Mimic Cellular Scaffolding

by
\end{abstract}

Colby M. James

Intravascular devices, such as stents, must be rigorously tested before they can be approved by the FDA. This includes bench top in vitro testing to determine biocompatibility, and animal model testing to ensure safety and efficacy. As an intermediate step, a blood vessel mimic (BVM) testing method has been developed that mimics the three dimensional structure of blood vessels using a perfusion bioreactor system, human derived endothelial cells, and a biocompatible polymer scaffold used to support growth of the blood vessel cells. The focus of this thesis was to find an in-house fabrication method capable of making cellular scaffolding for use in the BVM. Research was conducted based on three aims. The first aim was to survey possible fabrication methods to choose a technique most appropriate for producing BVM scaffolding. The second aim was to set up the selected fabrication method (electrospinning) in-house at Cal Poly and gain understanding of the process. The third aim was to evaluate consistency of the technique. 
The work described in this thesis determined that electrospinning is a viable fabrication technique for producing scaffolding for BVM use. Electrospun scaffolding is highly tailorable, and a structure that mimics the natural organization of nano sized collagen fibers is especially desirable when culturing endothelial cells. An electrospinning apparatus was constructed in house and a series of trial experiments was conducted to better understand the electrospinning process. A consistency study evaluated scaffold reproducibility between different spins and within individual spins while setting a baseline that can be used for comparison in future work aimed at electrospinning. 


\section{ACKNOWLEDGEMENTS}

First of all, I would like to thank my thesis committee members. I really appreciate your time and support, not to mention all of your classes I had the fortune of being a part of!

To both the Biomedical and General Engineering Department and Material Engineering Department at Cal Poly, I cannot thank you enough. The list of faculty I could name that helped me tremendously could go on and on. If I have to name one person, it must be Dave Laiho, without whom I might still be in lab waiting for things to get fixed.

My fellow graduate students and undergraduate students who worked along side me during my thesis made this experience not just rewarding, but fun as well. To the other two members of Run DMC (Dimitri Delagrammaticas and Marc Dawson) - I really couldn't have asked to jump into a lab group with two smarter, more dedicated, and awesome individuals. Thanks for all your help! To Tiffany Flint/Pena - I was extremely happy when you decided to research electrospinning for your M aster's. The work you did was amazing, and I thank you for all the hours you spent talking to me in "electrospinning speak" and for all the editing you did for me. To Sara Leifer - you paved the way for us by being the first one to take the leap and defend. I have tried to follow your stellar example; hopefully, I will do half as well as you! To Aubrey Smith I really appreciate your help with histology, and for hanging out with me in lab! To Yvette Castillo - The hours you spent taking tedious measurements for me are greatly appreciated. Good luck with your electrospinning endeavors! To Chris Miracle - The tensile testing program you wrote literally saved me a day of my life. Thank you! To all other BVM lab group members - Thank you for being such a cool group of people to work with. Carry on the BVM research, and do so with passion. Also, have fun while you do so!

There are no words to express how grateful I am to my family, but I will try. Mom and Dad - thanks for always being supportive and reminding me that no matter what I do in life, I should enjoy it. After supporting me for these past years, I will do my best in the future to show you it was worth it. Dad, thanks for steering me towards engineering, and being genuinely interested whenever I talked about electrospinning. To my brother Jeff, I am so grateful for your support and approval. It's as much as a little brother ever needs. To my grandmother, I have to thank you for just being you. It's impossible for me to be in a bad mood when I am around you, and I truly think I am lucky to have such a great grandmother in my life. I also would like to thank my grandfather, who passed away from Parkinson's while I was in college. He taught me about hard work, and I work every day to make him proud. It is the possibility of tissue engineering to help with diseases like Parkinson's that makes me passionate about its potential and my role in making treatment real someday.

To Kristen - The opportunity you have given me has been the most rewarding of my life. Your constant encouragement and faith in what I am capable of have made me realize just how much potential I have. It does not surprise me that the BVM Lab Group is so popular. After all, it has an amazing advisor. 


\section{TABLE OF CONTENTS}

LIST OF TABLES .............................................................................................

LIST OF FIGURES................................................................................ xiv

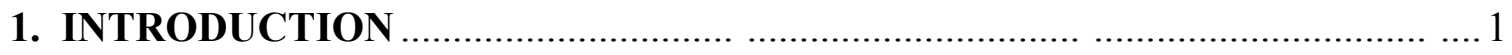

Overview .................................................................................................. 1

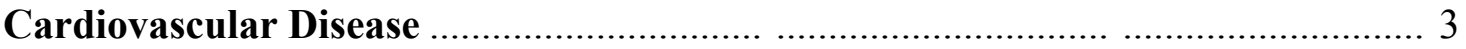

Statistics and Background Information........................................................... 3

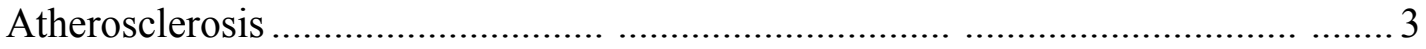

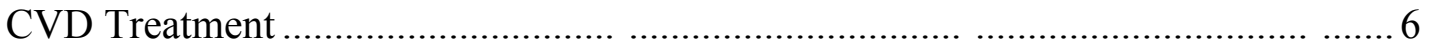

Percutaneous Transluminal Coronary Angioplasty ....................................... 6

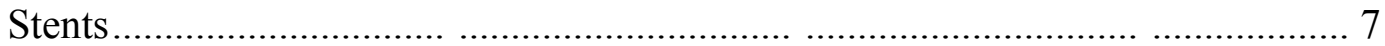

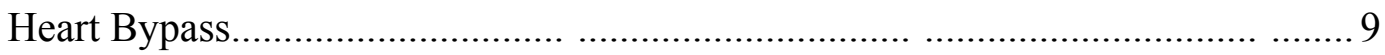

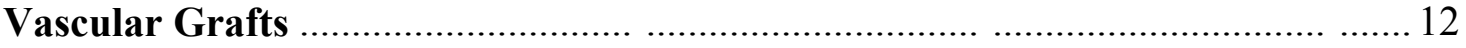

Synthetic Vascular Grafts ....................................................................... 12

Biologic Vascular Grafts......................................................................... 14

Tissue Engineered Vascular Grafts (TEVGs) .............................................. 15

Modified ePTFE Scaffolding ..................................................................... 18

Completely Biological TEVGs.............................................................. 20

Biodegradable Polymer TEVGs ....................................................... 22

An Alternate Use for Established TEVG Techniques: Blood Vessel Mimic ........... 24

Intermediate In Vitro Device Testing Using the BVM ................................... 25

Pulsatile Flow and Shear Stress Optimization ........................................... 28

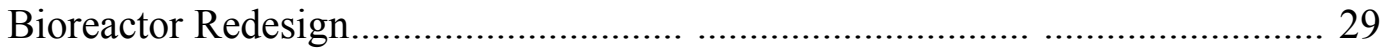


Cell Source and Sodding Procedure ………………..........................................2 29

Physiologically Appropriate and Cost Effective Scaffolding .............................. 30

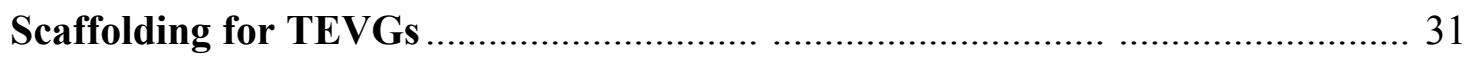

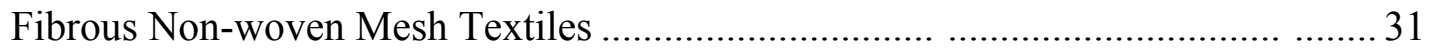

Solvent Casting and Particulate Leaching (SCPL) ............................................... 32

Thermally Induced Phase Separation (TIPS) ………….......................................... 32

Solid Freeform Fabricated Scaffolds (SFF) ........................................................ 35

Three-Dimensional Printing ...................................................................... 36

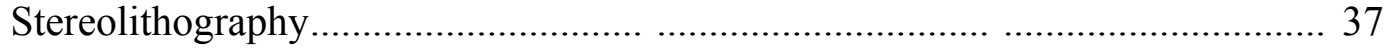

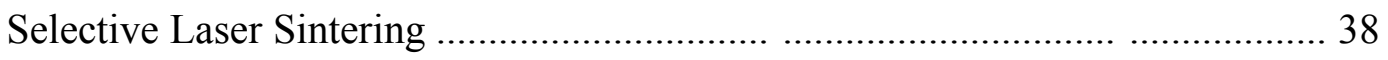

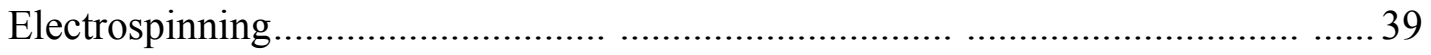

Scaffold Fabrication for the BVM ………………....................................... 40

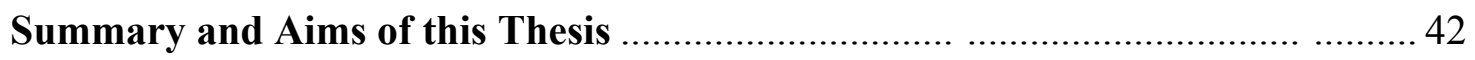

2. FABRICATION METHOD SELECTION AND ELECTROSPINNING............ 43

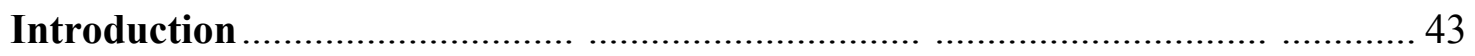

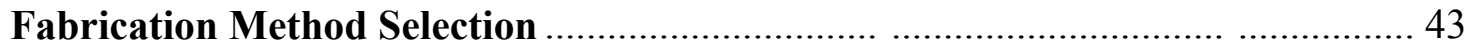

Controllable and Consistent Final Structure ……………...................................... 46

Compatible with a Variety of Materials ............................................................. .48

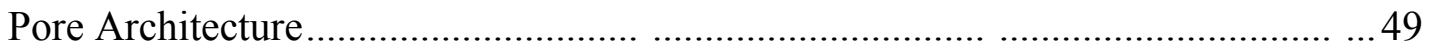

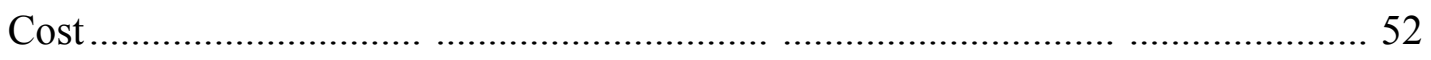

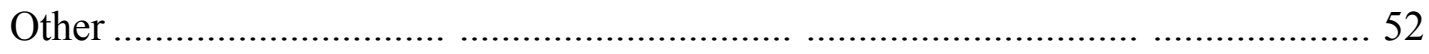

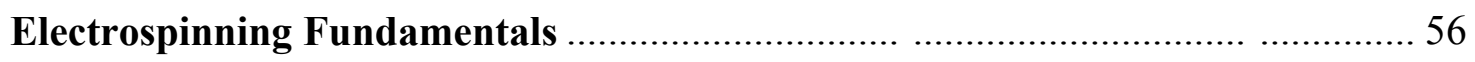

Brief History of Electrospinning ....................................................................... 56 


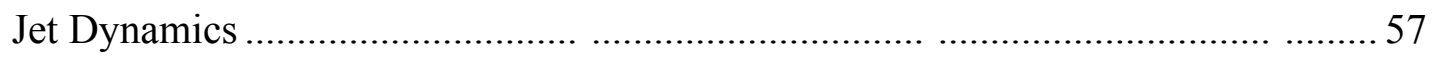

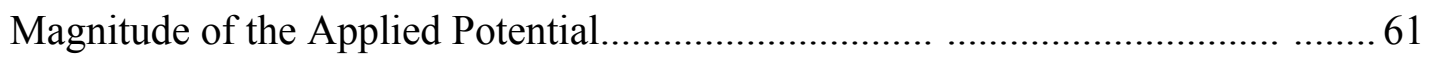

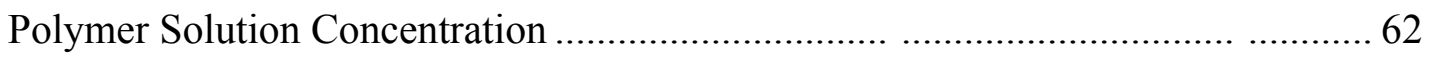

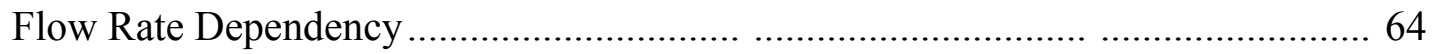

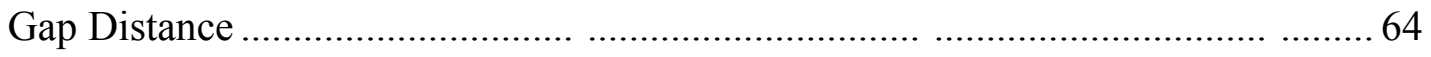

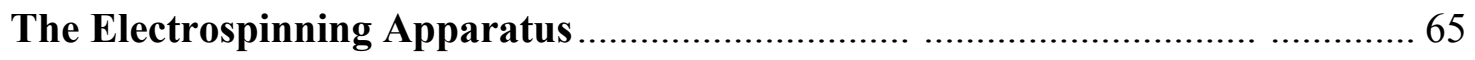

High Voltage Power Supply......................................................................... 66

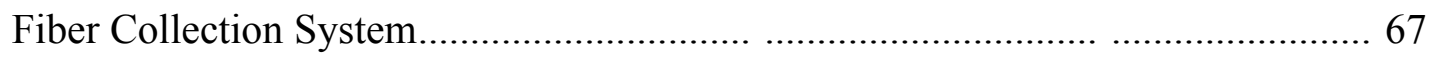

Polymer Infusion System .................................................................................. 68

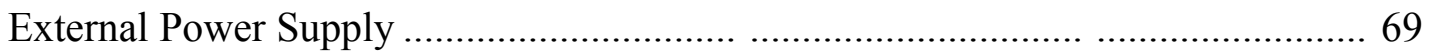

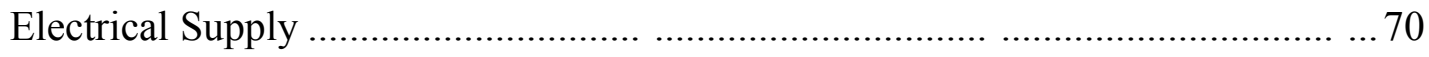

Containment Chamber …………………................................................... 70

3. ELECTROSPINNING TRIAL EXPERIMENTS ……….............................. 72

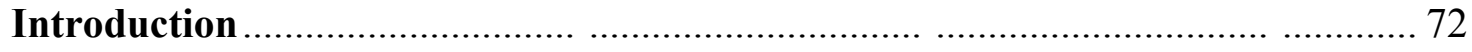

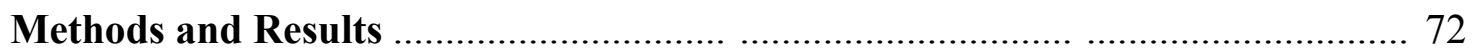

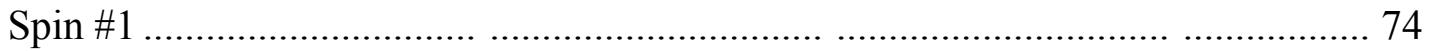

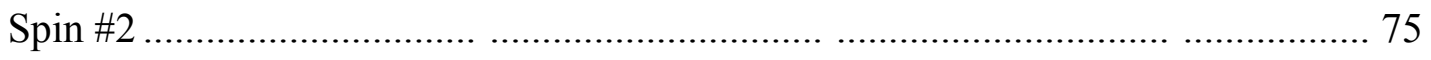

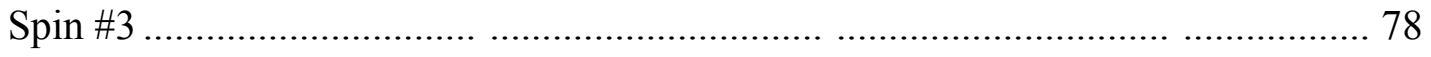

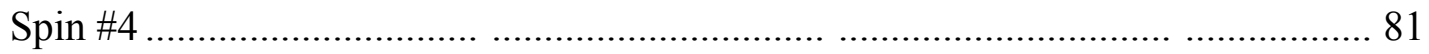

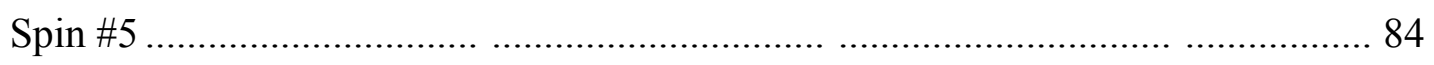

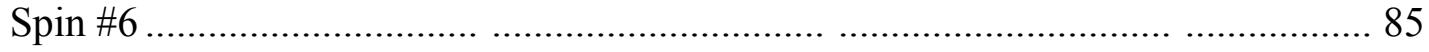

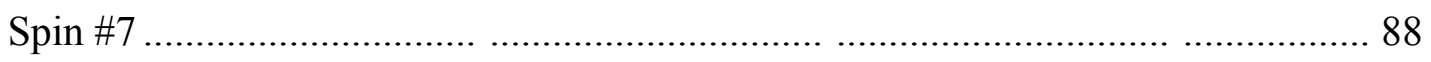

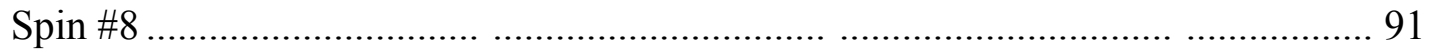




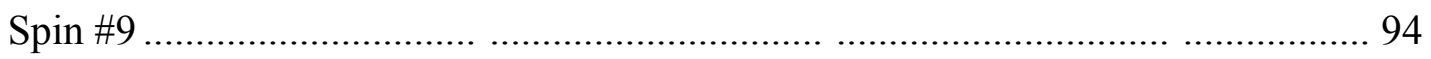

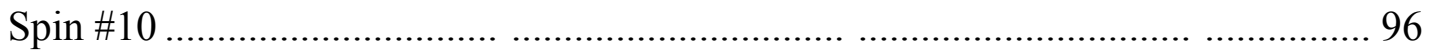

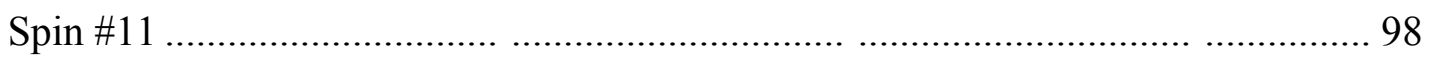

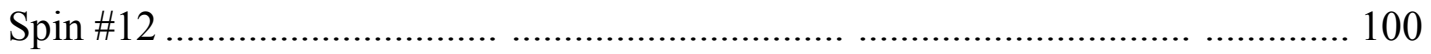

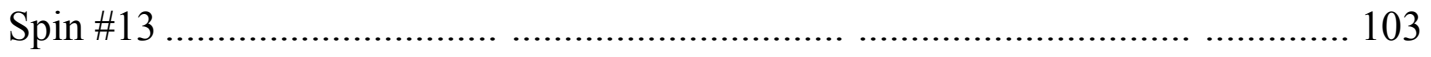

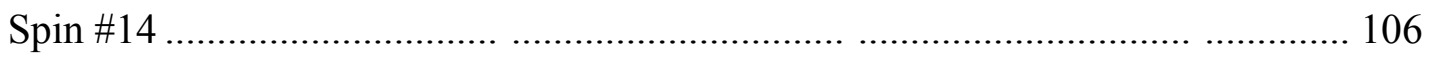

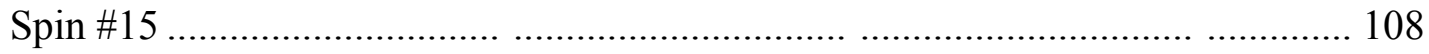

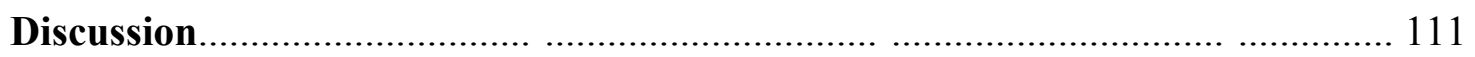

4. ELECTROSPINNING CONSISTENCY STUDY ……………...................... 112

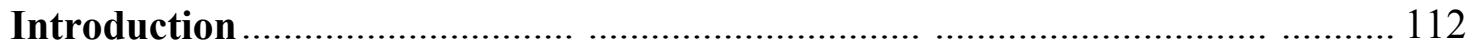

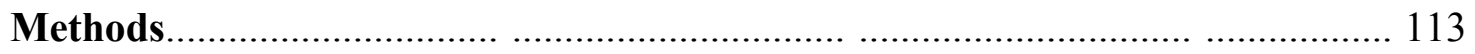

Electrospinning Parameters for the Consistency Study ……….......................... 113

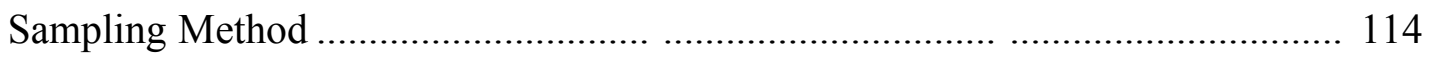

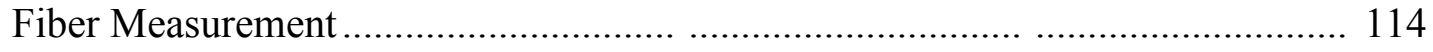

Fiber Measurement Method ................................................................ 115

Thickness Measurement........................................................................... 116

Thickness Measurement Method............................................................... 117

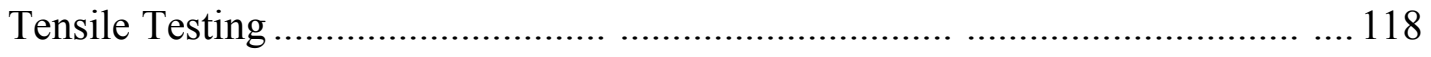

Tensile Testing Methods ……………................................................... 119

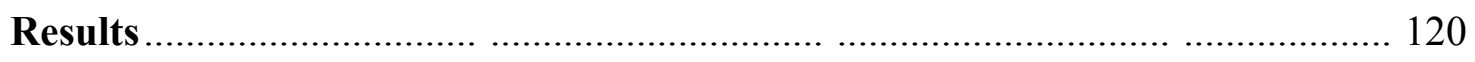

Statistical Analysis of Fiber Diameter .................................................................. 120

Results and Statistical Analysis of Scaffold Wall Thickness ................................ 124

Results and Statistical Analysis of Tensile Testing .............................................. 126 


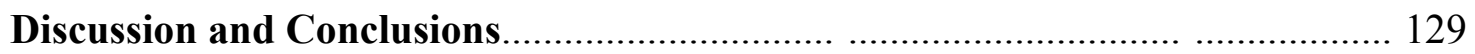

5. DISCUSSION AND FUTURE WORK …………………............................ 133

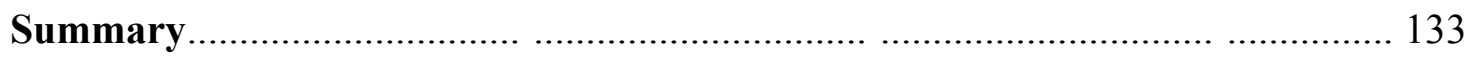

Electrospun Scaffolds: Novel Alternatives in the Blood Vessel Mimic System ... 134

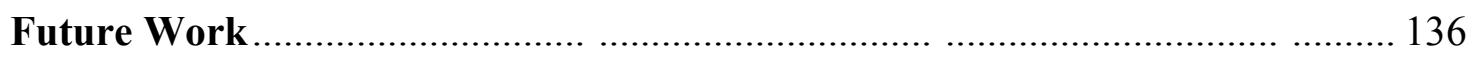

Improved Consistency: Further Exploration of Process Parameters ....................... 136

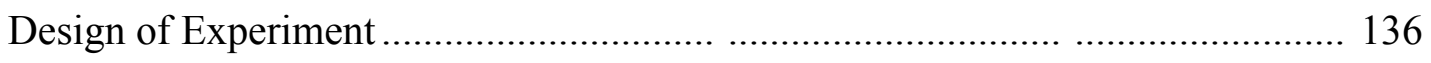

Improving the Electrospinning Apparatus ......................................................... 137

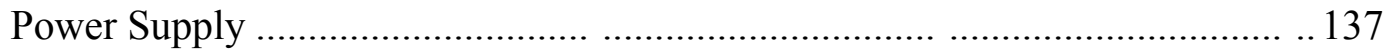

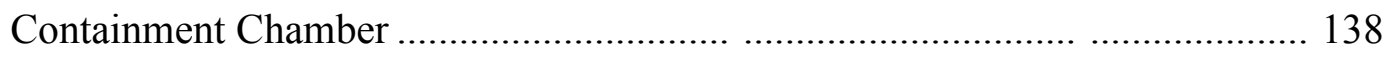

Characterization of Constructs .................................................................... 138

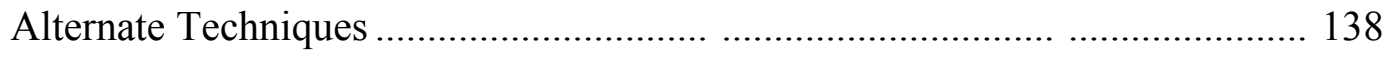

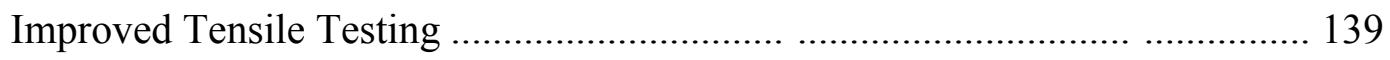

Improved Thickness Measurement .................................................................. 140

Physiologically Accurate Fiber Production ......................................................... 141

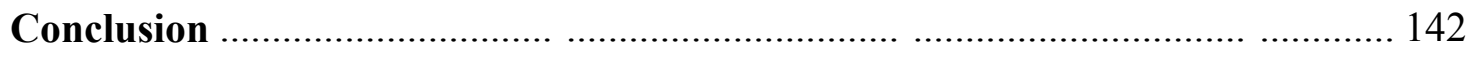

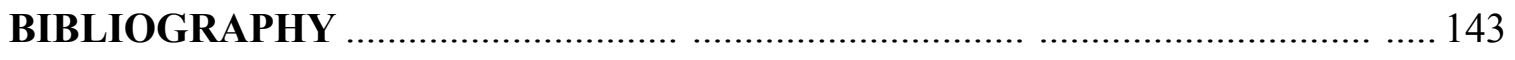

APPENDIX A: LIST OF ABBREVIATIONS ……….................................. 159

APPENDIX B: MIXING P(LLA-CL) SOLUTIONS FOR ELECTROSPINNING 160

APPENDIX C: ELECTROSPINNING PROTOCOL ……………..................... 165

APPENDIX D: HIGH CONTRAST SURFACE POROSIMETRY …………….... 174

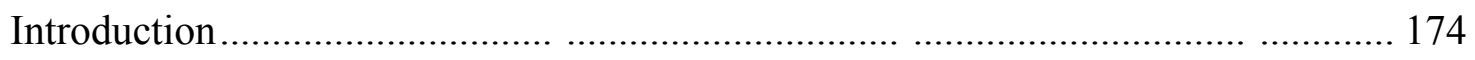

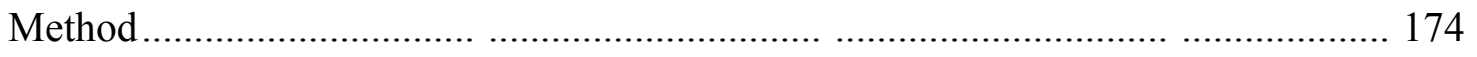


Conclusion

Introduction

Introduction

Introduction

APPENDIX H: INTRASCAFFOLD FIBER DIAMETER COMPARISON 324

Introduction 324

APPENDIX I: INTRASCAFFOLD THICKNESS COMPARISON 329

Introduction 329

\section{APPENDIX J: PRELIM INARY CELLULAR RESPONSE TO ELECTROSPUN}

P(LLA-CL) SCAFFOLDING 336

Introduction 336

Methods and Materials .... 336

Scaffold Fabrication and Preparation 336

Cell Sodding and Implentation Into the BVM 337

Imaging of Scaffolds. 337

Results 338

Discussion 341 


\section{LIST OF TABLES}

Table 1 - Summary of Candidate Scaffold Fabrication Methods................................. 45

Table 2 - Intrascaffold ANOVA Results .......................................................... 124 


\section{LIST OF FIGURES}

Figure 1 - The progression of atherosclerosis........................................................... 5

Figure 2 - Stenting following percutaneous transluminal coronary angioplasty ............... 8

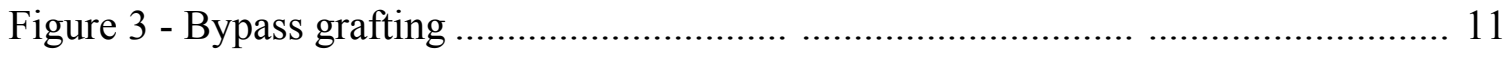

Figure 4 - Microstructure of ePTFE .................................................................... 13

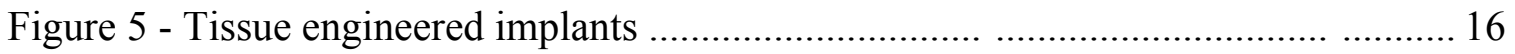

Figure 6 - The three layered structure of a native blood vessel ................................. 18

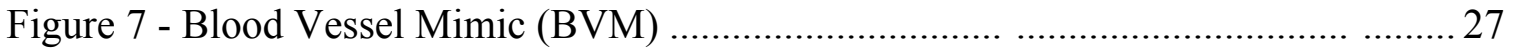

Figure 8 - Binary phase diagram for a polymer in a solvent .................................. 34

Figure 9 - Solid freeform fabrication methods ..................................................... 36

Figure 10 - Electrospinning apparatus diagram ............................................. 40

Figure 11 - Fabrication technique decision matrix ................................................ 54

Figure 12 - Electrospinning bending perturbations .......................................... 58

Figure 13 - Path of an electrospinning jet .......................................................... 60

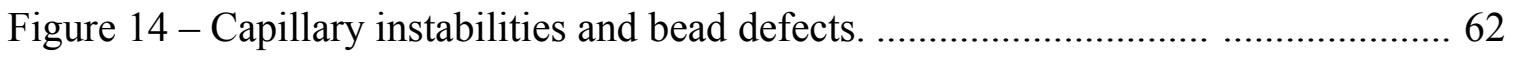

Figure 15 - Polymer concentration effects on surface tension and viscosity ................. 63

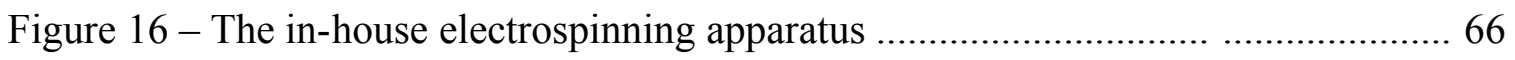

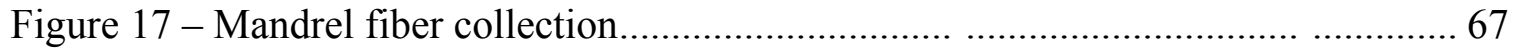

Figure 18 - Polymer solution injection and applied voltage ................................. 69

Figure 19 - Interior surface of Spin $\# 1$......................................................... 74

Figure 20 - Interior surface of Spin $\# 2$...................................................... 76

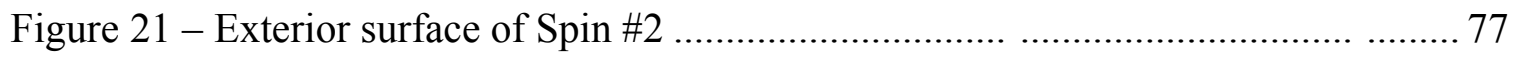

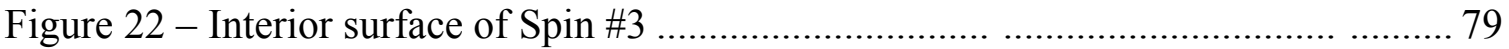


Figure 23 - Cross-sectional view of Spin \#3 ........................................................... 80

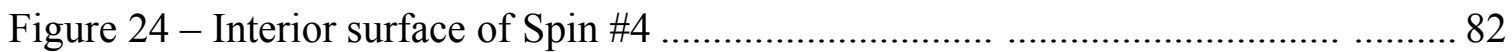

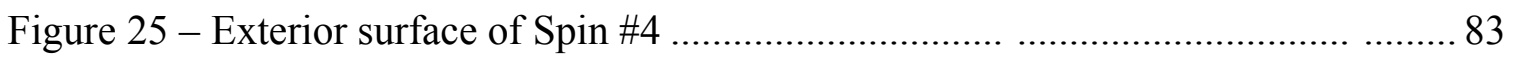

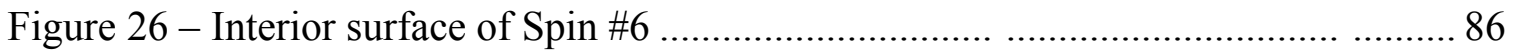

Figure 27 - Exterior surface of Spin \#6 ………………...................................... 87

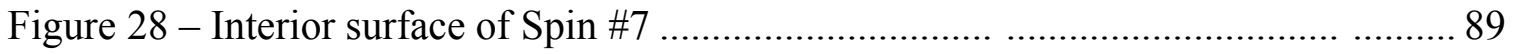

Figure 29 - Exterior surface of Spin \#7 ………………......................................... 90

Figure 30 - Interior surface of Spin \#8 ………………................................... 92

Figure 31 - Exterior surface of Spin \#8 ………………......................................... 93

Figure 32 - Interior surface of Spin \#9 ……………….................................... 95

Figure 33 - Interior surface of Spin \#10 ................................................................ 97

Figure 34 - Interior surface of Spin \#11 _............................................................ 99

Figure 35 - Interior surface of Spin \#12 ………………................................... 101

Figure 36 - Exterior surface of Spin \#12 ……………............................................. 102

Figure 37 - Interior surface of Spin \#13 .............................................................. 104

Figure 38 - Exterior surface of Spin \#13 …………………..................................... 105

Figure 39 - Interior surface of Spin \#14 ........................................................... 106

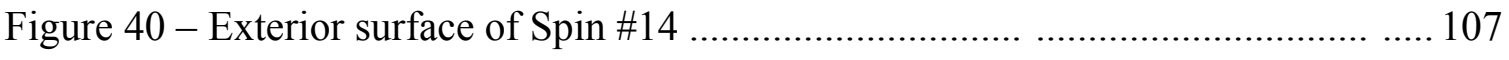

Figure 41 - Interior surface of Spin \#15 ............................................................. 109

Figure 42 - Exterior surface of Spin \#15 ……………......................................... 110

Figure 43 - Consistency study sample sectioning ................................................... 114

Figure 44 - Fiber diameter measurement images ..................................................... 116

Figure 45 - Cross-sectional thickness sampling method ............................................ 117 
Figure 46 - Cross-sectional thickness measurement images................................. 118

Figure 47 - Histogram of fiber diameter .................................................... 121

Figure 48 - Boxplots of fiber diameter data ...................................................... . 122

Figure 49 - ANOVA comparing fiber diameter and spin .................................. 123

Figure 50 - Boxplots of cross-sectional thickness data....................................... 125

Figure 51 - ANOVA comparing scaffold wall thickness and spin.......................... 126

Figure 52 - Histogram of Young's modulus ..................................................... 127

Figure 53 - ANOVA comparing Young's Modulus and spin ................................. 127

Figure 54 - ANOVA comparing Young's Modulus and testing section ..................... 128

Figure 55 - ANOVA comparing Young's modulus (E) and sample orientation .......... 129

Figure 56 - Tensile testing alternative to rectangular samples............................... 140 


\section{INTRODUCTION}

\section{Overview}

The purpose of this thesis was to develop new scaffolds for growing tissue engineered blood vessels. Specifically, the new scaffolds were intended to replace the existing scaffolding in an in vitro blood vessel mimic (BVM) system used to test intravascular devices. This Introduction aims to explain the relevancy of the research conducted.

Before describing the specifics of the work, background information is required to understand the motivation for this work. Therefore, this Introduction starts by explaining cardiovascular disease (CVD) in section 1, including the available treatments for CVD. The information in section 1 emphasizes the need for improved CVD treatment methods, which includes better device testing methods. The BVM is such a testing method, and continued improvement to the system could make it invaluable in the device approval process.

One avenue of CVD treatment, tissue engineered vascular grafts (TEVGs), is a promising new field of study that was used as the basis for the BVM. In section 2, a review of TEVG research is included to introduce the available methods for creating blood vessels in a laboratory and the different types of scaffolding employed. After TEVG information is presented, the BVM is explained in detail, including the current research being conducted to improve the system. 
As the focus of this thesis was to explore alternative scaffolding for the BVM, section 3 introduces candidate fabrication methods to produce scaffolds for use in the BVM. 


\section{Cardiovascular Disease}

Statistics and Background Information

Cardiovascular disease (CVD) is a class of diseases that affect the heart or blood vessels, and is the leading cause of death in the United States [1]. In 2006, CVD killed 829,072 people, accounting for $34.2 \%$ of all deaths [2]. In 2009 , CVD is expected to directly and indirectly cost the United States approximately $\$ 475.3$ billion, and current health trends among Americans signal the potential for future devastation [3]. For example, statistics show a trend towards an increasingly overweight America, a key risk factor for CVD. For children between the ages of 6 and 11 years, $17.5 \%$ were overweight in 2001-2004, in contrast to just 4.0\% in 1971-1974. For adolescents 12 to 17 years of age in the same time periods, the prevalence of overweight individuals increased from $6.1 \%$ to $17.0 \%$ [4]. Also, diabetes mellitus, another key CVD risk factor, is an increasingly problematic disease in the United States [5]. In 2006, diabetes mellitus affected $10.6 \%$ of adults 20 years and older [6].

\section{Atherosclerosis}

A major subset of patients with CVD suffer from atherosclerosis, a chronic, progressive, multifocal disease that affects the intimal layer of blood vessel walls through the formation of fatty plaques (Figure 1). Generally, atherosclerosis targets large elastic arteries and medium to large muscular arteries, most often at points of branching, sharp curvatures, and bifurcations. Advanced atherosclerotic plaques, or atheromatous plaques, 
are composed of a central core of lipid and cholesterol crystals, as well as cells such as macrophages and smooth muscle cells (SMCs). Necrotic debris, proteins, and degenerated blood cells are also contained in the plaque cores. The core of the plaque is separated from the lumen of the blood vessel by a fibrous cap rich in collagen [7]. Atherosclerotic plaques are dangerous for several reasons. First, they can block the vascular lumen, restricting blood flow distally. Second, if the plaque is disrupted, a thrombus can form. Third, the presence of plaques can degrade the contacting vascular wall, increasing the risk for aneurysm [8]. 

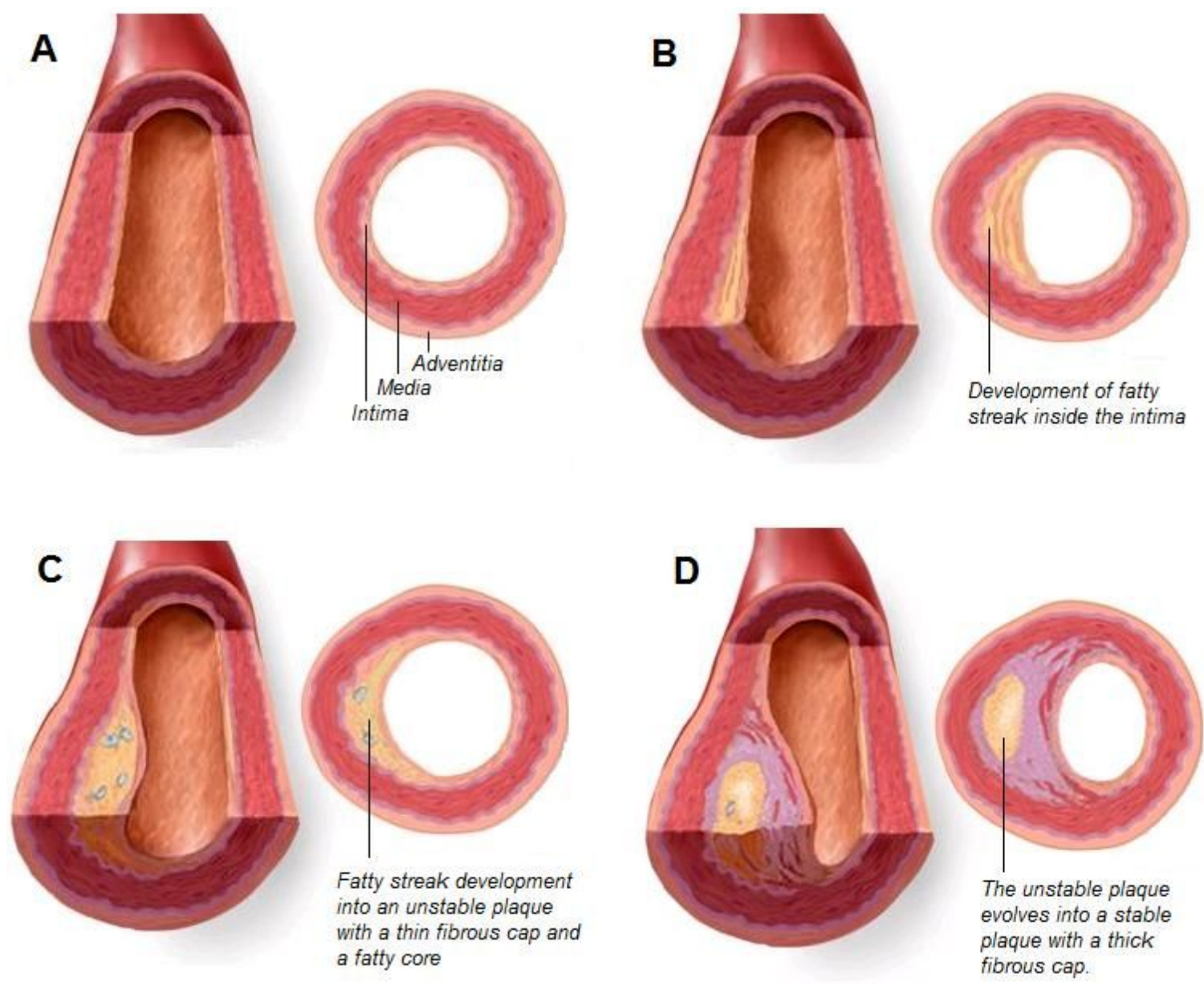

Figure 1 - The progression of atherosclerosis from a healthy blood vessel (A) to the start (B) and development (C) of plaque formation. Advanced plaque development results in significant vessel narrowing (D). [American Heart Association, "Cholesterol Media Library-Atherosclerosis," 2009 [9].] 
Of all the diseases classified as atherosclerotic CVD, coronary artery disease (CAD), also known as ischemic heart disease (IHD), is the most problematic. Not only is IHD the most common cause of myocardial ischemia and infarction, it is also the most serious, chronic, life-threatening illness in the United States [7]. One in every five deaths in the United States in 2005 was the result of CAD, and in 2009 it is estimated that 785,000 Americans will suffer from a first coronary attack, while 470,000 will have a recurrent attack [6].

Cardiovascular Disease Treatment

Percutaneous Transluminal Coronary Angioplasty

Minimally invasive methods are available for the restoration of proper blood flow through coronary arteries. One method termed percutaneous transluminal coronary angioplasty (PTCA), or balloon angioplasty, involves passing a long catheter from the femoral artery up the aorta to the openings of the coronary arteries, running a deflated balloon up the catheter, and inflating the balloon to compress against plaque deposits. PTCA widens the lumen, and increases blood flow through the vessel [10]. Plaque reduction occurs by compression, embolization, or redistribution of the plaque contents and by expansion of the vessel wall $[8,11]$. PTCA success is limited by long term restenosis of the artery which is known to occur within the first four to six months in 30$40 \%$ of patients. Restenosis occurs due to fibrous tissue formation in the lumen, which is caused by excessive smooth muscle cell proliferation in response to angioplasty induced 
injury [12]. In order to combat restenosis after PTCA, a majority of patients undergo a process known as stenting.

Stents

Stents are tube shaped cages or meshes most commonly made of 316 stainless steel or nickel titanium shape memory alloys that expand and support a larger, more uniform lumen (Figure 2). Specifically, the role stents play as scaffolding in a blood vessel act to support the disrupted lumen and minimize thrombus formation [13]. Stenting immediately after balloon angioplasty has been shown to be superior to angioplasty alone in several situations, including: blood vessels greater than $3 \mathrm{~mm}$ in diameter, occluded vein grafts, recurring total occlusions, myocardial infarction, and in restenotic vessels following PTCA [14]. However, complications from stent implantation do arise. Subacute stent thrombosis has occurred in 1 to $3 \%$ of patients within 7 to 10 days after implantation, but has been virtually overcome by antiplatelet drugs [8]. A longer term complication called in-stent restenosis occurs among $50 \%$ of stented patients within 6 months of the procedure. In-stent restenosis starts as damage to the intimal layer and stretching of the vessel wall, leading to a series of biological responses that results in luminal narrowing and restenosis from increased smooth muscle cell (SMC) migration and proliferation [15]. 

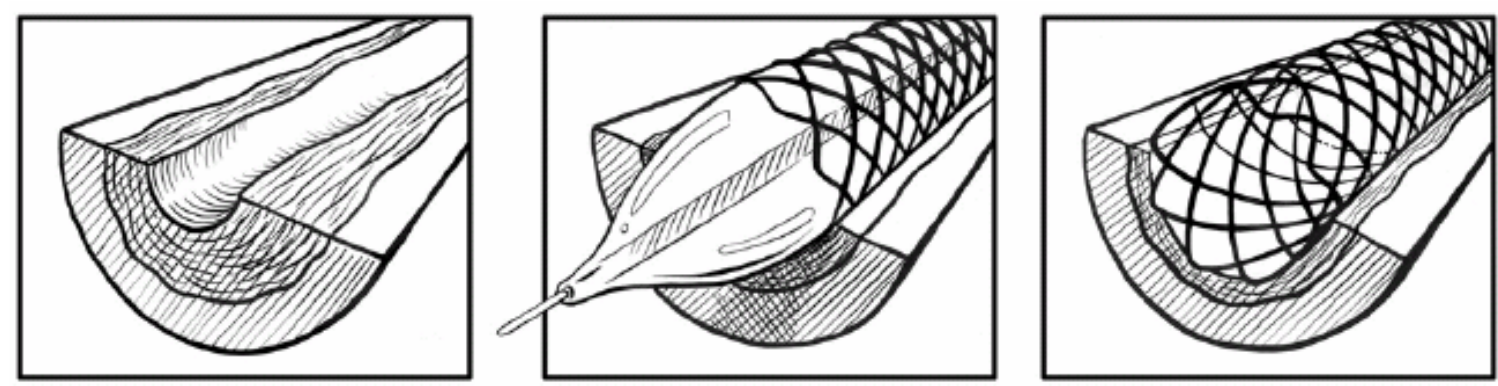

Figure 2 - Following percutaneous transluminal coronary angioplasty, a stent is used to prevent recoil of the luminal wall. [American Heart Association, "What is a Stent?" 2007 [16]] 
Reduction of in-stent restenosis has been most successful with the introduction of drug-eluting stents. Drug-eluting stents are coated with a polymer that can contain a variety of drugs that act to prevent SMC action, avoiding restenosis. While the ultimate goal of any drug utilized in a drug-eluting stent is to prevent restenosis, the prevention pathway varies. One example, sirolimus, prevents the degradation of p27kipl, a kinase inhibitor that plays an important role in regulating vascular SMC migration and proliferation [17]. Another drug called paclitaxel is a cytotoxic drug that suppresses SMC and endothelial cell (EC) migration and proliferation by disrupting microtubule action [18]. Drugs are released through diffusion or polymer degradation, both of which can be controlled through the engineering of specific polymer/drug systems.

While drug-eluting stents beneficially inhibit in-stent restenosis, they may also disrupt EC activity, thus delaying arterial healing, and cause late thrombosis (any platelet rich thrombus occupying greater than $25 \%$ of the lumen after more that 30 days of implantation) [19]. Future drug-eluting stents seeking to alleviate the late thrombosis problem will require extensive preclinical testing, both in vitro and in vivo. The BVM has potential to provide a physiologically accurate in vitro testing system.

\section{Heart Bypass}

Many ischemic patients have atherosclerotic constrictions or blockages in a few discrete locations along the artery, necessitating surgical intervention in the form of a heart bypass operation. This involves bypassing blockages using a graft attached from the aorta to a site on the peripheral coronary artery beyond atherosclerotic plaque 
blockages as shown in Figure 3 [20]. Grafts are mostly sections of subcutaneous vein from an arm or leg, which account for 400,000 bypass operations annually in the U.S. $[21,22]$. These grafts, however, have a tendency to occlude over time due to accelerated atherosclerosis, resulting in 50\% patency at 10 years [23]. Thus, there is an effort underway to engineer a suitable vascular graft for coronary artery applications. 


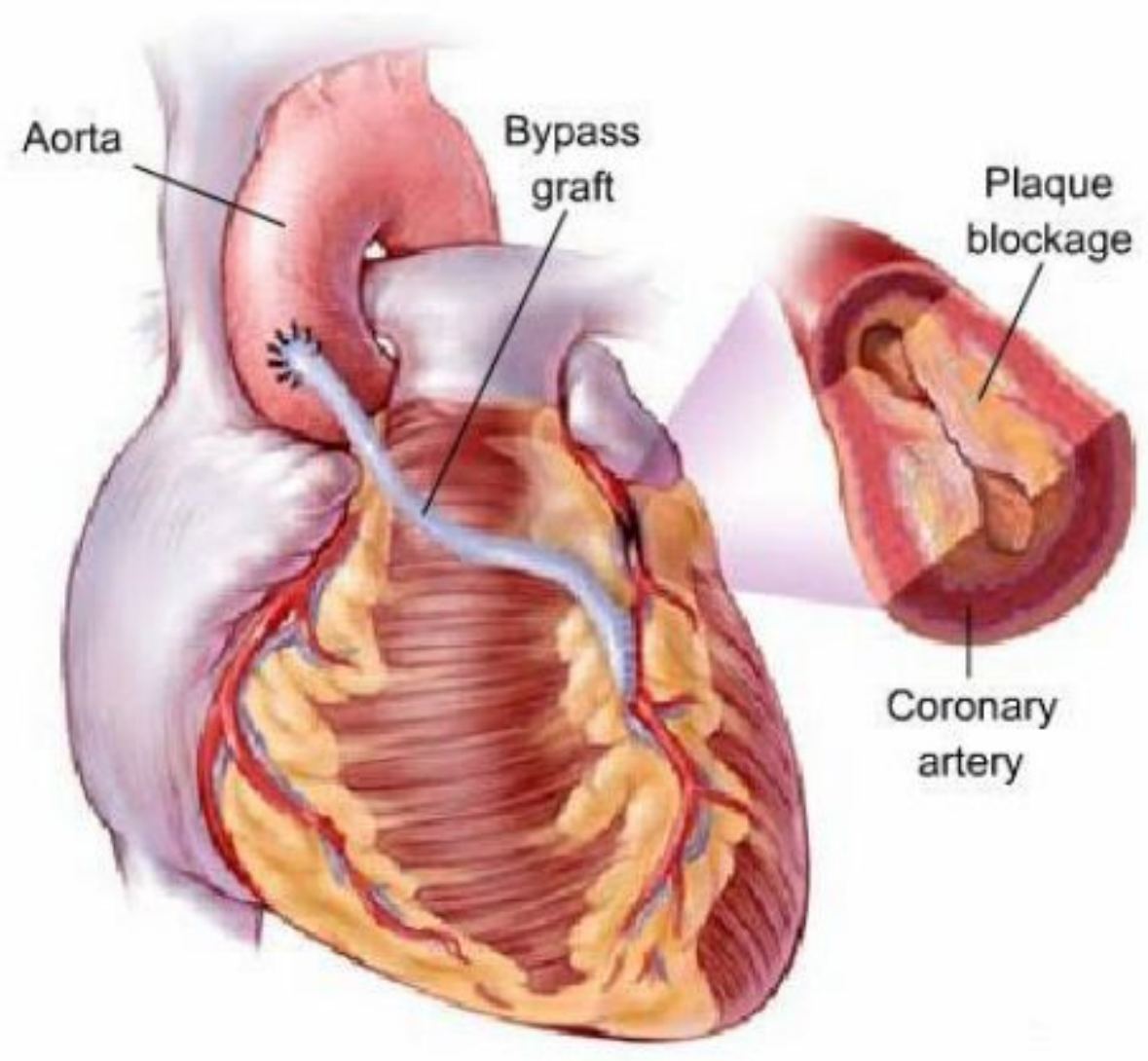

Figure 3 - Bypass grafts restore blood flow to distal portions of arteries by circumventing blockages. [American Heart Association/American Stroke Association, "Heart Attack Media Library - Coronary Artery Bypass Grafting (CABG), " 2009 [24]] 


\section{Vascular Grafts}

Grafting was introduced briefly in the preceding section during the discussion of coronary bypass procedures. The autologous bypass veins are used as substitute conduits for blood to flow through, which is the general function of vascular grafts. Although autologous vein grafting is the most prevalent grafting procedure, other synthetic and biological grafts have been developed. The research on grafting is relevant to the work in this thesis as it was used to develop the BVM, and can be used to further improve the system. Grafting research is described in the following section.

Synthetic Vascular Grafts

As early as the beginning of the $20^{\text {th }}$ century, researchers and doctors were conducting animal experiments to test the viability of vascular grafts constructed of many different materials, including aluminum, silver, glass, and Lucite tubes [8]. Currently, synthetic grafts are typically fabricated from poly(ethylene terephthalate) (Dacron) or expanded polytetrafluoroethylene (ePTFE) (Figure 4). Both materials can be used to

produce highly tailored grafts, varying not just length and diameter, but also porosity to promote healing and endothelialization. In large diameter vessel applications (greater than $6 \mathrm{~mm}$ ), where there is high flow and low resistance, synthetic grafts have a $90 \%$ patency rate at 5 to 10 years [25]. When used as small caliber arterial replacements (inner diameter less than $6 \mathrm{~mm}$ ), they are prone to thrombus induction, embolism, and occlusion [26]. 


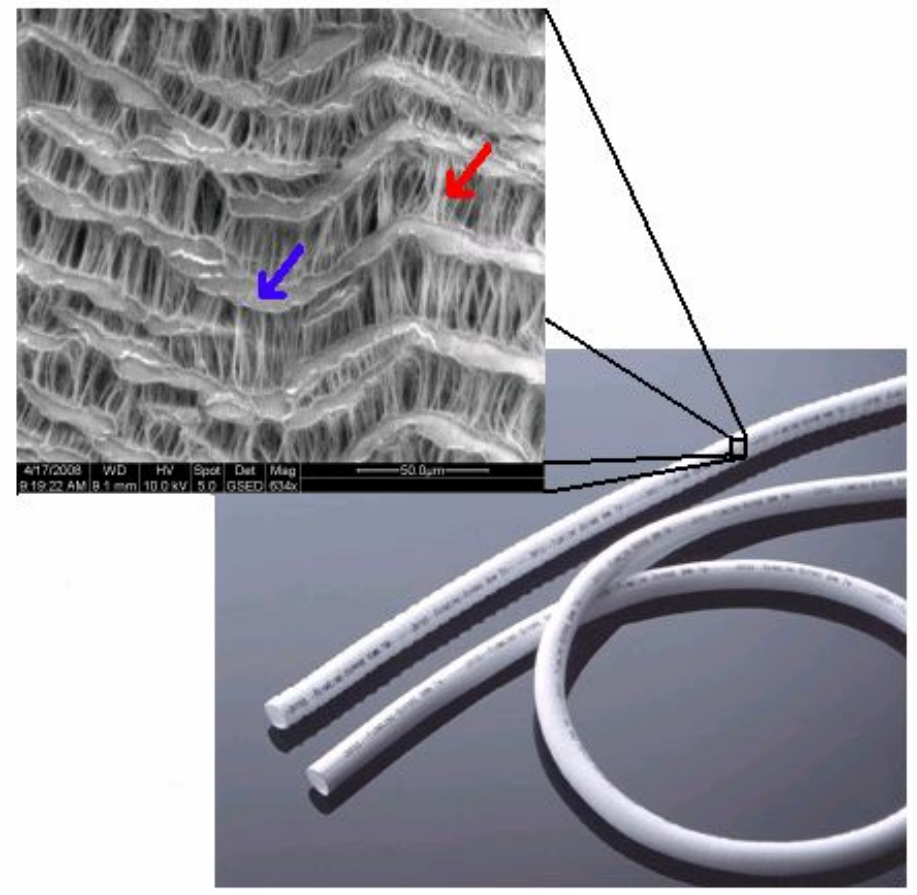

Figure 4 - Tubular geometry synthetic ePTFE with scanning electron micrograph of its microstructure $(634 \mathrm{x})$. Nodes (blue arrow) run parallel to the long axis of the graft, while internodal fibers (red arrow) run in the circumferential direction.

The mechanism behind synthetic graft failure involves the series of biological responses that occurs after implantation. First, the luminal surfaces of implanted grafts become coated in a layer of plasma proteins consisting primarily of fibrinogen. Second, this layer develops over time into a platelet-fibrin aggregate called the pseudointima. Finally, ECs cover this layer, thereby imitating the lumen of a native blood vessel and serving as a nonthrombogenic surface. Due to the human body's limited ability to endothelialize vascular grafts, this new intima, or neointima, is confluent only over a 15 $\mathrm{mm}$ zone adjacent to the anastomosis, or site of sutured connection between the graft and native artery. Thus, even after long term implantation, only the ends of implanted 
vascular grafts have endothelialized lumens while the inner lumen is covered by the platelet-fibrin pseudointima. This inner layer may not adhere tenaciously to the graft, and may become dislodged, resulting in distal embolization or formation of a flap-valve that causes acute obstruction [8].

\section{Biologic Vascular Grafts}

Grafts classified as biological are the result of natural fabrication within a living system and must be explanted from a donor. No external fabrication techniques, such as the use of scaffolding for cellular support, are necessary. Biological grafts can be classified as autografts, allografts, or xenografts. Autografts come from the receiving individual, such as the sections of subcutaneous vein used for coronary bypass described earlier. These are readily accepted by the host, eliciting little immunogenic or inflammatory response [20]. Allografts are donated from one individual to another of the same species. Due to allelic variants of genes of the major histocompatibility complex, allografts run the risk of rejection by the host. In the case that donor and recipient belong to different species, the graft is classified as a xenograft. Rejection is high with xenografts due to maximal genetic disparity [27]. Autografts are the best choice for biological vascular grafts, but are in short supply due to a limited amount of selfharvestable blood vessel conduit. Around $60 \%$ of patients in need of bypass surgery do not have a vessel suitable for grafting [28]. One solution is to create autologous vascular grafts using techniques from the relatively new field of tissue engineering. 
Tissue Engineered Vascular Grafts (TEVGs)

A field still in its infancy, tissue engineering is the melding of biology, chemistry, physics, materials science, engineering, and many other disciplines, to design and build tissues and organs at least partially outside the body. The goal of tissue engineering is the ability to start with cells from a host and transform them into an implantable construct that will improve the hosts quality of life by curing or alleviating the symptoms of a disease. Figure 5 shows the typical steps necessary to create a tissue engineered implant. Tissues produced in this manner could eliminate the need for donors, as well as suppress the need for immunosuppressant therapy due to an autologous cell source [29, 30]. 


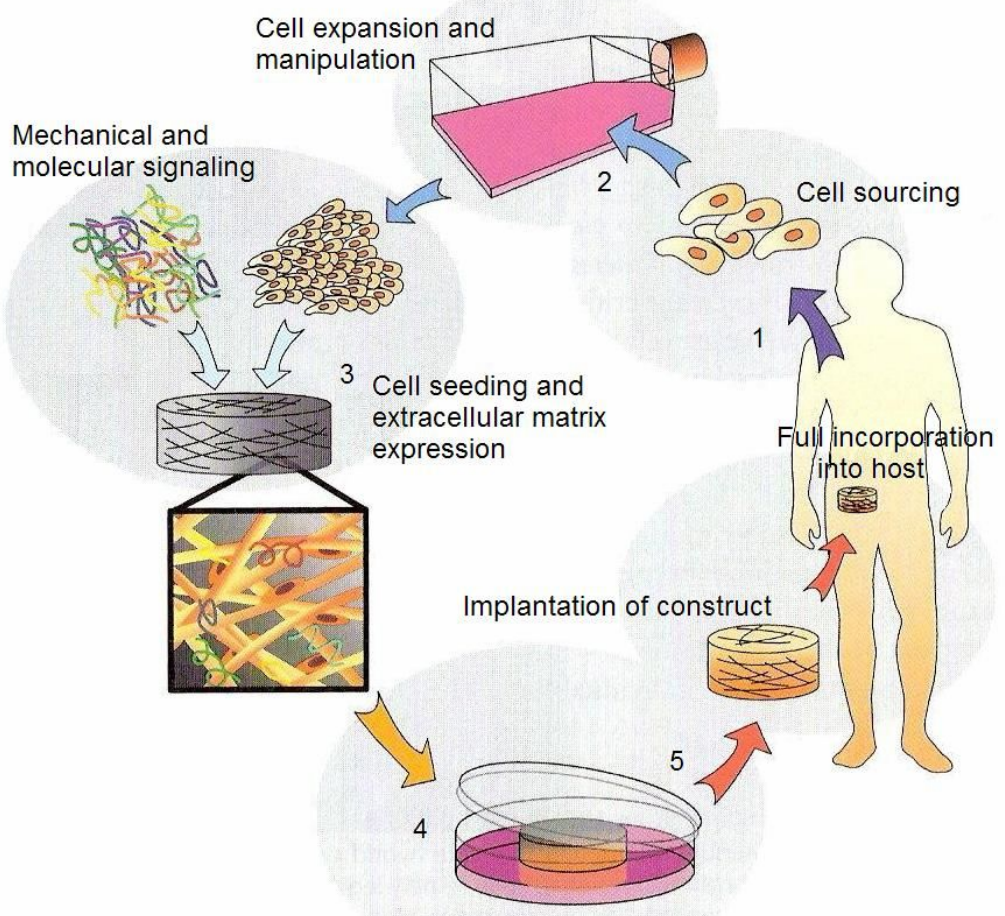

Figure 5 - A typical tissue engineered implant starts with autologous cell sourcing (1). The explanted cells are expanded in vitro (2) and seeded onto a support scaffold (3). Further culturing of the seeded construct may include a variety of chemical and mechanical stimulation $(3,4)$ to induce normal physiological cellular activity. Finally, constructs are implanted into the host with the hope that immune response will be minimal (5). [Blitterswijk, “The Central Tissue Engineering Paradigm,” 2008 [31]] 
With respect to vascular grafts, tissue engineering may potentially replace synthetic materials, like ePTFE, in small diameter vessel applications. The ideal graft would meet a long list of criteria, including: easy to handle, good suture retention, flexible and kink resistant, biocompatible, compliance matched to connecting artery, resistant to aneurysm, easily manufactured, economical, easily stored, and producible in many sizes [32].

The basic strategy behind most attempts to produce tissue engineered vascular grafts (TEVGs) is to mimic the natural tri-layer structure and/or function of a blood vessel. Figure 6 illustrates the tri-layer structure of native blood vessels, which TEVGs strive to at least partially replicate. Different methods exist to attempt achieving this, from simple modification of ePTFE to building a completely biological graft. Due to the focus of this thesis on vessel scaffolds, a review of these various methods is presented in the following sections. 


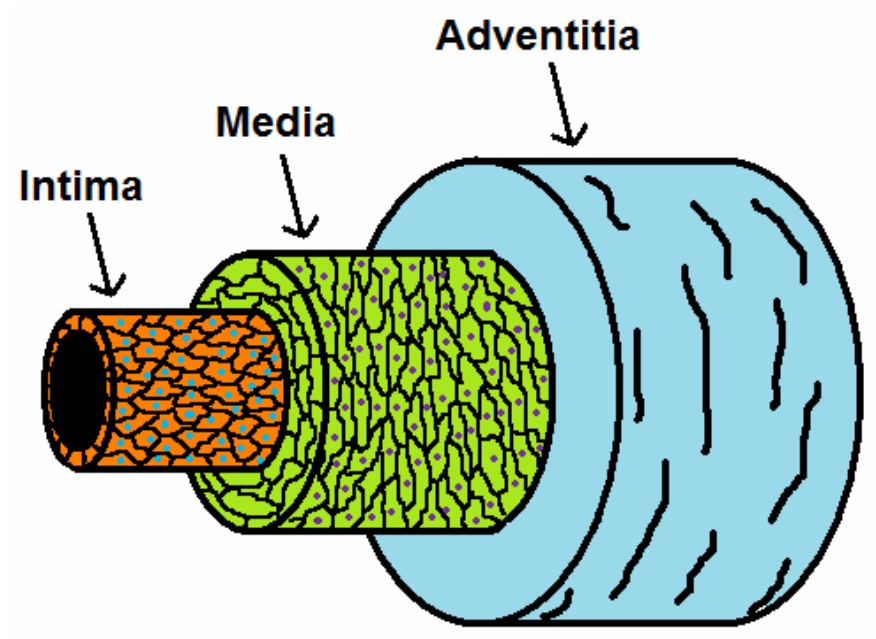

Figure 6 - The three layered structure of a native blood vessel. The intima consists of a single layer of blood contacting ECs that prevent platelet adhesion. Below the intima lies a much thicker layer called the media comprised of multiple layers of SMCs as well as sheets of elastin and collagen occurring at regular intervals. The pitch of these intervals is determined by vessel size and flow conditions [33]. The medial layer is largely responsible for blood vessel mechanical response to chemical signals, such as vasoconstriction and vasodilation, as well as mechanical integrity, such as resistance to bursting. Composed largely of collagen and interspersed fibroblasts, the outer adventitia is a supportive connective tissue $[1,29]$.

\section{Modified ePTFE Scaffolding}

Endothelial cells comprising the intimal layer of blood vessels are known to inhibit thrombosis as well as act as an anticoagulant surface for blood flow [34]. With this in mind, researchers have produced small-diameter ePFTE vascular grafts with luminal endothelial linings in hopes to prevent thrombus induction, embolism, and occlusion. This is a logical first step in the evolution of tissue engineered blood vessels since it involves simply altering the existing ePTFE grafts. 
Zilla et al developed and clinically applied an in vitro endothelialization procedure for ePTFE grafts. Patients that required infrainguinal grafts but had unsuitable veins for grafting participated in the clinical study. Autologous ECs were isolated from segments of the patients' cephalic, brachial, external jugular, or, if unsuitable as a conduit material, saphenous veins. ECs were cultured until the required number of cells, approximately $16 \times 10^{6}$, was reached. Sections of ePTFE were coated with a fibrinolytically inhibited fibrin glue to provide a surface for EC adherence. ECs were seeded onto the luminal surface of the grafts and allowed to cultivate for approximately 6 to 11 days to ensure maturation of the cytoskeleton for structural integrity before implantation [35].

A long term study from the same research group showed that patency rates for these endothelialized infrainguinal grafts at both 5 and 10 years improved compared to bare ePTFE grafts, and all retrieved samples had a confluent endothelium after $38.9 \pm$ 17.8 months [36]. However, while a confluent endothelium addresses part of the problem of vascular graft failure, the mechanical behavior of ePTFE is still vastly different from native artery. Specifically, ePTFE has poor radial compliance. Compliance in this case is vessel distension caused by increased blood pressure resulting in a temporary, reversible increase in diameter. The inability of ePTFE vascular grafts to stretch in the radial direction has been shown to contribute to distal anastomotic intimal hyperplasia in vivo as a result of shear stress abnormalities [37]. Also, some functions of ECs are interrupted by a flow altering lack of radial compliance. For example, ECs will produce various substances in response to shear stress and cyclic radial stretch, including 
prostacyclins and nitric oxide, important substances in blood platelet aggregation and vasodilation, respectively [38].

Synthetic ePTFE graft material has another arguably disadvantageous property in that it is permanent. ECs signal arterial remodeling in order to maintain a shear stress in most arteries of $15 \mathrm{dynes} / \mathrm{cm}^{2}$, but the non-degradable nature of ePTFE prevents this remodeling from occurring, resulting in non-ideal intimal shear stress [1, 39]. This has lead some researchers to avoid synthetic, non-biodegradable materials in the search for the ideal TEVG.

\section{Completely Biological TEVGs}

By abstaining from using synthetic materials, L'Heureux et al hoped to produce a vascular graft that would be superior to any synthetic graft in three important ways. First, an autologous, completely biological graft could be healed by the body. Second, a biological matrix could be remodeled, as opposed to synthetic material that only degrades. Finally, the absence of synthetic material could prevent foreign body reaction that results in inflammation and/or infection.

The result was a fused 3 layer biological construct similar to a native blood vessel consisting of the following: acellular inner membrane sodded with ECs to mimic the intima; middle layer of SMCs as a replacement for the media; and an outer layer of fibroblasts to act as the adventitia. Histological examination of the lumen of the graft confirmed von Willebrand factor expression and acetylated-low density lipoprotein uptake characteristic of ECs $[40,41]$. The middle "media" layer stained positive for $\alpha$ - 
smooth muscle actin, a SMC marker [42]. The outer adventitial layer contained vimentin and small fibers of elastin assembled in large circular arrays similar to native blood vessel adventitial layers. Burst pressure of the engineered vessels reached a plateau of $2232 \pm$ $251 \mathrm{mmHg}$, compared to $1680 \pm 307 \mathrm{mmHg}$ for human saphenous vein. While this ultimate strength is more than adequate, L'Heureux et al noted that compliance of the engineered biological grafts was much less than that of saphenous vein, a problem known to cause anastomotic intimal hyperplasia, resulting in decreased patency rates [43]. In vivo analysis in a canine model yielded a 50\% patency rate at 7 days, even though the grafts were produced using human cells making them xenografts, which have severe problems maintaining patency. The promising results from initial testing of biological TEVGs were tempered by the 3-month production time. In cases where an alternate blood conduit is required immediately, these grafts would not be available [44].

Another type of completely biological TEVG pioneered by Weinberg and Bell in 1986 used scaffolding made from natural collagen type I, a component of the ECM. The collagen scaffold was produced by denaturing bovine collagen, which was subsequently seeded with cultured bovine ECs, SMCs, and fibroblasts. The resultant graft had inadequate burst strength $(\sim 325 \mathrm{mmHg})$ compared to native coronary artery $(\sim 5000$ $\mathrm{mmHg})$ or saphenous vein $(\sim 2000 \mathrm{mmHg})$, necessitating the incorporation of a Dacron mesh sleeve for extra radial strength [45].

To increase the mechanical properties of collagen based vascular grafts, Girton et al. subjected collagen gel constructs seeded with smooth muscle cells to glycation. This is the nonenzymatic crosslinking of ECM proteins by reducing sugars. Once crosslinked, the constructs showed a 16-fold increase in circumferential tensile stiffness. Despite the 
similarity in compliance between these grafts and native arteries, the tensile strength and burst pressure still fell below the required values [46, 47].

Completely biological TEVGs offer the advantage of easy incorporation into the host, but the long production time and lack of mechanical strength have led some researchers to pursue other avenues, including incorporation of synthetic, biodegradable polymers.

\section{Biodegradable Polymer TEVGs}

One of the pervasive themes of extracellular (ECM ) based scaffolds, such as collagen, is their lack of mechanical strength. Many research groups have focused on hybrid grafts, or those that incorporate a variety of synthetic materials for structural support and natural materials to interact with blood. A majority of the synthetic structural support materials are biodegradable in hopes that remodeling processes within the body will eventually produce a true blood vessel, thus negating the need for synthetic support. Based on this, research groups have pursued synthetic, biodegradable scaffolds that have sufficient mechanical strength, and other advantages over naturally derived scaffolding. For example, degradation rate can be tailored in manufactured scaffolds. The body can use the scaffold as a guide for reconstruction, and by the time the healing process is complete, the scaffold can be completely degraded. In addition, scaffold properties can be controlled, such as molecular and macroscopic structure, mechanical properties, and porosity.[29] 
Some degradable polyesters have been known for many years to be biocompatible, and as such provided a good starting point for TEVGs [48]. Mooney et al investigated the mechanical viability of spray treated polyglycolic acid (PGA) based tubular meshes for tissue engineering purposes in the mid 1990's. PGA meshes that were spray treated with a solution of poly(L-lactic acid) (PLLA) showed only a slight compression with the application of a $200 \mathrm{mN}$ radial compressive force, a significant improvement on the performance of untreated PGA meshes, which were completely crushed by a force of $5 \mathrm{mN}$. The study not only demonstrated the mechanical potential of biodegradable vascular scaffolding, but also showed a positive cellular response to the scaffolds by seeding SMCs and ECs on the lumen of a PLLA sprayed PGA mesh.[49]

Tissue engineered pulmonary artery grafts utilizing PGA/polyglactin copolymer scaffolds were produced by Shinoka et al. in 1998. Autologous ECs, SMCs, and fibroblasts were expanded in culture, seeded onto the scaffolds, and cultured in vitro for seven days. The finished grafts were used to replace $20 \mathrm{~mm}$ long x $15 \mathrm{~mm}$ diameter sections of pulmonary artery in lambs. One control graft of unseeded PGA/polyglactin scaffolding was also implanted. Results showed that all 7 cell-seeded grafts remained patent for up to 24 weeks, but the acellular polymer scaffold occluded due to thrombus formation. Polymer scaffolds completely degraded by the $11^{\text {th }}$ week, but the replacement tissue had insufficient burst strength to be used in high pressure systemic circulation [50].

In 1999, Niklason et al used a PGA scaffold and a pulsatile bioreactor to conduct a study that looked more closely at the effects of culturing conditions in vitro. Tubular PGA scaffolds were treated with sodium hydroxide to cause ester hydrolysis on the surface of the fibers, increasing hydrophilicity and cellular attachment. SMCs were 
seeded on the luminal surface and cultured under pulsatile and static conditions for 8 weeks. Histological examination revealed infiltration of SMCs into the wall of the PGA scaffold among the pulsed constructs, resulting in a smooth luminal surface suitable for EC seeding. Static conditioned SMC constructs showed no infiltration and a luminal surface characterized by uneven polymer fragments, an unsuitable surface for EC seeding. A layer of ECs was seeded on the lumen of pulsed constructs, followed by continuous perfusion of the vessel lumens for 3 days. The final grafts showed a confluent endothelium expressing von Willebrand factor and platelet endothelial cell adhesion molecule. They also attained a suitable burst strength at $2150 \pm 709 \mathrm{mmHg}$, but a suture retention strength of $91 \pm 26 \mathrm{~g}$ was still less than the strength of $273 \pm 31 \mathrm{~g}$ reached by native arteries [51].

The results from the studies presented in this section show how biodegradable polymer scaffolding has contributed to the field of TEVGs. Grafts with mechanical properties more suited for implantation have been created, while immunogenic response has been minimized. In addition, other scaffold properties can be controlled using synthetic, biodegradable polymers, including molecular properties and porosity. Using a variety of techniques, it is possible to create grafts that continue to perform more and more like natural, human blood vessels.

An Alternate Use for Established TEVG Techniques: Blood Vessel Mimic

The methods and research described in the preceding sections were focused on pursuing a TEVG for implantation into a human host. However, TEVGs can potentially 
serve an alternate purpose. Inspired by these established techniques, the blood vessel mimic (BVM) creates TEVGs not for implantation, but for in vitro device testing. The following section introduces the BVM and current research being conducted to make the system more physiologically accurate.

Intermediate In Vitro Device Testing Using the BVM

Based on the challenges of animal studies, such as cost, lack of facilities and number of personnel required, and the leap that is made from in vitro static-culturing response to animal testing, a three dimensional BVM was developed to test intravascular devices in vitro. Based on tissue engineered blood vessel techniques described earlier, the blood vessel mimic (BVM) provides a more efficient means of initial assessment in order to direct animal and clinical studies toward the most promising devices [52].

The BVM system (Figure 7) seeks to create a physiologically accurate environment for testing and evaluating intravascular devices prior to clinical trials. At the start, the BVM consists of human ECs seeded on the luminal surface of an ePTFE support scaffold. The system is placed in a perfusion bioreactor system, consisting of a peristaltic pump, gas permeable tubing, a chamber used as a media reservoir, and another chamber containing the BVM. The perfusion bioreactor maintains flow of media through the BVM to cultivate the ECs until confluency, effectively making the luminal surface of the BVM similar to the luminal surface of a human blood vessel. Once the ECs are confluent on the luminal surface of the ePTFE scaffold, a stent can be "implanted" by way of the inlet port. Media perfusion is resumed through the stented graft to encourage 
EC proliferation and migration. Removal of the grafts from the system and subsequent histological examination provides information on $\mathrm{EC}$ response to the implanted stent. Based on these results, the goal is that researchers can make a more informed decision on which products to move on to animal testing. 


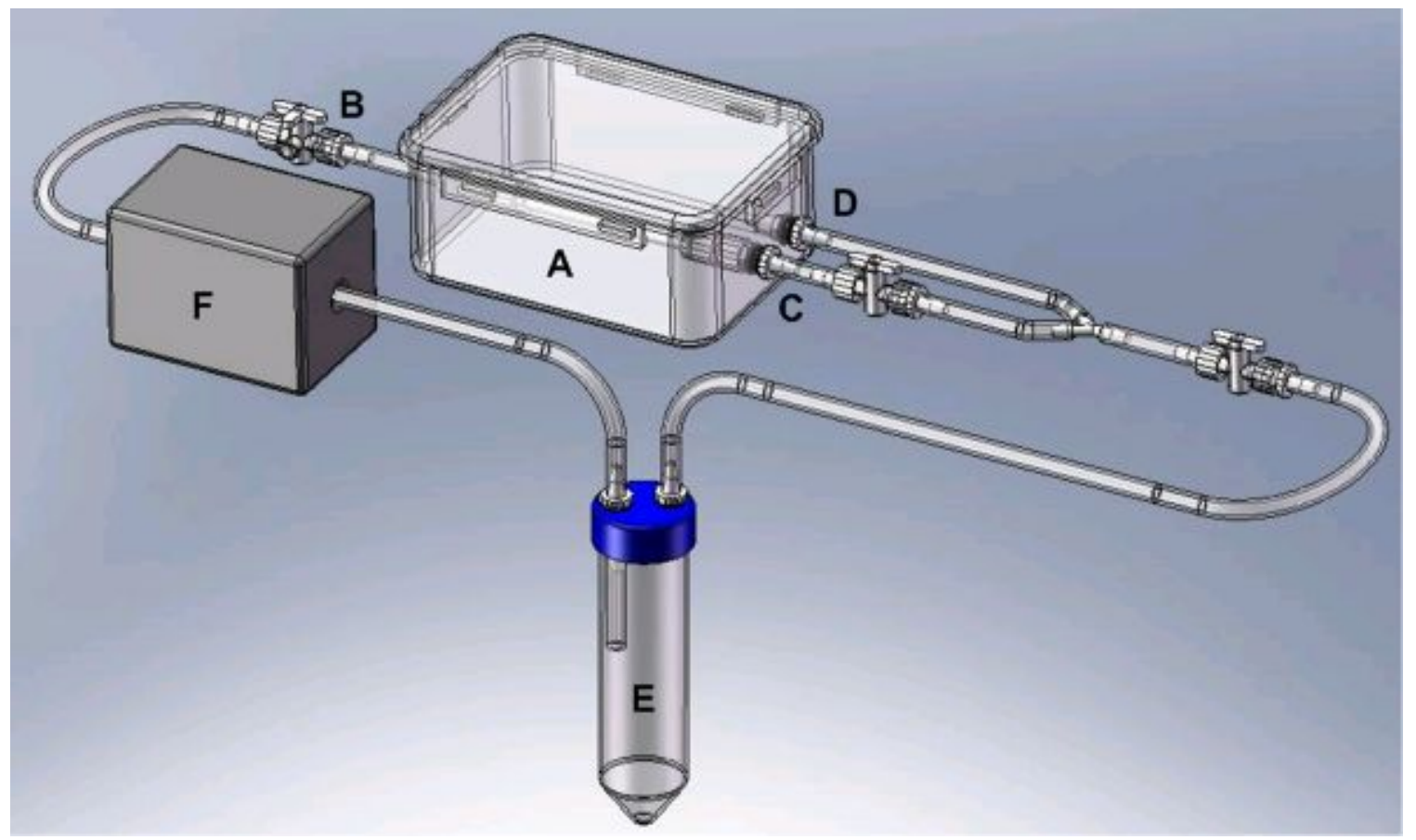

Figure 7 - Bioreactor system containing the Blood Vessel Mimic (BVM). The BVM chamber (A) consists of three ports: luminal inlet port (B), luminal outlet port (C), and extraluminal outlet port (D). The luminal inlet port allows media to flow into the system and implantation of stents. The luminal outlet port allows media to flow out of the system. The extraluminal outlet port, when unclamped and coupled with clamped luminal outlet and inlet ports, facilitates flow through the ePTFE vessel wall for cell sodding. A separate reservoir is attached for easy media changing (E), and a peristaltic pump drives the flow of media through the system $(\mathrm{F})$. The entire bioreactor system is placed in an incubator at $37^{\circ} \mathrm{C}$ and $5 \%$ carbon dioxide.

The high throughput BVM is cost effective and expedient compared to preliminary animal testing. Also, the effectiveness of animal models in intravascular device testing has been a concern for researchers, specifically the rate and extent of endothelialization [53]. An improved cost effective, high-throughput, physiologically 
accurate BVM could help alleviate the dependency on expensive animal studies, and eventually provide a more appropriate testing environment for intravascular devices. The following sections describe research aimed at making the BVM more cost effective and physiologically accurate, while maintaining the BVM's ability to perform as a highthoughput testing environment.

Pulsatile Flow and Shear Stress Optimization

Endothelial cells on the BVM luminal surface are currently exposed to steady flow of low viscosity media. This is not physiologically accurate, so media flow dynamics are being changed to more accurately imitate conditions found in native arteries. Conditions of flow determine the differentiation path and properties of ECs, so it is important to recreate physiologic flow conditions as closely as possible. To do this, the viscosity of the media must be changed to tune the shear stress experienced by the ECs to normal arterial shear stress. In addition, the steady flow should be replaced with a pulsating flow since this is what occurs in the body [29]. Altering the media viscosity and introducing pulsatile flow into the BVM system are currently under development [54]. 


\section{Bioreactor Redesign}

A new BVM bioreactor is being designed for several reasons. First, installing and removing scaffolds from the BVM bioreactor is difficult, which causes operator frustration. Also, the BVM must remain sterile at all times, and the difficulty of accessing the scaffolds makes maintaining sterility a challenge in itself. A redesign of the system is necessary to make accessing the scaffolds more user-friendly and, thus, easier to maintain sterility [55]. Second, stents come in many shapes and sizes to fit the variations of coronary arteries, so the bioreactor should accommodate a BVM suitable for testing a wide range of stent sizes. The current bioreactor limits testable stents to short, straight segments. Finally, the bioreactor should be constructed of readily available, cost effective components so scale up is possible. The current bioreactor vessel chambers are specially machined and not cost-effective, and should be replaced. This work is currently underway [56].

\section{Cell Source and Sodding Procedure}

In order to make the BVM more physiologically accurate, research on the cellular component of the system is also underway. Currently, human microvessel endothelial cells (HMVECs) derived from liposuction fat are currently used to form the intima-like monolayer of ECs. A more appropriate cell line sourced from large blood vessels is commercially available, and substitution of these large vessel cells for the HMVECs is under investigation. In addition, the feasibility and development of protocols necessary 
to construct a SMC/EC dual layer BVM is being researched. The addition of a medial layer composed of SMCs adds physiological accuracy to the BVM.

Physiologically Appropriate and Cost Effective Scaffolding

The BVM utilizes ePTFE because it is biocompatible, readily available, has adequate porosity, and does not degrade. However, the mechanical properties and high cost of ePTFE make its use in the BVM less than optimal. As mentioned in Section 2.3.1, when implanted in vivo as a conduit, ePTFE tends to have poor long term patency due to its poor radial compliance. But, since media is used in place of blood, intimal hyperplasia is not a concern. The radial distension of vessels does, however, directly affect ECs in other ways. The cyclic pumping of the cardiac cycle alters the shear stress imposed on endothelial cells, by both changing the fluid flow rate, and varying the caliber of the vessel. Stress causes several endothelial cell reactions. First, stress fibers of the actin cytoskeleton at the base of endothelial cells extend and align with the direction of shear stress, causing an elongated, cobblestone morphology. Second, endothelial cells will produce various substances in response to shear stress and cyclic radial stretch, including prostacyclins and nitric oxide, important substances in blood platelet aggregation and vasodilation, respectively [57]. Third, endothelial cells signal arterial remodeling in order to maintain a shear stress in most arteries of 15 dynes $/ \mathrm{cm}^{2}[1,39]$. If the goal is modifying the BVM to be as physiologically accurate as possible, including the response of endothelial cells to induced stress, then ePTFE must be replaced with a 
more compliant scaffold. The cost of any replacement scaffolding should be equal to or less than the current cost of ePTFE, which is currently priced at $\$ 10 / \mathrm{cm}$.

As the purpose of this thesis is to explore more physiologically appropriate, inhouse fabricated scaffolding, a viable fabrication method for scaffold production based on established tissue engineering scaffold fabrication techniques must be identified. The next section introduces and reviews possible fabrication methods that could produce appropriate scaffolding for the BVM. Chapter 2 contains an evaluation of the fabrication methods for use in the BVM.

\section{Scaffolding for TEVGs}

Fibrous Non-woven Mesh Textiles

Micron-diameter fibers can be produced by extruding or gravity spinning a polymer solution or melt through a spinneret, followed by a drawing out process between a series of winders. By collecting and compressing the fibers, a nonwoven textile scaffold is produced. Depending on process parameters such as extrusion and drawing rate, scaffolds with varying fiber diameters and porosities are attainable [58, 59]. Early attempts at tissue engineering vascular grafts used non-woven mesh textile scaffolds made of polyurethane (PU) and PGA [49, 60, 61]. PGA mesh textiles have also provided scaffolding for tissue engineered cartilage [62]. 
Solvent Casting and Particulate Leaching (SCPL)

Introduced by Mikos et al. in the early 1990s, solvent casting and particulate leaching (SCPL) produces scaffolds that are mechanically and morphologically tunable. In SCPL, salts are sieved to control for particle size and evenly distributed in a solution of dissolved polymer. When the solvent evaporates the resulting solid polymer is impregnated with salt particles, which can be leached out using another solvent, usually high purity water. The removal of the salt leaves a porous, degradable polymer scaffold, the pore size and percent porosity of which depend upon the amount of salt suspended in solution and the size of individual salt particles, determined by the sieving parameters [63]. SCPL scaffolds can be highly porous with pores large enough for complete cellular infiltration and vascularization. For example, Mooney et al. used SCPL scaffolds made of PLLA to support the growth of hepatocytes and allow for vascularization in tissue engineered liver [64].

Thermally Induced Phase Separation (TIPS)

When a single homogenous polymer solution mixed at elevated temperatures is subsequently cooled, the solution converts into a two-phased system composed of domains of polymer-rich and polymer-poor regions. Freeze drying of liquid-liquid phase-separated polymer solution produces microcellular structures as a result of solvent removal. By controlling conditions such as polymer concentration, quenching route, quenching depth, solvent to non-solvent composition, and the presence of additives, the 
final pore size distribution and interconnectivity can be tailored $[65,66]$. A binary phase diagram is used to determine the solution concentration that results in spinoidal decomposition as shown in Figure 8. More complicated methods of TIPS fabrication have been used as well; Dalton et al. produced poly(2-hydroxyethyl methacrylate-comethyl methacrylate) TIPS hydrogel tubes for in vivo nerve regeneration using a tertiary diagram of 2-hydroxyethyl methacrylate, methyl methacrylate, and water [66]. 


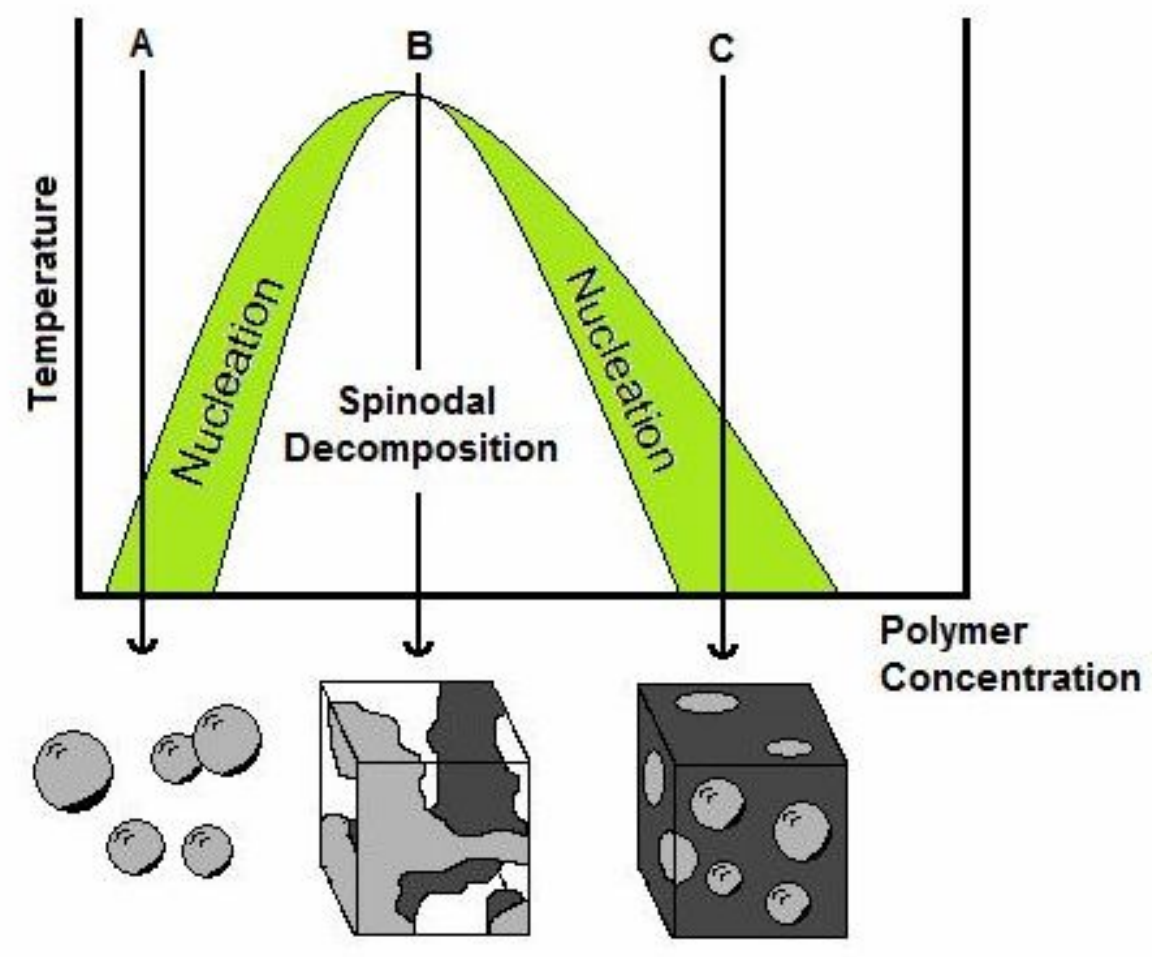

Figure 8 - Typical binary phase diagram for a polymer in a solvent. Quenching with too little polymer in solution results in disconnected microspheres of solidified polymer (A), while using a high concentration solution produces a porous macroscopic polymer matrix with no interconnecting pores (C). By controlling quenching so that the polymer undergoes spinodal decomposition, a porous matrix with $100 \%$ pore interconnectivity is produced (B).

If freezing occurs prior to liquid-liquid demixing, the polymer is expelled to the boundaries of the solvent crystallites, resulting in a solid-liquid phase separated polymer system. Liu et al. demonstrated the variability of this technique using various solutions of polyurethane in 1,4-Dioxane frozen at different temperatures. By keeping the concentration of the solution constant at $10 \%$ by weight polymer and freezing at temperatures ranging from $-196{ }^{\circ} \mathrm{C}$ to $0{ }^{\circ} \mathrm{C}$, scaffolds with pore sizes ranging from $4.7 \pm$ 
$2.1 \mu \mathrm{m}$ to $70 \pm 40 \mu \mathrm{m}$ were produced. Conversely, using a constant freezing temperature of $-80{ }^{\circ} \mathrm{C}$ and varying solution concentration from $5 \%$ to $15 \%$ by weight polymer, scaffold pore architecture was affected in two ways. First, lower concentrations $(5 \%$ by weight polymer) produced pore sizes of $75 \pm 29 \mu \mathrm{m}$ and higher concentrations ( $15 \%$ by weight polymer) resulted in $20 \pm 9 \mu \mathrm{m}$ pores. In addition, porosity was affected by varying solution concentration. Lower concentrations yielded scaffolds with $51 \pm 4 \%$ porosity, while higher concentration solutions created $10 \pm 2 \%$ porosity scaffolds [67].

Solid Freeform Fabricated Scaffolds (SFF)

A high degree of control over the final structure of a scaffold is possible using solid freeform fabrication (SFF). Several forms of SFF have been used to create scaffolds for tissue engineering, three of which are presented in this section and shown below in Figure 9. 


\section{Three-Dimensional Printing}

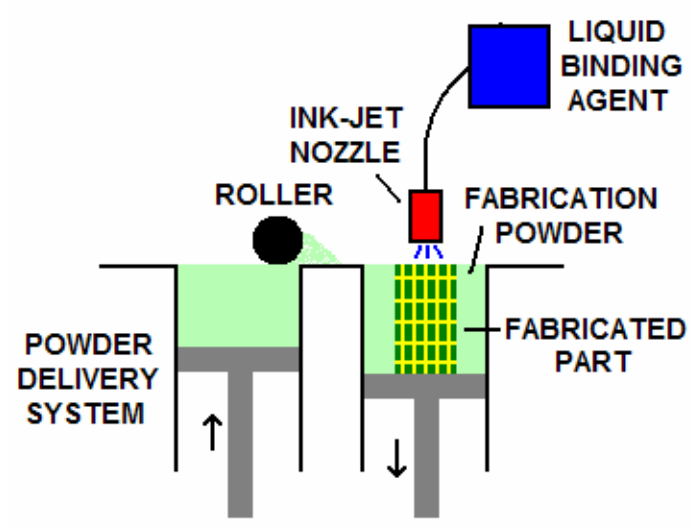

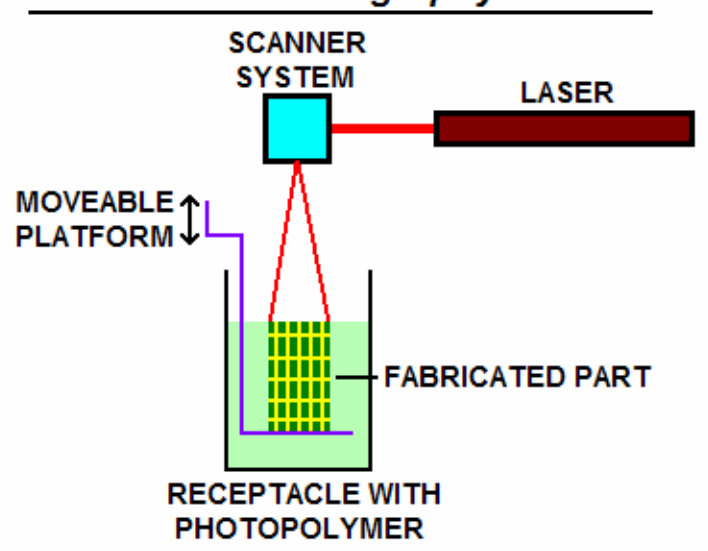

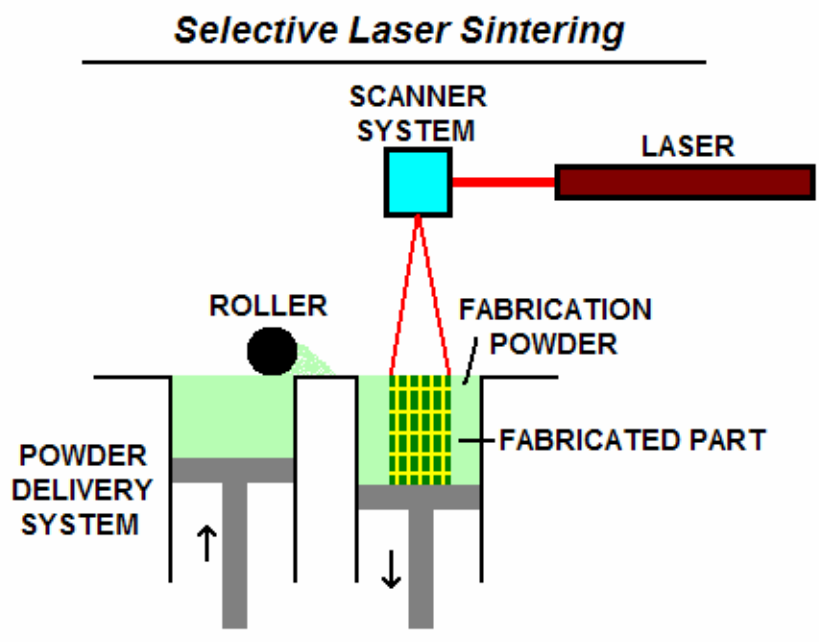

Figure 9 - Equipment diagrams for three solid freeform fabrication methods. All three systems construct three dimensional parts by fusing many two dimensional layers.

Three-Dimensional Printing

Using various powders and a liquid binder, three dimensional printing builds constructs from the bottom up. A three dimensional computer model is sliced into two dimensional layers, and each layer is built and fused with the previous layer by spraying 
fresh powder with a binding agent [68]. Natural powdered materials have been used, such as cornstarch, dextran, and gelatin [69]. Synthetic polymers, like polycaprolactone, have also been used, but most biomaterials are not usually available in powder form and require processing [70]. A process called indirect three-dimensional printing involves creating scaffold molds using three dimensional printing, followed by solvent casting using a biopolymer. Using this method, extra processing to manufacture powdered biomaterials is unnecessary, but complete removal of the powdered mold can be difficult [71].

Resolution of three dimensional printing is limited in several ways. First, the nozzle size and position controller introduce limits on particle placement. Second, particle size limits the layer thickness to a minimum of $80-250 \mu \mathrm{m}$. Also, internal unbound powder is difficult to remove from porous parts, resulting in a lower bound on pore size $[58,72]$. Kim et al. utilized three dimensional printing for macroscopic part construction, and coupled the technique with particulate leaching to introduce microporosity into the construct [73]. In this manner, the resolution limit of threedimensional printing was avoided while taking advantage of the macroscopic accuracy of the technique.

\section{Stereolithography}

Stereolithography uses a focused ultraviolet laser that is scanned over a liquid bath of photopolymerizable polymer. When the laser hits the bath, it causes low molecular weight monomers to chain react and polymerize at the laser-monomer 
interface, resulting in a solid polymer. By lowering the polymerized solid sections and striking the liquid on top with the laser, a construct is built layer by layer [74]. Since stereolithography requires a photopolymerizable polymer, the materials available for this process are limited [72]. In a similar fashion to indirect three-dimensional printing, stereolithogaphy has been used to produce scaffold molds, which were subsequently filled with powdered hydroxyapatite and sintered for bone scaffolding applications [75]. A major limitation of stereolithography is that equipment required for this fabrication process is expensive [58].

\section{Selective Laser Sintering}

Similar to stereolithography, selective laser sintering (SLS) also uses a focused laser beam, but the beam's function is not to polymerize. The laser is selectively scanned over the surface of a powder, causing particles to locally melt and fuse together, creating a two dimensional cross section of a CAD designed part that can be built upon to create a three dimensional structure. Tan et al. demonstrated the ability to incorporate the bioactive material hydroxyapatite into poly(etheretherketone) SLS processed scaffolds, an attractive fabrication technique for bone tissue engineering [76]. 


\section{Electrospinning}

The electrohydrodynamical phenomena known as electrospinning can be used to produce an interconnecting fiber network with fiber diameters ranging from millimeters to nanometers. Electrospinning is based on the complex interplay between surface energy, rheology, and electrical charge. The basic elements required for electrospinning are a polymer source, high voltage power supply, and grounded collector as shown below in Figure 10. A high electric potential around 8,000 to 30,000 volts applied between the polymer solution being extruded through a metal syringe needle and the collector causes charge to build up in a polymeric droplet at the tip of the metal needle. This charge creates a force large enough to overcome the surface tension of the solution causing the droplet to erupt towards the collector. After eruption, the strand of polymer solution that extends from the needle tip to the collector is called a jet. As it travels towards the collector, electrostatic forces within the jet cause physical reactions called bending instabilities that result in elongation of the fiber and solvent evaporation. The result is a solid fiber network that takes the shape of the grounded collector [77]. 


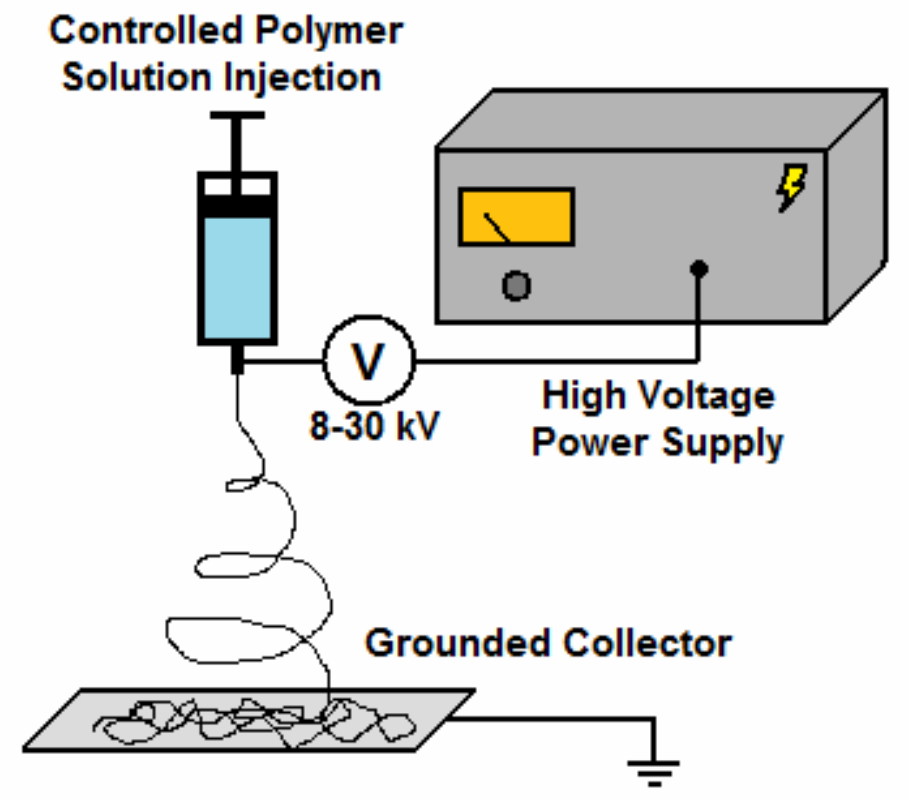

Figure 10 - A simple electrospinning apparatus. Variable parameters include polymer solution concentration and injection rate, power supply setting, distance from polymer source to grounded collector (gap distance), and collector geometry.

Still in its infancy, electrospinning is gaining popularity as a fabrication process for producing scaffolds with microstructures that mimic ECM. The process is compatible with a number of materials, including synthetic biodegradable polymers like PGA, PLA, and PCL, and natural ECM components including collagen and elastin $[32,58,78]$.

Scaffold Fabrication for the BVM

A variety of scaffold microstructures are possible using the techniques presented in the preceding sections. Tubular scaffolds can be composed of a solid matrix with pores randomly suffused throughout (SCPL and TIPS), a solid matrix with carefully 
controlled porous structure (SFF), or micro-fibers layered to form a macroscopic construct (Mesh textiles and electrospinning). Chapter 2 will evaluate the candidate fabrication techniques, and their resultant microstructures, as replacements for ePTFE in the BVM. 


\section{Summary and Aims of this Thesis}

Cardiovascular disease is the number one cause of mortality in the United States. There is a need to develop treatments for this epidemic, whether the treatments take the form of improved stent technology for revascularization or better conduits for bypass operations. Using tissue engineering techniques, a blood vessel mimic was created to expedite the development of cardiovascular devices and therapies. The current scaffolding used to support the growth, migration, and proliferation of endothelial cells on the lumen of the blood vessel mimic is expanded polytetrafluoroethylene, which exhibits a lack of radial compliance and cannot be modified in-house. Thus, there exists a need to replace the expanded polytetrafluoroethylene with a more compliant scaffold that can be fabricated and modified in house.

The overall goal of this thesis was to pursue the replacement of the expanded polytetrafluoroethylene scaffolding used in the BVM. To accomplish this goal, three aims were identified and pursued. First, methods for scaffold fabrication were compared and the most promising fabrication technique was selected to make scaffolds for use in the BVM. Second, the apparatus for the chosen fabrication process was constructed. Third, the capabilities of the new system were evaluated. Additionally, a preliminary proof of concept that the new fabricated scaffolds would support placement within a BVM bioreactor and subsequent cell deposition was performed. 


\section{FABRICATION METHOD SELECTION AND ELECTROSPINNING}

\section{Introduction}

The end of Chapter 1 introduced various scaffold fabrication techniques that were candidates to replace commercially obtained ePTFE in the BVM. The first section of Chapter 2 evaluates these techniques based on their appropriateness for use in the BVM. Criteria used for evaluation included: consistency and controllability, material compatibility, pore architecture, cost, and other special considerations. Using a decision matrix, electrospinning was selected as the fabrication technique to pursue for BVM scaffold fabrication. Section 2 will examine the process of electrospinning more closely by presenting a brief history, the physics involved, and some of the governing parameters of the fabrication technique. Finally, section 3 concludes Chapter 2 by examining the electrospinning apparatus constructed in-house.

\section{Fabrication Method Selection}

To choose the most appropriate method for BVM scaffold fabrication in-house at Cal Poly, candidate techniques were compared and a fabrication method was chosen using a decision matrix. The decision matrix was based on both fabrication process and physical properties of scaffolds specific to the BVM. The first aspect that methods were evaluated on was the controllability/reproducibility of the process. Specifically, fabrication process inconsistencies were explored, such as resolution, final pore structure, and negative residual effects. Second, fabrication methods were evaluated based on their 
compatibility with a variety of materials; the more materials a process was compatible with, the higher it scored. Third, pore architecture was evaluated. In this case, pore architecture included pore size, percent porosity, and pore interconnectivity. Fourth, monetary cost of each method was taken into consideration; the higher the cost of a fabrication method, the lower it scored in the decision matrix. Finally, extra points were awarded to methods that had other desirable traits that did not fit into one of the first four evaluation criterion.

A table summarizing key features of all fabrication methods is provided (Table 1), followed by a discussion of each aspect. 
Table 1 - Summary of Candidate Scaffold Fabrication Methods

\begin{tabular}{|c|c|c|c|c|c|}
\hline FABRICATION & CONTROLLABLE & & & & \\
\hline METHOD & STRUCTURE & PORE ARCHITECTURE & MATERIAL SELECTION & $\underline{\operatorname{cosT}}$ & $\underline{\text { OTHER }}$ \\
\hline \multirow[t]{2}{*}{ Fiber Mesh } & Controllable fiber & Interconnected, variable & Good & Low & - \\
\hline & diameter & $>100$ microns & & & \\
\hline & & & & & \\
\hline \multirow[t]{3}{*}{ SCPL } & Controllable pore size; & Semi-interconnected, & Good & Very Low & Simple \\
\hline & random interstitial pore & non-uniform distribution & & & \\
\hline & formation & $5-600$ microns & & & \\
\hline & & & & & \\
\hline \multirow[t]{2}{*}{ TIPS } & Controllable pore size & Interconnected & Good & Very Low & Simple \\
\hline & & 5-600 microns & & & \\
\hline \multirow[t]{5}{*}{ Electrospinning } & Controllable fiber & Dependent on fiber & Good & Low & Mimics ECM \\
\hline & diameter (smaller than & architecture & & & Fiber size from \\
\hline & fiber mesh) & Interconnected & & & nano to micro \\
\hline & & $<1$ to 100 microns & & & Prevents EC \\
\hline & & & & & infiltration \\
\hline \multirow{3}{*}{ 3D Printing } & & & Reguires nowdered & & \\
\hline & $\begin{array}{l}\text { CAD controlled } \\
\text { architecture }\end{array}$ & $\begin{array}{c}\text { Great unitormity at } \\
\text { larger sizes }\end{array}$ & $\begin{array}{l}\text { Requires powdered } \\
\text { biomaterials }\end{array}$ & High & - \\
\hline & & $>100$ microns & Good for ceramics (bone) & & \\
\hline \multirow[t]{3}{*}{ Stereolithography } & CAD controlled & Superior uniformity at & Requires UV curable & High & - \\
\hline & architecture; highest & larger sizes & photopolymers & & \\
\hline & SFF resolution & $>20$ microns & & & \\
\hline Selective Laser & CAD controlled & Superior uniformity at & Requires powdered & High & - \\
\hline \multirow[t]{2}{*}{ Sintering } & architecture & larger sizes & biomaterials with & & \\
\hline & & $>30$ microns & controlled particle size & & \\
\hline
\end{tabular}


Controllable and Consistent Final Structure

Any fabrication method used for manufacturing scaffolds for the BVM must be consistent and controllable; the final product should be predictably reproducible. For example, pore architecture and mechanical properties should be consistent between scaffolds that are produced using identical processes.

SCPL has the lowest rating in this category because interstitial pore size, or the size of pores not formed by salt particles, and wall thickness have been difficult to control [79]. In addition, in SCPL scaffolds thicker than $2 \mathrm{~mm}$ it is difficult to completely remove the salt from the center of the scaffold [79]. TIPS utilizes spinodal decomposition to produce an interconnected porous network, but the exact architecture of the pores is uncontrollable [58]. While exact architecture is still random, electrospinning offers a slightly more predictable outcome in that fiber diameter size is controllable [78]. In addition, fiber alignment is possible, as opposed to random orientation, both by collection using a grounded rotating drum or a dual ring collection system that alters the electromagnetic field around the collector $[80,81]$. Fiber mesh fabrication is similar to electrospinning; fiber diameter is controllable and different collection methods can be used to generate scaffolds with varying geometry [82]. However, fiber mesh scaffolds exhibit random orientation and a lack of interconnecting structure, preventing them from load bearing applications [83].

Solid freeform fabrication methods excel as controllable processes because the porous structure of scaffolding is planned using computer aided design software prior to fabrication [84]. However, the individual SFF methods are not equal in this category. For example, the resolution of three dimensional printing is limited in several ways. 
First, the nozzle size and position controller introduce limits on particle placement. Second, powder particle size limits the layer thickness to a minimum of $80-250 \mu \mathrm{m}$. Also, internal unbound powder is difficult to remove from porous parts, resulting in a lower bound on pore size $[58,72]$. However, these shortcomings of three-dimensional printing have been alleviated; Kim et al. utilized three dimensional printing for macroscopic part construction, and coupled the technique with particulate leaching to introduce microporosity into the construct [75]. In this manner, the resolution limit of three-dimensional printing was avoided while taking advantage of the macroscopic accuracy of the technique. Similar to three-dimensional printing, selective laser sintering also has a lower bound on resolution and pore size due to powder particle size [58].

While three-dimensional printing, stereolithography, and selective laser sintering all offer a high degree of control, stereolithography is especially controllable because it is not limited in resolution by powder particle size, and the use of a laser curing system is more accurate than the liquid binder spraying system used in three-dimensional printing. Heat transfer and unintentional polymerization in the polymer bath do, however, limit the resolution of stereolithography [74].

The relative scoring of these techniques with regard to consistency and controllability is provided in the decision matrix (Figure 11). 
Compatible with a Variety of Materials

The ability to fabricate scaffolds using a multitude of materials imparts flexibility into the morphological and mechanical properties attainable for the BVM. Any restrictions on the materials compatible with a particular fabrication method limit the possibilities for scaffold production, and these limited methods should be considered after more versatile methods.

Fiber mesh scaffold fabrication, SCPL, TIPS, and electrospinning are all compatible with the traditional polyester biomaterials poly(L-lactic acid), poly(glycolic acid), poly(caprolactone), and copolymers of these three [49, 63, 65, 80, 82, 85-90]. Solution preparation for these methods usually involves an organic solvent, but electrospinning and fiber mesh fabrication by melt spinning are also possible using a polymer melt, obviating the need for a solvent $[59,91]$.

Of the SFF techniques, stereolithography is the most limited fabrication method since it requires photopolymerizable biomaterials. Examples of these materials include polyethylene glycol acrylate, polyethylene glycol methacrylate, polyvinyl alcohol and modified polysaccharides [72]. For this reason, stereolithography scored the lowest on the decision matrix in the "Material Selection" category. Three dimensional printing and SLS both require powdered biomaterials, which include the traditional polyesters, and even powdered ECM $[92,93]$. However, suitable powdered material requires processing, so three dimensional printing and SLS score lower than all other methods besides stereolithography [70]. Furthermore, SLS powders must also be controlled for particle size (more processing), so SLS scores lower than three dimensional printing [58]. 
The relative scoring of these techniques with regard to material compatibility is provided in the decision matrix (Figure 11).

Pore Architecture

Matsuda et al. reported the ideal pore size for vascular graft scaffolding as 15 to $80 \mu \mathrm{m}$ [94]. This pore size is meant to support cellular attachment and migration through a scaffold, as well as vascularization of the tissue. BVM scaffolding is unique because there is no need for cellular infiltration into or vascularization of the scaffold. The only requirement is the ability to support a monolayer of endothelial cells. As a result, fabrication methods that result in macroporous structures, or pores that are larger than 50 $\mu \mathrm{m}$ and suited to influence macroscopic tissue formation and vascularization, are not necessary. Of interest are microporous structures $(<50 \mu \mathrm{m})$ that affect cellular attachment, migration, and proliferation [29]. Specifically, a porous structure that prevents cellular infiltration while supporting a monolayer of ECs is desired.

In addition to pore size, another structural property called porosity, or the total volume of a scaffold that pores occupy, plays a role in cellular development. High porosities allow greater cellular infiltration and vascularization, as well as nutrient, waste, and oxygen transport. An important aspect of porosity is pore interconnectivity. Without interconnectivity between adjacent pores, increasing porosity does not result in the aforementioned transport benefits since interconnectivity results in paths from the scaffolding matrix to the surface. Also, pores that are not connected to any other pores are voids that lower the mechanical strength of scaffolds [58]. An ideal value for 
porosity in the BVM is unknown at this time, but fluid transfer from the lumen through the scaffold wall must be possible.

For SCPL and TIPS, pore sizes of 5 to $600 \mu \mathrm{m}$ and porosity values of up to $90 \%$ have been reported $[84,95]$ SCPL is rated lower than TIPS because SCPL results in a random dispersion of pores can result in incomplete pore interconnectivity [96]. When processed correctly, TIPS scaffolds go through spinoidal decomposition and achieve $100 \%$ pore interconnectivity [95]. This interconnectivity would allow for media transfer through the BVM scaffold, while low pore sizes could prevent EC infiltration. In addition, research has shown that porous structure integrity of TIPS scaffolds is superior to SCPL scaffolds. Cao et al. compared poly(lactic-co-glycolic acid) (PLGA) scaffolds produced by both SCPL and TIPS by examining in vitro degradation. In vitro immersion in an aqueous environment showed PLGA scaffolds produced by SCPL went through more pronounced changes in volume than TIPS scaffolds. SCPL scaffolds lost about $15 \%$ of their total mass by week six, whereas no noticeable mass loss was reported for TIPS scaffolds. Morpholigically, TIPS scaffolds showed some deformation of their walls, but an overall porous and interconnected structure after 4 weeks of in vitro testing. The porous structure of SCPL scaffolds had completely collapsed by week 4 [79]. This lack of integrity translates to a SCPL score lower than TIPS on the decision matrix, however both methods score relatively well due to the pore sizes attainable.

Non-woven fiber mesh textile scaffolds exhibit fiber sizes with lower bounds of approximately 10-20 $\mu \mathrm{m}$, allowing for a fully interconnected porous structure; however, the pore sizes $(>100 \mu \mathrm{m})$ are too large to prevent EC infiltration if used in the BVM [82, 
97-99]. Due to the large pore size, non-woven fiber mesh fabrication scores the lowest on the decision matrix.

Scaffolds composed of sub-micron electrospun fibers are of interest since they can be permeable or impermeable to cellular infiltration depending on material selection or fiber size. Electrospun collagen scaffolds with submicron fiber diameters have been shown to promote cellular infiltration and vascularization, while biodegradable polyester electrospun scaffolds with similar fiber morphologies and porosities prevented any infiltration [100]. Electrospun PCL scaffolds fabricated with varying fiber diameters and evaluated for cellular infiltration showed a correlation between larger fiber size and greater cellular infiltration since larger fibers resulted in larger pore sizes [101]. Kwon et al. demonstrated that reducing fiber diameter to 0.5 to $1 \mu \mathrm{m}$ created an impermeable scaffold surface that supported EC migration, proliferation, and growth [102]. As stated at the beginning of this section, this type of scaffold architecture is attractive since it encourages the formation of a monolayer of ECs, so electrospinning scores the highest on the decision matrix in the "Pore Architecture" category.

As explained earlier, the resolution of solid freeform fabrication methods is limited; due to this resolution limitation, pore size and porosity are limited. Threedimensional printing does not have the resolution required to produce pores smaller than 100 microns, and even stereolithography can only achieve pore sizes as small as 20 microns $[58,74,84]$. These pore sizes are too large for use in the BVM, but SFF techniques can be combined with other fabrication techniques, such as SCPL, to produce suitable BVM scaffolds. 
The relative scoring of all techniques with regard to porosity is provided in the decision matrix (Figure 11).

Cost

The goal of the BVM is a high throughput, low cost model for intravascular device testing, so BVM scaffolding should be inexpensive to fabricate. Methods of fabrication were evaluated for cost based primarily on the cost of required equipment. For example, fiber mesh scaffold fabrication requires an infusion system, a spinneret, and a collector. In contrast, stereolithography requires more expensive equipment such as a laser, a computer control system, and a photopolymer receptacle [58]. Since the exact cost of each fabrication method is ultimately dependent upon the specific system used, methods were evaluated for cost relative to other methods.

The relative scoring of these techniques with regard to cost is provided in the decision matrix (Figure 11).

Other

There are special aspects of certain fabrication methods that are important to consider when deciding on a final BVM scaffold fabrication method. SCPL and TIPS both have the advantage of being simple compared to all other methods, and due to their simplicity both were awarded extra points $[58,84]$. As mentioned in the Introduction, an 
important feature of electrospun fibers is their mimicry of the ECM. Sub-micron fibers are beneficial for cell deposition and proliferation, similar to the function collagen plays in the ECM [103]. Also, the ability of electrospun scaffolds with small fiber diameters to act as an impermeable sieve to cells allows the creation of a BVM with a monolayer of EC on the scaffold surface. Preventing EC infiltration saves cells and time, important inputs in the high throughput BVM. For these benefits, electrospinning was given several extra points in the decision matrix (Figure 11).

Decision Matrix and Fabrication Method Choice

The summary of all candidate fabrication methods presented in Table 1 was used to help generate the decision matrix shown in Figure 11. Both consistent/controllable final structure and pore architecture were considered paramount for BVM scaffolding, so there criteria were rated on a scale of 1 to 10 , where a score of 1 was the worst and 10 the best. A method that is uncontrollable and completely inconsistent, and produces scaffolds with no porous structure would receive a score of 1 in both categories. Material selection and cost were rated on a scale of 1 to 5 , with a score of 1 again corresponding to a technique inappropriate for BVM scaffolding and 5 with techniques that are desirable for BVM scaffolding fabrication. Extra points were awarded in the "Other" category for fabrication methods with desirable characteristics that did not fit in other categories. Scores were awarded based on personal opinion. 


\begin{tabular}{|c|c|c|c|c|c|c|}
\hline FABRICATION & CONTROLLABLE & PORE & MATERIAL & & & \\
\hline METHOD & STRUCTURE & ARCHITECTURE & SELECTION & COST & OTHER & TOTAL \\
\hline RATING & $1-10$ & $1-10$ & $1-5$ & $1-5$ & Varied & \\
\hline Fiber Mesh & 5 & 4 & 5 & 4 & & 18 \\
\hline SCPL & 4 & 6 & 5 & 5 & 1 & 21 \\
\hline TIPS & 6 & 8 & 5 & 5 & 1 & 25 \\
\hline Electrospinning & 6 & 10 & 5 & 4 & 3 & 28 \\
\hline 3D Printing & 9 & 4 & 3 & 2 & & 18 \\
\hline Stereolithography & 10 & 6 & 1 & 1 & & 18 \\
\hline SLS & 9 & 6 & 2 & 1 & & 18 \\
\hline
\end{tabular}

Figure 11 - The decision matrix used to determine the most desirable fabrication technique for producing BVM scaffolds was generated using the summary information shown in Table 1. 
According to the decision matrix, electrospinning emerged as the fabrication choice for BVM scaffolding. This technique is inexpensive and can be performed inhouse at Cal Poly, and the resulting scaffolding can be made of many materials. By changing process parameters, fibrous scaffolds with fiber diameters ranging from tens of microns to hundreds of nanometers can be consistently fabricated. In addition, electrospun fibrous scaffolding can mimic the natural structure of ECM, and prevent EC infiltration into BVM scaffolding walls while supporting an endothelial monolayer on the lumen. The next section will introduce electrospinning in more detail. 


\title{
Electrospinning Fundamentals
}

\author{
Brief History of Electrospinning
}

In the late $19^{\text {th }}$ century, Lord Rayleigh noticed disturbances in liquids placed in electric fields. Later, liquids were observed to discharge in reaction to excess charge after metals had been tested for discharge based on their point geometry and material composition, and it was found that point discharge characteristics are independent of the kind of metal [104]. It was observed that eruption of liquid from a drop of water occurred when charged using a high potential. Using glycerine in order to observe the effects of increased viscosity, threads formed from the drop surface and extended $15 \mathrm{~mm}$ toward ground before breaking up into individual droplets [105]. In 1934, the process of electrospinning was patented, but work pioneering the fundamentals behind the phenomenon did not begin until the 1960s with the work of Sir Geoffrey Taylor [106]. Taylor furthered the understanding of electrified liquid jets with his calculation of the conical shape of the droplet where a jet may originate [107]. Interest in the process of electrospinning to produce nano and micro fibers, and to explain and quantify electrospinning phenomena, has only been seriously pursued since the 1990s [108].

The following sections explain the dynamic electrospinning process, and look at four key parameters in the electrospinning process: applied voltage, solution concentration, volumetric flow rate, and gap distance. 


\section{Jet Dynamics}

A droplet of polymeric solution from which a jet originates is stable largely due to surface tension. When subjected to an electric field, ions form within the droplet and distribute on the surface. This creates a repulsive force, and results in a droplet in the shape of a characteristic Taylor cone [107]. If the force is large enough, surface tension is overcome and a jet erupts from the droplet. After a jet is established, ions carry excess electrical charge away from droplet, resulting in fewer surface charges on the droplet than the polymer jet. Radial components of the Coulomb forces cause these ions to move to the surface of the jet, while the repulsive Coulomb forces between charges of like sign on the jet surface work against viscoelastic stress and cause jet elongation and thinning. The surface charge density of the jet increases as it moves away from the drop, eventually causing a bending perturbation (Figure 12). The bending perturbation grows outwards radially, resulting in an erratic, whipping jet known as a bending instability [78]. 


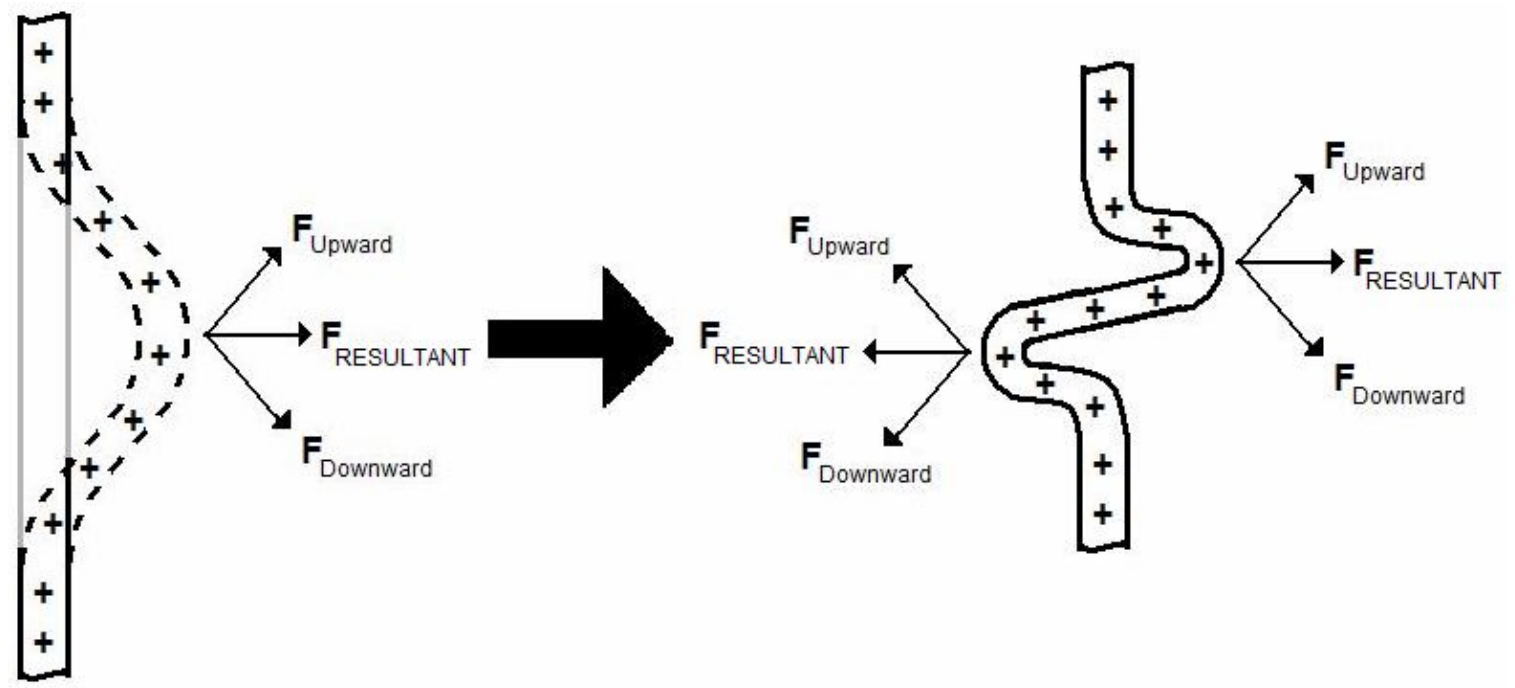

Figure 12 - When surface charge density is large enough, charges will interact to cause the jet to move slightly off its axis, a feature known as a bending perturbation. The bent section is forced downward and outward ( $\mathrm{F}_{\text {Downward }}$, as well as upward $\left(\mathrm{F}_{\text {Upward }}\right)$ and outward ( $\left.\mathrm{F}_{\text {Resultant }}\right)$ by charges above and below the region, respectively. Due this repulsive Coulomb force, the bending perturbation grows, later becoming a three dimensional bending instability. [Fig. 5 from Reneker, D.H. et al.. "Electrospinning jets and polymer nanofibers." 2008 [78]] 
A bending instability is a growing, perturbed path of charged jet that forms as a three-dimensional coil carried downstream. Continued forces from Coulomb repulsion causes the coil to increase in diameter as it travels further from the droplet, allowing the jet to elongate by large factors in a small region of space. Surface charge density continues to increase as the jet approaches the collector, resulting in a second bending perturbation and subsequent bending instability within the first coil. Smaller diameter bending instabilities form in this manner until the jet strikes the collector, or solidifies into a fiber. Without the large amount of elongation in a small space that takes place because of the bending instabilities, kinetic energy would be required to keep the leading sections of the jet ahead of the following sections. Instead, the electrical energy is used by the jet to elongate, decrease in diameter, and increase in surface area per unit mass of solution (Figure 13) [109]. 


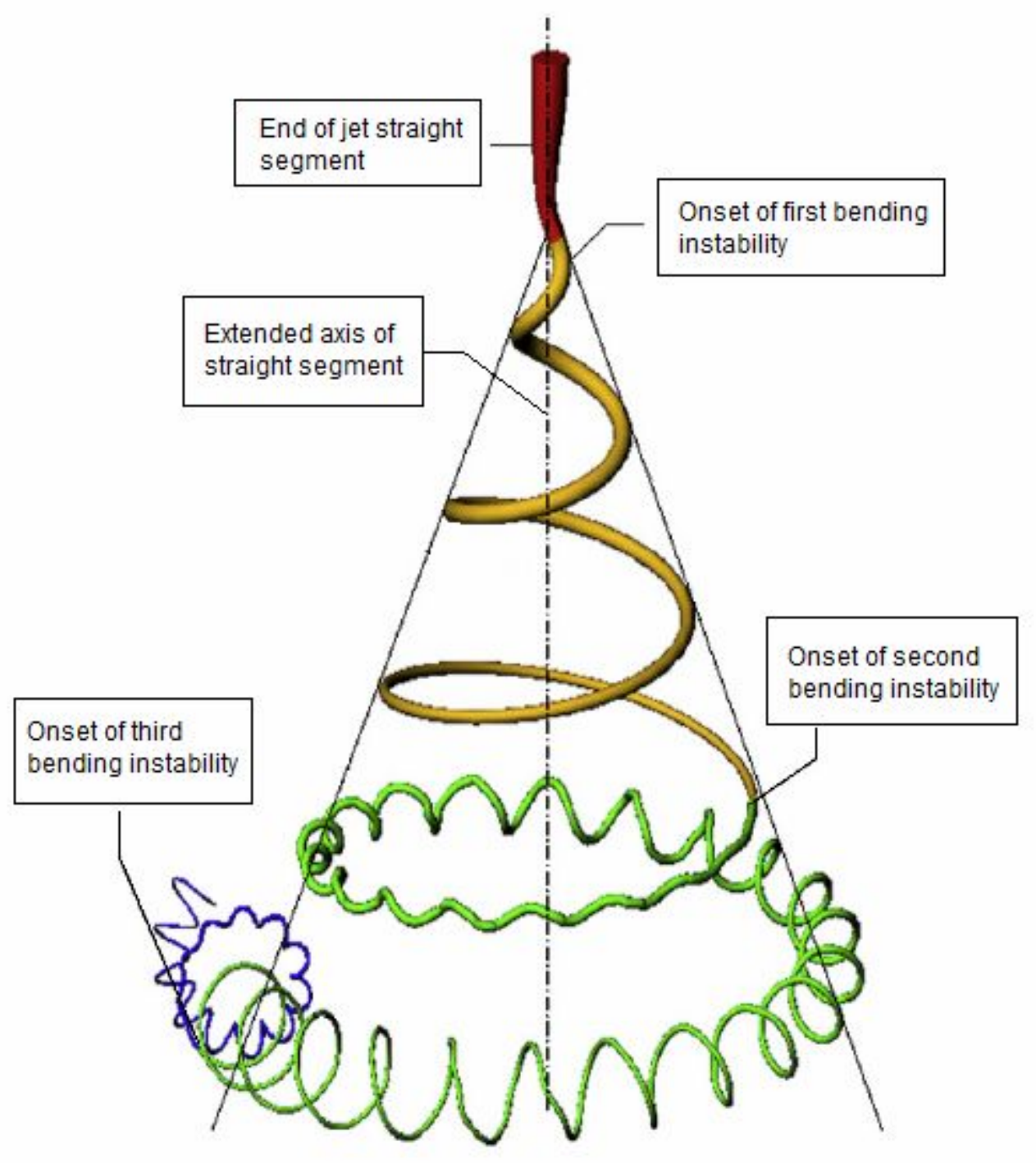

Figure 13 - The path of an electrospinning jet is complicated, but not random. The first bending instability coils outward, forming a conical flow path. The second bending instability follows the coiled path of the first, coiling around its instantaneous path of travel. The third bending instability forms in the same manner, coiling around the path of travel of the second bending instability, and also outward with the trajectory of the first bending instability. [Fig. 6 from Reneker, D.H. et al., "Electrospinning jets and polymer nanofibers." 2008 [78]] 
Magnitude of the Applied Potential

As stated previously, at a low applied potential difference, surface charges and surface tension on a droplet act against each other, distorting the shape of the droplet into the characteristic Taylor cone. Above a critical voltage, surface tension is overcome and a jet forms. With a low viscosity solution, the jet breaks apart into micro droplets due to surface tension, resulting in the process of electrospraying. If the solution has a sufficiently high viscosity, the jet does not break apart and electrospinning results [110].

When electrospinning voltage is varied, but all other variables are kept constant, the surface charge density on the polymer jet changes, which affects the final morphology of the electrospun fibers. Deitzel et al. found that above a certain potential difference between the needle and collector, bead defects began to form rapidly [111]. This is attributed to the capillary instability of polymer jets; surface tension within the cylindrical jet drives the formation of spheres, which have less surface free energy per unit volume than cylinders. Excess surface charges carried with the jet create a strong elongational flow, and may either stabilize or destabilize the capillary instability [112]. This means either increasing or decreasing the magnitude of the applied voltage can decrease bead defect density, depending on the electrospinning system. Bead defects are repeated at intervals, and the fibers that connect adjacent beads decrease in diameter as the bead density increases (Figure 14). 

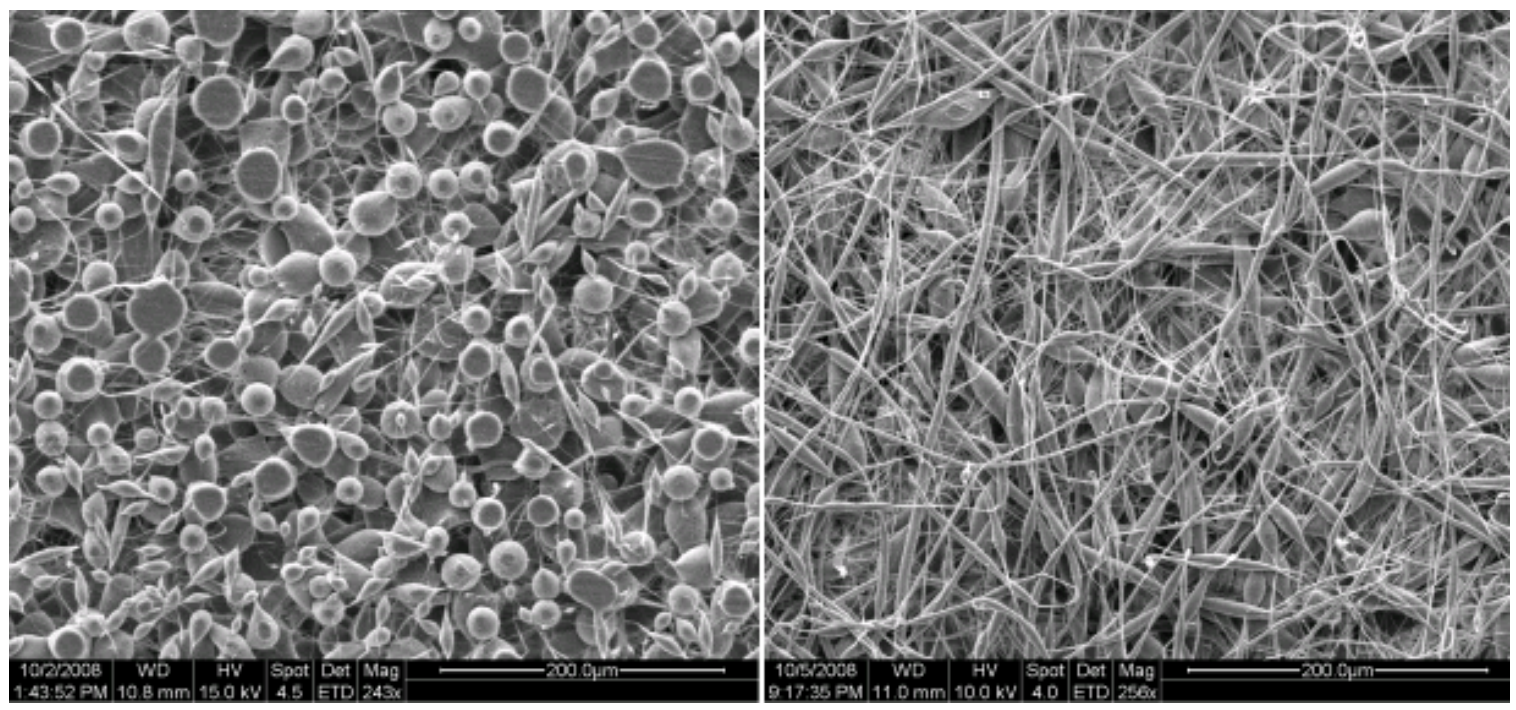

Figure 14 - Capillary instabilities drove the formation of a bead-dominated electrospun fiber mat with small diameter connecting fibers (left, 243X). Increasing the applied voltage decreased the bead defect density and increased the diameter of the connecting fibers (right, 256X).

Polymer Solution Concentration

Surface tension and viscosity are directly related to polymer solution concentration (Figure 15). This means solution concentration has a significant influence on the electrospinning process. Low viscosity solutions show surface tension as the dominating factor, and concentrations below a threshold value will result in drops instead of fibers. Higher concentrations that result in solutions with high viscosities can cause processing problems; polymer solution flow to the needle tip becomes difficult to control, and the cohesive nature of the viscoelastic solution resists jet elongation [111].

There is a range of concentrations that produces solutions with appropriate viscosity for fiber formation and easy processing. Variation of solution concentration 
within this range causes variation in final fiber morphology. A power law relationship exists between fiber diameter and solution concentration, with higher concentrations correlating to larger fiber diameters $[101,111]$. This is because higher solution concentration leads to higher viscosity, which resists jet elongation and thinning. The exact exponent of the power relationship depends on the polymer/solvent system used in the electrospinning process [109].

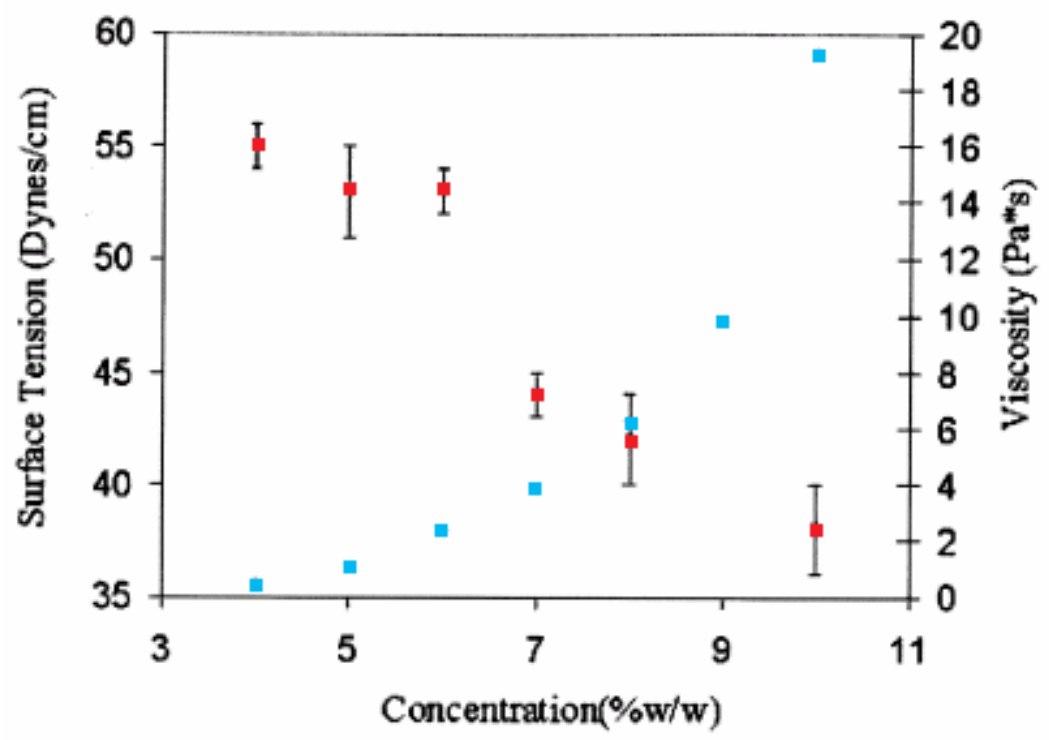

Figure 15 - Increasing polymer concentration causes a simultaneous decrease in surface tension (red) and increase in viscosity (blue). [Fig. 6 from Deitzel et al., "The effect of processing variables on the morphology of electrospun nanofibers and textiles." 2001 


\section{Flow Rate Dependency}

Volumetric flow of polymer solution into a charged Taylor cone affects the electrospinning process in two ways. First, the rate must be tuned so that a stable Taylor cone is formed. A low flow rate results in the formation of a vacuum in the needle, causing the Taylor cone to disappear and temporarily stop the electrospinning process. Conversely, high flow rates cause build up of solution at the needle tip. Second, as flow rate increases, surface charge density decreases. The rate of charge withdrawl into the solution is dependent upon the residence time of ions in contact with the needle, and at higher flow rates solution spends less time in contact with the needle [113]. Since surface charge density is the driving force behind electrospinning, and flow rate directly affects surface charge density, flow rate is an important parameter in the process.

\section{Gap Distance}

Thompson et al. showed that distance from the charged Taylor cone to the collector and final fiber diameter follow a negative power relationship [114]. The increase in distance allowed bending instabilities and whipping action to elongate and decrease the diameter of the polymer jet. However, gap distances that are too great have negative results. Gap distance shares a negative exponential relationship with surface charge density, so when gap distance increases, surface charge density drops [113]. As distance between the charged solution and collector increases, the magnitude of the electric field between the two decreases, resulting in the formation of fewer charged ions. 
This can cause the jet to fail to collect on the grounded collector, or introduce bead defects into collected fibers.

\section{The Electrospinning Apparatus}

Following selection of electrospinning as the best technique to pursue in-house fabrication of BVM scaffolding, the next aim of this work was to implement the new method. Previously, to the best of our knowledge, no electrospinning systems or research had been pursued on the Cal Poly campus. An electrospinning device was constructed piece-wise from components supplied by various companies. The final configured system is illustrated below in Figure 16. The following sections describe the function of each component. 


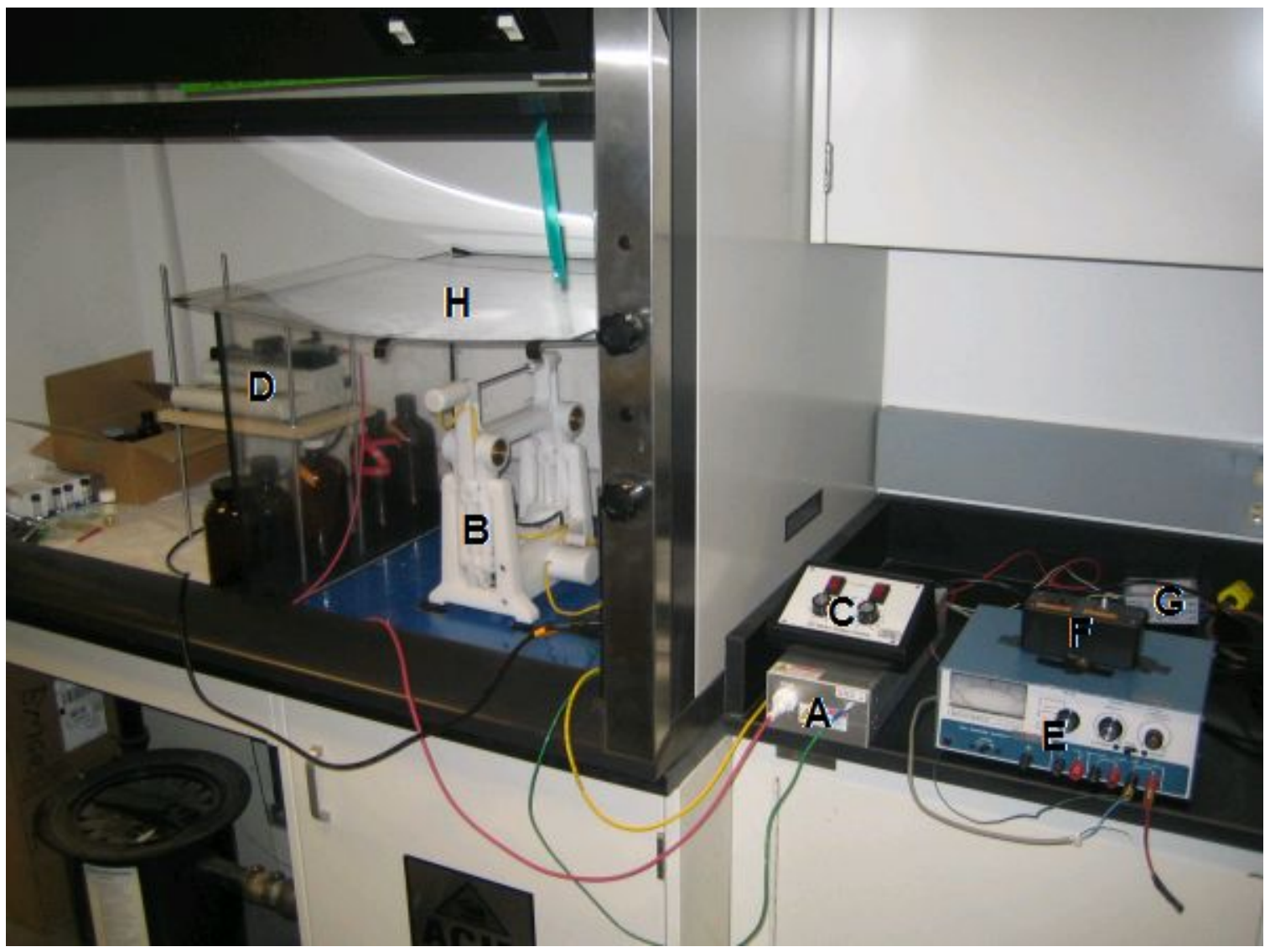

Figure 16 - The electrospinning apparatus incorporates a high voltage power supply (A), a rotating/translating mandrel collection system (B) with dual speed control box (C), a syringe pump for polymer solution infusion (D), an external power supply (E), a dual switch array $(\mathrm{F})$, an $\mathrm{AC} / \mathrm{DC}$ power converter, and an isolation chamber $(\mathrm{H})$.

High Voltage Power Supply

The driving force behind the electrospinning phenomenon is a high potential difference between a polymer solution and a collection system. A K7-30R high voltage power supply, from Matsusada Precision Inc., provided this driving force. The K7-30R is capable of output voltages from 0 to $30,000 \mathrm{~V}$ at a current of $250 \mu \mathrm{A}$ with a ripple of only $150 \mathrm{mV}$. The output voltage is controlled via an external power supply amplifier 
ranging from 0 to $10 \mathrm{~V}$, which will be explained later. The polarity of the K7-30R is reversible, however the feature was unnecessary for the application presented in this thesis.

Fiber Collection System

To produce tubular geometries of electrospun fibers, a rotating/translating mandrel collection system is used. Machined and assembled by Custom Design and Fabrication (Richmond, Virginia), the collector has speed controls for rotation and translation of the metallic mandrel where the fibers are collected, as well as adjustable pitch for the swing arms (Figure 17). By rotating continuously in one direction and translating back forth over a set distance, the electrospun fiber builds up a tubular geometry on the mandrel.

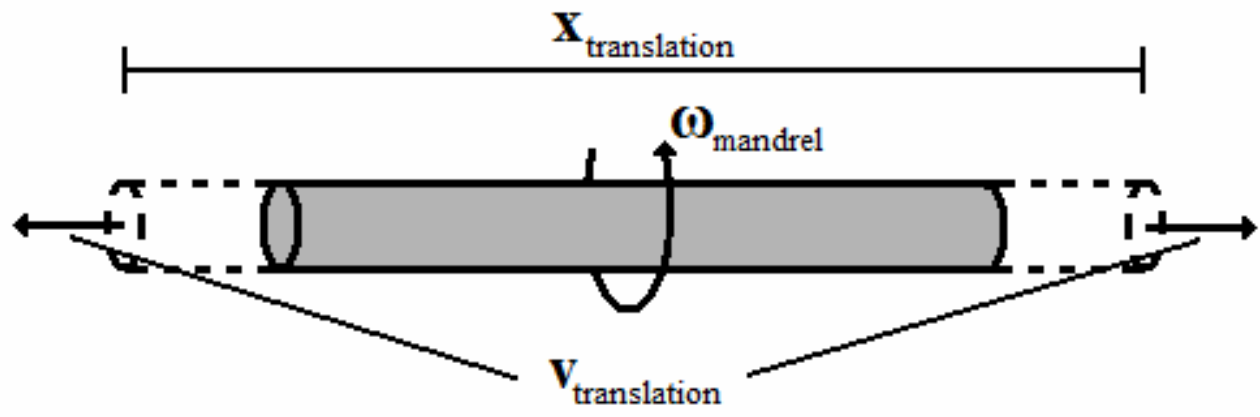

Figure 17 - Mandrel angular velocity $\left(\omega_{\text {mandrel }}\right)$ and translational velocity $\left(\mathrm{v}_{\text {translation }}\right)$ were adjusted on the speed control box. The translation distance $\left(\mathrm{x}_{\text {translation }}\right)$ was set on a swing arm wheel attached to the collector. 


\section{Polymer Infusion System}

High precision infusion of polymer into the electrospinning apparatus is performed with a KD Scientific Model 100 syringe pump, syringes, and metallic needles. The lead from the high voltage power source is attached to the needle while ground is connected to the collector (Figure 18). As the polymer solution is pushed through the needle by the syringe pump at a controlled volumetric rate, the high potential difference between the needle and collector causes ion formation in the polymer solution, leading to the formation of a Taylor cone and subsequent polymer jet, as described earlier. 


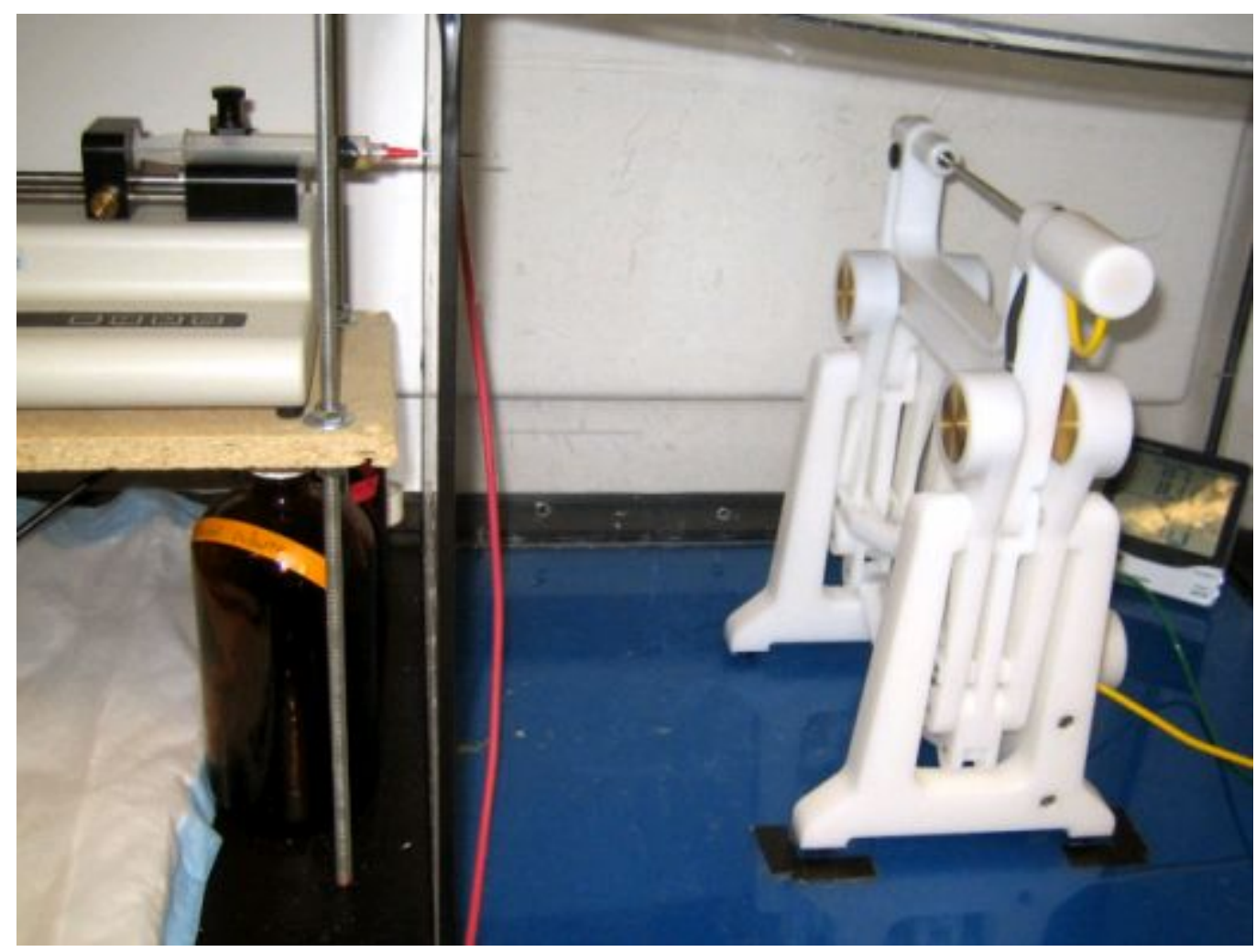

Figure 18 - Polymer solution was injected through the needle from the syringe by force from the syringe pump (left). The high voltage and grounding leads (red and green wires, respectively) from the high voltage power supply provided the electrostatic force necessary for electrospinning to occur.

External Power Supply

The K7-30R high voltage power supply requires a separate DC power supply with a 1 to $10 \mathrm{~V}$ range to act as an amplifier for high voltage output. The amplifying factor is 3000. This means a setting of $3 \mathrm{~V}$ on the external power supply correlates to a high voltage output of 9,000 V. A Heathkit IP-2718 DC power supply provides the amplification. 


\section{Electrical Supply}

Power from the laboratory where the electrospinner is housed comes from a wall receptacle at around $110 \mathrm{~V}_{\mathrm{AC}}$. Since the K7-30R high voltage power supply requires 24 $\mathrm{V}_{\mathrm{DC}}$ at $1.3 \mathrm{~A}$, a Kele PS5R-SC AC/DC transformer is used to convert the power. A two switch array was also built to control power input into the high voltage power supply. One switch provides the $\mathrm{K} 7-30 \mathrm{R}$ with $24 \mathrm{~V}_{\mathrm{DC}}$ at $1.3 \mathrm{~A}$, and the other starts the high voltage output.

\section{Containment Chamber}

Electrospun fibers have a tendency to settle not only on the collection surface, but also on any other surrounding surfaces because the fiber motion during bending instabilities is erratic. To prevent material build up on the surrounding surfaces in the fume hood, a containment chamber was constructed using $1 / 8$ " and $1 / 4$ " thick transparent acrylic sheets. The collector sits within the containment chamber, and the necessary wires pass through a small opening cut out of the corner of the front panel. The front panel is attached only with adhesive tape, making it removable to allow access to the collector. A small hole was drilled in the left wall panel to slide the needle through and into the chamber. Four 1/4" diameter holes were drilled into the top of the back panel to allow solvent vapor to escape the containment chamber. The entire chamber is placed in a fume hood to safely remove solvent vapor from the electrospinning process. 
Following successful construction of the electrospinning apparatus, the next step of this project was to use the system to create tubular polymer scaffolds. 


\section{ELECTROSPINNING TRIAL EXPERIMENTS}

\section{Introduction}

Chapter 2 explained how electrospinning may be an appropriate scaffold fabrication technique for use in the BVM. The aim of the work in this chapter was to use the newly constructed electrospinning system to create polymer tubes through a series of Spin experiments. The Spin experiments presented in this chapter served two important functions. First, these experiments provided experience with and understanding of the inhouse electrospinning apparatus. This included getting familiar with the various process parameters and how they affected the electrospun cylindrical constructs. Second, the Spin series was used to determine an electrospinning protocol for a particular polymer, $\mathrm{P}(\mathrm{LLA}-\mathrm{CL})$, that resulted in continuous, uniform fibers with diameters as close to the nanoscale as possible. The resulting protocol was implemented for consistency studies that will be described in the next chapter.

\section{Methods and Results}

Methods and results from each Spin will be presented in the following manner; the purpose behind the experiment is explained, process parameters are specified, observations made during the process are given, microstructural construct images are included, and an explanation behind the resulting structure is provided. Polymer solutions for all experiments in the Spin series varied in concentration, but the process for mixing and using it followed the Solution Mixing Protocol (see Appendix B). The 
selected polymer in all cases was P(LLA-CL) with a monomer to monomer molar ratio of 90:10 (L-lactic acid to caprolactone) obtained from Lakeshore Biomaterials

(Birmingham, Alabama). This particular polymer was selected due to advice from Dr. Gene Boland (Tissue Genesis, Inc., Honolulu, HI; currently at Cardiovascular Innovation Institute, Louisville, KY) asserting that P(LLA-CL) was simple to electrospin and degradation rates could be tailored by changing the monomer to monomer molar ratio. The selected solvent in all cases was chloroform (99.8\% min., BDH), also recommended by Dr. Boland based on its effectiveness and low cost. All constructs were spun (see Appendix C), immediately removed from the collector, allowed to sit in a desiccator for 24 hours, sputter coated with gold, and examined using a scanning electron microscope (SEM). The interior surface (surface that contacted the mandrel collector) of all constructs was examined, and some constructs had other surfaces (exterior or crosssectional) examined as well. All SEM pictures were taken at random locations on the indicated scaffold surfaces.

Probable explanations are presented for the variations noticed between spins, but flawless theoretical explanation was not the purpose of the Spin series. It is simply worthwhile to use knowledge gained from literature to try to explain the science behind changes in morphology. 


\section{Spin \#1}

Purpose:

These initial process parameters were recommended by Dr. Gene Boland.

SOLUTION:

FLOW RATE:

APPLIED VOLTAGE:

GAP DISTANCE:
$5.3 \%$ by weight $\mathrm{P}(\mathrm{LLA}-\mathrm{CL})$ in chloroform

$6 \mathrm{ml} / \mathrm{hr}$ (3 $\mathrm{ml}$ of solution used) through $18 \mathrm{G}$ needle $16.5 \mathrm{kV}$

10 in

\section{Process Observations:}

The solution droplet originating at the needle tip grew over time until it was a $1 \mathrm{~cm}$ long cylindrical shaped mass hanging vertically from the needle tip.

\section{SEM Imaging:}

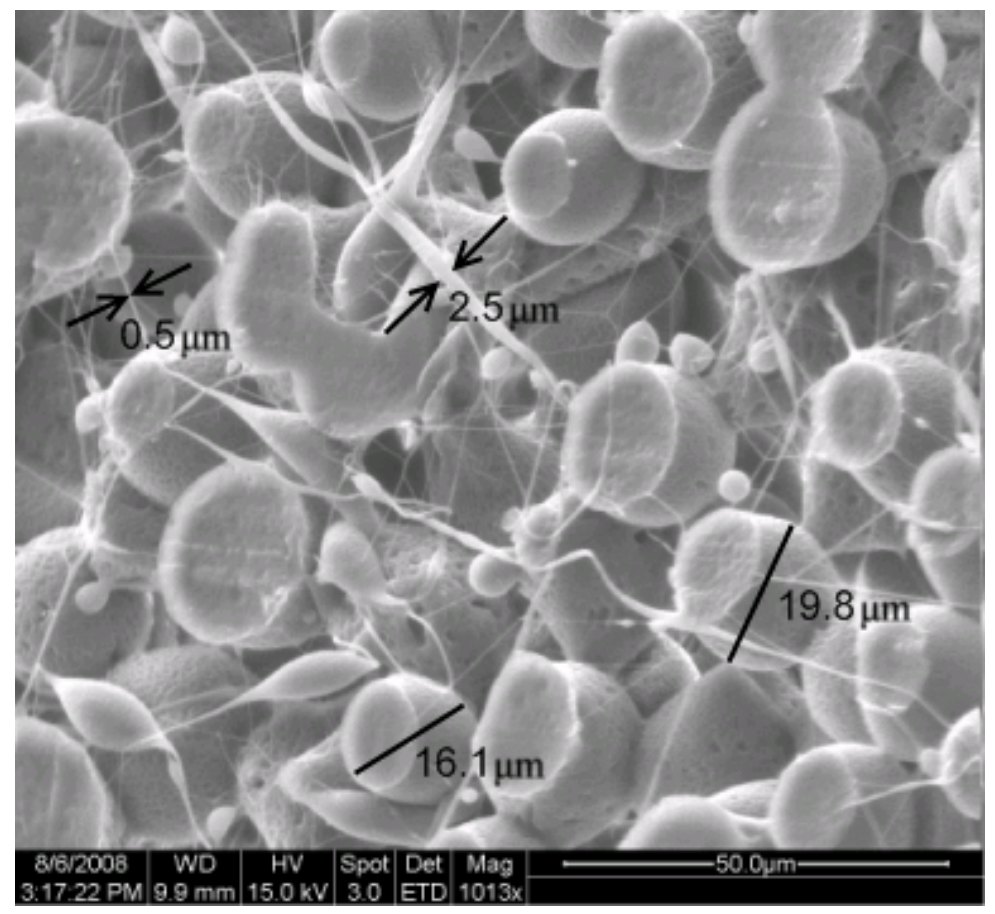

Figure 19 - The interior surface of the cylindrical scaffold from Spin \#1 shows beads that are 15 to $20 \mu \mathrm{m}$ in diameter (black lines) and fibers that connect the beads with diameters of hundreds of nanometers to several microns (black arrows). (1013x) 
Spin \#1 resulted in bead-on-fiber defects with a large ratio of polymer contained in the beads. As shown in Figure 19, the fiber diameters ranged in size from hundreds of nanometers to several microns. Spherical bead diameters were an order of magnitude larger than fiber diameters. Some of the beads flattened, which likely resulted from insufficient solvent evaporation upon striking the collector.

\section{Spin \#2}

Purpose:

This experiment looked at the effects of decreasing gap distance on the electrospinning product. The decrease in gap distance should have increased the electric field strength between needle tip and collector, and also restricted the space available for polymer jet bending instability formation.

SOLUTION: $\quad 5.3 \%$ by weight P(LLA-CL) in chloroform FLOW RATE: $\quad 6 \mathrm{ml} / \mathrm{hr}$ (3 ml of solution used) through $18 \mathrm{G}$ needle APPLIED VOLTAGE: $\quad 16.5 \mathrm{kV}$ GAP DISTANCE: $\quad 5$ in

\section{Process Observations:}

After $1.6 \mathrm{ml}$ of solution had been spun, the droplet had formed a partially solidified polymer mass stretching the needle tip to within 1 in of the collector. The process was stopped, the mass was removed, and then the process was restarted.

The stretched mass returned after $2.3 \mathrm{ml}$ of solution had been spun, but the process was not stopped. 
The Taylor cone had moved away from the needle tip by approximately $1 \mathrm{~cm}$ by the end of the process.

Cross-sectional thickness of the product from Spin \#2 was greater than Spin \#1.

\section{SEM Imaging:}

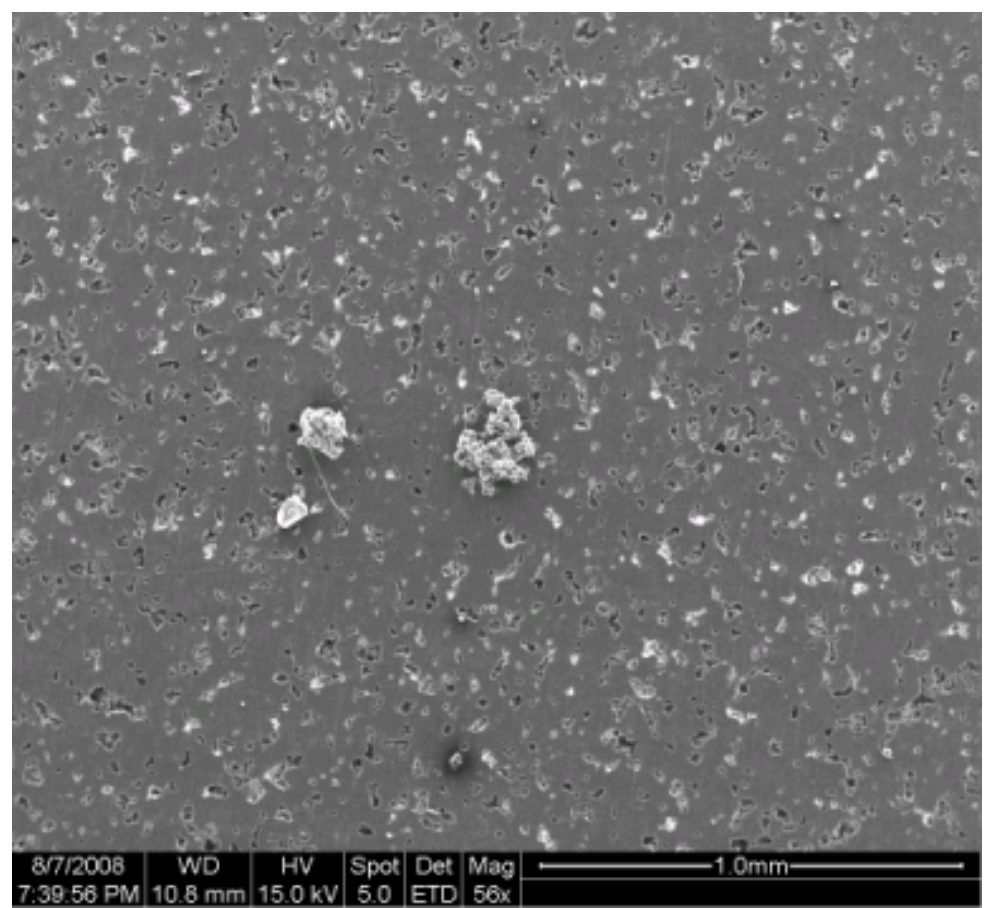

Figure 20 - The interior surface of Spin \#2 appeared to be a fused, solid surface with very little porosity and no visible fibers. (56x) 


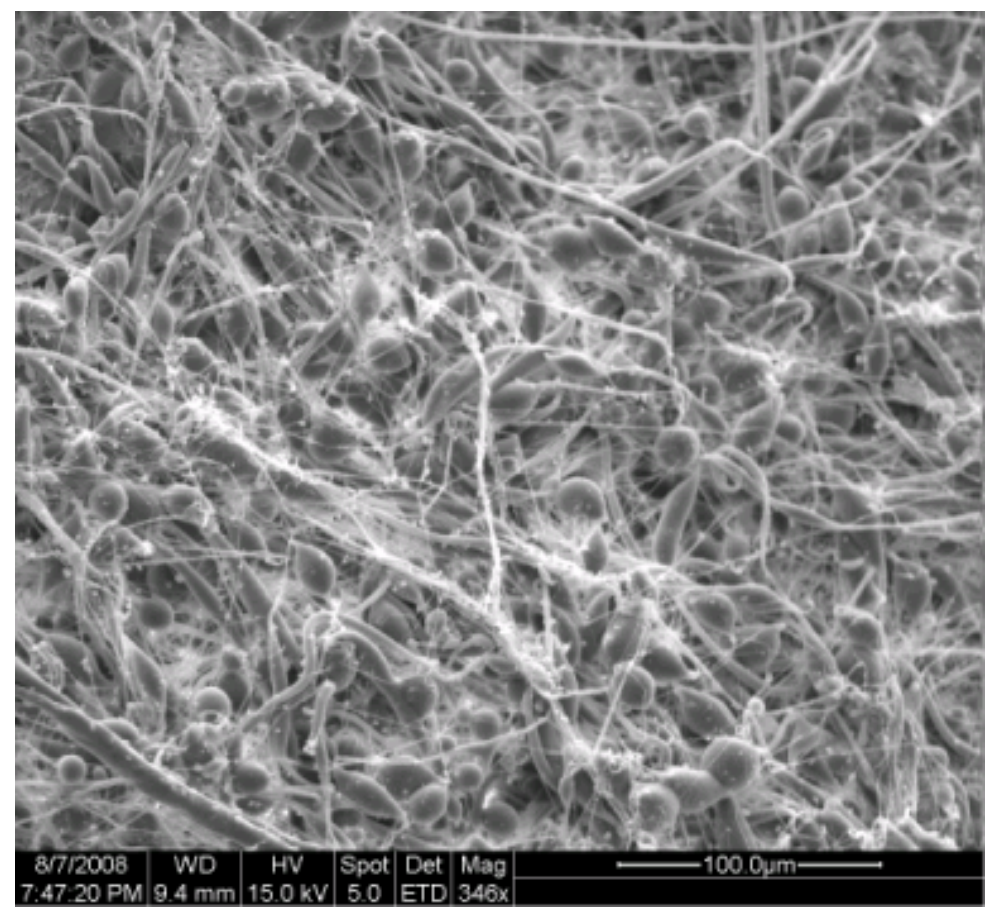

Figure 21 - The exterior surface of Spin \#2 was comprised of a bead-on-fiber defect morphology. (346x)

Looking at the interior surface of Spin \#2 (Figure 20), the nearly flat surface could have been caused by electrosprayed droplets fusing together on the collector surface, indicating an incorrect accelerating voltage or low solution concentration. The flat, fused surface could also have been caused by electrospinning of either uniform fibers or bead-on-fiber fibers that did not reach the bending instability phase that causes extensive solvent evaporation, thus resulting in viscous fibers that fused together on contacting the collector surface.

The outside surface of Spin \#3 (Figure 21), however, shows the same bead-onfiber morphology as Spin \#1 (Figure 19). This is strange behavior, but may be explained by the movement of the Taylor cone away from the needle tip. Since the solution had to 
travel $1 \mathrm{~cm}$ in open air before erupting through the Taylor cone, evaporation of the solvent could have caused an increase in solution concentration. If the process started out as electrospraying, an increase in solution concentration would explain why the process ended as electrospinning. If the process was electrospinning throughout, an unintended change in concentration could have changed the distance the jet had to travel before the start of bending instabilities, resulting in solidified fibers that did not fuse together.

For electrospun BVM scaffolding, the interior surface should be composed of continuous fibers free of bead defects [89]. Further Spin experiments attempted to fabricate continuous fiber scaffolds.

\section{Spin \#3}

Purpose:

The gap distance of Spin \#3 was changed to 15 in to examine the effects of increased gap distance. This should have weakened the electric field while allowing more room for bending instabilities to form.

SOLUTION: FLOW RATE: APPLIED VOLTAGE: GAP DISTANCE:

\section{Process Observations:}

The Taylor cone stayed close to the needle tip the entire time, but a silken thread of polymer 1 in long formed and was hanging from the needle tip by the end of the process. 
There was noticeable build up on the containment chamber walls.

Cross-sectional thickness of this spin was less than Spin \#1.

\section{SEM Imaging:}

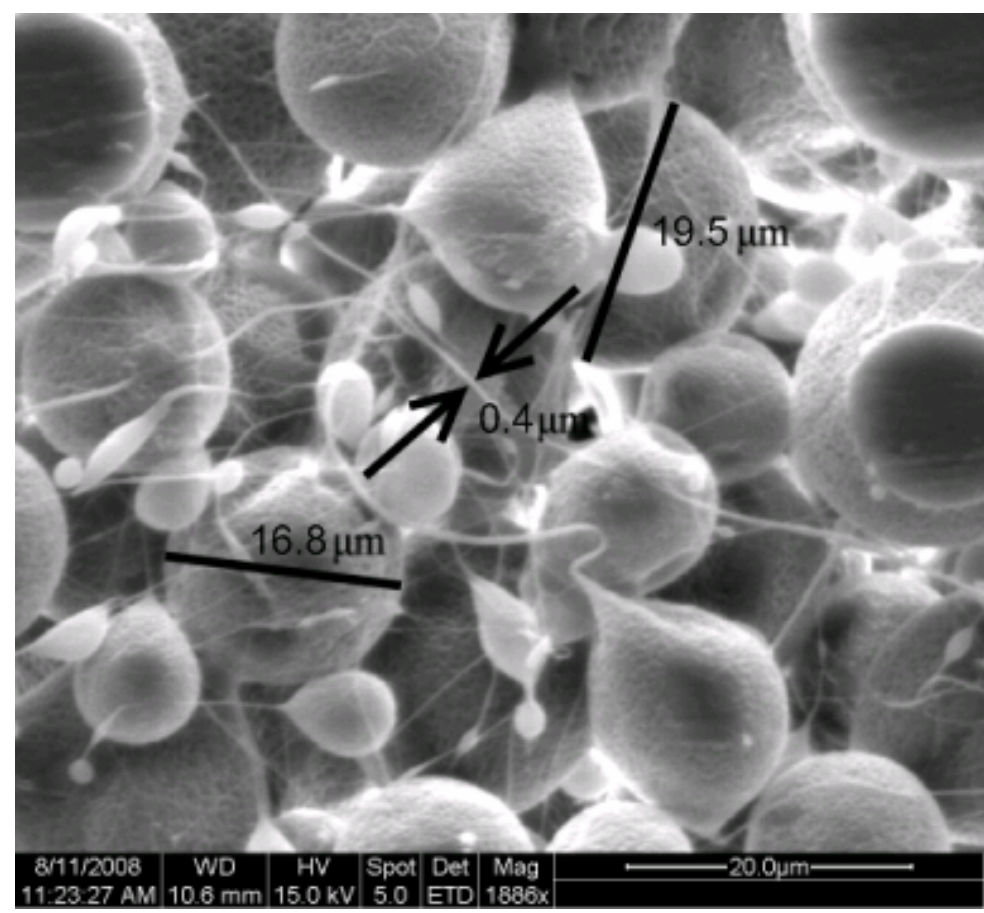

Figure 22 - The interior surface of Spin \#3 shows a bead-on-fiber morphology with bead diameters (black lines) similar to those of Spin \#1 (Figure 19). Fiber diameters do not appear to rise above 1 micron. (1886x) 


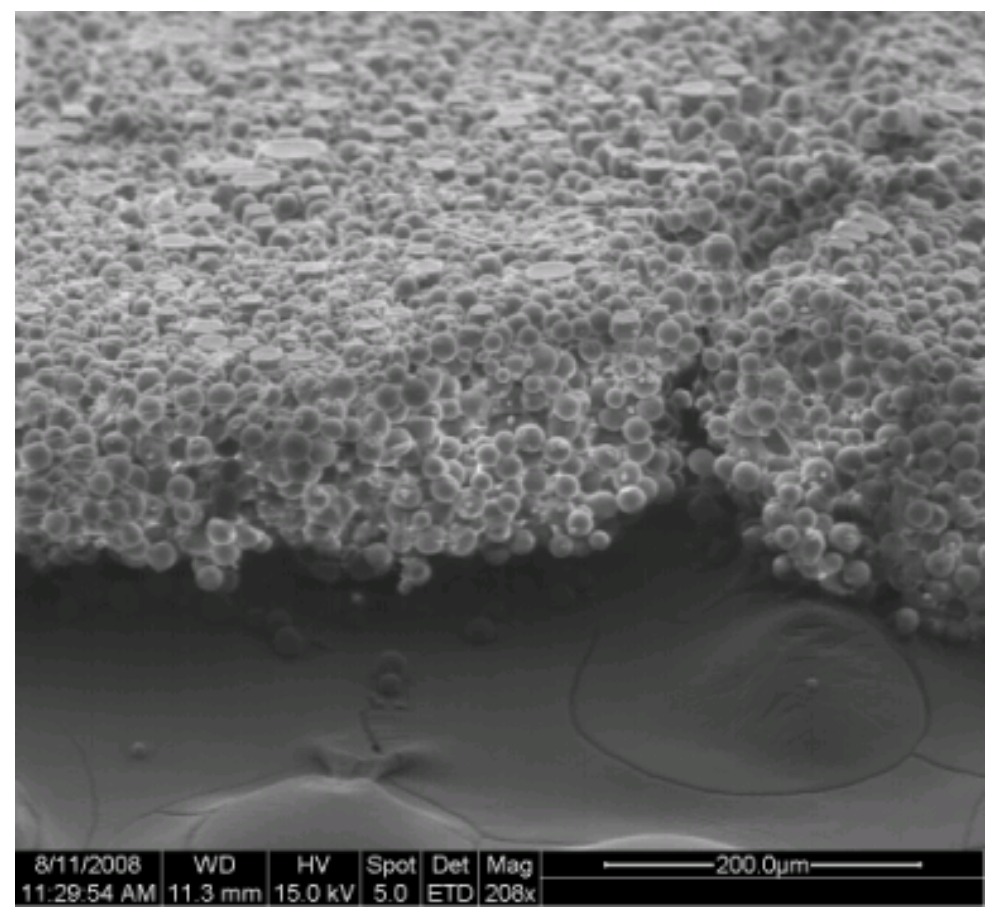

Figure 23 - A cross-sectional view of Spin \#3 shows a structure formed predominately of beads.

The interior surface of Spin \#3 (Figure 22) showed fewer flattened beads than Spin \#1, but the bead-on-fiber morphology remained the same. The bead connecting fibers may have slightly smaller diameters, indicating continued elongation made possible by the increase in gap distance. The reduction in the appearance of flattened beads is probably due to the extra distance the jet had to travel. Most of the beads had enough time to have the solvent completely diffuse out. Only the larger beads deformed upon impact with the collector because not all the solvent was able to diffuse out.

A cross-sectional examination of this spin (Figure 23) reveals a consistent structure throughout, as opposed to the drastic difference seen in the structure of Spin \#2. This may be explained by the location of the Taylor cone. Since the Taylor cone stayed 
close to the needle tip, the solution concentration stayed constant, resulting in a uniform structure. The cross-sectional thickness was also less, indicating that less solution ended up actually striking the collector. Akin to this, the walls of the containment chamber had noticeable build up on them. Two explanations for the build up on the chamber walls are probable. First, the decrease in the electric field may explain this; surface charge causes jet elongation and bending instability, but the electric field attracts the jet towards the collector. A weaker field may have caused the jet to stray from the needle-to-collector path and strike other surfaces, including the containment chamber walls. Second, the deviation of the jet from the straight line path from needle tip to collector caused by the expanding radius of the conical bending instabilities may have increased to the point that the jet struck the chamber walls before reaching the collector.

The formation of silken threads on the needle tip were probably the result of the electrospinning process stopping and starting intermittently as a consequence of the increased gap distance. The extra distance the jet had to travel might have allowed more opportunities for the jet to break apart between the beads, stopping the electrospinning process for a short time. Silken fibers would be the remnants of broken jets.

\section{Spin \#4}

\section{Purpose:}

Generally, decreasing the applied voltage should decrease the surface charge density on the polymer jet, decreasing elongational flow and affecting capillary instability in either a positive (decrease in the occurrence of beads) or negative (increase in the 
occurrence of beads) manner. The purpose of this experiment was to decrease the applied voltage to observe if bead defect density increased or decreased. The needle was also changed to a smaller internal diameter for the remainder of the Spins.

SOLUTION:

FLOW RATE:

APPLIED VOLTAGE: GAP DISTANCE:
$5.3 \%$ by weight $\mathrm{P}(\mathrm{LLA}-\mathrm{CL})$ in chloroform

$6 \mathrm{ml} / \mathrm{hr}$ ( $3 \mathrm{ml}$ of solution used) through $27 \mathrm{G}$ needle

$13 \mathrm{kV}$

10 in

\section{Process Observations:}

After $2.0 \mathrm{ml}$ of solution had been spun, the polymer mass had formed again and the Taylor cone and jet were based at the tip of the mass. The needle was not cleaned.

\section{SEM Imaging:}

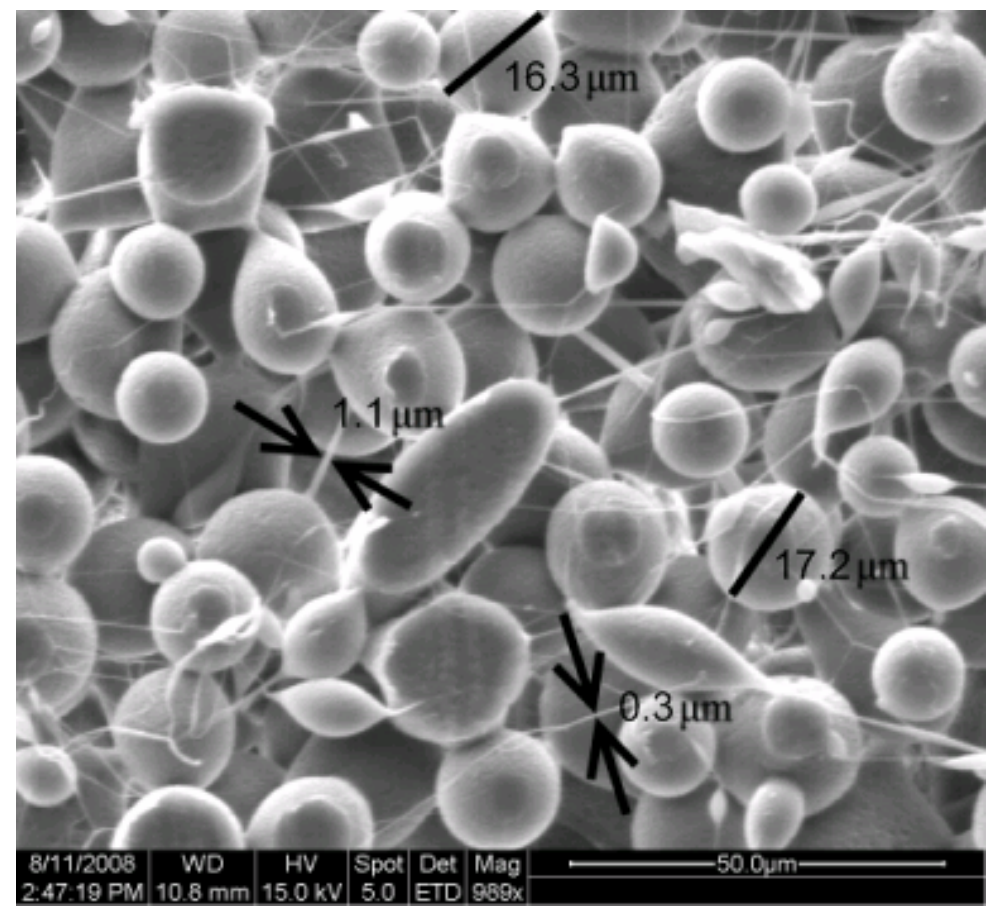

Figure 24 - This interior surface image of Spin \#4 shows a bead-on-fiber morphology similar to the interior surface of Spin \#1 (Figure 19). Black lines show designate the diameter of selected beads, while black arrows mark the diameter of selected fibers.

$(989 x)$ 


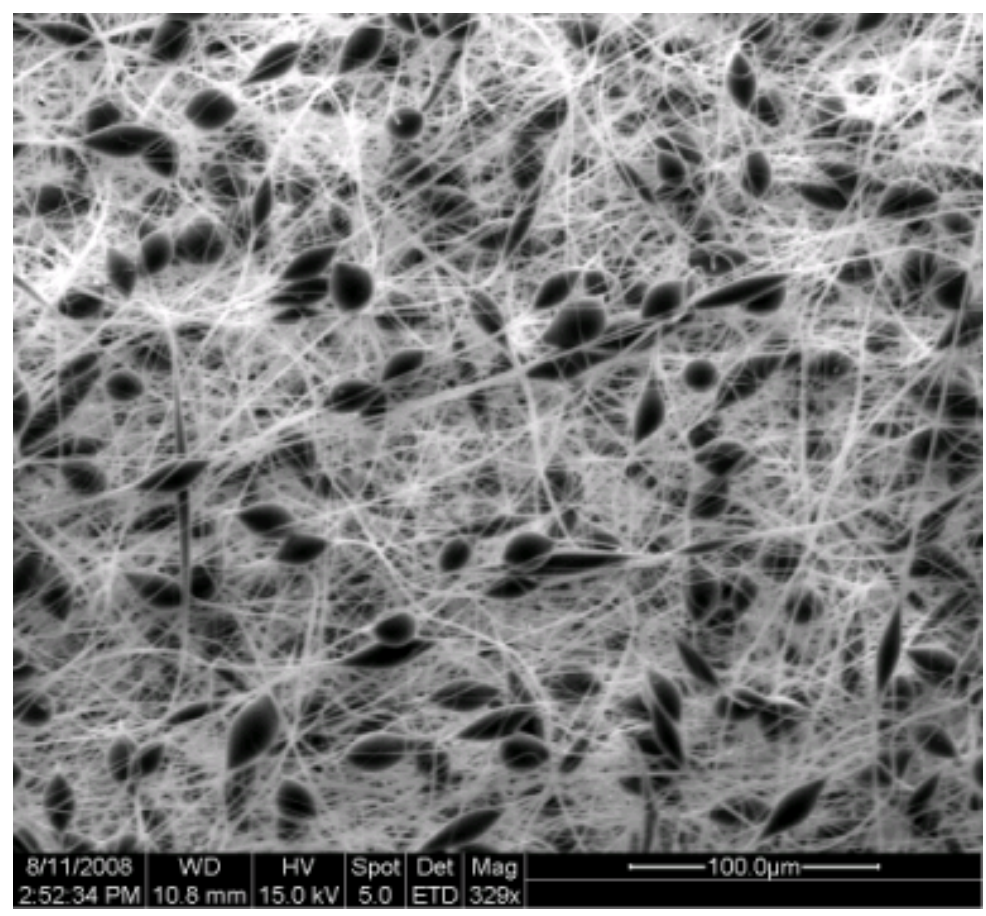

Figure 25 - Bead defect density significantly decreased on the exterior surface of Spin \#4 compared to the interior surface (Figure 24) as shown above. This structure was observed on the exterior of Spin \#2 as well (Figure 21). (329x)

The main purpose of Spin \#4 was to test the effects of decreasing the applied voltage, but since the needle size was changed from $18 \mathrm{G}$ to $27 \mathrm{G}$ as well, the results are mostly inconclusive. The smaller needle diameter was used in subsequent spins, so more conclusions can be made from later experiments.

The lumen of this construct looks similar to that of Spin \#1, with a large amount of polymer contained in bead defects connected by small diameter fibers (Figure 24). During the electrospinning process, the Taylor cone moved away from the needle tip, 
which may explain the fiber dominated exterior surface of the construct (Figure 25) in the same manner as Spin \#2 (Figure 21).

\section{Spin \#5}

\section{Purpose:}

This experiment attempted to increase the applied voltage to observe the effects on the final morphology of the construct.

SOLUTION:

FLOW RATE:

APPLIED VOLTAGE:

GAP DISTANCE:

\section{Process Observations:}

Shortly after the process was started the external amplifying power supply started making a clicking noise. The process was stopped immediately.

The external amplifying power supply was examined to discover what malfunctioned. The fuse was not blown, but the output would not change from $30 \mathrm{~V}$ (the max output of the power supply). This means the voltage regulating device within the power supply was damaged. An alternate external amplifying source would need to be obtained.
$5.3 \%$ by weight $\mathrm{P}$ (LLA-CL) in chloroform

$6 \mathrm{ml} / \mathrm{hr}$ ( $3 \mathrm{ml}$ of solution used) through $27 \mathrm{G}$ needle $25 \mathrm{kV}$

10 in 


\section{Spin \#6}

Purpose:

A temporary external amplifying power source was located that provided a set voltage of $5 \mathrm{~V}$ at $1.5 \mathrm{~A}$, equating to a high output voltage of $15 \mathrm{kV}$. Until another variable external power supply could be located, the series of experiments looking at varying applied voltage was put on hold. Spin \#6 experiment looked at the effect of increasing the solution concentration.

SOLUTION: FLOW RATE: APPLIED VOLTAGE: GAP DISTANCE:

Process Observations:
$7.8 \%$ by weight $P(L L A-C L)$ in chloroform $6 \mathrm{ml} / \mathrm{hr}$ ( $3 \mathrm{ml}$ of solution used) through 27 gauge needle $15 \mathrm{kV}$ (channel set at $5 \mathrm{~V}, 1.5 \mathrm{~A}$ ) 10 in

The droplet grew downward as the process continued until it reached $2 \mathrm{~cm}$ long, at which time $3 \mathrm{ml}$ of solution had been used and the process ended. The Taylor cone was located near the needle tip during the entire process. 
SEM Imaging:

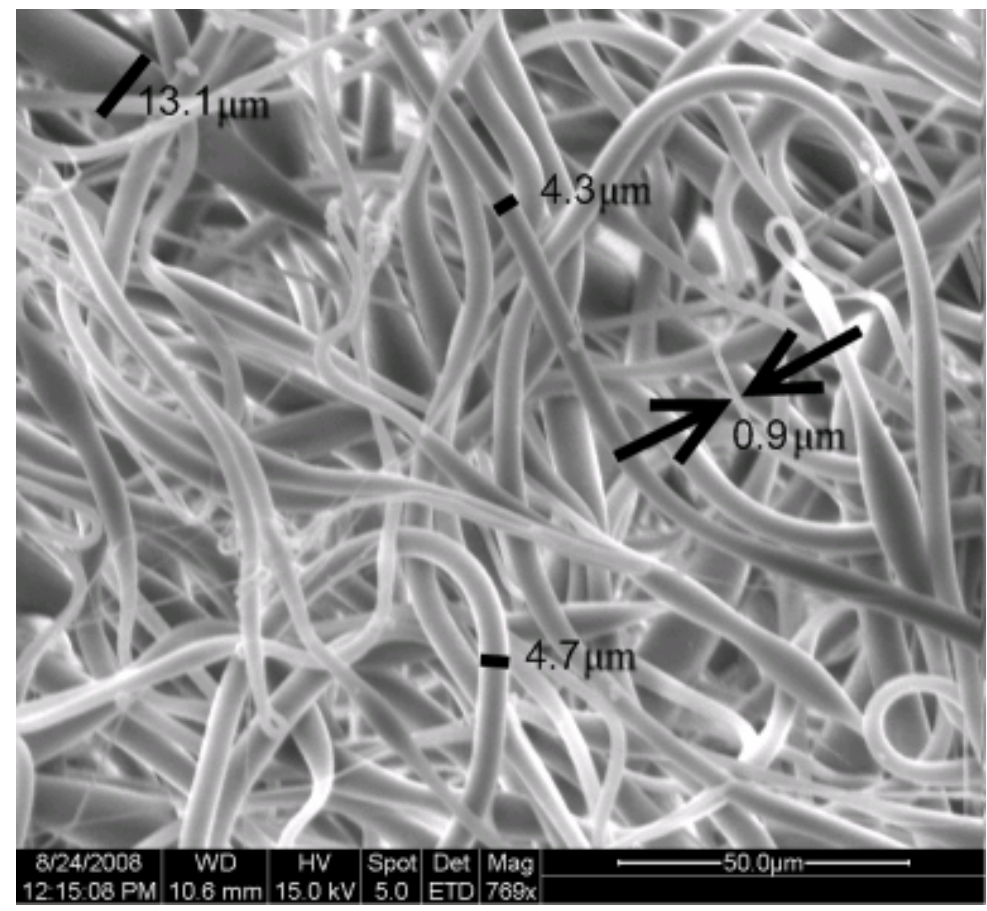

Figure 26 - Continuous fibers were present on the interior surface of Spin \#6 shown above, but fiber diameter appears to be distributed over a range $(<1$ to $5 \mu \mathrm{m})$ as indicated by the measured fibers (black lines and arrows). Periodic large-diameter sections characteristic of capillary instability are present as well (top left). (769x) 


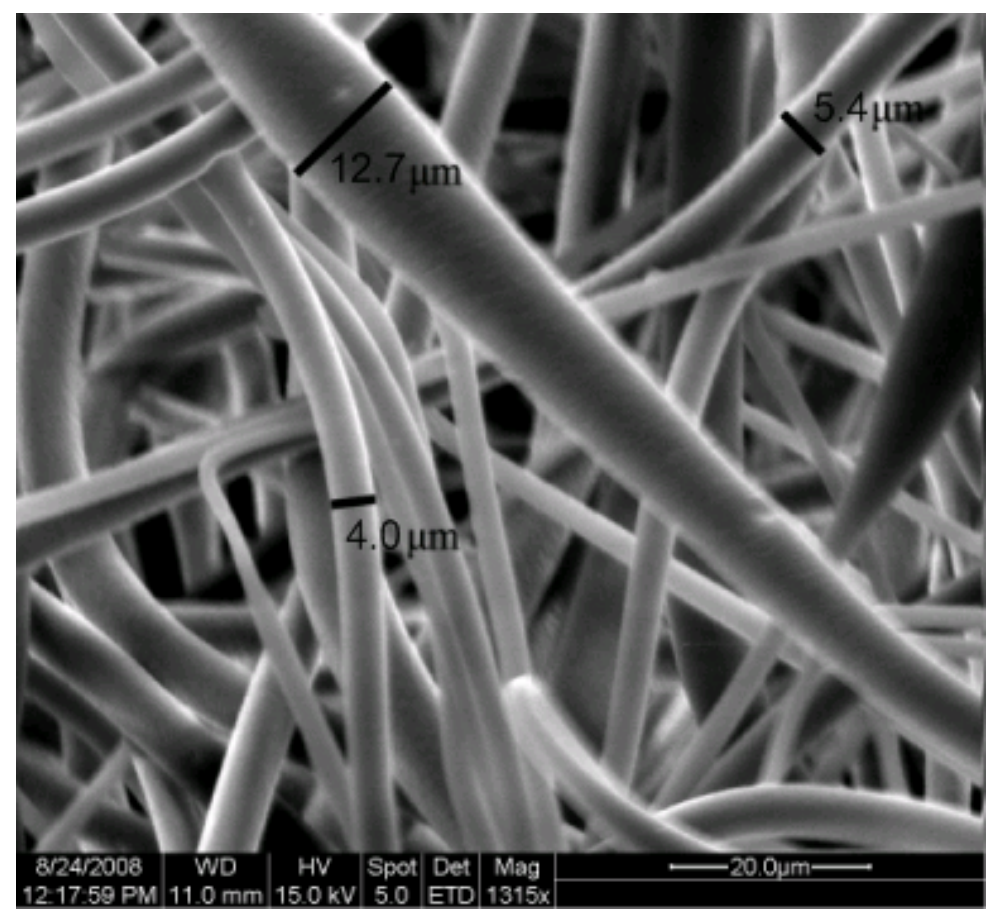

Figure 27 - The exterior surface of Spin \#6 appeared similar to the interior surface (Figure 26), but fiber diameter distribution was tighter. The thick sections characteristic of capillary instability were still present (top middle). The diameters of three random fibers are shown above (black lines). (1315x)

With this experiment, continuous fiber morphology was achieved for the first time. This success was likely due to the increase in solution concentration. The variation in fiber diameter seen on the interior surface (Figure 26) might have been due to jet behavior at start-up, prior to the formation of a completely stable jet. On the exterior surface (Figure 27), fiber diameter is more uniform, indicating that the jet had changed somehow by the end of the process. Both surfaces have periodic larger diameter sections of fiber indicative of capillary instability.

Since the external power source had to be switched, the applied voltage was varied in addition to the solution concentration. But, although inconclusive due to the 
variation in applied voltage, this experiment demonstrated the probable importance of solution concentration on the final structure of the P(LLA-CL) electrospun constructs.

\section{Spin \#7}

Purpose:

Since Spin \#6 resulted in continuous fibers with a small defect presence, the effects of an even higher polymer weight percentage solution were investigated in Spin \#7.

SOLUTION:

FLOW RATE: needle APPLIED VOLTAGE: GAP DISTANCE:
$10.1 \%$ by weight $P(L L A-C L)$ in chloroform

$6 \mathrm{ml} / \mathrm{hr}$ (3 $\mathrm{ml}$ of solution used) through $27 / 18^{*}$ gauge

$15 \mathrm{kV}$ (channel set at $5 \mathrm{~V}, 1.5 \mathrm{~A}$ )

10 in

\section{Process Observations:}

Similar to the previous spin, the droplet grew while the Taylor cone stayed near the needle tip.

The droplet grew faster during this spin.

*After $1.25 \mathrm{ml}$ of solution had been spun, the syringe pump's release switch engaged, indicating the pump could not provide enough force to feed the solution through the needle.

*The 27 gauge needle was replaced with an 18 gauge needle.

The droplet did not grow after the needle was replaced, and the spin finished without complications. 
SEM Imaging:

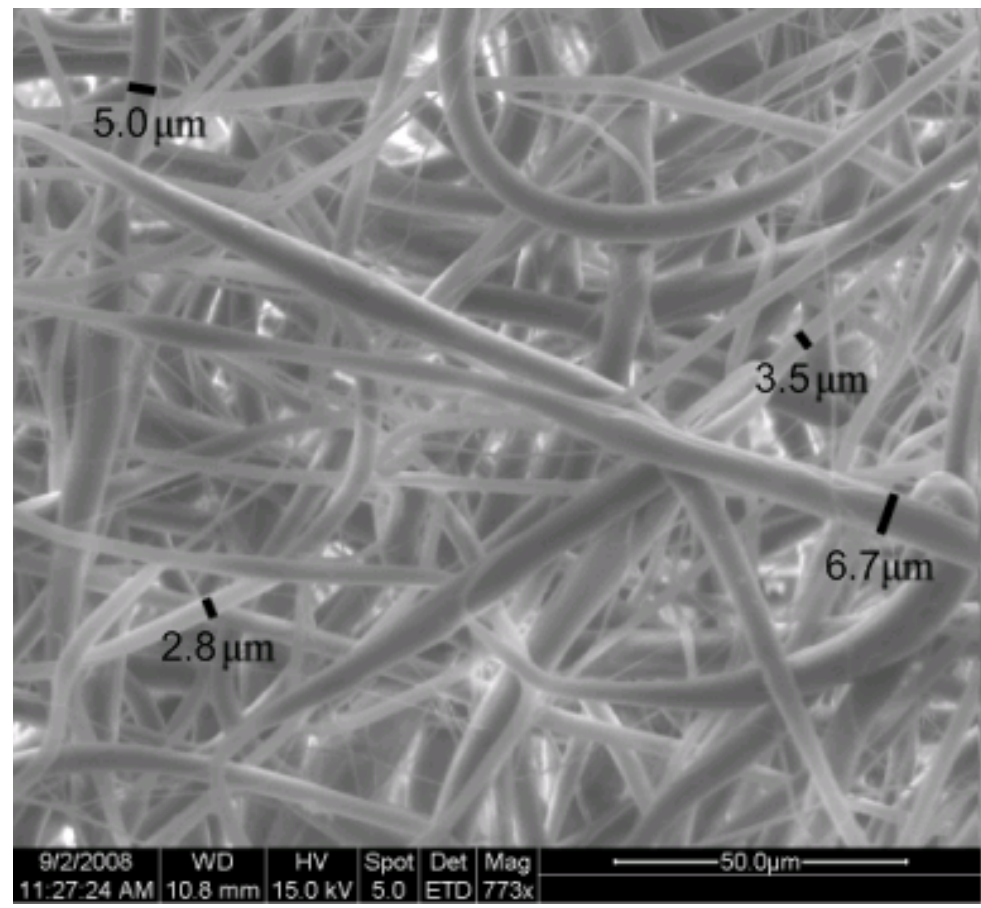

Figure 28 - Spin \#7 appeared to have greater variation among fiber diameters than Spin \#6 (Figure 26). Several of the larger fibers were measured (black lines), but sub-micron fibers were prevalent as well. (773x) 


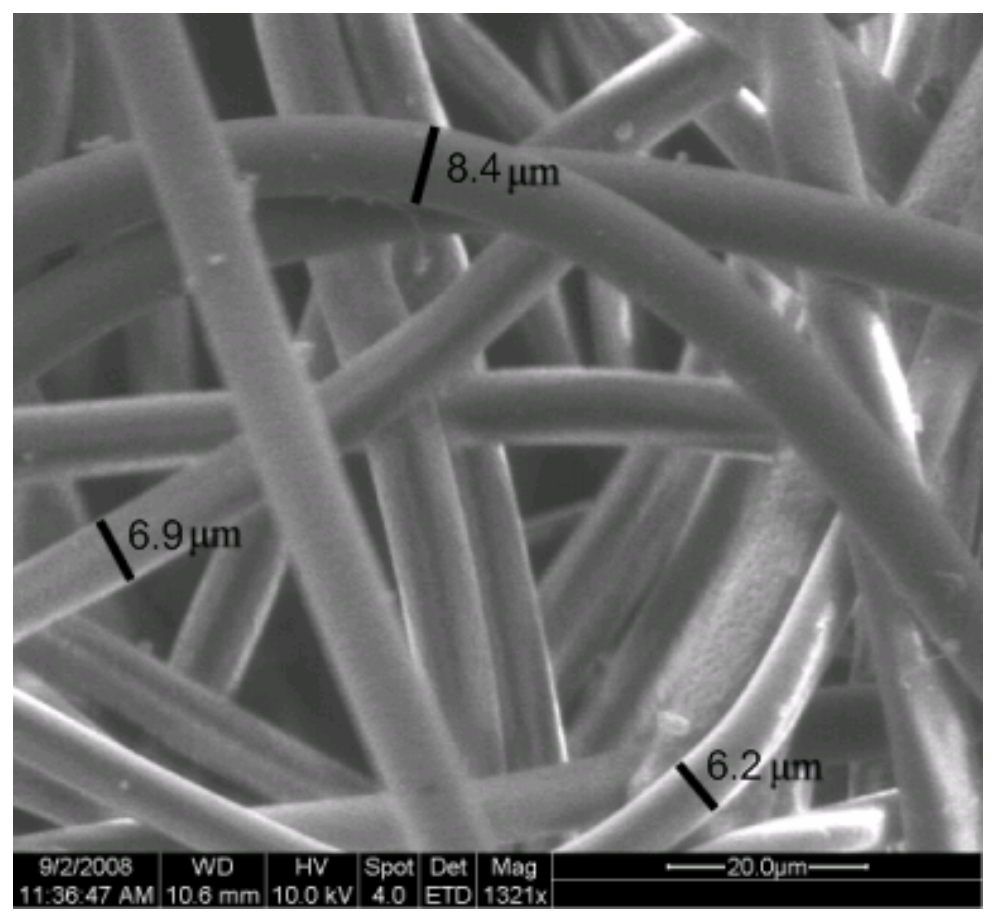

Figure 29 - Variation of fiber diameter decreased by the end of the process, evidenced by this figure showing the exterior surface of Spin \#7. Three random fiber diameters are shown above (black lines). Sub-micron fibers were not present on the exterior surface. $(1321 x)$

Similar to Spin \#6, the interior surface of Spin \#7 contained fibers with varying diameters (Figure 28). At first approximation, it appears that the variation of fiber diameter was greater than that of Spin \#6 (Figure 26). It also appears that fiber diameter on the interior surface stayed the same between Spins \#6 and \#7. Increasing the solution concentration should have increased the average fiber diameter, but in this case it resulted in a larger distribution of fiber sizes. This result went unexplained in terms of controllable variables. Other undocumented variables could have been responsible such as temperature, humidity, and age of the polymer solution. 
An unexpected complication with the syringe pump and needle required a switch to a larger diameter needle during the process. This lead to the unintended discovery that switching to a larger needle may have produced more uniform fibers with slightly larger fiber diameters on the exterior surface (Figure 29).

\section{Spin \#8}

Purpose:

With previous spins, it was discovered that increasing the solution concentration resulted in continuous fibers. Also, increased viscosity caused by the increase in solution concentration restricted the needle size to $18 \mathrm{G}$. This experiment tested a solution concentration in between the concentrations used in Spin \#6 and \#7 while using the larger diameter needle to see if variability of fiber diameter could be reduced.

SOLUTION:

FLOW RATE: APPLIED VOLTAGE: GAP DISTANCE:

\section{Process Observations:}

Using a flashlight, it was possible to see the onset of the first bending instability approximately 4 inches from the needle tip.

By the end of the process, a small mass of polymer was hanging from the needle tip, but the Taylor cone never shifted position.
9.0\% by weight P(LLA-CL) in chloroform

$6 \mathrm{ml} / \mathrm{hr}$ ( $3 \mathrm{ml}$ of solution used) through 18 gauge needle $15 \mathrm{kV}$ (channel set at $5 \mathrm{~V}, 1.5 \mathrm{~A})$ 10 in 
SEM Analysis:

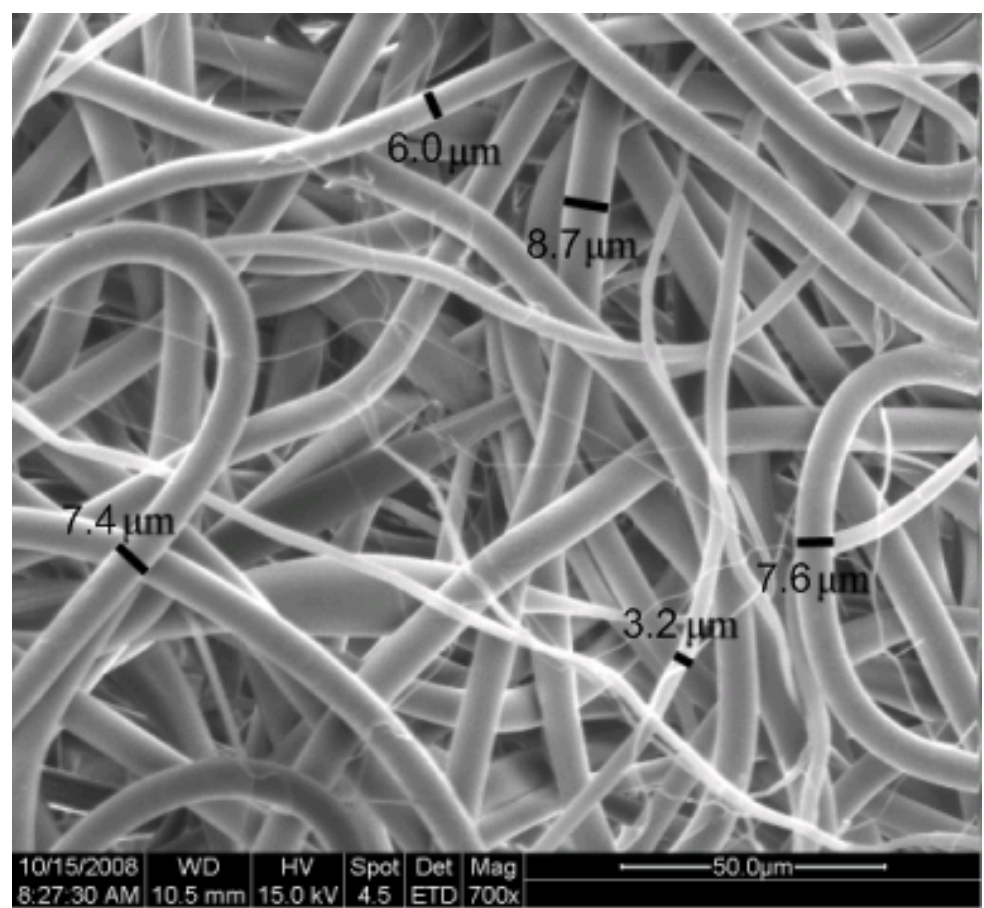

Figure 30 - Fiber diameters from the interior surface of Spin \#8 varied (black lines), but the distribution appeared tighter than the interior surfaces of Spin \#6 (Figure 26) and Spin \#7 (Figure 28). (700x) 


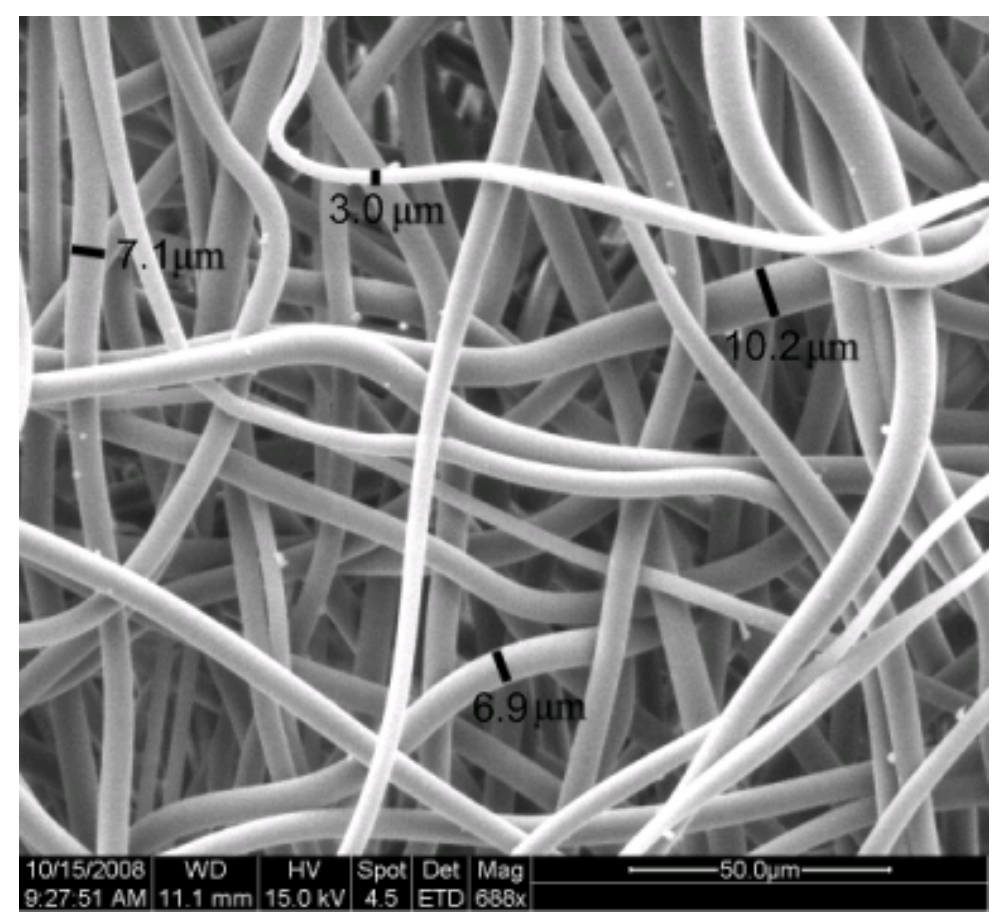

Figure 31 - The exterior surface of Spin \#8 showed continuous fibers with slightly smaller diameters (black lines) than those of the interior surface. (688x)

The interior surface of Spin \#8 was composed of uniform fibers with less variation in diameter (Figure 30), similar to that of the exterior surface of Spin \#7. Average fiber diameter looked to have decreased slightly by the end of the process, although this observation was a first approximation based on the appearance of the exterior surface image (Figure 31). This structure was deemed appropriate for the consistency study, although the fiber size was larger than desired. Further experiments were conducted in an attempt to reduce fiber diameter. 


\section{Spin \#9}

Purpose:

Continuous fibers were first produced after increasing the solution concentration and switching to an external power source set at $5 \mathrm{~V}, 1.5 \mathrm{~A}$. The current from the alternate external power source increased from approximately $0.2 \mathrm{~A}$ in early experiments to $1.5 \mathrm{~A}$ for experiments that yielded fibrous structures, so it may have been this equipment switch that resulted in a fibrous structure as opposed to the increase in solution concentration. Spin \#9 recreated Spin \#1 using the new external amplifying power source to see if a fibrous structure resulted.

SOLUTION:

FLOW RATE: APPLIED VOLTAGE: GAP DISTANCE:

\section{Process Observations:}

$5.3 \%$ by weight $\mathrm{P}(\mathrm{LLA}-\mathrm{CL})$ in chloroform

$6 \mathrm{ml} / \mathrm{hr}$ ( $3 \mathrm{ml}$ of solution used) through 18 gauge needle $15 \mathrm{kV}$ (channel set at $5 \mathrm{~V}, 1.5 \mathrm{~A}$ )

10 in

No complications were observed during this spin. 
SEM Analysis:

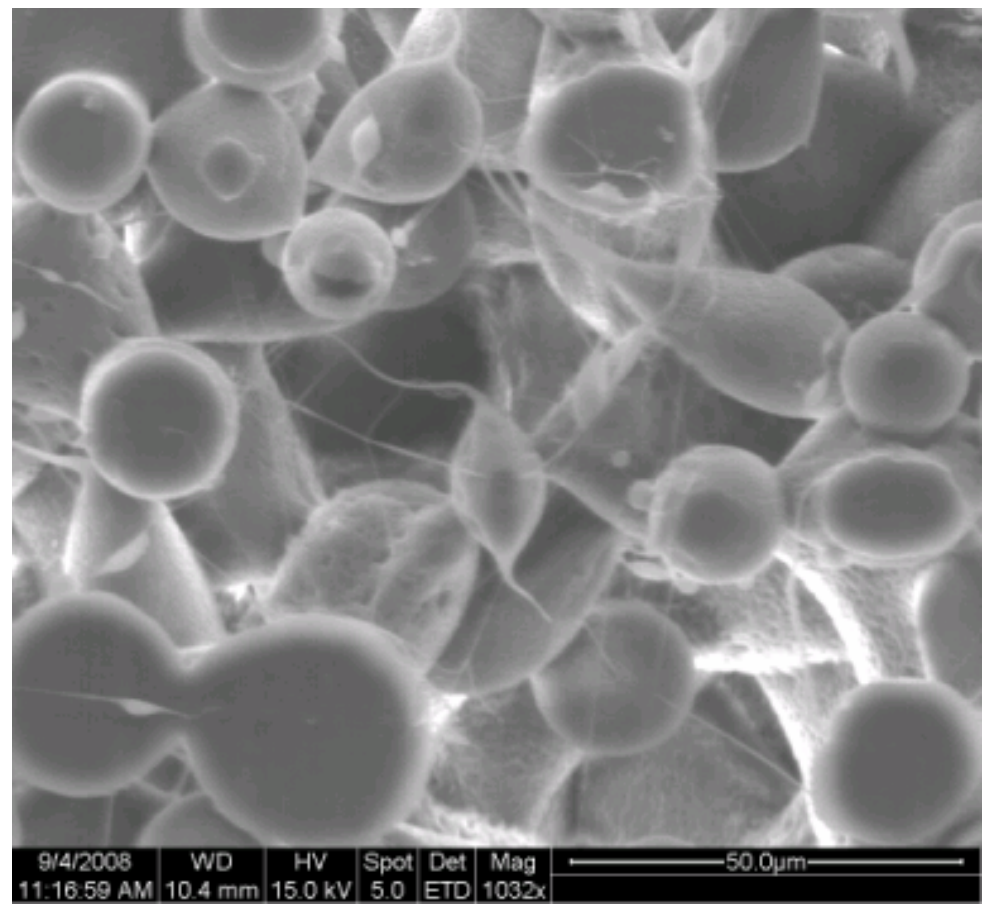

Figure 32 - Bead-on-fiber defects were still present on the interior surface of Spin \#9 despite using the alternate external power source. $(1032 \mathrm{x})$

The external power source was simply an amplifier used to control the output from the high voltage power source, and this was accomplished by varying voltage, not current. In other words, the current from the external source should have had no effect on the final morphology of the construct. The interior surface of the construct from this trial (Figure 32) consists of a similar bead-on-fiber morphology as Spin \#1 (Figure 19). From this experiment, it was concluded that increasing solution concentration resulted in a continuous fiber morphology, not the change in external amplifying power source. 


\section{Spin \#10}

Purpose:

By increasing the gap distance on Spin \#6, it appeared that bead-connecting fibers had decreased in diameter. This experiment followed Spin \#6, but gap distance was increased in an attempt to decrease fiber diameter.

SOLUTION: FLOW RATE: APPLIED VOLTAGE: GAP DISTANCE:

Process Observations:
$7.8 \%$ by weight $\mathrm{P}(\mathrm{LLA}-\mathrm{CL})$ in chloroform $6 \mathrm{ml} / \mathrm{hr}$ ( $3 \mathrm{ml}$ of solution used) through 18 gauge needle $15 \mathrm{kV}$ (channel set at $5 \mathrm{~V}, 1.5 \mathrm{~A}$ ) $15 \mathrm{in}$

There were no complications during the spin, including no build up of polymer on the needle tip.

After the spin, a large amount of polymer coated the containment chamber walls and the plastic frame of the collector.

SEM sample preparation was more difficult than normal because the constructs tore easily. 
SEM Analysis:

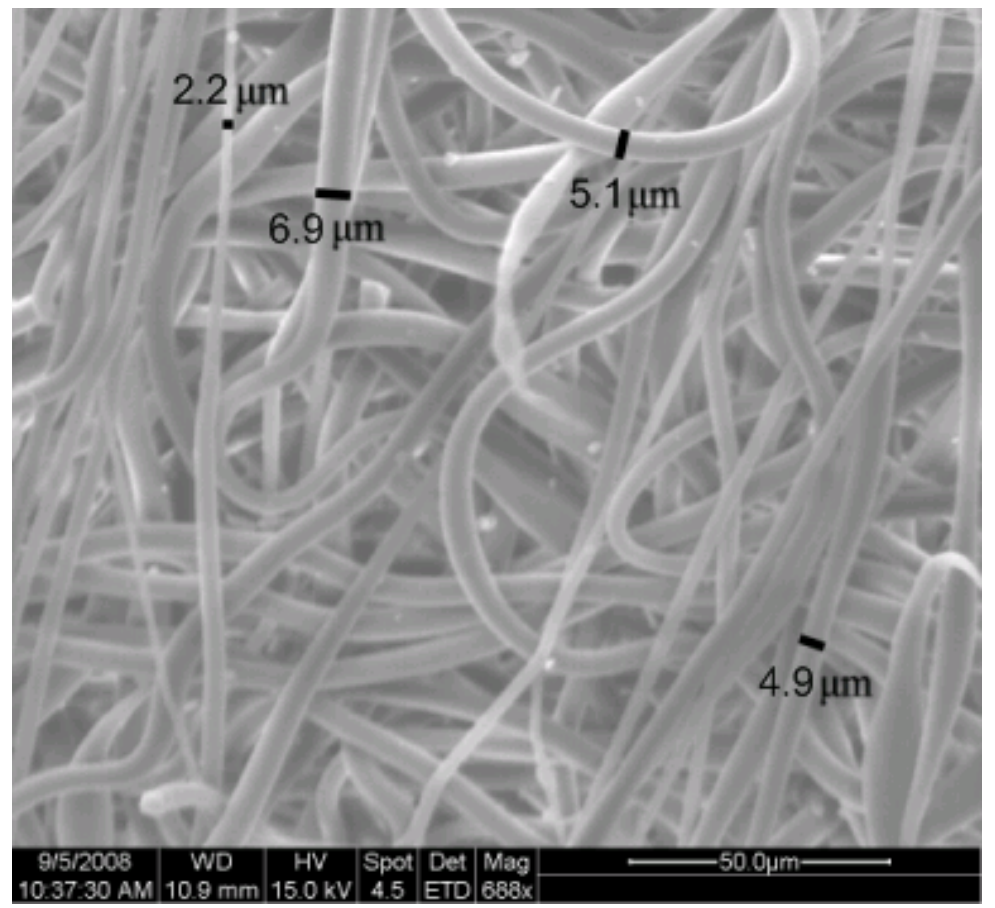

Figure 33 - As expected, increasing the gap distance decreased the average diameter of the fibers on the interior surface of Spin \#10 (black lines). (688x)

Although thinner fibers resulted from increasing the gap distance (Figure 33), less fiber collected on the mandrel, resulting in fragile constructs that tended to rip apart during SEM sample preparation. A more robust construct might be produced by allowing the process to continue until $6 \mathrm{ml}$ of polymer solution is spun. The tradeoff is the increased time and expense associated with scaffold production. Therefore, increased gap distance was not pursued as a method of reducing the average fiber diameter. 


\section{Spin \#11}

Purpose:

This experiment explored a completely new set of parameters used by X.M. Mo et al [89]. Fibers with diameters around 600 nanometers were fabricated with these parameters, which more closely mimics the size of ECM collagen.

$\begin{array}{ll}\text { SOLUTION: } & 5.3 \% \text { by weight P(LLA-CL) in chloroform } \\ \text { FLOW RATE: } & 2 \mathrm{ml} / \mathrm{hr}(3 \mathrm{ml} \text { of solution used }) \text { through } 27 \text { gauge needle } \\ \text { APPLIED VOLTAGE: } & 12 \mathrm{kV} \\ \text { GAP DISTANCE: } & 15 \mathrm{in}\end{array}$

\section{Process Observations:}

The process had to be stopped several times so the needle tip could be cleaned due to polymer build up. Cleaning was necessary because no Taylor cone issued from the mass of polymer at the needle tip. 
SEM Analysis:

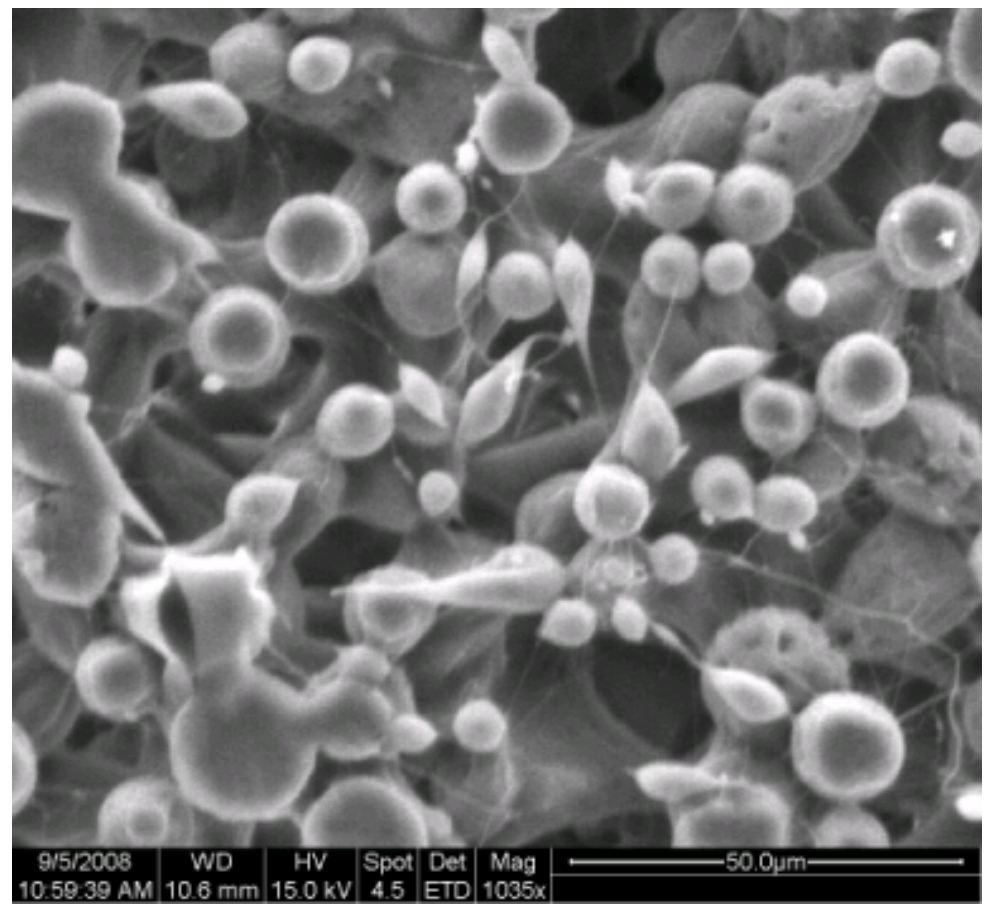

Figure 34 - The interior surface of Spin \#11 was almost completely void of fibers. The process appears to have been electrospraying, not electrospinning. (1035x)

Attempting to recreate the experiment performed by Mo et al. failed to produce a uniform fiber morphology (Figure 34). The familiar bead-on-fiber morphology resulted, with the beads less densely packed than in past experiments. There are several reasons this experiment failed to recreate electrospun fibers made by Mo et al. First, the polymer used in the established study was 75:25 P(LLA-CL), and despite its similarity to the 90:10 P(LLA-CL) used in the Spin series, the 75:25 required different parameters for a successful spin. Second, the 75:25 P(LLA-CL) was dissolved in acetone, not chloroform. Different solvents have inherently different properties, which will affect the final structure of electrospun constructs. Third, a flat, aluminum collector was used by Mo et 
al. instead of a rotating mandrel. The electric fields created by collectors varies based on their geometry, and influences jet dynamics.

Results from this spin were disappointing, so later experiments were conducted that altered the parameters used in Spin \#8 in order to fabricate continuous fiber scaffolds while trying to mimimize fiber diameter.

\section{Spin \#12}

Purpose:

This experiment was the start of a series of trials that attempted to find the lowest solution concentration that would result in a continuous fiber morphology. In this manner, fiber size could theoretically be decreased and P(LLA-CL) resin could be conserved during future studies. Spin \#6 with a lower solution concentration was the basis for this experiment.

SOLUTION: FLOW RATE: APPLIED VOLTAGE: GAP DISTANCE:

Process Observations:
$7.0 \%$ by weight $P(L L A-C L)$ in chloroform $6 \mathrm{ml} / \mathrm{hr}$ (3 $\mathrm{ml}$ of solution used) through 18 gauge needle $15 \mathrm{kV}$ 10 in

No complications were observed during the process. 
SEM Analysis:

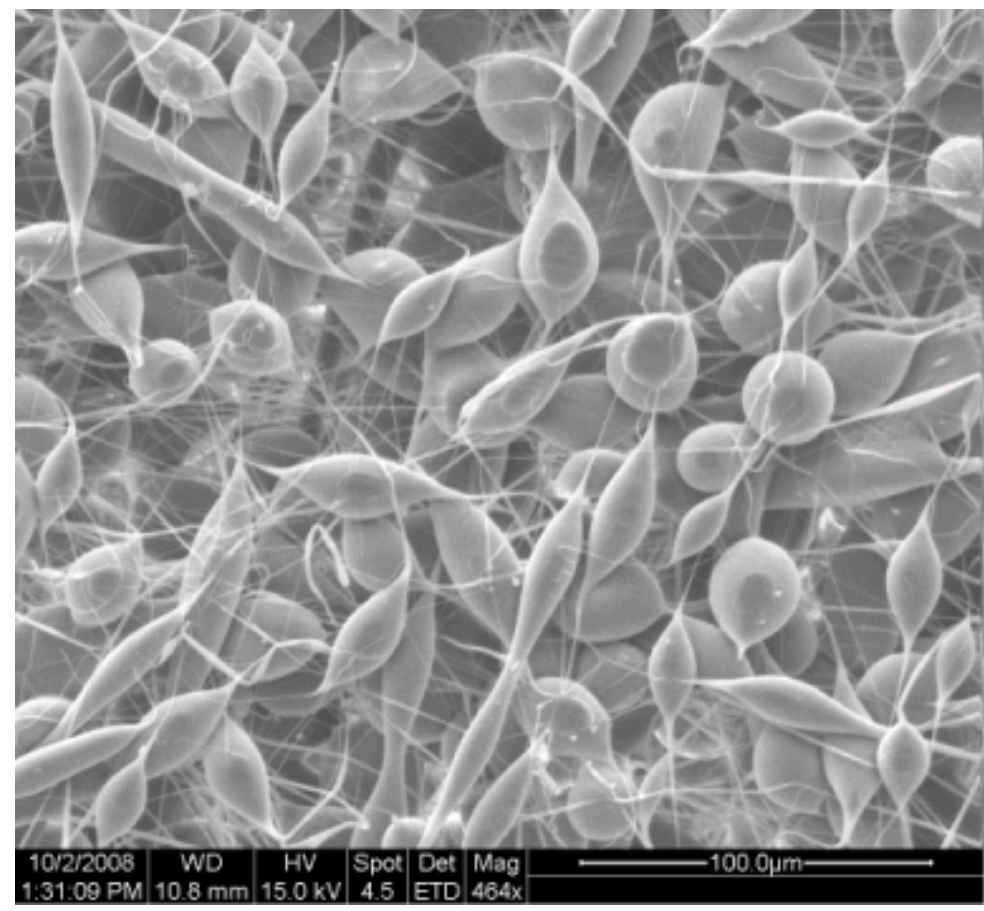

Figure 35 - The interior surface of Spin \#12 consisted of a bead-on-fiber morphology with most of the beads slightly elongated. (464x) 


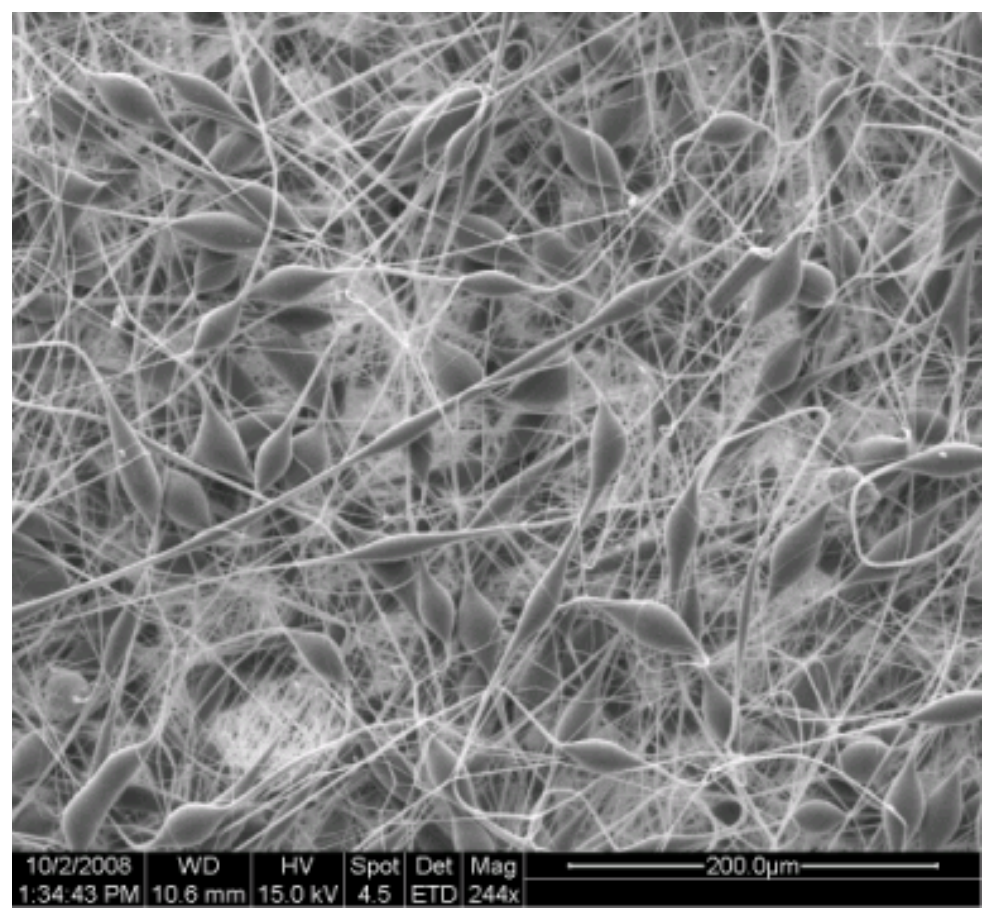

Figure 36 - Overall structure changed by the exterior surface of Spin \#12 to a more fibrous morphology. Beads are still present, but comprise much less of the structure than the interior surface (Figure 35). (244x)

Spin \#6, which used a solution concentration of $7.8 \%$ by weight polymer, resulted in continuous fibers (Figure 26), but this spin, which used a $7.0 \%$ solution, did not (Figure 35). This revealed that for this particular set of parameters (gap distance, applied voltage, flow rate, and needle size) there was a threshold solution concentration that would increase viscosity and decrease surface tension enough that continuous electrospun fibers would result. The next logical step in the process was to increase the solution concentration slightly to find this threshold concentration.

Similar to earlier trial experiments, the exterior structure was more fibrous than the interior (Figure 36). No movement of the Taylor cone was noted during the spinning 
process, so there was another variable at work that caused the structure to change. The interior structure may have had a greater presence of bead-on-fiber defects because it was created directly after the jet was formed. At this time, the full potential of the applied voltage to create surface charges may not have been realized. The lack of surface charge could have allowed capillary instability to cause the jet to form the bead-on-fiber structure.

\section{Spin \#13}

Purpose:

Solution concentration was increased slightly in an attempt to find the threshold concentration that would produce a continuous fiber morphology.

SOLUTION:

FLOW RATE:

APPLIED VOLTAGE: GAP DISTANCE:

Process Observations:
$7.4 \%$ by weight $P(L L A-C L)$ in chloroform $6 \mathrm{ml} / \mathrm{hr}$ ( $3 \mathrm{ml}$ of solution used) through 18 gauge needle $15 \mathrm{kV}$

10 in

No complications were observed during the process. 
SEM Analysis:

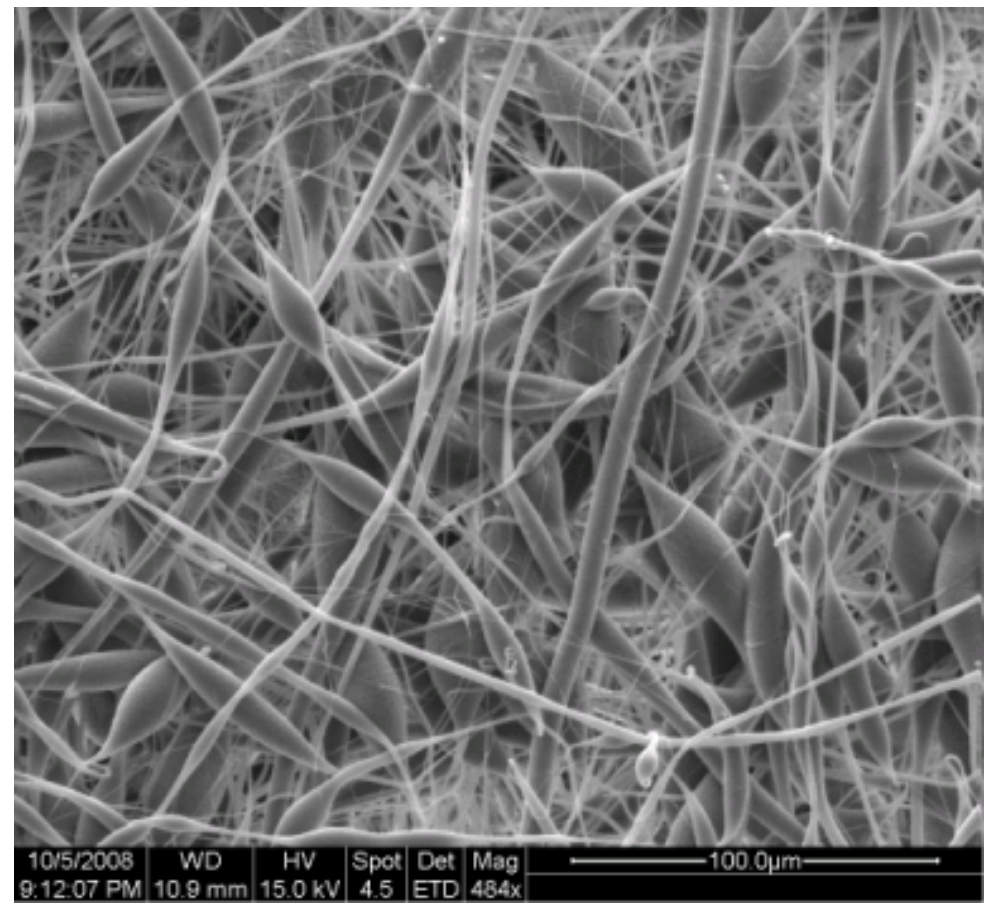

Figure 37 - The beads elongated on the interior surface of Spin \#13 as compared to Spin \#12 (Figure 35), but continuous fibers were not produced. (484x) 


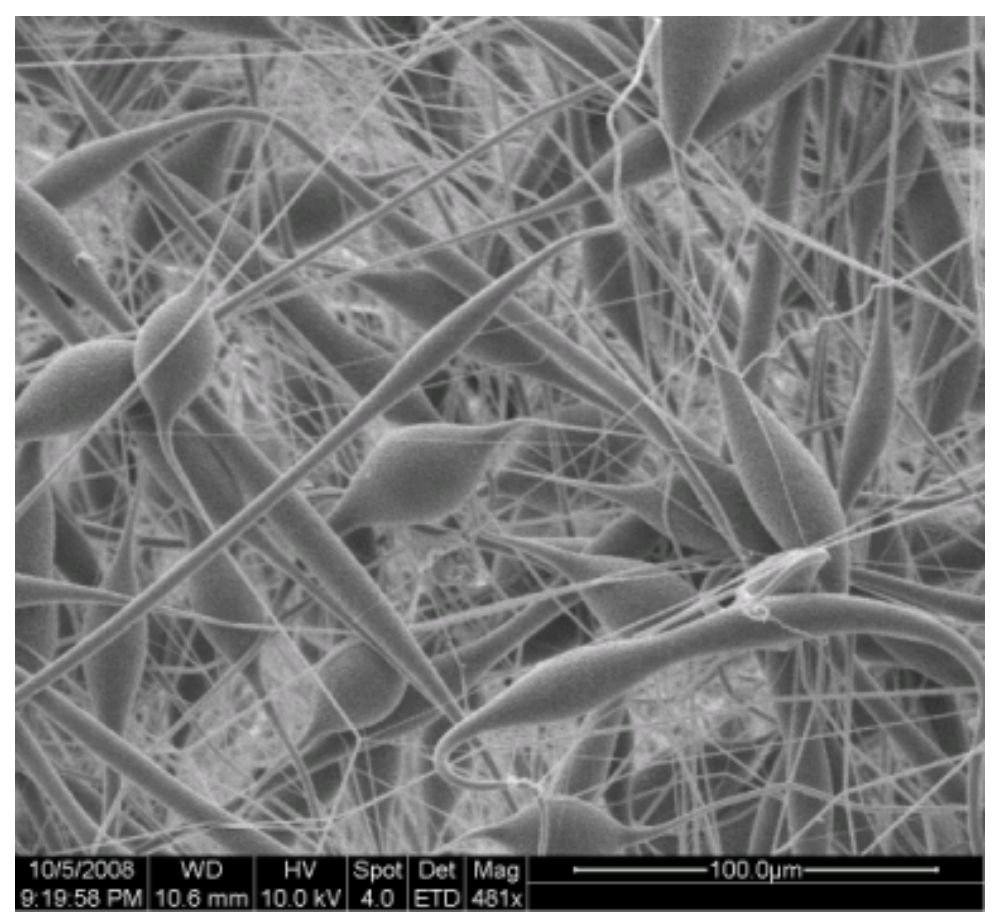

Figure 38 - The exterior surface of Spin \#13 also showed a bead-on-fiber morphology. $(481 x)$

The increased solution concentration of this trial resulted in a more fibrous structure, but bead defects were still present (Figure 37). Interestingly, the exterior surface morphology was the same as that of the interior surface (Figure 38). This was different behavior than Spin \#12, and may be explained by a slightly different start-up procedure that resulted in quicker establishment of a fully charged jet. Specifically, the electrospinning apparatus was supplied with power, the external amplifier, and the high voltage power supply were all turned on within 5 seconds of each other. In past experiments, no attempt was made to start the process in this manner. 


\section{Spin \#14}

Purpose:

Spin \#14 tested a slightly increased solution concentration to continue the search for a threshold concentration.

SOLUTION:

FLOW RATE: APPLIED VOLTAGE: GAP DISTANCE:

\section{Process Observations:}

Polymer built up at the needle tip at the beginning of the spin. The process was stopped after $0.5 \mathrm{ml}$ was spun, the needle was cleaned, and the process was restarted.

SEM Analysis:

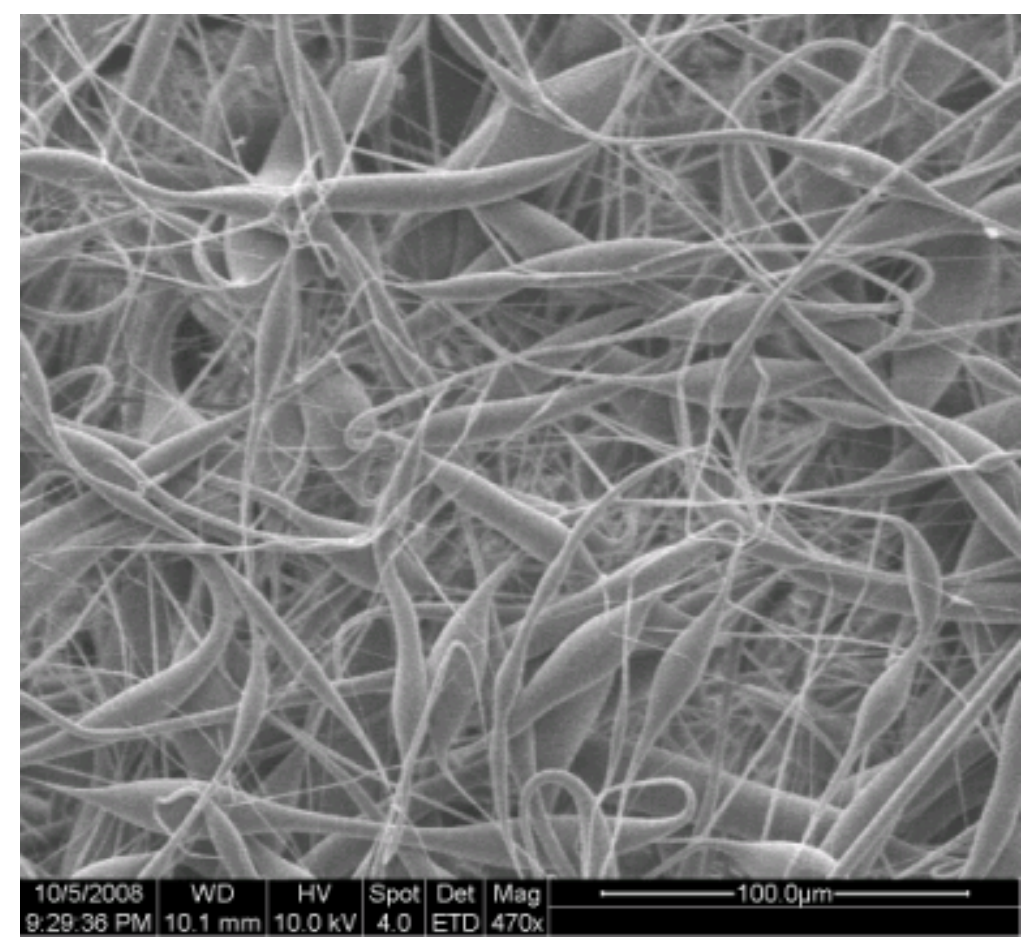

Figure 39 - Beads on the interior surface of Spin \#14 were elongated similar to those on the interior surface of Spin \#13 (Figure 37). (470x) 


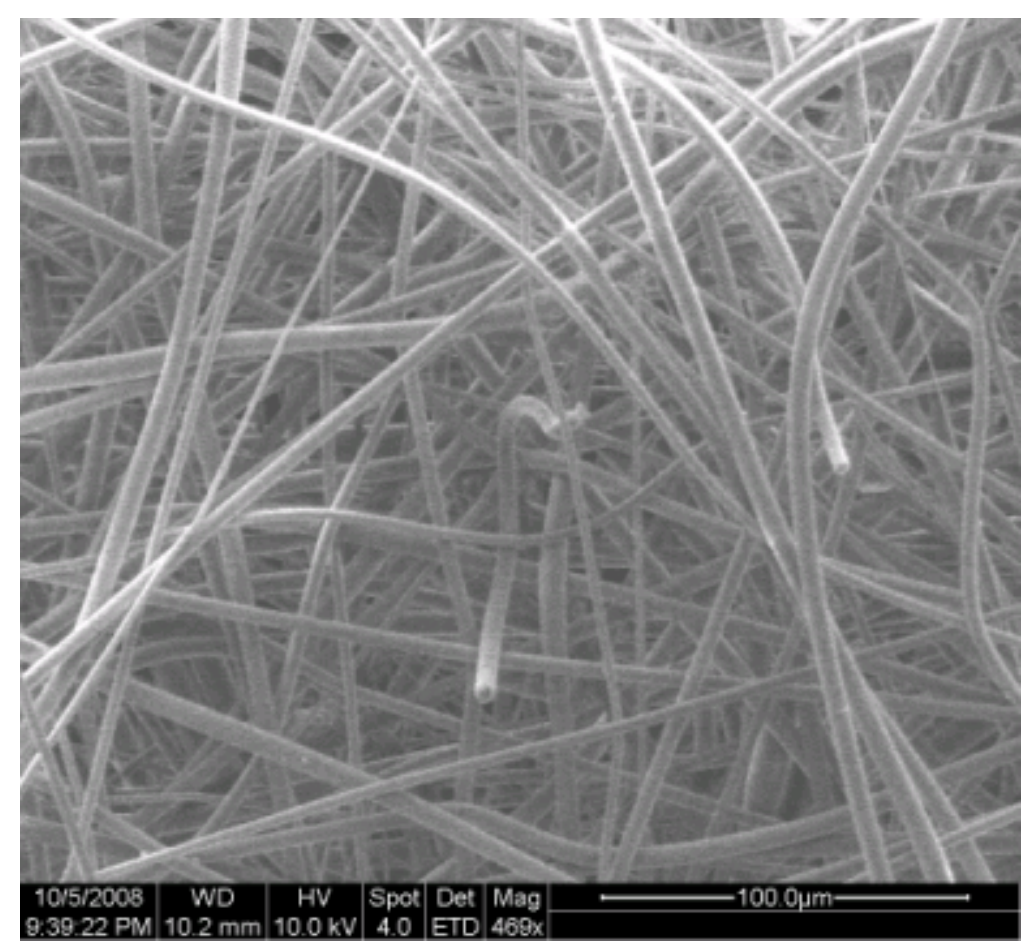

Figure 40 - The exterior surface of Spin \#14 consisted of uniform fibers. (489x)

Bead defects on the interior surface of this trial elongated slightly compared to Spin \#13 (Figure 39). By the end of the process, the morphology was again different from the interior surface (Figure 40), similar to Spin \#12 (Figure 36). Since the exact start-up procedure was not recorded for this or any previous experiment, it cannot be determined if start-up sequence played a role in the change in structure. For the consistency study, the start-up procedure was the same for all spins to eliminate this as a source of variability. 


\section{Spin \#15}

Purpose:

By this time, enough trials had been conducted that the electrospinning process was familiar. Although nano-sized uniform fibers were not produced, a suitable structure for the consistency study had been fabricated (Spin \#8). This experiment was a repeat of Spin \#8 with a set start-up procedure.

SOLUTION: FLOW RATE: APPLIED VOLTAGE: GAP DISTANCE:

\section{Process Observations:}

After $1.7 \mathrm{ml}$ of solution had been spun, polymer build-up started to appear and hang from the needle tip, but the Taylor cone appeared unaffected. The needle tip was not cleaned because the consistency study protocol called for an uninterrupted process, and this spin was used as a consistency study trial. 
SEM Analysis:

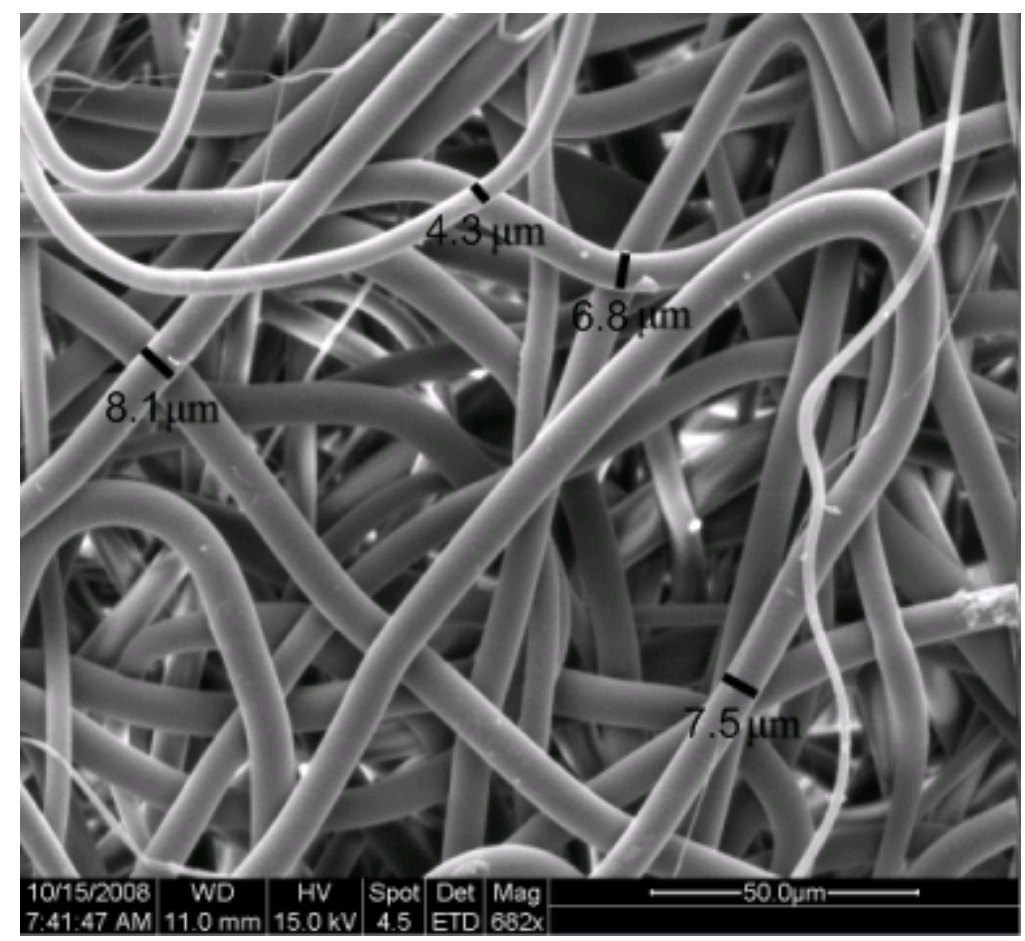

Figure 41 - Fiber diameter sizes from the interior surface of Spin \#15 (black lines) were similar to sizes seen from Spin \#8 (Figure 30). (682x) 


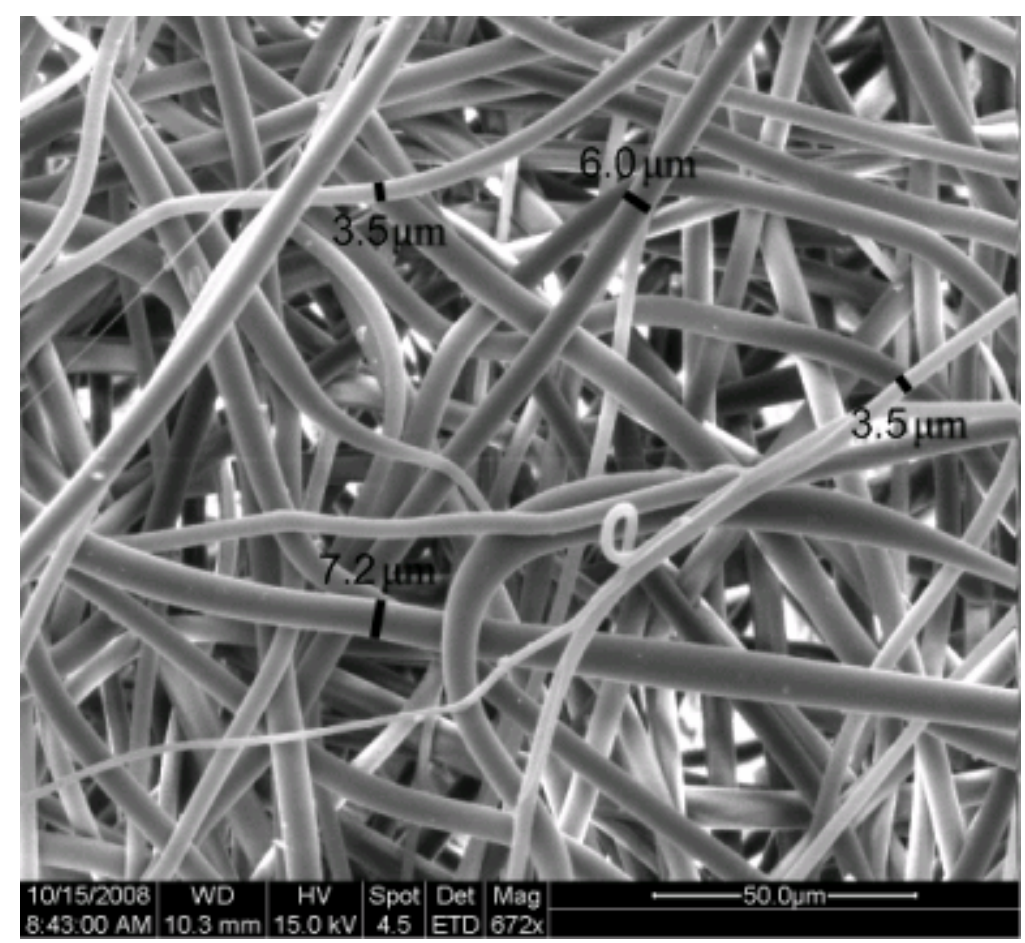

Figure 42 - Fiber size on the exterior surface of Spin \#15 was slightly smaller than interior surface fibers.

Implementing an electrospinning start-up protocol produced an interior surface with less variability in fiber diameter (Figure 41). However, fiber sizes on the interior surface were slightly larger than fiber sizes on the exterior surface (Figure 42). The reason for this morphological discrepancy is unknown, but somehow the process changed from the start to the finish of the spin. Exploring this phenomenon was not the purpose of this work, and the interior surface of Spin \#15 was appropriate for the consistency study. 


\section{Discussion}

Overall, these 15 trial spins met the goals stated at the beginning of this chapter. After gaining an understanding for how electrospinning parameters affect fiber morphology, a protocol was established to fabricate scaffolds composed of continuous diameter fibers (see Appendix C). The trial spins explored parameters such as applied voltage, solution concentration, gap distance, and needle size. In general it was noted that concentration of the P(LLA-CL) solution had the most effect on fiber diameter; the higher the concentration, the larger the fiber diameter would be. In addition, below a certain solution concentration ( $\sim \%$ by weight $90: 10$ P(LLA-CL) in chloroform), the process becomes more like electrospraying than electrospinning. Increasing the applied voltage appeared to slightly decrease the average fiber diameter, as did decreasing the needle diameter. Increasing gap distance also resulted in a decrease in fiber diameter, but less polymer collected on the grounded mandrel. 


\section{ELECTROSPINNING CONSISTENCY STUDY}

\section{Introduction}

In order for electrospun scaffolds to be a viable replacement for ePTFE, they must meet qualifications which include the following: biologically acceptable, mechanically appropriate, lower cost than ePTFE, and producible in a high throughput manner. The fourth qualification, high throughput, involves the speed and ease with which the scaffolds are produced. In the case of the electrospun scaffolds, in-house production allows for quick manufacturing time and the process itself is relatively simple. However, high throughput scaffolds are inappropriate if there is not at least some level of consistency between scaffolds produced with the same controllable parameters. It is with this in mind that the same spinning parameters were used to manufacture multiple scaffolds to establish consistency data. Consistency was examined by looking at fiber diameter size, wall thickness, and Young's modulus of individual spins. Porosity testing was initially intended, but lack of a suitable method dictated that no porosity data be included in consistency evaluations. See Appendix D for the method originally intended to collect porosity measurement. 


\section{Methods}

Electrospinning Parameters for the Consistency Study

All consistency study scaffolds were fabricated using the electrospinning

parameters shown below and the electrospinning protocol in Appendix C. A total of 11 spins were performed using this protocol.

Polymer: $\quad 9.0 \mathrm{wt} \% \mathrm{P}(\mathrm{LLA}-\mathrm{CL})$ in chloroform

Feed Rate: $\quad 6 \mathrm{ml} / \mathrm{hr}$, using a total of $3 \mathrm{ml}$ per spin

Needle: $\quad 18$ gauge, beveled, blunt (BD 305180)

Gap: $\quad \underline{10 \text { in }}$

Voltage: $\quad 15000 \mathrm{~V}$ (external source set to $5 \mathrm{~V}$ )

Translate: $\quad$ Distance set at $16 \mathrm{~cm}$, speed set at 2

Rotate: $\quad$ Speed set at 6

After fabrication, scaffolds were placed in a desiccator for at least 24 hours to remove residual solvent. 
Sampling Method

After desiccation, all spins were sectioned as shown below (Figure 43). Sections were cut while the scaffold was on the mandrel using a No. 11 razor blade.

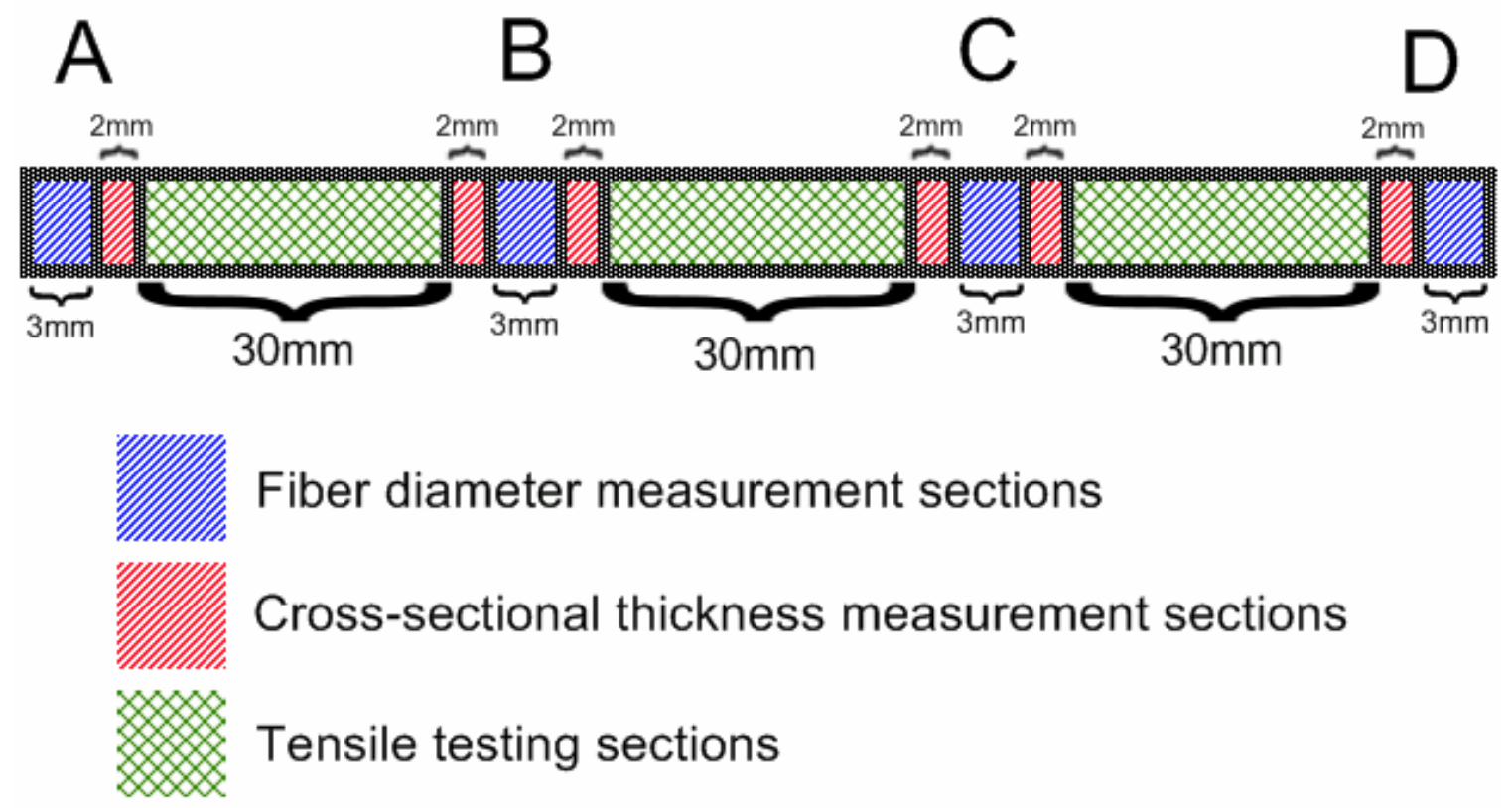

Figure 43 - Individual scaffolds fabricated in the consistency study were all sectioned for examination in a similar manner. Section A represented the area of the scaffold furthest away from the collector's rotation motor, while section D was always the area of the scaffold closest to the collector's rotation motor.

Fiber Measurement

The diameter of electrospun fibers can be used to characterize the electrospinning process [80], and this study used fiber diameter to evaluate the morphological consistency of the in-house electrospinning process. 
Fiber Measurement Method

For each scaffold, fiber diameter measurement sections A, B, C, and D (Figure 43) were mounted with luminal surfaces up on SEM sample stages using adhesive graphite tape and sputter coated with gold using a Pelco Model 3 Sputter Coater. All sections were imaged using an FEI Model 200 Scanning Electron Microscope at approximately 700x magnification. Three random locations were selected on each section and imaged for fiber diameter analysis. To choose the fibers to be measured, a fixed fiber selection mask was applied to each image, resulting in 9 measurements per image (Figure 44). This resulted in 27 fiber diameter measurements per section, and 108 measurements per scaffold. Measurements were made using ImageJ 1.410 image analysis software (National Institutes of Health, USA). All images and collected fiber size measurements are compiled in Appendix E. 


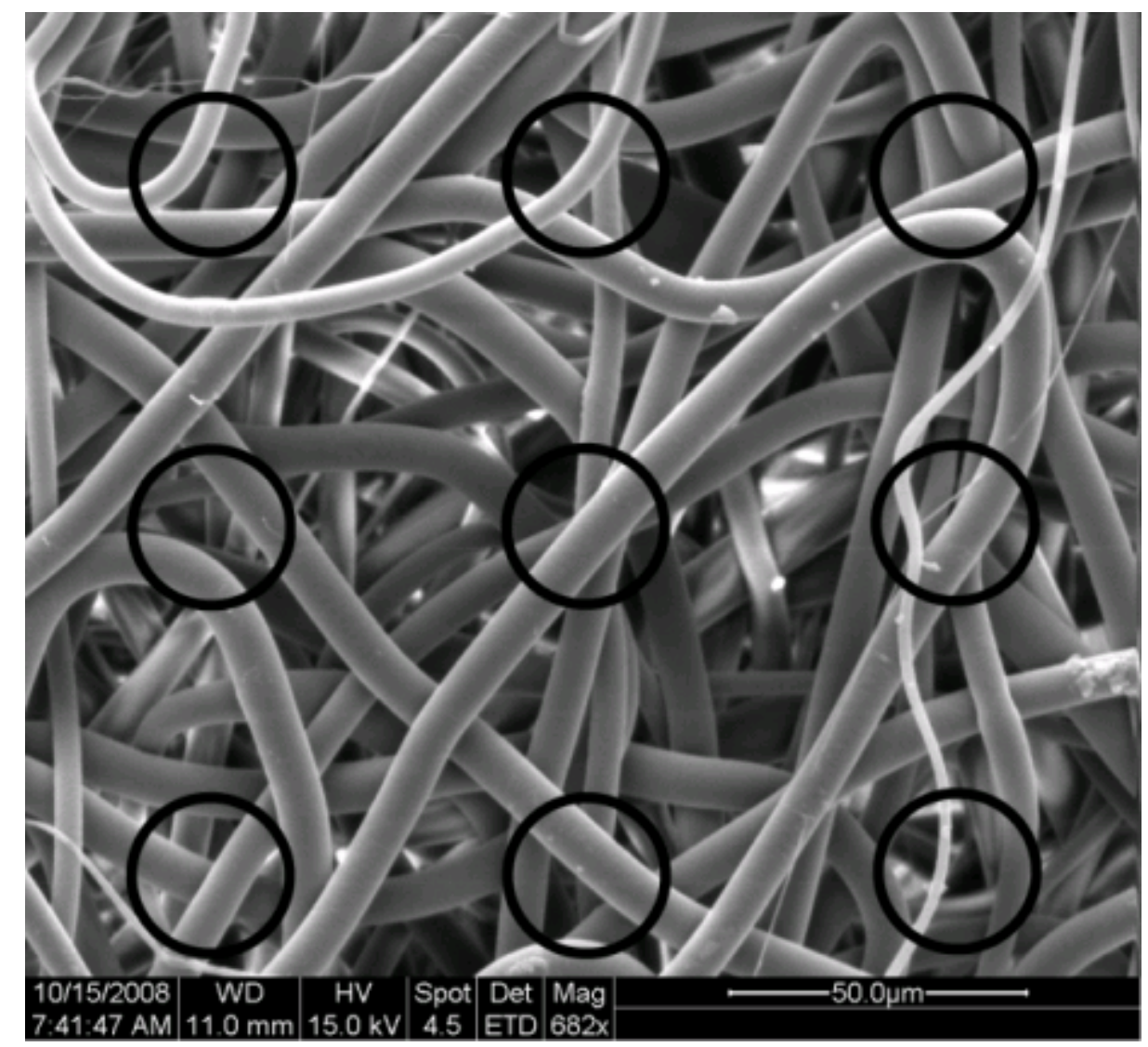

Figure 44 - All fiber diameter measurement images appeared similar to this figure. The nine circles were the fixed fiber selection mask used to pick individual fibers to measure. Fibers closest to the center of the circles had their diameters measured and recorded for analysis.

\section{Thickness Measurement}

Scaffold wall thickness was also measured to evaluate the consistency of the electrospinning process. Wall thickness is important because different thicknesses equate to different mechanical responses; thicker scaffolds will be less compliant than scaffolds with thinner walls. In addition, accurate tensile testing requires careful measurement of 
sample wall thickness. Values for sample thickness were obtained from cross-sectional images of the cylindrical scaffolds by the method described in the next section.

Thickness Measurement Method

Each scaffold was measured for thickness at 6 different locations (Figure 45). Samples were prepared in the same manner as the fiber measurement samples, including mounting on SEM stages and sputter coating with gold. The FEI Model 200 Scanning Electron Microscope was used to capture images of wall thickness at approximately 20x magnification (Figure 46). Twelve thickness measurements were made for each image at locations corresponding to numbers on a clock ( 1 o'clock, 2 o'clock, etc.) using the ImageJ 1.410 image analysis software. All images and collected thickness measurements are compiled in Appendix F.

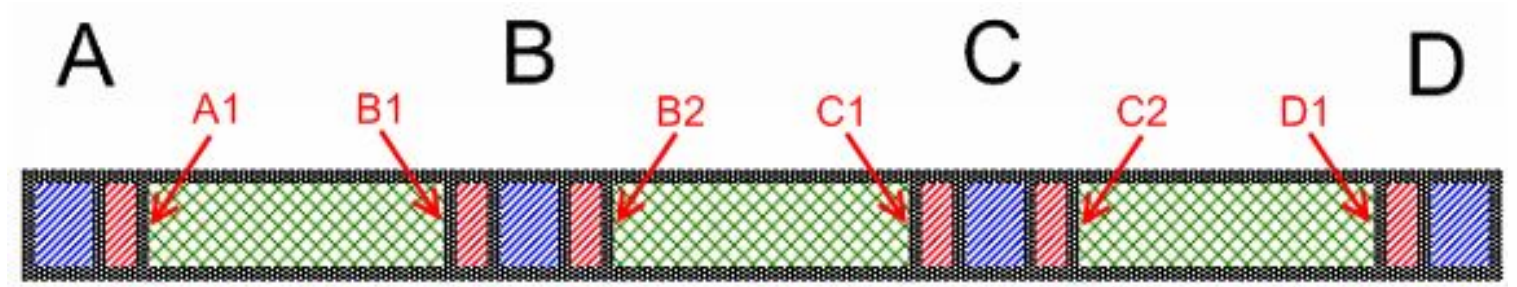

Figure 45 - Six cross-sectional surface thicknesses were measured (A1, B1, B2, C1, C2, and D1). Tensile testing sample thicknesses were calculated by averaging the thicknesses of the cross sectional surfaces on either end of the tensile testing sample. 


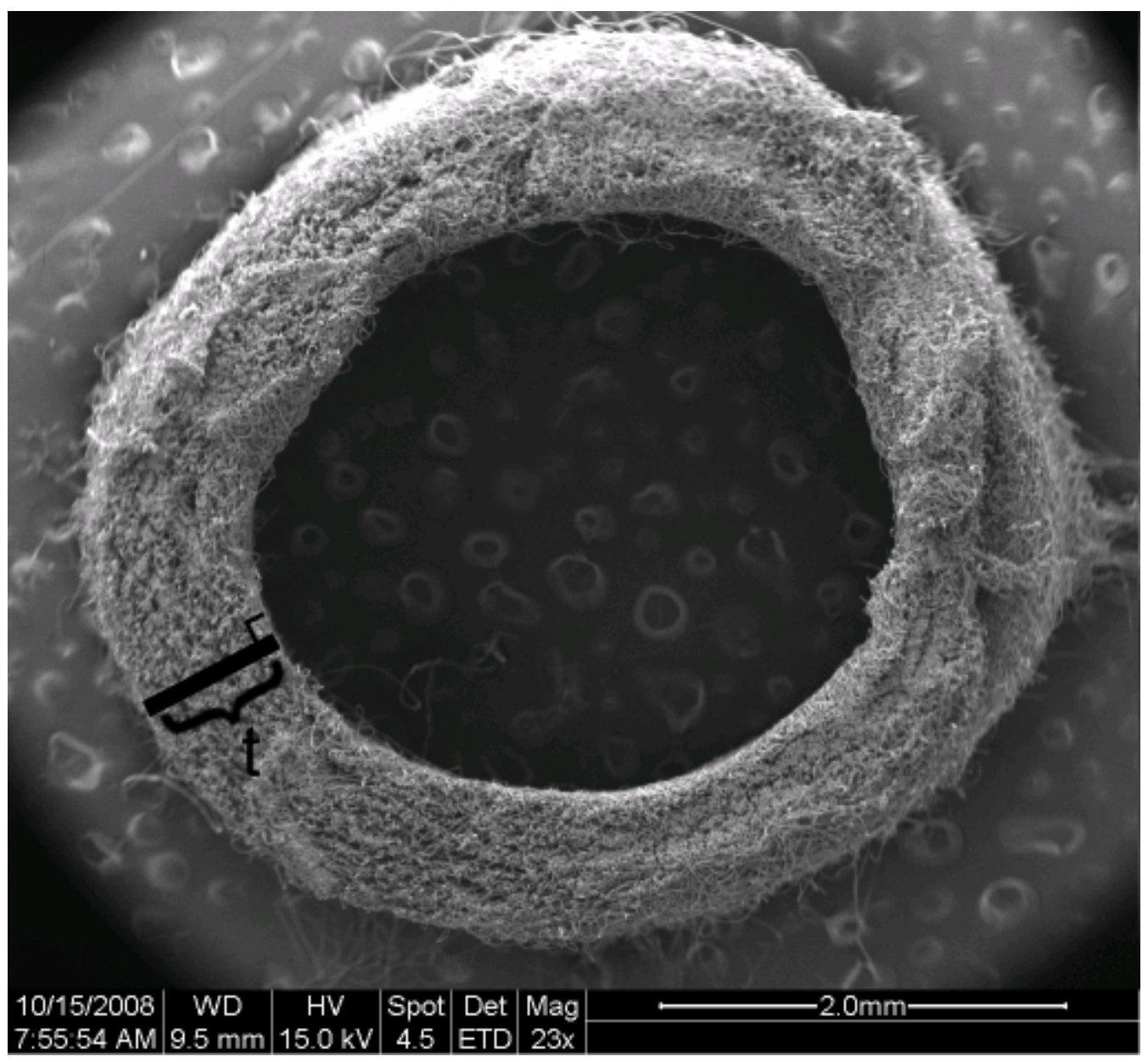

Figure 46 - All images acquired for cross-sectional thickness analysis appeared similar to this picture. Thickness measurements $(\mathrm{t})$ were made perpendicularly from the interior surface to the exterior surface of each scaffold at 12 separate locations.

Tensile Testing

Uniaxial tensile testing was performed on the consistency study spins to evaluate the similarity of the mechanical responses. Young's modulus was the property investigated for two reasons. First, Young's modulus is easily obtained from a stress versus strain plot. Second, in a pulsing BVM system, compliant scaffolding would 
ideally stretch circumferentially, then return to its original dimensions. This denotes stress in the linear elastic region of the scaffolding material, characterized by Young's modulus. Thus, it is Young's modulus, not other parameters such as ultimate tensile strength that is of interest in this study.

Accurate tensile testing requires careful measurement of sample thickness. Since cross-sectional thicknesses of the consistency spins varied, individually estimating the thickness for each tensile testing sample was necessary, as opposed to using an average value pooled from all thickness measurements. By averaging the two thicknesses measured at the ends of each tensile testing sample, an estimate for thickness appropriate for calculating stress in the tensile testing sample was obtained. For example, thickness for the tensile testing sample between sections A and B was calculated as the average thickness between cross-sections A1 and B1.

\section{Tensile Testing Methods}

An Instron In-Spec 2200 benchtop tensile tester was used to test all tensile testing samples at a pulling speed of $5.6 \mathrm{~mm} / \mathrm{min}$. Samples were cut into two rectangular sheets for testing in both the longitudinal and circumferential directions, resulting in six tests per scaffold. Each of the tensile testing samples was measured for width and gauge length prior to testing. Using the measured dimensions (thickness, width, and gauge length), collected data (pulling force and extension), and a Microsoft Excel macro program, stress versus strain plots were generated. The aforementioned Excel macro was created to automatically convert all tensile testing inputs into stress versus strain data, plot the data, 
isolate the linear elastic region of the plot, and calculate Young's modulus. All tensile testing figures are contained in Appendix G.

\section{Results}

Results and Statistical Analysis of Fiber Diameter

Fiber diameter data was analyzed using Minitab 15 statistical analysis software, and the figures and tests presented in this section were generated by the same program. The data did not form a normal distribution, but a majority of the fibers had diameters between 6 and 9 microns (Figure 47). Boxplots are shown that summarize the data based on each of the 11 scaffolds and 4 sections sampled on each scaffold (Figure 48). A oneway analysis of variance (ANOVA) test was also performed to test for statistical difference between the spins, with a Tukey's method of comparison test to highlight the differences between individual tests (Figure 49). Table 2 shows P-value results for intrascaffold ANOVA testing which indicate the scaffolds that had significantly different fiber diameters at the different sections tested $(\mathrm{P}>0.05)$. Appendix $\mathrm{H}$ contains the full intrascaffold ANOVA test results. 


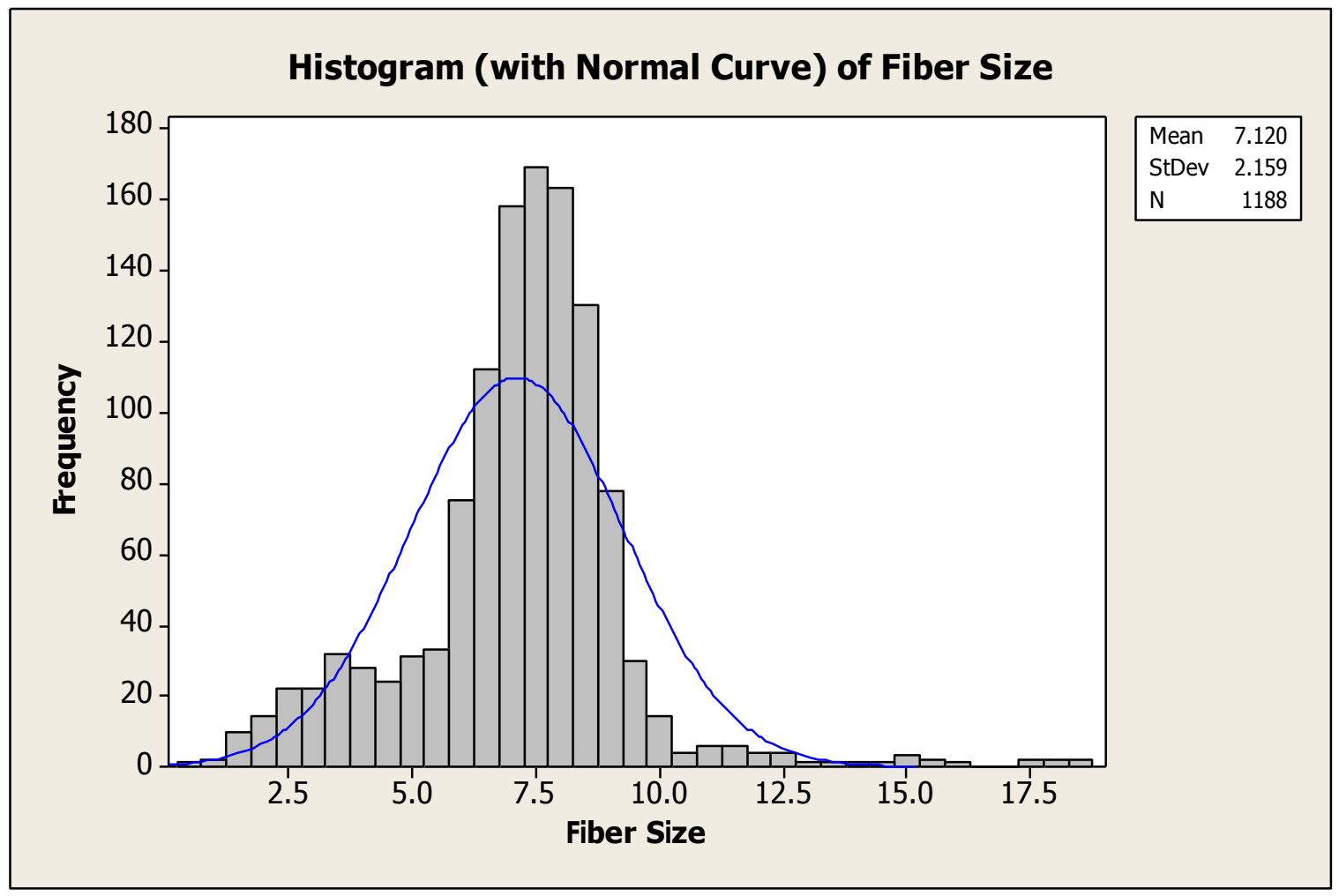

Figure 47 - A histogram of all 1188 data points shows that the average fiber diameter was $7.12 \mu \mathrm{m}$ with a standard deviation of $2.159 \mu \mathrm{m}$. A normal distribution curve with the same mean and standard deviation (7.12 and 2.159, respectively) is superimposed on the image. 


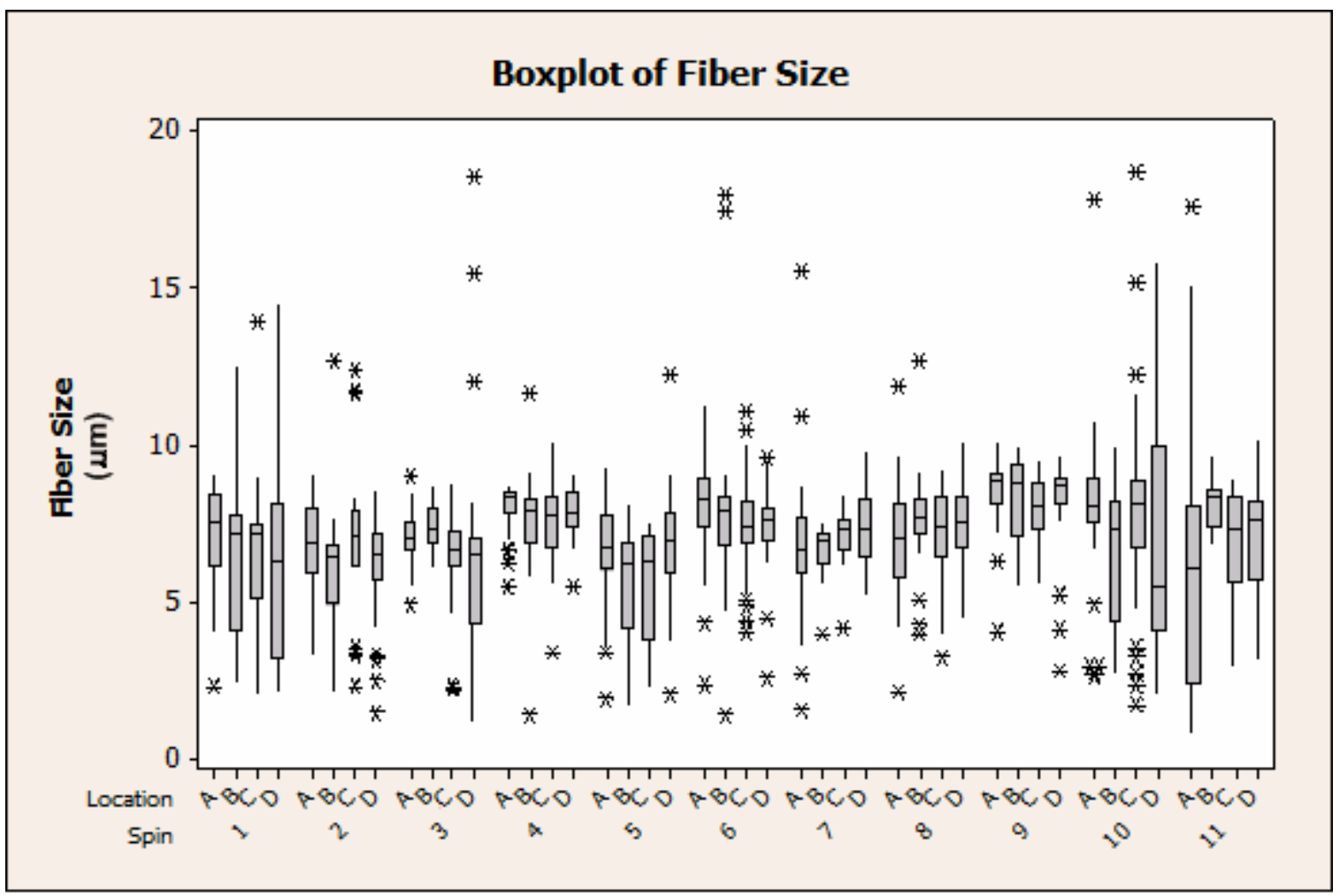

Figure 48 - Boxplots of all fiber diameter measurements separated into individual spins and sections provide a summary of the data. An asterisk indicates an outlier, the vertical lines show the non-outlier data range, and the rectangle on each line represents the interquartile range of each data set. The horizontal line through each interquartile range box marks the median of each data set. 
One-way ANOVA: Fiber Size versus Spin

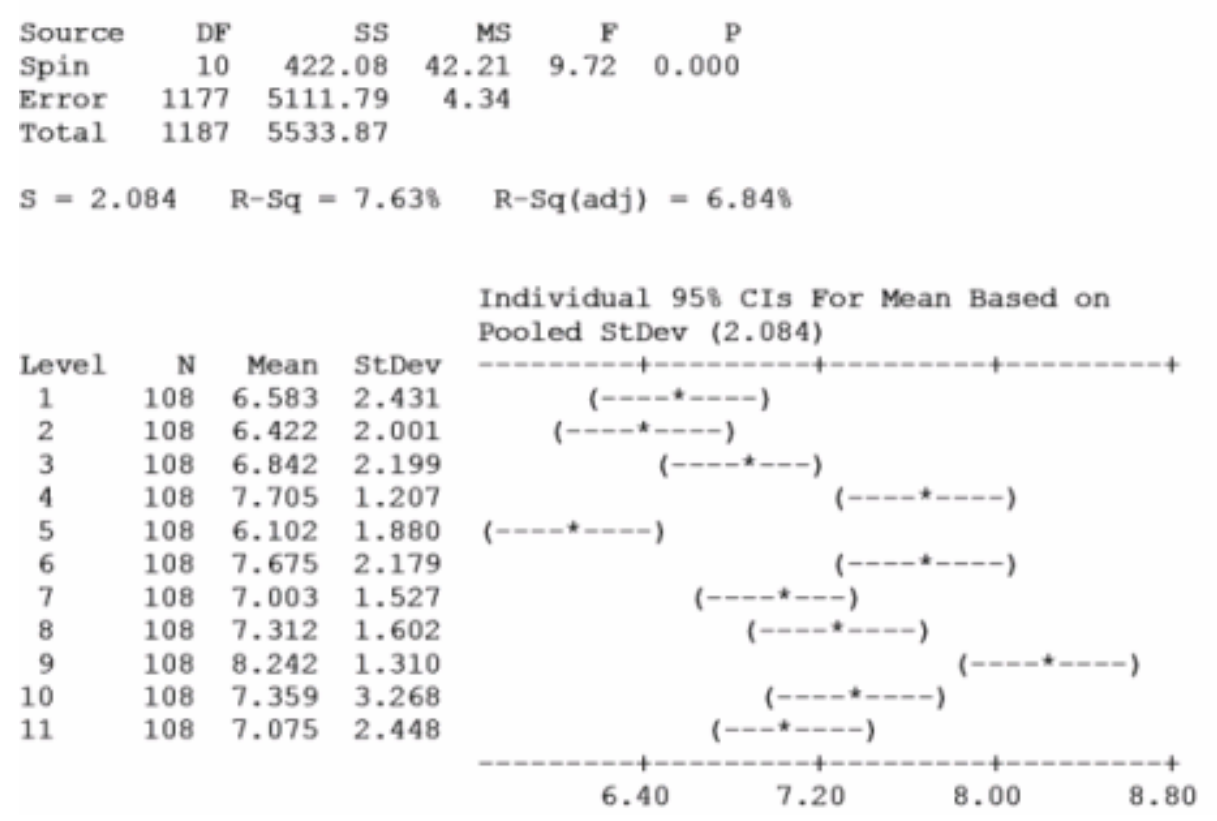

Figure 49 - An analysis of variance model comparing fiber diameter between spins (top) indicated that at least one spin was significantly different from the rest $(\mathrm{P}<0.05)$. Tukey's method of comparison was used to generate 95\% confidence intervals for individual spins (bottom). The confidence intervals can be used to determine which spins were significantly different from each other. For example, the confidence interval of spin 3 does not overlap the confidence interval of spin 9, so the fiber diameter of the two is statistically different. 
Table 2 - Intrascaffold ANOVA Results

$\begin{array}{cc}\text { SPIN } & \text { P-VALUE } \\ 1 & 0.741 \\ 2 & 0.219 \\ 3 & 0.336 \\ 4 & 0.439 \\ 5 & 0.047 \\ 6 & 0.503 \\ 7 & 0.344 \\ 8 & 0.287 \\ 9 & 0.490 \\ 10 & 0.450 \\ 11 & 0.028\end{array}$

Statistical analysis of fiber diameter indicates that differences in fiber diameter did exist between individual spins. In addition, intrascaffold ANOVA testing revealed that the sections imaged for Spin 5 and Spin 11 contained fibers of statistically different diameter (Table 2).

Results and Statistical Analysis of Scaffold Wall Thickness

Wall thickness data was analyzed in the same manner as fiber diameter. ANOVA testing using Minitab 15 showed that wall thickness between spins varied, with thinner walls produced for the first 4 spins than the remaining 7 (Figure 51). Wall thickness along the length of scaffolds (or intrascaffold wall thickness) was tested using one-way ANOVA testing. A table of P-values is not provided since the only spin with statistically similar wall thickness data was Spin \#10. All 11 intrascaffold wall thickness ANOVA tests can be found in Appendix I. 


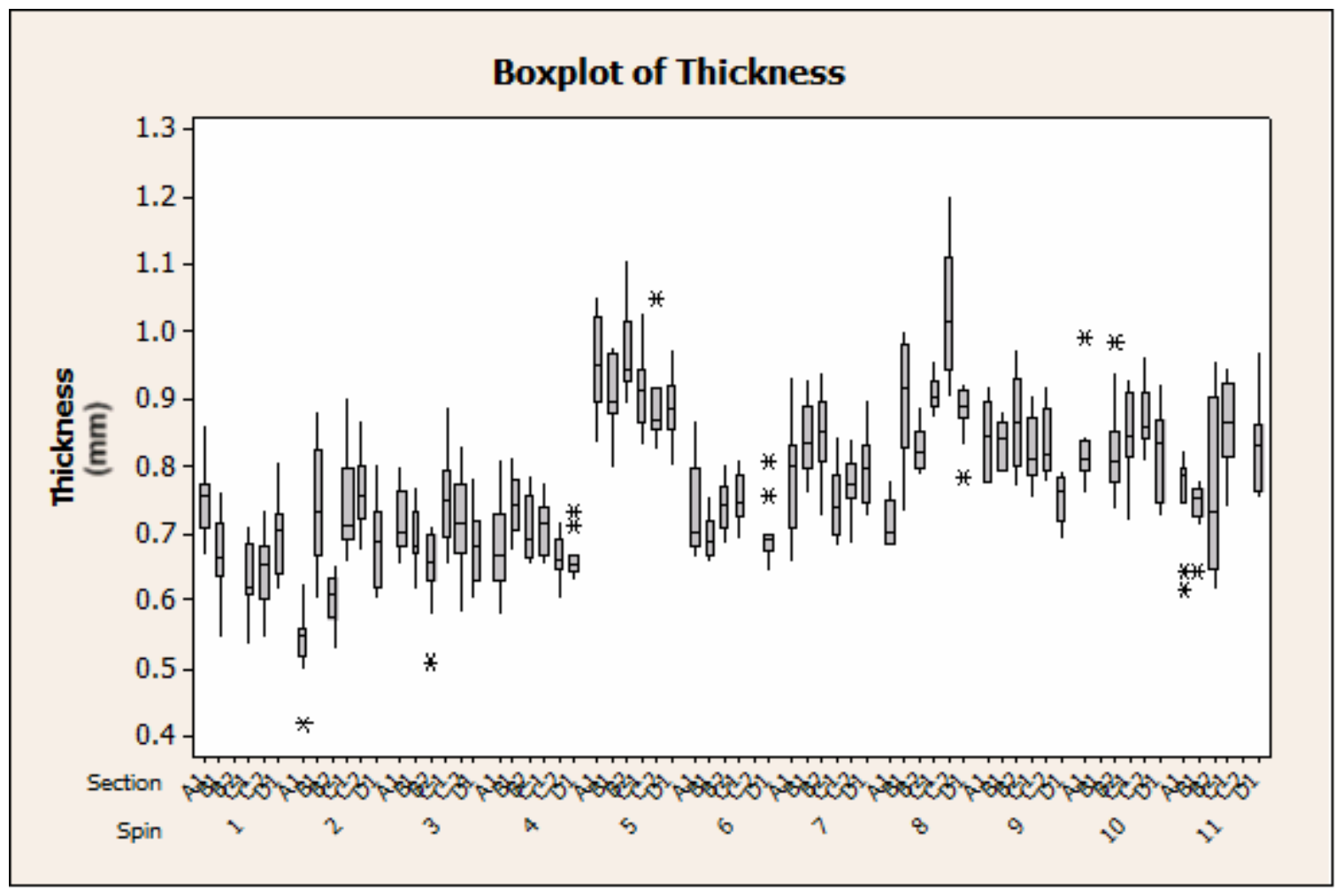

Figure 50 - A boxplot of the cross-sectional thickness data gathered shows how thickness varied. An asterisk indicates an outlier, the vertical lines show the non-outlier data range, and the rectangle on each line represents the interquartile range of each data set. The horizontal line through each interquartile range box marks the median of each data set. 
One-way ANOVA: Thickness versus Spin

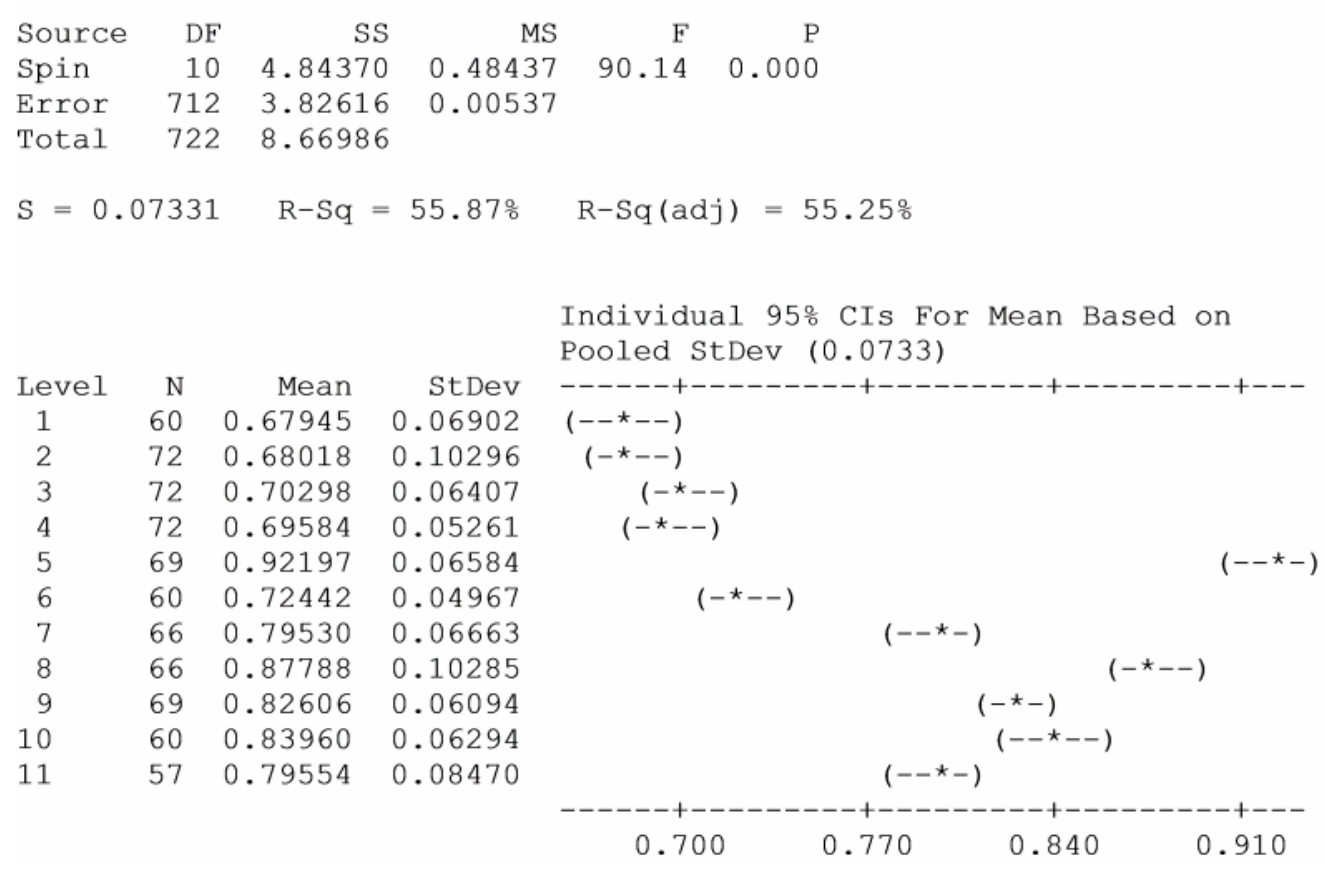

Figure 51 - The ANOVA results comparing scaffold wall thickness between Spins indicates that thickness varied from spin to spin.

Results and Statistical Analysis of Tensile Testing

Minitab 15 was also used to analyze tensile testing data and generate all figures. A histogram of all recorded values for Young's modulus shows a range of values from 2 to $17 \mathrm{MPa}$ (Figure 52). An ANOVA test of Young's modulus data versus spin number indicates that despite the wide range of the data, all consistency spins produced scaffolds that were mechanically similar (Figure 53). 


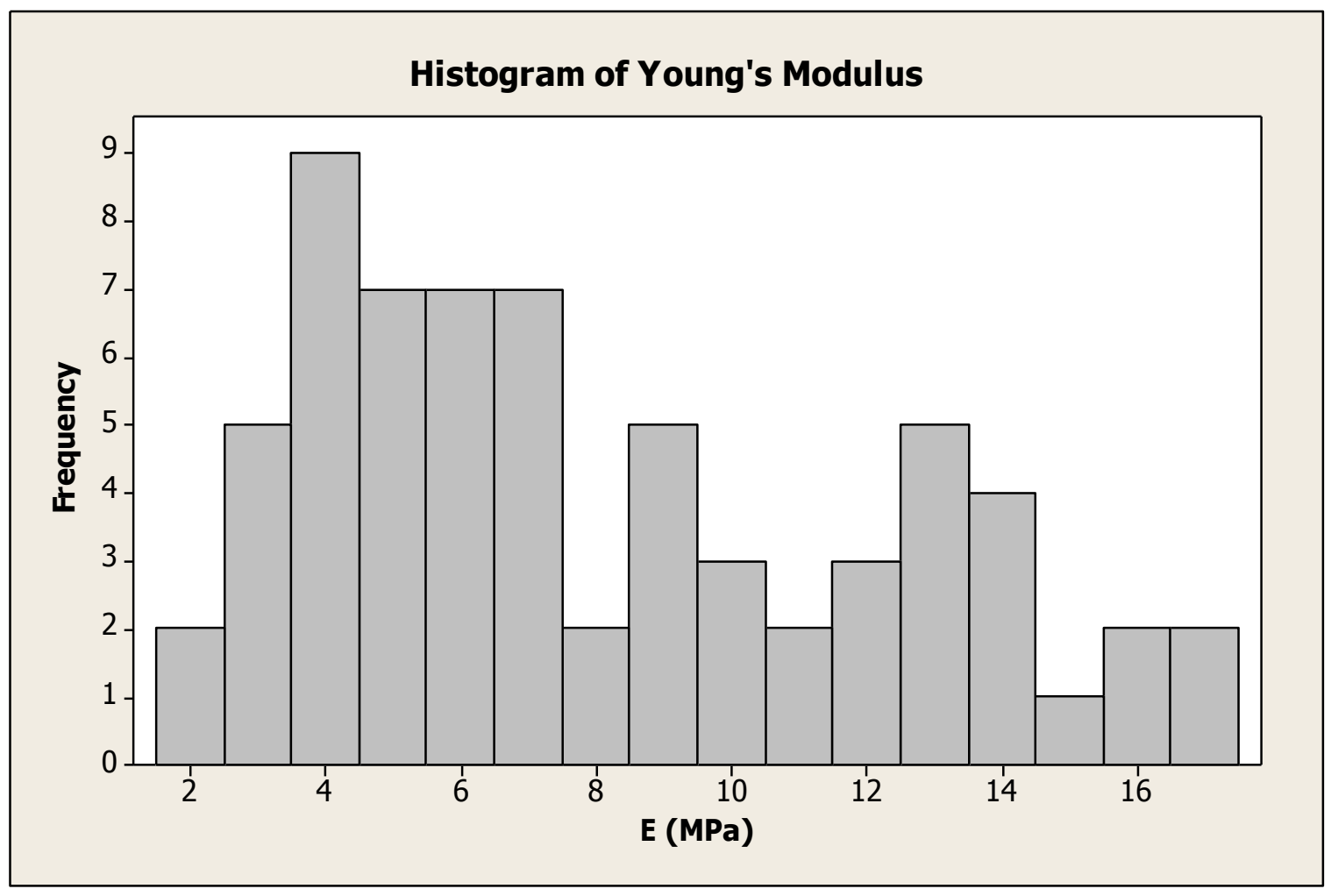

Figure 52 - The range of the data shown in this histogram of Young's modulus values yielded a mean value of $8.1 \mathrm{MPa}$ with a standard deviation of $4.2 \mathrm{MPa}$.

\section{One-way ANOVA: E versus Spin}

$\begin{array}{lrrrrr}\text { Source } & \text { DF } & \text { SS } & \text { MS } & \text { F } & \text { P } \\ \text { Spin } & 10 & 148.2 & 14.8 & 0.82 & 0.608 \\ \text { Error } & 55 & 989.9 & 18.0 & & \\ \text { Total } & 65 & 1138.1 & & & \\ \text { S }=4.242 & \text { R-Sq }=13.02 \% & \text { R-Sq(adj) }=0.00 \%\end{array}$

Figure 53 - An ANOVA analysis of Young's Modulus (E) based on individual spins suggested that all scaffolds were statistically similar $(\mathrm{P}>0.05)$. This analysis does not take into account the effects of testing section $(\mathrm{AB}, \mathrm{BC}$, and $\mathrm{CD})$ or sample orientation (longitudinal or radial pull). 
To better understand what affected the Young's modulus values, several other ANOVA tests were performed. An ANOVA test of Young's modulus versus sample section showed that different sections had no effect on mechanical properties (Figure 54). Sample orientation, however, had a clear effect on Young's modulus values (Figure 55).

\section{One-way ANOVA: E versus Section}

$\begin{array}{lrrrrr}\text { Source } & \text { DF } & \text { SS } & \text { MS } & \text { F } & \text { P } \\ \text { Section } & 2 & 6.1 & 3.1 & 0.17 & 0.843 \\ \text { Error } & 63 & 1131.9 & 18.0 & & \\ \text { Total } & 65 & 1138.1 & & & \\ \text { S }=4.239 & \text { R-Sq }=0.54 \% & \text { R-Sq (adj) }=0.00 \%\end{array}$

Figure 54 - The large P-value from this ANOVA test of Young's Modulus (E) versus testing section $(\mathrm{AB}, \mathrm{BC}$, and $\mathrm{CD})$ indicates that separate sections from all scaffolds were statistically similar. 


\section{One-way ANOVA: E versus Direction}

$\begin{array}{lrrrrr}\text { Source } & \text { DF } & \text { SS } & \text { MS } & \text { F } & \text { P } \\ \text { Direction } & 1 & 631.39 & 631.39 & 79.75 & 0.000 \\ \text { Error } & 64 & 506.67 & 7.92 & & \\ \text { Total } & 65 & 1138.05 & & & \\ \text { S }=2.814 & \text { R-Sq }=55.48 \% & \text { R-Sq (adj) }=54.78 \%\end{array}$

Figure 55 - The wide range of the tensile testing data was caused by mixing data from both testing directions (longitudinal and radial) as indicated by this ANOVA test of Young's modulus (E) versus sample direction (orientation). The P-value is essentially zero, meaning that sample orientation had a definite effect on the tensile testing data.

\section{Discussion and Conclusions}

The goal of the consistency study was to evaluate the reproducibility of the electrospinning process. In summary:

Fiber diameter between scaffolds was inconsistent, but the data indicates a majority of fiber sizes between 6 and 9 microns. The reason behind the variation in fiber diameter among certain consistency spins is unknown. Variation in fiber diameter has been shown to decrease with decreasing solution concentration, though below the threshold solution concentration, the process ceases to be electrospinning and become electrospraying [89, 115]. By lowering the concentration of the P(LLA-CL) solution, as well as adjusting other electrospinning parameters, variation in fiber diameter can be reduced. 
Within individual scaffolds, fiber diameter was consistent, except in the cases of Spin \#5 and Spin \#11. This indicates that fiber diameter across the length of the tubular P(LLA-CL) scaffolds is mostly consistent. Improved electrospinning technique and equipment set-up may increase the intrascaffold consistency.

Wall thickness was inconsistent between spins; thickness values ranged from $0.4 \mathrm{~mm}$ to $1.2 \mathrm{~mm}$. Sample preparation may have contributed to the inconsistent data gathered in this study.

Wall thickness within individual scaffolds was also inconsistent in all but one scaffold (Spin \#10). This is believed to have been caused by areas of lower resistance along the length of the grounded mandrel, resulting in preferential fiber collection sites.

Pooled together, all the data obtained from tensile testing suggests no statistical difference in the mechanical response between consistency spins. However, when the data is examined based on sample orientation, a surprising result emerges. Since the polymer collector is a rotating drum, and this rotation should produce a scaffold composed of circumferentially aligned fibers, it is natural to assume that the scaffold would be mechanically anisotropic with a higher Young's modulus in the radial direction. The results from this study, however, suggest that the scaffolds were actually stiffer in the longitudinal direction. The first possible reason for this is that fibers somehow managed to align themselves longitudinally, not circumferentially. Examination of the SEM images used to gather fiber diameter data showed 
that this was probably not the case. Rather than having one clear direction of fiber orientation, the fibers appeared to be randomly oriented, as if they had been collected on a flat sheet collector. Thus, the probable reason for the discrepancy between data collected from longitudinal pulls and radial pulls lies in the testing method. Specifically, when radial samples were loaded into the tensile tester, they had to be unfurled to create flat sheets. This unfurling action caused internal stresses that severed the connections between many fibers, resulting in a weaker material than the longitudinal samples.

Despite the indicated difference in fiber diameter and wall thickness between scaffolds, mechanical testing data indicated the scaffolds were all the same. These results are counterintuitive since structure (in this case fiber diameter) determines properties (Young's modulus). In other words, scaffolds with different fiber diameters should have different values for Young's modulus.

In conclusion, the question of whether or not electrospinning is a reproducible process in-house at Cal Poly (and thus applicable to the BVM system) has a mixed answer. Morphologically, the process has thus-far proven statistically inconsistent; however, mechanically, the process is statistically consistent. In addition, preliminary cell viability testing on the electrospun $\mathrm{P}(\mathrm{LLA}-\mathrm{CL})$ scaffolding has shown promising results (see Appendix J). With further experimentation into changing parameters such as solution concentration, accelerating voltage, solution flow rate, gap distance, and needle size, scaffolds with tighter fiber diameter, wall thickness, and Young's modulus distributions can be produced. The data presented in this thesis provides a benchmark for 
comparison for future in-house electrospinning research. With further research and

improvements to the process, electrospinning should provide appropriate scaffolding for the BVM. 


\section{DISCUSSION AND FUTURE WORK}

\section{Summary}

This thesis describes work completed to improve the existing in vitro blood vessel mimic (BVM) system used to test and evaluate intravascular devices - specifically, the need to improve the scaffolding used to support the attachment, proliferation, migration, and growth of endothelial cells. This thesis hypothesized that more appropriate scaffolding could be manufactured in-house using an established fabrication technique without increasing scaffolding cost. Research was conducted in two phases. First, using peer reviewed literature to identify and evaluate established candidate fabrication techniques, a method for scaffold production was chosen for further research. The selection was based on a list of criteria which included: process consistency, pore architecture and microstructure, material compatibility, cost, and BVM -specific benefits. This first phase of research was presented in Chapter 2, and results led to the selection of electrospinning as the fabrication method to pursue. The second phase of research tested the hypothesis that the chosen fabrication method (electrospinning) could produce highthroughput scaffolding with consistent morphology and mechanical properties. Chapter 3 presented the series of electrospinning trial experiments used to obtain a basic understanding of the electrospinning process and arrive at a set of fabrication process parameters used to evaluate scaffold consistency. By using the same set of electrospinning parameters to produce multiple scaffolds and examining the structural morphology and mechanical properties of each, process consistency was evaluated. Chapter 4 presented the methods used to gather the consistency data, as well as the 
resulting statistical analysis of the data. This work determined that electrospinning is a viable fabrication technique for producing scaffolding for BVM use. Results from the consistency study showed that average diameter of the microfibers composing the 11 electrospun scaffolds was not statistically similar from scaffold to scaffold, but fibers from the same scaffold were. Scaffold wall thickness proved to be inconsistent both between scaffolds and within the same scaffold. Mechanical response in the form of Young's modulus indicated that the 11 scaffolds were statistically similar. Results from the consistency study set a baseline that could be used for comparison in future work aimed at electrospinning BVM scaffolding.

\section{Electrospun Scaffolds: Novel Alternatives in the Blood Vessel Mimic System}

Tissue engineering was introduced in Chapter 1 of this thesis as a means to potentially replace synthetic materials, like ePTFE, in small diameter vessel applications. Methods used to create small caliber blood vessels using tissue engineering vary widely including various cell sodding procedures, culturing conditions, and scaffolding (see Chapter 1, Section 2). Using established techniques from past research, a blood vessel mimic (BVM) was previously developed. The system utilizes ePTFE as scaffolding, a popular material in large diameter blood vessel replacements. Much research has been conducted on ePTFE and its effectiveness in various applications in the human body. In the case of the BVM, ePTFE is not necessarily the ideal scaffolding; high cost, lack of radial compliance, and necessary out-of-house production are all reasons that scaffolding in the BVM could be optimized. 
With this need in mind, electrospinning was pursued as a possible scaffold fabrication method for replacing ePTFE. Electrospinning has been studied on both a physical phenomenon front and as a method for producing tissue engineering scaffolding. In the case of tissue engineering, the nanoscale fibrous structures that are possible through electrospinning mimic the collagen rich ECM of native blood vessels, and as such may prove to be the scaffolding of choice in future tissue engineered blood vessels. This work was unique in that it did not attempt to produce an implantable tissue engineered blood vessel, as many other groups strive to do [32, 77, 116-123]. Rather, electrospun scaffolding was studied as a method for creating in-vitro blood vessel models never meant to be implanted into hosts. For this reason, the consistency and scalability of electrospinning techniques was the primary focus, which is a unique contribution to the tissue engineering and electrospinning field.

Ultimately, electrospun scaffolding was implemented in the BVM system in a small, preliminary study, and results indicated that cells could adhere to P(LLA-CL) scaffolds in the BVM (see Appendix J). The future work necessary to produce electrospun scaffolding that can be successfully implemented in the BVM is described in the next section. 


\section{Future Work}

Improved Consistency: Further Exploration of Process Parameters

Experimental data on fiber morphology, wall thickness, and mechanical properties of the electrospun P(LLA-CL) was analyzed to set a base line for the consistency of the in-house electrospinning process. Information obtained on the initial consistency of the electrospinning process is not meant to be used as a finishing point. Rather, further research should be conducted and the elctrospinning apparatus modified as needed to increase the consistency of the resulting constructs. Improving consistency may involve many aspects of the electrospinning process, including: making more precise measurements (such gap distance and amount of solution used), exploring other variables in the process (solution feed rate, needle geometry, temperature, humidity, time in solution, and collector to power source resistance), and experimenting with alternate equipment (power supply, collector, and containment chamber). It may also prove worthwhile to repeat the experiments presented in this thesis to determine if operator skill played a role in the consistency of the electrospinning process.

\section{Design of Experiment}

After an electrospinning procedure has been developed that results in desirable scaffolds with respect to microstructure and mechanical properties, process parameters for that specific system should be evaluated using a design of experiment (DOE). A DOE would simplify the process of understanding how the electrospinning process 
parameters affect the final scaffold structure and mechanical properties. Other electrospinning systems have been characterized in this manner, including P(LLA-CL) [89]. A good example of a DOE comes from work by Thompson et al. An in depth analysis of 13 process parameters was performed for several polymer systems to determine which treatments had the most effect on final fiber diameter [114]. While an analysis of 13 parameters may be excessive for electrospun BVM scaffolding, a DOE involving 5 or 6 primary parameters for the in-house electrospinning system would make it easier to tailor scaffolds to desired specifications in the future.

Improving the Electrospinning Apparatus

\section{Power Supply}

The driving force behind the electrospinning process is electrostatic repulsion produced by a high voltage power supply [77]. The current power supply consists of both a high voltage power converter (Matsusada K7-30R) and an external amplifying unit (Heathkit IP-2718). During the trial spin series of experiments, the external amplifier broke when turned past 6 volts due to the amount of current being drawn through it. This limits the accelerating voltage possible to $18 \mathrm{kV}$. As future scaffolding may require higher accelerating voltages, a replacement power supply may be necessary. A replacement power supply should be a self contained power converter and amplification unit with a voltage output meter and, possibly, a current output meter. The output current should be kept as low as possible to minimize the hazardousness of the electrospinning 
process, but the output voltage possible should be at least $30 \mathrm{kV}$ to allow for a variety of possible electrospun constructs. A good replacement would be ES30R-5W from Gamma High Voltage Research, or the CZE1000R from Spellman High Voltage.

Containment Chamber

Minimizing the influence of external electromagnetic fields and grounded surfaces is important in the electrospinning process in order to generate consistent jet dynamics between spins and to ensure that the jet strikes the collector surface [124]. With this in mind, the containment chamber could be improved. For example, the access panel should seal better to prevent perturbation of the jet. Also, research has shown that carefully oriented electrodes can influence jet dynamics [81, 125]; a new containment chamber could incorporate a series of electrodes designed to influence the jet in flight, the orientation of collected fibers, or both.

\title{
Characterization of Constructs
}

\author{
Alternate Techniques
}

Alternate testing methods may also improve the validity and accuracy of a consistency rating for these particular constructs without changing any aspect of the process. Consider, for example, a process such as mercury porosimetry, which is based on the principle that the pressure required for a non-wetting liquid like mercury to 
overcome liquid surface tension and be forced into pores is indicative of pore size [126]. Mercury porosimetry could be used to find a value for average pore size of electropsun scaffolds, and also to compare the pressures required to completely infiltrate all pores. Statistical analysis of these pressures is another method that could be used to evaluate consistency.

Improved Tensile Testing

As explained in the Chapter 4, tensile testing samples were rectangular sheets cut from the tubular constructs. In retrospect, using rectangular samples probably contributed to a lack of precision in the mechanical testing data for several reasons. First, while thickness measurements were carefully conducted using SEM analysis, width measurements of the sample were obtained using a simple ruler with an accuracy of \pm $1 \mathrm{~mm}$. Second, the action of unfolding the electrospun constructs into rectangular sheets appeared to disrupt the cohesive structure of the construct matrix. The flattening of tubular samples results in a compressive force on the exterior surface of the constructs and a tensile force on the interior surface. These opposing forces most likely caused separation of fibers in the constructs below the midpoint of the thickness. The effect was more pronounced in radially-oriented samples because the amount of unrolling needed to produce a flat, rectangular tensile testing sample was much greater than that of longitudinally oriented samples. In the future, rectangular samples should not be used for tensile testing mainly due to the probable damage done to electrospun constructs during 
sample preparation. Rather, tensile tests should be conducted on unaltered tubular samples (Figure 56).

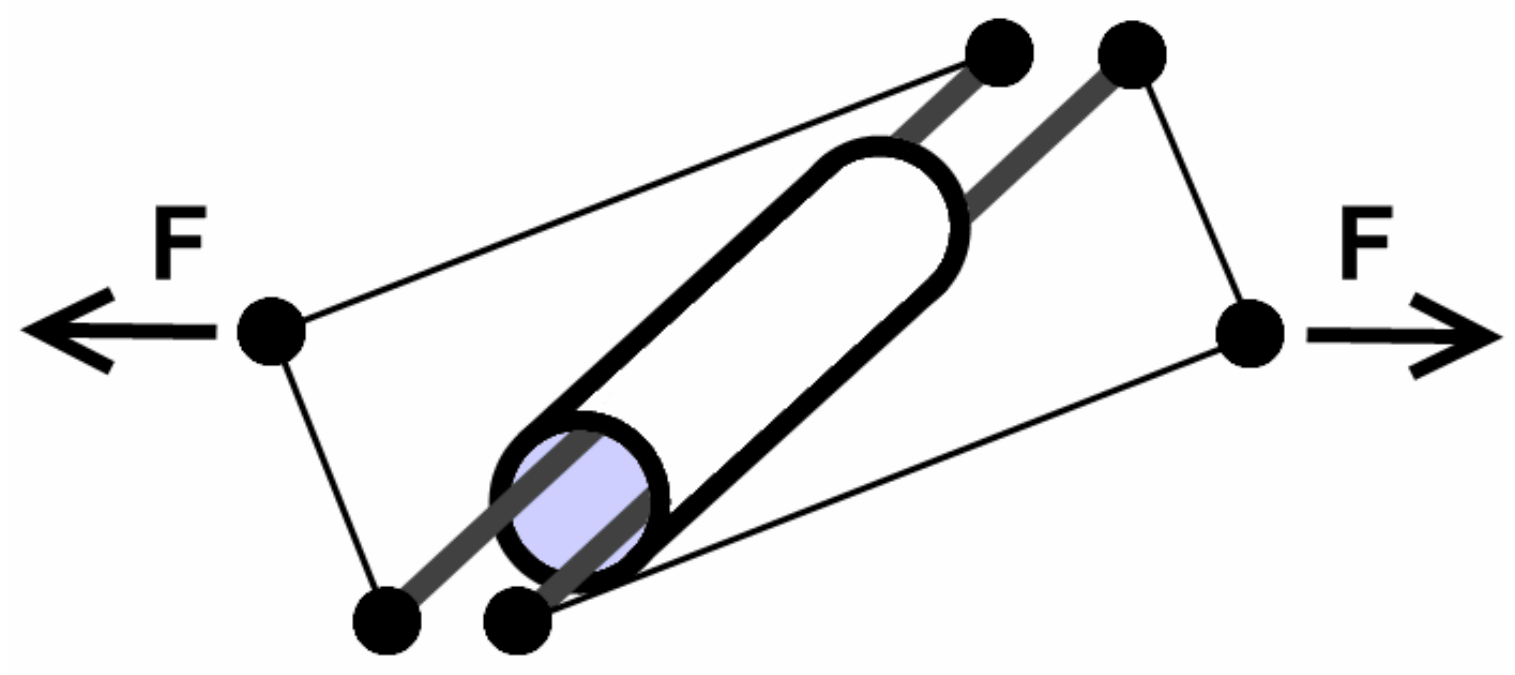

Figure 56 - One possible tensile testing alternative to rectangular samples. A custom fixture would be constructed that uses two small rods to pull the electrospun tubular construct apart.

Improved Thickness Measurement

Sample preparation methods used for wall thickness samples involved an excess of contact with the scaffold. Accidental pressure from contact with the scaffold could have decreased the true cross-sectional thickness. This problem seems probable due to the lower values for wall thickness gathered from earlier spins; at this stage, sample preparation was not as refined as in later spins. To alleviate this problem, alternate 
thickness measurement techniques should be explored. Ideally, thickness could be quickly measured with the scaffold still on the mandrel using a method like ultrasonic imaging. This would not only save time and increase the accuracy of wall thickness data, but the thickness at any point along the scaffold length could be easily obtained.

Physiologically Accurate Fiber Production

Although this work successfully produced fibers with micron-scale crosssectional diameters, electrospinning is capable of producing nanoscale fibers. Literature indicates that nanoscale fibers may be ideal for cell incorporation since they mimic the natural structure of the ECM $[89,123]$. The parameters necessary to electrospin fibers that mirror the size of collagen is not discrete; small fibers may be produced by changing electrospinning process parameters while using the same polymer, or by experimenting with different polymers $[80,87,111,125,127,128]$. Future work should focus on both approaches in order to build a library of available scaffolds for use in the BVM. In addition, incorporation of biomacromolecules such as collagen and elastin is an avenue worth exploring that might improve the physiological appropriateness of electrospun scaffolding for the BVM $[122,129]$. 


\section{Conclusion}

This thesis has identified, created, and utilized a new fabrication technique for creating blood vessel mimic scaffolding that can potentially replace ePTFE. As the goal of the BVM is to provide an in-vitro intravascular device testing environment as similar to native human blood vessel as possible, highly tailorable scaffolding is desirable in order to produce blood vessel mimics with various geometries and mechanical properties. Information presented here is meant as a starting point for future research that seeks to improve the in-house electrospinning process and its role in the BVM. Electrospinning as a scaffold fabrication technique has the potential to produce consistent, tailorable, and inexpensive constructs made in-house for use in the BVM. 


\section{BIBLIOGRAPHY}

1. Marieb, E.N., Anatomy and Physiology. 2 ed. 2005, San Francisco, CA: Pearson Benjamin Cummings.

2. Association, A.H. Cardiovascular Disease Statistics. 2009 [cited 2009;

Available from: http://www.americanheart.org/presenter.jhtml?identifier=4478.

3. Association, A.H. Cardiovascular Disease Cost. 2009 [cited 2009; Available from: http://www.americanheart.org/presenter.jhtml?identifier=4475.

4. Arrone, L.J., W.V. Brown, and K.K. Isoldi, Cardiovascular disease in obesity: A review of related risk factors and risk reduction strategies. Journal of Clinical Lipidology, 2007. 1(6): p. 575-582.

5. Wagner, A.M. and e. al, Diabetes mellitus and cardiovascular disease. European Journal of Internal Medicine, 2002. 13: p. 15-30.

6. Lloyd-Jones, D., et al., Heart Disease and Stroke Statistics 2009 Update: A report from the American Heart Association Statistics Committee and Stroke Statistics Subcommittee. Circulation, 2009. 119: p. 21-181.

7. Braunwald, E., et al., Principles of Internal Medicine. 15 ed. 2001, New York, NY: McGraw-Hill.

8. Ratner, B.D., et al., Biomaterials Science: An Introduction to Materials in Medicine. 2 ed. 2004, San Diego, CA: Elsevier Academic Press.

9. Association, A.H. Cholesterol Media Library-Atherosclerosis. 2009 [cited 2009; Available from:

http://www.medmovie.com/mmdatabase/MediaPlayer.aspx?ClientID=67\&TopicI $\underline{\mathrm{D}=573}$ 
10. Landau, C., R.A. Lange, and D.L. Hillis, Percutaneous transluminal coronary angioplasty. New England Journal of Medicine, 1994. 330(14): p. 981-993.

11. Virmani, R., A. Farb, and A.P. Burke, Coronary angioplasty from the perspective of the atherosclerotic plaque: morphological predictors of immediate success and restenosis. American Heart Journal, 1994. 127: p. 163-179.

12. Haudenschild, C.C., Pathiobiology of restenosis after angioplasty. American Journal of Medicine, 1993. 94(supplement): p. 40-44.

13. Surruys, P.W., et al., A comparison of balloon-expandable-stent implantation with balloon angioplasty in patients with coronary artery disease. New England Journal of Medicine, 1994. 331: p. 489-495.

14. Stone, G.W., et al., Comparison of angioplasty with stenting, with or without abciximab, in acute myocardial infarction. New England Journal of Medicine, 2002. 346: p. 957-966.

15. Virmani, R. and A. Farb, Pathology of in-stent restenosis. Current Opinion in Lipidology, 1999. 10: p. 499-506.

16. Association, A.H. What is a Stent? 2007 [cited 2009; Available from: http://www.americanheart.org/downloadable/heart/1196103176378\%206\%20Wh atIsaStent.pdf

17. Sun, J., et al., Role for p27(Kipl) in vascular smooth muscle cell migration. Circulation, 2001. 103: p. 2967-2972.

18. Abal, M., J.M. Andreu, and I. Barasoain, Taxanes: Microtubule and centrosome targets, and cell cycle dependent mechanisms of action. Current Cancer Drug Targets, 2003. 3: p. 193-203. 
19. Finn, A.V., et al., Vascular responses to drug eluting stents: Importance of delayed healing. Arteriosclerotic Thrombotic Vascular Biology, 2007. 27: p. $1500-1510$.

20. Guyton, A.C. and J.E. Hall, Textbook of Medical Physiology. 11 ed. 2006, Philadelphia, PA: Elsevier Saunders.

21. Baim, D.S., Percutaneous treatment of saphenous vein graft disease: the ongoing challenge. Journal of the American College of Cardiology, 2003. 42: p. 13701372.

22. Cooper, G.J., P.B. Deverall, and M.J. Underwood, Arterial and venous conduits for coronary artery bypass: A current review. European Journal of CardioThoracic Surgery, 1996. 10(2): p. 129-140.

23. Suma, H., Arterial grafts in coronary bypass surgery. Annals of Thoracic and Cardiovascular Surgery, 1999. 5: p. 141-145.

24. Association, A.H.A.A.S. Heart Attack Media Library - Coronary Artery Bypass Grafting (CABG). 2009 [cited 2009; Available from: http://www.medmovie.com/mmdatabase/MediaPlayer.aspx?ClientID=68\&TopicI $\underline{\mathrm{D}=557}$

25. Clagett, G.P., What's new in vascular surgery? Journal of the American College of Surgeons, 2002. 194: p. 165-201.

26. Bos, G.W., et al., Small diameter vascular graft prosthesis: Current status. Archives of Physiological Biochemistry, 1998. 106: p. 100-115.

27. Bianco, P., et al., Cell Source, in Tissue Engineering, C.V. Blitterswijk, Editor. 2008, Elsevier Academic Press: London, UK. p. 279-306. 
28. Moneta, G.L. and J.M. Porter, Arterial substitutes in peripheral vascular surgery: a review. Journal of Long-Term Effects of Medical Implants, 1995. 5: p. 47-67.

29. Fisher, J.P., A.G. Mikos, and J.D. Bronzino, eds. Tissue Engineering. 2007, CRC Press: Boca Raton, FL.

30. Tissue Engineering. 1 ed, ed. C.v. Blitterswijk. 2008, London, UK: Elsevier Academic Press.

31. Blitterswijk, C.A.v., et al., Tissue Engineering - an Introduction, in Tissue Engineering, C.A.v. Blitterswijk, Editor. 2008, Elsevier Academic Press: London. p. XV.

32. Boland, E.D., et al., Electrospinning collagen and elastin: preliminary vascular tissue engineering. Front Biosci, 2004. 9: p. 1422-32.

33. Wolinsky, H. and S. Glagov, A lamellar unit of aortic medial structure and function in mammals. Circulation Research, 1967. 20(1): p. 99-111.

34. Heyligers, J.M.M., et al., Improving Small-Diameter Vascular Grafts: From the Application of an Endothelial Cell Lining to the Construction of a TissueEngineered Blood Vessel. Annals of Vascular Surgery, 2005. 19: p. 1-9.

35. Meinhart, J.G., et al., Clinical autologous in vitro endothelialization of 153 infrainguinal ePTFE grafts. Annals of Thoracic Surgery, 2001. 71: p. S327-331.

36. Deutsch, M., et al., Clinical autologous in vitro endothelialization of infrainguinal ePTFE grafts: Long-term experience in 318 patients. Journal of Vascular Surgery, 2008. 
37. Sarkar, S., et al., The mechanical properties of infrainguinal vascular bypass grafts: Their role in influencing patency. European Journal of Vascular and Endovascular Surgery, 2006. 31(6): p. 627-636.

38. Casey, P.J., et al., The effect of combined arterial hemodynamics on saphenous venous endothelial nitric oxide production. Journal of Vacular Surgery, 2001. 33: p. $1199-1205$.

39. Ethier, C.R. and C.A. Simmons, Introductory Biomechanics: From Cells to Organisms, ed. W.M. Saltzman. 2007, New York, NY: Cambridge University Press.

40. Wagner, D.D., J.B. Olmstead, and V.J. Marder, Immunolocalization of von Willebrand protein in Weibel-Palade bodies of human endothelial cells. Journal of Cell Biology, 1982. 95: p. 355-360.

41. Voyta, J.C., et al., Identification and isolation of endothelial cells based on their increased uptake of acetylated-low density lipoprotein. Journal of Cell Biology, 1984. 99: p. 2034-2040.

42. Skalli, O., et al., A monoclonal antibody against alpha-smooth muscle actin: A new probe for smooth muscle differentiation. The Journal of Cell Biology, 1986. 103: p. 2787-2796.

43. Trubel, W., et al., Compliance mismatch and formation of distal anastomotic intimal hyperplasia in externally stiffened and lumen-adapted venous grafts. European Journal of Vascular and Endovascular Surgery, 1995. 10: p. 415-423.

44. L'Heureux, N., et al., A completely biological tissue-engineered human blood vessel. FASEB Journal, 1998. 12: p. 47-56. 
45. Weinberg, C.B. and E. Bell, A blood vessel model constructed from collagen and cultured vascular cells. Science, 1986. 231: p. 397-400.

46. Girton, T.S., T.R. Oegema, and R.T. Tranquillo, Exploiting glycation to stiffen and strengthen tissue equivalents for tissue engineering. Journal of Biomedical Materials Research, 1999. 46: p. 87-92.

47. Girton, T.S., et al., Mechanisms of stiffening and strengthening in mediaequivalents fabricated using glycation. Journal of Biomedical Engineering, 2000. 122: p. 216-223.

48. Athanasiou, K.A., G.G. Niederauer, and C.M. Agrawal, Sterilization, toxicity, biocompatibility and clinical applications of polylactic acid/polyglycolic acid copolymers. Biomaterials, 1996. 17: p. 93-102.

49. Mooney, D.J., et al., Stabilized polyglycolic acid fibre-based tubes for tissue engineering. Biomaterials, 1996. 17: p. 115-124.

50. Shinoka, T., et al., Creation of viable pulmonary artery autografts through tissue engineering. Journal of Thoracic Cardiovascular Surgery, 1998. 115: p. 536-546.

51. Niklason, L.E., et al., Functional arteries grown in vitro. Science, 1999. 284: p. 489-493.

52. Cardinal, K.O.H., et al., Tissue-engineered vascular grafts as in vitro blood vessel mimics for the evaluation of endothelialization of intravascular devices. Tissue Engineering, 2006. 12(12): p. 1-8.

53. Zilla, P., D. Bezuidenhout, and P. Human, Prosthetic vascular grafts: Wrong models, wrong questions and no healing. Biomaterials, 2007. 28: p. 5009-5027. 
54. Dawson, M., Implementation of physiologic flow conditions in a blood vessel mimic bioreactor system for the evaluation of intravascular devices, in Biomedical Engineering. 2009, California Polytechnic State University: San Luis Obispo. p. 151.

55. Alvarez-Barreto, J.F. and V.I. Sikavitsas, Tissue Engineering Bioreactors, in Tissue Engineering, J.P. Fisher, A.G. Mikos, and J.D. Bronzino, Editors. 2007, CRC Press: Boca Raton, FL.

56. Leifer, S., Design and optimization of a blood vessel mimic bioreactor system for the evaluation of intravascular devices in simple and complex vessel geometries, in Biomedical Engineering. 2008, California Polytechnic State University: San Luis Obispo.

57. Casey, P.J., et al., The effect of combined arterial hemodynamics on saphenous venous endothelial nitric oxide production. Journal of Vascular Surgery, 2001. 33: p. 1199-1205.

58. Hutmacher, D., et al., Scaffold Design and Fabrication, in Tissue Engineering, C.V. Blitterswijk, Editor. 2008, Elsevier Academic Press: London, UK. p. 403454.

59. Schmack, G., et al., Biodegradable fibers of poly(L-lactide) produced by highspeed melt spinning and spin drawing. Journal of Applied Polymer Science, 1999. 73: p. 2785-2797.

60. Leidner, J., et al., A novel process for the manufacturing of porous grafts: Process description and product evaluation. Journal of Biomedical Materials Research, 1983. 17: p. 229-247. 
61. Mikos, A.G., et al., Preparation of poly (glycolic acid) bonded fiber structures for cell attachment and transplantation. Journal of Biomedical Materials Research, 1993. 27(2): p. 183-189.

62. Ma, P.X., et al., Development of biomechanical properties and morphogenesis of in-vitro tissue engineered cartilage. Journal of Biomedical Materials Research, 1995. 29(12): p. 1587-1595.

63. Mikos, A.G., et al., Preparation and characterization of poly(L-lactic acid) foams. Polymer, 1994. 35(5): p. 1068-1077.

64. Mooney, D.J., et al., Biodegradable sponges for hepatocyte transplantation. Journal of Biomedical Materials Research, 1995. 29(8): p. 959-965.

65. Nam, Y.S. and T.G. Park, Biodegradable polymeric microcellular foams by modified thermally induced phase separation method. Biomaterials, 1999. 20: p. 1783-1790.

66. Dalton, P.D., L. Flynn, and M.S. Shoichet, Manufacture of poly(2-hydroxyethyl methacrylate-co-methyl methacrylate) hydrogel tubes for use as nerve guidance channels. Biomaterials, 2002. 23: p. 3843-3851.

67. Liu, S.Q. and M. Kodama, Porous polyurethane vascular prostheses with variable compliances. Journal of Biomedical Materials Research, 1992. 26(11): $\mathrm{p}$. $1489-1502$.

68. Cima, M., et al., Three-dimensional printing techniques, in Google Patent, U.P. Office, Editor. 1995: United States of America.

69. Lam, C.X.F., et al., Scaffold development using 3D printing with a starch-based polymer. Materials Science and Engineering C, 2002. 20: p. 49-56. 
70. Tay, B.Y., et al., Processing of polycaprolactone porous structure for scaffold development. Journal of Materials Processing Technology, 2007. 182: p. 117-121.

71. Lee, M., J.C.Y. Dunn, and B.M. Wu, Scaffold fabrication by indirect threedimensional printing. Biomaterials, 2005. 26: p. 4281-4289.

72. Hutmacher, D.W., M. Sittinger, and M.V. Risbud, Scaffold-based tissue engineering: rationale for computer-aided design and solid free-form fabrication systems. Trends in Biotechnology, 2004. 22(7): p. 354-362.

73. Leong, K.F. and C.K. Chua, Solid freeform fabrication of three-dimensional scaffolds for engineering replacement tissues and organs. Biomaterials, 2003. 24(13): p. 2362-2378.

74. Lee, J.W., et al., Fabrication and characteristic analysis of a poly(propylene fumarate) scaffold using micro-stereolithography technology. Journal of Biomedical Materials Research Part B: Applied Biomaterials, 2008. 87B: p. 1-9.

75. Kim, J.Y., et al., Development of a bone scaffold using HA nanopowder and micro-steroelithography technology. Microelectronic Engineering, 2007. 84(5-8): p. $1762-1765$.

76. Tan, K.H., et al., Scaffold development using selective laser sintering of polyetheretherketone-hydroxyapatite biocomposite blends. Biomaterials, 2003. 24: p. 3115-3123.

77. Lannutti, J., et al., Electrospinning for tissue engineering scaffolds. Materials Science and Engineering C, 2007. 27: p. 504-509.

78. Reneker, D.H. and A.L. Yarin, Electrospinning jets and polymer nanofibers. Polymer, 2008. 49: p. 2387-2425. 
79. Cao, Y., et al., The influence of architecture on degradation and tissue ingrowth into three-dimensional poly(lactic-co-glycolic acid) scaffolds in vitro and in vivo. Biomaterials, 2006. 27: p. 2854-2864.

80. Boland, E.D., et al., Tailoring tissue engineering scaffolds using electrostatic processing techniques: a study of poly(glycolic acid) electrospinning. Journal of Macromolecular Science - Pure Applied Chemistry, 2001. A38(12): p. 12311243.

81. Dalton, P.D., D. Klee, and M. Moller, Electrospinning with dual collection rings. Polymer, 2005. 46: p. 611-614.

82. Williamson, M.R. and A.G.A. Coombes, Gravity spinning of polycaprolactone fibres for applications in tissue engineering. Biomaterials, 2004. 25: p. 459-465.

83. Hutmacher, D.W., Scaffolds in tissue engineering bone and cartilage. Biomaterials, 2000. 21: p. 2529-2543.

84. Dalton, P.D., T. Woodfield, and D.W. Hutmacher, Snapshot: Polymer scaffolds for tissue engineering. Biomaterials, 2009. 30(4): p. 701-702.

85. Fambri, L., et al., Biodegradable fibres: Poly L-lactic fibres produced by solution spinning. Journal of Materials Science: Materials in Medicine, 1994. 5: p. 679683.

86. Widmer, M.S., et al., Manufacture of porous biodegradable polymer conduits by an extrusion process for guided tissue regeneration. Biomaterials, 1998. 19: $\mathrm{p}$. 1945-1955. 
87. Mo, X., H.J. Weber, and S. Ramakrishna, PCL-PGLA composite tubular scaffold preparation and biocompatibility investigation. International Journal of Artificial Organs, 2006. 8: p. 790-799.

88. Hua, F.J., T.G. Park, and D.S. Lee, A facile preparation of highly interconnected macroporous poly(D,L-lactic acid-co-glycolic acid) (PLGA) scaffolds by liquidliquid phase separation of a PLGA-dioxane-water ternary system. Polymer, 2003. 44: p. 1911-1920.

89. Mo, X.M., et al., Electrospun P(LLA-CL) nanofiber: a biomimetic extracellular matrix for smooth muscle cell and endothelial cell proliferation. Biomaterials, 2004. 25(10): p. 1883-90.

90. Reneker, D.H., et al., Nanofiber garlands of polycaprolactone by electrospinning. Polymer, 2002. 43: p. 6785-6794.

91. Dalton, P.D., et al., Electrospinning of polymer melts: Phenomenological observations. Polymer, 2007. 48: p. 6823-6833.

92. Williams, J.M., et al., Bone tissue engineering using polycaprolactone scaffolds fabricated via selective laser sintering. Biomaterials, 2005. 26(23): p. 4817-4827.

93. Gilbert, T.W., et al., Production and characterization of ECM powder: implications for tissue engineering applications. Biomaterials, 2005. 26(12): $\mathrm{p}$. $1431-1435$.

94. Matsuda, T. and Y. Nakayama, Surface microarchitectural design in biomedical applications: in vitro transmural endothelialization on microporous segmented polyurethane films fabricated using an excimer laser. Journal of Biomedical Materials Research, 1996. 31(2): p. 235-242. 
95. Heijkants, R.G.J.C., et al., Polyurethane scaffold formation via a combination of salt leaching and thermally induced phase separation. Journal of Biomedical Materials Research Part A, 2008. 87(4): p. 921-932.

96. Lickorish, D., L. Guan, and J.E. Davies, A three-phase, fully resorbable, polyester/calcium phosphate scaffold for bone tissue engineering: Evolution of scaffold design. Biomaterials, 2007. 28: p. 1495-1502.

97. Williamson, M.R., R. Black, and C. Kielty, PCL-PU composite vascular scaffold production for vascular tissue engineering: attachment, proliferation and bioactivity of human vascular endothelial cells. Biomaterials, 2006. 27(19): p. 3608-16.

98. Hokugo, A., T. Takamoto, and Y. Tabata, Preparation of hybrid scaffold from fibrin and biodegradable polymer fiber. Biomaterials, 2006. 27: p. 61-67.

99. Mezghani, K. and J.E. Spruiell, High Speed Melt Spinning of Poly(L-lactic acid) Filaments. Journal of Polymer Science: Part B: Polymer Physics, 1998. 36: p. 1005-1012.

100. Telemeco, T.A., et al., Regulation of cellular infiltration into tissue engineering scaffolds composed of submicron diameter fibrils produced by electrospinning. ActaBiomaterialia, 2005. 1: p. 376-384.

101. Balguid, A., et al., Tailoring fiber diameter in electrospun poly(E-caprolactone) scaffolds for optimal cellular infiltration in cardiovascular tissue engineering. Tissue Engineering: Part A, 2009. 15(2): p. 437-444. 
102. Kwon, I.K., S. Kidoaki, and T. Matsuda, Electrospun nano- to microfiber fabrics made of biodegradable copolyesters: structural characteristics, mechanical properties and cell adhesion potential. Biomaterials, 2005. 26: p. 3929-3939.

103. Huang, Z.-M., et al., A review on polymer nanofibers by electrospinning and their applications in nanocomposites. Composites Science and Technology, 2003. 63: p. $2223-2253$.

104. Zeleny, J., The electrical discharge from liquid points, and a hydrostatic method of measuring the electric intensity at their surfaces. The Physical Review, 1914. 3(2): p. 69-91.

105. Zeleny, J., Instability of electrified liquid surfaces. The Physical Review, 1917. 10(1): p. 1-7.

106. Formhals, A., Process and apparatus for preparing artificial threads, U.S.P. Office, Editor. 1934: United States.

107. Taylor, S.G., Electrically driven jets. Proceedings of the Royal Society of London A, 1969. 313: p. 453-475.

108. Warner, S.B., et al., A fundamental invesitigation of the formation and properties of electrospun fibers, in National Textile Center Annual Report. 1998, National Textile Center. p. 83-90.

109. Reneker, D.H., et al., Bending instability of electrically charged liquid jets of polymer solutions in electrospinning. Journal of Applied Physics, 2000. 87(9): p. $4531-4547$. 
110. Taylor, G., Disintegration of water drops in an electric field. Proceedings of the Royal Society of London. Series A, Mathematical and Physical Sciences, 1964. 280: p. 383-397.

111. Deitzel, J.M., et al., The effect of processing variables on the morphology of electrospun nanofibers and textiles. Polymer, 2001. 42: p. 261-272.

112. Huebner, A.L. and H.N. Chu, Instability and breakup of charged liquid jets. Journal of Fluid Mechanics, 1971. 49: p. 361-372.

113. Theron, S.A., E. Zussman, and A.L. Yarin, Experimental investigation of the governing parameters in the electrospinning of polymer solutions. Polymer, 2004. 45: p. 2017-2030.

114. Thompson, C.J., et al., Effects of parameters on nanofiber diameter determined from electrospinning model. Polymer, 2007. 48: p. 6913-6922.

115. Deitzel, J.M., et al., The effect of processing variables on the morphology of electrospun nanofibers and textiles. Polymer, 2001. 42: p. 261-272.

116. Bhattarai, S.R., et al., Novel biodegradable electrospun membrane: scaffold for tissue engineering. Biomaterials, 2004. 25(13): p. 2595-602.

117. Boland, E.D., et al., Electrospinning of tissue engineered scaffolds, in ACS. 2001.

118. Chen, M., et al., Role of fiber diameter in adhesion and proliferation of NIH 3 T3 fibroblast on electrospun polycaprolactone scaffolds. Tissue Eng, 2007. 13(3): p. $579-87$.

119. Hutmacher, D.W., Scaffold design and fabrication technologies for engineering tissues - state of the art and future perspectives. Journal of Biomaterials Science Polymer Edition, 2001. 12(1): p. 107-124. 
120. Inoguchi, H., et al., Mechanical responses of a compliant electrospun poly $(L-$ lactide-co-epsilon-caprolactone) small-diameter vascular graft. Biomaterials, 2006. 27(8): p. 1470-8.

121. Jeong, S.I., et al., Tissue-engineered vascular grafts composed of marine collagen and PLGA fibers using pulsatile perfusion bioreactors. Biomaterials, 2007. 28(6): p. $1115-22$.

122. Kwon, I.K. and T. Matsuda, Co-electrospun nanofiber fabrics of poly(L-lactideco-epsilon-caprolactone) with type I collagen or heparin. Biomacromolecules, 2005. 6(4): p. 2096-105.

123. Li, W.-J., et al., Electrospun nanofibrous structure: A novel scaffold for tissue engineering. Journal of Biomedical Materials Research, 2002. 60: p. 613-621.

124. Frenot, A. and I. Chronakis, Polymer nanofibers assembled by electrospinning. Current Opinion in Colloid and Interface Science, 2003. 8: p. 64-75.

125. Deitzel, J.M., et al., Controlled deposition of electrospun poly(ethylene oxide) fibers. Polymer, 2001. 42: p. 8163-8170.

126. Ho, S.T. and D.W. Hutmacher, A comparison of micro CT with other techniques used in the characterization of scaffolds. Biomaterials, 2006. 27: p. 1362-1376.

127. Matsuda, T., et al., Mechano-active scaffold design of small-diameter artificial graft made of electrospun segmented polyurethane fabrics. Journal of Biomedical Materials Research, 2005. 73A: p. 125-131.

128. Yao, L., et al., Electrospinning and Stabilization of Fully Hydrolyzed Poly(Vinyl Alcohol) Fibers. Chemical Materials, 2003. 
129. Wnek, G.E., et al., Electrospinning of Nanofiber Fibrinogen Structures. Nano Letters, 2002. 0. 


\title{
APPENDIX A: LIST OF ABBREVIATIONS
}

\author{
ANOVA - Analysis of Variance \\ BVM - Blood Vessel Mimic \\ CAD - Computer Aided Design \\ CAD - Coronary Artery Disease \\ CVD - Cardiovascular Disease \\ DOE - Design of Experiment \\ EC - Endothelial Cell \\ ECM - Extracellular Matrix \\ ePTFE - Expanded Polytetrafluoroethylene \\ FDA - Food and Drug Administration \\ HMVECS - Human Microvessel Endothelial Cells \\ IHD - Ischemic Heart Disease \\ P(LLA-CL) - Poly(L-Lactic-co-Caprolactone) \\ PCL - Polycaprolactone \\ PGA - Polyglycolic Acid \\ PLGA - Poly(Lactic-co-Glycolic Acid) \\ PLLA - Poly(L-Lactic Acid) \\ PTCA - Percutaneous Transluminal Coronary Angioplasty \\ PU - Polyurethane \\ SCPL - Solvent Casting Particulate Leaching \\ SEM - Scanning Electron Microscope \\ SFF- Solid Freeform Fabrication \\ SLS - Selective Laser Sintering \\ SMC - Smooth Muscle Cell \\ TEVG - Tissue Engineered Vascular Graft \\ TIPS - Thermally Induced Phase Separation
}


APPENDIX B: MIXING P(LLA-CL) SOLUTIONS FOR ELECTROSPINNING

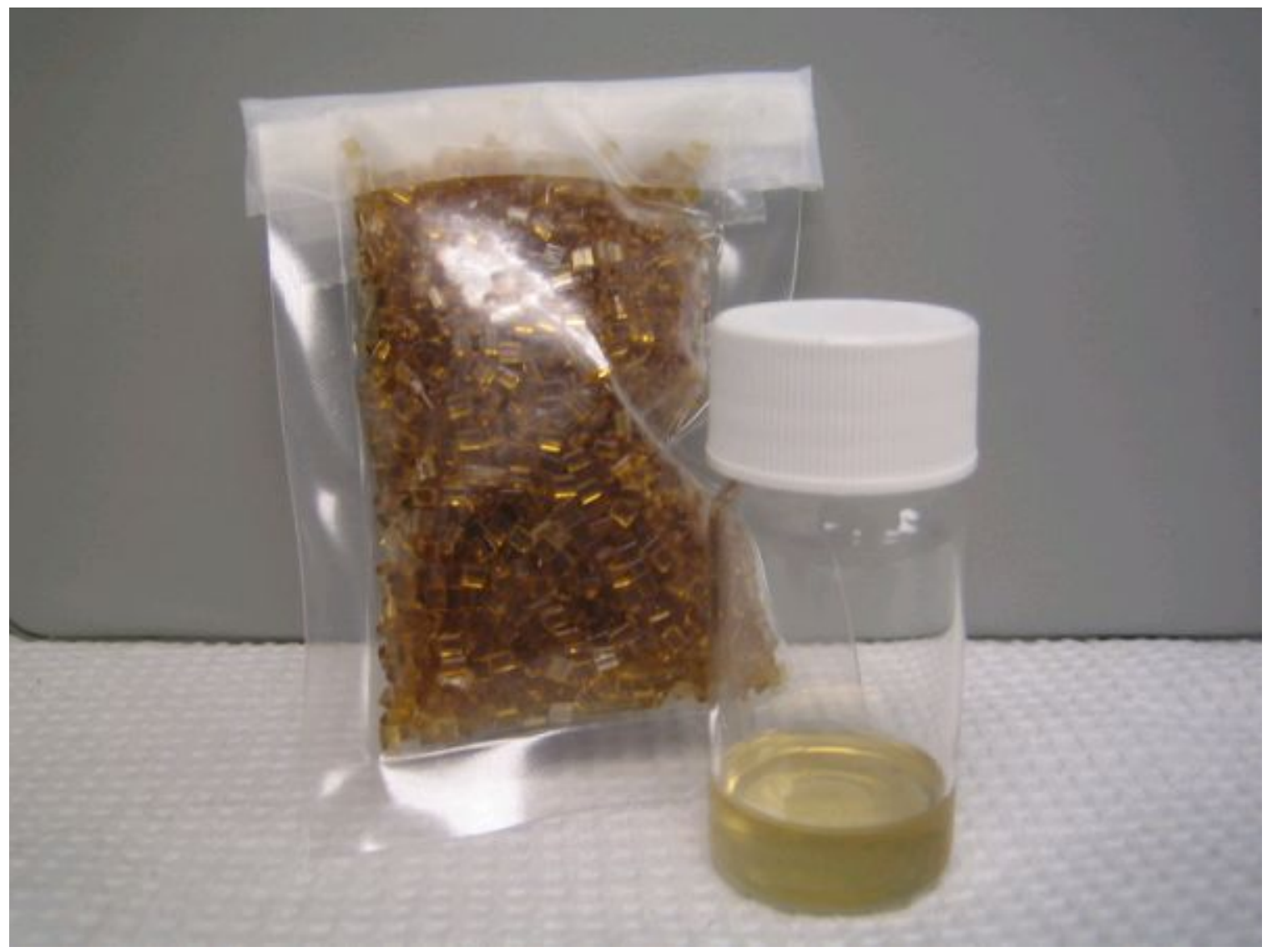




\begin{tabular}{|l|c|c|c|}
\hline \multicolumn{1}{|c|}{ Materials/Equipment } & Vendor & Part Number & Quantity \\
\hline $\begin{array}{l}\text { Poly(L-lactide-co-caprolactone) [P(LLA-CL)] } \\
\text { (90:10) Monomer Mole \% L-Lactide:Caprolactone }\end{array}$ & $\begin{array}{c}\text { Lakeshore } \\
\text { Biomaterials }\end{array}$ & $90: 10$ LCL 11E & 50 grams \\
\hline Chloroform, extra dry, water <50ppm, stabilized & $\begin{array}{c}\text { Fisher } \\
\text { Scientific, Inc }\end{array}$ & 326820010 & 1 Liter \\
\hline 10 ml Syringe, Luer-Lok tip & BD & 309604 & $100 /$ Pack \\
\hline Blunt Fill Needle, 18G 1/2 (1.2 mm x 40 mm) & BD & 305180 & $100 /$ Pack \\
\hline Analytical Balance & Acculab & ALC-80.4 & 1 \\
\hline Orbital Shaker & FinePCR & SH130 & 1 \\
\hline Vaccum-Pressure Pipette Aid & $\begin{array}{c}\text { Drummond } \\
\text { Scientific Co. }\end{array}$ & P-80991 & 1 \\
\hline Serological Pipet 5 x 1/10 ml & $\begin{array}{c}\text { VWR } \\
\text { International }\end{array}$ & $53283-706$ & NA \\
\hline Clear Glass Vial $20 \mathrm{ml}$ & $\begin{array}{c}\text { VWR } \\
\text { International }\end{array}$ & $15900-002$ & $72 / \mathrm{CS}$ \\
\hline
\end{tabular}

1.) Put on safety gloves. (WARNING: Chloroform can have serious side-effects if it comes in contact with skin, eyes or is inhaled or swallowed. Target organs to be effected are kidneys, heart, central nervous system, liver, eyes, reproductive system and skin. Always open chloroform in a hood and wear protective clothing!!)

2.) Remove the $\mathrm{P}(\mathrm{LLA}-\mathrm{CL}$ ) resin (Figure $\mathrm{B} 1$ ) from the freezer and allow it to sit at room temperature for at least 10 minutes. This prevents condensation on the resin when exposed to the air, reducing the amount of atmospheric degradation.

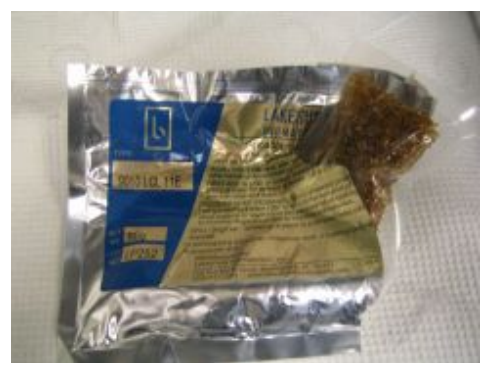

Figure B1 - 90:10 P(LLACL) resin from Lakeshore Biomaterials.

3.) While the resin is warming, calculate the weight percent polymer of the finished solution based on quantity P(LLA-CL) being used. See below for this calculation.

a. Polymer solutions are quantified by weight percent polymer (WPP), and there are two components to the solution: P(LLA-CL) and chloroform. The polymer 
can be weighed directly, but the chloroform must be converted from volume to mass using density $(1.48 \mathrm{~g} / \mathrm{ml})$.

$$
W P P=100\left(\frac{a}{a+b}\right)
$$

where:

$$
\begin{aligned}
& a=\text { mass of polymer }(\mathrm{g}) \\
& b=\text { mass of solvent }(\mathrm{g})
\end{aligned}
$$

Consider: $1.10 \mathrm{~g}$ of $\mathrm{P}(\mathrm{LLA}-\mathrm{CL})$ is dissolved in $8 \mathrm{ml}$ of chloroform (1.48 $\mathrm{g} / \mathrm{ml}$ ), the weight percent polymer of the solution would be calculated as follows:

$$
\begin{aligned}
& \left.W P P=100\left(\frac{1.10 g}{1.10 g+8 m l[1.48 g / m l}\right]\right) \\
& W P P=100\left(\frac{1.10 g}{12.94 g}\right) \\
& W P P=8.50 \%
\end{aligned}
$$

Conversely, by rearranging the equation, the mass of polymer required for a specific WPP solution can be determined. 
4.) Weigh out the $\mathrm{P}(\mathrm{LLA}-\mathrm{CL})$ and put it in a $20 \mathrm{ml}$ glass vial (Figure B2). Set the vial aside for now and return the P(LLA-CL) resin to the freezer.

Figure B2 - A glass scintillation vial used to mix and store solutions.

5.) Retrieve the chloroform (Figure B3) from the hazardous chemical cabinet and place it in a fume hood immediately.

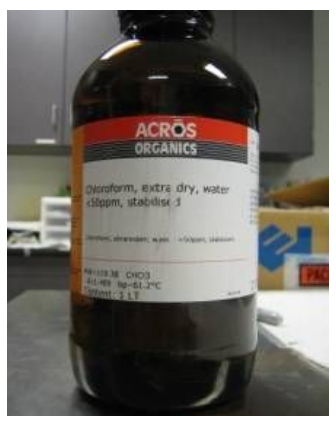

Figure B3 - A bottle of chloroform.

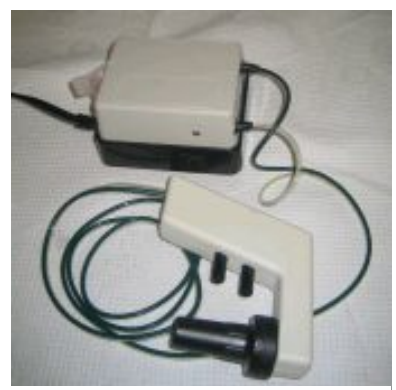

Figure B4 - A pipette aid used to pipette solvent.

7.) Properly label the solution vial with the WPP, date, and your initials. 
8.) Wrap the labeled vial in aluminum foil. Prohibiting light exposure can prevent solution degradation.

9.) Place the vial on an orbital shake table (Figure B5). Secure the vial using tape to ensure the vial will not tip over when mixing. Set the shake table speed to 3 revolutions per second.

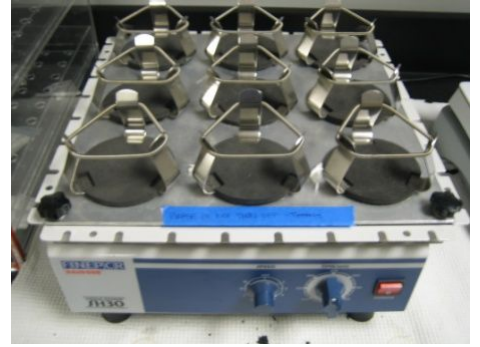

Figure B5 - The orbital shake table used to mix solutions.

10.) Allow the P(LLA-CL) solution to mix for 24 hours. After mixing is complete, the solution is usable for up to 48 hours. 


\section{APPENDIX C: ELECTROSPINNING PROTOCOL}

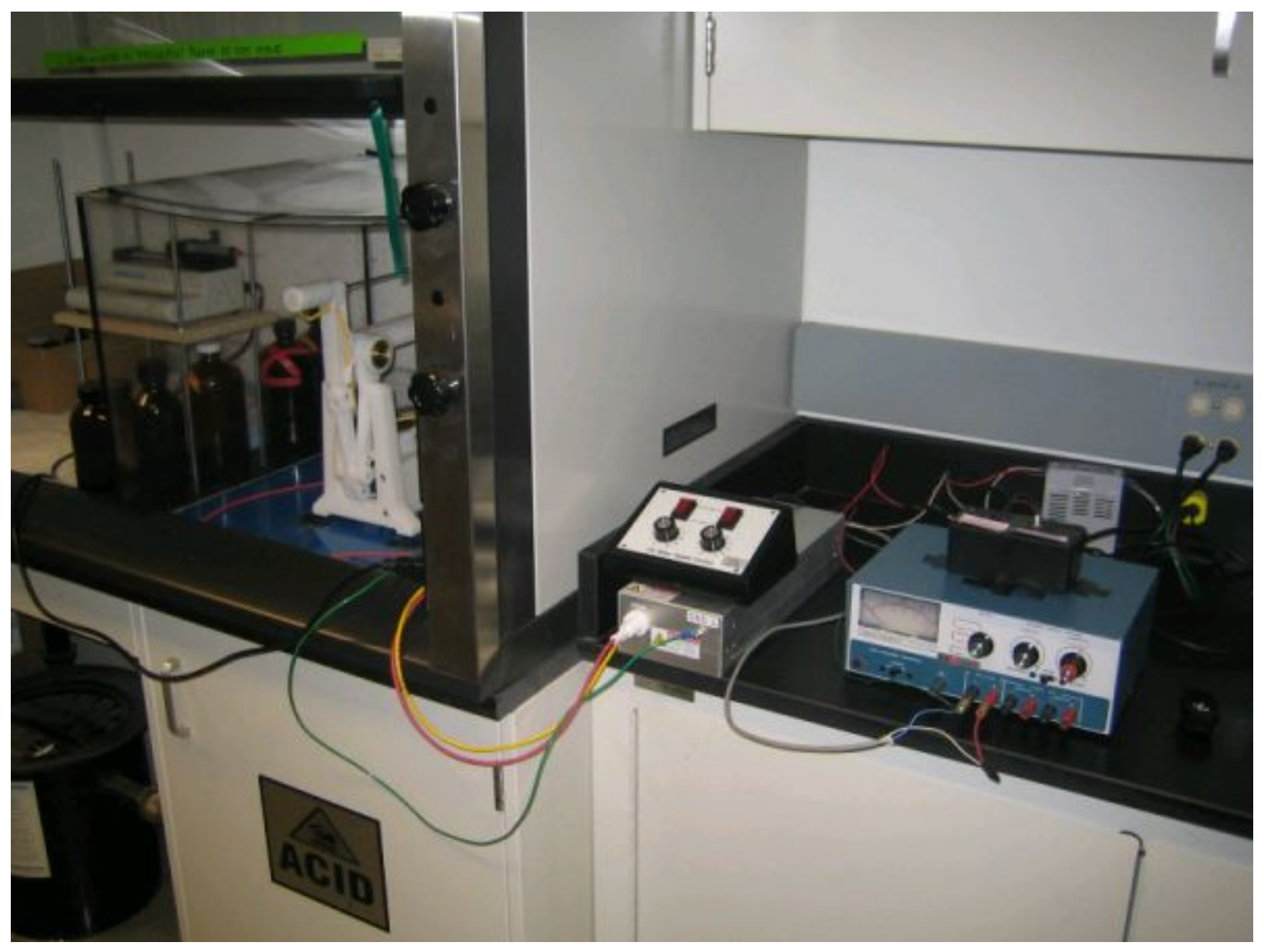

WARNING: This process requires extremely high voltages! Always wear shoes, gloves, and be mindful of what you are touching. To use the electrospinner, you must be trained by a qualified user and cleared by an appropriate faculty member. 
1.) Put on gloves gloves.

2.) Prepare to clean the polymer collector (Figure C1) by getting paper towels and isopropal alcohol.

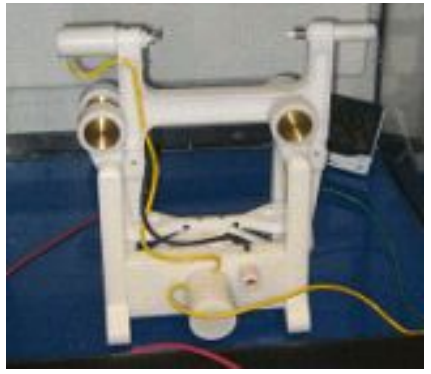

Figure C1 - Collector for the electrospinning apparatus.

3.) Unplug the green ground wire from the collector (Figure C2).

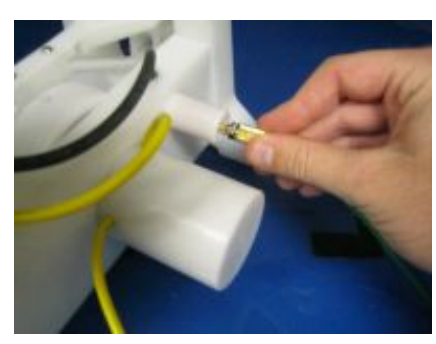

Figure C2 - Ground input for the collector is located to the right of the engine casing.

4.) Unplug the yellow power wire from the DC motor control box (Figure C3).

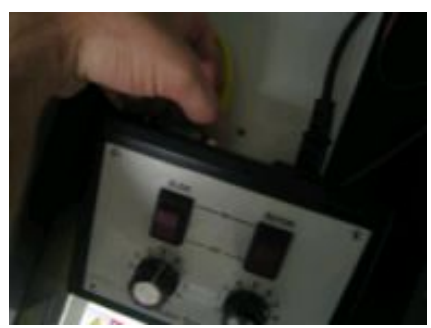

Figure C3 - The yellow power wire connects into the rear panel of the motor control box. 
5.) Clean debris from previous spins using isopropyl alcohol. Stray polymer preferentially builds up on exposed metal, wires, and motor casings, but polymer build-up can occur anywhere on the collector, even the side not facing the electrified needle (Figure C4).

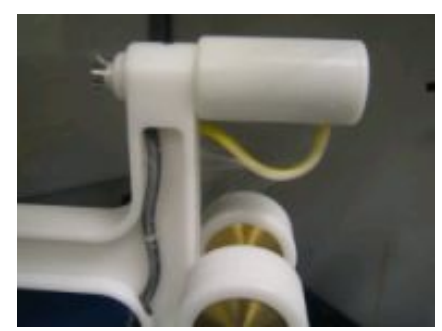

Figure C4 - Polymer fiber build up on the collector.

6.) Replace the collector in the containment chamber and reconnect the ground and power wires.

7.) Plug in all equipment (syringe pump, main power, external power supply, and DC motor control box).

8.) Using a multimeter, check the resistance between the ground connection and the aluminum mandrel (Figure C5). Verify that there is some conductivity.

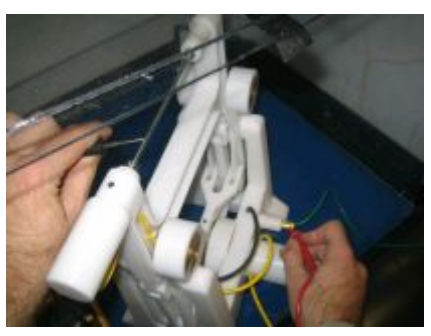

Figure C5 - If resistance between the mandrel and the ground connection is too high, electrospinning will not occur.

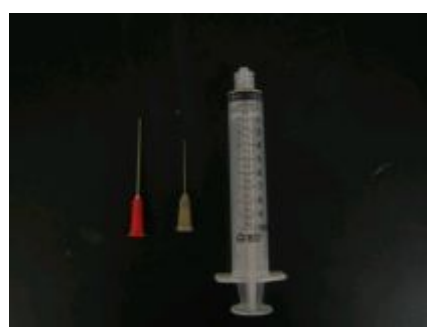

Figure C6 - A variety of needles can be attached to the $10 \mathrm{ml}$ syringes. 18 and 27 gauge needles are shown above. 
10.) In the fume hood, pull just over the desired amount of polymer solution into the syringe (Figure C7). The solution may be very thick, so this could take some time. Make sure there is no air in the syringe.

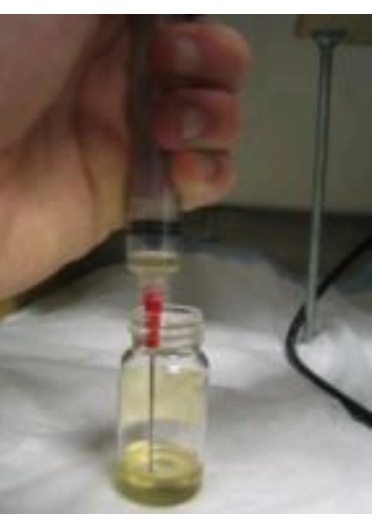

Figure C7 - Loading polymer solution into a syringe.

11.) Put the needle through the small hole drilled in the containment chamber and set the syringe into the syringe pump (Figure C8).

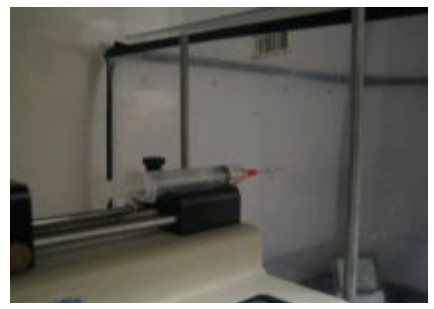

Figure C8 - The syringe should sit on the syringe pump while the needle enters the containment chamber via the access hole.

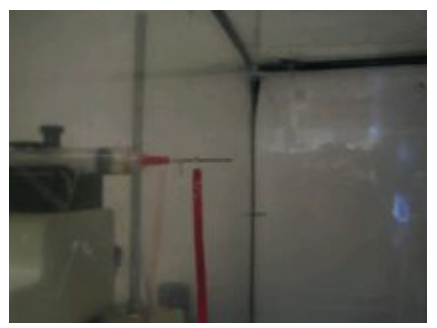

Figure C9 - The high voltage wire hangs on the needle inside the containment chamber to reduce the risk of shock. 
13.) Position the polymer collector in the containment chamber so that the mandrel and needle tip are at the desired gap distance apart and perpendicular to each other (Figure C10). The ground wire input should face away from the needle.

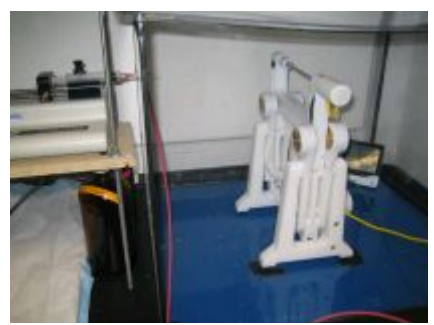

Figure C10 - The correct alignment of the needle and collector is necessary to ensure a uniform spin.

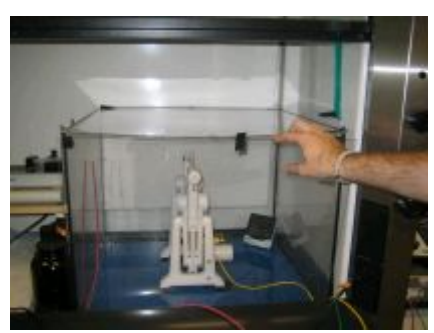

Figure C11 - The front panel must be secure prior to electrospinning.

15.) Switch on the syringe pump and enter the desired settings (ie - syringe, volume, flow rate, etc.). If you previously used the electrospinner and want to run with the same syringe pump settings (and no one else had used the apparatus since), there is no need to make any changes to the syringe pump programming.

Syringe pump instructions should be located in a cabinet close to the electrospinner if you need instructions on how to operate it. 
16.) Turn on the "Rotate" and "Slide" functions of the collector at the DC motor control box (Figure C12). The speed at which the mandrel rotates and translates can be adjusted here.

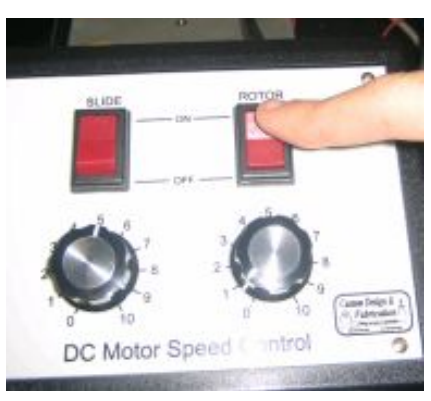

Figure C12 - The DC motor control box.

17.) Turn on the external power source and set it at the desired voltage $(0-10 \mathrm{~V})$, then turn the power source off (Figure C13).

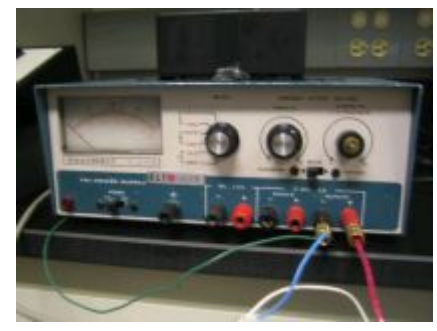

Figure C13- The external power source that acts as an amplifier control for the high voltage power converter.

18.) Press the "Run" button on the syringe pump. The solution is now being slowly pushed through the needle.

19.) When a droplet forms on the end of the needle, the process is ready to begin. The following three steps should be performed in order, as quickly as possible. 
20.) Turn on the "Main Power" (Figure C14). Power is on if the light on the $\mathrm{AC} / \mathrm{DC}$ power converter turns green.

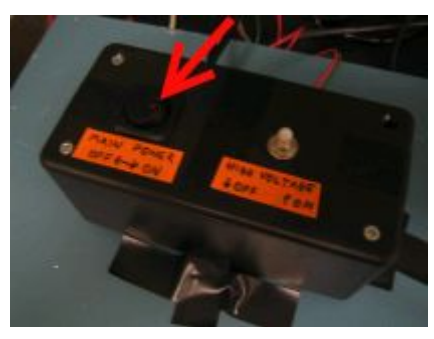

Figure C14 - The main power switch is located on the left side of power control switch box (red arrow).

21.) Turn on the external source.

22.) Be ready to turn the "HV" on. Look at the droplet on the end of the syringe, and turn "HV" on (Figure C15). The droplet should disappear.

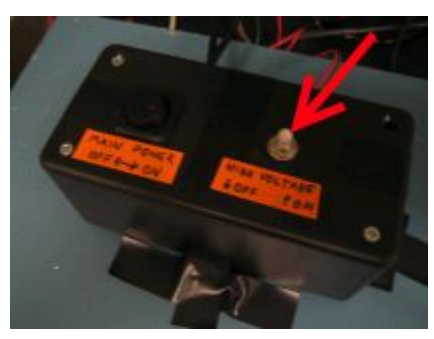

Figure C15 - The HV switch is located on the right side of the power control switch box (red arrow).

23.) Look and listen for any problems with the equipment. If you hear clicking coming from either of the power supplies, stop the process immediately.

24.) Observe the process for the entire spin. The aluminum mandrel should slowly start to become coated with polymer, turning it white. 
25.) When the desired amount of solution has been spun, the process should be shut down as follows:

a.) Press the "Run/Stop" button on the syringe pump to stop the pump. Turn off the syringe pump.

b.) Turn the "HV" switch to off.

c.) Turn off the external power supply.

d.) Turn "Main Power" off.

e.) Turn off "Rotate" and "Slide" for the collector.

26.) Allow the evaporated solvent to leak out of the containment chamber for a few minutes.

27.) Remove the front window of the containment chamber and detach the coated mandrel from the collector.

28.) Touch the red high voltage wire to the green ground wire to remove residual charge.

29.) Using gauze, gently twist the scaffold to break it loose from the mandrel. The scaffold should now easily slide off the mandrel. Finished scaffolds should be stored in a desiccator.

30.) Unplug all equipment. 
31.) Remove the syringe and needle from the syringe pump and dispose of properly, and close the hood. 


\section{APPENDIX D: HIGH CONTRAST SURFACE POROSIMETRY}

\section{Introduction}

Porosity, as referred to in this thesis, is defined as the percent space not occupied by electrospun fibers. Porosity is normally reported in terms of void space in a volume, but appropriate methodology for gathering volumetric data was not available. The method developed instead measured the percent void space on the surface of samples. This method, intended to collect surface porosity data on the electrospun P(LLA-CL) scaffolds, was named high contrast surface porosimetry.

\section{Method}

The same sampled sections that were used to take fiber diameter measurement images were used to capture porosity images. The FEI Model 200 Scanning Electron Microscope was also used for imaging. Voltage bias on the Everhart-Thornley electron detector was set to $-200 \mathrm{~V}$. This prevented detection of lower energy electrons from subsurface fibers while highlighting the surface fibers. Contrast and brightness were adjusted on the SEM until surface fibers were clearly defined (Figure D1). High contrast images were then converted black and white images using the "Threshold" command in ImageTool V3.0 image analysis software (The University of Texas Health Science Center in San Antonio) (Figure D2). Finally, percent white/void space was calculated using the "Count White/Black Pixels" command in ImageTool. The value for white pixels represented the percent surface void space. 


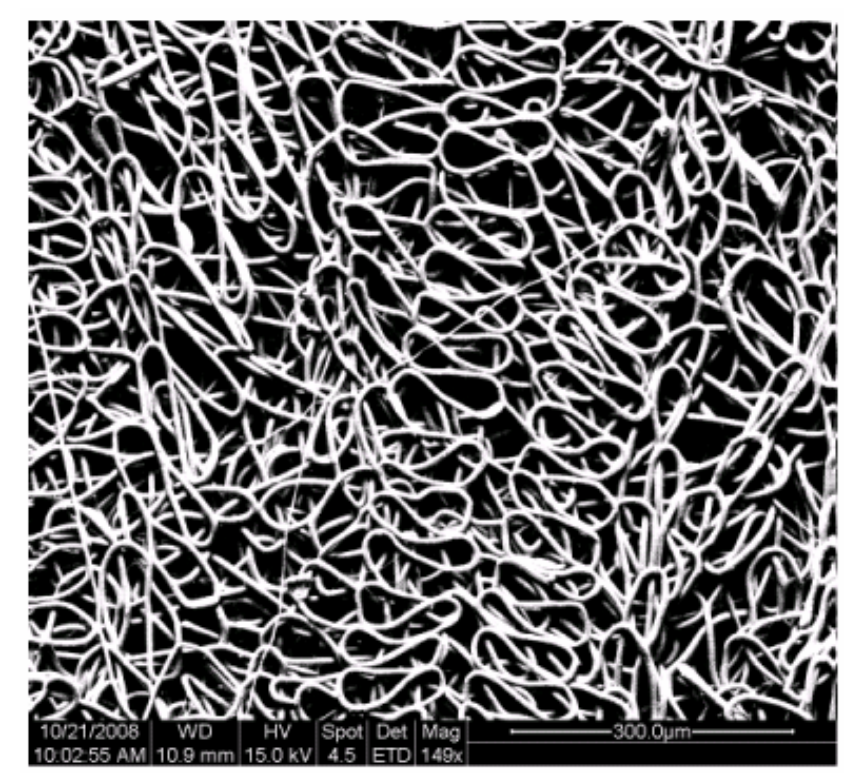

Figure D1- A high contrast, backscattered electron image taken using the FEI Model 200 Scanning Electron Microscope. Surface fibers are highlighted, while subsurface fiber presence is minimized. (149x) 


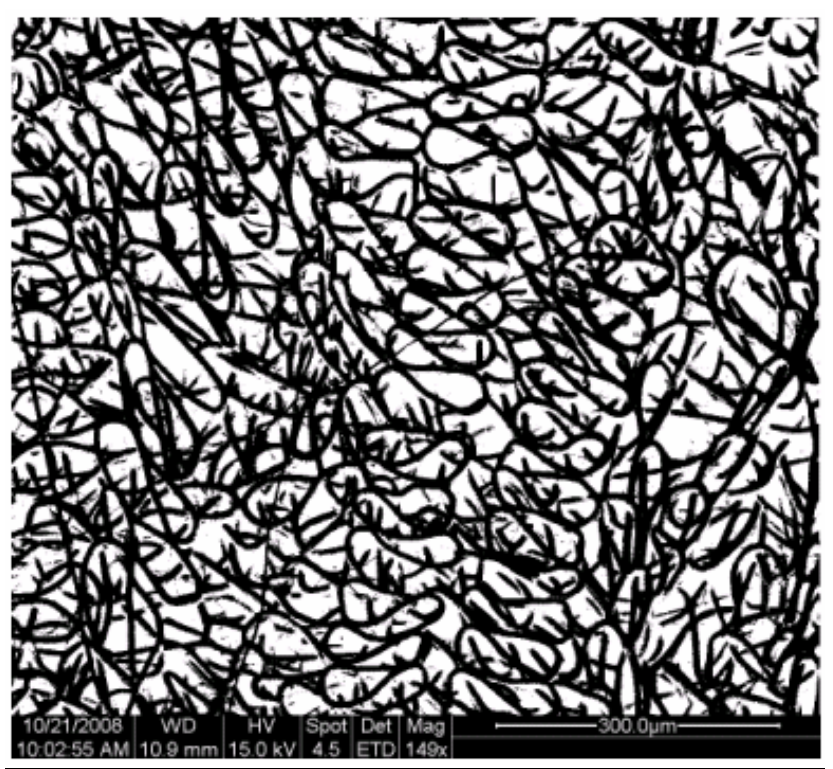

Figure D2 - The "Threshold" command in ImageTool converts all pixels into either white or black. Whether a pixel translates to white or black depends on a user controlled threshold scale.

\section{Conclusion}

No results are reported due to concerns that the method is prone to operator subjectivity during two important steps. First, when capturing the image using the SEM, contrast and brightness are controlled by the operator. Since it is difficult to set these parameters the same for every image, this is a source of error. Second, the "Threshold" command is also operator controlled. Although the threshold was adjusted until it appeared that only solid fibers were highlighted, this method still depended on operator opinion. After these two concerns were raised, it was decided that gathering porosity data in this matter would not give accurate results, and so the method was abandoned. 
Although as of now the method has the issues mentioned above, future

improvements may make it an acceptable method for quickly gathering surface porosity data. 


\section{APPENDIX E: FIBER DIAMETER MEASUREMENTS}

\section{Introduction}

As stated in Chapter 4, fiber diameters were measured using a combination of SEM images and the Image J software. Using the program's line length measurement tool (ANALYZE > MEASURE), the scale bar on all images was measured. This length corresponded to 50 microns on all images. The sample selection mask was then used to measure 9 separate fiber thicknesses. A simple calculation was then used in Microsoft Excel to convert the collected fiber thicknesses into fiber diameter in microns. In the data shown below, entries under Diameter are in microns. All other values are unitless.

$$
D=\left(\frac{t}{S}\right) \bullet 50 \text { microns }
$$

Where: $\quad D$ is fiber diameter in microns

$t$ is fiber thickness measured on Image $\mathrm{J}$

$S$ is length of the scale bar measured on Image $\mathrm{J}$ 


\section{SPIN 1}

Section A1

Scale Bar: 50 microns is

2.792

$\begin{array}{rrr}\text { Sample } & \text { Length } & \text { Diameter } \\ 1 & 0.343 & 6.14 \\ 2 & 0.226 & 4.05 \\ 3 & 0.393 & 7.04 \\ 4 & 0.459 & 8.22 \\ 5 & 0.441 & 7.90 \\ 6 & 0.347 & 6.21 \\ 7 & 0.42 & 7.52 \\ 8 & 0.509 & 9.12 \\ 9 & 0.452 & 8.09\end{array}$

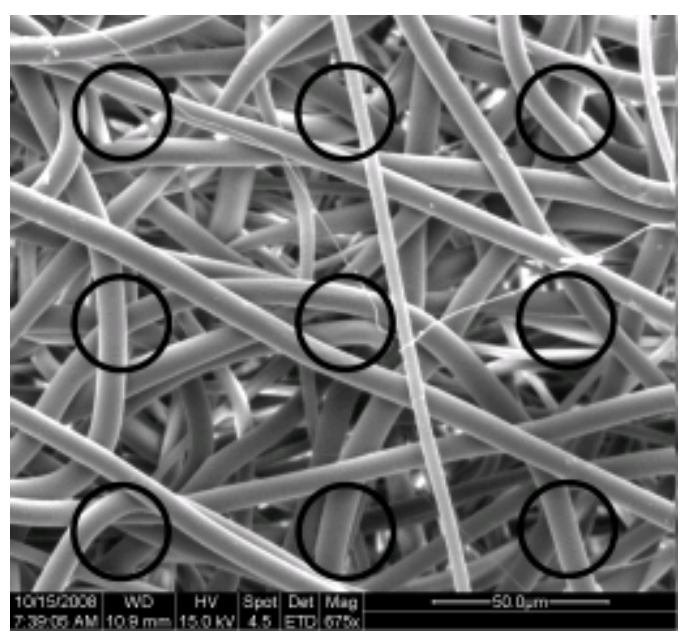

Section A2

Scale Bar: 50 microns is

2.834

$\begin{array}{rrr}\text { Sample } & \text { Length } & \text { Diameter } \\ 1 & 0.488 & 8.61 \\ 2 & 0.458 & 8.08 \\ 3 & 0.251 & 4.43 \\ 4 & 0.444 & 7.83 \\ 5 & 0.237 & 4.18 \\ 6 & 0.506 & 8.93 \\ 7 & 0.4 & 7.06 \\ 8 & 0.483 & 8.52 \\ 9 & 0.409 & 7.22\end{array}$

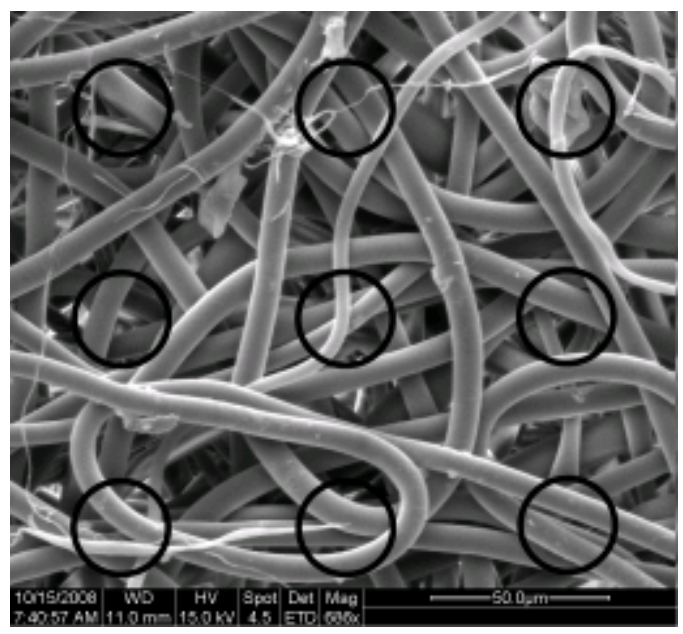

Section A3

Scale Bar: 50 microns is

2.834

$\begin{array}{rrr}\text { Sample } & \text { Length } & \text { Diameter } \\ 1 & 0.253 & 4.46 \\ 2 & 0.284 & 5.01 \\ 3 & 0.4 & 7.06 \\ 4 & 0.496 & 8.75 \\ 5 & 0.479 & 8.45 \\ 6 & 0.446 & 7.87 \\ 7 & 0.427 & 7.53 \\ 8 & 0.497 & 8.77 \\ 9 & 0.131 & 2.31\end{array}$

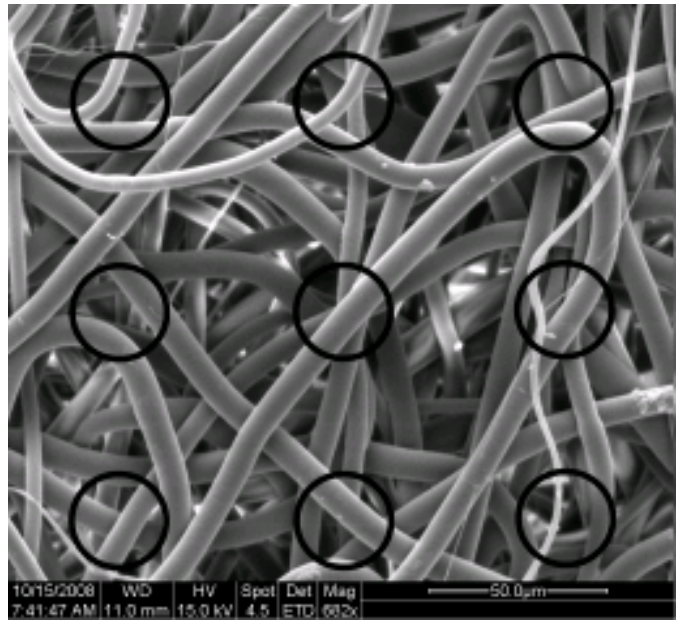


Section B1

Scale Bar: 50 microns is

2.807

$\begin{array}{rrr}\text { Sample } & \text { Length } & \text { Diameter } \\ 1 & 0.427 & 7.61 \\ 2 & 0.428 & 7.62 \\ 3 & 0.457 & 8.14 \\ 4 & 0.439 & 7.82 \\ 5 & 0.138 & 2.46 \\ 6 & 0.168 & 2.99 \\ 7 & 0.352 & 6.27 \\ 8 & 0.174 & 3.10 \\ 9 & 0.432 & 7.70\end{array}$

Section B2

Scale Bar: 50 microns is

2.958

$\begin{array}{rrr}\text { Sample } & \text { Length } & \text { Diameter } \\ 1 & 0.471 & 7.96 \\ 2 & 0.154 & 2.60 \\ 3 & 0.241 & 4.07 \\ 4 & 0.262 & 4.43 \\ 5 & 0.512 & 8.65 \\ 6 & 0.41 & 6.93 \\ 7 & 0.409 & 6.91 \\ 8 & 0.218 & 3.68 \\ 9 & 0.741 & 12.53\end{array}$

Section B3

Scale Bar: 50 microns is

2.889

$\begin{array}{rrr}\text { Sample } & \text { Length } & \text { Diameter } \\ 1 & 0.34 & 5.88 \\ 2 & 0.401 & 6.94 \\ 3 & 0.416 & 7.20 \\ 4 & 0.416 & 7.20 \\ 5 & 0.448 & 7.75 \\ 6 & 0.418 & 7.23 \\ 7 & 0.448 & 7.75 \\ 8 & 0.464 & 8.03 \\ 9 & 0.214 & 3.70\end{array}$
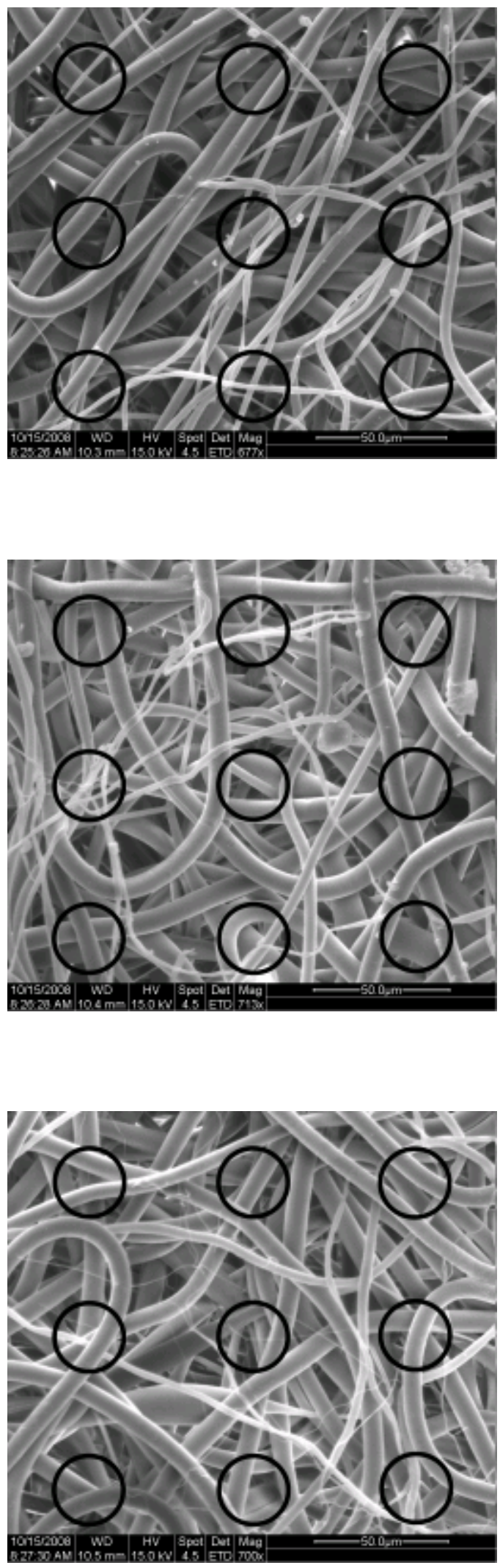
Section C1

Scale Bar: 50 microns is

2.862

$\begin{array}{rrr}\text { Sample } & \text { Length } & \text { Diameter } \\ 1 & 0.427 & 7.46 \\ 2 & 0.437 & 7.63 \\ 3 & 0.421 & 7.35 \\ 4 & 0.179 & 3.13 \\ 5 & 0.471 & 8.23 \\ 6 & 0.478 & 8.35 \\ 7 & 0.113 & 1.97 \\ 8 & 0.798 & 13.94 \\ 9 & 0.402 & 7.02\end{array}$

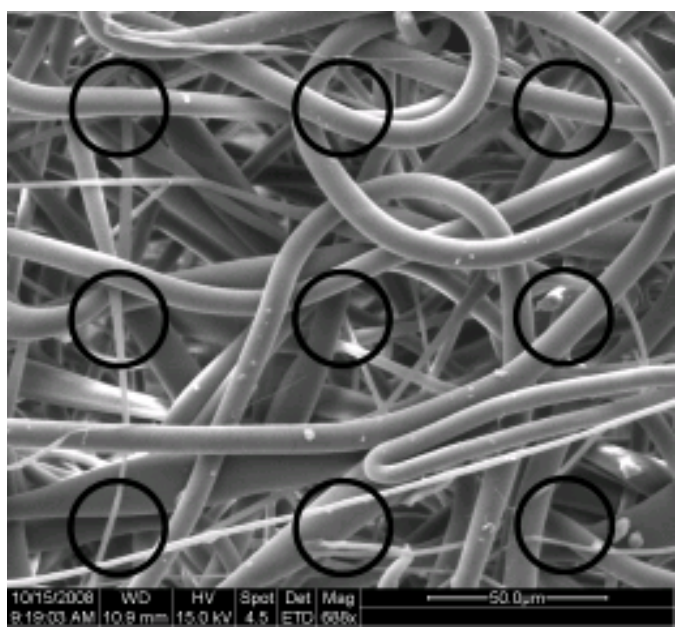

Section C2

Scale Bar: 50 microns is

2.834

$\begin{array}{rrr}\text { Sample } & \text { Length } & \text { Diameter } \\ 1 & 0.441 & 7.78 \\ 2 & 0.224 & 3.95 \\ 3 & 0.379 & 6.69 \\ 4 & 0.151 & 2.66 \\ 5 & 0.244 & 4.30 \\ 6 & 0.414 & 7.30 \\ 7 & 0.394 & 6.95 \\ 8 & 0.406 & 7.16 \\ 9 & 0.41 & 7.23\end{array}$

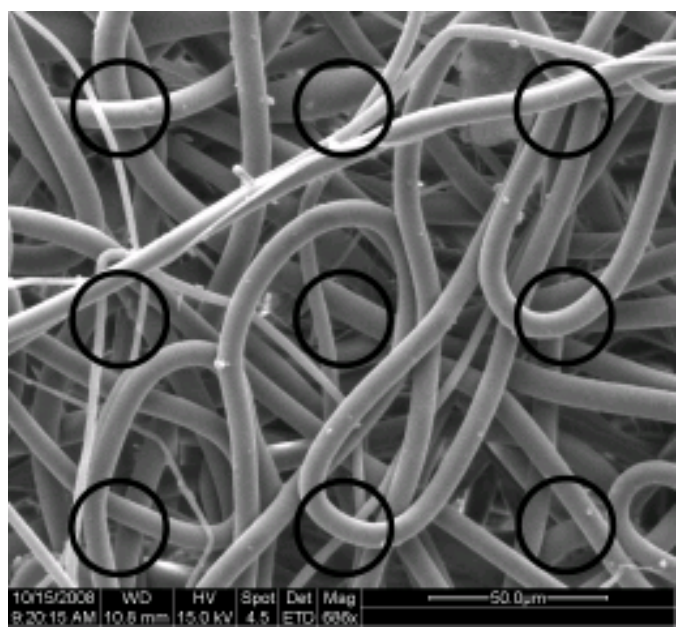

Section C3

Scale Bar: 50 microns is

2.875

$\begin{array}{rrr}\text { Sample } & \text { Length } & \text { Diameter } \\ 1 & 0.372 & 6.47 \\ 2 & 0.166 & 2.89 \\ 3 & 0.425 & 7.39 \\ 4 & 0.346 & 6.02 \\ 5 & 0.424 & 7.37 \\ 6 & 0.418 & 7.27 \\ 7 & 0.394 & 6.85 \\ 8 & 0.517 & 8.99 \\ 9 & 0.294 & 5.11\end{array}$

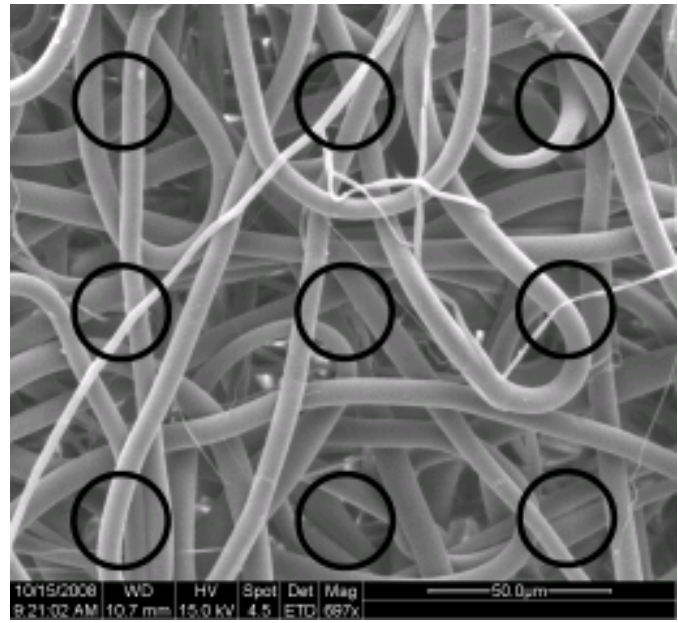


Section D1

Scale Bar: 50 microns is

2.972

Sample Length Diameter

$\begin{array}{rrr}1 & 0.21 & 3.53 \\ 2 & 0.485 & 8.16 \\ 3 & 0.396 & 6.66 \\ 4 & 0.654 & 11.00 \\ 5 & 0.504 & 8.48 \\ 6 & 0.358 & 6.02 \\ 7 & 0.175 & 2.94 \\ 8 & 0.557 & 9.37 \\ 9 & 0.439 & 7.39\end{array}$

Section D2

Scale Bar: 50 microns is

2.875

$\begin{array}{rrr}\text { Sample } & \text { Length } & \text { Diameter } \\ 1 & 0.335 & 5.83 \\ 2 & 0.181 & 3.15 \\ 3 & 0.437 & 7.60 \\ 4 & 0.127 & 2.21 \\ 5 & 0.432 & 7.51 \\ 6 & 0.138 & 2.40 \\ 7 & 0.461 & 8.02 \\ 8 & 0.179 & 3.11 \\ 9 & 0.263 & 4.57\end{array}$

Section D3

Scale Bar: 50 microns is

3.027

$\begin{array}{rrr}\text { Sample } & \text { Length } & \text { Diameter } \\ 1 & 0.641 & 10.59 \\ 2 & 0.322 & 5.32 \\ 3 & 0.14 & 2.31 \\ 4 & 0.338 & 5.58 \\ 5 & 0.381 & 6.29 \\ 6 & 0.507 & 8.37 \\ 7 & 0.478 & 7.90 \\ 8 & 0.878 & 14.50 \\ 9 & 0.124 & 2.05\end{array}$
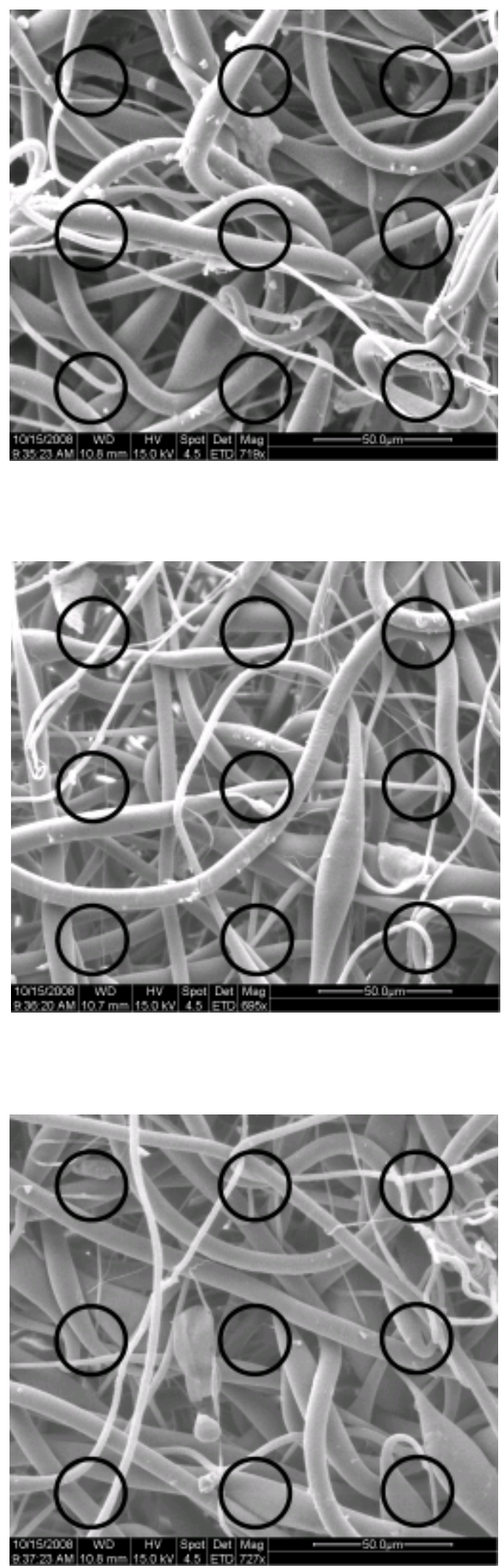


\section{SPIN 2}

Section A1

Scale Bar: 50 microns is

2.903

$\begin{array}{rrr}\text { Sample } & \text { Length } & \text { Diameter } \\ 1 & 0.346 & 5.96 \\ 2 & 0.392 & 6.75 \\ 3 & 0.487 & 8.39 \\ 4 & 0.338 & 5.82 \\ 5 & 0.473 & 8.15 \\ 6 & 0.453 & 7.80 \\ 7 & 0.195 & 3.36 \\ 8 & 0.462 & 7.96 \\ 9 & 0.4 & 6.89\end{array}$

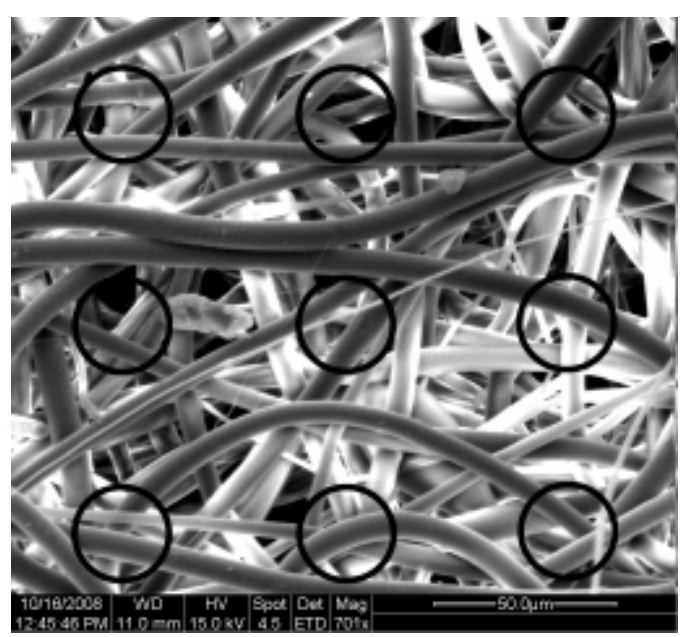

Section A2

Scale Bar: 50 microns is

2.917

$\begin{array}{rrr}\text { Sample } & \text { Length } & \text { Diameter } \\ 1 & 0.44 & 7.54 \\ 2 & 0.348 & 5.97 \\ 3 & 0.418 & 7.16 \\ 4 & 0.531 & 9.10 \\ 5 & 0.424 & 7.27 \\ 6 & 0.357 & 6.12 \\ 7 & 0.489 & 8.38 \\ 8 & 0.372 & 6.38 \\ 9 & 0.468 & 8.02\end{array}$

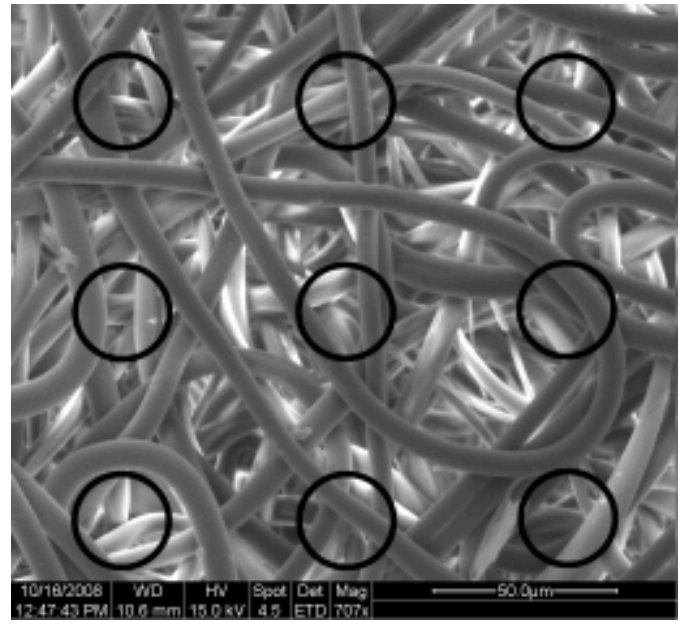

Section A3

Scale Bar: 50 microns is

2.917

$\begin{array}{rrr}\text { Sample } & \text { Length } & \text { Diameter } \\ 1 & 0.386 & 6.62 \\ 2 & 0.187 & 3.21 \\ 3 & 0.345 & 5.91 \\ 4 & 0.195 & 3.34 \\ 5 & 0.467 & 8.00 \\ 6 & 0.275 & 4.71 \\ 7 & 0.443 & 7.59 \\ 8 & 0.245 & 4.20 \\ 9 & 0.418 & 7.16\end{array}$

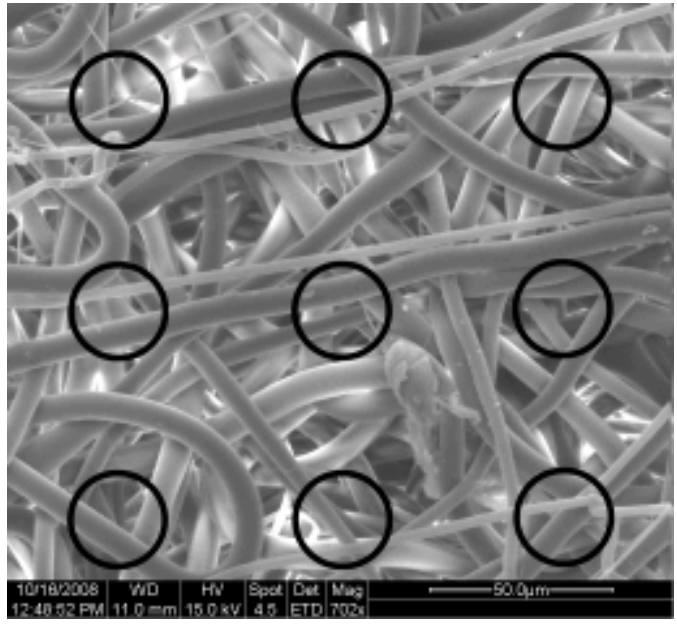


Section B1

Scale Bar: 50 microns is

2.93

Sample Length Diameter

$\begin{array}{lll}1 & 0.184 & 3.14 \\ 2 & 0.337 & 5.75 \\ 3 & 0.391 & 6.67 \\ 4 & 0.358 & 6.11 \\ 5 & 0.376 & 6.42 \\ 6 & 0.376 & 6.42 \\ 7 & 0.361 & 6.16 \\ 8 & 0.381 & 6.50 \\ 9 & 0.358 & 6.11\end{array}$

Section B2

Scale Bar: 50 microns is

2.958

$\begin{array}{rrr}\text { Sample } & \text { Length } & \text { Diameter } \\ 1 & 0.751 & 12.69 \\ 2 & 0.241 & 4.07 \\ 3 & 0.405 & 6.85 \\ 4 & 0.317 & 5.36 \\ 5 & 0.439 & 7.42 \\ 6 & 0.157 & 2.65 \\ 7 & 0.406 & 6.86 \\ 8 & 0.394 & 6.66 \\ 9 & 0.387 & 6.54\end{array}$

Section B3

Scale Bar: 50 microns is

2.862

$\begin{array}{rrr}\text { Sample } & \text { Length } & \text { Diameter } \\ 1 & 0.44 & 7.69 \\ 2 & 0.384 & 6.71 \\ 3 & 0.427 & 7.46 \\ 4 & 0.118 & 2.06 \\ 5 & 0.294 & 5.14 \\ 6 & 0.282 & 4.93 \\ 7 & 0.206 & 3.60 \\ 8 & 0.267 & 4.66 \\ 9 & 0.428 & 7.48\end{array}$
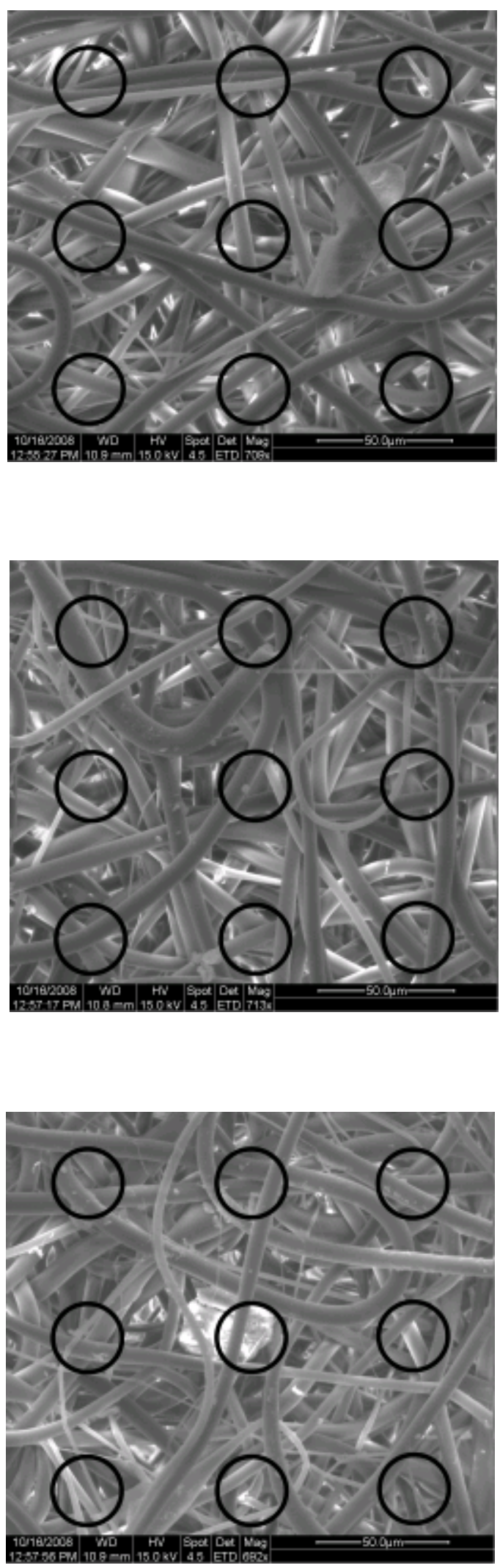
Section C1

Scale Bar: 50 microns is

2.889

$\begin{array}{rrr}\text { Sample } & \text { Length } & \text { Diameter } \\ 1 & 0.431 & 7.46 \\ 2 & 0.427 & 7.39 \\ 3 & 0.196 & 3.39 \\ 4 & 0.461 & 7.98 \\ 5 & 0.443 & 7.67 \\ 6 & 0.388 & 6.72 \\ 7 & 0.388 & 6.72 \\ 8 & 0.482 & 8.34 \\ 9 & 0.45 & 7.79\end{array}$

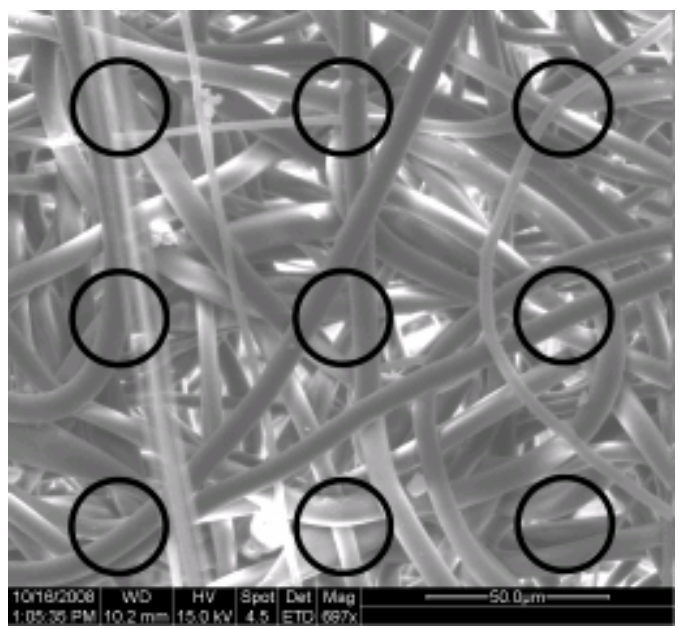

Section C2

Scale Bar: 50 microns is

2.889

$\begin{array}{rrr}\text { Sample } & \text { Length } & \text { Diameter } \\ 1 & 0.428 & 7.41 \\ 2 & 0.453 & 7.84 \\ 3 & 0.677 & 11.72 \\ 4 & 0.203 & 3.51 \\ 5 & 0.372 & 6.44 \\ 6 & 0.675 & 11.68 \\ 7 & 0.406 & 7.03 \\ 8 & 0.394 & 6.82 \\ 9 & 0.193 & 3.34\end{array}$

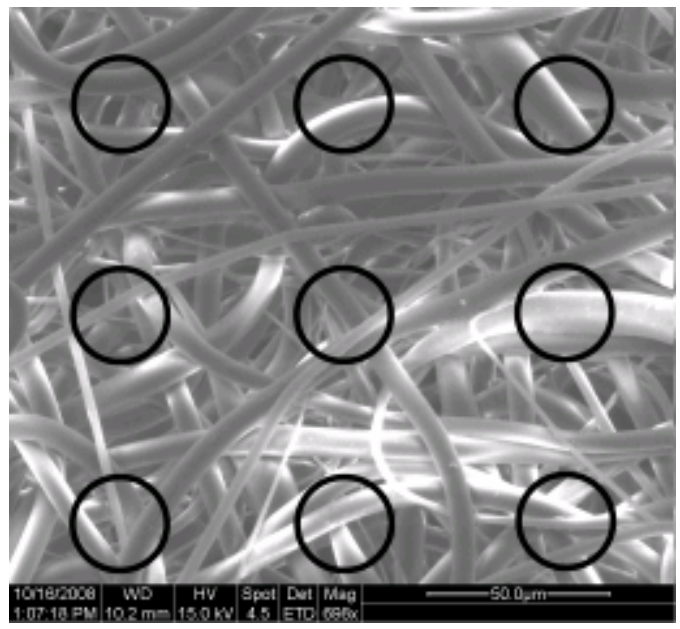

Section C3

Scale Bar: 50 microns is

$\begin{array}{rrr}\text { Sample } & \text { Length } & \text { Diameter } \\ 1 & 0.497 & 8.21 \\ 2 & 0.477 & 7.88 \\ 3 & 0.43 & 7.10 \\ 4 & 0.402 & 6.64 \\ 5 & 0.748 & 12.36 \\ 6 & 0.2 & 3.30 \\ 7 & 0.374 & 6.18 \\ 8 & 0.138 & 2.28 \\ 9 & 0.37 & 6.11\end{array}$

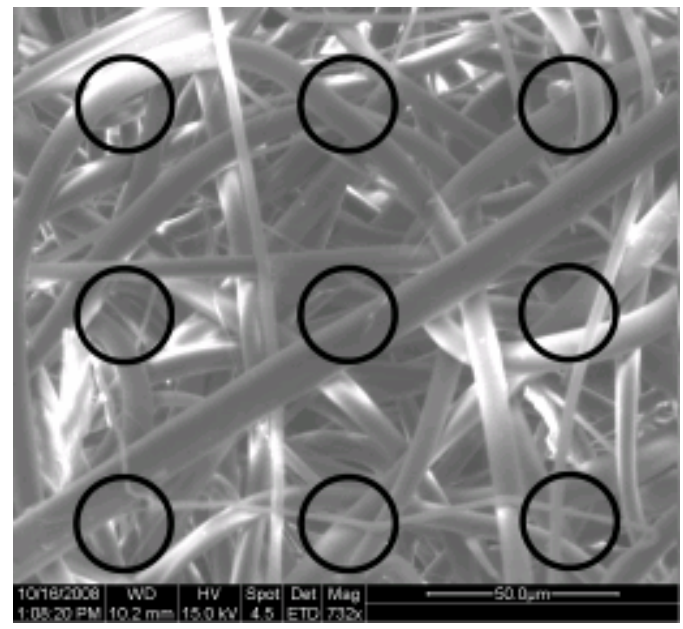


Section D1

Scale Bar: 50 microns is

3.013

$\begin{array}{rrr}\text { Sample } & \text { Length } & \text { Diameter } \\ 1 & 0.388 & 6.44 \\ 2 & 0.394 & 6.54 \\ 3 & 0.37 & 6.14 \\ 4 & 0.352 & 5.84 \\ 5 & 0.087 & 1.44 \\ 6 & 0.427 & 7.09 \\ 7 & 0.378 & 6.27 \\ 8 & 0.471 & 7.82 \\ 9 & 0.482 & 8.00\end{array}$

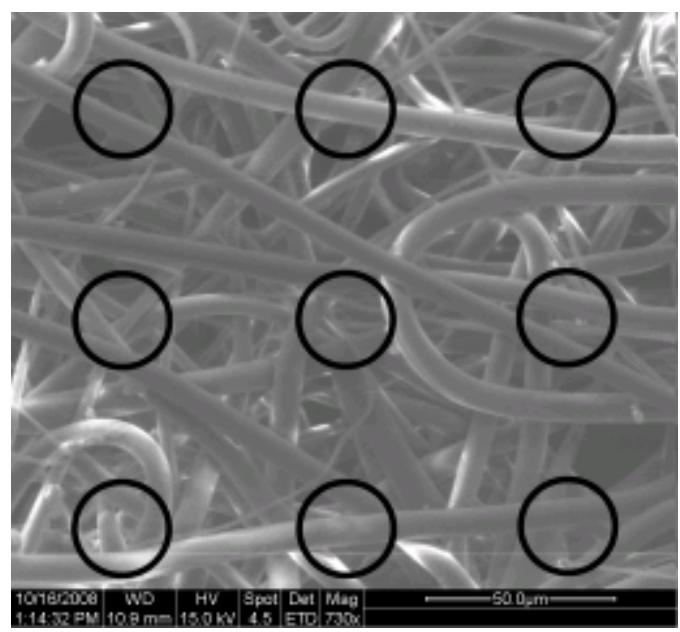

Section D2

Scale Bar: 50 microns is

3.068

$\begin{array}{rrr}\text { Sample } & \text { Length } & \text { Diameter } \\ 1 & 0.451 & 7.35 \\ 2 & 0.258 & 4.20 \\ 3 & 0.442 & 7.20 \\ 4 & 0.43 & 7.01 \\ 5 & 0.44 & 7.17 \\ 6 & 0.422 & 6.88 \\ 7 & 0.418 & 6.81 \\ 8 & 0.4 & 6.52 \\ 9 & 0.527 & 8.59\end{array}$

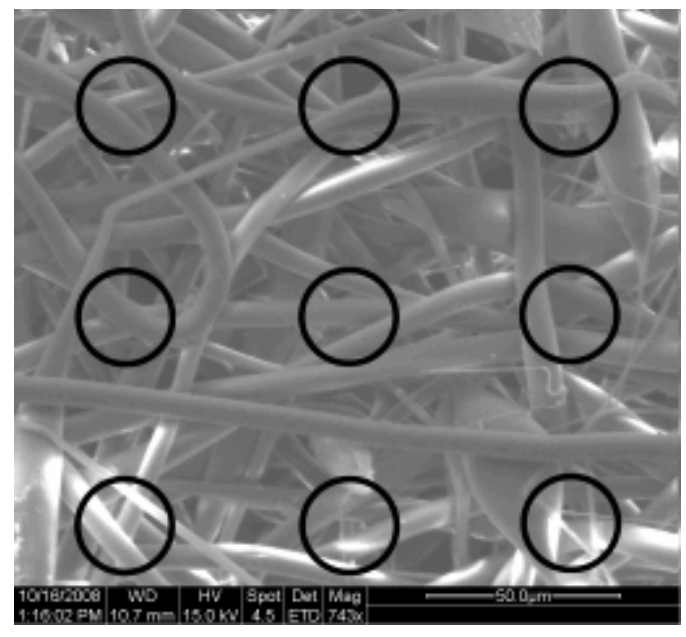

Section D3

Scale Bar: 50 microns is

2.944

$\begin{array}{rrr}\text { Sample } & \text { Length } & \text { Diameter } \\ 1 & 0.187 & 3.18 \\ 2 & 0.148 & 2.51 \\ 3 & 0.418 & 7.10 \\ 4 & 0.336 & 5.71 \\ 5 & 0.357 & 6.06 \\ 6 & 0.196 & 3.33 \\ 7 & 0.448 & 7.61 \\ 8 & 0.331 & 5.62 \\ 9 & 0.351 & 5.96\end{array}$

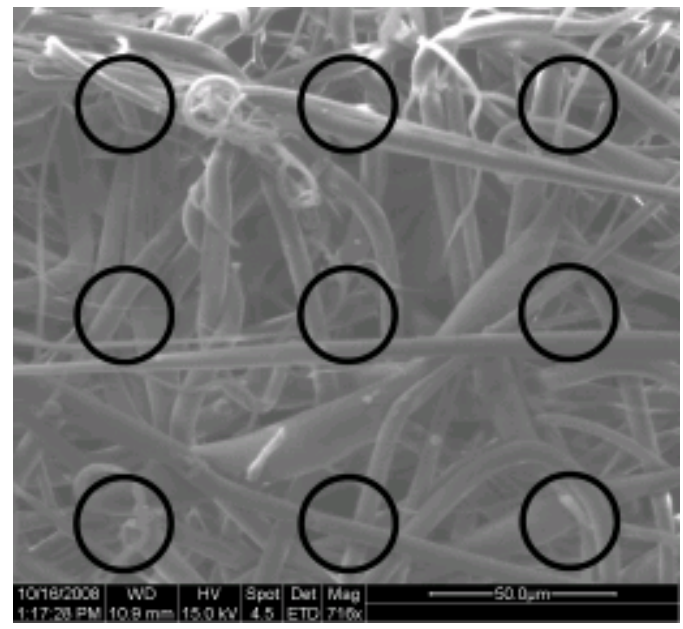




\section{SPIN 3}

Section A1

Scale Bar: 50 microns is

2.999

$\begin{array}{rrr}\text { Sample } & \text { Length } & \text { Diameter } \\ 1 & 0.406 & 6.77 \\ 2 & 0.403 & 6.72 \\ 3 & 0.414 & 6.90 \\ 4 & 0.51 & 8.50 \\ 5 & 0.418 & 6.97 \\ 6 & 0.406 & 6.77 \\ 7 & 0.448 & 7.47 \\ 8 & 0.399 & 6.65 \\ 9 & 0.464 & 7.74\end{array}$

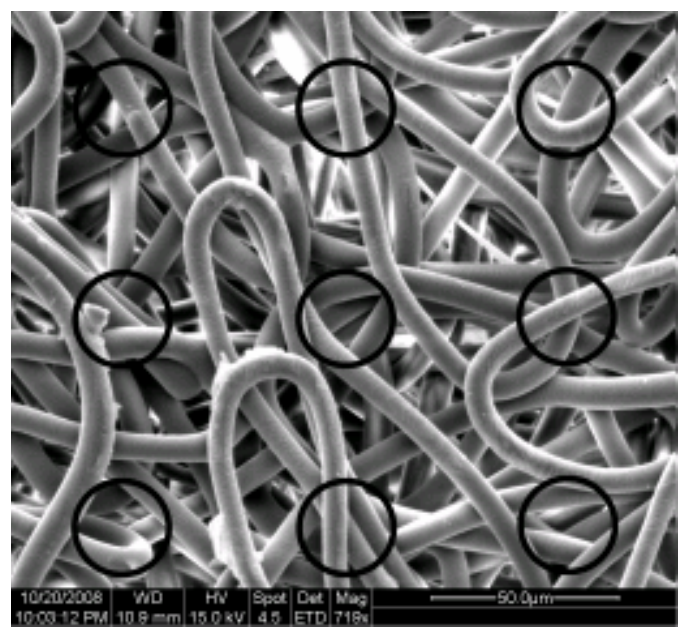

Section A2

Scale Bar: 50 microns is

2.917

$\begin{array}{rrr}\text { Sample } & \text { Length } & \text { Diameter } \\ 1 & 0.44 & 7.54 \\ 2 & 0.427 & 7.32 \\ 3 & 0.385 & 6.60 \\ 4 & 0.454 & 7.78 \\ 5 & 0.434 & 7.44 \\ 6 & 0.424 & 7.27 \\ 7 & 0.443 & 7.59 \\ 8 & 0.318 & 5.45 \\ 9 & 0.492 & 8.43\end{array}$

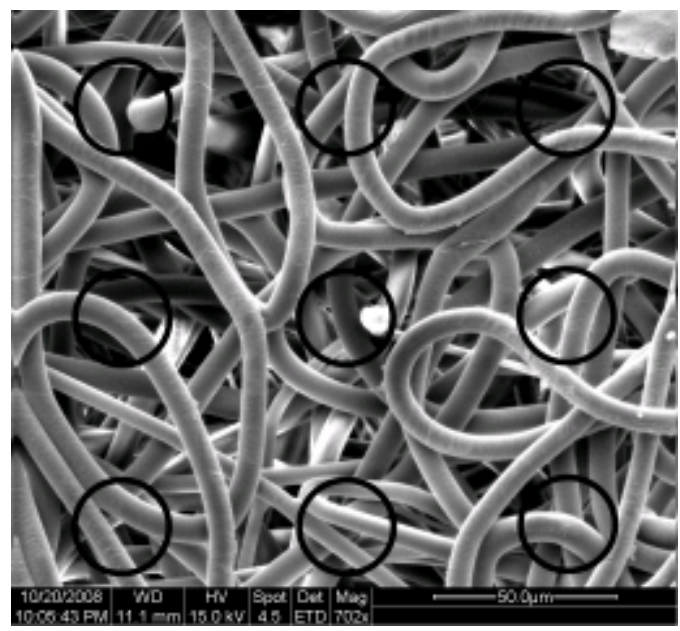

Section A3

Scale Bar: 50 microns is

2.972

$\begin{array}{rrr}\text { Sample } & \text { Length } & \text { Diameter } \\ 1 & 0.385 & 6.48 \\ 2 & 0.405 & 6.81 \\ 3 & 0.379 & 6.38 \\ 4 & 0.292 & 4.91 \\ 5 & 0.34 & 5.72 \\ 6 & 0.44 & 7.40 \\ 7 & 0.425 & 7.15 \\ 8 & 0.418 & 7.03 \\ 9 & 0.535 & 9.00\end{array}$

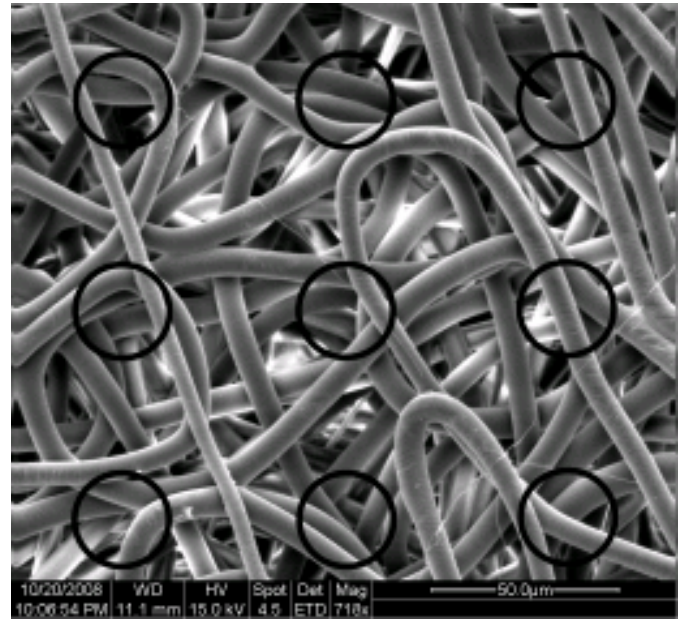


Section B1

Scale Bar: 50 microns is

2.999

$\begin{array}{rrr}\text { Sample } & \text { Length } & \text { Diameter } \\ 1 & 0.467 & 7.79 \\ 2 & 0.37 & 6.17 \\ 3 & 0.439 & 7.32 \\ 4 & 0.431 & 7.19 \\ 5 & 0.523 & 8.72 \\ 6 & 0.389 & 6.49 \\ 7 & 0.367 & 6.12 \\ 8 & 0.418 & 6.97 \\ 9 & 0.44 & 7.34\end{array}$

Section B2

Scale Bar: 50 microns is

2.917

$\begin{array}{rrr}\text { Sample } & \text { Length } & \text { Diameter } \\ 1 & 0.435 & 7.46 \\ 2 & 0.51 & 8.74 \\ 3 & 0.416 & 7.13 \\ 4 & 0.468 & 8.02 \\ 5 & 0.422 & 7.23 \\ 6 & 0.473 & 8.11 \\ 7 & 0.494 & 8.47 \\ 8 & 0.427 & 7.32 \\ 9 & 0.449 & 7.70\end{array}$

Section B3

Scale Bar: 50 microns is

2.862

$\begin{array}{rrr}\text { Sample } & \text { Length } & \text { Diameter } \\ 1 & 0.418 & 7.30 \\ 2 & 0.457 & 7.98 \\ 3 & 0.438 & 7.65 \\ 4 & 0.358 & 6.25 \\ 5 & 0.391 & 6.83 \\ 6 & 0.396 & 6.92 \\ 7 & 0.37 & 6.46 \\ 8 & 0.413 & 7.22 \\ 9 & 0.457 & 7.98\end{array}$
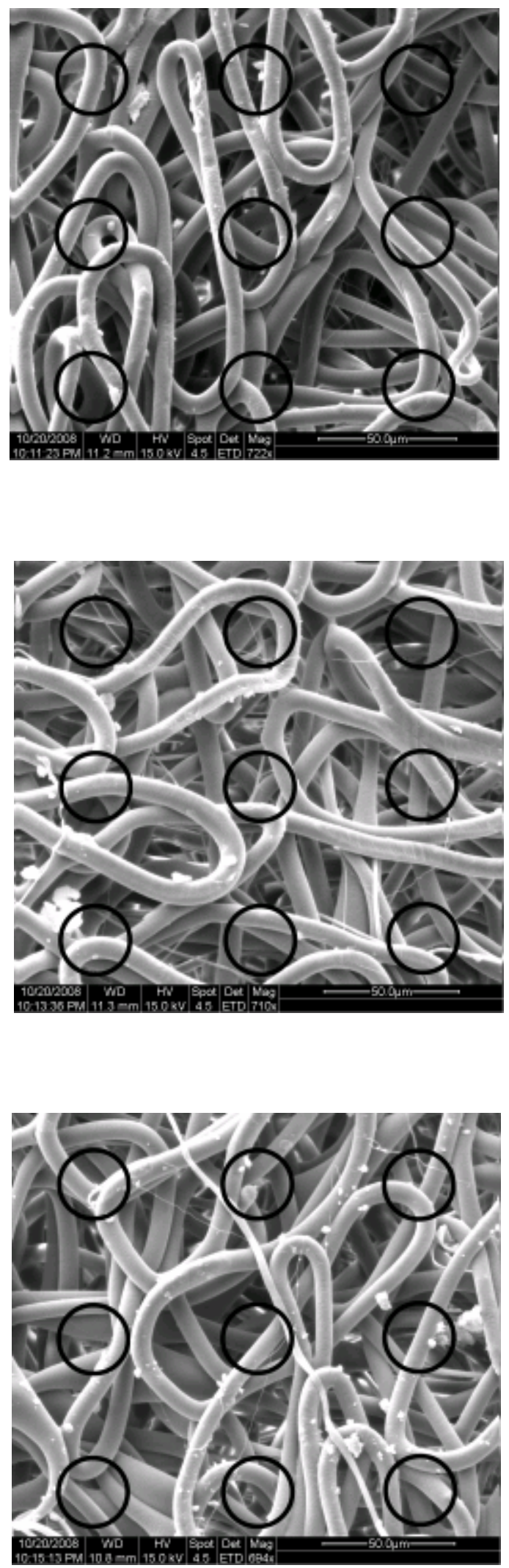
Section C1

Scale Bar: 50 microns is

2.999

$\begin{array}{rrr}\text { Sample } & \text { Length } & \text { Diameter } \\ 1 & 0.448 & 7.47 \\ 2 & 0.492 & 8.20 \\ 3 & 0.427 & 7.12 \\ 4 & 0.338 & 5.64 \\ 5 & 0.382 & 6.37 \\ 6 & 0.407 & 6.79 \\ 7 & 0.138 & 2.30 \\ 8 & 0.444 & 7.40 \\ 9 & 0.486 & 8.10\end{array}$

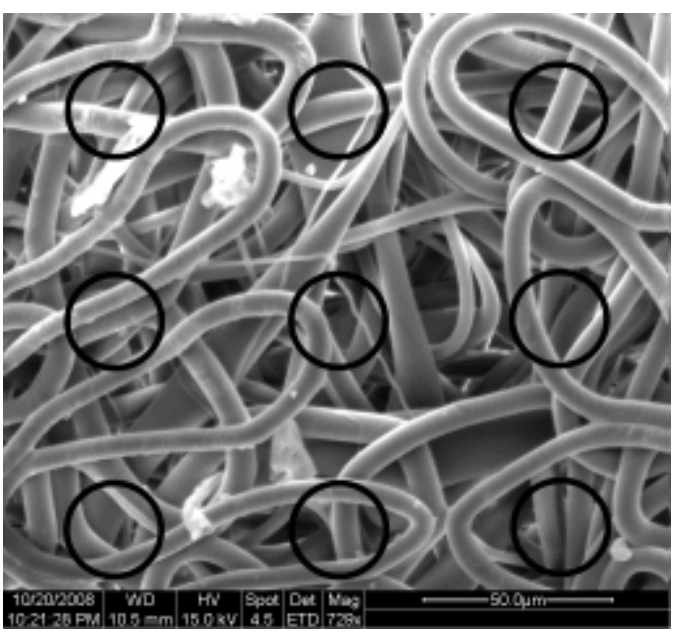

Section C2

Scale Bar: 50 microns is

2.862

$\begin{array}{rrr}\text { Sample } & \text { Length } & \text { Diameter } \\ 1 & 0.263 & 4.59 \\ 2 & 0.502 & 8.77 \\ 3 & 0.374 & 6.53 \\ 4 & 0.399 & 6.97 \\ 5 & 0.296 & 5.17 \\ 6 & 0.401 & 7.01 \\ 7 & 0.286 & 5.00 \\ 8 & 0.455 & 7.95 \\ 9 & 0.127 & 2.22\end{array}$

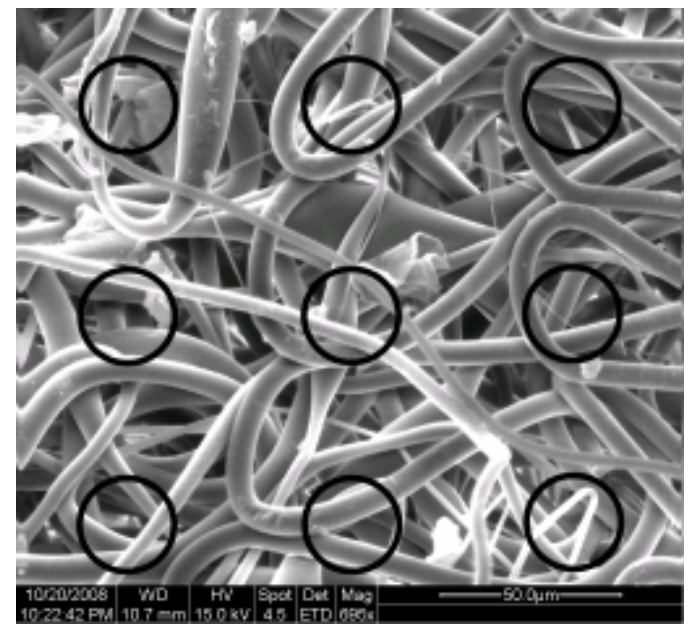

Section C3

Scale Bar: 50 microns is

$\begin{array}{rrr}\text { Sample } & \text { Length } & \text { Diameter } \\ 1 & 0.41 & 7.23 \\ 2 & 0.347 & 6.12 \\ 3 & 0.379 & 6.69 \\ 4 & 0.376 & 6.63 \\ 5 & 0.354 & 6.25 \\ 6 & 0.372 & 6.56 \\ 7 & 0.409 & 7.22 \\ 8 & 0.361 & 6.37 \\ 9 & 0.359 & 6.33\end{array}$

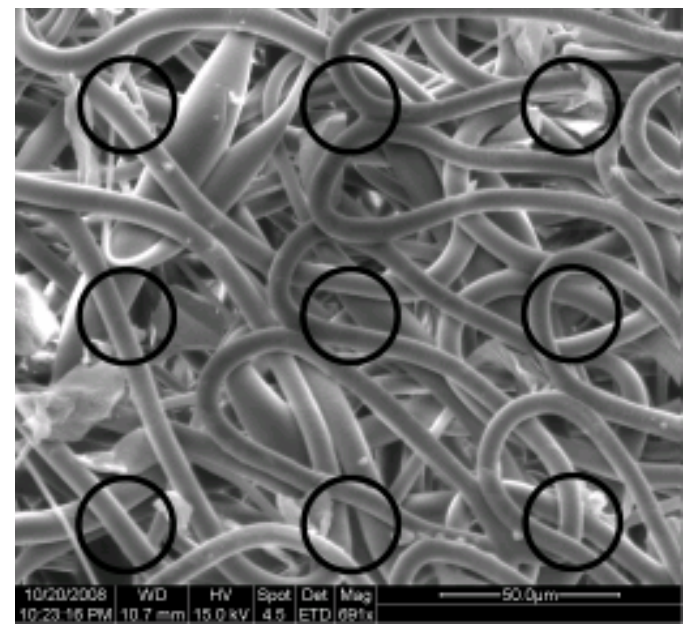


Section D1

Scale Bar: 50 microns is

2.903

$\begin{array}{rrr}\text { Sample } & \text { Length } & \text { Diameter } \\ 1 & 0.41 & 7.06 \\ 2 & 0.346 & 5.96 \\ 3 & 0.088 & 1.52 \\ 4 & 0.069 & 1.19 \\ 5 & 0.392 & 6.75 \\ 6 & 0.374 & 6.44 \\ 7 & 0.696 & 11.99 \\ 8 & 0.105 & 1.81 \\ 9 & 0.424 & 7.30\end{array}$

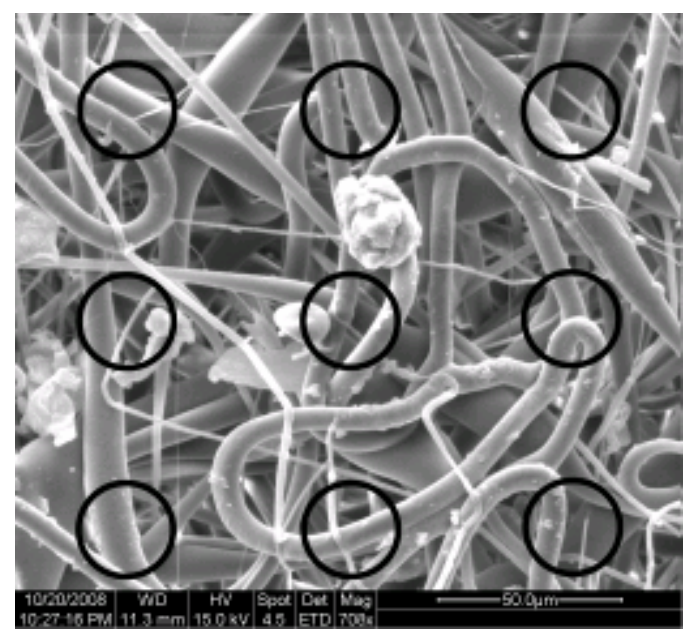

Section D2

Scale Bar: 50 microns is

2.889

$\begin{array}{rrr}\text { Sample } & \text { Length } & \text { Diameter } \\ 1 & 0.474 & 8.20 \\ 2 & 0.36 & 6.23 \\ 3 & 0.388 & 6.72 \\ 4 & 0.474 & 8.20 \\ 5 & 0.074 & 1.28 \\ 6 & 0.389 & 6.73 \\ 7 & 0.157 & 2.72 \\ 8 & 0.388 & 6.72 \\ 9 & 0.376 & 6.51\end{array}$

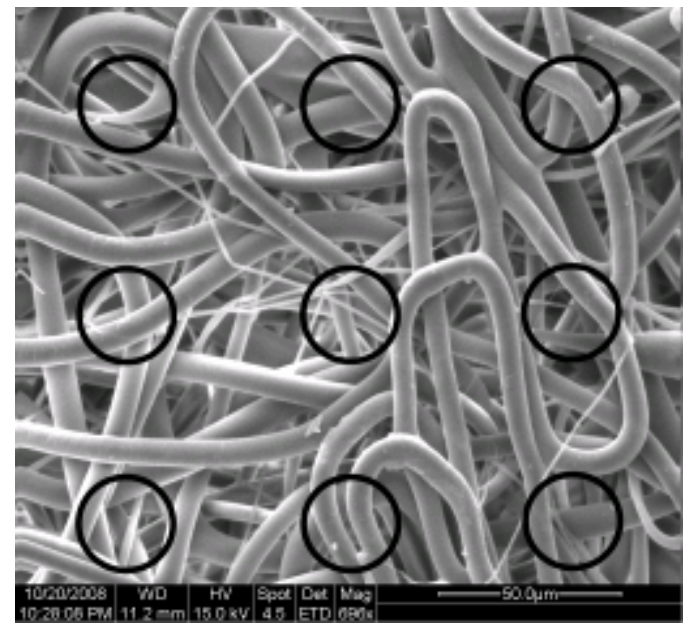

Section D3

Scale Bar: 50 microns is

2.944

$\begin{array}{rrr}\text { Sample } & \text { Length } & \text { Diameter } \\ 1 & 0.113 & 1.92 \\ 2 & 0.251 & 4.26 \\ 3 & 0.364 & 6.18 \\ 4 & 0.911 & 15.47 \\ 5 & 1.092 & 18.55 \\ 6 & 0.381 & 6.47 \\ 7 & 0.415 & 7.05 \\ 8 & 0.39 & 6.62 \\ 9 & 0.381 & 6.47\end{array}$

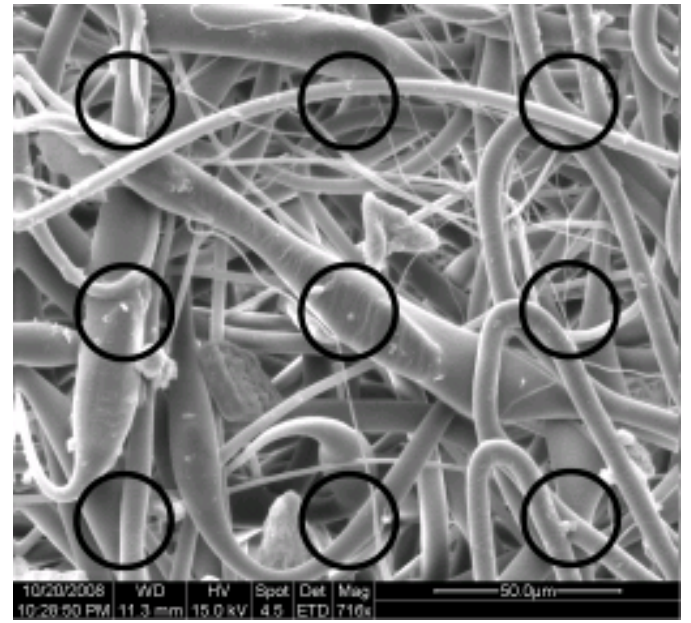




\section{SPIN 4}

Section A1

Scale Bar: 50 microns is

2.889

$\begin{array}{rrr}\text { Sample } & \text { Length } & \text { Diameter } \\ 1 & 0.387 & 6.70 \\ 2 & 0.481 & 8.32 \\ 3 & 0.319 & 5.52 \\ 4 & 0.486 & 8.41 \\ 5 & 0.38 & 6.58 \\ 6 & 0.453 & 7.84 \\ 7 & 0.489 & 8.46 \\ 8 & 0.481 & 8.32 \\ 9 & 0.501 & 8.67\end{array}$

Section A2

Scale Bar: 50 microns is

2.93

$\begin{array}{rrr}\text { Sample } & \text { Length } & \text { Diameter } \\ 1 & 0.492 & 8.40 \\ 2 & 0.479 & 8.17 \\ 3 & 0.506 & 8.63 \\ 4 & 0.461 & 7.87 \\ 5 & 0.496 & 8.46 \\ 6 & 0.409 & 6.98 \\ 7 & 0.464 & 7.92 \\ 8 & 0.462 & 7.88 \\ 9 & 0.511 & 8.72\end{array}$

Section A3

Scale Bar: 50 microns is

2.889

$\begin{array}{rrr}\text { Sample } & \text { Length } & \text { Diameter } \\ 1 & 0.358 & 6.20 \\ 2 & 0.482 & 8.34 \\ 3 & 0.489 & 8.46 \\ 4 & 0.486 & 8.41 \\ 5 & 0.486 & 8.41 \\ 6 & 0.443 & 7.67 \\ 7 & 0.497 & 8.60 \\ 8 & 0.462 & 8.00 \\ 9 & 0.458 & 7.93\end{array}$
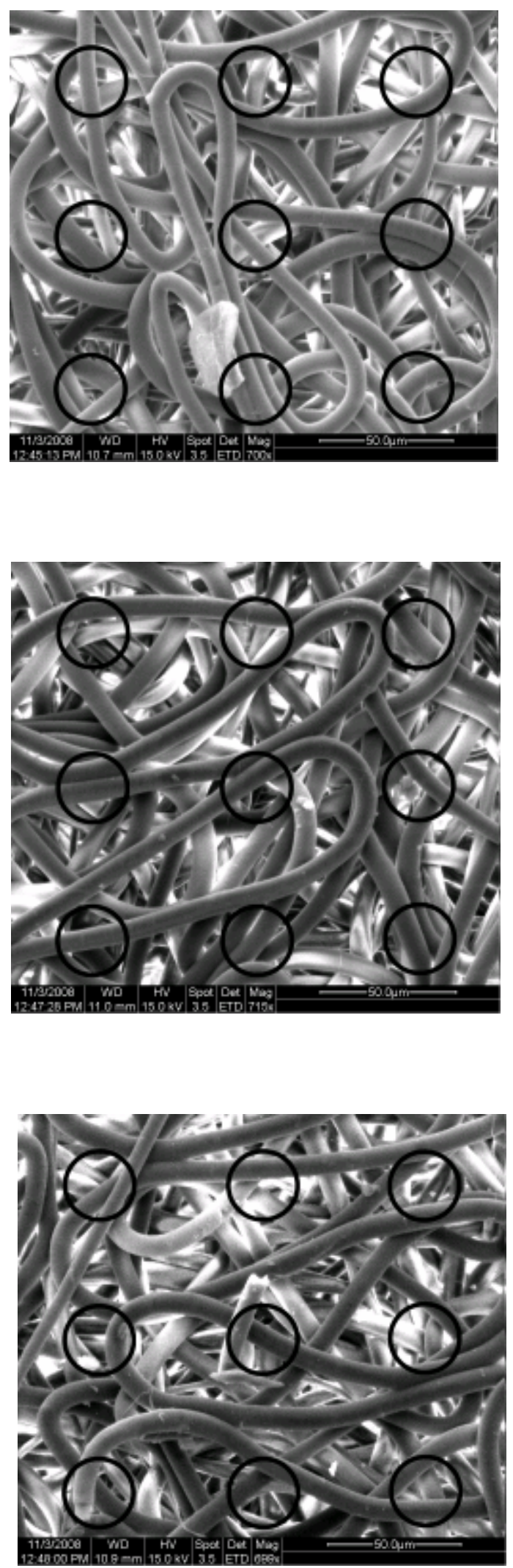
Section B1

Scale Bar: 50 microns is

2.889

$\begin{array}{rrr}\text { Sample } & \text { Length } & \text { Diameter } \\ 1 & 0.08 & 1.38 \\ 2 & 0.466 & 8.07 \\ 3 & 0.477 & 8.26 \\ 4 & 0.363 & 6.28 \\ 5 & 0.672 & 11.63 \\ 6 & 0.461 & 7.98 \\ 7 & 0.333 & 5.76 \\ 8 & 0.353 & 6.11 \\ 9 & 0.462 & 8.00\end{array}$

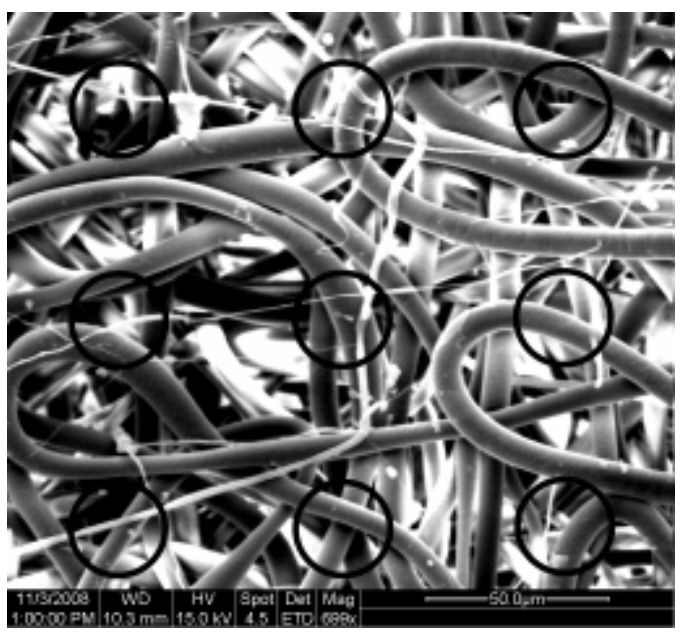

Section B2

Scale Bar: 50 microns is

2.834

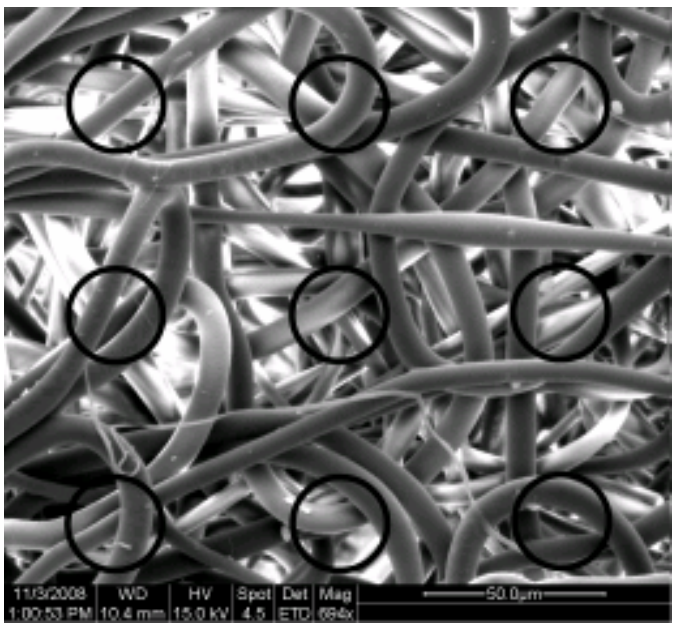

Section B3

Scale Bar: 50 microns is

2.82

$\begin{array}{rrr}\text { Sample } & \text { Length } & \text { Diameter } \\ 1 & 0.446 & 7.91 \\ 2 & 0.412 & 7.30 \\ 3 & 0.363 & 6.44 \\ 4 & 0.389 & 6.90 \\ 5 & 0.419 & 7.43 \\ 6 & 0.448 & 7.94 \\ 7 & 0.448 & 7.94 \\ 8 & 0.374 & 6.63 \\ 9 & 0.462 & 8.19\end{array}$

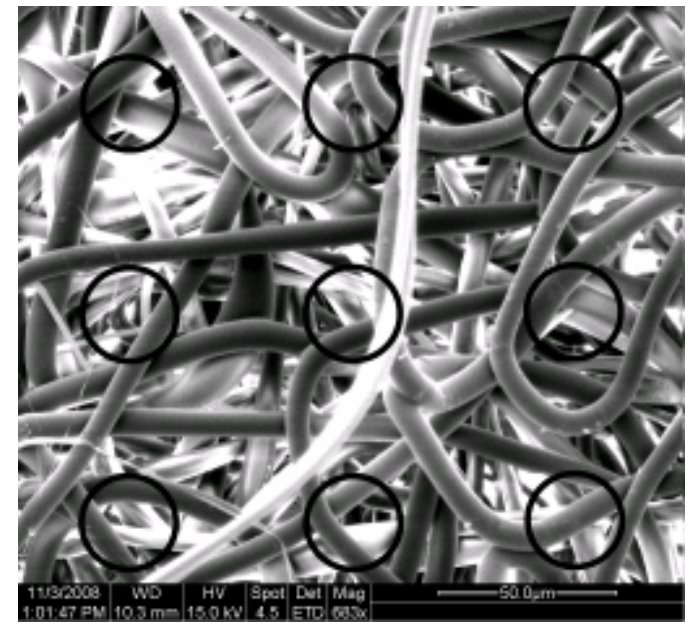


Section C1

Scale Bar: 50 microns is

2.93

\begin{tabular}{rrr} 
Sample & Length & \multicolumn{1}{c}{ Diameter } \\
1 & 0.514 & 8.77 \\
2 & 0.488 & 8.33 \\
3 & 0.363 & 6.19 \\
4 & 0.493 & 8.41 \\
5 & 0.508 & 8.67 \\
6 & 0.427 & 7.29 \\
7 & 0.501 & 8.55 \\
8 & 0.471 & 8.04 \\
9 & 0.593 & 10.12
\end{tabular}

Section C2

Scale Bar: 50 microns is

2.889

$\begin{array}{rrr}\text { Sample } & \text { Length } & \text { Diameter } \\ 1 & 0.43 & 7.44 \\ 2 & 0.193 & 3.34 \\ 3 & 0.321 & 5.56 \\ 4 & 0.388 & 6.72 \\ 5 & 0.448 & 7.75 \\ 6 & 0.437 & 7.56 \\ 7 & 0.467 & 8.08 \\ 8 & 0.451 & 7.81 \\ 9 & 0.44 & 7.62\end{array}$

Section C3

Scale Bar: 50 microns is

2.944

$\begin{array}{rrr}\text { Sample } & \text { Length } & \text { Diameter } \\ 1 & 0.358 & 6.08 \\ 2 & 0.389 & 6.61 \\ 3 & 0.478 & 8.12 \\ 4 & 0.456 & 7.74 \\ 5 & 0.399 & 6.78 \\ 6 & 0.467 & 7.93 \\ 7 & 0.432 & 7.34 \\ 8 & 0.372 & 6.32 \\ 9 & 0.5 & 8.49\end{array}$
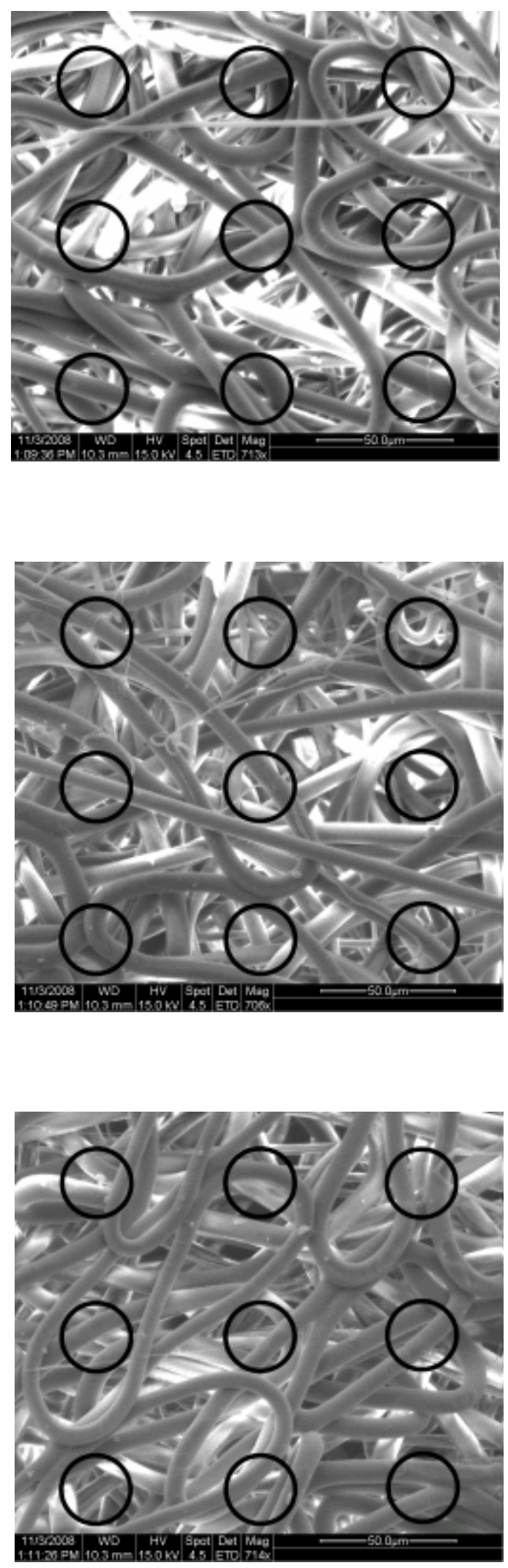
Section D1

Scale Bar: 50 microns is

2.875

$\begin{array}{rrr}\text { Sample } & \text { Length } & \text { Diameter } \\ 1 & 0.485 & 8.43 \\ 2 & 0.505 & 8.78 \\ 3 & 0.488 & 8.49 \\ 4 & 0.478 & 8.31 \\ 5 & 0.497 & 8.64 \\ 6 & 0.479 & 8.33 \\ 7 & 0.464 & 8.07 \\ 8 & 0.437 & 7.60 \\ 9 & 0.47 & 8.17\end{array}$

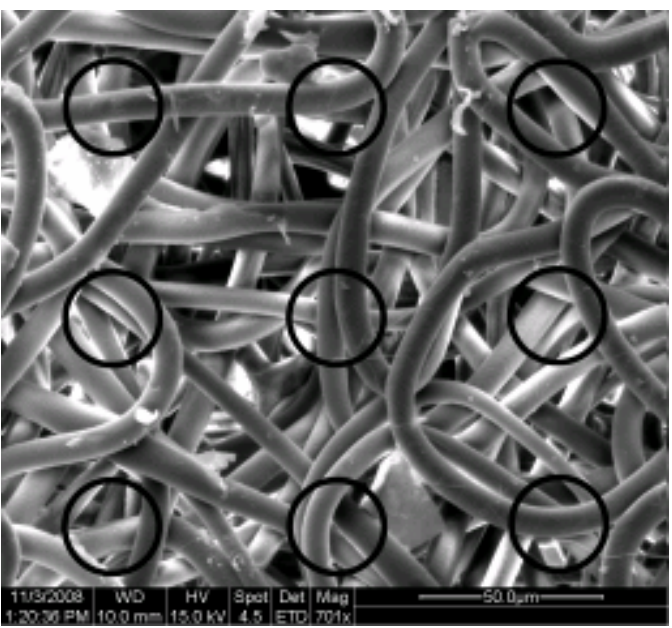

Section D2

Scale Bar: 50 microns is

2.917

$\begin{array}{rrr}\text { Sample } & \text { Length } & \text { Diameter } \\ 1 & 0.514 & 8.81 \\ 2 & 0.414 & 7.10 \\ 3 & 0.437 & 7.49 \\ 4 & 0.456 & 7.82 \\ 5 & 0.421 & 7.22 \\ 6 & 0.418 & 7.16 \\ 7 & 0.44 & 7.54 \\ 8 & 0.453 & 7.76 \\ 9 & 0.385 & 6.60\end{array}$

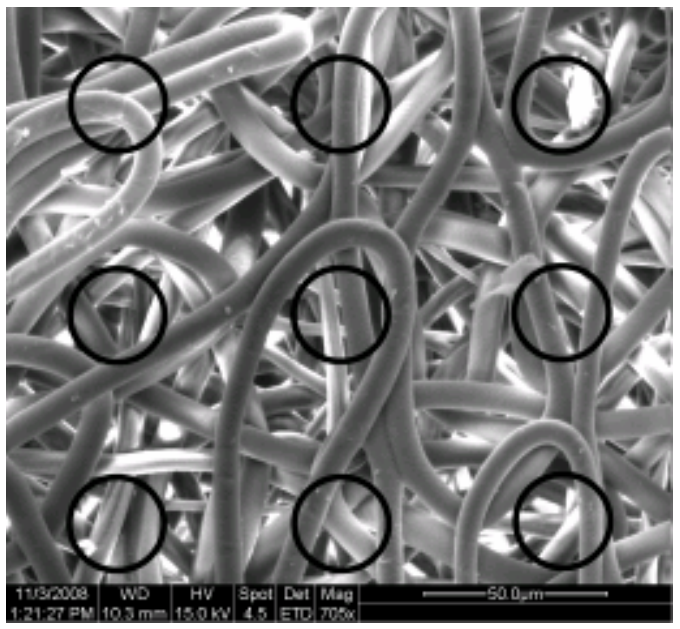

Section D3

Scale Bar: 50 microns is

2.875

$\begin{array}{rrr}\text { Sample } & \text { Length } & \text { Diameter } \\ 1 & 0.427 & 7.43 \\ 2 & 0.457 & 7.95 \\ 3 & 0.448 & 7.79 \\ 4 & 0.493 & 8.57 \\ 5 & 0.442 & 7.69 \\ 6 & 0.523 & 9.10 \\ 7 & 0.317 & 5.51 \\ 8 & 0.409 & 7.11 \\ 9 & 0.509 & 8.85\end{array}$

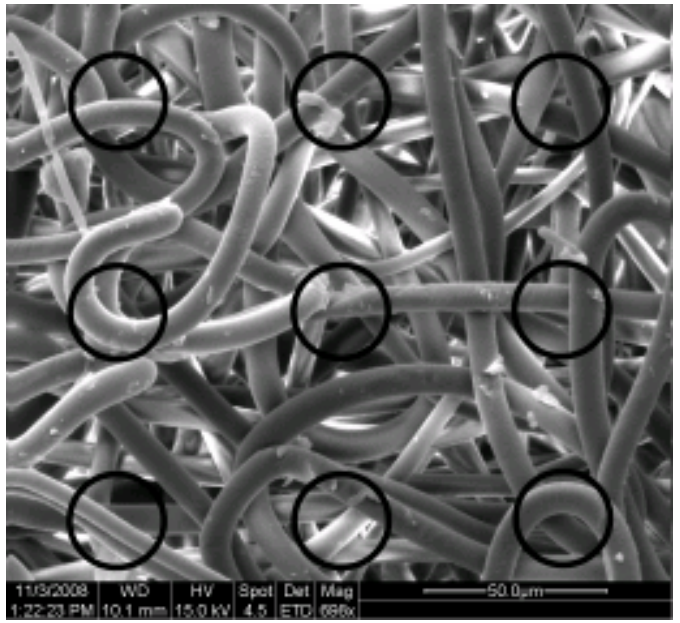




\section{SPIN 5}

Section A1

Scale Bar: 50 microns is

2.82

$\begin{array}{rrr}\text { Sample } & \text { Length } & \text { Diameter } \\ 1 & 0.191 & 3.39 \\ 2 & 0.397 & 7.04 \\ 3 & 0.469 & 8.32 \\ 4 & 0.351 & 6.22 \\ 5 & 0.351 & 6.22 \\ 6 & 0.467 & 8.28 \\ 7 & 0.393 & 6.97 \\ 8 & 0.418 & 7.41 \\ 9 & 0.205 & 3.63\end{array}$

Section A2

Scale Bar: 50 microns is

2.903

$\begin{array}{rrr}\text { Sample } & \text { Length } & \text { Diameter } \\ 1 & 0.218 & 3.75 \\ 2 & 0.366 & 6.30 \\ 3 & 0.203 & 3.50 \\ 4 & 0.451 & 7.77 \\ 5 & 0.468 & 8.06 \\ 6 & 0.413 & 7.11 \\ 7 & 0.54 & 9.30 \\ 8 & 0.468 & 8.06 \\ 9 & 0.499 & 8.59\end{array}$

Section A3

Scale Bar: 50 microns is

2.889

$\begin{array}{rrr}\text { Sample } & \text { Length } & \text { Diameter } \\ 1 & 0.111 & 1.92 \\ 2 & 0.447 & 7.74 \\ 3 & 0.358 & 6.20 \\ 4 & 0.195 & 3.37 \\ 5 & 0.389 & 6.73 \\ 6 & 0.364 & 6.30 \\ 7 & 0.358 & 6.20 \\ 8 & 0.348 & 6.02 \\ 9 & 0.389 & 6.73\end{array}$
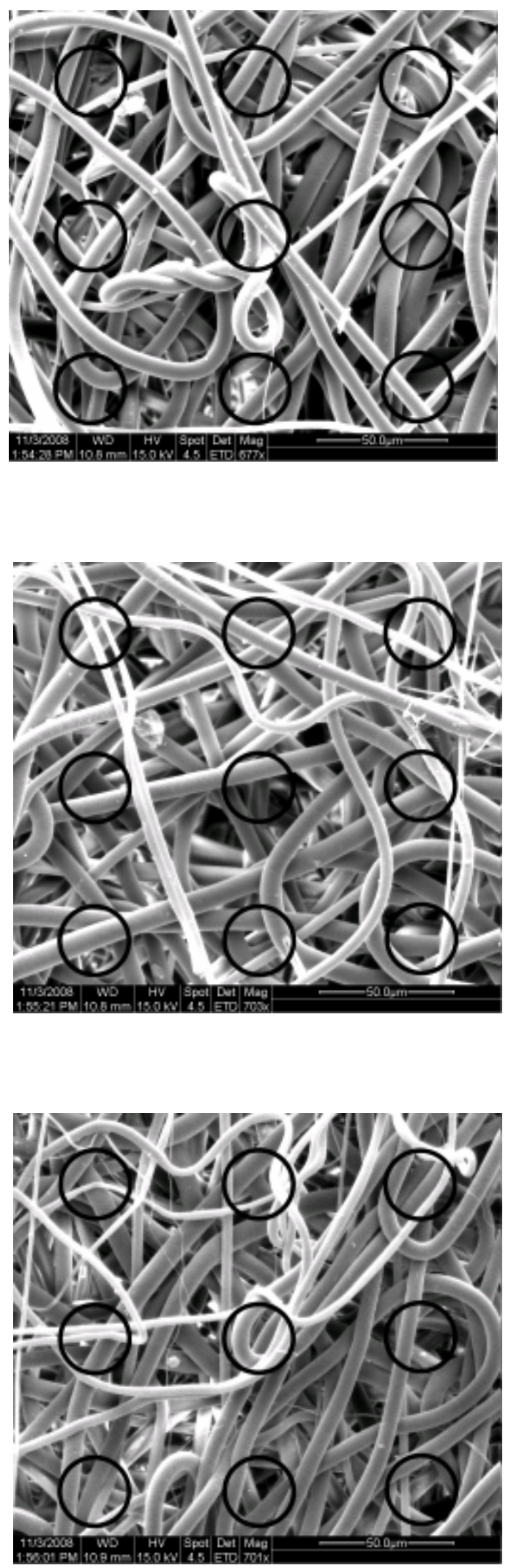
Section B1

Scale Bar: 50 microns is

2.862

$\begin{array}{rrr}\text { Sample } & \text { Length } & \text { Diameter } \\ 1 & 0.467 & 8.16 \\ 2 & 0.224 & 3.91 \\ 3 & 0.431 & 7.53 \\ 4 & 0.44 & 7.69 \\ 5 & 0.314 & 5.49 \\ 6 & 0.191 & 3.34 \\ 7 & 0.37 & 6.46 \\ 8 & 0.265 & 4.63 \\ 9 & 0.359 & 6.27\end{array}$

Section B2

Scale Bar: 50 microns is

2.889

$\begin{array}{rrr}\text { Sample } & \text { Length } & \text { Diameter } \\ 1 & 0.382 & 6.61 \\ 2 & 0.309 & 5.35 \\ 3 & 0.118 & 2.04 \\ 4 & 0.399 & 6.91 \\ 5 & 0.364 & 6.30 \\ 6 & 0.358 & 6.20 \\ 7 & 0.36 & 6.23 \\ 8 & 0.24 & 4.15 \\ 9 & 0.2 & 3.46\end{array}$

Section B3

Scale Bar: 50 microns is

2.903

$\begin{array}{rrr}\text { Sample } & \text { Length } & \text { Diameter } \\ 1 & 0.393 & 6.77 \\ 2 & 0.097 & 1.67 \\ 3 & 0.416 & 7.17 \\ 4 & 0.326 & 5.61 \\ 5 & 0.444 & 7.65 \\ 6 & 0.265 & 4.56 \\ 7 & 0.193 & 3.32 \\ 8 & 0.396 & 6.82 \\ 9 & 0.408 & 7.03\end{array}$
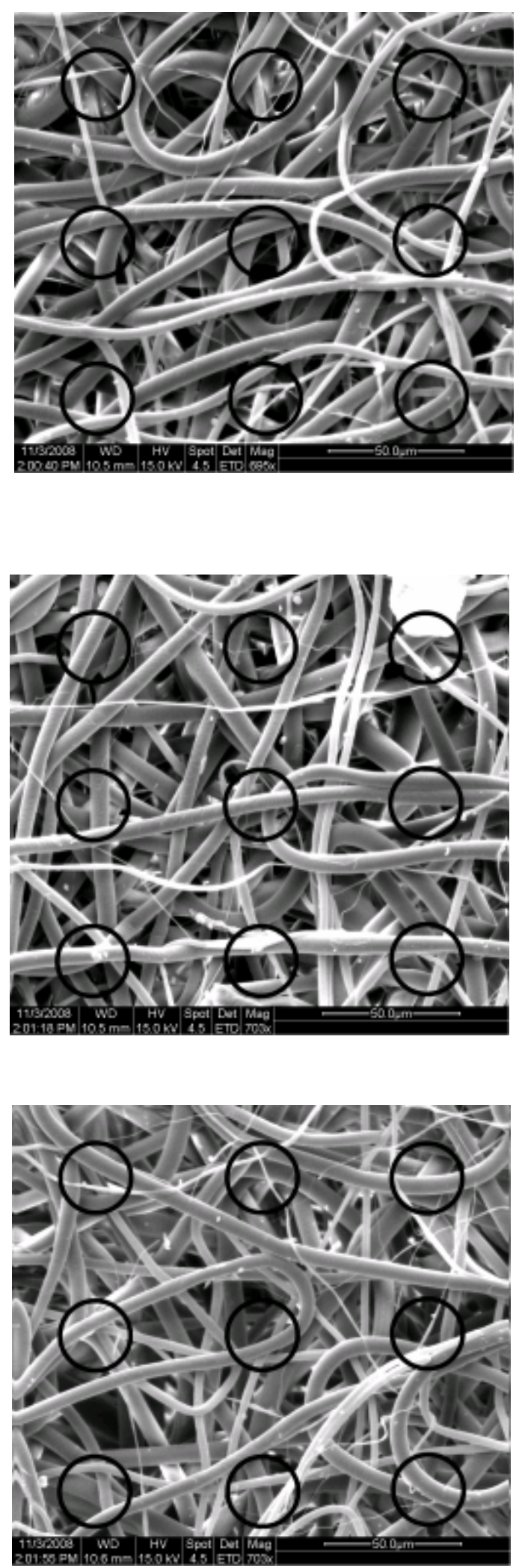
Section C1

Scale Bar: 50 microns is

2.848

$\begin{array}{rrr}\text { Sample } & \text { Length } & \text { Diameter } \\ 1 & 0.131 & 2.30 \\ 2 & 0.166 & 2.91 \\ 3 & 0.403 & 7.08 \\ 4 & 0.24 & 4.21 \\ 5 & 0.382 & 6.71 \\ 6 & 0.258 & 4.53 \\ 7 & 0.197 & 3.46 \\ 8 & 0.246 & 4.32 \\ 9 & 0.401 & 7.04\end{array}$

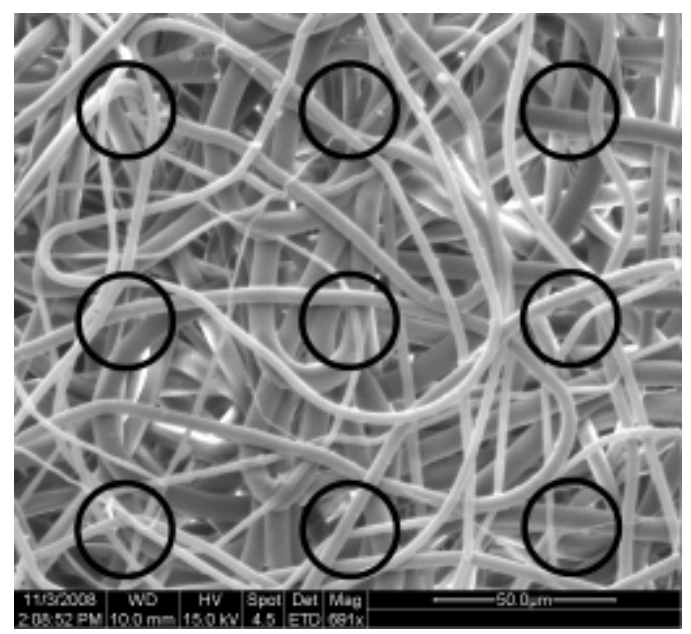

Section C2

Scale Bar: 50 microns is

2.848

$\begin{array}{rrr}\text { Sample } & \text { Length } & \text { Diameter } \\ 1 & 0.432 & 7.58 \\ 2 & 0.428 & 7.51 \\ 3 & 0.37 & 6.50 \\ 4 & 0.405 & 7.11 \\ 5 & 0.387 & 6.79 \\ 6 & 0.207 & 3.63 \\ 7 & 0.358 & 6.29 \\ 8 & 0.176 & 3.09 \\ 9 & 0.414 & 7.27\end{array}$

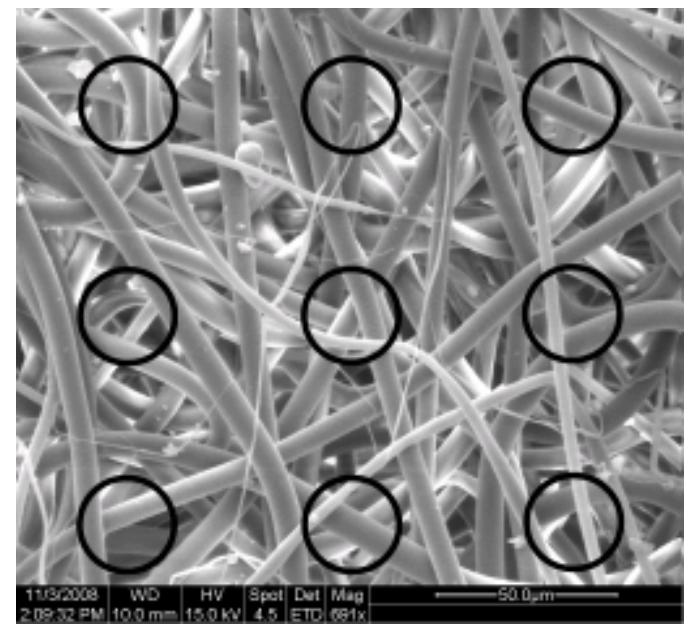

Section C3

Scale Bar: 50 microns is

2.875

$\begin{array}{rrr}\text { Sample } & \text { Length } & \text { Diameter } \\ 1 & 0.418 & 7.27 \\ 2 & 0.394 & 6.85 \\ 3 & 0.197 & 3.43 \\ 4 & 0.34 & 5.91 \\ 5 & 0.424 & 7.37 \\ 6 & 0.215 & 3.74 \\ 7 & 0.36 & 6.26 \\ 8 & 0.432 & 7.51 \\ 9 & 0.333 & 5.79\end{array}$

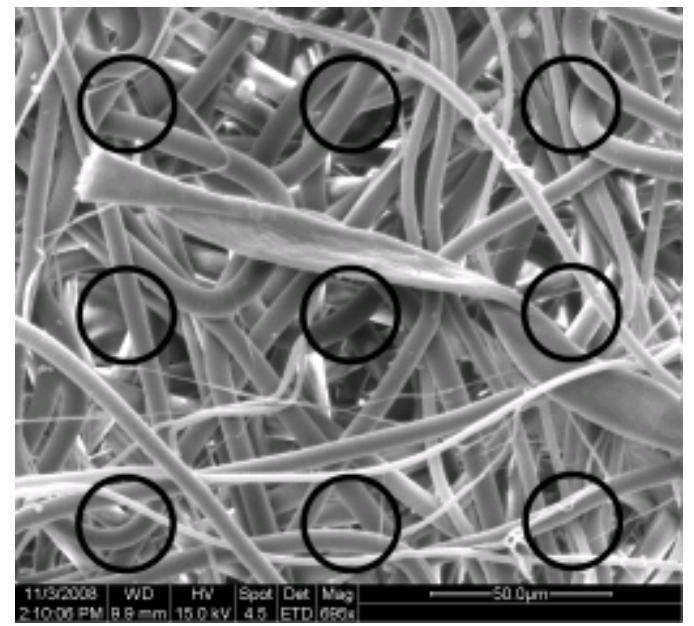


Section D1

Scale Bar: 50 microns is

2.889

$\begin{array}{rrr}\text { Sample } & \text { Length } & \text { Diameter } \\ 1 & 0.413 & 7.15 \\ 2 & 0.344 & 5.95 \\ 3 & 0.399 & 6.91 \\ 4 & 0.514 & 8.90 \\ 5 & 0.214 & 3.70 \\ 6 & 0.45 & 7.79 \\ 7 & 0.39 & 6.75 \\ 8 & 0.118 & 2.04 \\ 9 & 0.382 & 6.61\end{array}$

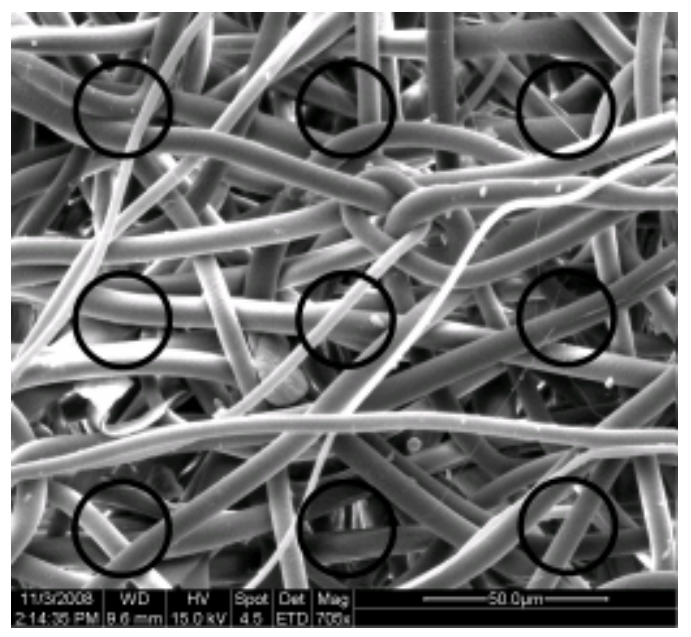

Section D2

Scale Bar: 50 microns is

2.889

$\begin{array}{rrr}\text { Sample } & \text { Length } & \text { Diameter } \\ 1 & 0.36 & 6.23 \\ 2 & 0.39 & 6.75 \\ 3 & 0.278 & 4.81 \\ 4 & 0.415 & 7.18 \\ 5 & 0.451 & 7.81 \\ 6 & 0.234 & 4.05 \\ 7 & 0.527 & 9.12 \\ 8 & 0.382 & 6.61 \\ 9 & 0.401 & 6.94\end{array}$

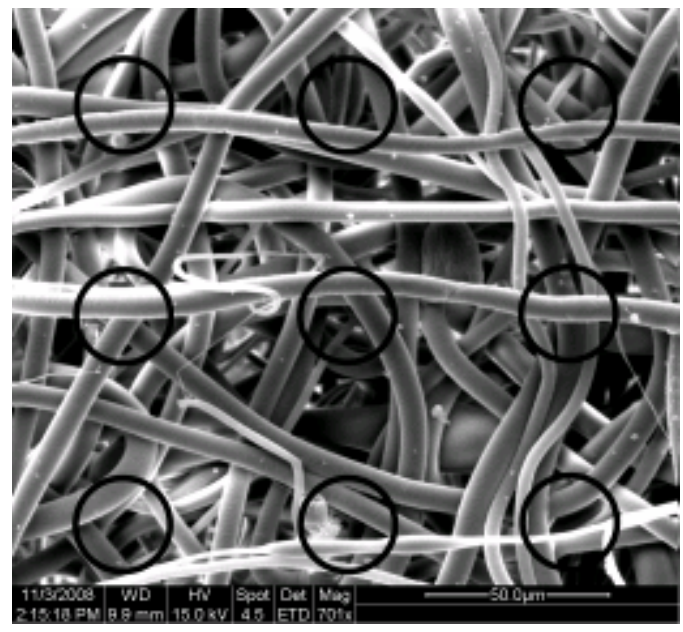

Section D3

Scale Bar: 50 microns is

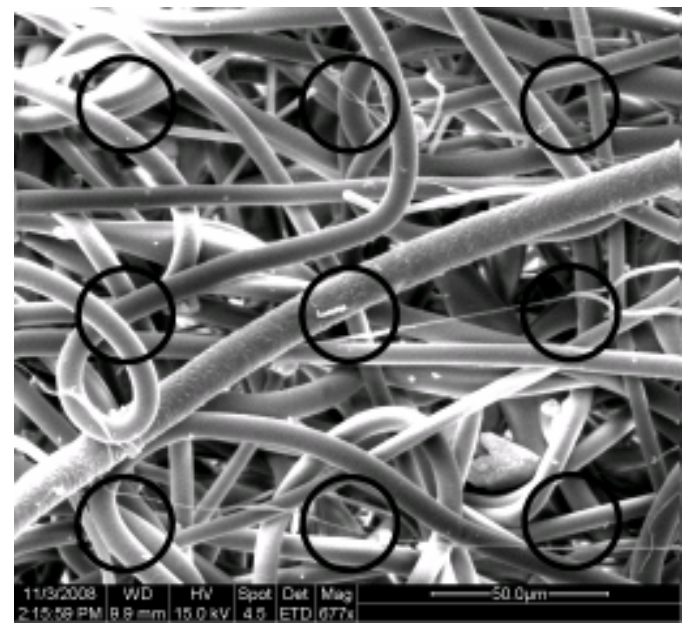




\section{SPIN 6}

Section A1

Scale Bar: 50 microns is

2.834

\begin{tabular}{rrr} 
Sample & Length & \multicolumn{1}{c}{ Diameter } \\
1 & 0.638 & 11.26 \\
2 & 0.469 & 8.27 \\
3 & 0.488 & 8.61 \\
4 & 0.489 & 8.63 \\
5 & 0.419 & 7.39 \\
6 & 0.486 & 8.57 \\
7 & 0.384 & 6.77 \\
8 & 0.361 & 6.37 \\
9 & 0.37 & 6.53
\end{tabular}

Section A2

Scale Bar: 50 microns is

2.903

$\begin{array}{rrr}\text { Sample } & \text { Length } & \text { Diameter } \\ 1 & 0.587 & 10.11 \\ 2 & 0.529 & 9.11 \\ 3 & 0.641 & 11.04 \\ 4 & 0.318 & 5.48 \\ 5 & 0.138 & 2.38 \\ 6 & 0.456 & 7.85 \\ 7 & 0.477 & 8.22 \\ 8 & 0.486 & 8.37 \\ 9 & 0.462 & 7.96\end{array}$

Section A3

Scale Bar: 50 microns is

2.917

$\begin{array}{rrr}\text { Sample } & \text { Length } & \text { Diameter } \\ 1 & 0.254 & 4.35 \\ 2 & 0.544 & 9.32 \\ 3 & 0.609 & 10.44 \\ 4 & 0.44 & 7.54 \\ 5 & 0.486 & 8.33 \\ 6 & 0.502 & 8.60 \\ 7 & 0.432 & 7.40 \\ 8 & 0.523 & 8.96 \\ 9 & 0.482 & 8.26\end{array}$
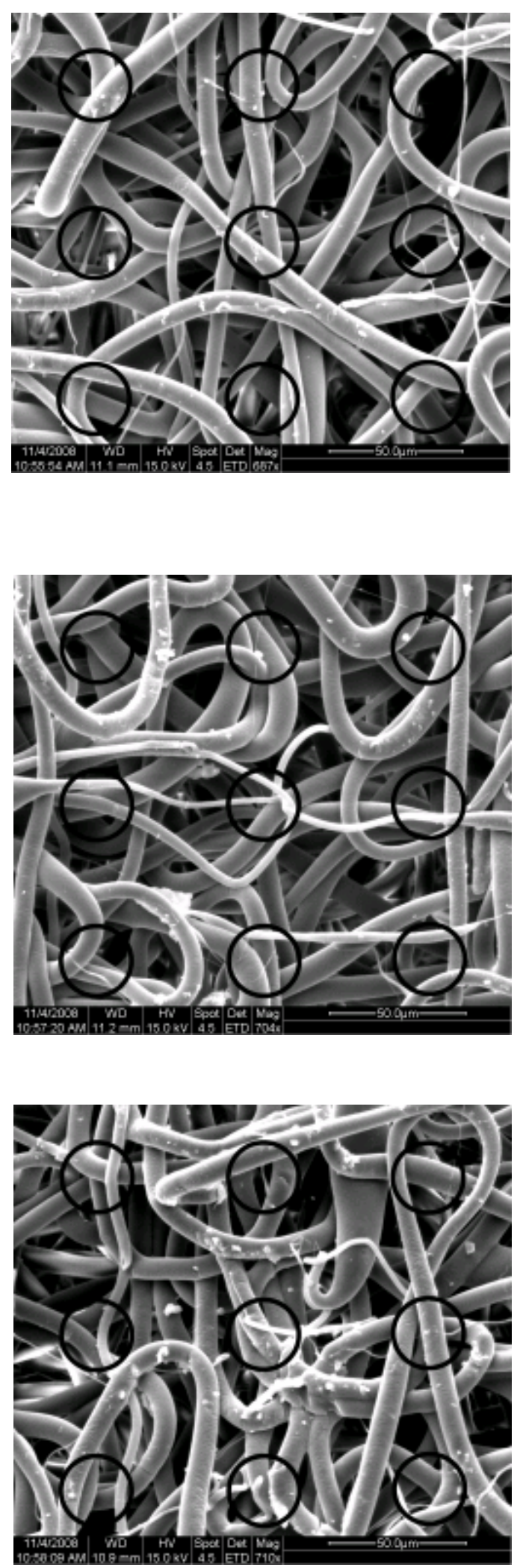
Section B1

Scale Bar: 50 microns is

$\begin{array}{rrr}\text { Sample } & \text { Length } & \text { Diameter } \\ 1 & 0.083 & 1.42 \\ 2 & 1.016 & 17.42 \\ 3 & 0.475 & 8.14 \\ 4 & 0.461 & 7.90 \\ 5 & 0.448 & 7.68 \\ 6 & 0.399 & 6.84 \\ 7 & 0.443 & 7.59 \\ 8 & 0.273 & 4.68 \\ 9 & 0.357 & 6.12\end{array}$

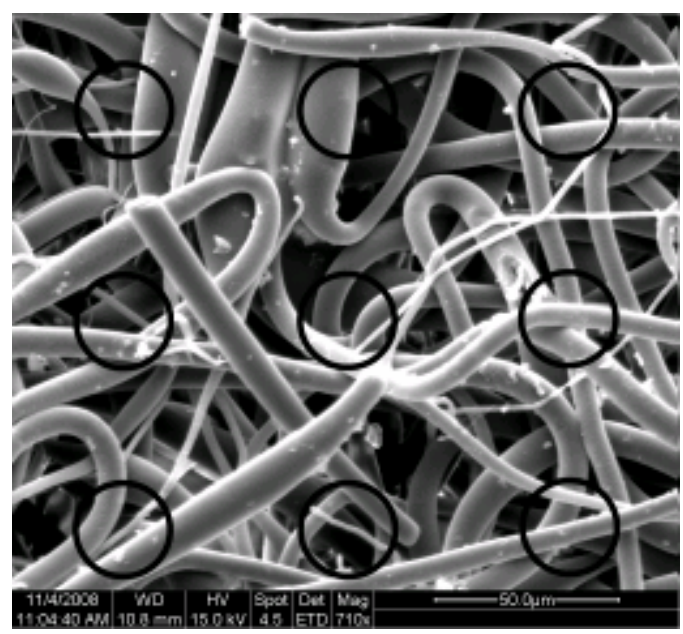

Section B2

Scale Bar: 50 microns is

2.82

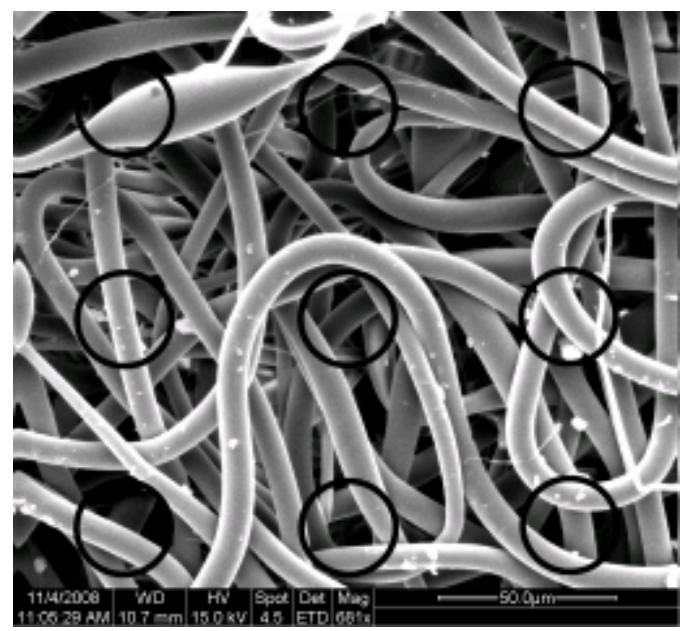

Section B3

Scale Bar: 50 microns is

$\begin{array}{rrr}\text { Sample } & \text { Length } & \text { Diameter } \\ 1 & 0.406 & 7.23 \\ 2 & 0.455 & 8.10 \\ 3 & 0.289 & 5.15 \\ 4 & 0.394 & 7.02 \\ 5 & 0.451 & 8.03 \\ 6 & 0.382 & 6.80 \\ 7 & 0.317 & 5.65 \\ 8 & 0.449 & 8.00 \\ 9 & 0.512 & 9.12\end{array}$

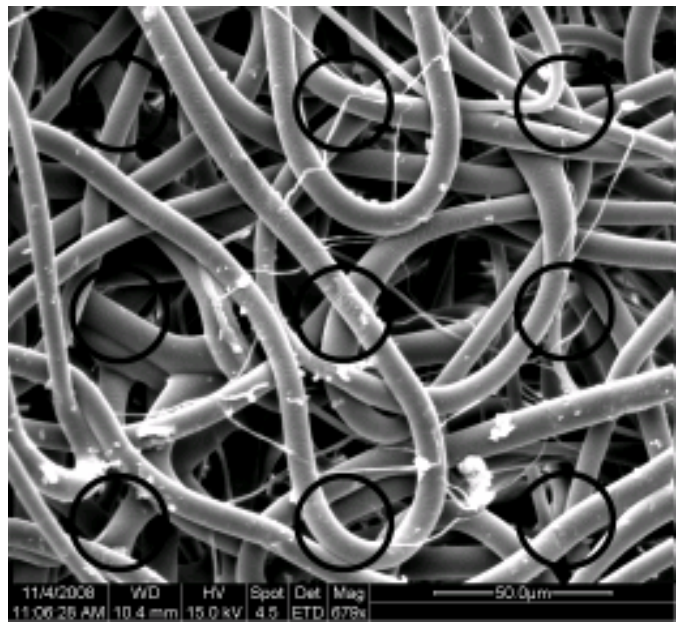


Section C1

Scale Bar: 50 microns is

2.889

Sample Length Diameter

$\begin{array}{rrr}1 & 0.5 & 8.65 \\ 2 & 0.234 & 4.05 \\ 3 & 0.446 & 7.72 \\ 4 & 0.418 & 7.23 \\ 5 & 0.449 & 7.77 \\ 6 & 0.427 & 7.39 \\ 7 & 0.473 & 8.19 \\ 8 & 0.418 & 7.23 \\ 9 & 0.485 & 8.39\end{array}$

Section C2

Scale Bar: 50 microns is

2.903

$\begin{array}{rrr}\text { Sample } & \text { Length } & \text { Diameter } \\ 1 & 0.446 & 7.68 \\ 2 & 0.641 & 11.04 \\ 3 & 0.402 & 6.92 \\ 4 & 0.448 & 7.72 \\ 5 & 0.435 & 7.49 \\ 6 & 0.292 & 5.03 \\ 7 & 0.418 & 7.20 \\ 8 & 0.451 & 7.77 \\ 9 & 0.254 & 4.37\end{array}$

Section C3

Scale Bar: 50 microns is

2.848

$\begin{array}{rrr}\text { Sample } & \text { Length } & \text { Diameter } \\ 1 & 0.419 & 7.36 \\ 2 & 0.413 & 7.25 \\ 3 & 0.296 & 5.20 \\ 4 & 0.275 & 4.83 \\ 5 & 0.474 & 8.32 \\ 6 & 0.409 & 7.18 \\ 7 & 0.57 & 10.01 \\ 8 & 0.597 & 10.48 \\ 9 & 0.314 & 5.51\end{array}$
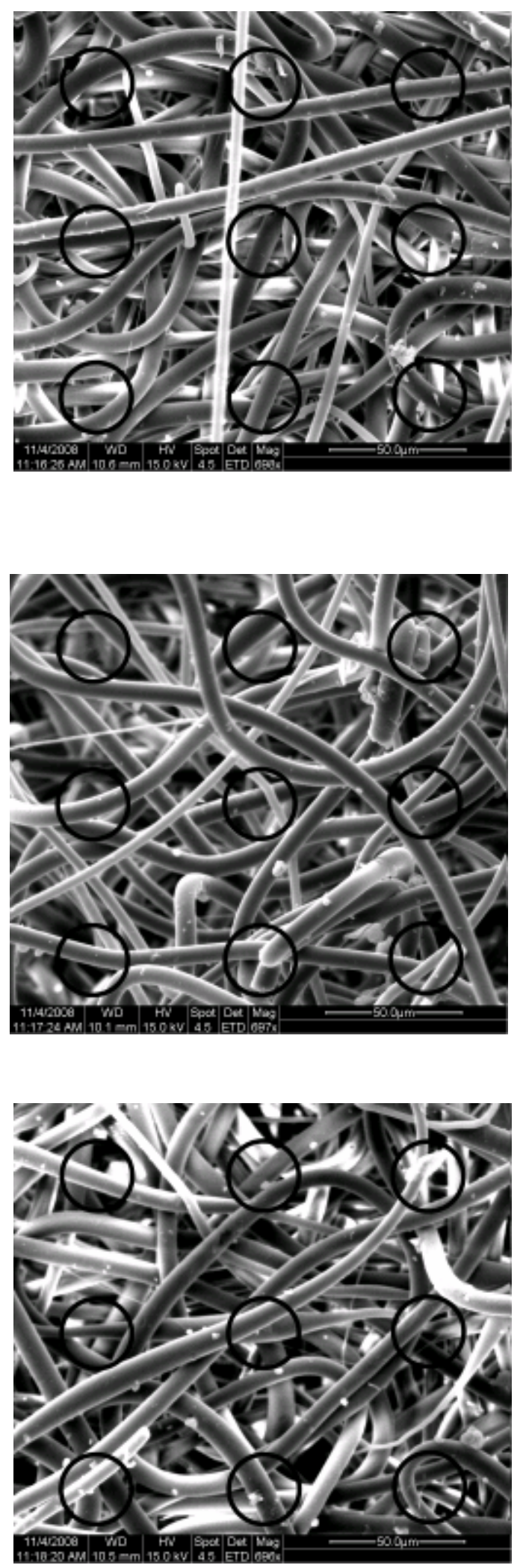
Section D1

Scale Bar: 50 microns is

2.917

$\begin{array}{rrr}\text { Sample } & \text { Length } & \text { Diameter } \\ 1 & 0.447 & 7.66 \\ 2 & 0.149 & 2.55 \\ 3 & 0.495 & 8.48 \\ 4 & 0.457 & 7.83 \\ 5 & 0.466 & 7.99 \\ 6 & 0.457 & 7.83 \\ 7 & 0.376 & 6.44 \\ 8 & 0.45 & 7.71 \\ 9 & 0.41 & 7.03\end{array}$

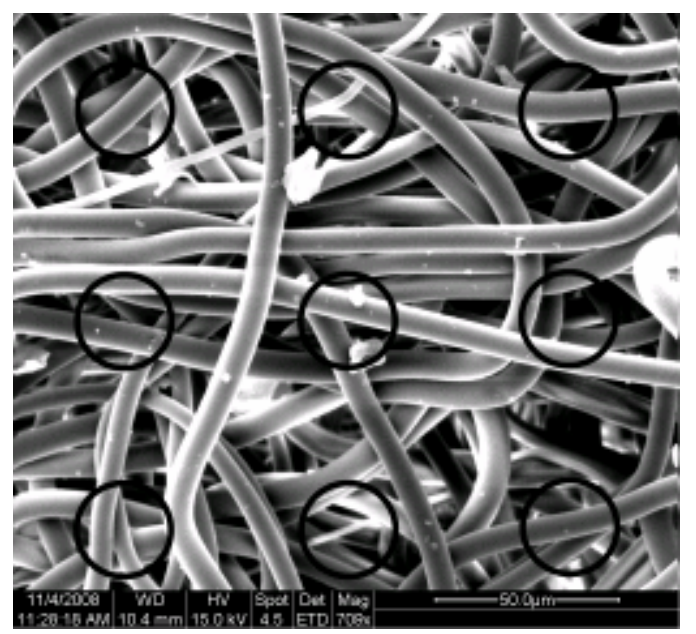

Section D2

Scale Bar: 50 microns is

2.875

$\begin{array}{rrr}\text { Sample } & \text { Length } & \text { Diameter } \\ 1 & 0.548 & 9.53 \\ 2 & 0.45 & 7.83 \\ 3 & 0.37 & 6.43 \\ 4 & 0.432 & 7.51 \\ 5 & 0.374 & 6.50 \\ 6 & 0.439 & 7.63 \\ 7 & 0.41 & 7.13 \\ 8 & 0.399 & 6.94 \\ 9 & 0.524 & 9.11\end{array}$

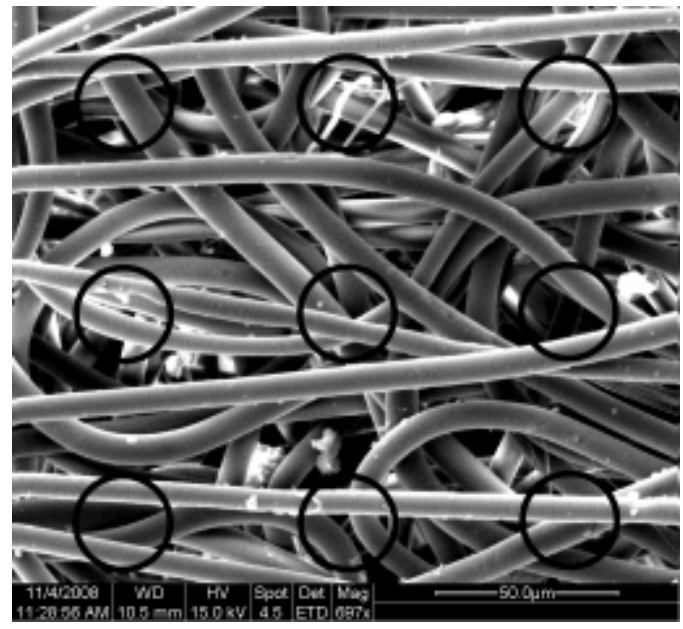

Section D3

Scale Bar: 50 microns is

2.807

$\begin{array}{rrr}\text { Sample } & \text { Length } & \text { Diameter } \\ 1 & 0.351 & 6.25 \\ 2 & 0.253 & 4.51 \\ 3 & 0.539 & 9.60 \\ 4 & 0.462 & 8.23 \\ 5 & 0.405 & 7.21 \\ 6 & 0.426 & 7.59 \\ 7 & 0.468 & 8.34 \\ 8 & 0.418 & 7.45 \\ 9 & 0.431 & 7.68\end{array}$

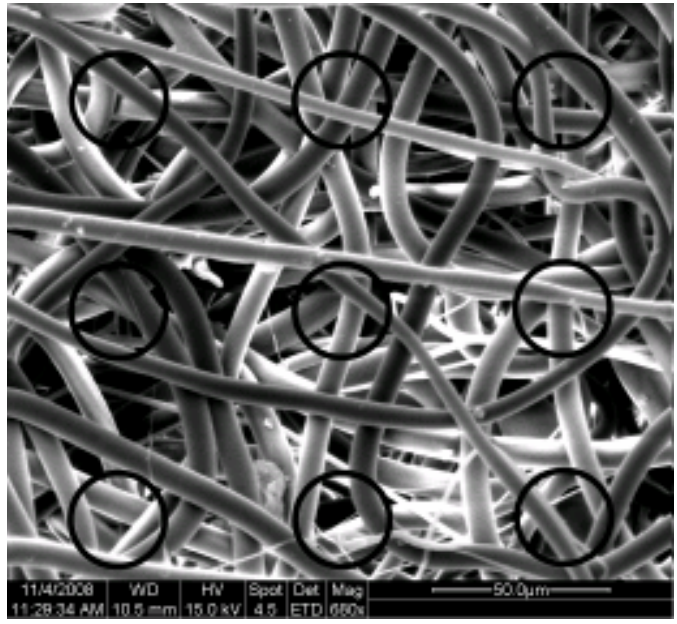




\section{SPIN 7}

Section A1

Scale Bar: 50 microns is

2.944

$\begin{array}{rrr}\text { Sample } & \text { Length } & \text { Diameter } \\ 1 & 0.328 & 5.57 \\ 2 & 0.361 & 6.13 \\ 3 & 0.439 & 7.46 \\ 4 & 0.335 & 5.69 \\ 5 & 0.401 & 6.81 \\ 6 & 0.413 & 7.01 \\ 7 & 0.35 & 5.94 \\ 8 & 0.462 & 7.85 \\ 9 & 0.399 & 6.78\end{array}$

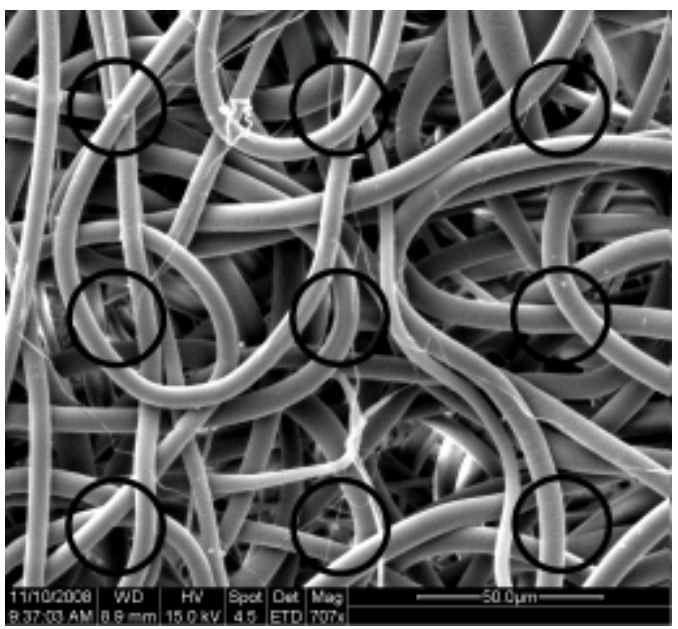

Section A2

Scale Bar: 50 microns is

2.862

$\begin{array}{rrr}\text { Sample } & \text { Length } & \text { Diameter } \\ 1 & 0.427 & 7.46 \\ 2 & 0.344 & 6.01 \\ 3 & 0.501 & 8.75 \\ 4 & 0.154 & 2.69 \\ 5 & 0.44 & 7.69 \\ 6 & 0.361 & 6.31 \\ 7 & 0.496 & 8.67 \\ 8 & 0.35 & 6.11 \\ 9 & 0.292 & 5.10\end{array}$

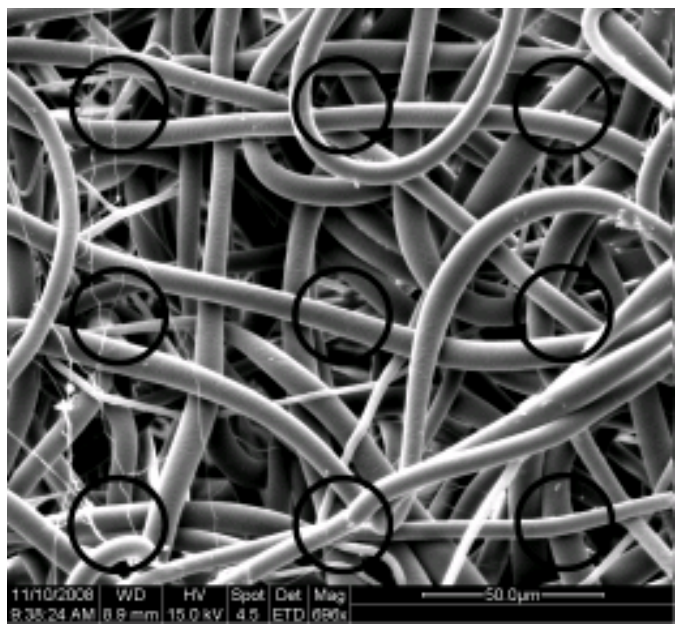

Section A3

Scale Bar: 50 microns is

2.875

$\begin{array}{rrr}\text { Sample } & \text { Length } & \text { Diameter } \\ 1 & 0.087 & 1.51 \\ 2 & 0.627 & 10.90 \\ 3 & 0.498 & 8.66 \\ 4 & 0.437 & 7.60 \\ 5 & 0.894 & 15.55 \\ 6 & 0.385 & 6.70 \\ 7 & 0.34 & 5.91 \\ 8 & 0.205 & 3.57 \\ 9 & 0.341 & 5.93\end{array}$

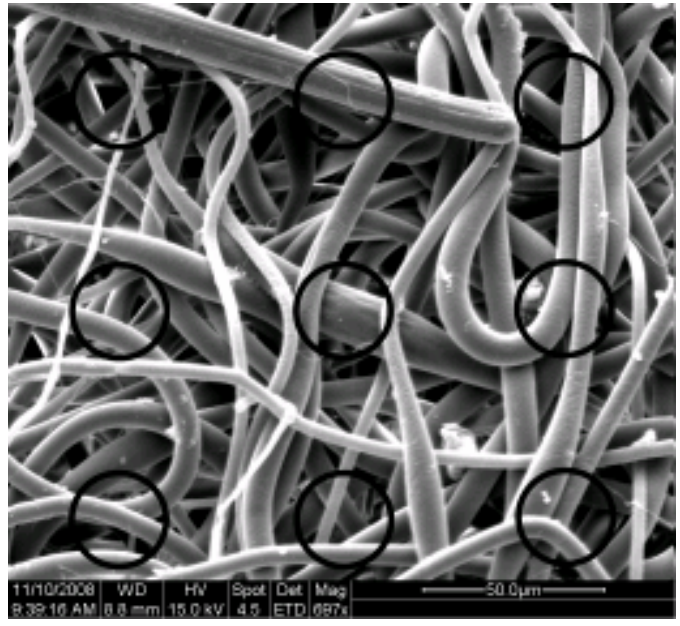


Section B1

Scale Bar: 50 microns is

2.903

Sample Length Diameter

$\begin{array}{lrl}1 & 0.406 & 6.99 \\ 2 & 0.415 & 7.15 \\ 3 & 0.37 & 6.37 \\ 4 & 0.419 & 7.22 \\ 5 & 0.351 & 6.05 \\ 6 & 0.424 & 7.30 \\ 7 & 0.419 & 7.22 \\ 8 & 0.382 & 6.58 \\ 9 & 0.383 & 6.60\end{array}$

Section B2

Scale Bar: 50 microns is

$\begin{array}{rrr}\text { Sample } & \text { Length } & \text { Diameter } \\ 1 & 0.361 & 6.13 \\ 2 & 0.432 & 7.34 \\ 3 & 0.405 & 6.88 \\ 4 & 0.235 & 3.99 \\ 5 & 0.401 & 6.81 \\ 6 & 0.42 & 7.13 \\ 7 & 0.409 & 6.95 \\ 8 & 0.418 & 7.10 \\ 9 & 0.413 & 7.01\end{array}$

Section B3

Scale Bar: 50 microns is

2.875

$\begin{array}{rrr}\text { Sample } & \text { Length } & \text { Diameter } \\ 1 & 0.428 & 7.44 \\ 2 & 0.36 & 6.26 \\ 3 & 0.401 & 6.97 \\ 4 & 0.351 & 6.10 \\ 5 & 0.366 & 6.37 \\ 6 & 0.35 & 6.09 \\ 7 & 0.435 & 7.57 \\ 8 & 0.412 & 7.17 \\ 9 & 0.322 & 5.60\end{array}$

2.944
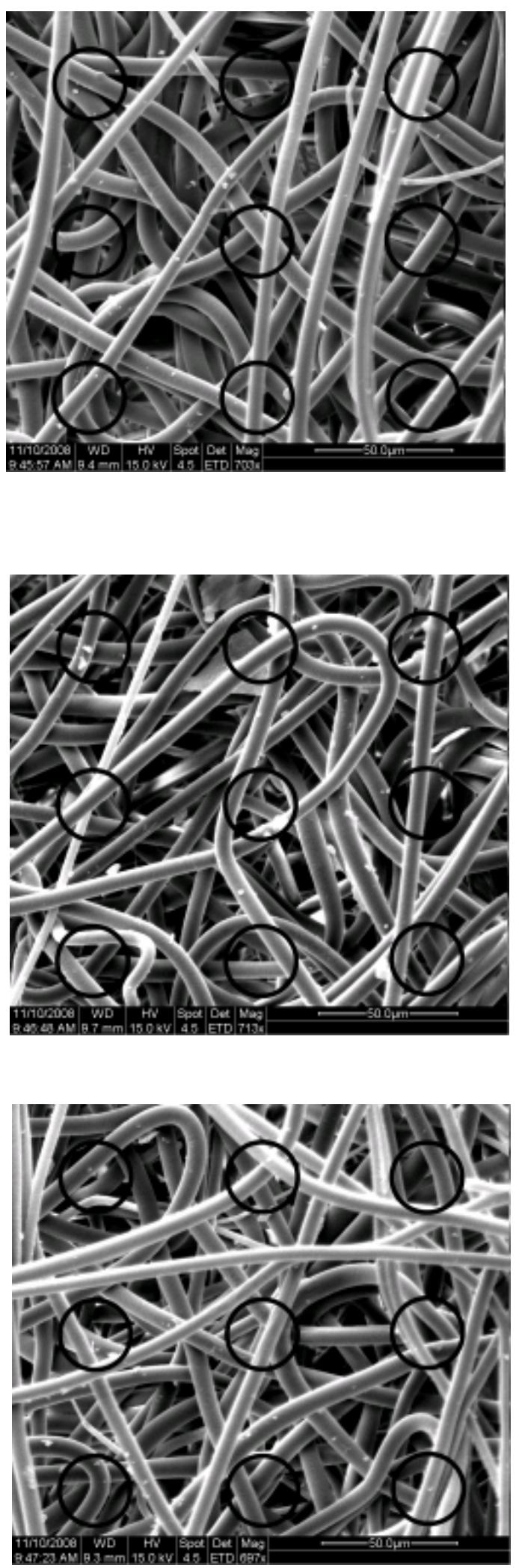
Section C1

Scale Bar: 50 microns is

2.848

$\begin{array}{rrr}\text { Sample } & \text { Length } & \text { Diameter } \\ 1 & 0.405 & 7.11 \\ 2 & 0.367 & 6.44 \\ 3 & 0.439 & 7.71 \\ 4 & 0.427 & 7.50 \\ 5 & 0.401 & 7.04 \\ 6 & 0.351 & 6.16 \\ 7 & 0.419 & 7.36 \\ 8 & 0.407 & 7.15 \\ 9 & 0.378 & 6.64\end{array}$

Section C2

Scale Bar: 50 microns is

2.862

$\begin{array}{rrr}\text { Sample } & \text { Length } & \text { Diameter } \\ 1 & 0.469 & 8.19 \\ 2 & 0.432 & 7.55 \\ 3 & 0.355 & 6.20 \\ 4 & 0.437 & 7.63 \\ 5 & 0.481 & 8.40 \\ 6 & 0.431 & 7.53 \\ 7 & 0.437 & 7.63 \\ 8 & 0.418 & 7.30 \\ 9 & 0.353 & 6.17\end{array}$

Section C3

Scale Bar: 50 microns is

2.958

$\begin{array}{rrr}\text { Sample } & \text { Length } & \text { Diameter } \\ 1 & 0.413 & 6.98 \\ 2 & 0.413 & 6.98 \\ 3 & 0.389 & 6.58 \\ 4 & 0.248 & 4.19 \\ 5 & 0.45 & 7.61 \\ 6 & 0.443 & 7.49 \\ 7 & 0.451 & 7.62 \\ 8 & 0.444 & 7.51 \\ 9 & 0.461 & 7.79\end{array}$
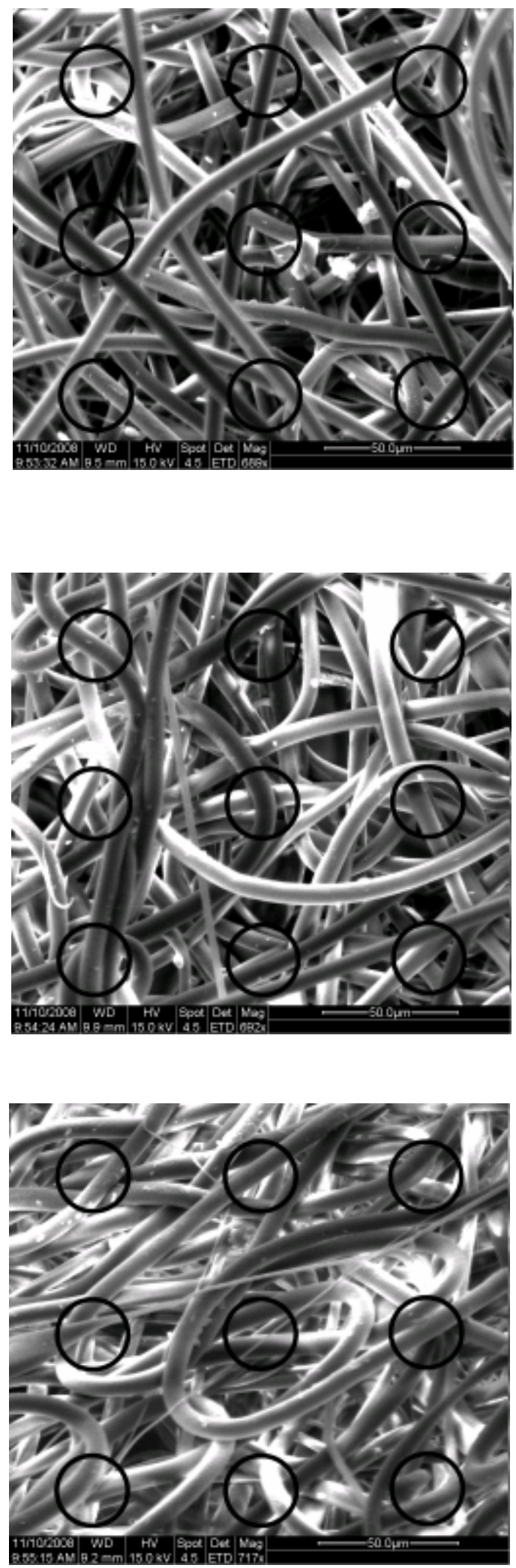
Section D1

Scale Bar: 50 microns is

2.848

\begin{tabular}{rrr} 
Sample & Length & \multicolumn{1}{c}{ Diameter } \\
1 & 0.419 & 7.36 \\
2 & 0.376 & 6.60 \\
3 & 0.44 & 7.72 \\
4 & 0.297 & 5.21 \\
5 & 0.47 & 8.25 \\
6 & 0.446 & 7.83 \\
7 & 0.353 & 6.20 \\
8 & 0.35 & 6.14 \\
9 & 0.351 & 6.16
\end{tabular}

Section D2

Scale Bar: 50 microns is

2.889

$\begin{array}{rrr}\text { Sample } & \text { Length } & \text { Diameter } \\ 1 & 0.567 & 9.81 \\ 2 & 0.469 & 8.12 \\ 3 & 0.385 & 6.66 \\ 4 & 0.536 & 9.28 \\ 5 & 0.372 & 6.44 \\ 6 & 0.405 & 7.01 \\ 7 & 0.394 & 6.82 \\ 8 & 0.515 & 8.91 \\ 9 & 0.405 & 7.01\end{array}$

Section D3

Scale Bar: 50 microns is

2.82

$\begin{array}{rrr}\text { Sample } & \text { Length } & \text { Diameter } \\ 1 & 0.467 & 8.28 \\ 2 & 0.431 & 7.64 \\ 3 & 0.316 & 5.60 \\ 4 & 0.332 & 5.89 \\ 5 & 0.522 & 9.26 \\ 6 & 0.482 & 8.55 \\ 7 & 0.418 & 7.41 \\ 8 & 0.389 & 6.90 \\ 9 & 0.457 & 8.10\end{array}$
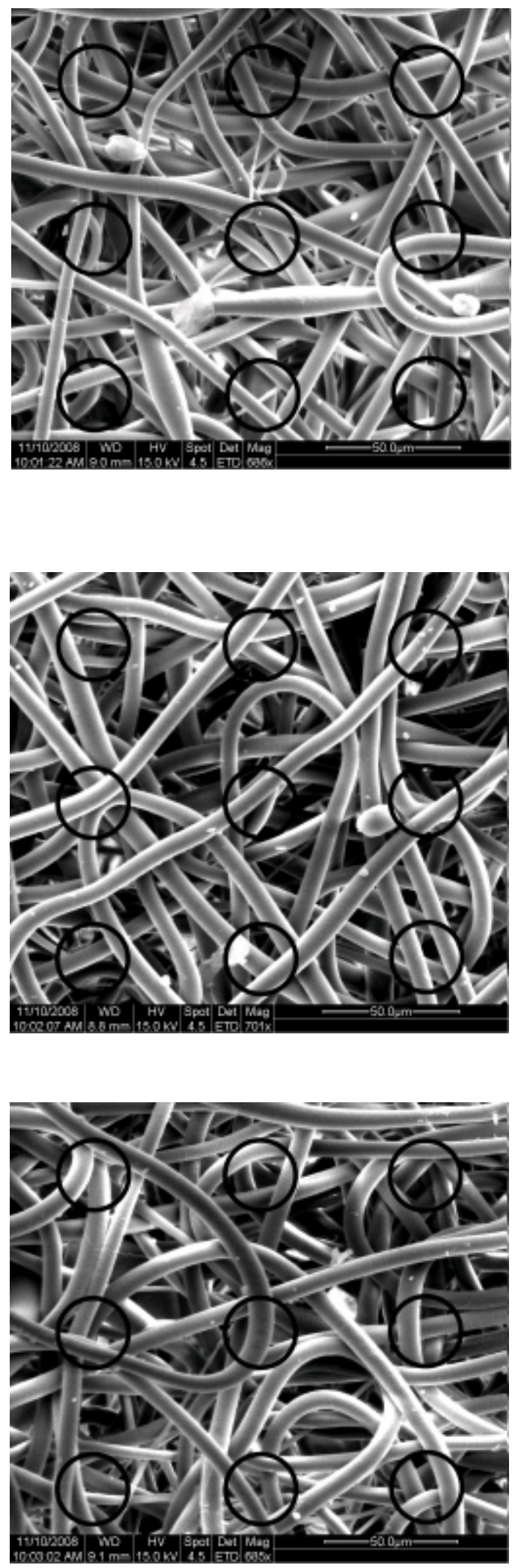


\section{SPIN 8}

Section A1

Scale Bar: 50 microns is

2.889

$\begin{array}{rrr}\text { Sample } & \text { Length } & \text { Diameter } \\ 1 & 0.426 & 7.37 \\ 2 & 0.344 & 5.95 \\ 3 & 0.415 & 7.18 \\ 4 & 0.687 & 11.89 \\ 5 & 0.489 & 8.46 \\ 6 & 0.471 & 8.15 \\ 7 & 0.418 & 7.23 \\ 8 & 0.425 & 7.36 \\ 9 & 0.56 & 9.69\end{array}$

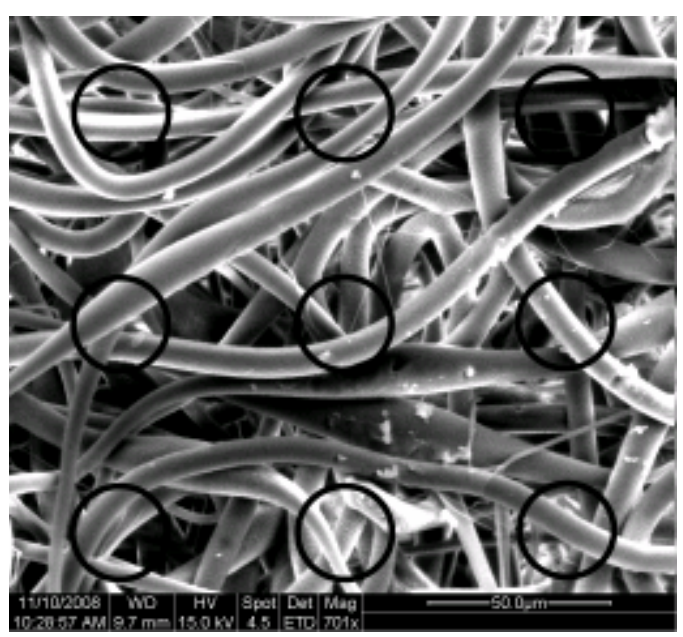

Section A2

Scale Bar: 50 microns is

2.986

$\begin{array}{rrr}\text { Sample } & \text { Length } & \text { Diameter } \\ 1 & 0.279 & 4.67 \\ 2 & 0.418 & 7.00 \\ 3 & 0.444 & 7.43 \\ 4 & 0.254 & 4.25 \\ 5 & 0.414 & 6.93 \\ 6 & 0.335 & 5.61 \\ 7 & 0.248 & 4.15 \\ 8 & 0.432 & 7.23 \\ 9 & 0.409 & 6.85\end{array}$

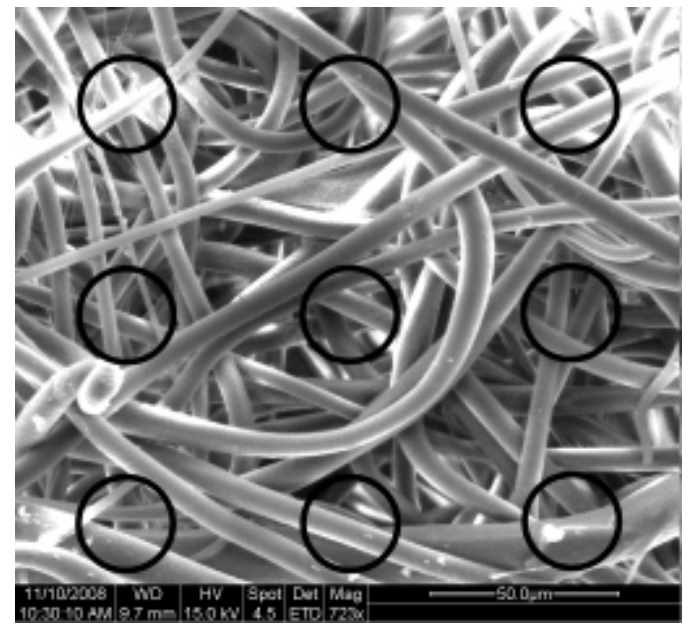

Section A3

Scale Bar: 50 microns is

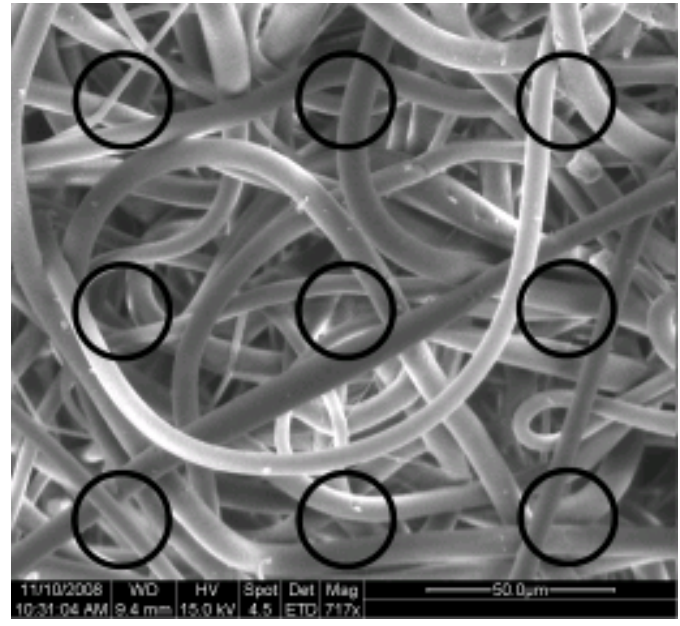

$\begin{array}{rrr}\text { Sample } & \text { Length } & \text { Diameter } \\ 1 & 0.127 & 2.13 \\ 2 & 0.488 & 8.17 \\ 3 & 0.511 & 8.56 \\ 4 & 0.388 & 6.50 \\ 5 & 0.5 & 8.38 \\ 6 & 0.37 & 6.20 \\ 7 & 0.303 & 5.08 \\ 8 & 0.418 & 7.00 \\ 9 & 0.345 & 5.78\end{array}$


Section B1

Scale Bar: 50 microns is

2.917

$\begin{array}{rrr}\text { Sample } & \text { Length } & \text { Diameter } \\ 1 & 0.42 & 7.20 \\ 2 & 0.458 & 7.85 \\ 3 & 0.535 & 9.17 \\ 4 & 0.461 & 7.90 \\ 5 & 0.485 & 8.31 \\ 6 & 0.492 & 8.43 \\ 7 & 0.441 & 7.56 \\ 8 & 0.519 & 8.90 \\ 9 & 0.43 & 7.37\end{array}$

Section B2

Scale Bar: 50 microns is

2.917

$\begin{array}{rrr}\text { Sample } & \text { Length } & \text { Diameter } \\ 1 & 0.428 & 7.34 \\ 2 & 0.418 & 7.16 \\ 3 & 0.231 & 3.96 \\ 4 & 0.449 & 7.70 \\ 5 & 0.491 & 8.42 \\ 6 & 0.464 & 7.95 \\ 7 & 0.408 & 6.99 \\ 8 & 0.443 & 7.59 \\ 9 & 0.46 & 7.88\end{array}$

Section B3

Scale Bar: 50 microns is

2.875

$\begin{array}{rrr}\text { Sample } & \text { Length } & \text { Diameter } \\ 1 & 0.244 & 4.24 \\ 2 & 0.728 & 12.66 \\ 3 & 0.444 & 7.72 \\ 4 & 0.462 & 8.03 \\ 5 & 0.47 & 8.17 \\ 6 & 0.29 & 5.04 \\ 7 & 0.475 & 8.26 \\ 8 & 0.409 & 7.11 \\ 9 & 0.374 & 6.50\end{array}$
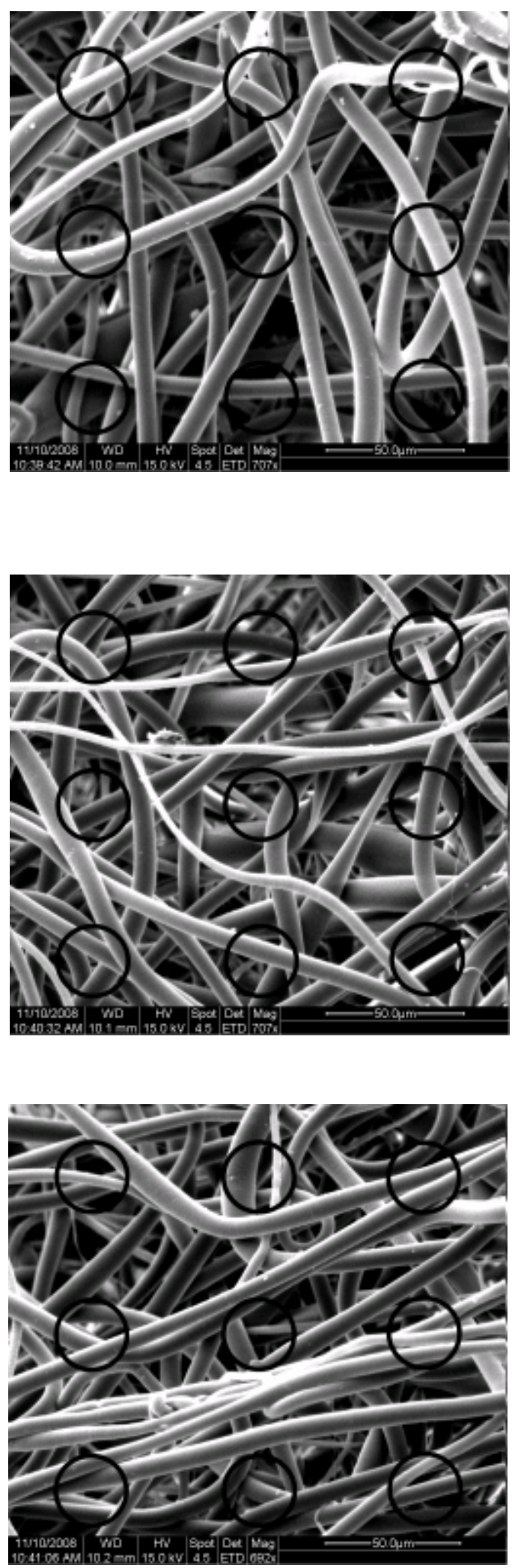
Section C1

Scale Bar: 50 microns is

2.917

\begin{tabular}{rrr} 
Sample & Length & \multicolumn{1}{c}{ Diameter } \\
1 & 0.456 & 7.82 \\
2 & 0.519 & 8.90 \\
3 & 0.497 & 8.52 \\
4 & 0.486 & 8.33 \\
5 & 0.289 & 4.95 \\
6 & 0.401 & 6.87 \\
7 & 0.372 & 6.38 \\
8 & 0.537 & 9.20 \\
9 & 0.409 & 7.01
\end{tabular}

Section C2

Scale Bar: 50 microns is

2.875

$\begin{array}{rrr}\text { Sample } & \text { Length } & \text { Diameter } \\ 1 & 0.425 & 7.39 \\ 2 & 0.416 & 7.23 \\ 3 & 0.387 & 6.73 \\ 4 & 0.457 & 7.95 \\ 5 & 0.414 & 7.20 \\ 6 & 0.401 & 6.97 \\ 7 & 0.372 & 6.47 \\ 8 & 0.481 & 8.37 \\ 9 & 0.473 & 8.23\end{array}$

Section C3

Scale Bar: 50 microns is

2.834

$\begin{array}{rrr}\text { Sample } & \text { Length } & \text { Diameter } \\ 1 & 0.467 & 8.24 \\ 2 & 0.184 & 3.25 \\ 3 & 0.496 & 8.75 \\ 4 & 0.426 & 7.52 \\ 5 & 0.224 & 3.95 \\ 6 & 0.357 & 6.30 \\ 7 & 0.477 & 8.42 \\ 8 & 0.366 & 6.46 \\ 9 & 0.43 & 7.59\end{array}$
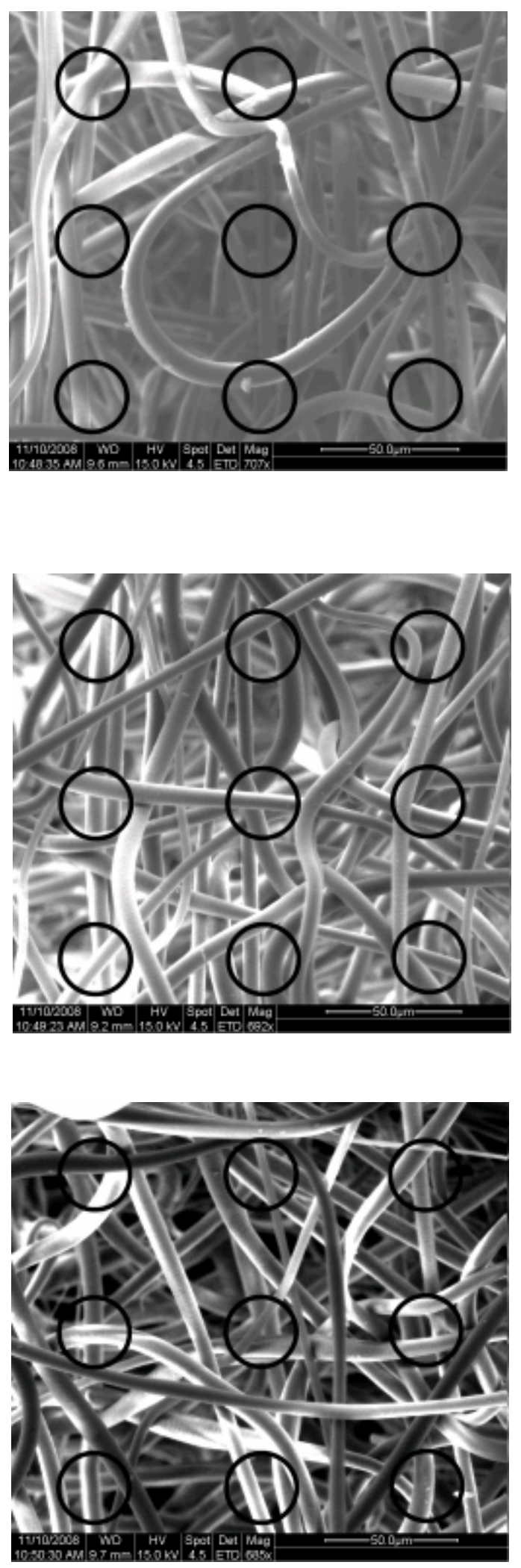
Section D1

Scale Bar: 50 microns is

2.903

$\begin{array}{rrr}\text { Sample } & \text { Length } & \text { Diameter } \\ 1 & 0.469 & 8.08 \\ 2 & 0.573 & 9.87 \\ 3 & 0.407 & 7.01 \\ 4 & 0.378 & 6.51 \\ 5 & 0.305 & 5.25 \\ 6 & 0.401 & 6.91 \\ 7 & 0.42 & 7.23 \\ 8 & 0.437 & 7.53 \\ 9 & 0.587 & 10.11\end{array}$

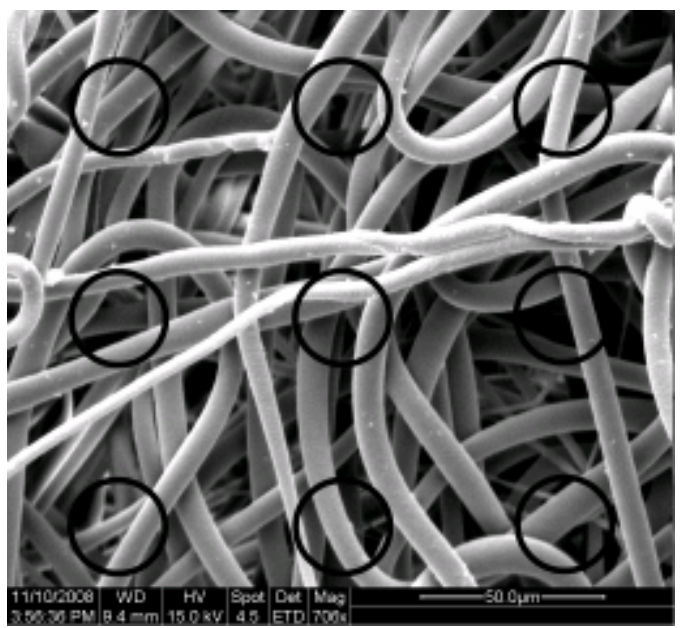

Section D2

Scale Bar: 50 microns is

2.93

$\begin{array}{rrr}\text { Sample } & \text { Length } & \text { Diameter } \\ 1 & 0.459 & 7.83 \\ 2 & 0.488 & 8.33 \\ 3 & 0.517 & 8.82 \\ 4 & 0.409 & 6.98 \\ 5 & 0.443 & 7.56 \\ 6 & 0.37 & 6.31 \\ 7 & 0.387 & 6.60 \\ 8 & 0.284 & 4.85 \\ 9 & 0.261 & 4.45\end{array}$

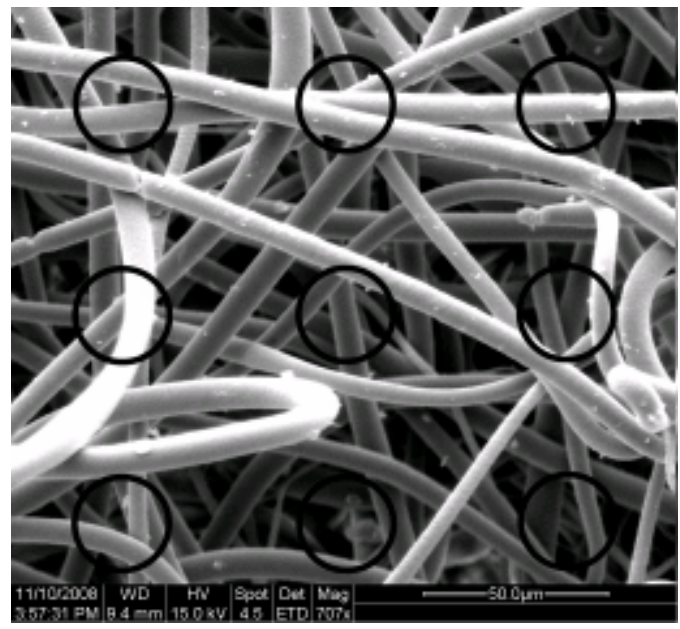

Section D3

Scale Bar: 50 microns is

$\begin{array}{rrr}\text { Sample } & \text { Length } & \text { Diameter } \\ 1 & 0.459 & 7.87 \\ 2 & 0.529 & 9.07 \\ 3 & 0.534 & 9.15 \\ 4 & 0.47 & 8.06 \\ 5 & 0.448 & 7.68 \\ 6 & 0.441 & 7.56 \\ 7 & 0.555 & 9.51 \\ 8 & 0.475 & 8.14 \\ 9 & 0.393 & 6.74\end{array}$

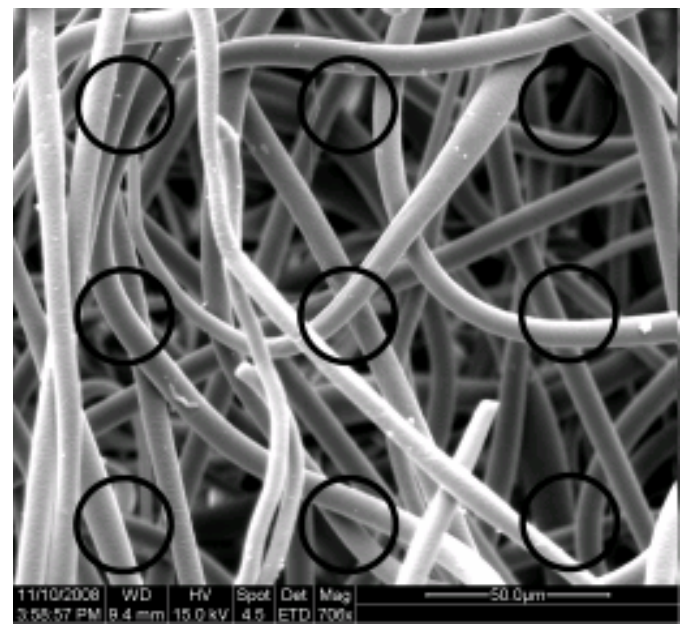




\section{SPIN 9}

Section A1

Scale Bar: 50 microns is

2.944

$\begin{array}{rrr}\text { Sample } & \text { Length } & \text { Diameter } \\ 1 & 0.531 & 9.02 \\ 2 & 0.554 & 9.41 \\ 3 & 0.514 & 8.73 \\ 4 & 0.595 & 10.11 \\ 5 & 0.479 & 8.14 \\ 6 & 0.469 & 7.97 \\ 7 & 0.524 & 8.90 \\ 8 & 0.501 & 8.51 \\ 9 & 0.499 & 8.47\end{array}$

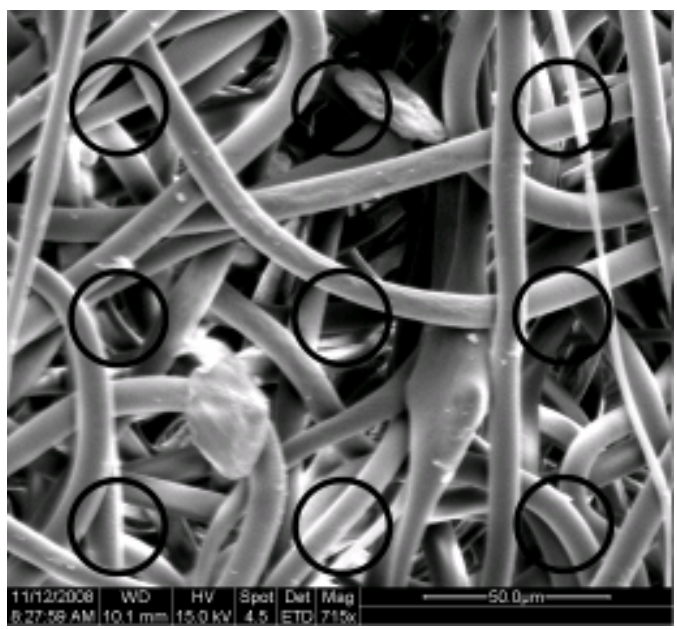

Section A2

Scale Bar: 50 microns is

2.875

$\begin{array}{rrr}\text { Sample } & \text { Length } & \text { Diameter } \\ 1 & 0.413 & 7.18 \\ 2 & 0.427 & 7.43 \\ 3 & 0.512 & 8.90 \\ 4 & 0.488 & 8.49 \\ 5 & 0.524 & 9.11 \\ 6 & 0.363 & 6.31 \\ 7 & 0.497 & 8.64 \\ 8 & 0.233 & 4.05 \\ 9 & 0.466 & 8.10\end{array}$

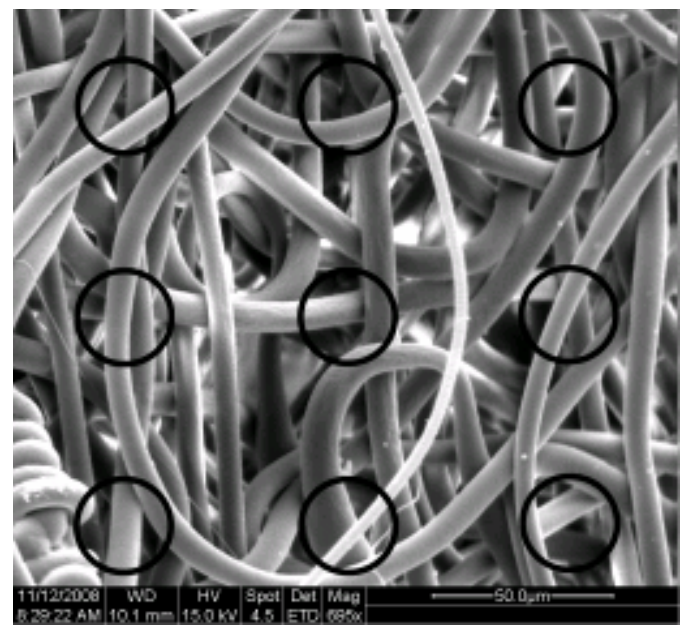

Section A3

Scale Bar: 50 microns is

2.875

$\begin{array}{rrr}\text { Sample } & \text { Length } & \text { Diameter } \\ 1 & 0.52 & 9.04 \\ 2 & 0.562 & 9.77 \\ 3 & 0.531 & 9.23 \\ 4 & 0.516 & 8.97 \\ 5 & 0.524 & 9.11 \\ 6 & 0.462 & 8.03 \\ 7 & 0.545 & 9.48 \\ 8 & 0.52 & 9.04 \\ 9 & 0.565 & 9.83\end{array}$

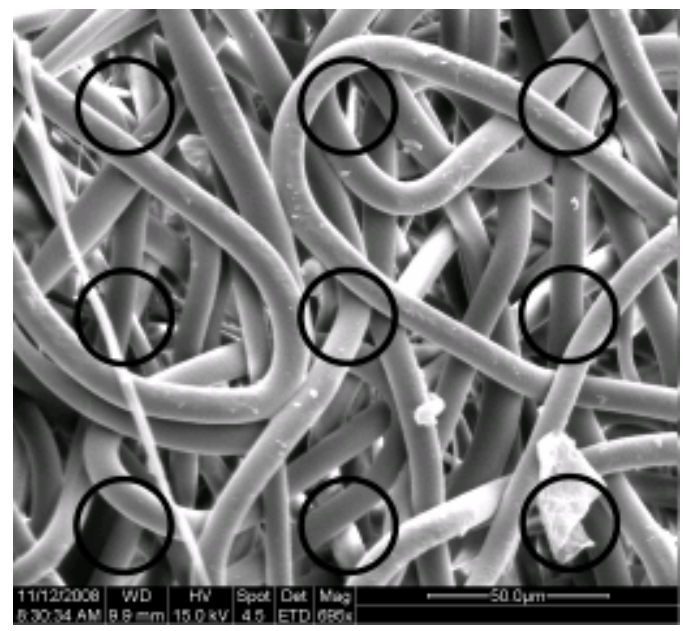


Section B1

Scale Bar: 50 microns is

2.875

$\begin{array}{rrr}\text { Sample } & \text { Length } & \text { Diameter } \\ 1 & 0.361 & 6.28 \\ 2 & 0.474 & 8.24 \\ 3 & 0.555 & 9.65 \\ 4 & 0.506 & 8.80 \\ 5 & 0.428 & 7.44 \\ 6 & 0.506 & 8.80 \\ 7 & 0.357 & 6.21 \\ 8 & 0.531 & 9.23 \\ 9 & 0.401 & 6.97\end{array}$

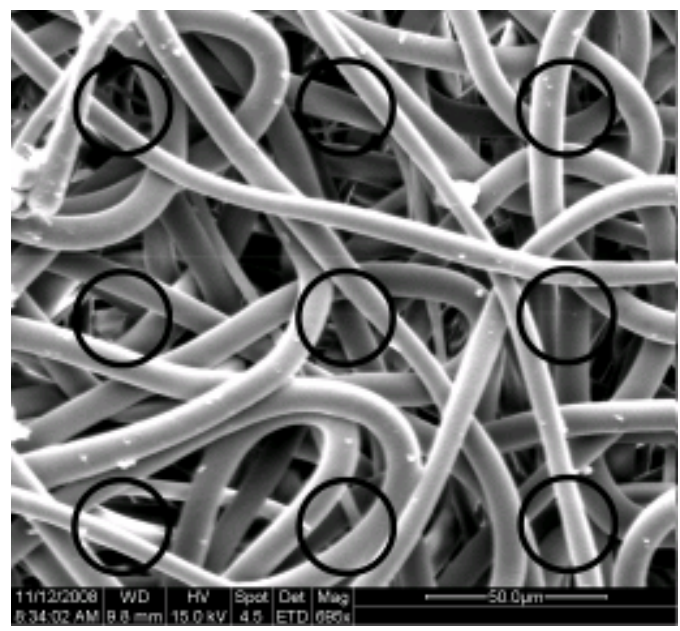

Section B2

Scale Bar: 50 microns is

2.862

$\begin{array}{rrr}\text { Sample } & \text { Length } & \text { Diameter } \\ 1 & 0.572 & 9.99 \\ 2 & 0.335 & 5.85 \\ 3 & 0.511 & 8.93 \\ 4 & 0.506 & 8.84 \\ 5 & 0.535 & 9.35 \\ 6 & 0.386 & 6.74 \\ 7 & 0.485 & 8.47 \\ 8 & 0.502 & 8.77 \\ 9 & 0.454 & 7.93\end{array}$

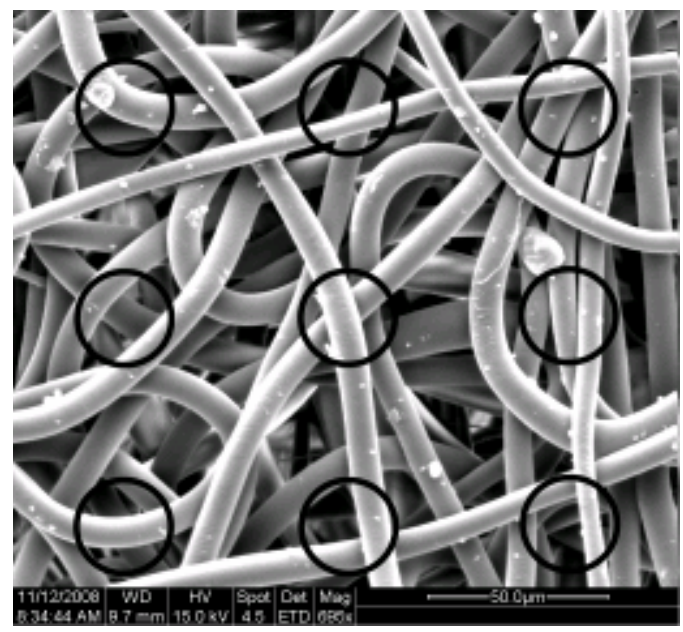

Section B3

Scale Bar: 50 microns is

$\begin{array}{rrr}\text { Sample } & \text { Length } & \text { Diameter } \\ 1 & 0.552 & 9.33 \\ 2 & 0.541 & 9.14 \\ 3 & 0.42 & 7.10 \\ 4 & 0.567 & 9.58 \\ 5 & 0.565 & 9.55 \\ 6 & 0.321 & 5.43 \\ 7 & 0.508 & 8.59 \\ 8 & 0.557 & 9.42 \\ 9 & 0.555 & 9.38\end{array}$

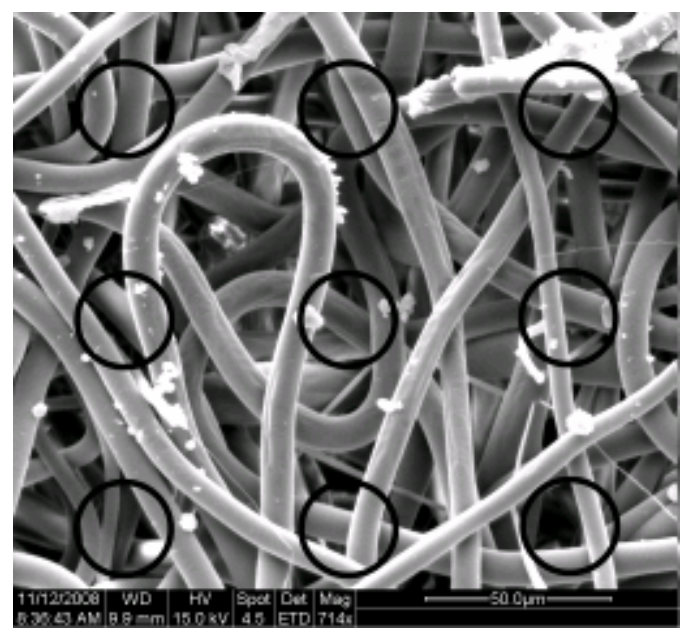


Section C1

Scale Bar: 50 microns is

2.889

$\begin{array}{rrr}\text { Sample } & \text { Length } & \text { Diameter } \\ 1 & 0.482 & 8.34 \\ 2 & 0.446 & 7.72 \\ 3 & 0.488 & 8.45 \\ 4 & 0.529 & 9.16 \\ 5 & 0.506 & 8.76 \\ 6 & 0.467 & 8.08 \\ 7 & 0.427 & 7.39 \\ 8 & 0.36 & 6.23 \\ 9 & 0.321 & 5.56\end{array}$

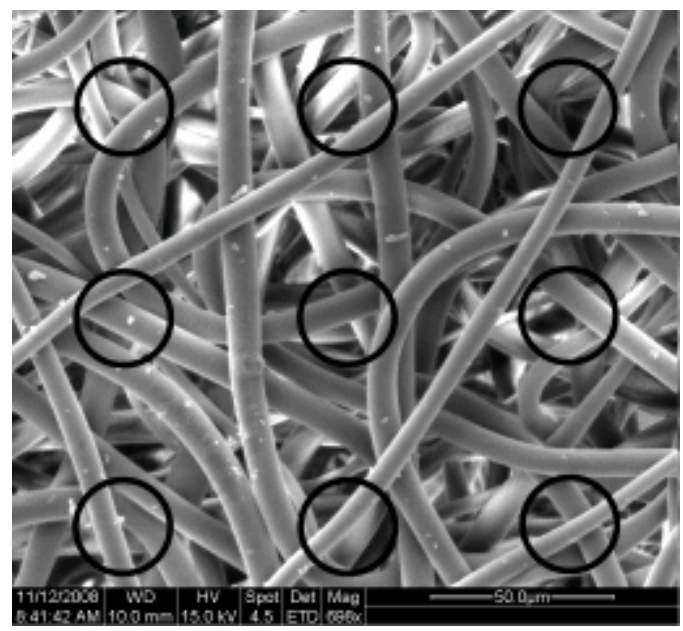

Section C2

Scale Bar: 50 microns is

2.903

$\begin{array}{rrr}\text { Sample } & \text { Length } & \text { Diameter } \\ 1 & 0.444 & 7.65 \\ 2 & 0.427 & 7.35 \\ 3 & 0.378 & 6.51 \\ 4 & 0.511 & 8.80 \\ 5 & 0.511 & 8.80 \\ 6 & 0.499 & 8.59 \\ 7 & 0.497 & 8.56 \\ 8 & 0.457 & 7.87 \\ 9 & 0.555 & 9.56\end{array}$

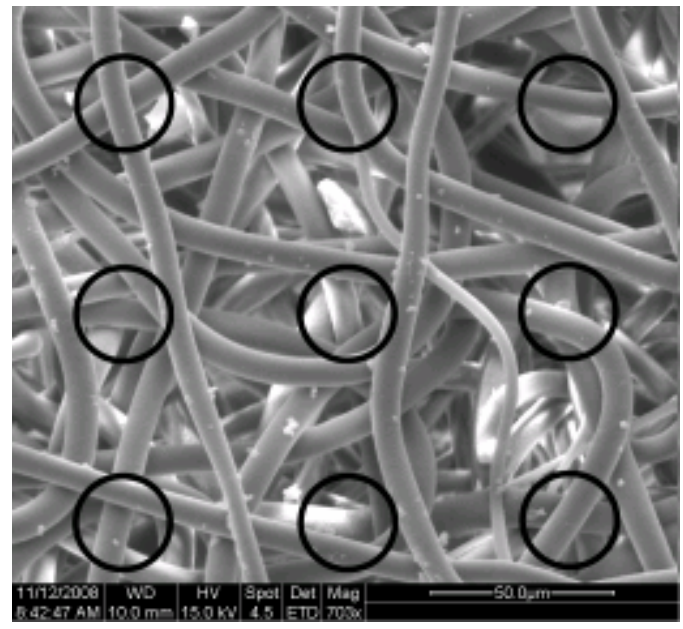

Section C3

Scale Bar: 50 microns is

$\begin{array}{rrr}\text { Sample } & \text { Length } & \text { Diameter } \\ 1 & 0.449 & 7.59 \\ 2 & 0.518 & 8.76 \\ 3 & 0.382 & 6.46 \\ 4 & 0.56 & 9.47 \\ 5 & 0.46 & 7.78 \\ 6 & 0.536 & 9.06 \\ 7 & 0.431 & 7.29 \\ 8 & 0.512 & 8.65 \\ 9 & 0.405 & 6.85\end{array}$

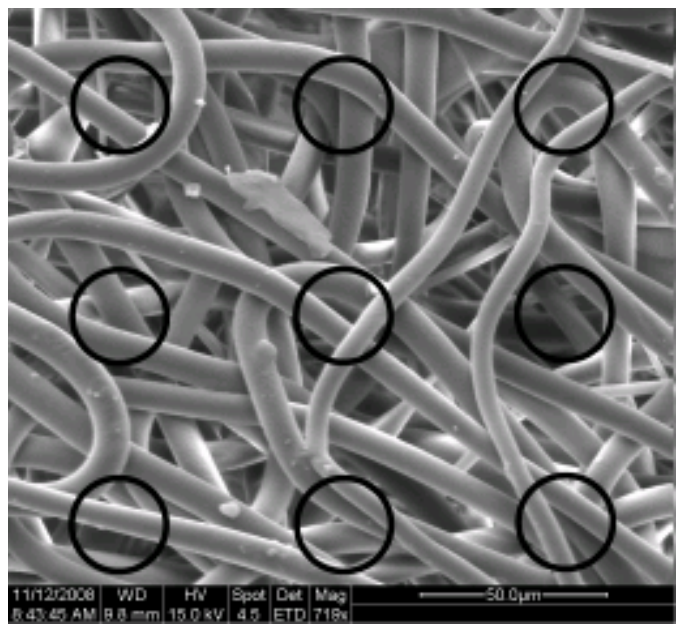


Section D1

Scale Bar: 50 microns is

2.903

$\begin{array}{rrr}\text { Sample } & \text { Length } & \text { Diameter } \\ 1 & 0.474 & 8.16 \\ 2 & 0.508 & 8.75 \\ 3 & 0.561 & 9.66 \\ 4 & 0.237 & 4.08 \\ 5 & 0.509 & 8.77 \\ 6 & 0.543 & 9.35 \\ 7 & 0.507 & 8.73 \\ 8 & 0.488 & 8.41 \\ 9 & 0.52 & 8.96\end{array}$

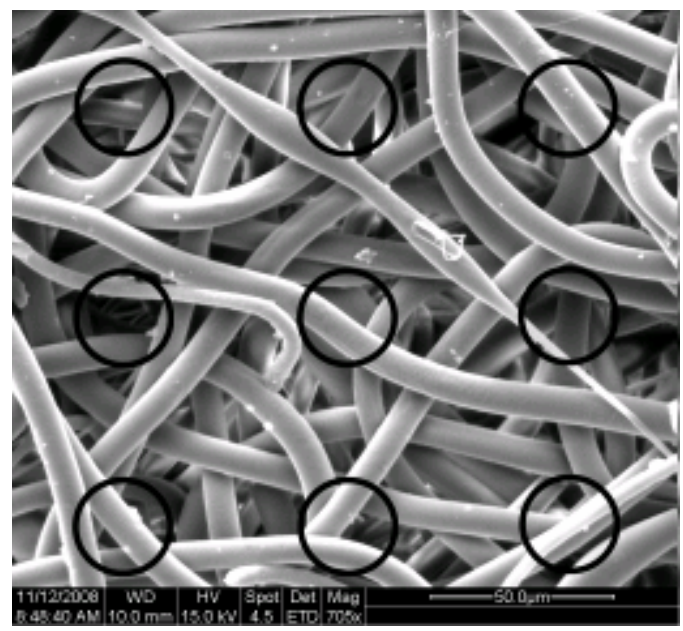

Section D2

Scale Bar: 50 microns is

2.972

$\begin{array}{rrr}\text { Sample } & \text { Length } & \text { Diameter } \\ 1 & 0.492 & 8.28 \\ 2 & 0.532 & 8.95 \\ 3 & 0.449 & 7.55 \\ 4 & 0.518 & 8.71 \\ 5 & 0.541 & 9.10 \\ 6 & 0.516 & 8.68 \\ 7 & 0.52 & 8.75 \\ 8 & 0.566 & 9.52 \\ 9 & 0.484 & 8.14\end{array}$

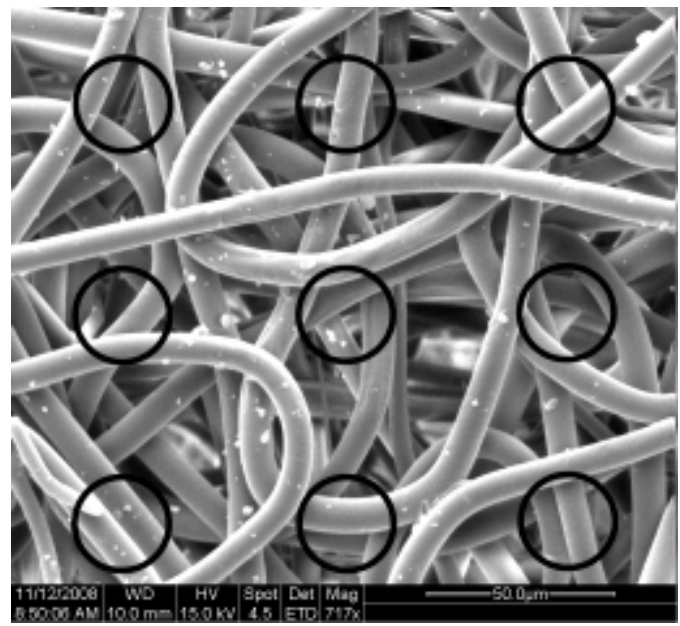

Section D3

Scale Bar: 50 microns is

2.972

$\begin{array}{rrr}\text { Sample } & \text { Length } & \text { Diameter } \\ 1 & 0.519 & 8.73 \\ 2 & 0.461 & 7.76 \\ 3 & 0.166 & 2.79 \\ 4 & 0.548 & 9.22 \\ 5 & 0.522 & 8.78 \\ 6 & 0.477 & 8.02 \\ 7 & 0.567 & 9.54 \\ 8 & 0.308 & 5.18 \\ 9 & 0.492 & 8.28\end{array}$

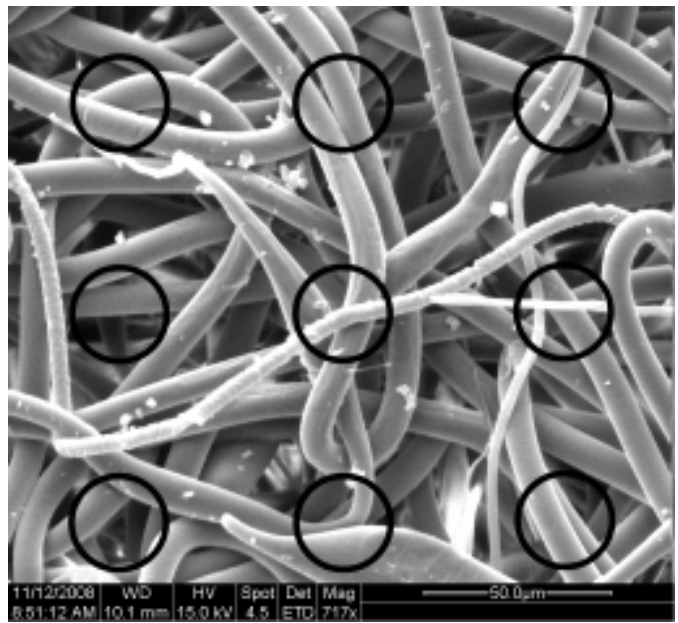




\section{SPIN 10}

Section A1

Scale Bar: 50 microns is

2.944

$\begin{array}{rrr}\text { Sample } & \text { Length } & \text { Diameter } \\ 1 & 0.541 & 9.19 \\ 2 & 0.454 & 7.71 \\ 3 & 0.174 & 2.96 \\ 4 & 0.174 & 2.96 \\ 5 & 0.481 & 8.17 \\ 6 & 0.161 & 2.73 \\ 7 & 0.636 & 10.80 \\ 8 & 0.466 & 7.91 \\ 9 & 0.388 & 6.59\end{array}$

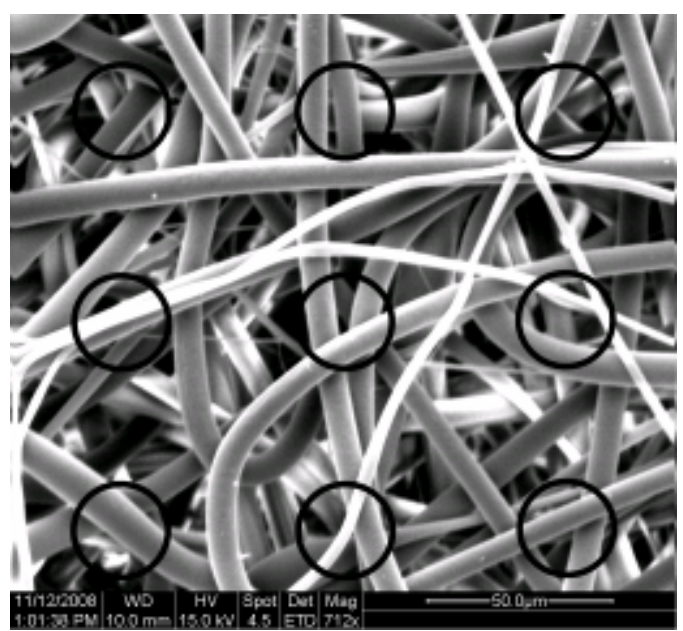

Section A2

Scale Bar: 50 microns is

2.889

$\begin{array}{rrr}\text { Sample } & \text { Length } & \text { Diameter } \\ 1 & 0.456 & 7.89 \\ 2 & 0.467 & 8.08 \\ 3 & 0.496 & 8.58 \\ 4 & 0.152 & 2.63 \\ 5 & 0.537 & 9.29 \\ 6 & 0.517 & 8.95 \\ 7 & 0.517 & 8.95 \\ 8 & 0.545 & 9.43 \\ 9 & 0.486 & 8.41\end{array}$

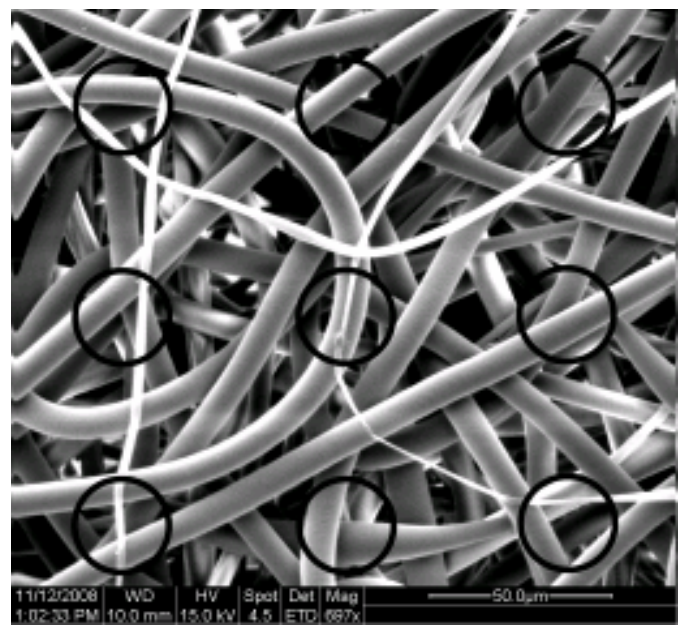

Section A3

Scale Bar: 50 microns is

2.903

$\begin{array}{rrr}\text { Sample } & \text { Length } & \text { Diameter } \\ 1 & 0.443 & 7.63 \\ 2 & 1.033 & 17.79 \\ 3 & 0.523 & 9.01 \\ 4 & 0.499 & 8.59 \\ 5 & 0.437 & 7.53 \\ 6 & 0.511 & 8.80 \\ 7 & 0.459 & 7.91 \\ 8 & 0.284 & 4.89 \\ 9 & 0.468 & 8.06\end{array}$

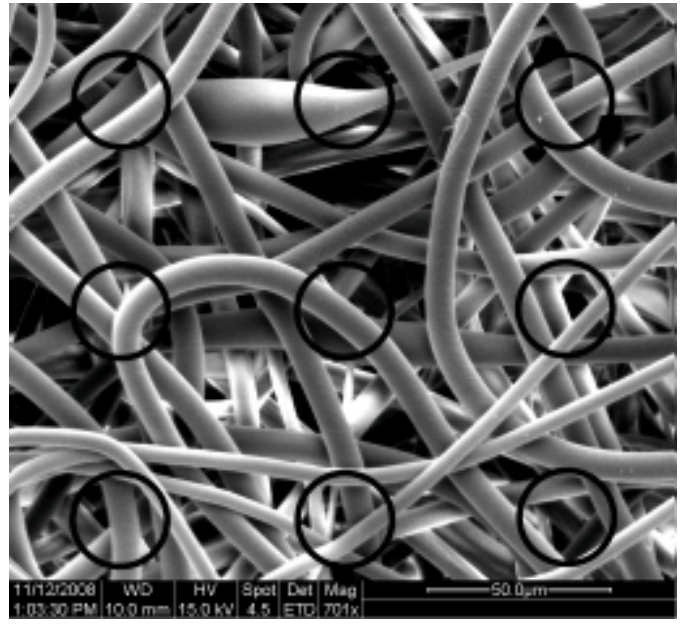


Section B1

Scale Bar: 50 microns is

2.903

$\begin{array}{rrr}\text { Sample } & \text { Length } & \text { Diameter } \\ 1 & 0.466 & 8.03 \\ 2 & 0.254 & 4.37 \\ 3 & 0.499 & 8.59 \\ 4 & 0.185 & 3.19 \\ 5 & 0.526 & 9.06 \\ 6 & 0.401 & 6.91 \\ 7 & 0.466 & 8.03 \\ 8 & 0.245 & 4.22 \\ 9 & 0.243 & 4.19\end{array}$

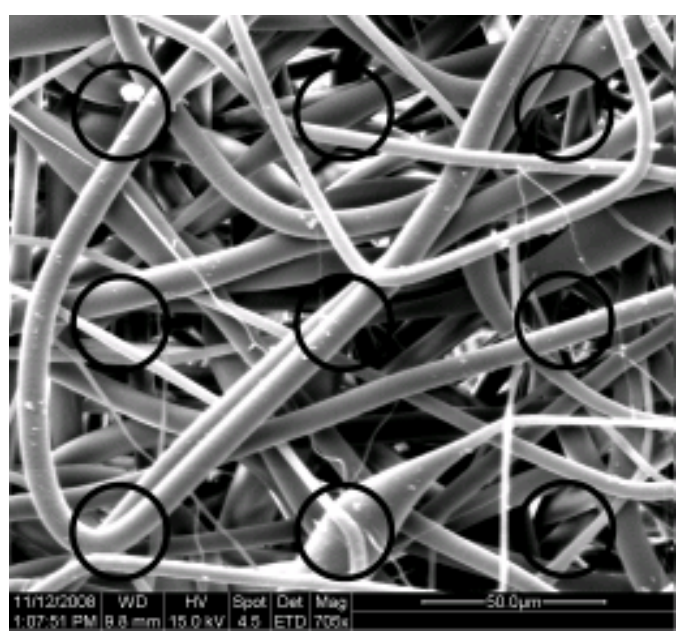

Section B2

Scale Bar: 50 microns is

2.93

$\begin{array}{rrr}\text { Sample } & \text { Length } & \text { Diameter } \\ 1 & 0.458 & 7.82 \\ 2 & 0.154 & 2.63 \\ 3 & 0.414 & 7.06 \\ 4 & 0.456 & 7.78 \\ 5 & 0.428 & 7.30 \\ 6 & 0.486 & 8.29 \\ 7 & 0.437 & 7.46 \\ 8 & 0.154 & 2.63 \\ 9 & 0.302 & 5.15\end{array}$

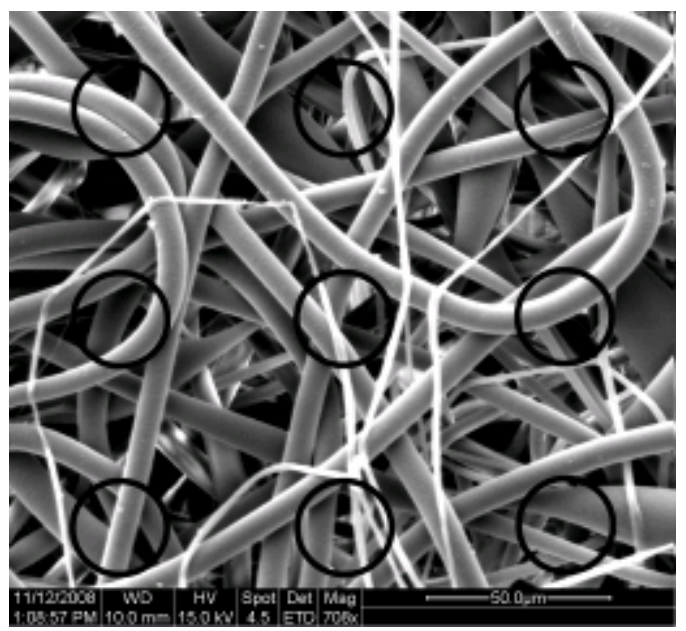

Section B3

Scale Bar: 50 microns is

2.903

$\begin{array}{rrr}\text { Sample } & \text { Length } & \text { Diameter } \\ 1 & 0.388 & 6.68 \\ 2 & 0.512 & 8.82 \\ 3 & 0.474 & 8.16 \\ 4 & 0.58 & 9.99 \\ 5 & 0.511 & 8.80 \\ 6 & 0.421 & 7.25 \\ 7 & 0.478 & 8.23 \\ 8 & 0.37 & 6.37 \\ 9 & 0.244 & 4.20\end{array}$

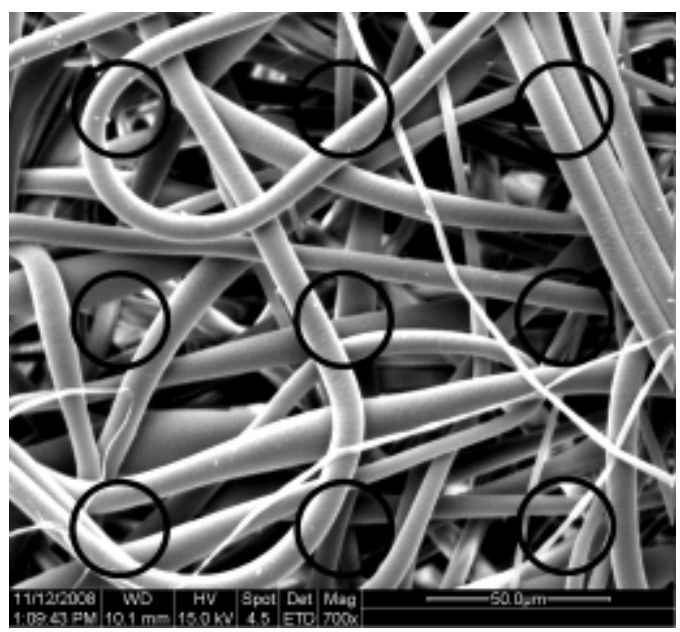


Section C1

Scale Bar: 50 microns is

2.972

$\begin{array}{rrr}\text { Sample } & \text { Length } & \text { Diameter } \\ 1 & 0.527 & 8.87 \\ 2 & 0.161 & 2.71 \\ 3 & 0.691 & 11.63 \\ 4 & 0.728 & 12.25 \\ 5 & 0.21 & 3.53 \\ 6 & 0.431 & 7.25 \\ 7 & 0.511 & 8.60 \\ 8 & 0.422 & 7.10 \\ 9 & 0.488 & 8.21\end{array}$

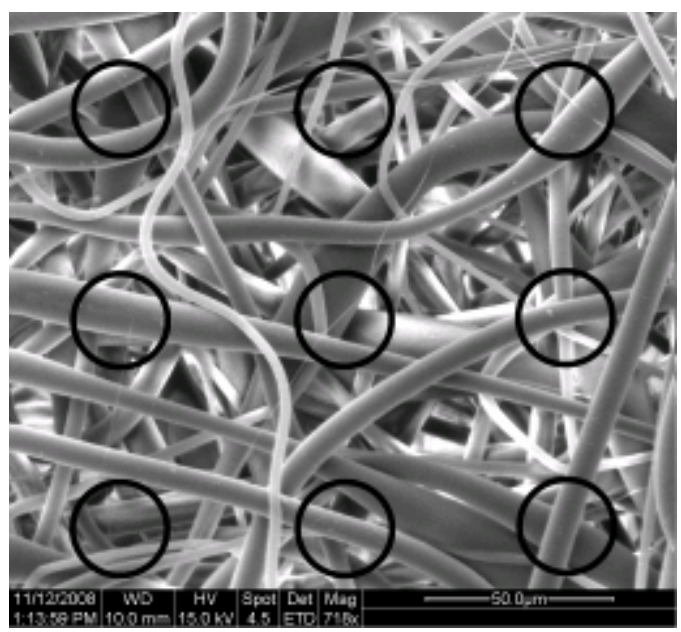

Section C2

Scale Bar: 50 microns is

2.972

$\begin{array}{rrr}\text { Sample } & \text { Length } & \text { Diameter } \\ 1 & 0.442 & 7.44 \\ 2 & 0.1 & 1.68 \\ 3 & 0.466 & 7.84 \\ 4 & 0.566 & 9.52 \\ 5 & 0.192 & 3.23 \\ 6 & 0.487 & 8.19 \\ 7 & 0.501 & 8.43 \\ 8 & 0.448 & 7.54 \\ 9 & 0.416 & 7.00\end{array}$

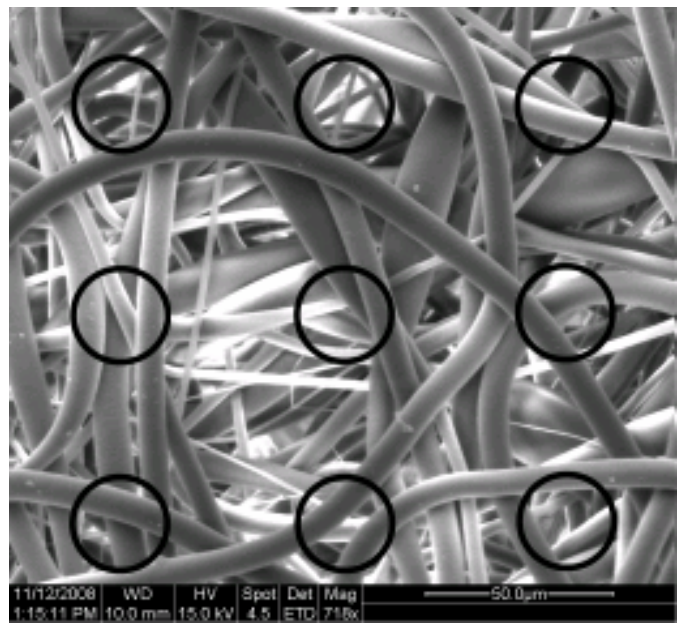

Section C3

Scale Bar: 50 microns is

$\begin{array}{rrr}\text { Sample } & \text { Length } & \text { Diameter } \\ 1 & 0.92 & 15.20 \\ 2 & 0.493 & 8.14 \\ 3 & 0.506 & 8.36 \\ 4 & 1.129 & 18.65 \\ 5 & 0.142 & 2.35 \\ 6 & 0.565 & 9.33 \\ 7 & 0.409 & 6.76 \\ 8 & 0.519 & 8.57 \\ 9 & 0.29 & 4.79\end{array}$

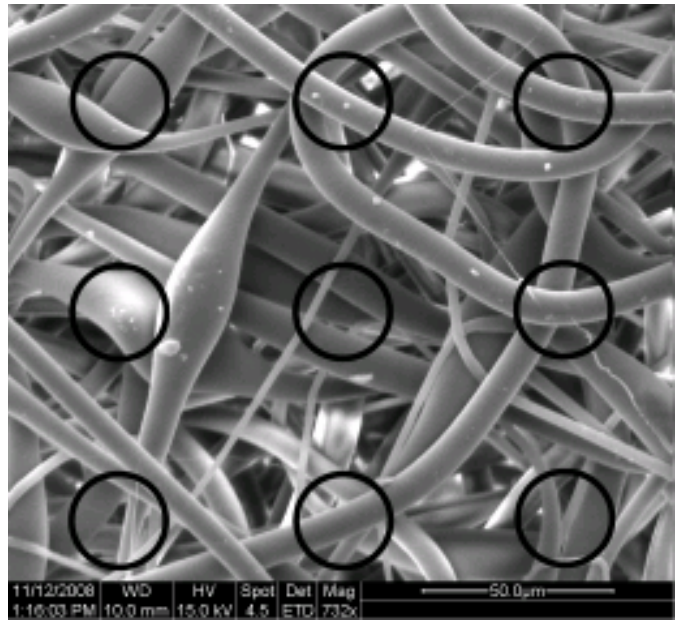


Section D1

Scale Bar: 50 microns is

2.917

$\begin{array}{rrr}\text { Sample } & \text { Length } & \text { Diameter } \\ 1 & 0.378 & 6.48 \\ 2 & 0.243 & 4.17 \\ 3 & 0.168 & 2.88 \\ 4 & 0.304 & 5.21 \\ 5 & 0.866 & 14.84 \\ 6 & 0.445 & 7.63 \\ 7 & 0.305 & 5.23 \\ 8 & 0.76 & 13.03 \\ 9 & 0.36 & 6.17\end{array}$

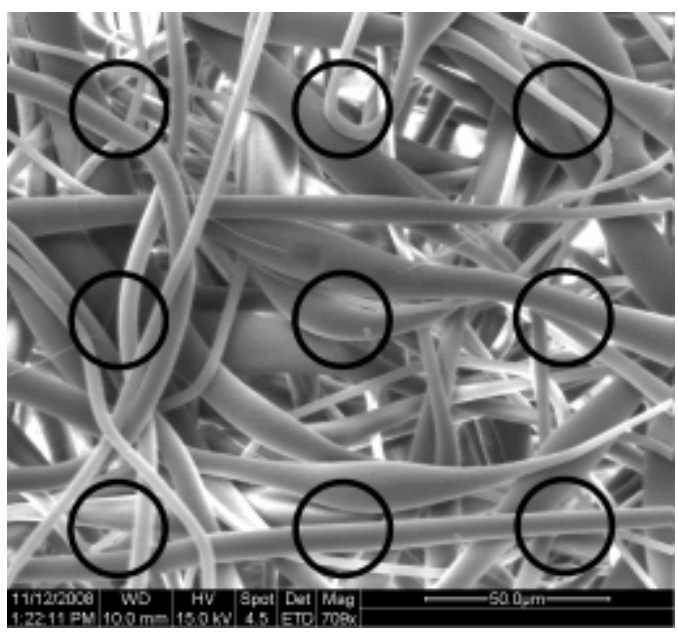

Section D2

Scale Bar: 50 microns is

2.903

$\begin{array}{rrr}\text { Sample } & \text { Length } & \text { Diameter } \\ 1 & 0.21 & 3.62 \\ 2 & 0.179 & 3.08 \\ 3 & 0.195 & 3.36 \\ 4 & 0.321 & 5.53 \\ 5 & 0.286 & 4.93 \\ 6 & 0.24 & 4.13 \\ 7 & 0.518 & 8.92 \\ 8 & 0.798 & 13.74 \\ 9 & 0.443 & 7.63\end{array}$

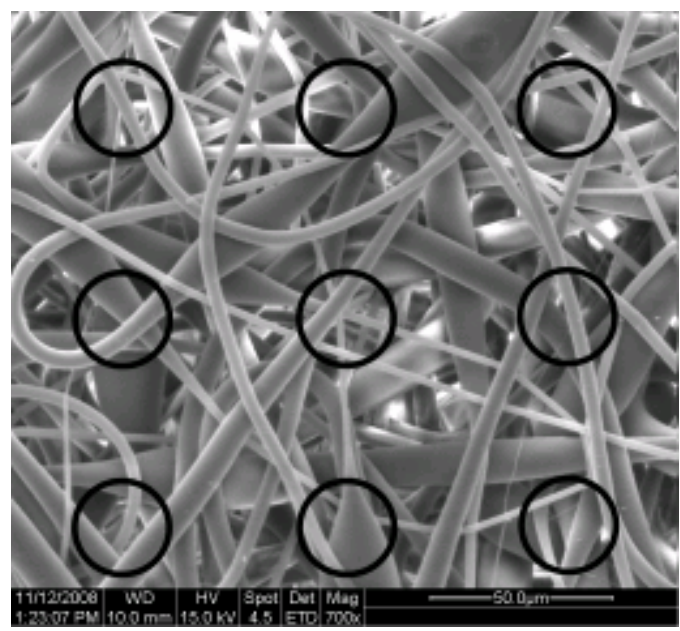

Section D3

Scale Bar: 50 microns is

2.958

$\begin{array}{rrr}\text { Sample } & \text { Length } & \text { Diameter } \\ 1 & 0.287 & 4.85 \\ 2 & 0.936 & 15.82 \\ 3 & 0.647 & 10.94 \\ 4 & 0.117 & 1.98 \\ 5 & 0.591 & 9.99 \\ 6 & 0.358 & 6.05 \\ 7 & 0.666 & 11.26 \\ 8 & 0.193 & 3.26 \\ 9 & 0.253 & 4.28\end{array}$

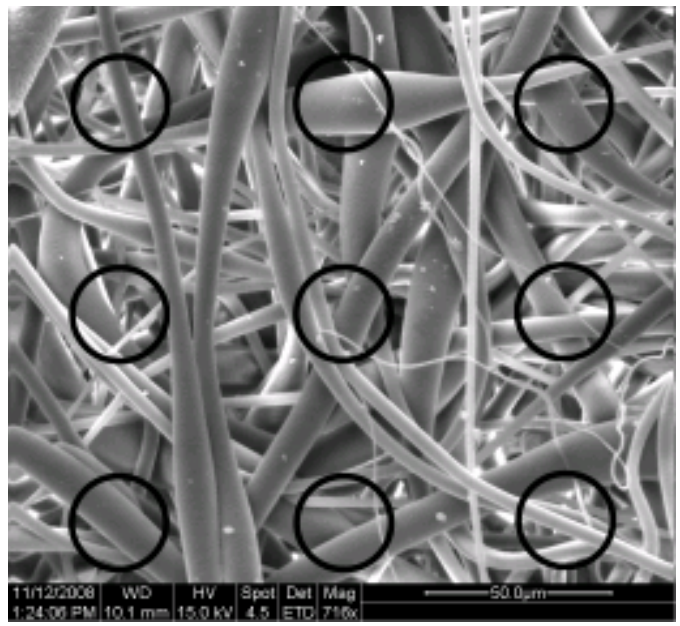




\section{SPIN 11}

Section A1

Scale Bar: 50 microns is

2.972

$\begin{array}{rrr}\text { Sample } & \text { Length } & \text { Diameter } \\ 1 & 0.586 & 9.86 \\ 2 & 0.198 & 3.33 \\ 3 & 0.069 & 1.16 \\ 4 & 0.142 & 2.39 \\ 5 & 0.898 & 15.11 \\ 6 & 0.391 & 6.58 \\ 7 & 0.363 & 6.11 \\ 8 & 0.481 & 8.09 \\ 9 & 0.044 & 0.74\end{array}$

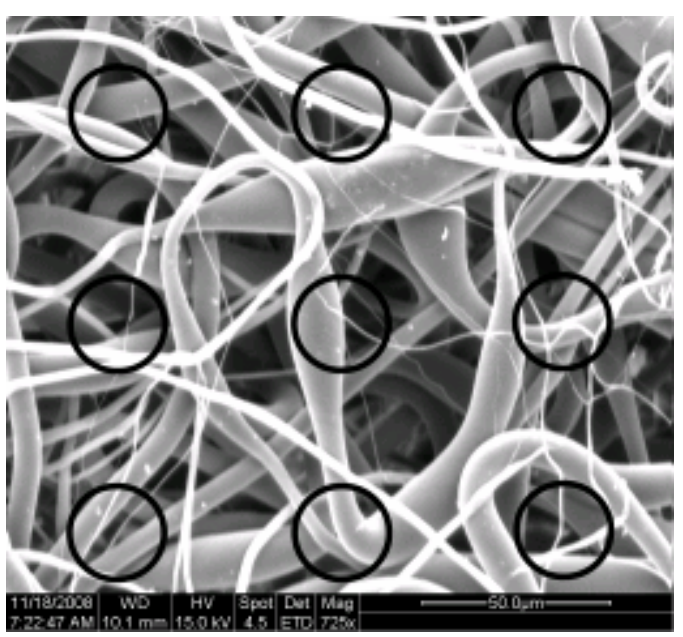

Section A2

Scale Bar: 50 microns is

2.917

$\begin{array}{rrr}\text { Sample } & \text { Length } & \text { Diameter } \\ 1 & 0.305 & 5.23 \\ 2 & 1.027 & 17.60 \\ 3 & 0.348 & 5.97 \\ 4 & 0.473 & 8.11 \\ 5 & 0.435 & 7.46 \\ 6 & 0.087 & 1.49 \\ 7 & 0.445 & 7.63 \\ 8 & 0.461 & 7.90 \\ 9 & 0.125 & 2.14\end{array}$

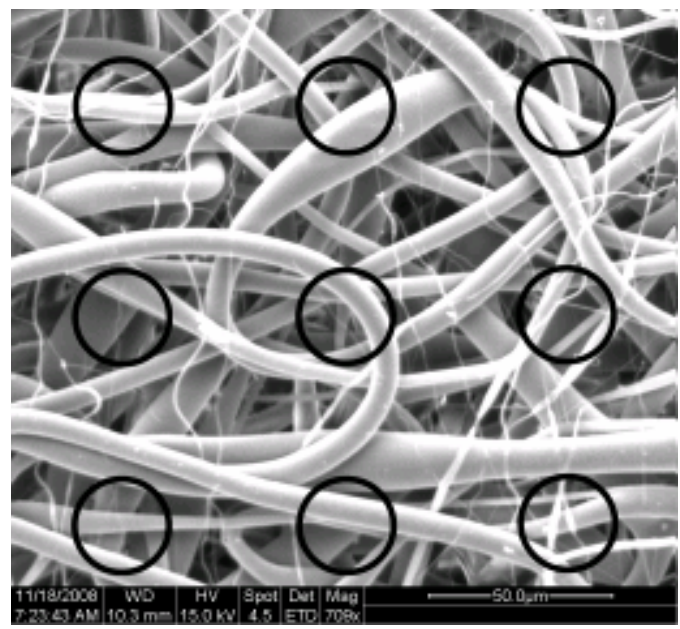

Section A3

Scale Bar: 50 microns is

$\begin{array}{rrr}\text { Sample } & \text { Length } & \text { Diameter } \\ 1 & 0.618 & 10.35 \\ 2 & 0.421 & 7.05 \\ 3 & 0.237 & 3.97 \\ 4 & 0.088 & 1.47 \\ 5 & 0.22 & 3.69 \\ 6 & 0.321 & 5.38 \\ 7 & 0.363 & 6.08 \\ 8 & 0.482 & 8.07 \\ 9 & 0.123 & 2.06\end{array}$

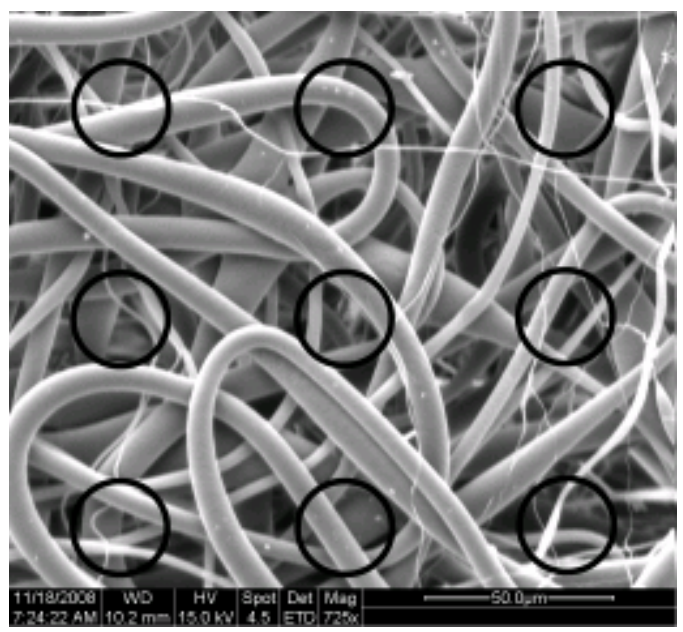


Section B1

Scale Bar: 50 microns is

2.738

$\begin{array}{rrr}\text { Sample } & \text { Length } & \text { Diameter } \\ 1 & 0.394 & 7.20 \\ 2 & 0.467 & 8.53 \\ 3 & 0.458 & 8.36 \\ 4 & 0.487 & 8.89 \\ 5 & 0.46 & 8.40 \\ 6 & 0.374 & 6.83 \\ 7 & 0.477 & 8.71 \\ 8 & 0.406 & 7.41 \\ 9 & 0.457 & 8.35\end{array}$

Section B2

Scale Bar: 50 microns is

2.944

$\begin{array}{rrr}\text { Sample } & \text { Length } & \text { Diameter } \\ 1 & 0.505 & 8.58 \\ 2 & 0.454 & 7.71 \\ 3 & 0.473 & 8.03 \\ 4 & 0.462 & 7.85 \\ 5 & 0.493 & 8.37 \\ 6 & 0.506 & 8.59 \\ 7 & 0.557 & 9.46 \\ 8 & 0.457 & 7.76 \\ 9 & 0.413 & 7.01\end{array}$

Section B3

Scale Bar: 50 microns is

$\begin{array}{rrr}\text { Sample } & \text { Length } & \text { Diameter } \\ 1 & 0.446 & 7.54 \\ 2 & 0.571 & 9.65 \\ 3 & 0.509 & 8.60 \\ 4 & 0.421 & 7.12 \\ 5 & 0.434 & 7.34 \\ 6 & 0.44 & 7.44 \\ 7 & 0.511 & 8.64 \\ 8 & 0.44 & 7.44 \\ 9 & 0.499 & 8.43\end{array}$
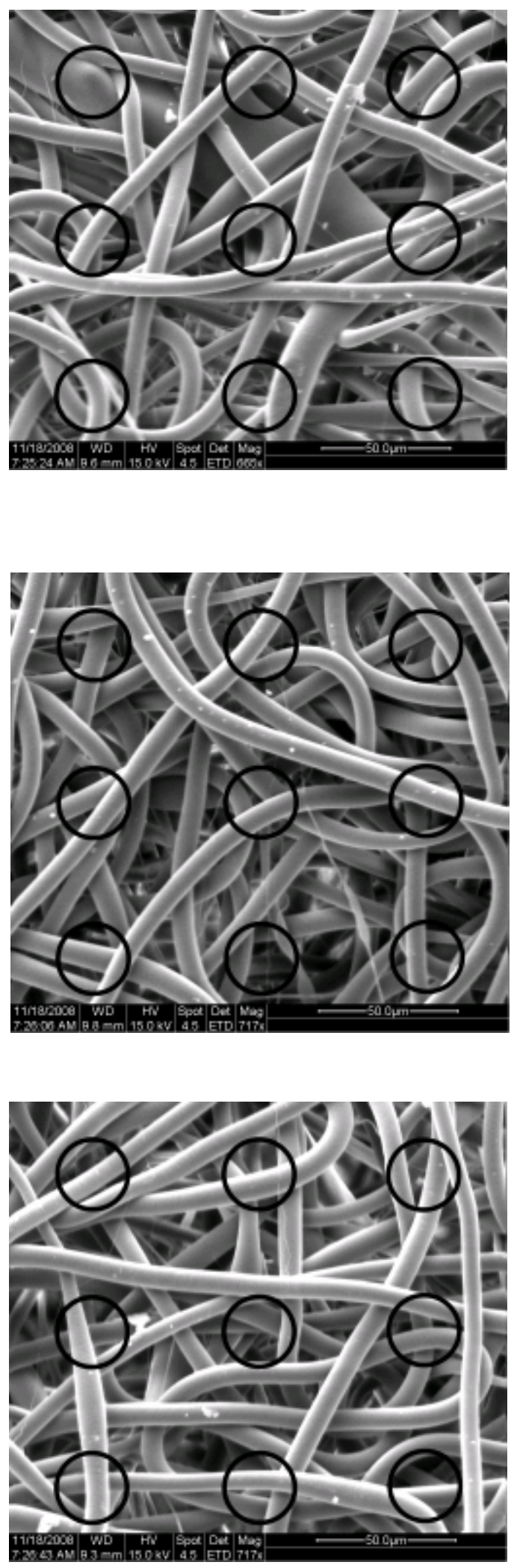
Section C1

Scale Bar: 50 microns is

2.875

$\begin{array}{rrr}\text { Sample } & \text { Length } & \text { Diameter } \\ 1 & 0.222 & 3.86 \\ 2 & 0.486 & 8.45 \\ 3 & 0.427 & 7.43 \\ 4 & 0.496 & 8.63 \\ 5 & 0.474 & 8.24 \\ 6 & 0.305 & 5.30 \\ 7 & 0.168 & 2.92 \\ 8 & 0.512 & 8.90 \\ 9 & 0.451 & 7.84\end{array}$

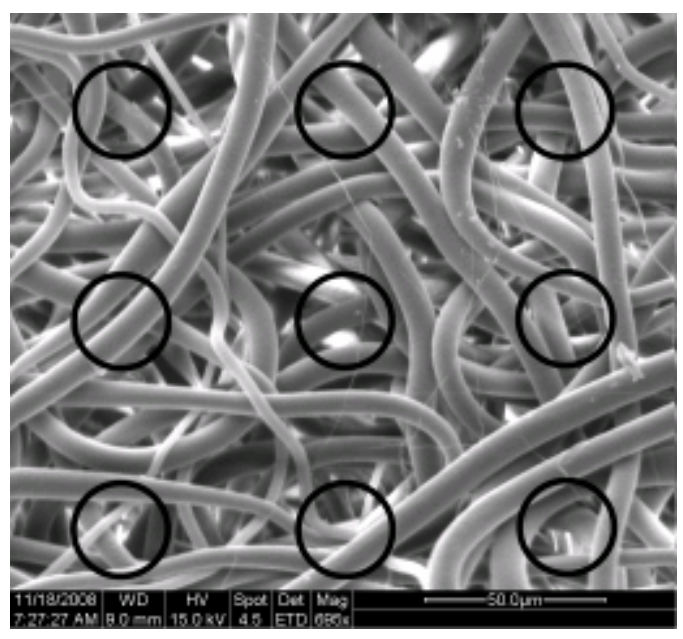

Section C2

Scale Bar: 50 microns is

2.862

$\begin{array}{rrr}\text { Sample } & \text { Length } & \text { Diameter } \\ 1 & 0.414 & 7.23 \\ 2 & 0.248 & 4.33 \\ 3 & 0.493 & 8.61 \\ 4 & 0.448 & 7.83 \\ 5 & 0.341 & 5.96 \\ 6 & 0.41 & 7.16 \\ 7 & 0.419 & 7.32 \\ 8 & 0.489 & 8.54 \\ 9 & 0.478 & 8.35\end{array}$

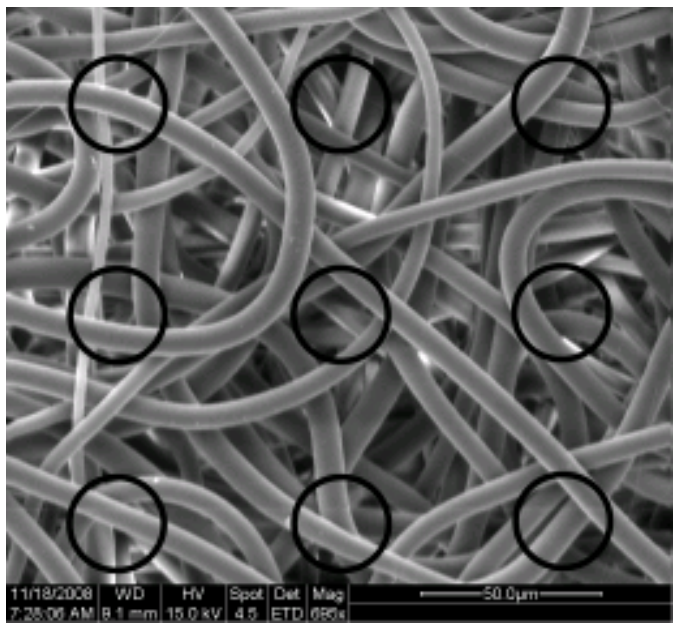

Section C3

Scale Bar: 50 microns is

2.779

$\begin{array}{rrr}\text { Sample } & \text { Length } & \text { Diameter } \\ 1 & 0.283 & 5.09 \\ 2 & 0.193 & 3.47 \\ 3 & 0.354 & 6.37 \\ 4 & 0.481 & 8.65 \\ 5 & 0.466 & 8.38 \\ 6 & 0.393 & 7.07 \\ 7 & 0.451 & 8.11 \\ 8 & 0.396 & 7.12 \\ 9 & 0.314 & 5.65\end{array}$

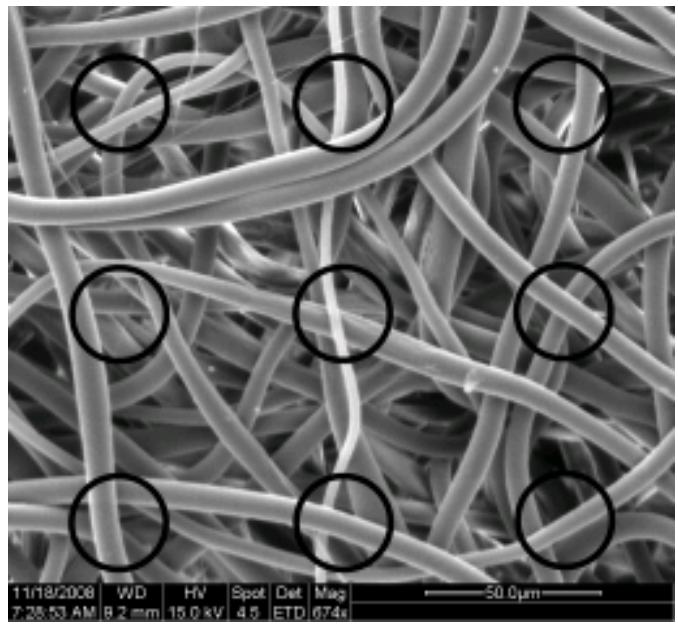


Section D1

Scale Bar: 50 microns is

2.82

$\begin{array}{rrr}\text { Sample } & \text { Length } & \text { Diameter } \\ 1 & 0.266 & 4.72 \\ 2 & 0.366 & 6.49 \\ 3 & 0.418 & 7.41 \\ 4 & 0.573 & 10.16 \\ 5 & 0.505 & 8.95 \\ 6 & 0.446 & 7.91 \\ 7 & 0.412 & 7.30 \\ 8 & 0.281 & 4.98 \\ 9 & 0.279 & 4.95\end{array}$

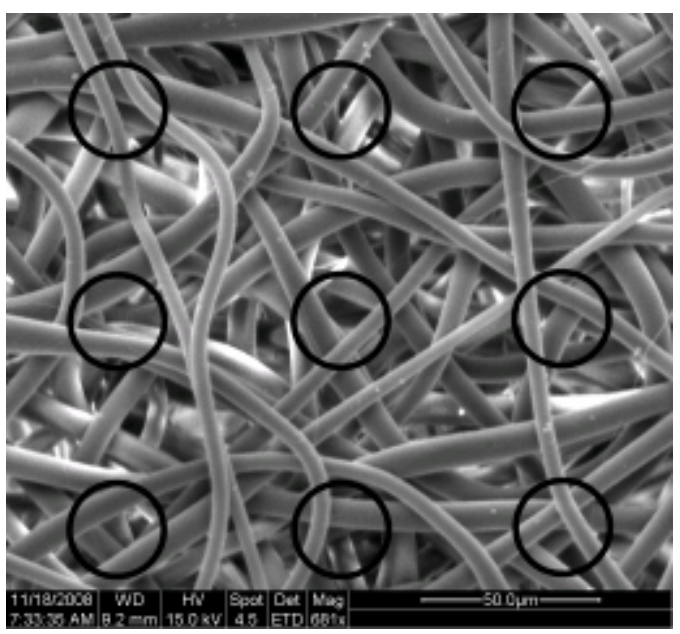

Section D2

Scale Bar: 50 microns is

2.807

\begin{tabular}{rrr} 
Sample & Length & \multicolumn{1}{c}{ Diameter } \\
1 & 0.428 & 7.62 \\
2 & 0.322 & 5.74 \\
3 & 0.312 & 5.56 \\
4 & 0.435 & 7.75 \\
5 & 0.172 & 3.06 \\
6 & 0.478 & 8.51 \\
7 & 0.469 & 8.35 \\
8 & 0.437 & 7.78 \\
9 & 0.409 & 7.29
\end{tabular}

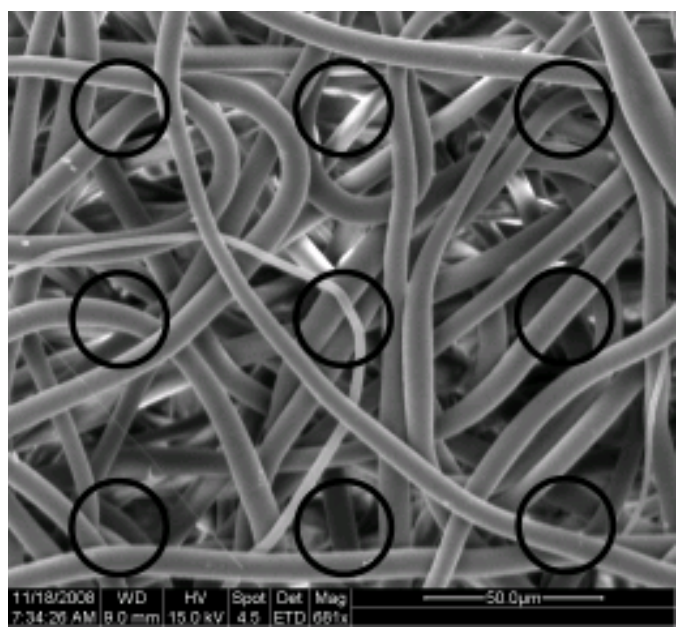

Section D3

Scale Bar: 50 microns is

2.889

$\begin{array}{rrr}\text { Sample } & \text { Length } & \text { Diameter } \\ 1 & 0.399 & 6.91 \\ 2 & 0.428 & 7.41 \\ 3 & 0.475 & 8.22 \\ 4 & 0.47 & 8.13 \\ 5 & 0.492 & 8.52 \\ 6 & 0.462 & 8.00 \\ 7 & 0.466 & 8.07 \\ 8 & 0.514 & 8.90 \\ 9 & 0.305 & 5.28\end{array}$

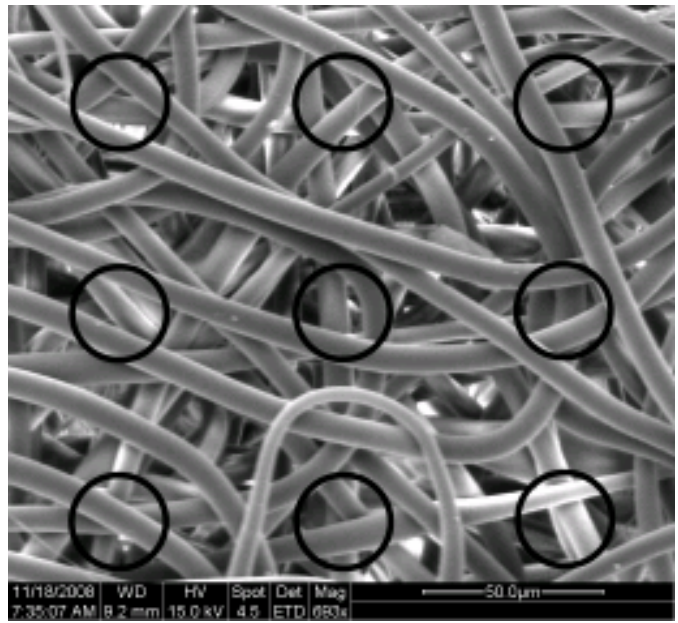




\section{APPENDIX F: THICKNESS MEASUREMENTS}

\section{Introduction}

Scaffold wall thicknesses were measured using a combination of SEM images and the Image J software. Using the program's line length measurement tool (ANALYZE > MEASURE), the scale bar on all images was measured. This length corresponded to 2 millimeters on all images. Twelve measurements corresponding to the numbers on a clock were made on each image, unless the sample had a corrupted section. In the case of corrupted sections or entire samples, no measurements were made. A simple calculation was then used in Microsoft Excel to convert the collected wall thickness into wall thickness in millimeters. In the data shown below, entries under Thickness are in millimeters. All other values are unitless. Below the thickness column, the mean and standard deviation of the data is given.

$$
D=\left(\frac{t}{S}\right) \bullet 2 m m
$$

Where: $\quad D$ is scaffold thickness in millimeters

$t$ is scaffold thickness measured on Image $\mathrm{J}$

$S$ is length of the scale bar measured on Image $\mathrm{J}$ 


\section{SPIN 1}

A1

Scale Bar: $2 \mathrm{~mm}$ is

365

Sample Length Thickness

Top 1

Top 2

Top 3

Right 1

Right 2

Right 3

Bottom 1

Bottom 2

Bottom 3

Left 1

Left 2

Left 3
138

157

142

139

140

132

122

129

126

133

145

138
0.758

0.863

0.778

0.762

0.769

0.724

0.670

0.705

0.694

0.731

0.797

0.759

$0.751 \pm \quad 0.051$

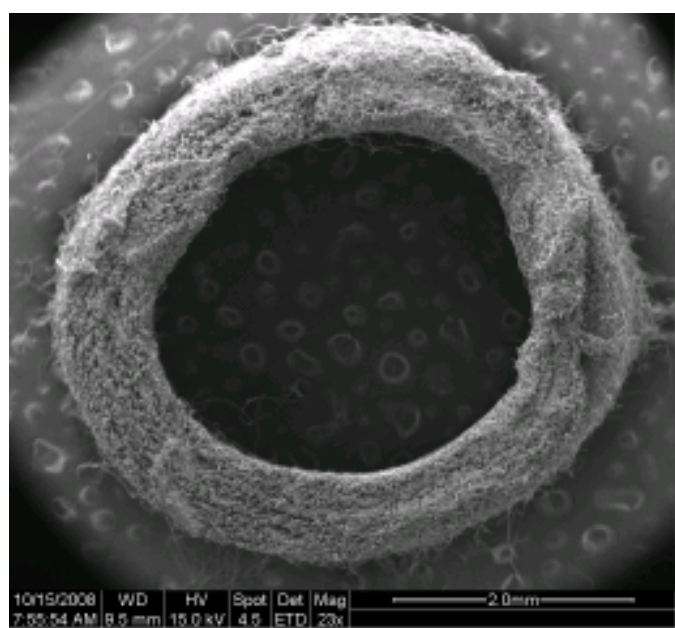

B1

Scale Bar: $2 \mathrm{~mm}$ is

343

Sample Length Size

Top 1

0.736

0.694

0.710

0.658

0.639

0.566

0.637

0.663

0.544

0.671

0.766

0.719

$0.667+$

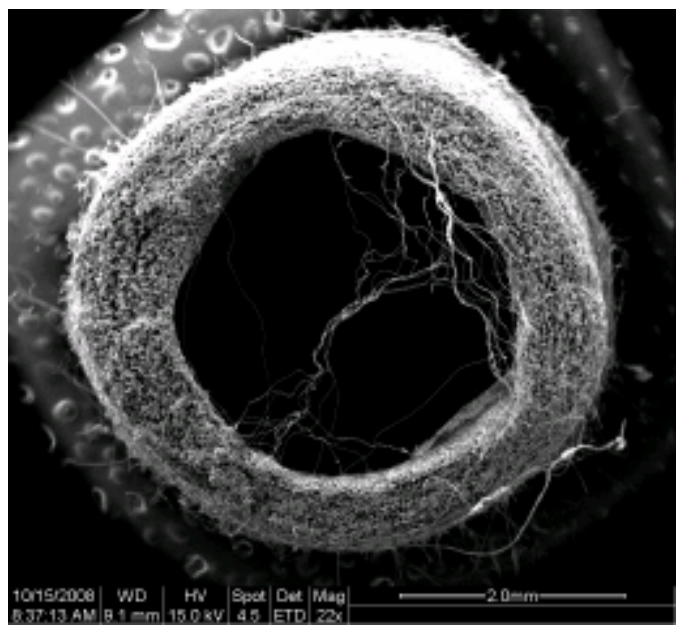

131.557

0.065 
B2

\section{BAD SAMPLE}

Sample Length Size

Top 1

Top 2

Top 3

Right 1

Right 2

Right 3

Bottom 1

Bottom 2

Bottom 3

Left 1

Left 2

Left 3
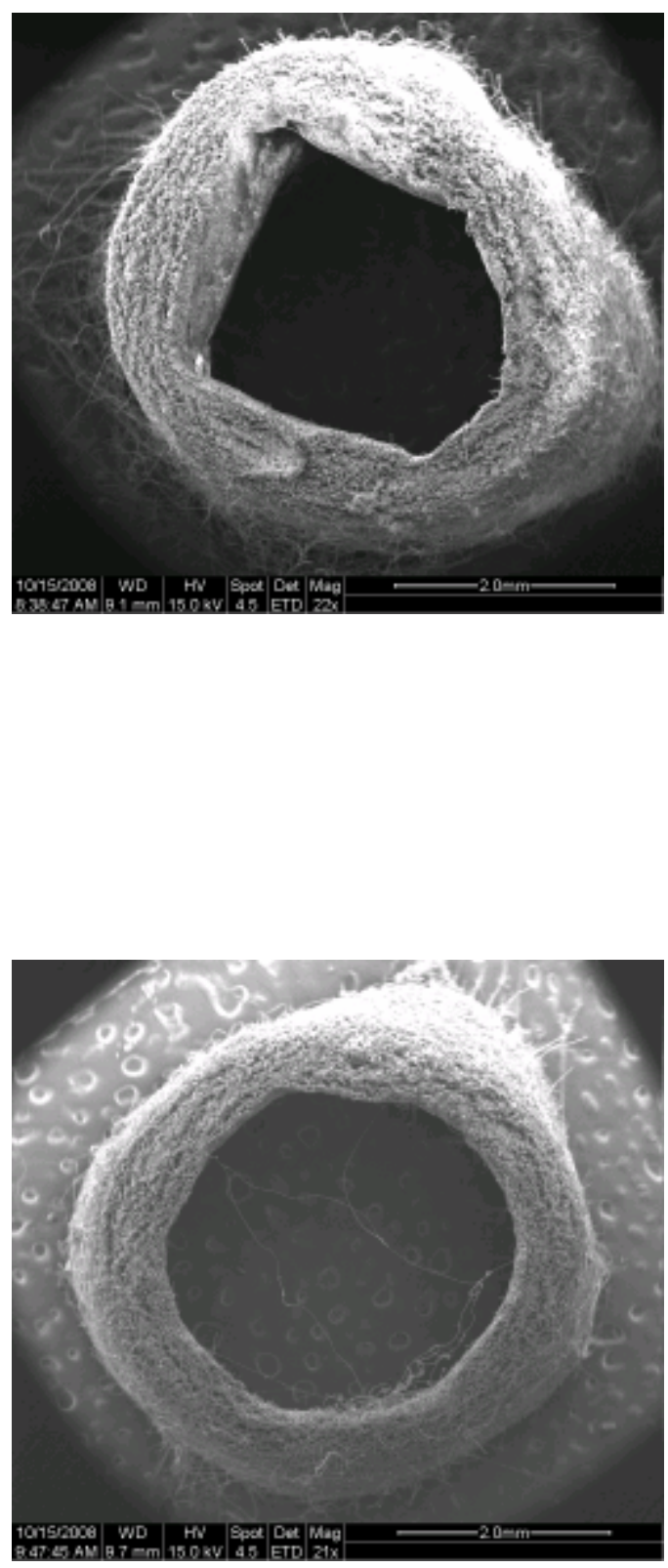

$0.633 \pm \quad 0.051$

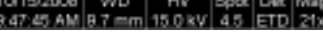

0.698

0.693

0.714

332

0.611

0.610

0.665

0.622

0.615

0.620

0.537

0.586

0.625

Bottom 3

Left 2

Left 3

118

89

104 


\section{C2}

Scale Bar: $2 \mathrm{~mm}$ is

Sample Length

Top 1

Top 2

Top 3

Right 1

Right 2

Right 3

Bottom 1

Bottom 2

Bottom 3

Left 1

Left 2

Left 3

108
101
115
90
99
100
104
108
122
108
114
108

$\underline{330}$

0.657

0.609

0.696

0.544

0.602

0.605

0.628

0.652

0.737

0.654

0.691

0.653

$0.644 \pm$

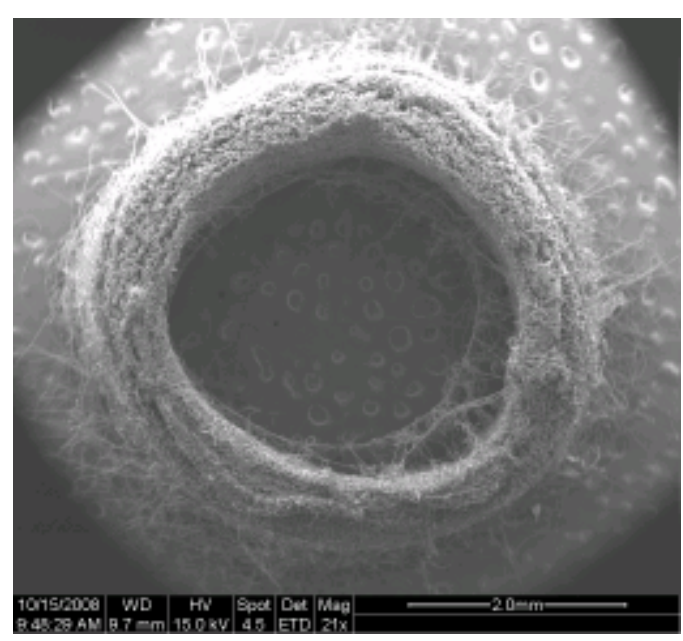

0.051

D1

Scale Bar: $2 \mathrm{~mm}$ is

Sample Length Size

Top 1

137.1

Top 2

Top 3

Right 1

Right 2

Right 3

Bottom 1

Bottom 2

Bottom 3

Left 1

Left 2

Left 3

127.8

142.7

127.2

129.4

123.7

109.9

112.0

123.8

125.4

109.3

118.2

$\underline{352.6}$

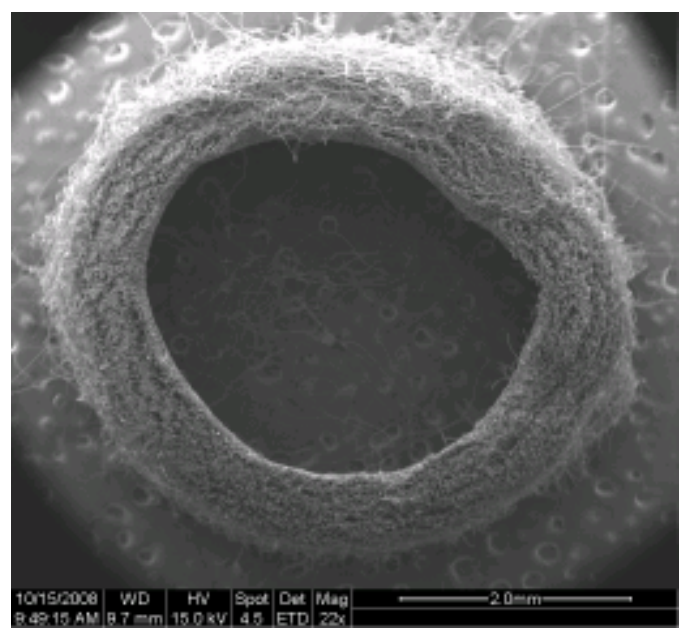

0.711

0.620

0.670

$0.703 \pm \quad 0.059$

TOTAL AVERAGE:

$0.679+0.069024$ 


\section{SPIN 2 Thickness Measurement Table}

A1

Scale Bar: $2 \mathrm{~mm}$ is

$\underline{322}$

Sample Length Thickness

Top 1

Top 2

Top 3

Right 1

Right 2

Right 3

Bottom 1

$80 \quad 0.498$

$67 \quad 0.417$

$88 \quad 0.545$

$97 \quad 0.600$

$89 \quad 0.550$

Bottom 2

101

0.627

0.554

0.515

0.542

0.560

Left 1

0.528

Left 2

0.563

$0.542 \pm$

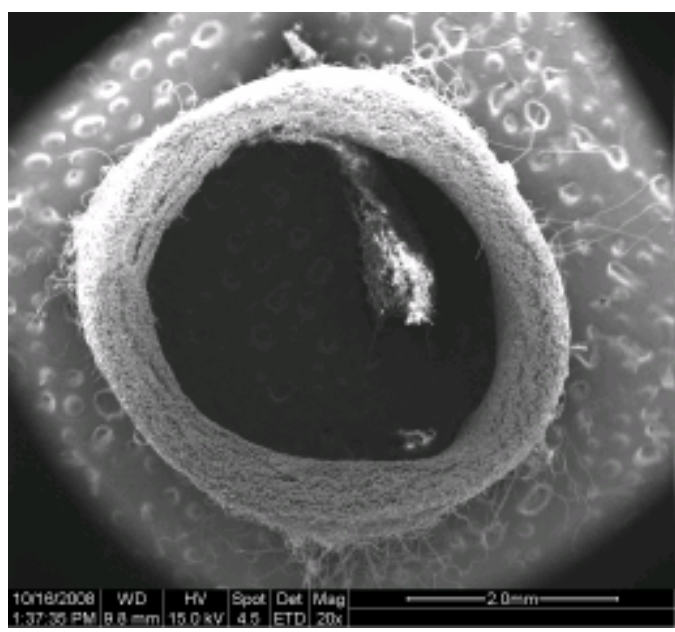

Left 3

91

B1

Scale Bar: $2 \mathrm{~mm}$ is

$\underline{305}$

Sample Length Size

Top 1

Top 2

134

Top 3

122

Right 1

135

Right 2

Right 3

Bottom 1

112

111

128

95

Bottom 2

92

Bottom 3

102

Left 1

108

Left 2

103

113

0.879

0.798

0.882

0.735

0.730

0.836

0.621

0.606

0.669

0.709

0.677

0.738

$0.740 \pm \quad 0.093$

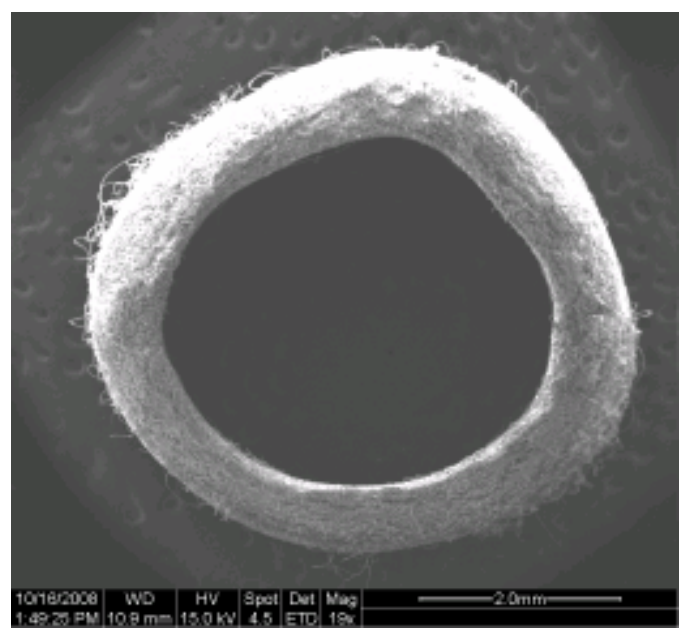

Left 3

0.052 
B2

Scale Bar: $2 \mathrm{~mm}$ is

Sample Length Size

Top 1

Top 2

Top 3

Right 1

Right 2

Right 3

Bottom 1

Bottom 2

Bottom 3

Left 1

Left 2

Left 3

100
95
96
83
81
93
99
93
97
94
92
87

$\underline{306}$

0.652

0.619

0.629

0.540

0.529

0.608

0.650

0.606

0.636

0.617

0.603

0.565

$0.604+$

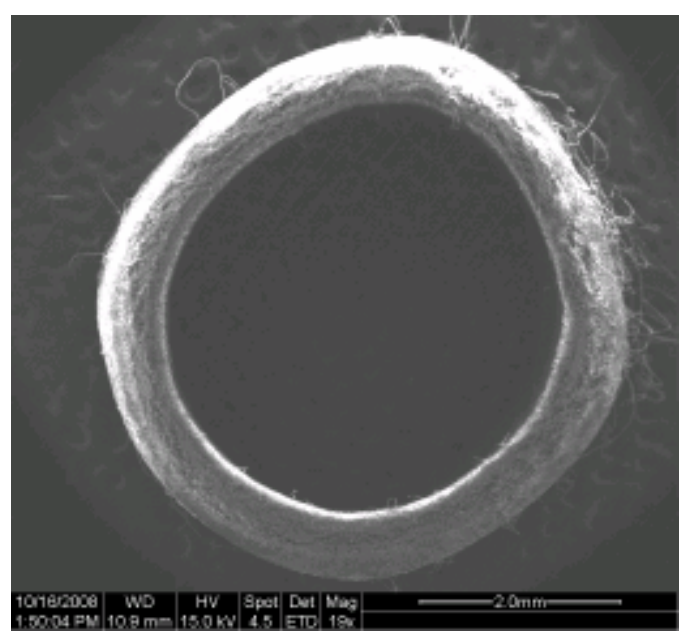

0.040

C1

Scale Bar: $2 \mathrm{~mm}$ is

Sample Length Size

Top 1

Top 2

Top 3

121

110

0.775

0.706

0.692

0.659

0.694

Right 2

108

103

109

0.807

0.705

0.719

0.754

0.691

0.858

0.903

$0.747 \pm \quad 0.075$

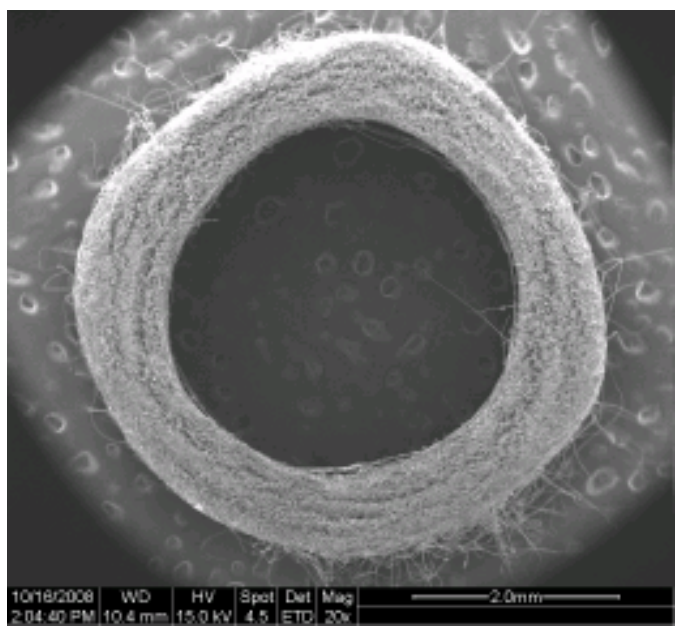

Left 2

134

141

112

118

Left 3 


\section{C2}

Scale Bar: $2 \mathrm{~mm}$ is

\begin{tabular}{lrr} 
Sample & Length & \multicolumn{1}{l}{ Size } \\
Top 1 & 119 & 0.76042 \\
Top 2 & 108 & 0.687573 \\
Top 3 & 122 & 0.776395 \\
Right 1 & 119 & 0.757225 \\
Right 2 & 126 & 0.807067 \\
Right 3 & 136 & 0.869051 \\
Bottom 1 & 113 & 0.722079 \\
Bottom 2 & 114 & 0.728469 \\
Bottom 3 & 106 & 0.675432 \\
Left 1 & 125 & 0.796843 \\
Left 2 & 126 & 0.803872 \\
Left 3 & 114 & 0.729747 \\
& & $\mathbf{0 . 7 6 0}$
\end{tabular}

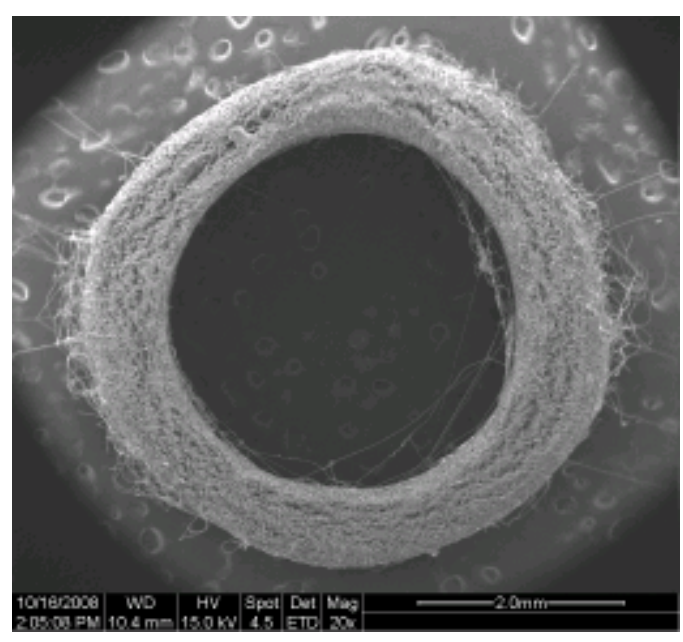

0.055

D1

Scale Bar: $2 \mathrm{~mm}$ is

313

$\begin{array}{lr}\text { Sample } & \text { Length } \\ \text { Top 1 } & 107 \\ \text { Top 2 } & 126 \\ \text { Top 3 } & 108 \\ \text { Right 1 } & 95 \\ \text { Right 2 } & 115 \\ \text { Right 3 } & 113 \\ \text { Bottom 1 } & 95 \\ \text { Bottom 2 } & 117 \\ \text { Bottom 3 } & 96 \\ \text { Left 1 } & 113 \\ \text { Left 2 } & 109 \\ \text { Left 3 } & 100\end{array}$

0.682

0.805

0.686

0.607

0.737

0.723

0.606

0.746

0.615

0.719

0.695

0.638

$0.688 \pm \quad 0.062$

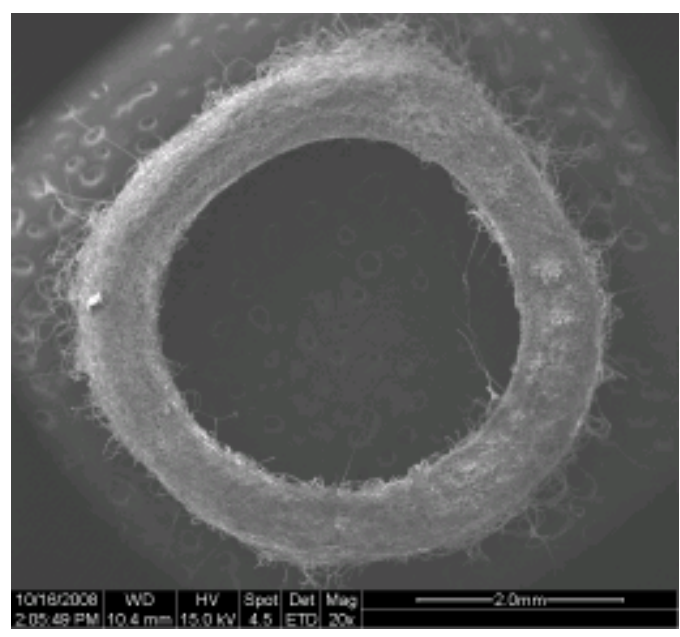

TOTAL AVERAGE:

$0.680 \pm \stackrel{0.102964}{ }$ 


\section{SPIN 3}

A1

Scale Bar: $2 \mathrm{~mm}$ is 321

Sample Length Thickness

Top 1

Top 2

Top 3

Right 1

Right 2

Right 3

Bottom 1

Bottom 2

Bottom 3

Left 1

Left 2

Left 3

113

129

123

109

112

122

105

110

108

110

119

123
0.705

0.769

0.681

0.699

0.759

0.652

0.686

0.674

0.688

0.739

0.765
0.802

$0.718 \pm$

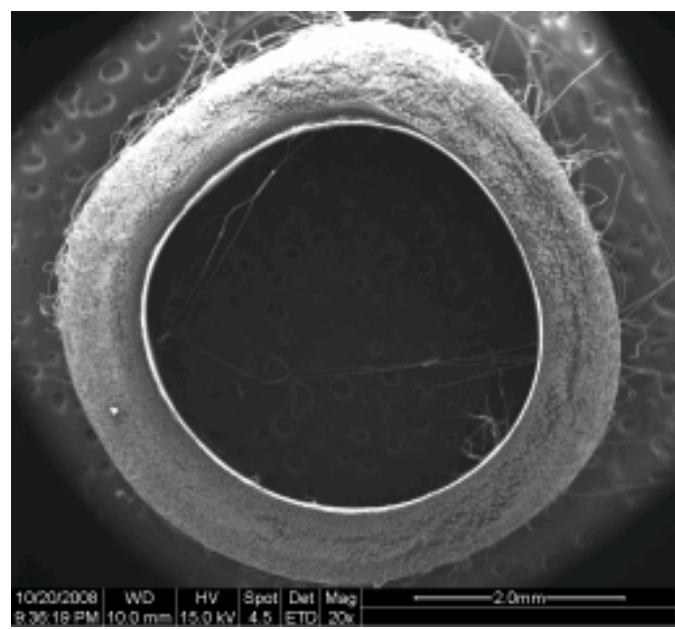

0.047

B1

Scale Bar: $2 \mathrm{~mm}$ is

Sample Length Size

Top 1

Top 2

Top 3

Right 1

Right 2

Right 3

Bottom 1

Bottom 2

Bottom 3

Left 1

Left 2

Left 3

Length
108
113
104
108
120
123
113
108
99
107
110
119

0.678

0.706

0.651

0.678

0.752

0.770

0.706

0.677

0.619

0.672

0.691

0.745

$0.695 \pm 0.043$

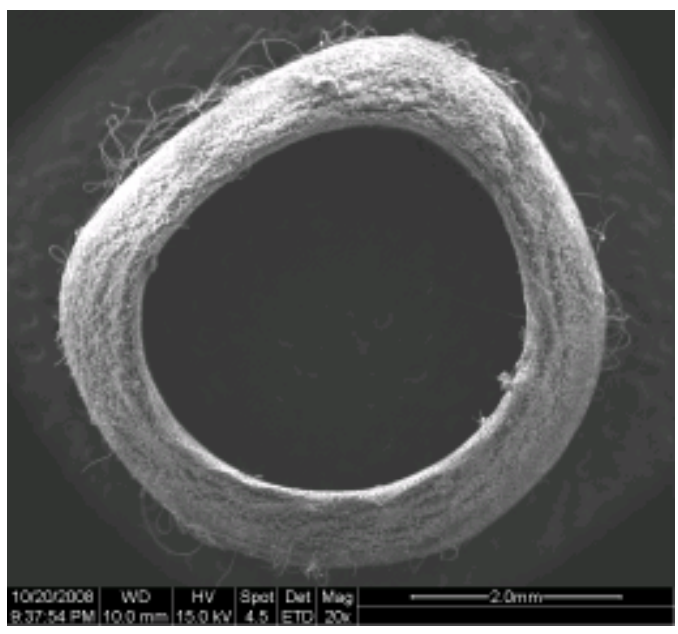


B2

Scale Bar: $2 \mathrm{~mm}$ is

Sample Length Size

Top 1103

Top $2 \quad 110$

Top $3 \quad 109$

Right 1

Right 2

Right 3

Bottom 1

Bottom 2

Bottom 3

Left 1

Left 2

Left 3
92

99

80

105

111

112

100

104

104 $\underline{314}$

0.656

0.702

0.696

0.582

0.630

0.508

0.666

0.707

0.713

0.639

0.661

0.660

$0.652 \pm$

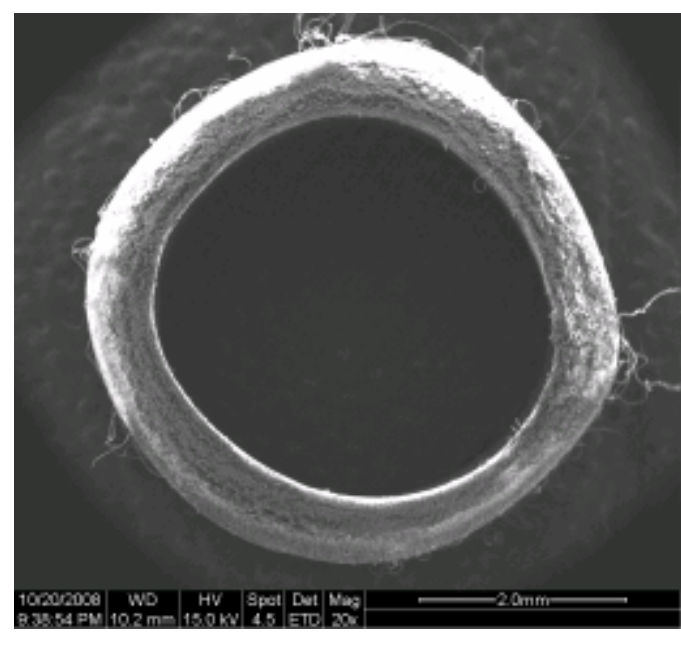

0.059

C1

Scale Bar: $2 \mathrm{~mm}$ is

$\underline{324}$

Sample Length Size

Top 1

Top 2

Top 3

Right 1

Right 2

Right 3

Bottom 1

Bottom 2

Bottom 3

Left 1

Left 2

Left 3

Length
106
111
124
106
116
132
128
119
128
129
118
144

0.657

0.687

0.766

0.654

0.720

0.817

0.793

0.735

0.791

0.795

0.729

0.891

$0.753 \pm \quad 0.070$

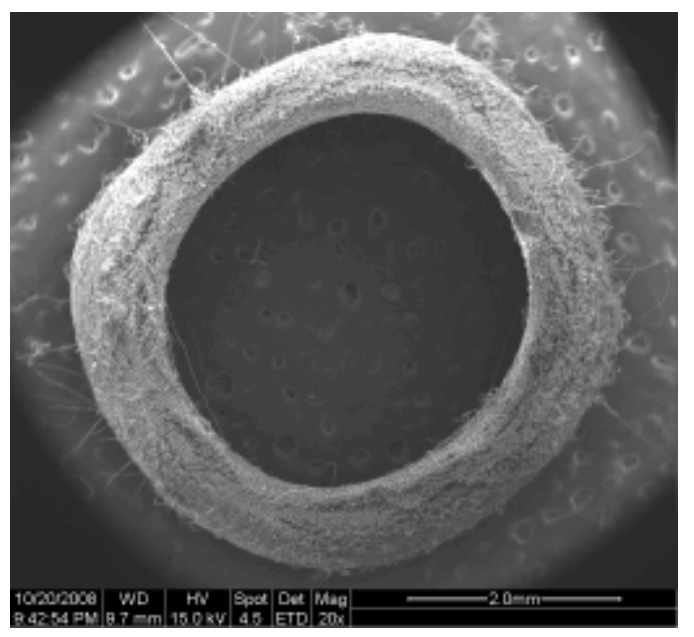




\section{C2}

Scale Bar: $2 \mathrm{~mm}$ is

\begin{tabular}{lrr} 
Sample & Length & \multicolumn{2}{l}{ Size } \\
Top 1 & 110 & 0.668404 \\
Top 2 & 128 & 0.780439 \\
Top 3 & 125 & 0.760048 \\
Right 1 & 121 & 0.733039 \\
Right 2 & 115 & 0.697456 \\
Right 3 & 115 & 0.699536 \\
Bottom 1 & 96 & 0.58331 \\
Bottom 2 & 109 & 0.664536 \\
Bottom 3 & 112 & 0.682404 \\
Left 1 & 124 & 0.75528 \\
Left 2 & 129 & 0.781595 \\
Left 3 & 137 & 0.832017 \\
& & $\mathbf{0 . 7 2 0} \pm \quad 0.067$
\end{tabular}

D1

Scale Bar: $2 \mathrm{~mm}$ is

$\begin{array}{lr}\text { Sample } & \text { Length } \\ \text { Top 1 } & \text { Size } \\ \text { Top 2 } & 100 \\ \text { Top 3 } & 110 \\ \text { Right 1 } & 116 \\ \text { Right 2 } & 113 \\ \text { Right 3 } & 126 \\ \text { Bottom 1 } & 117 \\ \text { Bottom 2 } & 111 \\ \text { Bottom 3 } & 106 \\ \text { Left 1 } & 101 \\ \text { Left 2 } & 96 \\ \text { Left 3 } & 102\end{array}$

$\underline{329}$

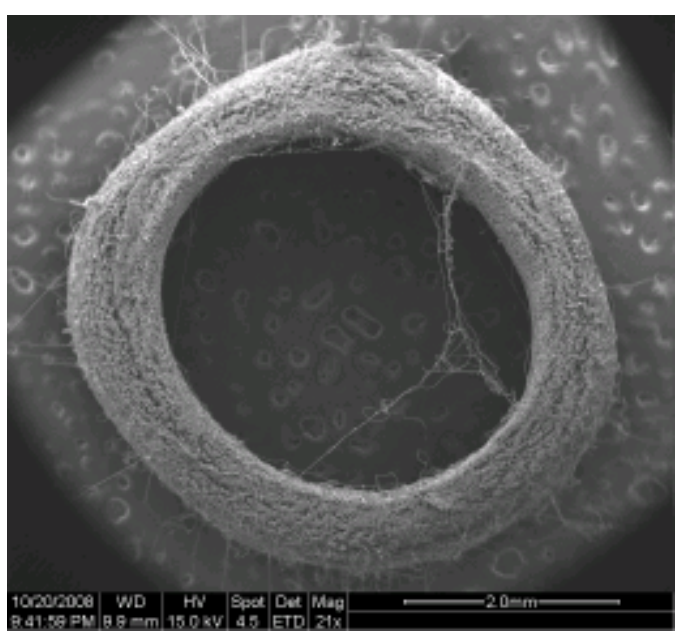

$0.720 \pm \quad 0.067$
320

0.625

0.665

0.690

0.725

0.706

0.786

0.732

0.694

0.666

0.632

0.602

0.638

$0.680 \pm \quad 0.053$

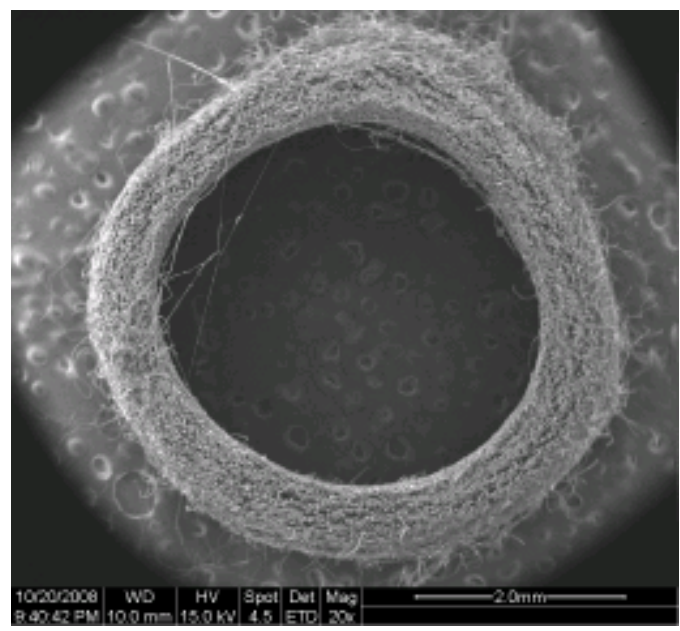

TOTAL AVERAGE:

$0.703 \pm 0.064068$ 


\section{SPIN 4}

A1

Scale Bar: $2 \mathrm{~mm}$ is

330

Sample Length Thickness

Top 1

Top 2

Top 3

Right 1

Right 2

Right 3

Bottom 1

Bottom 2

Bottom 3

Left 1

Left 2

Left 3 123

134

108

103

96

108

112

111

114

110

102

127
0.746

0.814

0.655

0.626

0.580

0.652

0.681

0.673

0.689

0.666

0.620

0.768
$0.681 \pm$

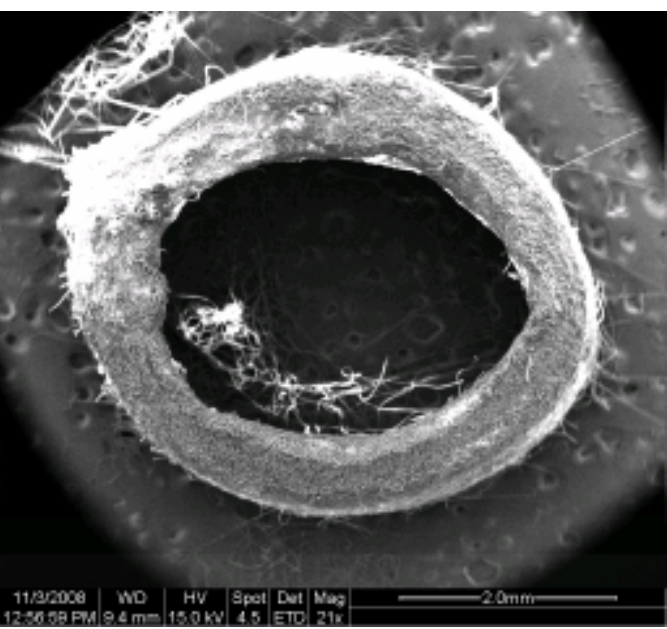

0.066

B1

Scale Bar: $2 \mathrm{~mm}$ is

Sample Length Size

Top 1

Top 2

Top 3

Right 1

Right 2

Right 3

Bottom 1

Bottom 2

Bottom 3

Left 1

Left 2

Left 3

Length
125
119
134
125
120
131
131
119
113
116
130
137

0.747

0.711

0.800

0.743

0.714

0.782

0.782

0.708

0.675

0.694

0.775

0.814

$0.745 \pm \quad 0.045$

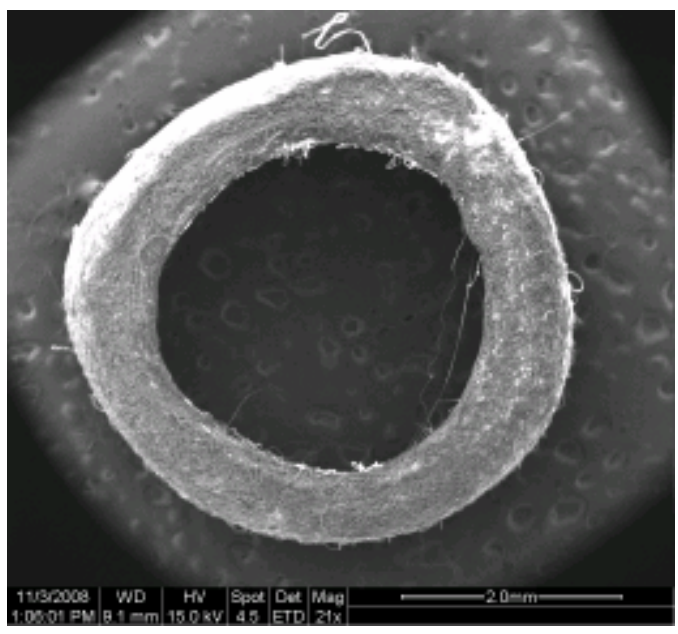


B2

Scale Bar: $2 \mathrm{~mm}$ is

Sample Length Size

Top $1 \quad 116$

Top $2 \quad 132$

Top $3 \quad 126$

Right $1 \quad 116$

Right $2 \quad 112$

Right $3 \quad 131$

Bottom 1

Bottom 2

Bottom 3

Left 1

Left 2

Left 3 $\underline{335}$

0.693

0.790

0.751

0.694

0.667

0.783

0.664

0.693

0.655

0.664

0.760

0.668

$0.707 \pm$

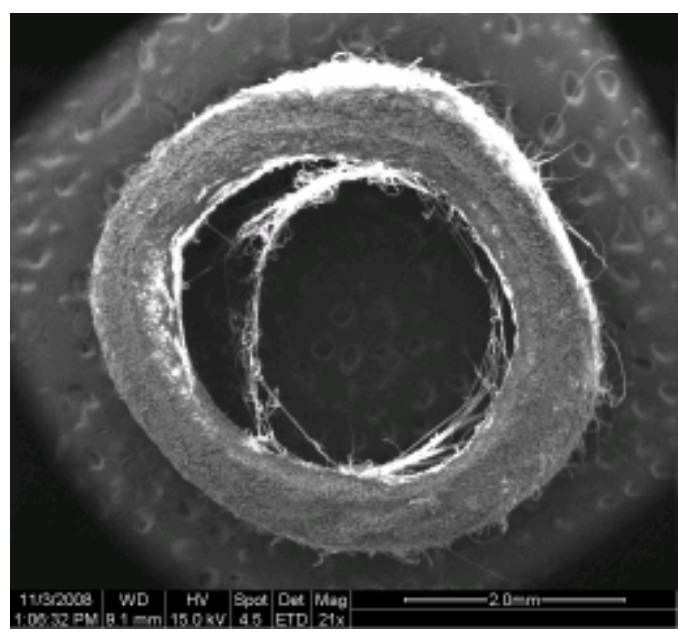

0.050

C1

Scale Bar: $2 \mathrm{~mm}$ is

Sample

Top 1

Top 2

Top 3

Right 1

Right 2

Right 3

Bottom 1

Length

Size

Bottom 2

Bottom 3

Left 1

Left 2

Left 3

108

123

120

110

115

119

109

111

120

128

127

118

0.655

0.745

0.725

0.669

0.697

0.718

0.657

0.672

0.725

0.778

0.769

0.715

$0.710 \pm$

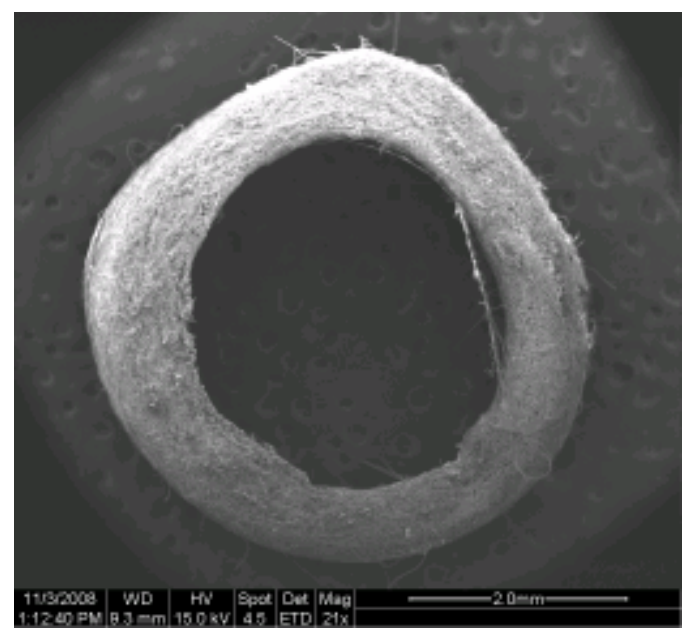

0.042 


\section{C2}

Scale Bar: $2 \mathrm{~mm}$ is

\begin{tabular}{lrr} 
Sample & Length & \multicolumn{1}{l}{ Size } \\
Top 1 & 100 & 0.605727 \\
Top 2 & 112 & 0.680182 \\
Top 3 & 119 & 0.720214 \\
Right 1 & 108 & 0.654869 \\
Right 2 & 112 & 0.675839 \\
Right 3 & 110 & 0.667601 \\
Bottom 1 & 108 & 0.655505 \\
Bottom 2 & 115 & 0.69604 \\
Bottom 3 & 106 & 0.641695 \\
Left 1 & 109 & 0.657213 \\
Left 2 & 107 & 0.64522 \\
Left 3 & 117 & 0.708797 \\
& & $\mathbf{0 . 6 6 7}$
\end{tabular}

$\underline{330}$

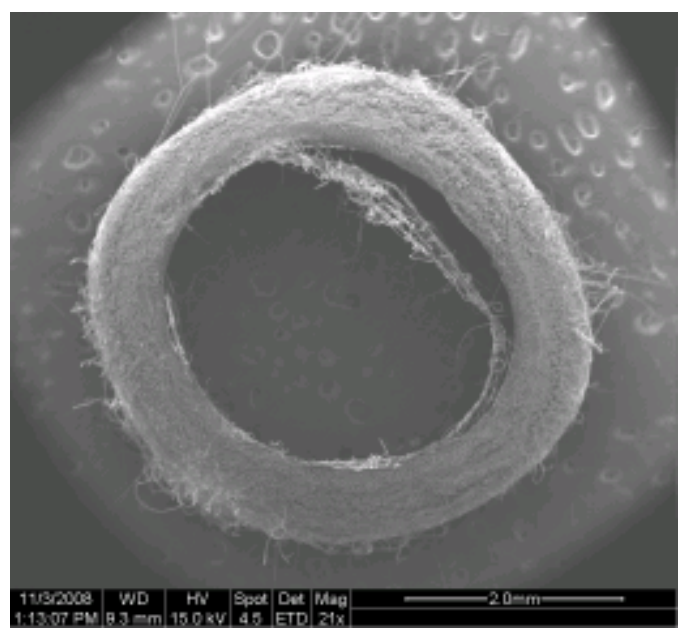

$0.667 \pm \quad 0.032$
D1

Scale Bar: $2 \mathrm{~mm}$ is

$\begin{array}{lrr}\text { Sample } & \text { Length } & \text { Size } \\ \text { Top 1 } & 104 \\ \text { Top 2 } & 102 \\ \text { Top 3 } & 100 \\ \text { Right 1 } & 102 \\ \text { Right 2 } & 104 \\ \text { Right 3 } & 103 \\ \text { Bottom 1 } & 106 \\ \text { Bottom 2 } & 116 \\ \text { Bottom 3 } & 104 \\ \text { Left 1 } & 101 \\ \text { Left 2 } & 113 \\ \text { Left 3 } & 106\end{array}$

317

0.657

0.644

0.632

0.645

0.654

0.652

0.670

0.734

0.657

0.640

0.715

0.669

$0.664 \pm \quad 0.031$

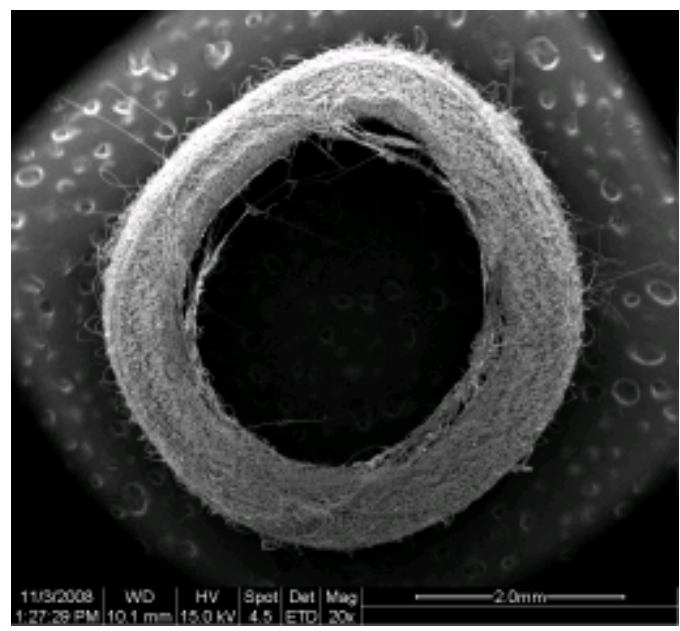

TOTAL AVERAGE:

$0.696 \pm 0.05261$ 


\section{SPIN 5}

A1

Scale Bar: $2 \mathrm{~mm}$ is

Sample Length Thickness

Top 1

Top 2

Top 3

Right 1

Right 2

Right 3

Bottom 1

Bottom 2

Bottom 3

Left 1

Left 2

Left 3

131

144

148

145

157

161

129

137

150

158

163

146
309.1

0.845

0.933

0.961

0.937

1.017

1.040

0.837

0.885

0.974

1.025

1.055

0.944

$0.954 \pm$

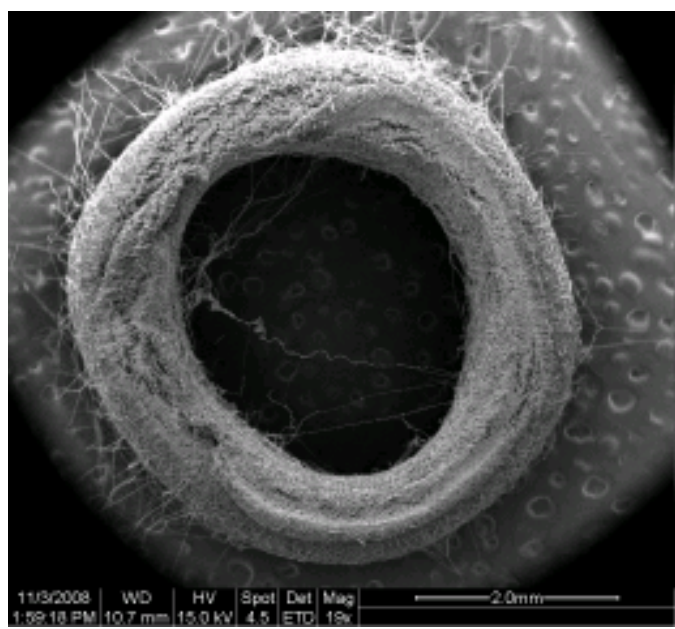

0.073

B1

Scale Bar: $2 \mathrm{~mm}$ is

313.0

Sample Length Size

Top 1

Top 2

Top 3

Right 1

Right 2

Right 3

Bottom 1

Bottom 2

Bottom 3

Left 1

Left 2

Left 3

$\begin{array}{ccl}\begin{array}{c}\text { Length } \\ -\end{array} & \text { Size } & \\ - & - \\ - & - \\ 153 & - \\ 141 & 0.975 \\ 140 & 0.898 \\ 153 & 0.892 \\ 151 & 0.977 \\ 139 & 0.963 \\ 136 & 0.891 \\ 147 & 0.868 \\ 125 & 0.940 \\ & 0.800 \\ & \mathbf{0 . 9 1 2} \pm\end{array}$

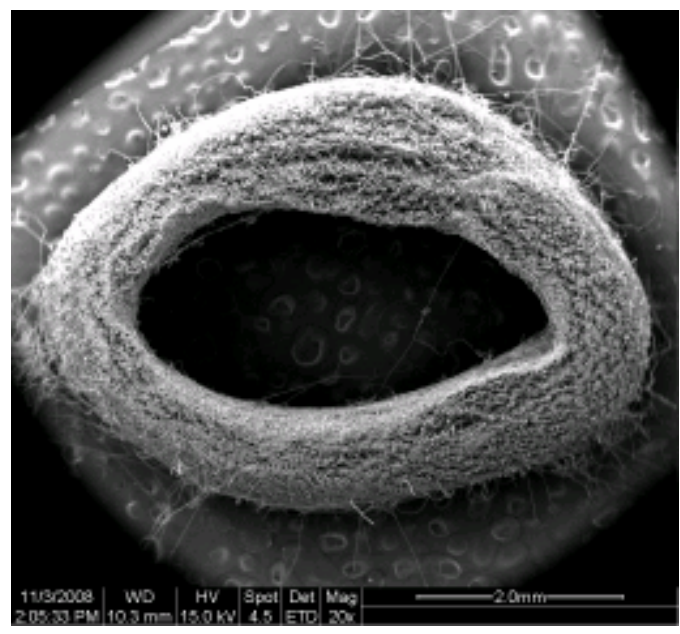

0.058 
B2

Scale Bar: $2 \mathrm{~mm}$ is

$\begin{array}{lr}\text { Sample } & \text { Length } \\ \text { Top 1 } & \text { Size } \\ \text { Top 2 } & 173 \\ \text { Top 3 } & 148 \\ \text { Right 1 } & 159 \\ \text { Right 2 } & 164 \\ \text { Right 3 } & 158 \\ \text { Bottom 1 } & 145 \\ \text { Bottom 2 } & 145 \\ \text { Bottom 3 } & 139 \\ \text { Left 1 } & 147 \\ \text { Left 2 } & 145 \\ \text { Left 3 } & 147\end{array}$

$\underline{311.7}$

0.960

1.108

0.952

1.019

1.052

1.015

0.929

0.932

0.894

0.941

0.928

0.941

$0.973 \pm$

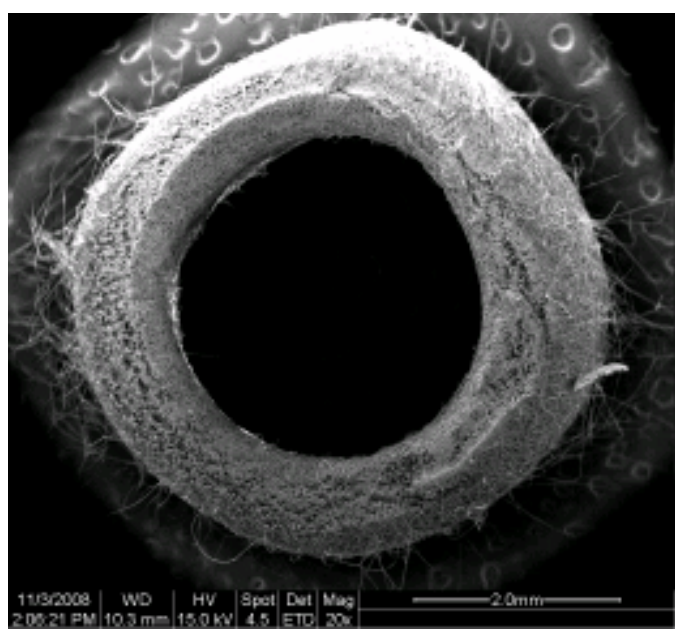

0.062

C1

Scale Bar: $2 \mathrm{~mm}$ is

313.0

Sample Length Size

Top 1

Top 2

Top 3

Right 1

Right 2

Right 3

Bottom 1

Bottom 2

Bottom 3

Left 1

Left 2

Left 3

Length
134
145
149
143
146
141
132
130
143
150
161
142

0.856

0.925

0.950

0.915

0.932

0.899

0.846

0.833

0.913

0.959

1.030

0.905

$0.913 \pm \quad 0.054$

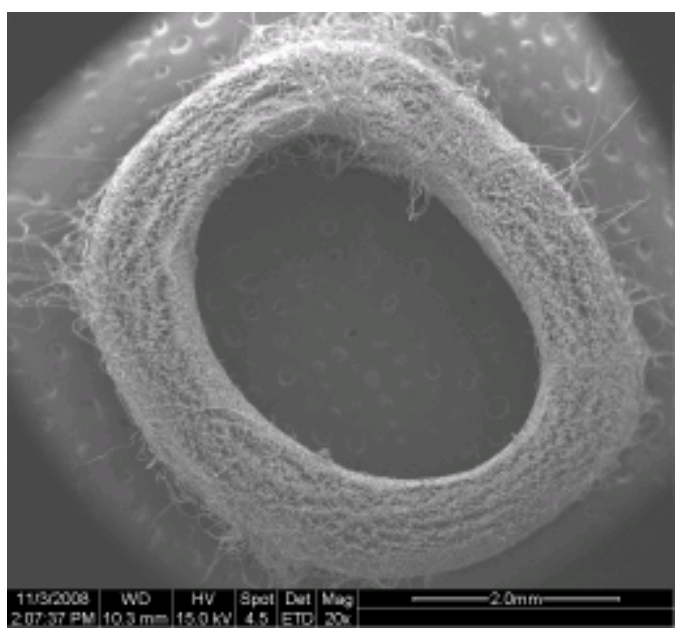




\section{C2}

Scale Bar: $2 \mathrm{~mm}$ is

Sample Length Size

Top 1

Top 2

Top 3

Right 1

Right 2

Right 3

Bottom 1

Bottom 2

Bottom 3

Left 1

Left 2

Left 3

144

135 $\underline{314.3}$

0.921

1.050

0.870

0.827

0.857

0.917

0.883

0.839

0.868

0.856

0.914

0.861

$0.889 \pm \quad 0.059$

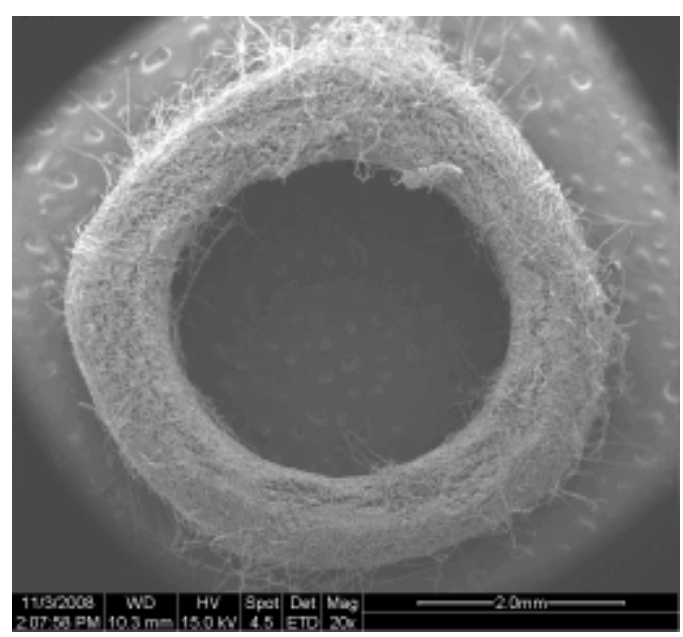

D1

Scale Bar: $2 \mathrm{~mm}$ is

\begin{tabular}{|c|c|}
\hline Sample & Length \\
\hline Top 1 & 145 \\
\hline Top 2 & 151 \\
\hline Top 3 & 143 \\
\hline Right 1 & 132 \\
\hline Right 2 & 151 \\
\hline Right 3 & 137 \\
\hline Bottom 1 & 140 \\
\hline Bottom 2 & 149 \\
\hline Bottom 3 & 144 \\
\hline Left 1 & 154 \\
\hline Left 2 & 160 \\
\hline Left 3 & 147 \\
\hline
\end{tabular}

$\underline{328.9}$

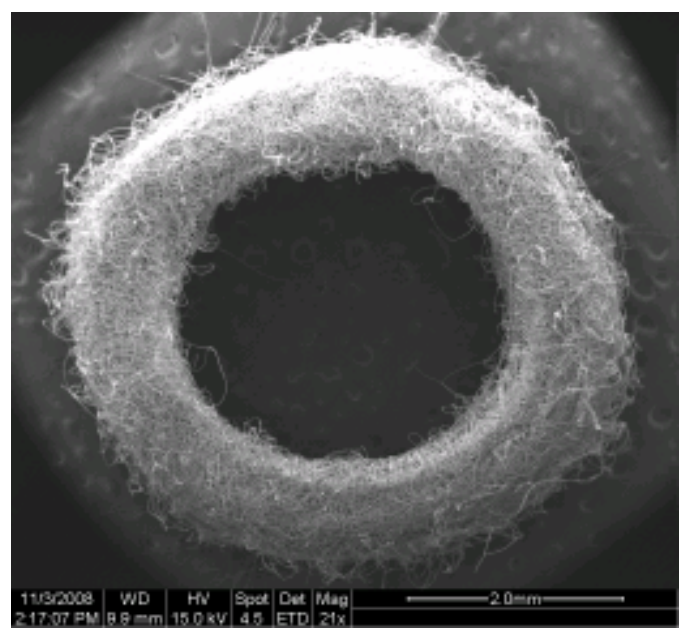

$0.889 \pm \quad 0.047$
0.975

0.892

0.884

0.920

0.869

0.803

0.917

0.833

0.852

0.909

0.875

0.936

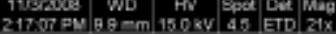

TOTAL AVERAGE:

$0.922+0.066$ 


\section{SPIN 6}

A1

Scale Bar: $2 \mathrm{~mm}$ is

328.9

Sample Length Thickness

Top 1

Top 2

Top 3

Right 1

Right 2

Right 3

Bottom 1

Bottom 2

Bottom 3

Left 1

Left 2

Left 3

143

123

121

110

112

113

112

110

113

118

135

134
0.870

0.751

0.738

0.682

0.686

0.684

0.667

0.689

0.716

0.819

0.813
0.672

$0.732 \pm$

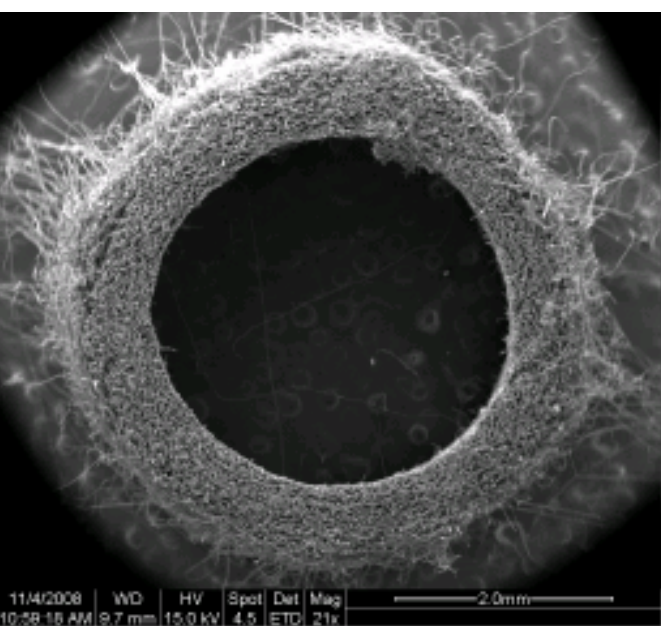

0.068

B1

Scale Bar: $2 \mathrm{~mm}$ is

324.9

Sample Length Size

Top 1

Top 2

Top 3

Right 1

Right 2

Right 3

Bottom 1

Bottom 2

Bottom 3

Left 1

Left 2

Left 3

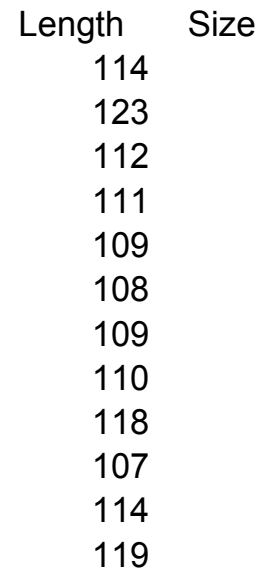

0.702

0.758

0.692

0.686

0.669

0.662

0.673

0.675

0.726

0.660

0.703

0.733

$0.695 \pm \quad 0.031$

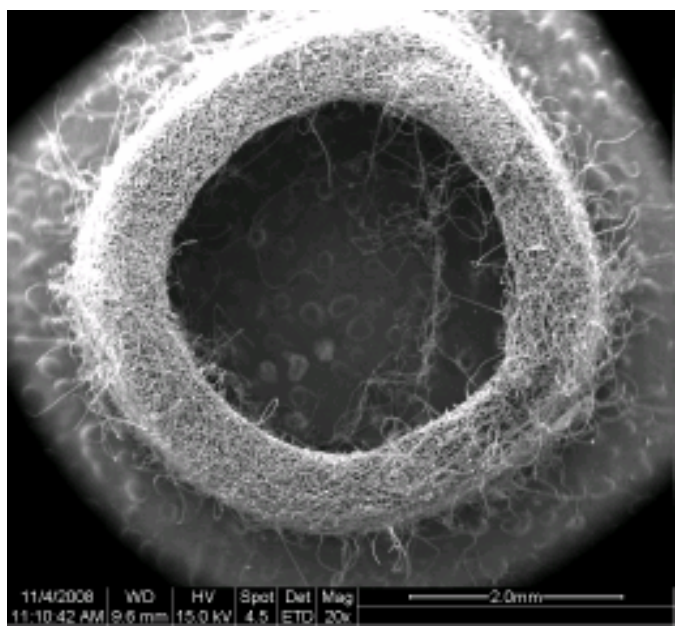




\section{B2}

Scale Bar: $2 \mathrm{~mm}$ is

324.9

Sample Length Size

Top $1 \quad 122$

Top $2 \quad 130$

Top 3

Right 1

Right 2

Right 3

Bottom 1

Bottom 2

Bottom 3

Left 1

Left 2

Left 3
126

122

131

114

111

113

119

119

120

124
0.752

0.797

0.776

0.753

0.805

0.701

0.686

0.695

0.731

0.731

0.740

0.761

$0.744 \pm$

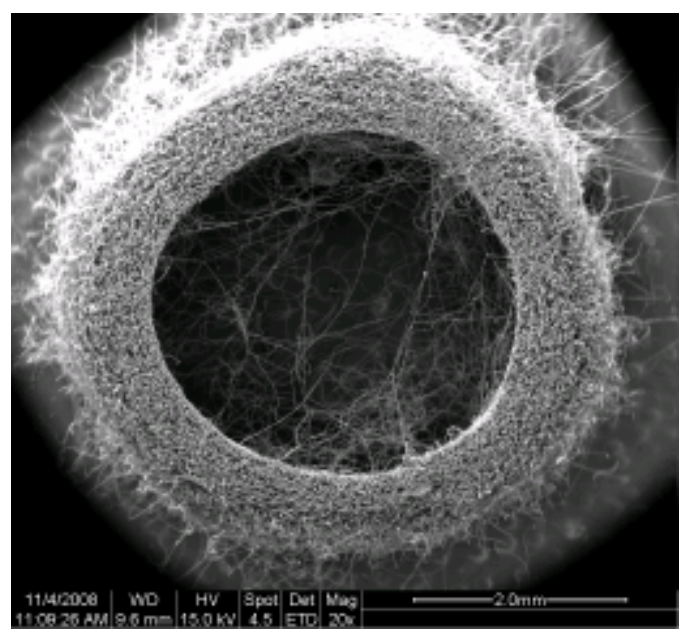

0.038

C1

Scale Bar: $2 \mathrm{~mm}$ is

320.9

Sample Length Size

Top 1

Top 2

Top 3

Right 1

Right 2

Right 3

Bottom 1

Bottom 2

Bottom 3

Left 1

Left 2

Left 3

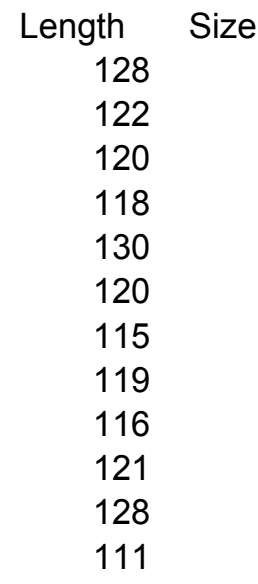

0.797

0.763

0.746

0.736

0.813

0.749

0.717

0.744

0.723

0.755

0.796

0.695

$0.753 \pm \quad 0.035$

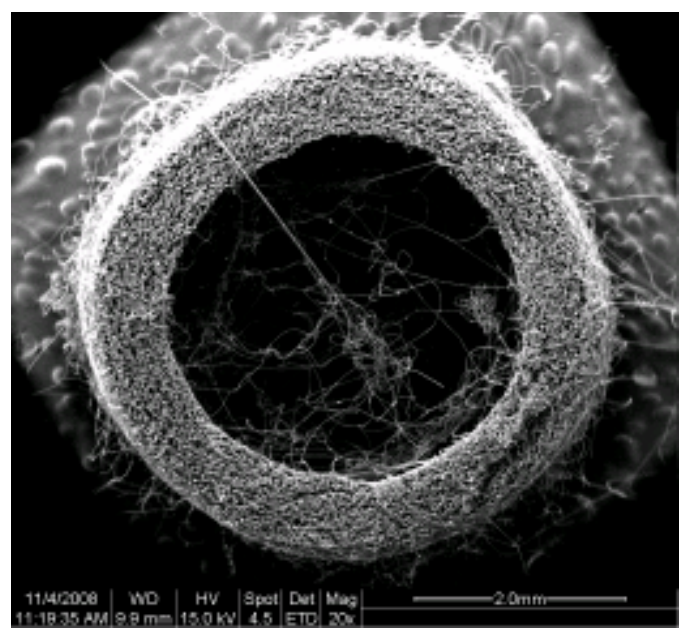




\section{C2}

BAD SAMPLE

$\begin{array}{lcc}\text { Sample } & \text { Length } & \text { Size } \\ \text { Top 1 } & - & - \\ \text { Top 2 } & - & - \\ \text { Top 3 } & - & - \\ \text { Right 1 } & - & - \\ \text { Right 2 } & - & - \\ \text { Right 3 } & - & - \\ \text { Bottom 1 } & - & - \\ \text { Bottom 2 } & - & - \\ \text { Bottom 3 } & - & - \\ \text { Left 1 } & - & - \\ \text { Left 2 } & - & - \\ \text { Left 3 } & - & -\end{array}$

\section{D1}

Scale Bar: $2 \mathrm{~mm}$ is

328.9

Sample Length Size

Top $1 \quad 115$

Top 2

Top 3

Right 1

Right 2

Right 3

Bottom 1

Bottom 2

Bottom 3

Left 1

Left 2

Left 3

\section{$0.698 \pm \quad 0.044$}

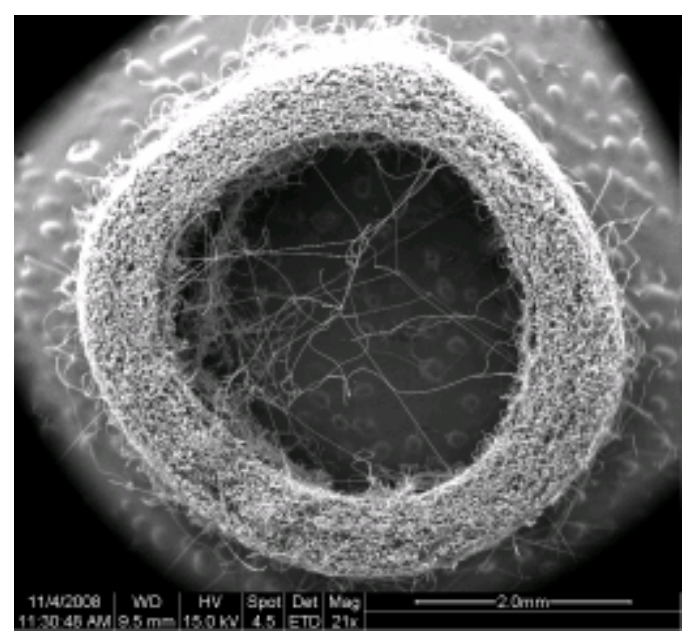




\section{SPIN 7}

A1

Scale Bar: $2 \mathrm{~mm}$ is

324.0

Sample Length Thickness

Top 1

Top 2

Top 3

Right 1

Right 2

Right 3

Bottom 1

Bottom 2

Bottom 3

Left 1

Left 2

Left 3

129

151

130

132

129

107

107

114

120

131

137

136
0.798

0.933

0.803

0.798

0.663

0.660

0.702

0.738

0.810

0.846

0.840
0.815

$0.784 \pm$

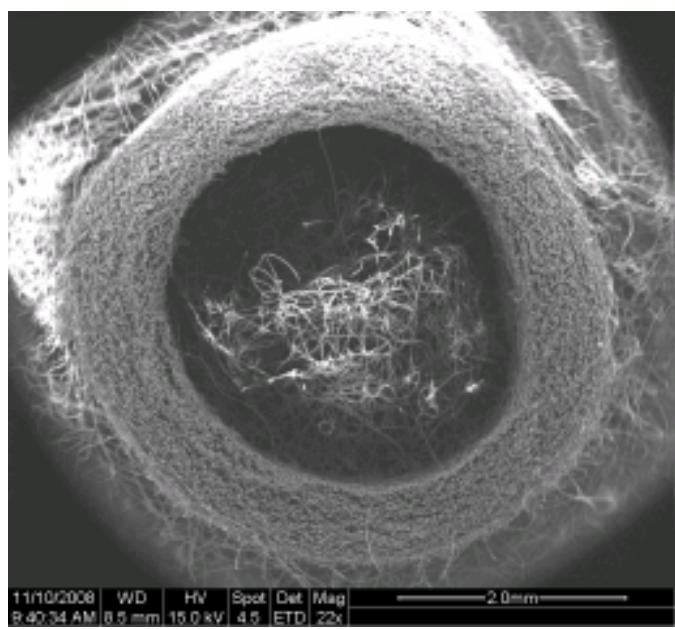

0.080

B1

Scale Bar: $2 \mathrm{~mm}$ is

336.8

Sample Length Size

Top 1

Top 2

Top 3 145

Right 1

Right 2

Right 3

Bottom 1

157

142

139

138

130

Bottom 2

128

139

Bottom 3

133

Left 1

Left 2

154

145

0.862

0.899

0.931

0.843

0.828

0.821

0.772

0.762

0.823

0.790

0.913

0.861

$0.842 \pm \quad 0.054$

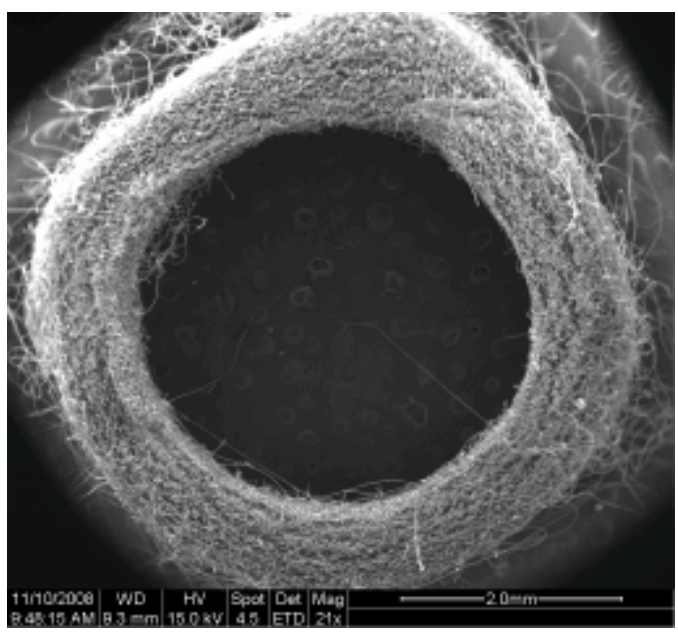

Left 3 
B2

Scale Bar: $2 m m$ is

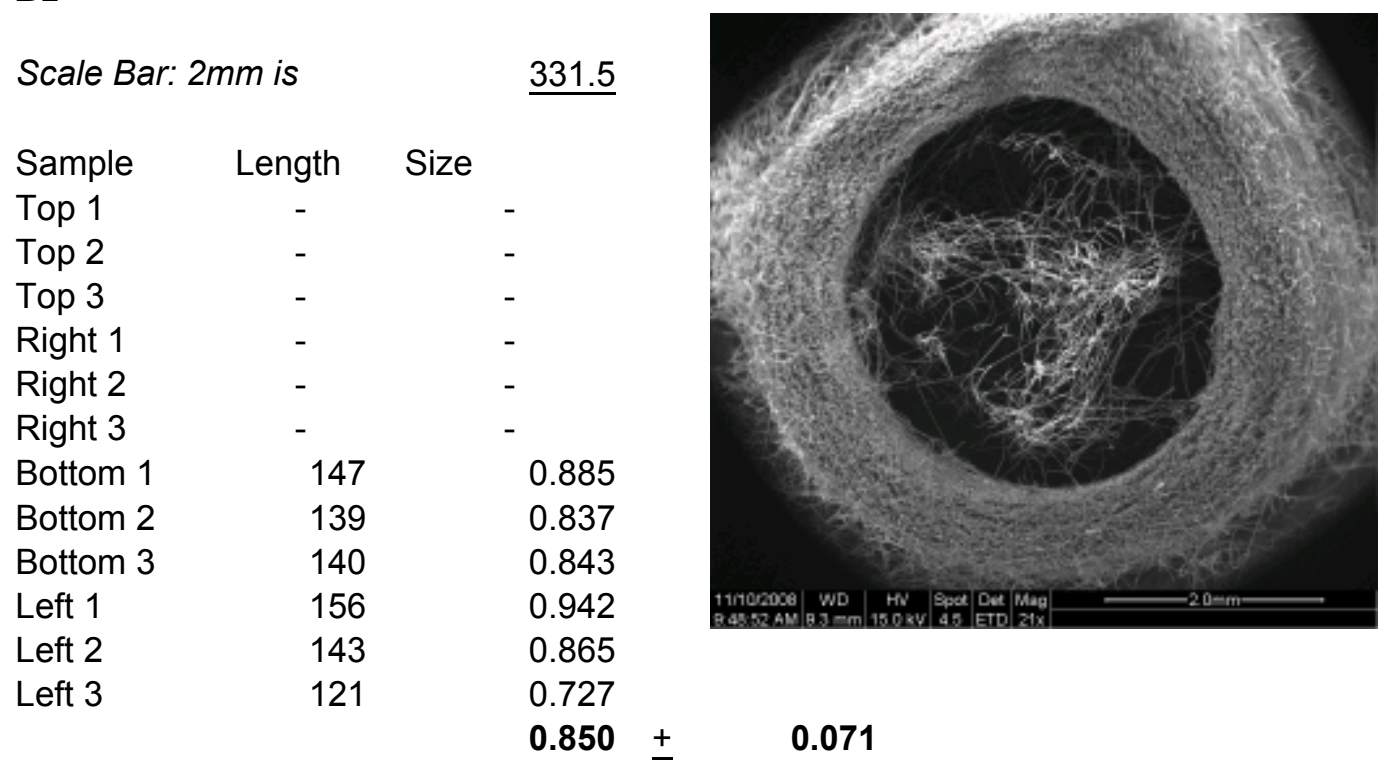

C1

Scale Bar: $2 \mathrm{~mm}$ is

$\underline{332.8}$

Sample Length Size

Top 1

Top 2

Top 3

Right 1

Right 2

Right 3

Bottom 1

Bottom 2

Bottom 3

Left 1

Left 2

Left 3

Length
123
132
113
123
135
129
116
115
122
125
141
119

331.5

0.071
0.740

0.795

0.678

0.740

0.809

0.772

0.696

0.690

0.735

0.752

0.847

0.713

$0.747 \pm \quad 0.051$

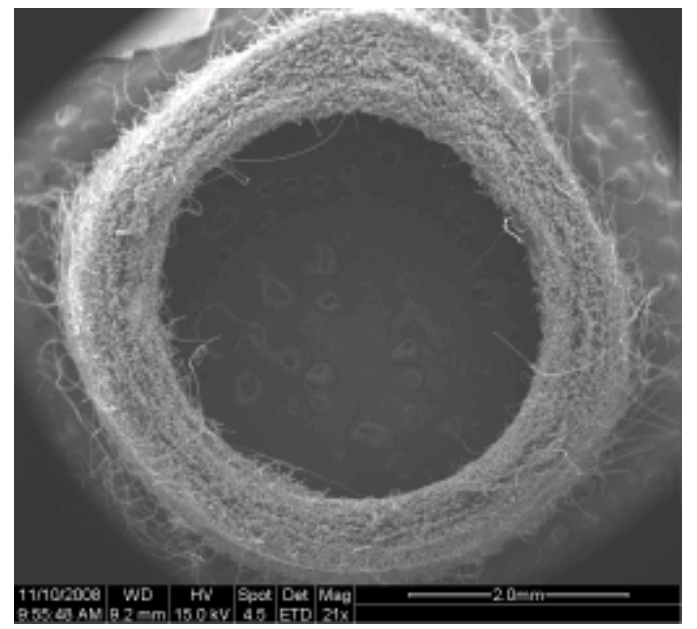




\section{C2}

Scale Bar: $2 \mathrm{~mm}$ is

Sample Length Size

Top 1

Top 2

Top 3

Right 1

Right 2

Right 3

Bottom 1

Bottom 2

Bottom 3

Left 1

Left 2

Left 3

\section{8}

0.763961

0.835403

0.752748

0.794992

0.844291

0.757255

0.773756

0.786068

0.773425

0.68479

0.754256

0.808507

$0.777 \pm$

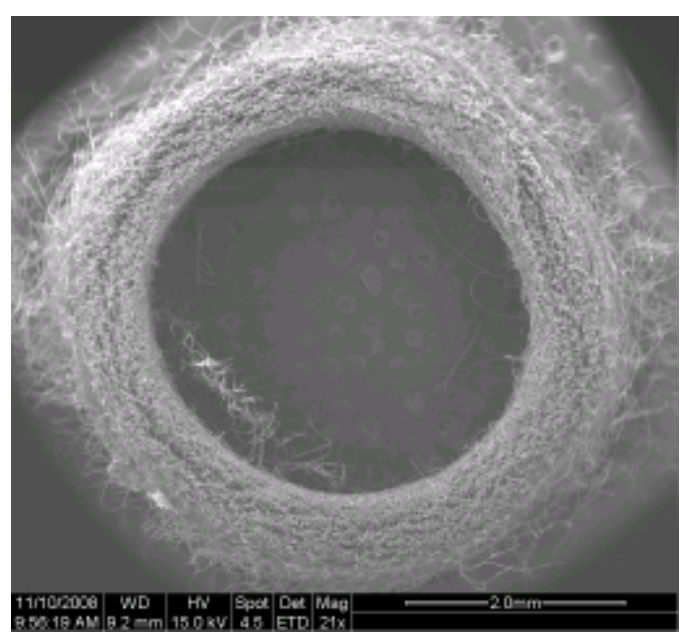

0.042

D1

Scale Bar: $2 \mathrm{~mm}$ is

$\underline{336.8}$

Sample Length Size

Top 1

Top 2

Top 3

Right 1

Right 2

Right 3

Bottom 1

Bottom 2

Bottom 3

Left 1

Left 2

Left 3

132

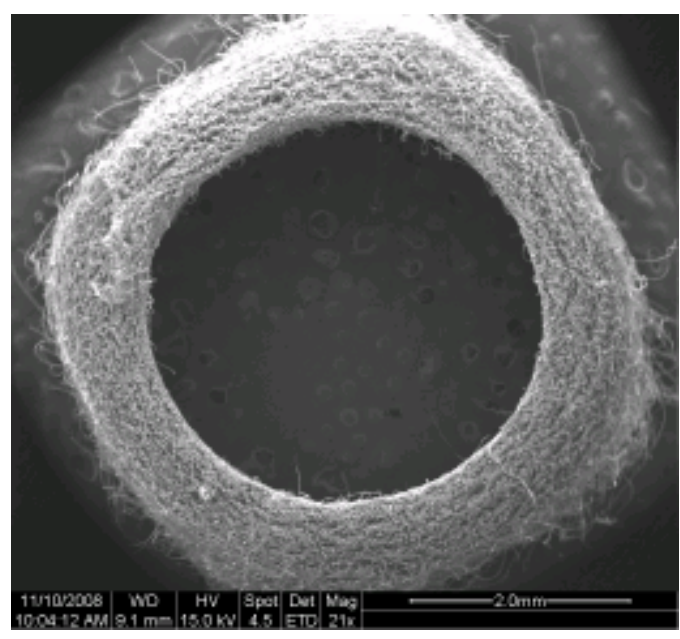

TOTAL AVERAGE:

$0.795+0.066627$




\section{SPIN 8}

A1

Scale Bar: $2 \mathrm{~mm}$ is

Sample Length Thickness

Top 1

Top 2

Top 3

Right 1

Right 2

Right 3

Bottom 1

Bottom 2

Bottom 3

Left 1

Left 2

Left 3

\section{-}

126
328.9

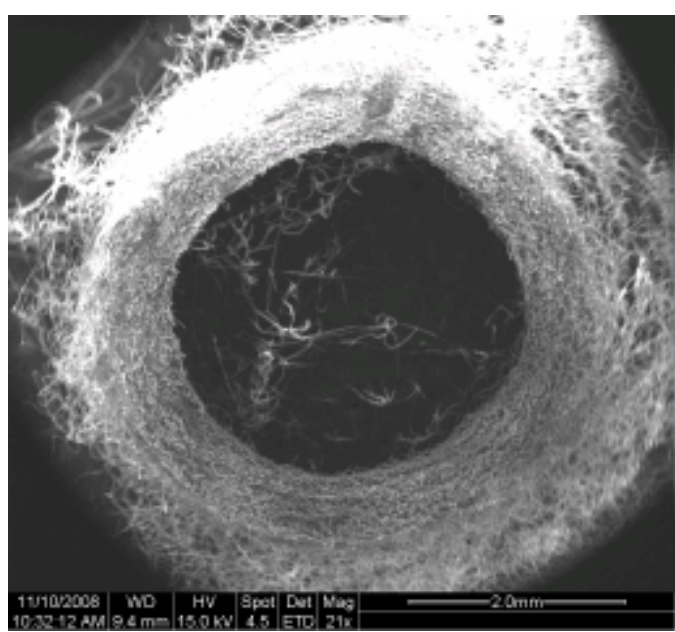

0.780

0.765

$0.717 \pm$

B1

Scale Bar: $2 \mathrm{~mm}$ is

317.0

Sample Length Size

Top 1

Top 2

Top 3

Right 1

Right 2

Right 3

Bottom 1

Bottom 2

Bottom 3

Left 1

Left 2

Left 3

157
159
144
147
134
136
129
116
131
155
151
156

0.988

1.003

0.908

0.926

0.844

0.860

0.817

0.734

0.823

0.978

0.951

0.984

$0.901 \pm$

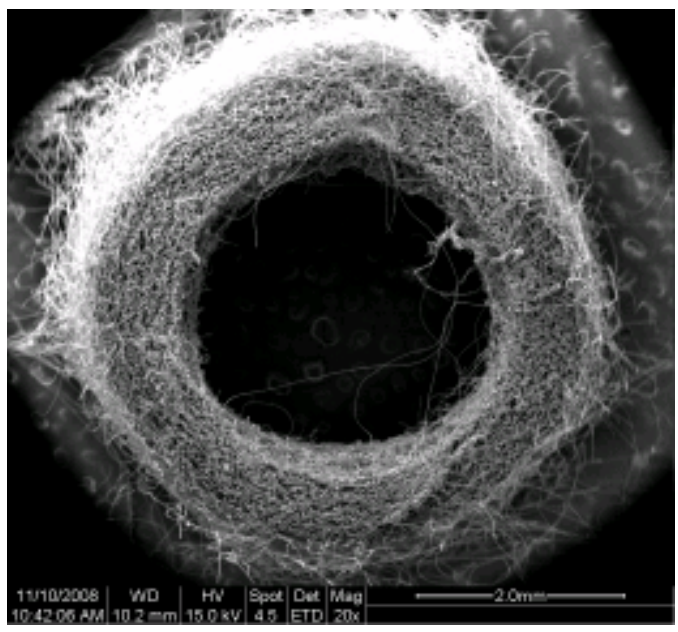

0.085 
B2

Scale Bar: $2 \mathrm{~mm}$ is

Sample Length Size

Top $1 \quad 132$

Top 2

Top $3 \quad 129$

Right 1

Right 2

Right 3

Bottom 1

Bottom 2

Bottom 3

Left 1

Left 2

Left 3
126

140

124

127

140

130

135

130

124 $\underline{315.7}$

0.838

0.811

0.819

0.795

0.889

0.788

0.804

0.887

0.824

0.857

0.824

0.789

$0.827 \pm$

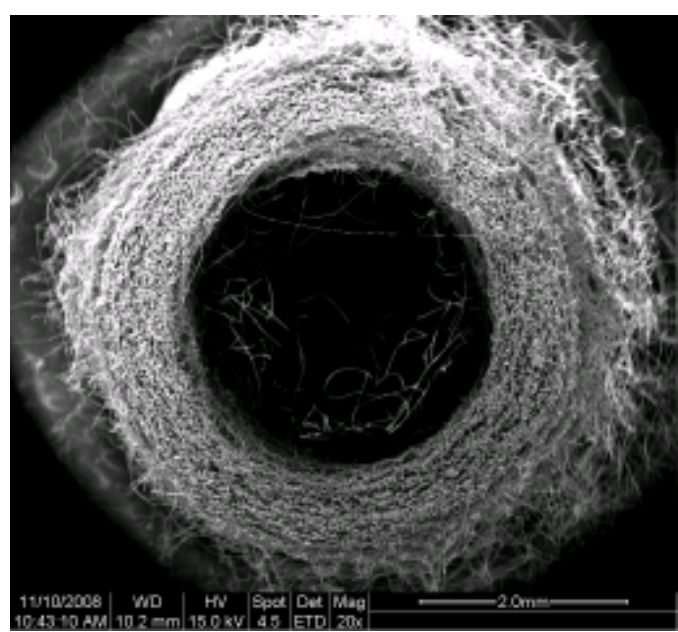

0.035

C1

Scale Bar: $2 \mathrm{~mm}$ is

323.6

Sample Length Size

Top 1

Top 2

Top 3

Right 1

Right 2

Right 3

Bottom 1

Bottom 2

Bottom 3

Left 1

Left 2

Left 3

\begin{tabular}{|c|c|}
\hline Length & \\
\hline 145 & \\
\hline 149 & \\
\hline 141 & \\
\hline 146 & \\
\hline 147 & \\
\hline 155 & \\
\hline 148 & \\
\hline 144 & \\
\hline 143 & \\
\hline 146 & \\
\hline 153 & \\
\hline 151 & \\
\hline
\end{tabular}

0.899

0.920

0.873

0.901

0.909

0.958

0.913

0.890

0.885

0.903

0.949

0.932

$0.911 \pm \quad 0.025$

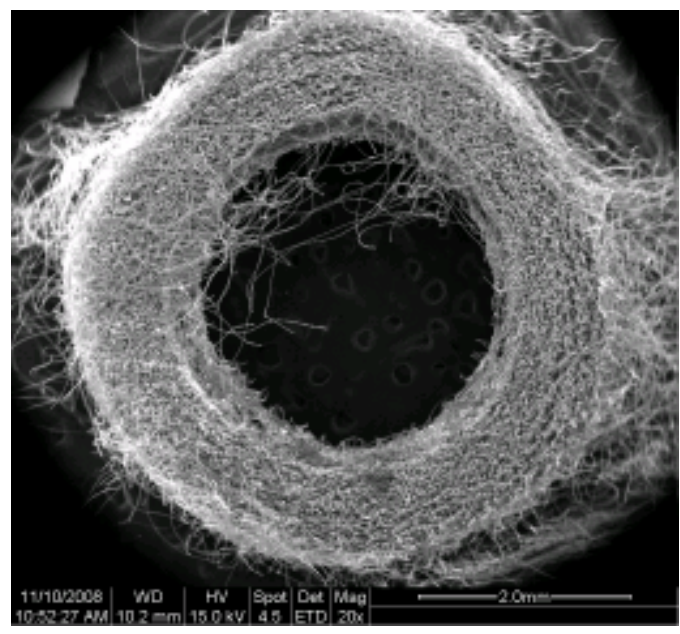




\section{C2}

Scale Bar: $2 \mathrm{~mm}$ is

Sample Length Size

Top 1

Top 2

Top 3

Right 1

Right 2

Right 3

Bottom 1

Bottom 2

Bottom 3

Left 1

Left 2

Left 3 $\underline{323.6}$

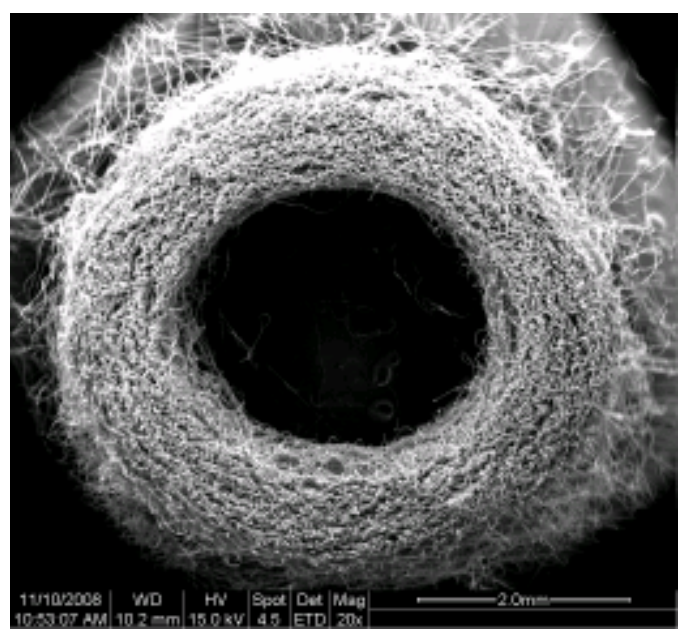

1.066519

1.026955

1.016515

$$
1.024 \pm \quad 0.102
$$

D1

Scale Bar: $2 \mathrm{~mm}$ is

$\begin{array}{lrr}\text { Sample } & \text { Length } & \text { Size } \\ \text { Top 1 } & 137 \\ \text { Top 2 } & 139 \\ \text { Top 3 } & 123 \\ \text { Right 1 } & 142 \\ \text { Right 2 } & 145 \\ \text { Right 3 } & 145 \\ \text { Bottom 1 } & 137 \\ \text { Bottom 2 } & 144 \\ \text { Bottom 3 } & 141 \\ \text { Left 1 } & 142 \\ \text { Left 2 } & 139 \\ \text { Left 3 } & 131\end{array}$

\section{$\underline{314.3}$}

0.875

0.886

0.786

0.902

0.926

0.923

0.872

0.916

0.898

0.906

0.882

0.832

$0.884 \pm \quad 0.040$

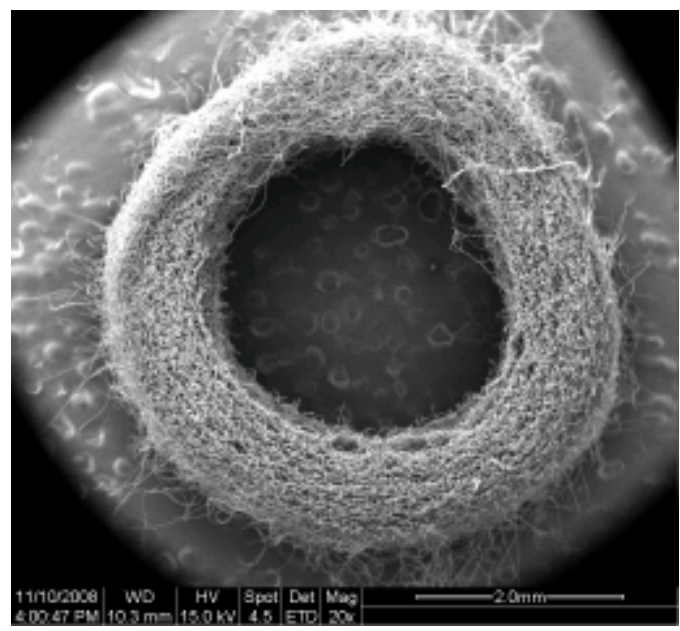

TOTAL AVERAGE:

$0.878 \pm 0.102846$ 


\section{SPIN 9}

A1

Scale Bar: $2 \mathrm{~mm}$ is

Sample Length Thickness

Top 1

Top 2

Top 3

Right 1

Right 2

Right 3

Bottom 1

Bottom 2

Bottom 3

Left 1

Left 2

Left 3

143

138

138

123

129

124

123

123

131

146

145

140
317.0

0.902

0.872

0.871

0.778

0.813

0.784

0.778

0.775

0.825

0.921

0.917

0.882

$0.843 \pm$

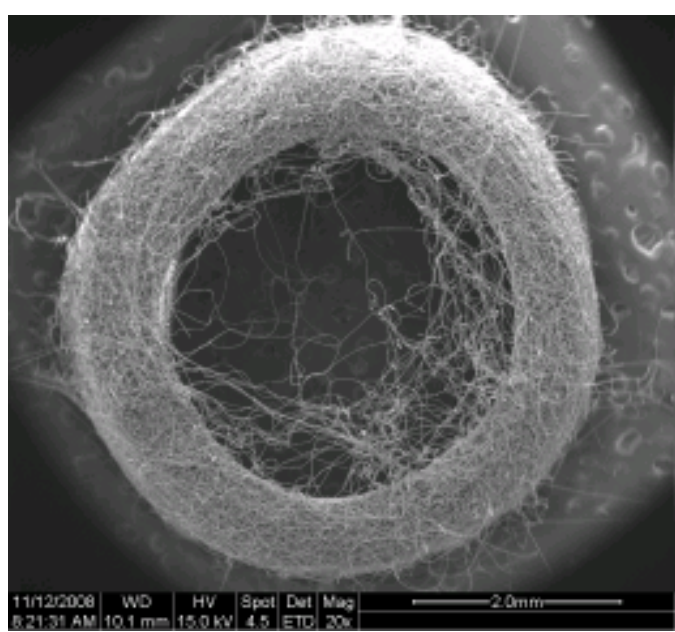

0.057

B1

Scale Bar: $2 m m$ is

326.2

Sample Length Size

Top 1

Top 2

Top 3

138

0.848

0.878

0.792

Right 1

Right 2

Right 3

Bottom 1

Bottom 2

Bottom 3

Left 1

Left 2

Left 3

$\begin{array}{ll}- & - \\ - & - \\ - & - \\ 130 & 0.794 \\ 131 & 0.805 \\ 130 & 0.798 \\ 138 & 0.844 \\ 144 & 0.884 \\ 139 & 0.855 \\ & \mathbf{0 . 8 3 3}\end{array}$

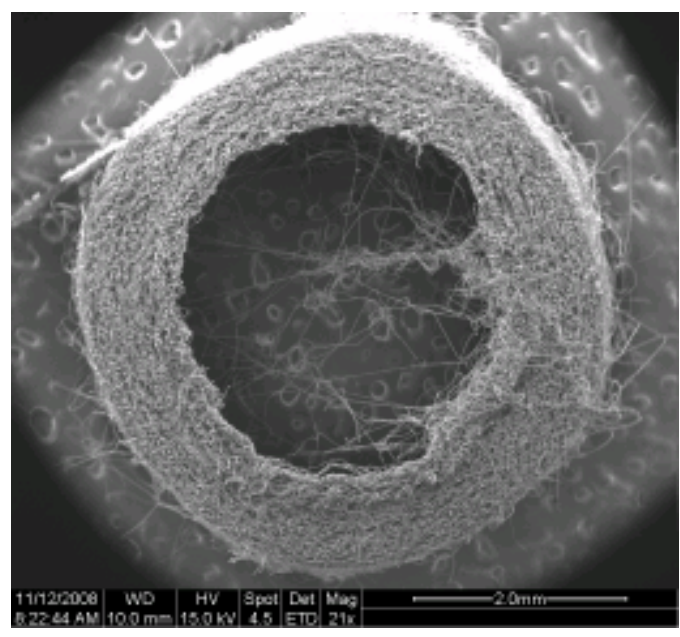

0.036 


\section{B2}

Scale Bar: $2 \mathrm{~mm}$ is

Sample Length Size

Top $1 \quad 137$

Top 2

Top 3

Right 1

Right 2

Right 3

Bottom 1

Bottom 2

Bottom 3

Left 1

Left 2

Left 3

145

145

155

150

149

126

127

123

131

132

139

143
318.3

0.860

0.913

0.976

0.941

0.937

0.794

0.798

0.772

0.822

0.832

0.876

0.898

$0.868 \pm$

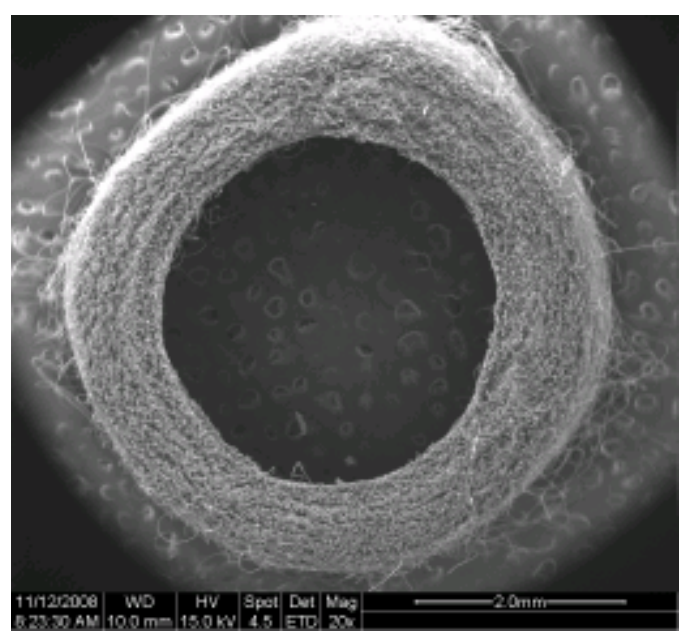

0.066

C1

Scale Bar: $2 \mathrm{~mm}$ is

320.9

Sample Length Size

Top 1

Top 2

Top 3

Right 1

Right 2

Right 3

Bottom 1

Bottom 2

Bottom 3

Left 1

Left 2

Left 3

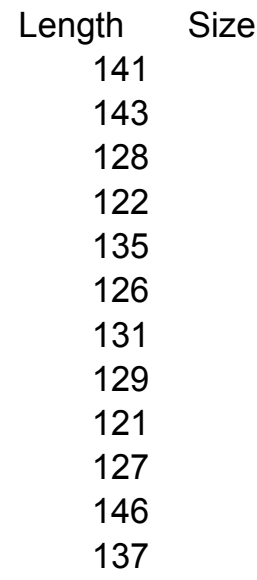

0.881

0.890

0.795

0.760

0.843

0.785

0.818

0.807

0.756

0.794

0.909

0.852

$0.824 \pm \quad 0.051$

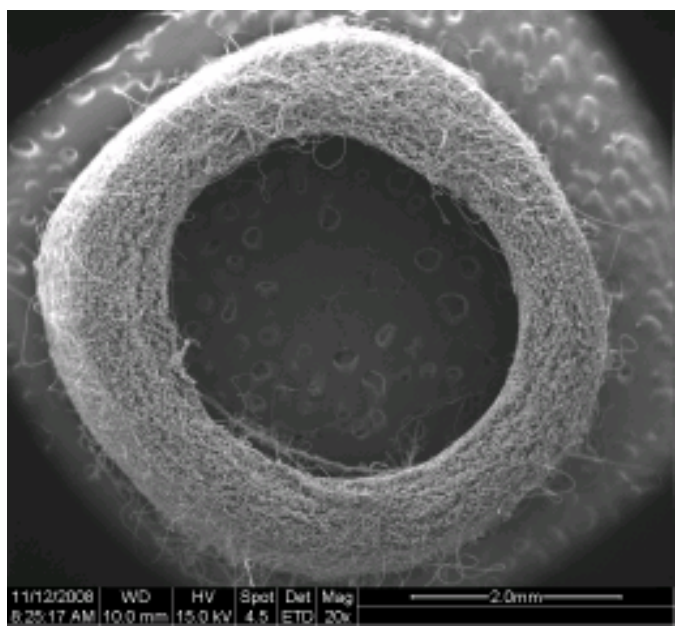




\section{C2}

Scale Bar: $2 \mathrm{~mm}$ is

Sample Length Size

Top 1

Top 2

Top 3

Right 1

Right 2

Right 3

Bottom 1

Bottom 2

Bottom 3

Left 1

Left 2

Left 3

127

145

129

132

132

134

127

129

128

125

146

147 $\underline{319.6}$

0.797

0.905

0.807

0.826

0.826

0.836

0.795

0.809

0.798

0.780

0.916

0.920

$0.835 \pm \quad 0.050$

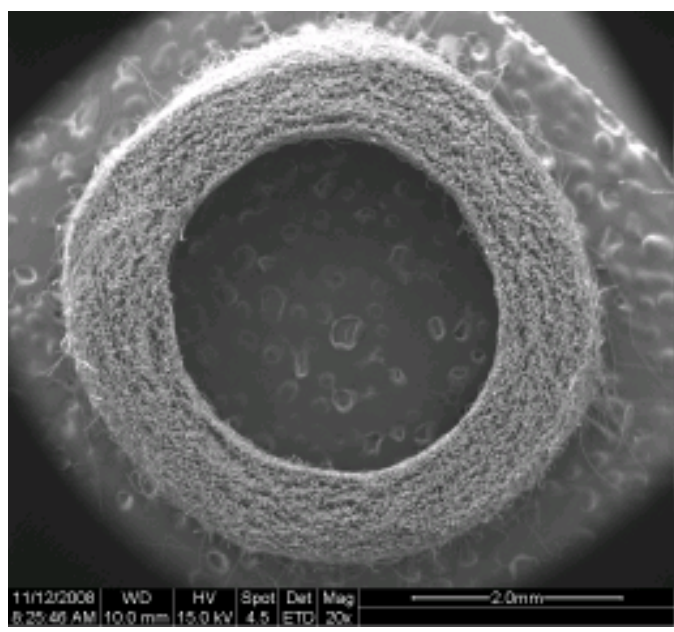

0.050

D1

Scale Bar: $2 \mathrm{~mm}$ is

$\underline{319.6}$

Sample Length Size

Top 1

Top 2

Top 3

Right 1

Right 2

Right 3

Bottom 1

Bottom 2

Bottom 3

Left 1

Left 2

Left 3

0.787

0.777

0.761

0.748

0.777

0.793

0.693

0.694

0.713

0.748

0.795

0.771

$0.755 \pm \quad 0.037$

TOTAL AVERAGE:

$0.826 \pm 0.06094$ 


\section{SPIN 10}

A1

Scale Bar: $2 \mathrm{~mm}$ is

332.8

Sample Length Thickness

Top 1

Top 2

Top 3

Right 1

Right 2

Right 3

Bottom 1

Bottom 2

Bottom 3

Left 1

Left 2

Left 3

140

165

134

130

136

134

134

140

141

126

136

132
0.839

0.993

0.808

0.779

0.818

0.803

0.806

0.839

0.845

0.760

0.818

0.792

$0.825 \pm$

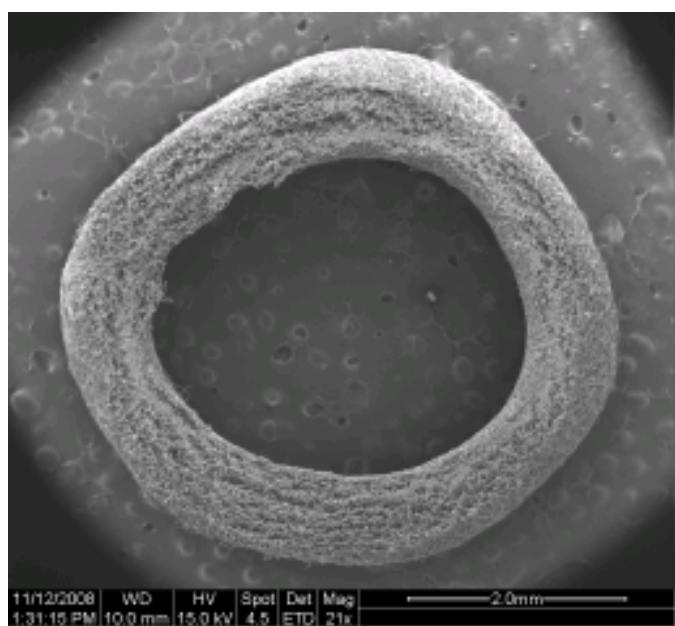

0.059

B1

Bad Sample

Sample Length Size

Top 1

Top 2

Top 3

Right 1

Right 2

Right 3

Bottom 1

Bottom 2

Bottom 3

Left 1

Left 2

Left 3 


\section{B2}

Scale Bar: $2 \mathrm{~mm}$ is

Sample Length Size

Top $1 \quad 125$

Top $2 \quad 138$

Top $3 \quad 125$

Right 1

Right 2

Right 3

Bottom 1

Bottom 2

Bottom 3

Left 1

Left 2

Left 3

C1

Scale Bar: $2 \mathrm{~mm}$ is

Sample Length Size

Top 1

Top 2

Top 3

Right 1

Right 2

Right 3

Bottom 1

Bottom 2

Bottom 3

Left 1

Left 2

Left 3

$\underline{319.6}$

319.6

0.780

0.862

0.780

0.942

0.987

0.796

0.737

0.827

0.818

0.830

0.780

0.752

$0.824 \pm$

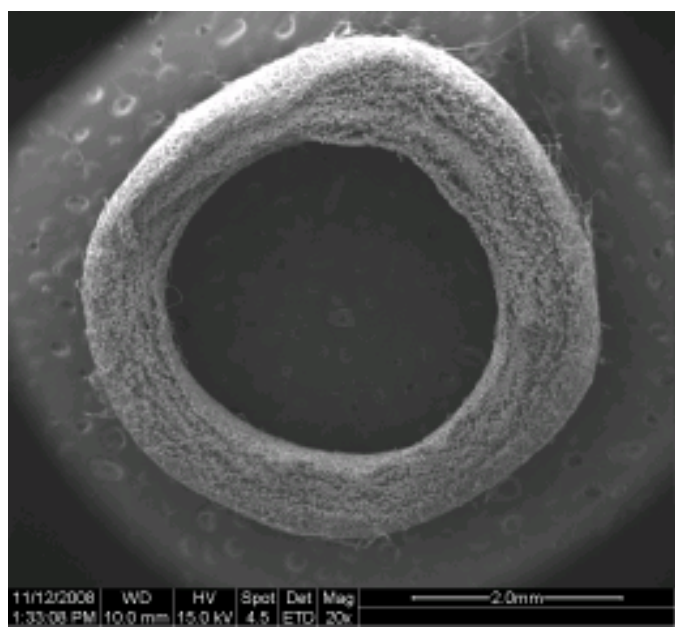

\subsection{5}

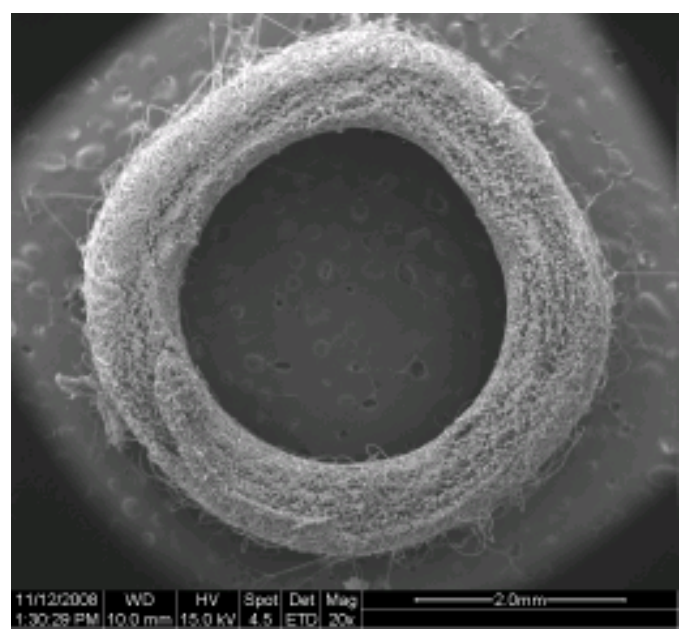

$0.851 \pm \quad 0.061$
0.832

0.886

0.931

0.794

0.919

0.864

0.850

0.926

0.830

0.721

0.843

0.812

130

142

149 


\section{C2}

Scale Bar: $2 \mathrm{~mm}$ is

Sample Length Size

Top 1

Top 2

Top 3

Right 1

Right 2

Right 3

Bottom 1

Bottom 2

Bottom 3

Left 1

Left 2

Left 3

129

142

144

135

154

146

135

136

138

136

151

135 $\underline{319.6}$

0.808

0.886

0.900

0.844

0.965

0.915

0.845

0.850

0.865

0.854

0.943

0.842

$0.876 \pm$

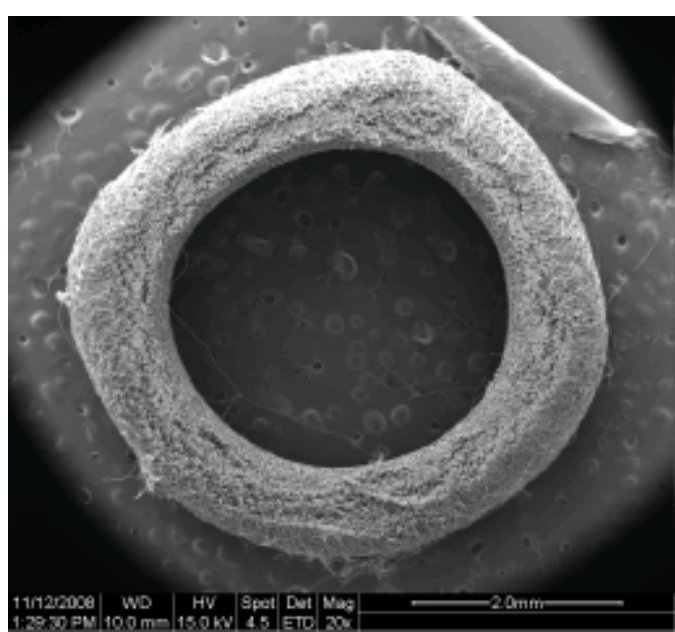

0.046

D1

Scale Bar: $2 \mathrm{~mm}$ is

320.9

Sample Length Size

Top 1

Top 2

Top 3

Right 1

Right 2

Right 3

Bottom 1

Bottom 2

Bottom 3

Left 1

Left 2

Left 3

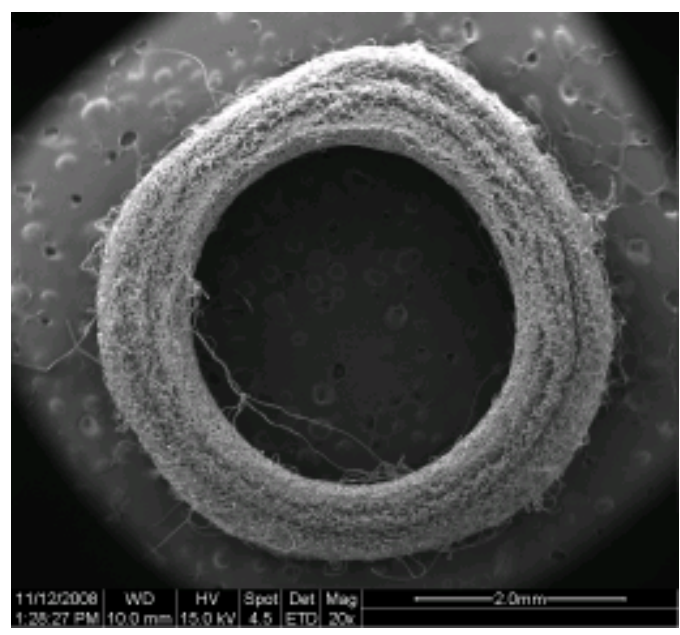

TOTAL AVERAGE:

$0.840 \pm 0.062941$ 


\section{SPIN 11}

A1

Scale Bar: $2 \mathrm{~mm}$ is

335.5

Sample Length Thickness

Top 1

Top 2

Top 3

Right 1

Right 2

Right 3

Bottom 1

Bottom 2

Bottom 3

Left 1

Left 2

Left 3

134

133

139

133

133

132

128

108

104

127

137

125
0.800

0.793

0.827

0.791

0.793

0.786

0.762

0.646

0.618

0.757

0.814

0.743
$0.761 \pm$

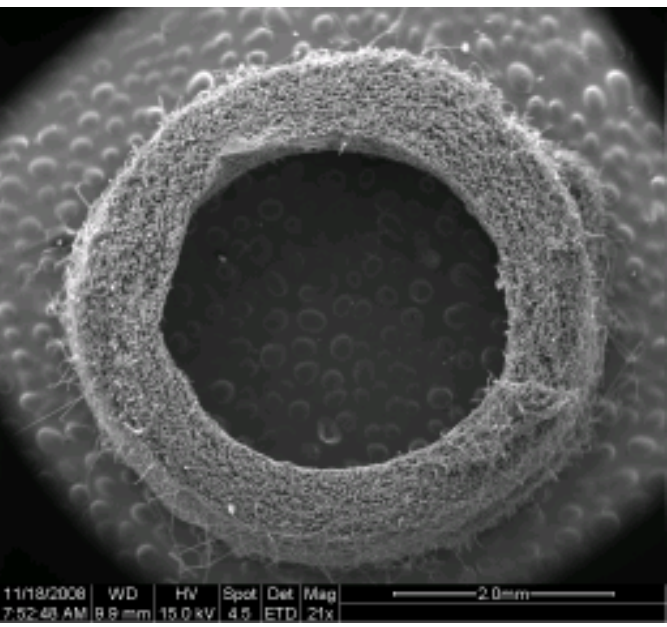

0.065

B1

Scale Bar: $2 \mathrm{~mm}$ is

319.6

Sample Length Size

Top 1

Top 2

Top 3

Right 1

Right 2

Right 3

Bottom 1

Bottom 2

Bottom 3

Left 1

Left 2

Left 3

Length
103
123
121
125
123
121
120
121
122
115
114
119
132

0.646

0.768

0.755

0.782

0.770

0.758

0.750

0.757

0.765

0.719

0.714

0.747

$0.744 \pm \quad 0.037$

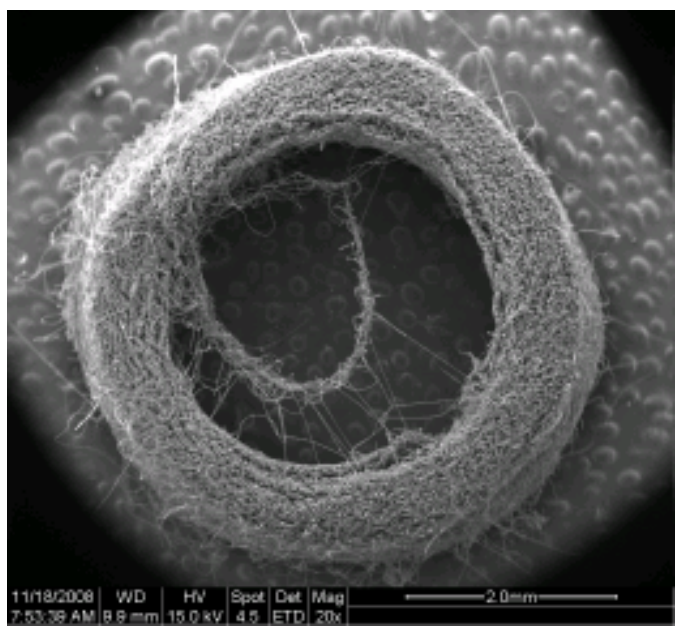




\section{B2}

Scale Bar: $2 m m$ is

Sample Length Size

Top 1

Top 2

Top 3

Right 1

Right 2

Right 3

Bottom 1

Bottom 2

Bottom 3

Left 1

Left 2

Left 3

-
-
154
145
145
118
106
99
103
116
139

320.9

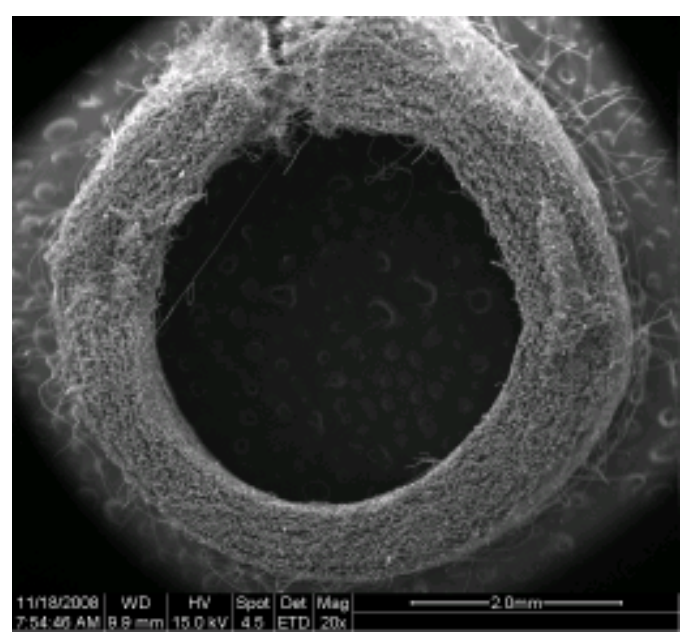

0.721

0.865

$0.778+$

0.130

C1

Scale Bar: $2 \mathrm{~mm}$ is

320.9

Sample Length Size

Top 1

Top 2

Top 3

Right 1

Right 2

Right 3

Bottom 1

Bottom 2

Bottom 3

Left 1

Left 2

Left 3

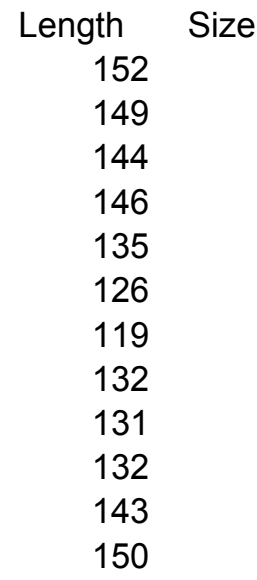

0.950

0.931

0.896

0.909

0.843

0.787

0.741

0.820

0.814

0.825

0.890

0.933

$0.862 \pm$

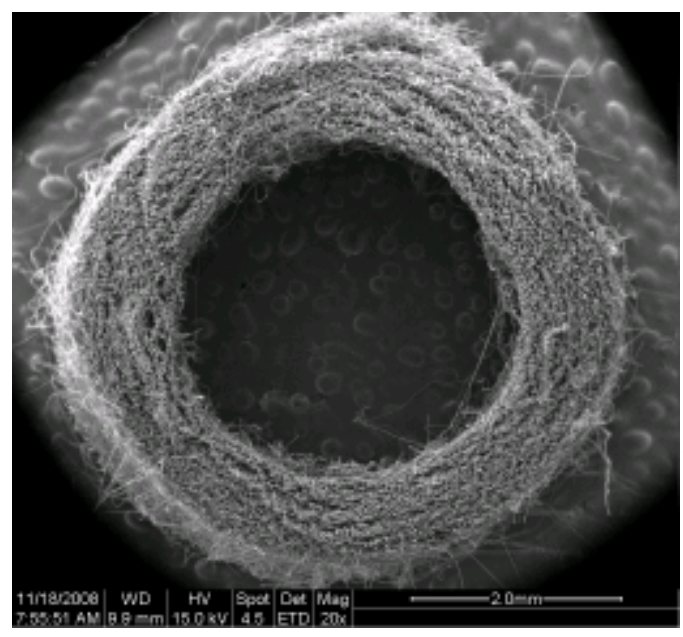

0.066 


\section{C2}

Bad Sample

$\begin{array}{lcc}\text { Sample } & \text { Length } & \text { Size } \\ \text { Top 1 } & - & - \\ \text { Top 2 } & - & - \\ \text { Top 3 } & - & - \\ \text { Right 1 } & - & - \\ \text { Right 2 } & - & - \\ \text { Right 3 } & - & - \\ \text { Bottom 1 } & - & - \\ \text { Bottom 2 } & - & - \\ \text { Bottom 3 } & - & - \\ \text { Left 1 } & - & - \\ \text { Left 2 } & - & - \\ \text { Left 3 } & - & -\end{array}$

\section{D1}

Scale Bar: $2 \mathrm{~mm}$ is

328.9

Sample Length Size

Top 1124

Top 2

Top 3

Right 1

Right 2

Right 3

Bottom 1

Bottom 2

Bottom 3

Left 1

Left 2

Left 3

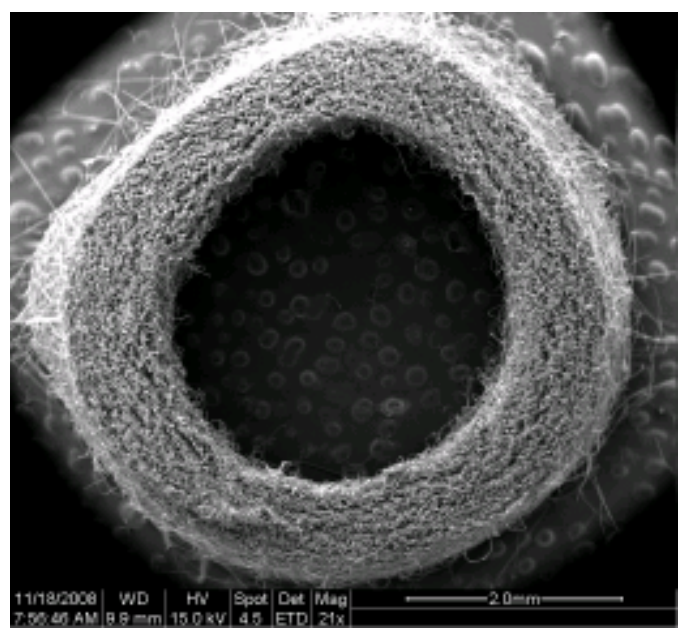

0.063 


\title{
APPENDIX G: TENSILE TESTING FIGURES
}

\author{
Introduction
}

In this appendix, tensile testing results for all consistency constructs are included. For an explanation of how each construct was sectioned and tested, see Chapter 4. The first graph presented for individual sections shows stress versus strain behavior for the entire tensile test. $\mathrm{Y}$-axis stress values are all in units of $\mathrm{MPa}$, while $\mathrm{x}$-axis strain values are unitless $(\mathrm{mm} / \mathrm{mm})$. The second graph shows linear stress behavior, in which the slope of the best-fit linear line represents Young's modulus according to Hooke's Law. Microsoft Excel was used to generate all figures and equations. 


\section{Spin 1}

Section AB: Longitudinal Test
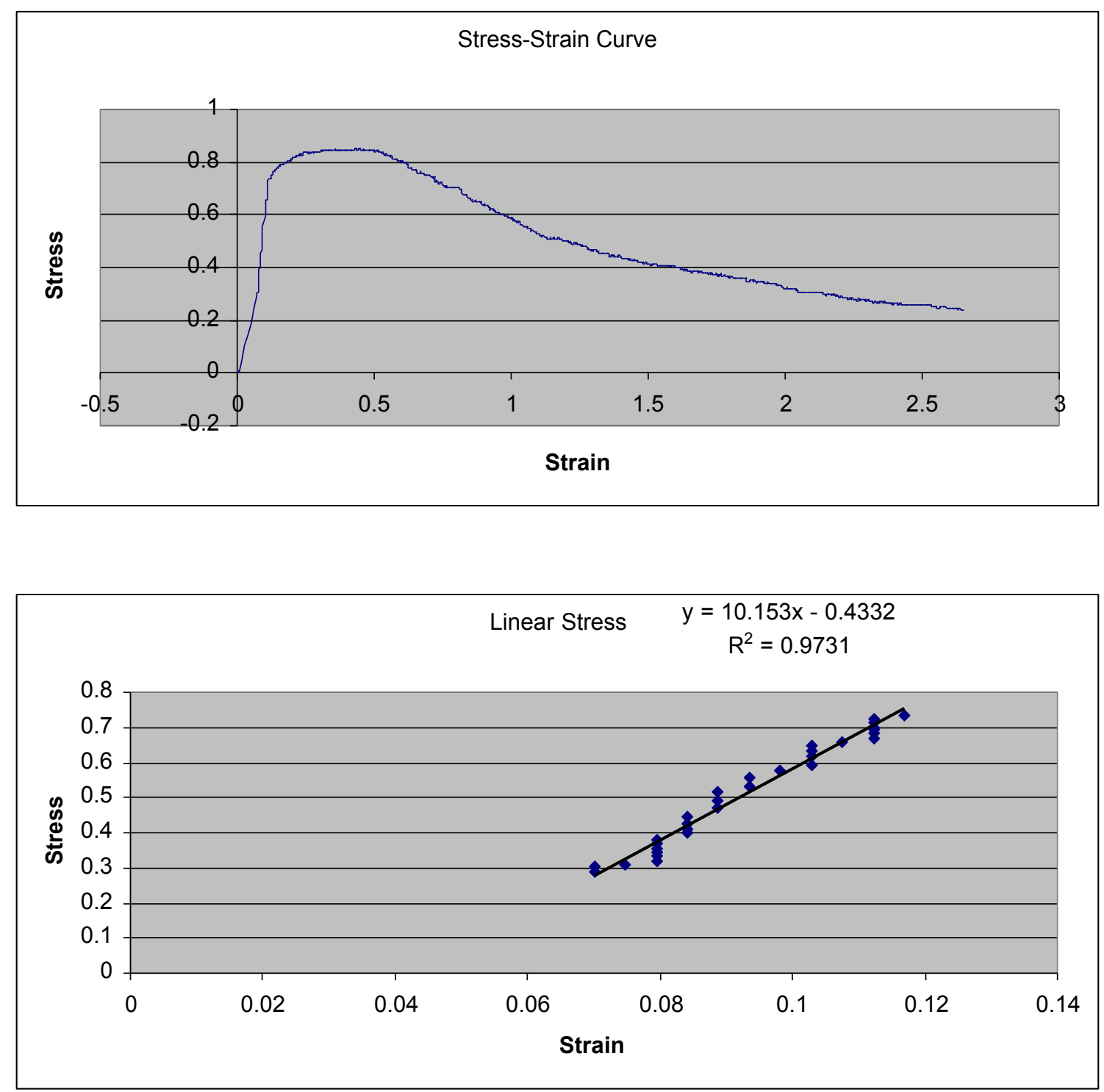


\section{Spin 1}

Section AB: Radial Test
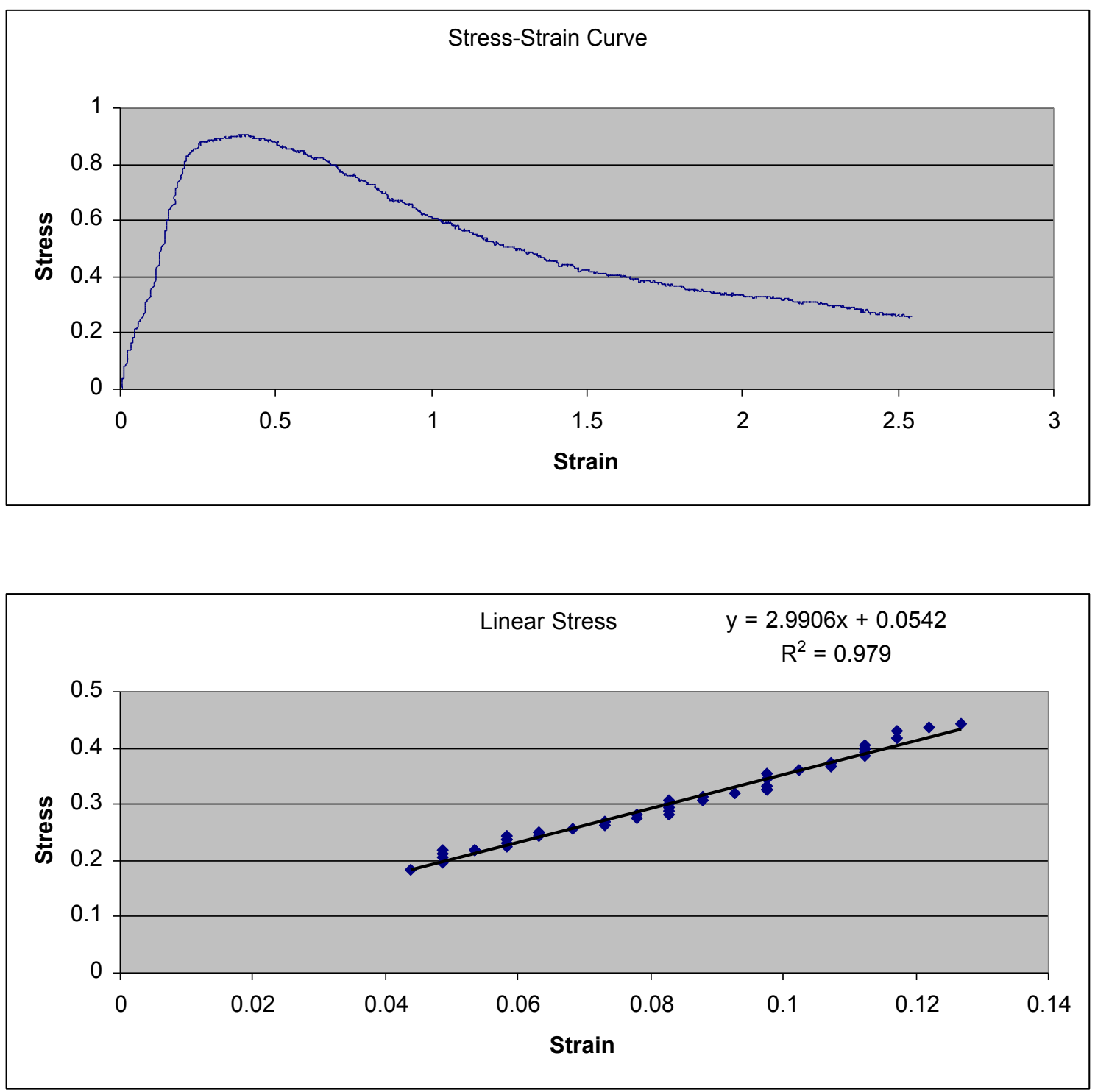


\section{Spin 1}

Section BC: Longitudinal Test
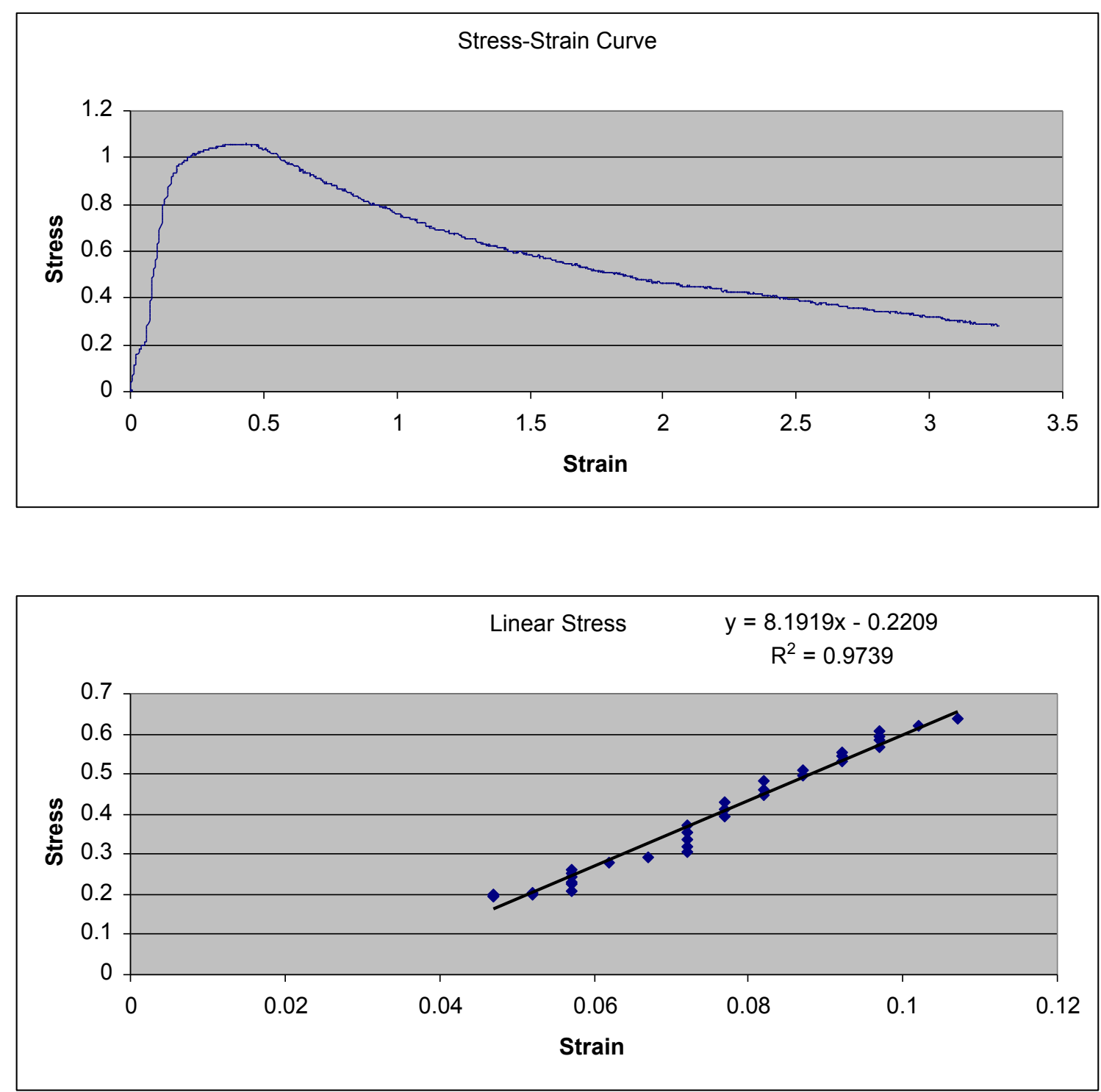


\section{Spin 1}

Section BC: Radial Test
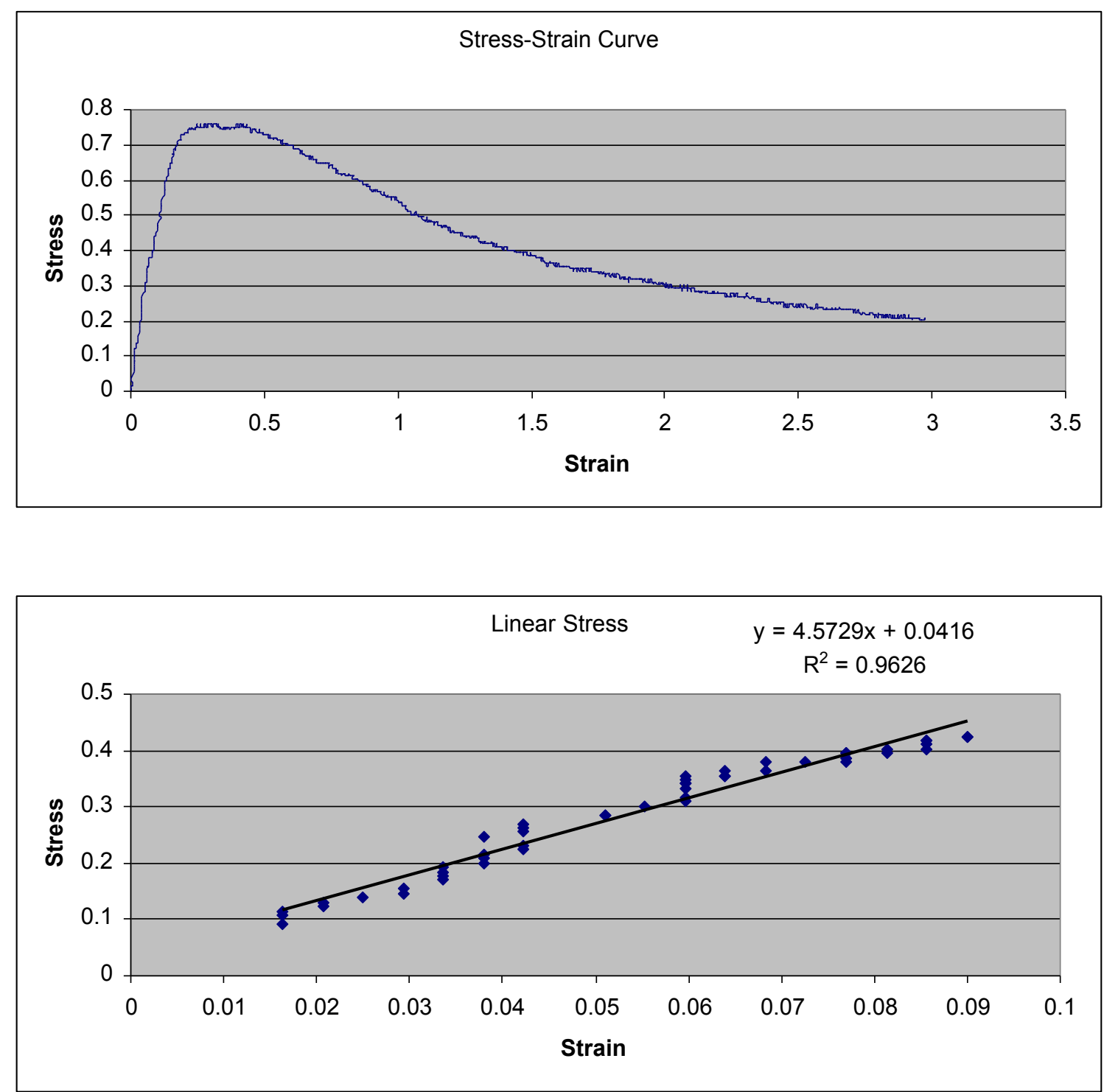


\section{Spin 1}

Section CD: Longitudinal Test
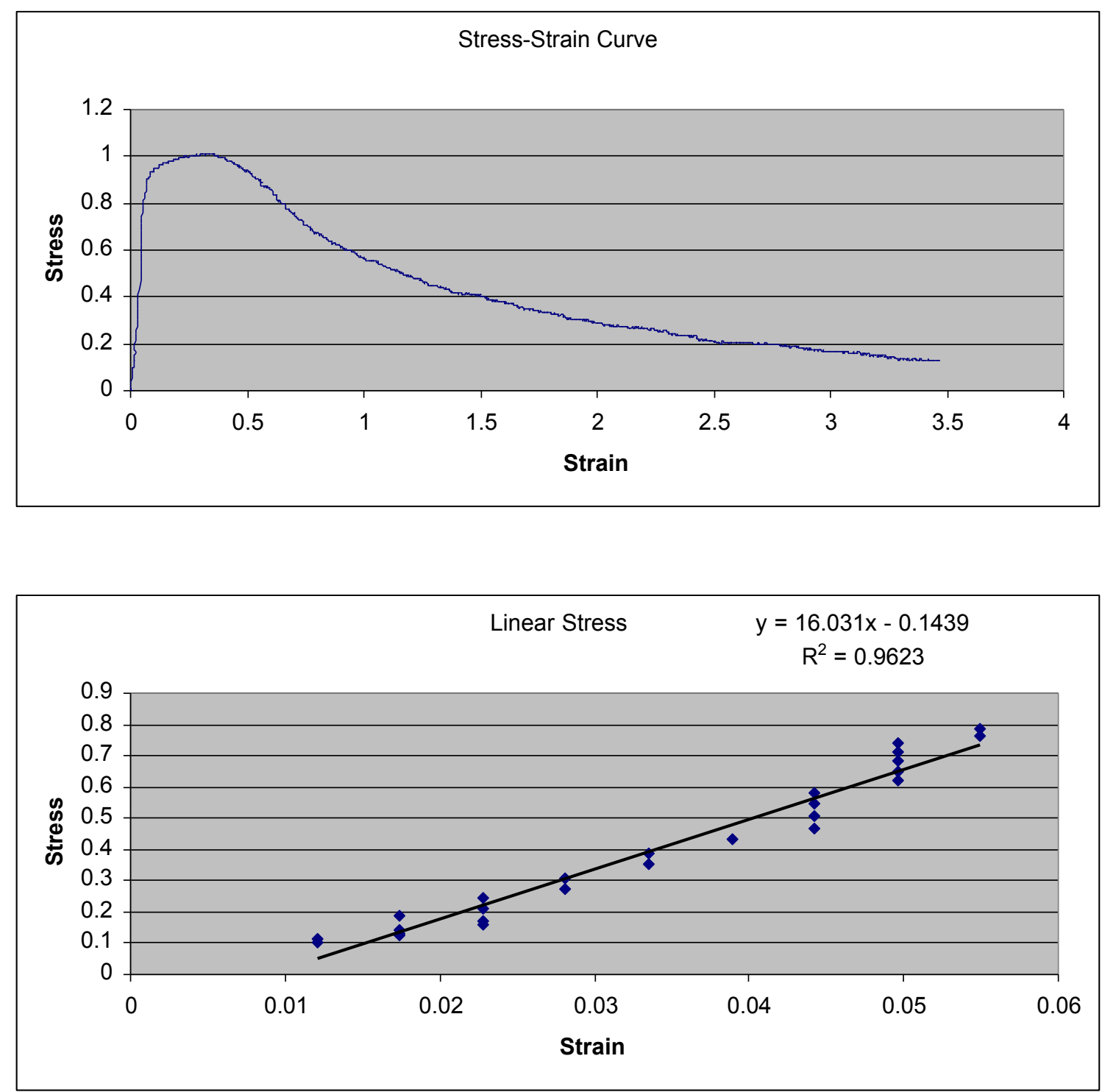


\section{Spin 1}

Section CD: Radial Test
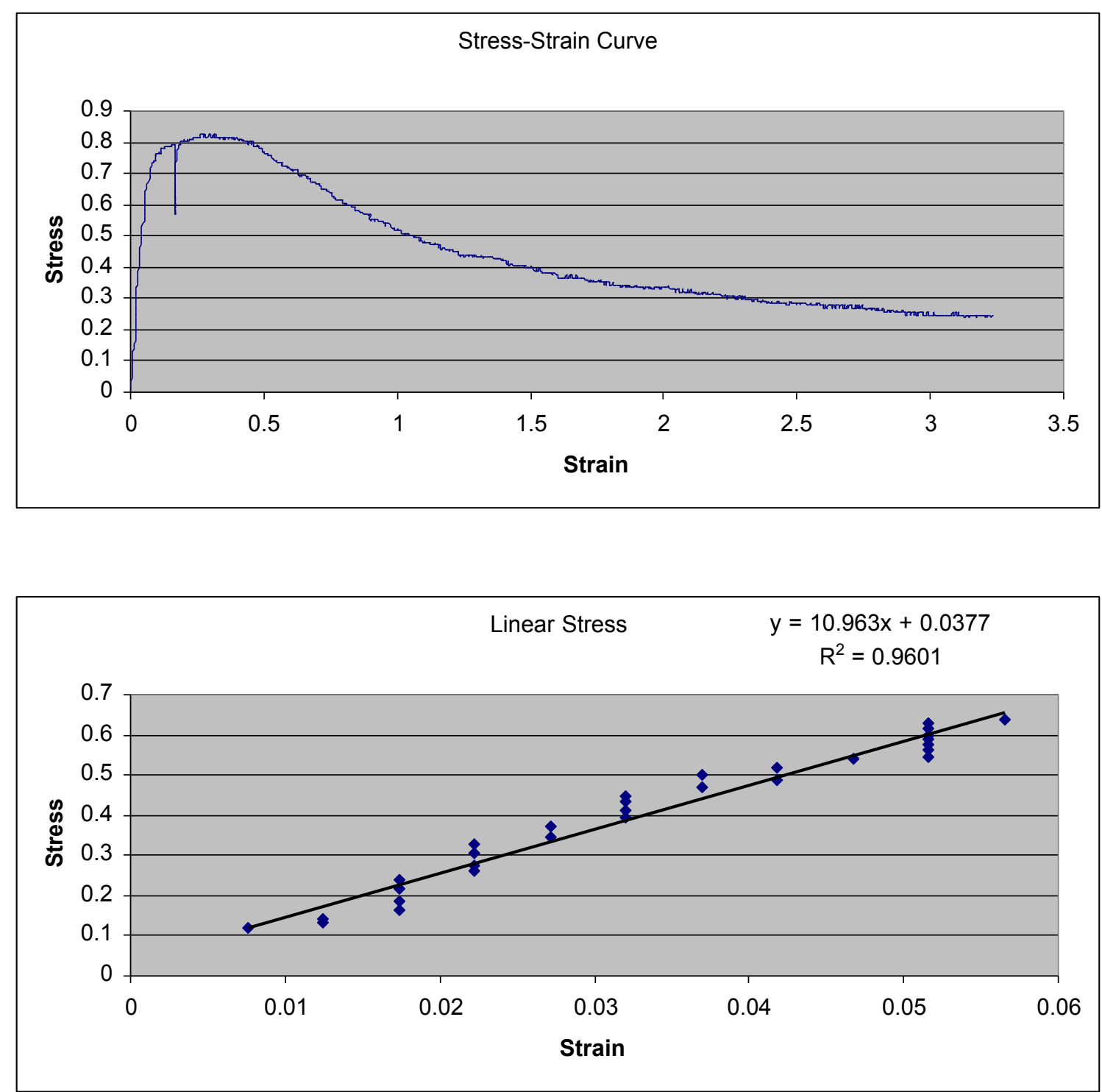


\section{Spin 2}

Section AB: Longitudinal Test
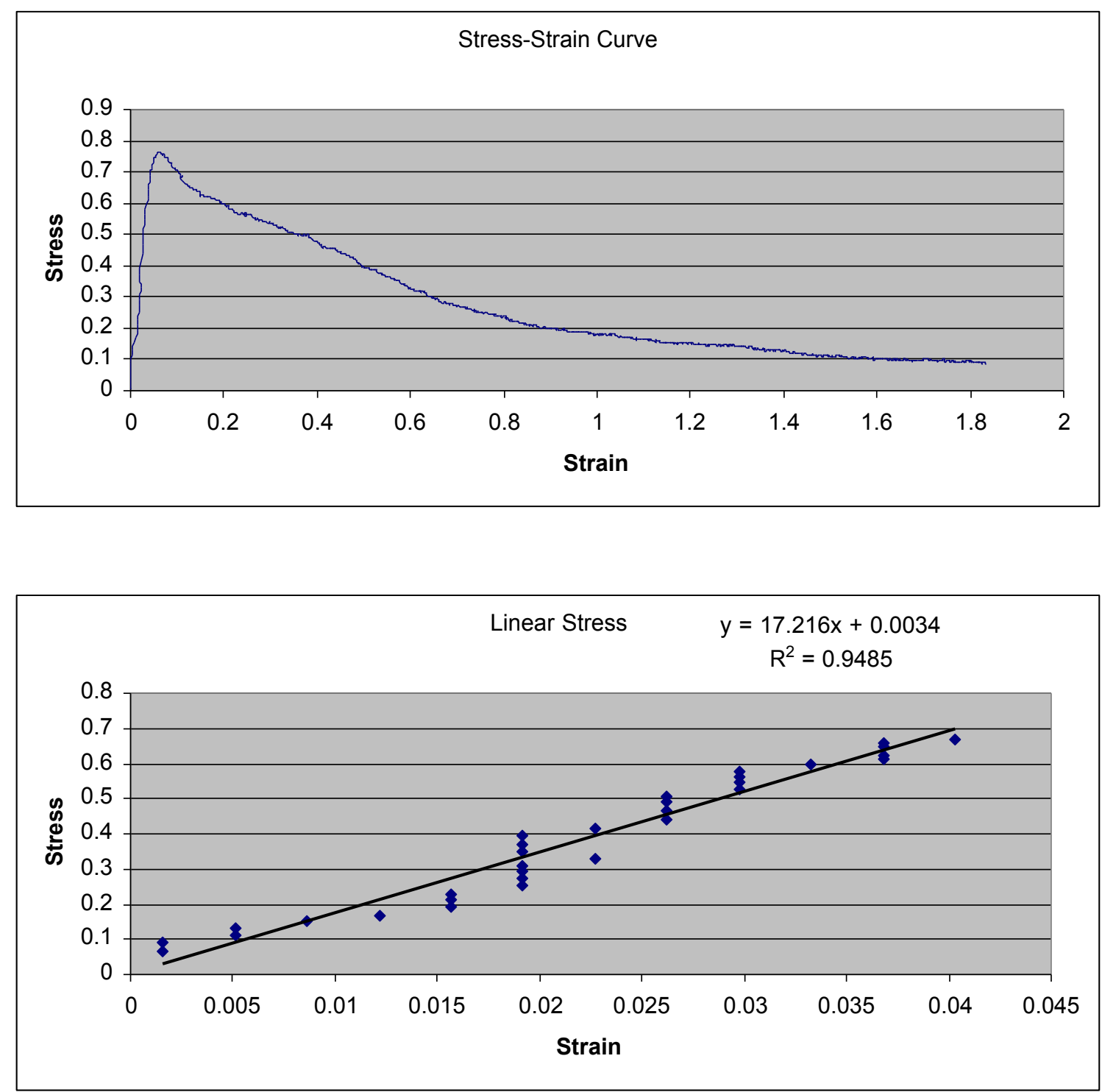
Spin 2

Section AB: Radial Test
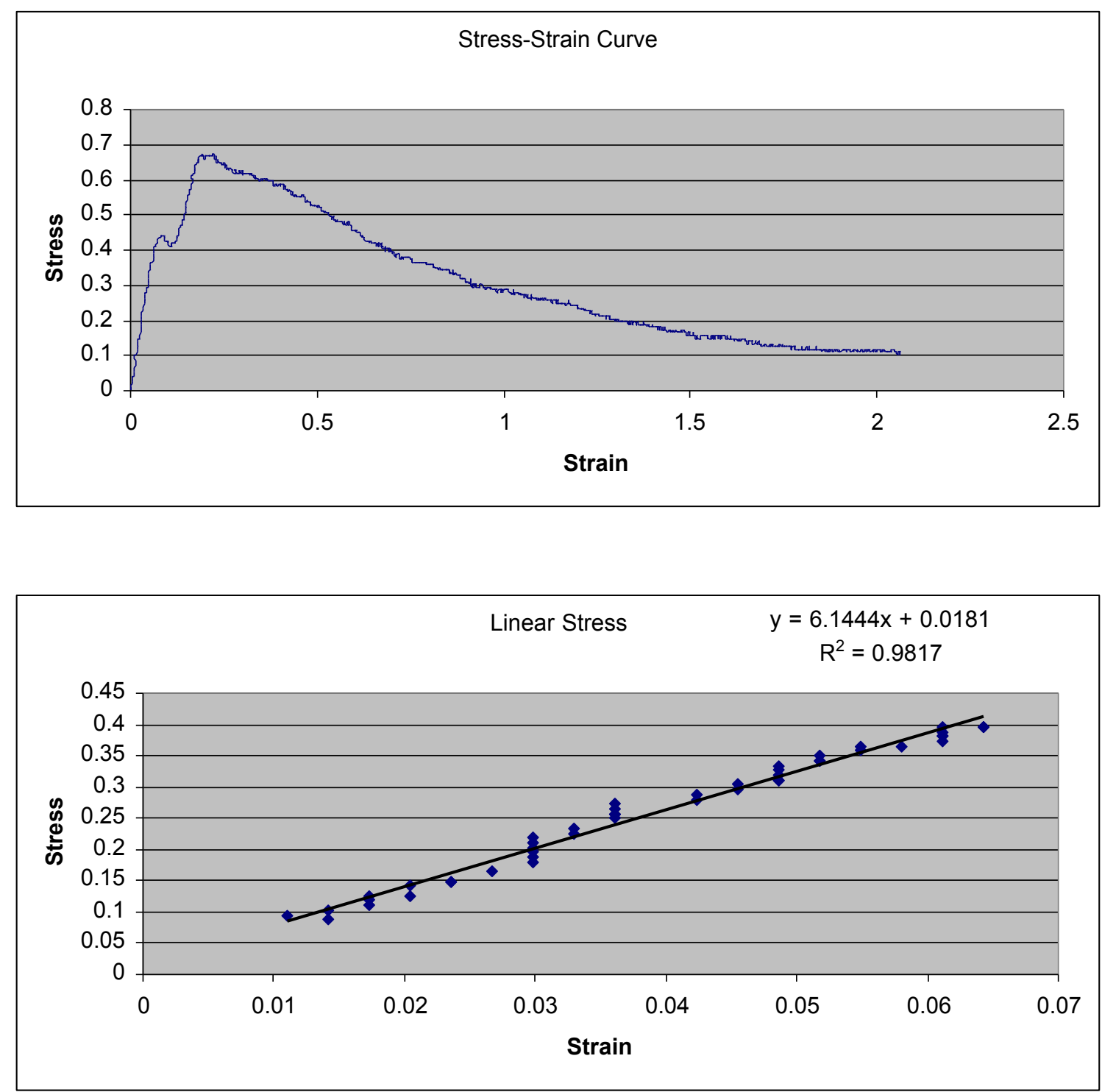
Spin 2

Section BC: Longitudinal Test
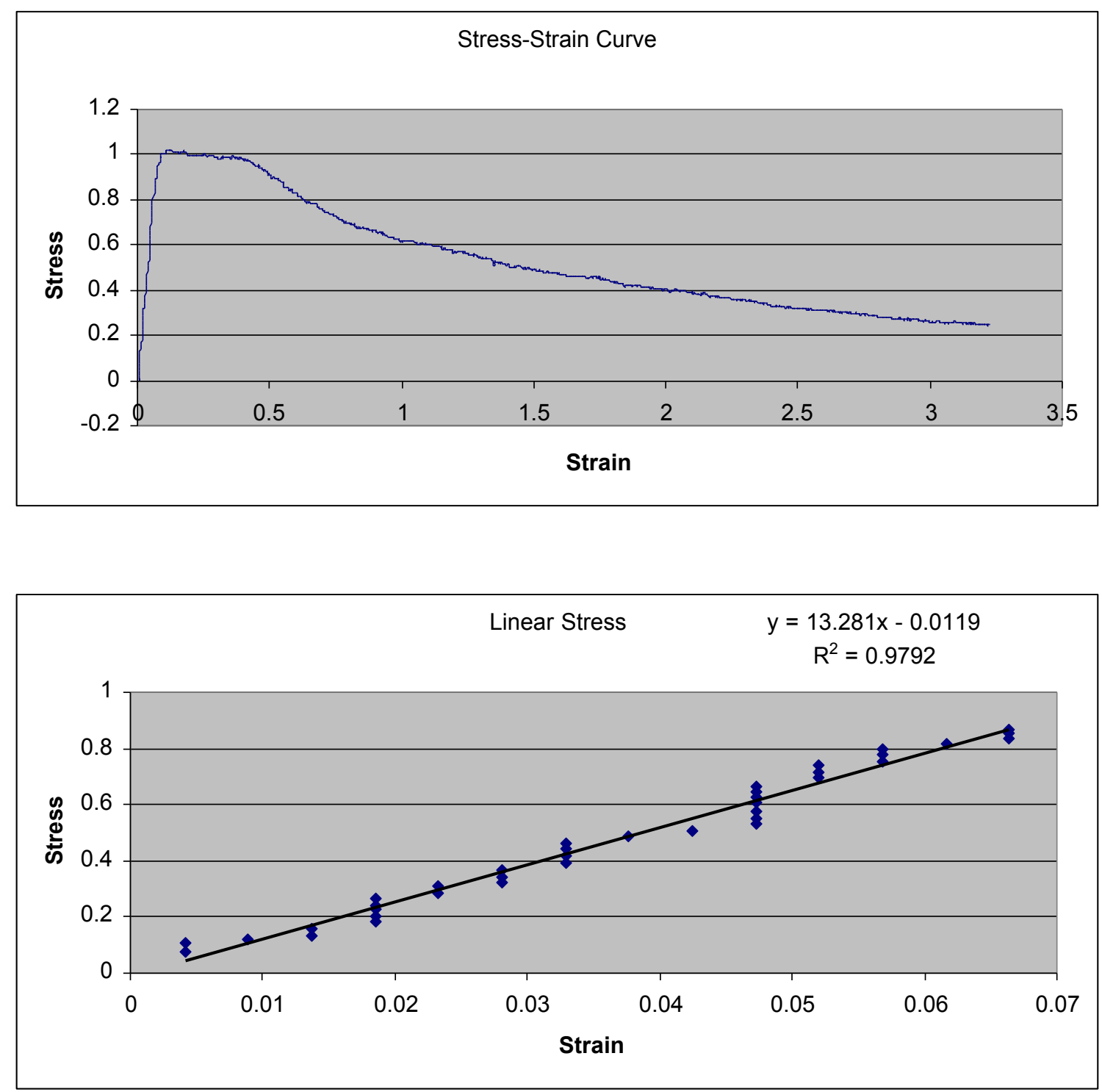


\section{Spin 2}

Section BC: Radial Test
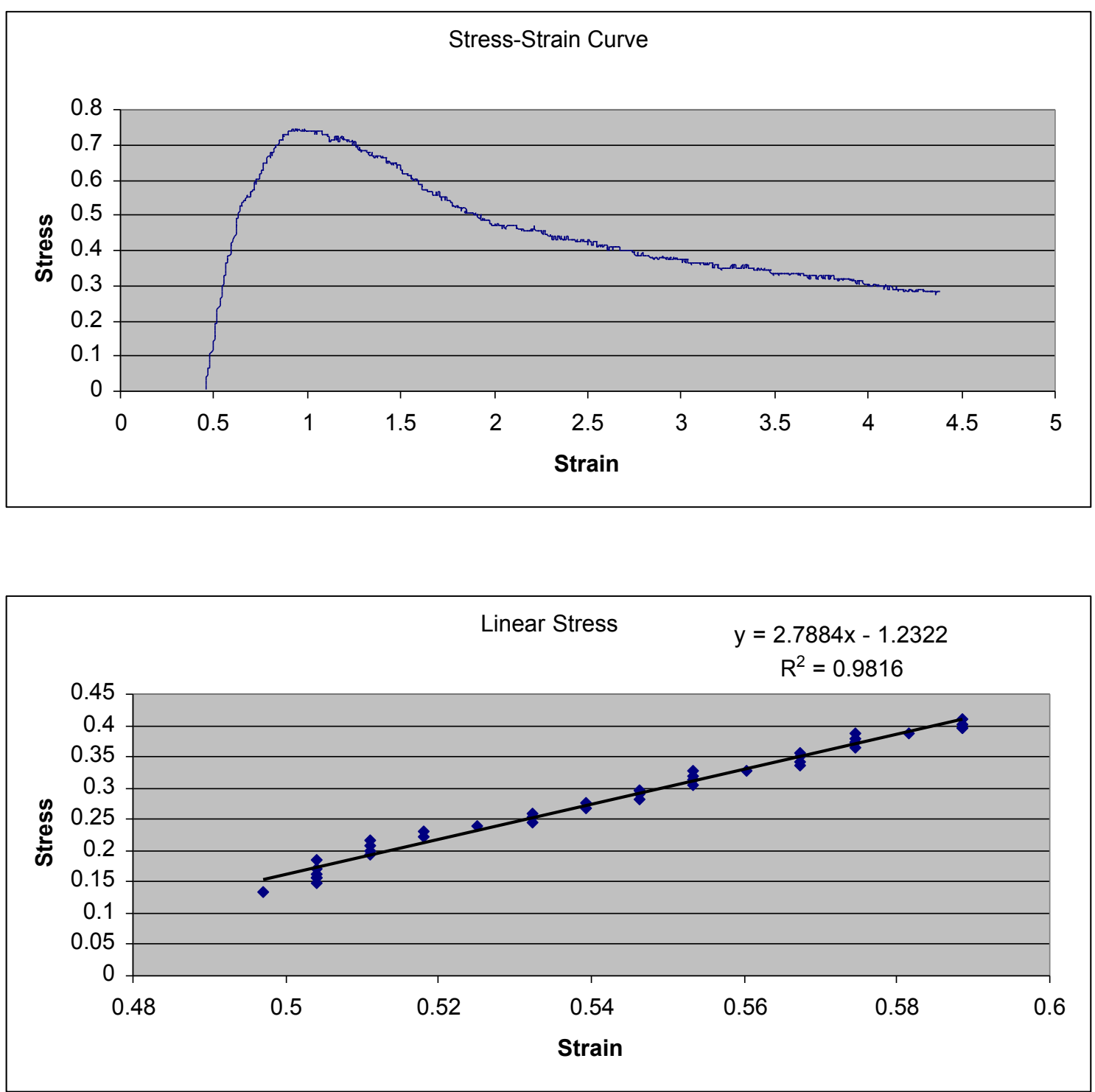
Spin 2

Section CD: Longitudinal Test
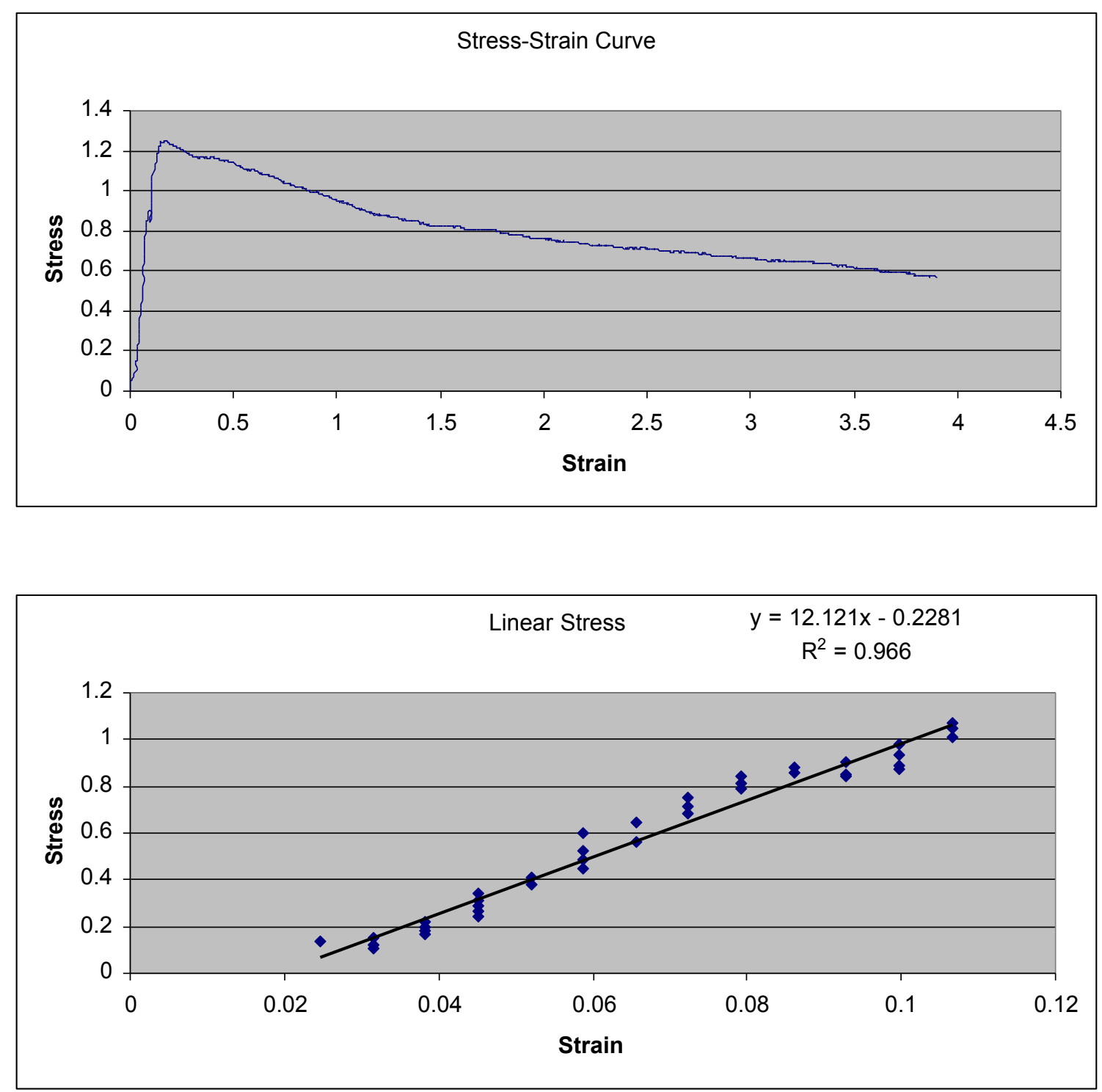
Spin 2

Section CD: Radial Test
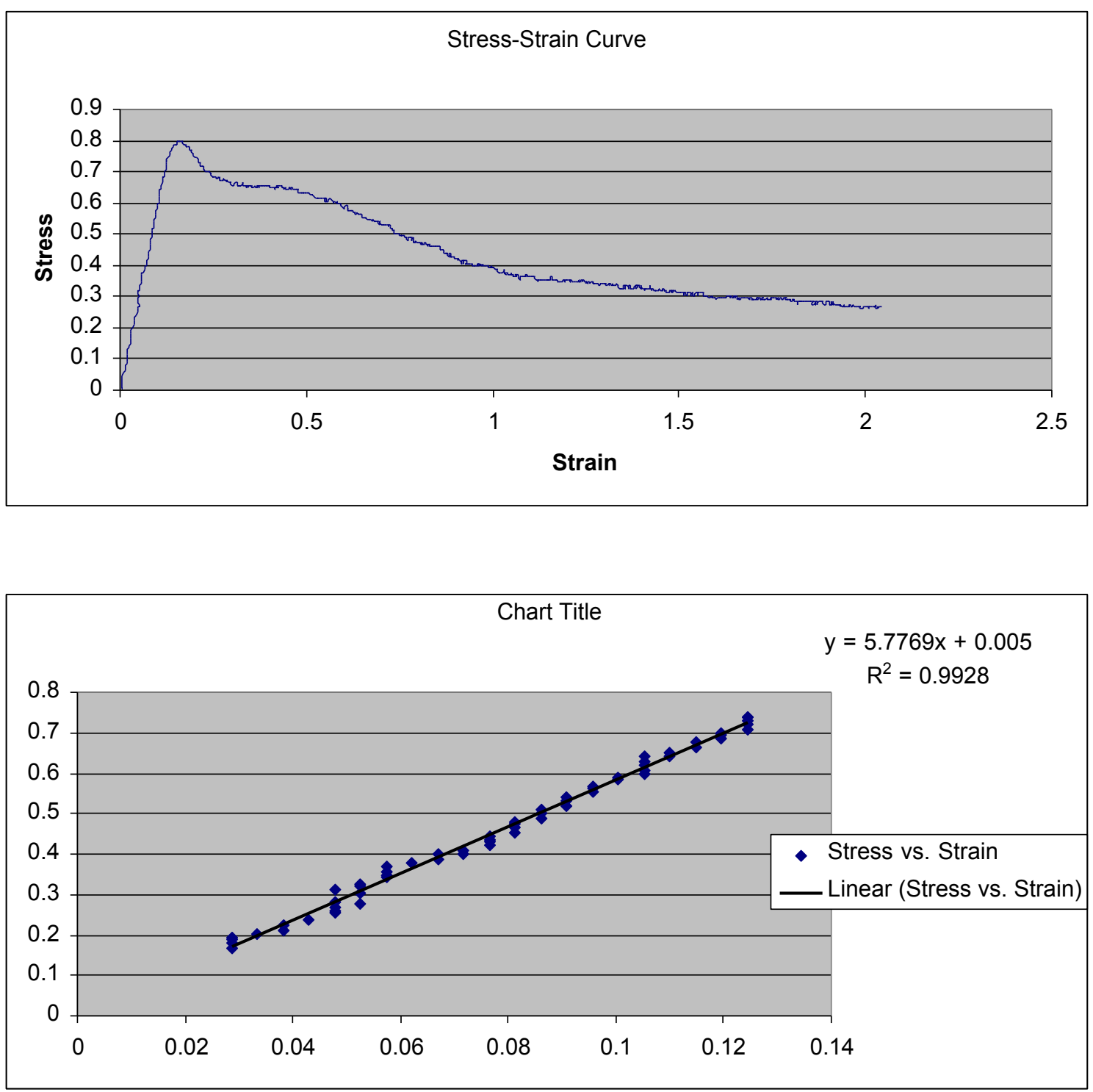
Spin 3

Section AB: Longitudinal Test
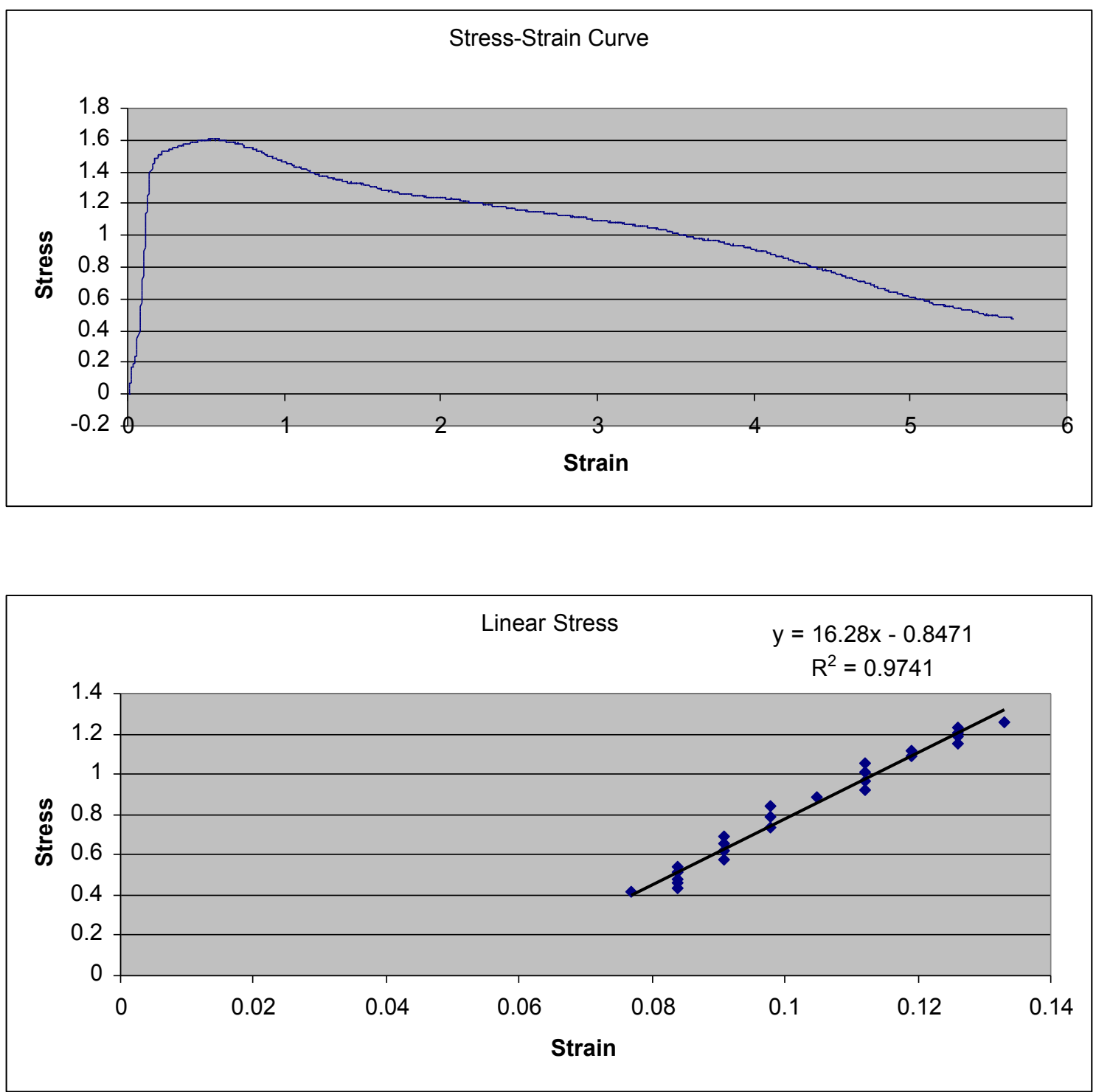
Spin 3

Section AB: Radial Test
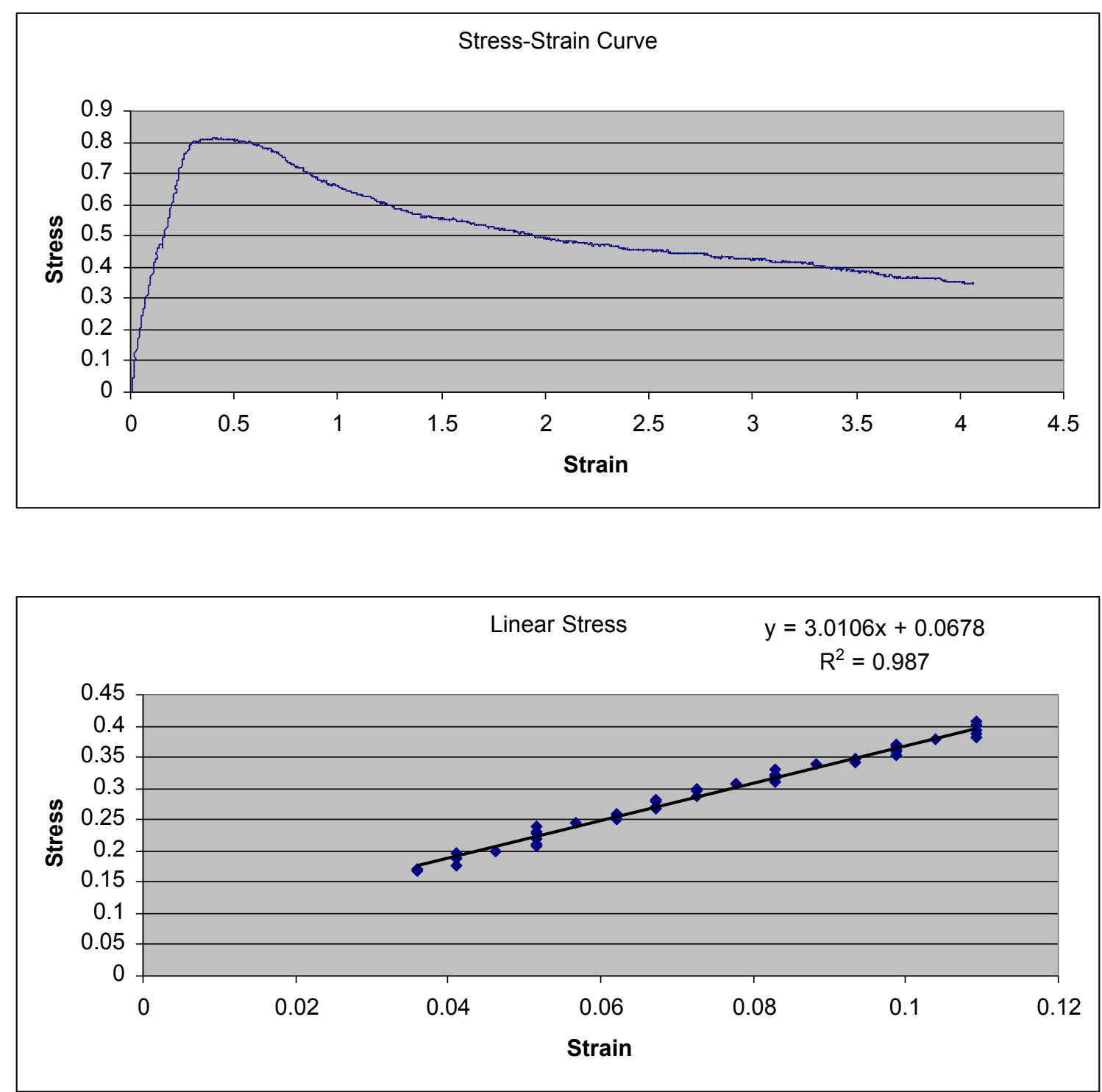


\section{Spin 3}

Section BC: Longitudinal Test
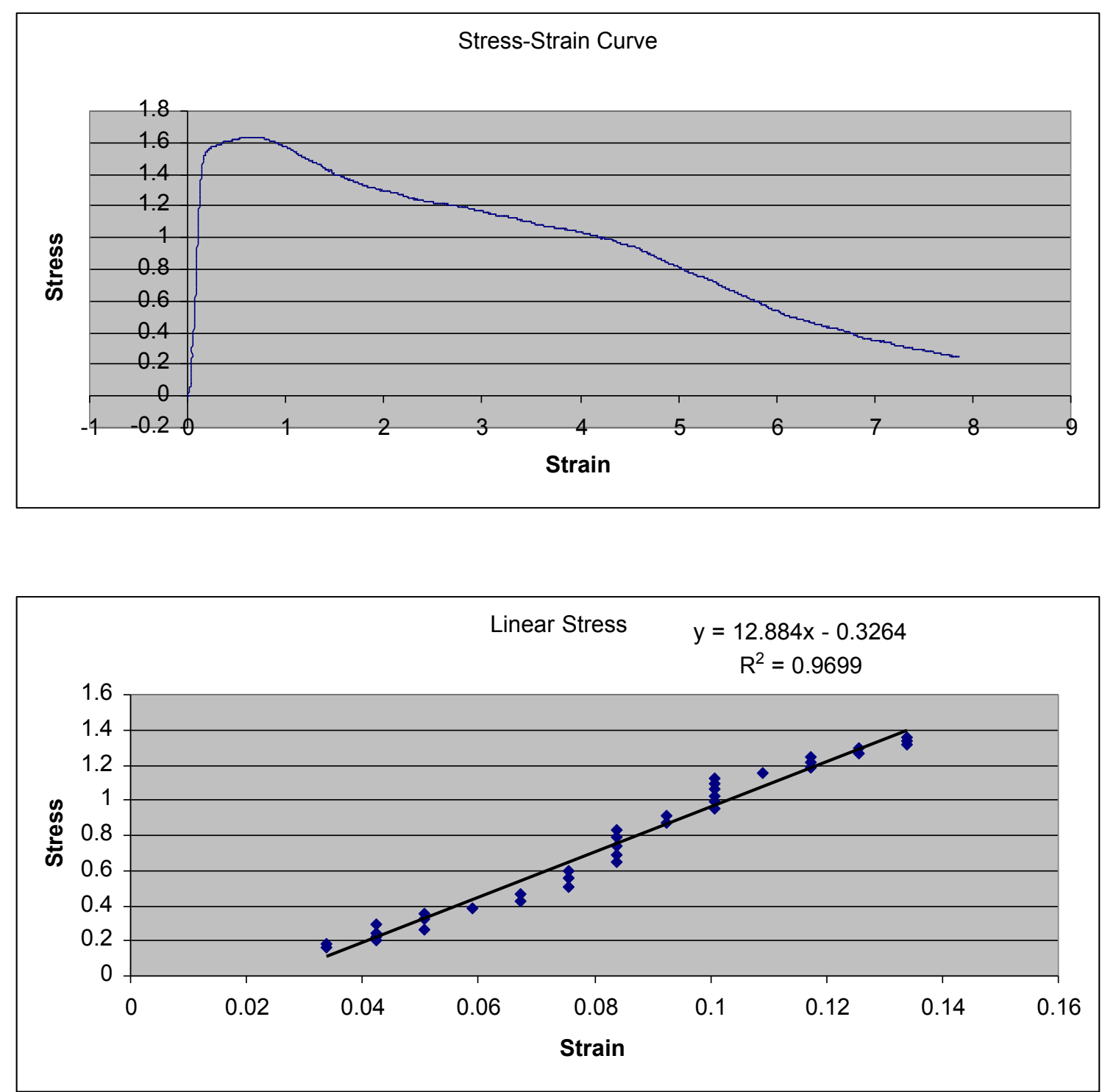
Spin 3

Section BC: Radial Test
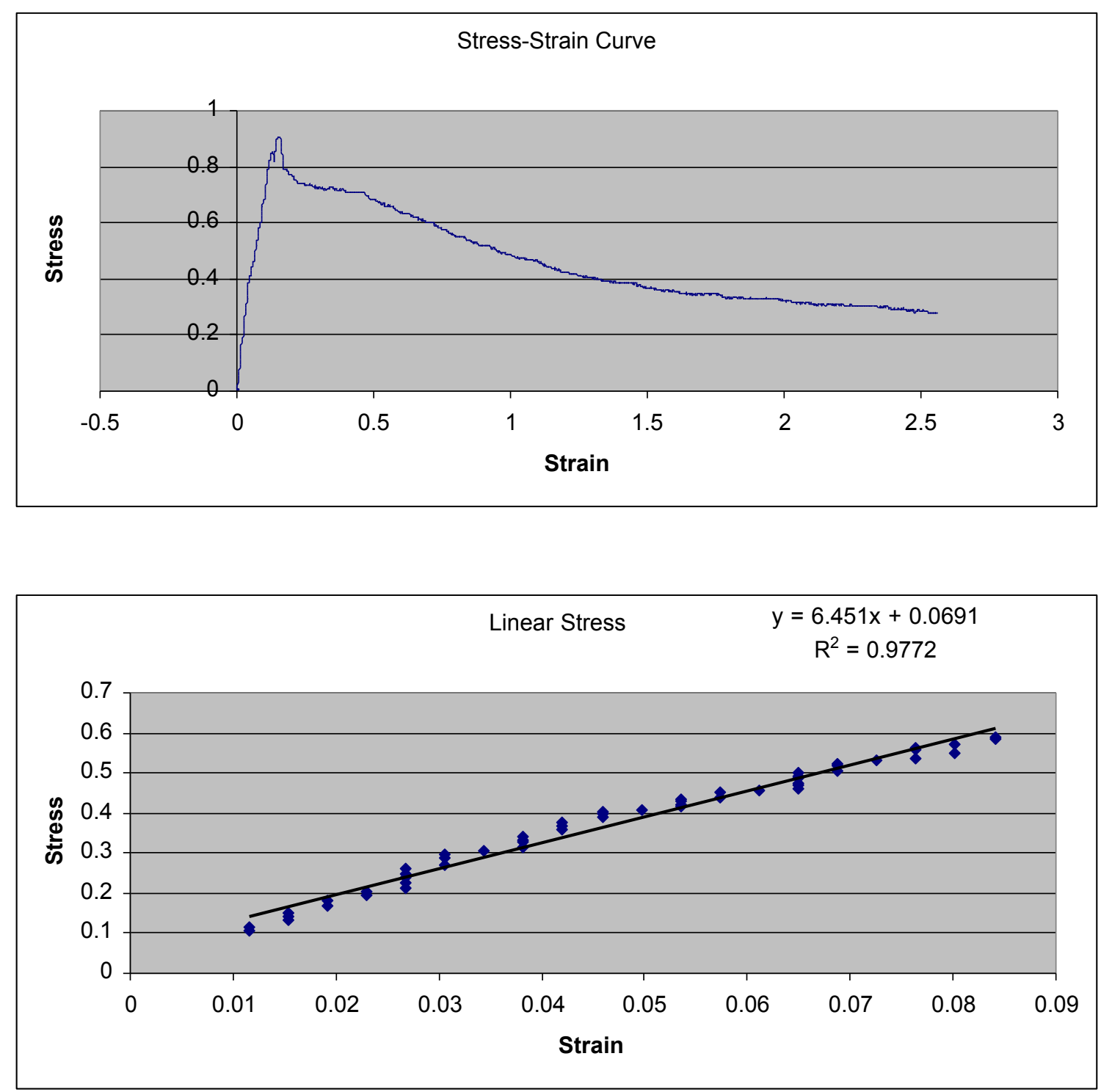
Spin 3

Section CD: Longitudinal Test
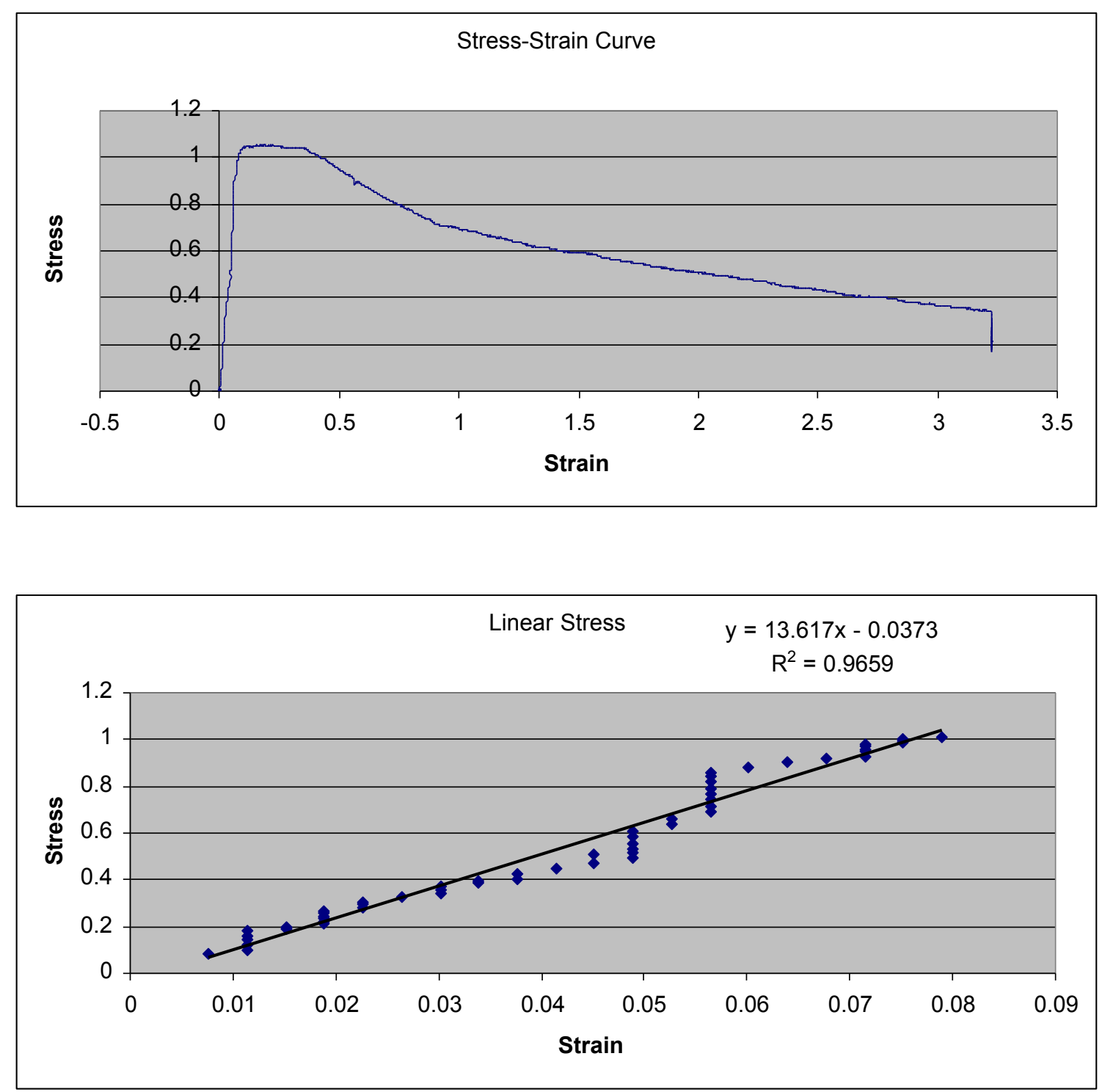
Spin 3

Section CD: Radial Test
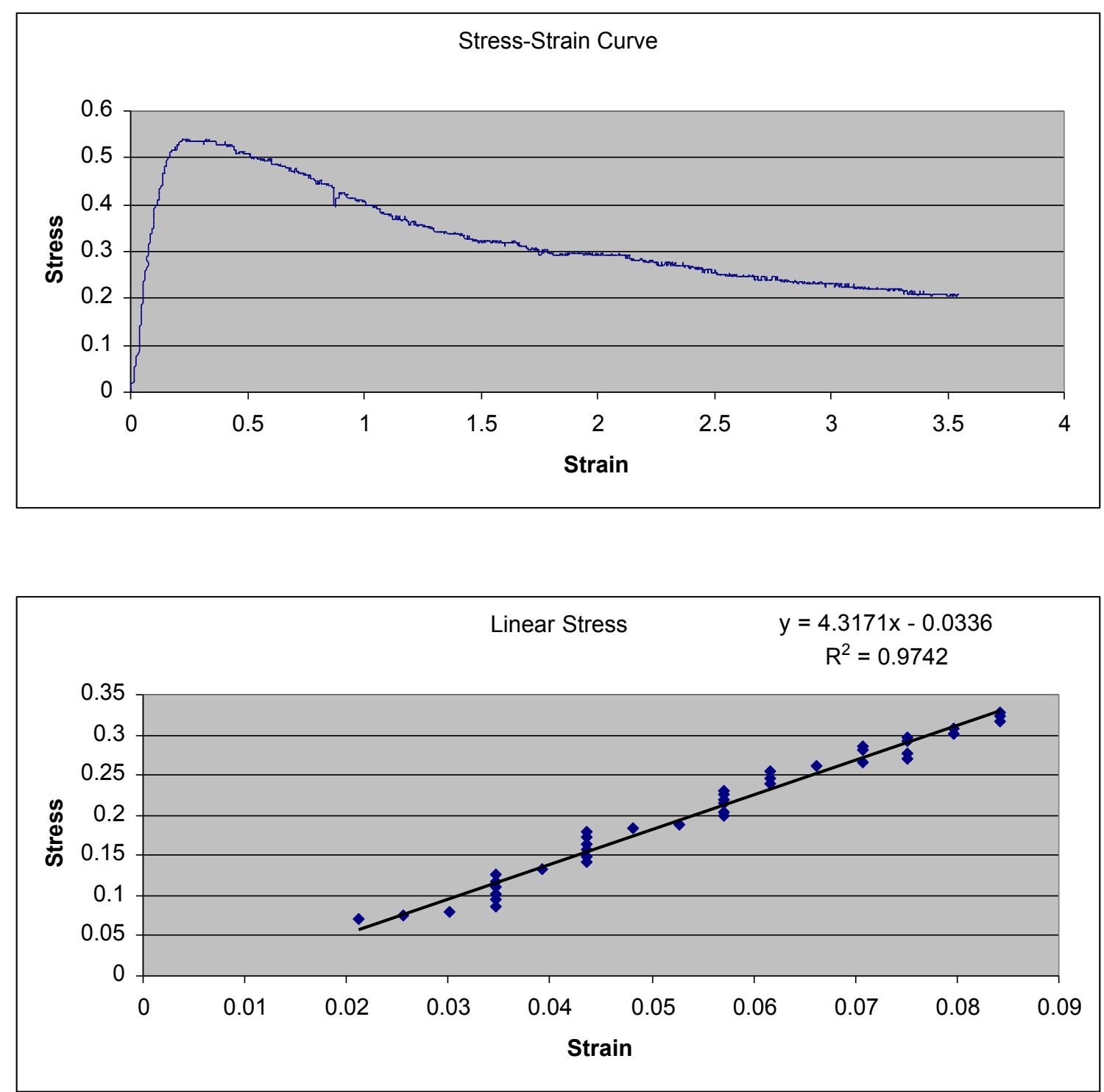


\section{Spin 4}

Section AB: Longitudinal Test
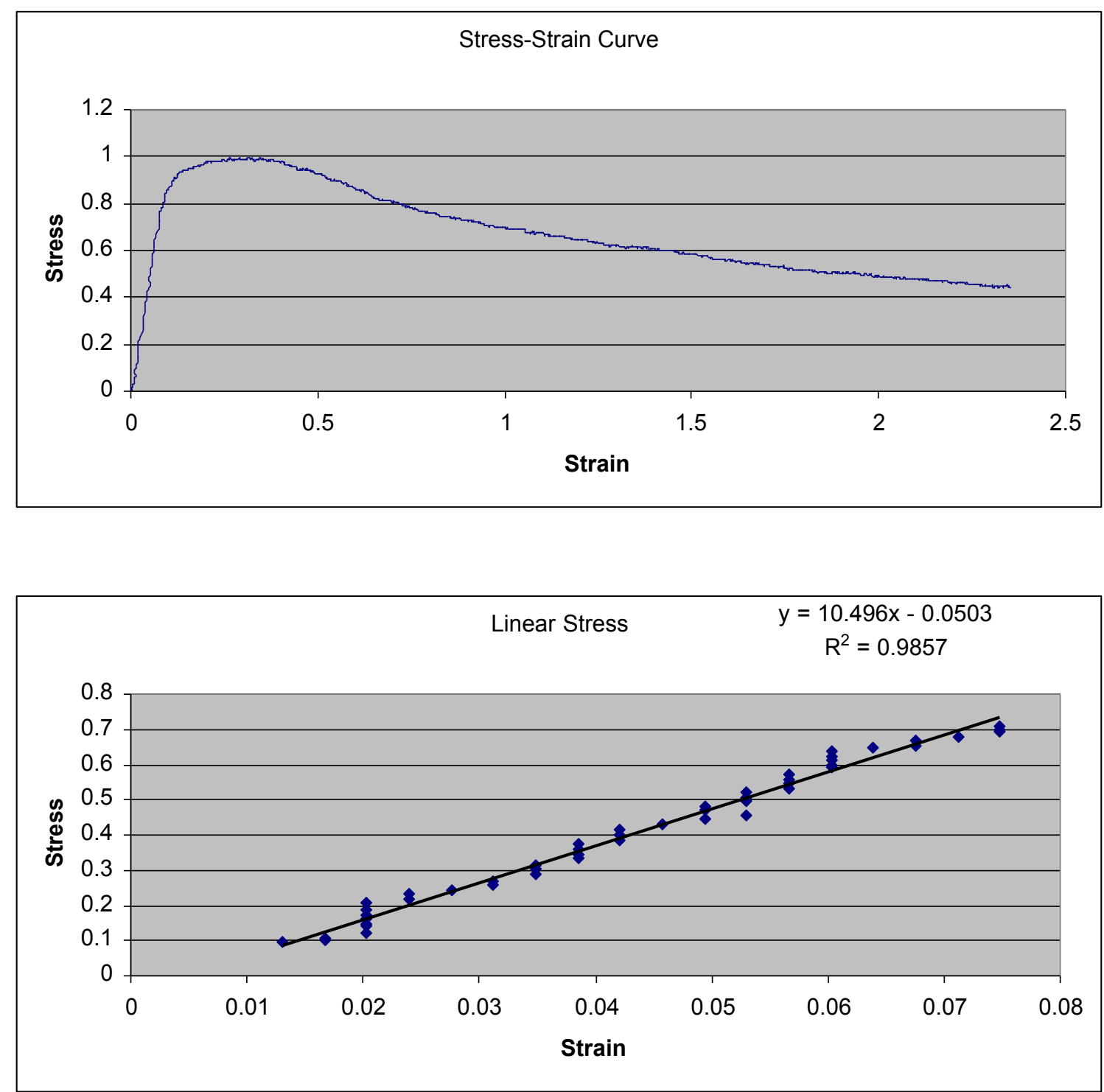


\section{Spin 4}

Section AB: Radial Test
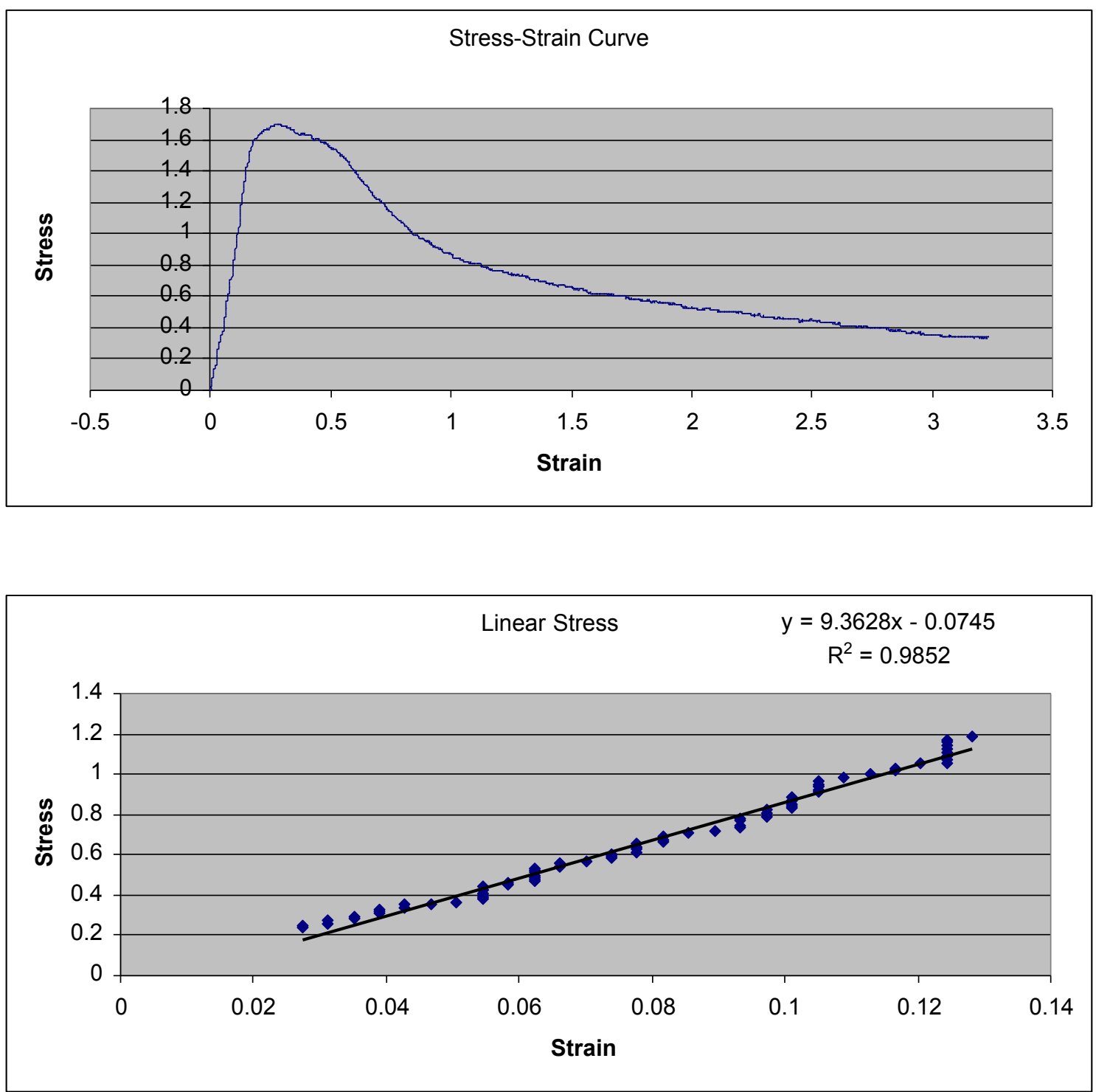


\section{Spin 4}

Section BC: Longitudinal Test
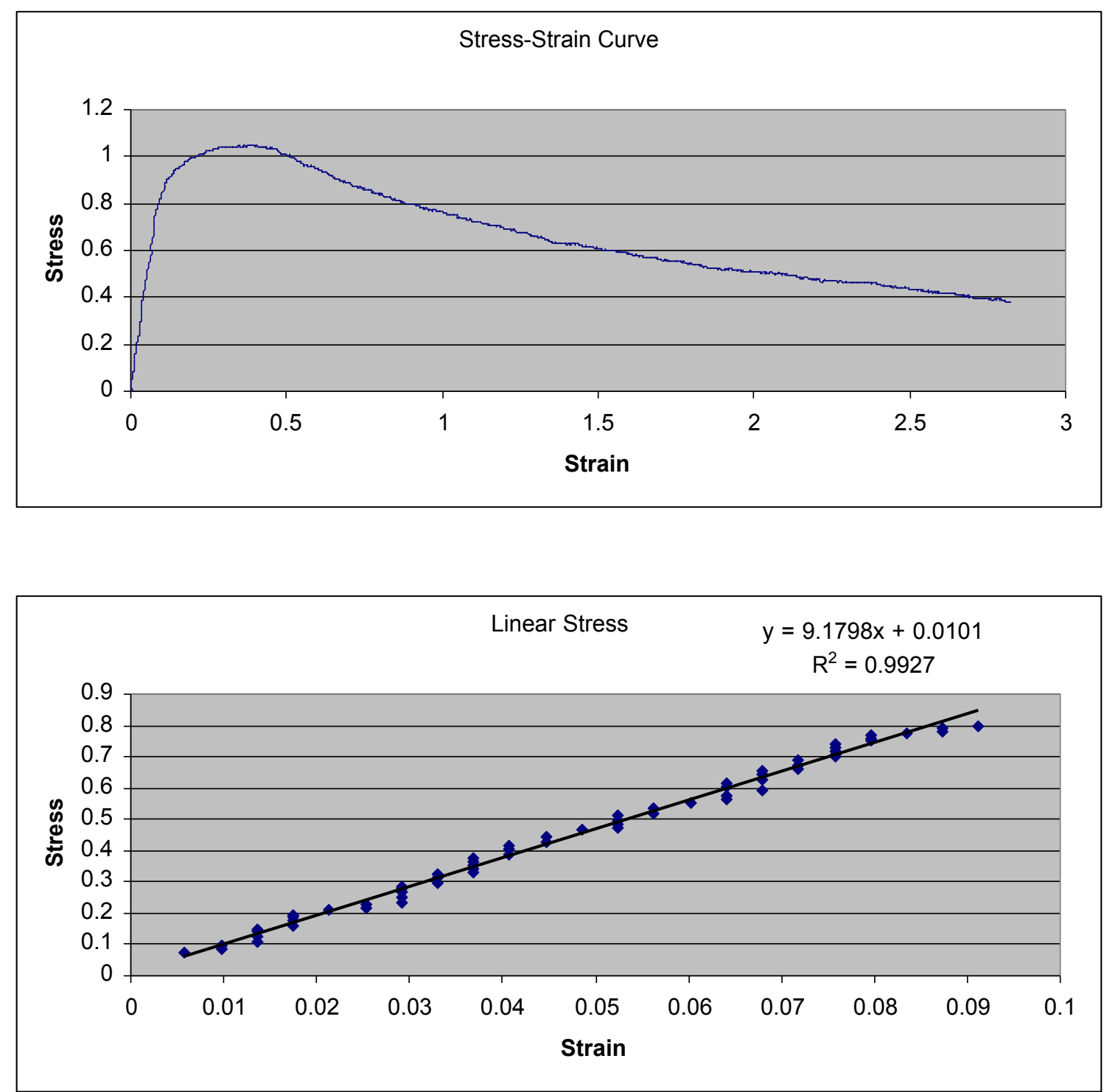


\section{Spin 4}

Section BC: Radial Test
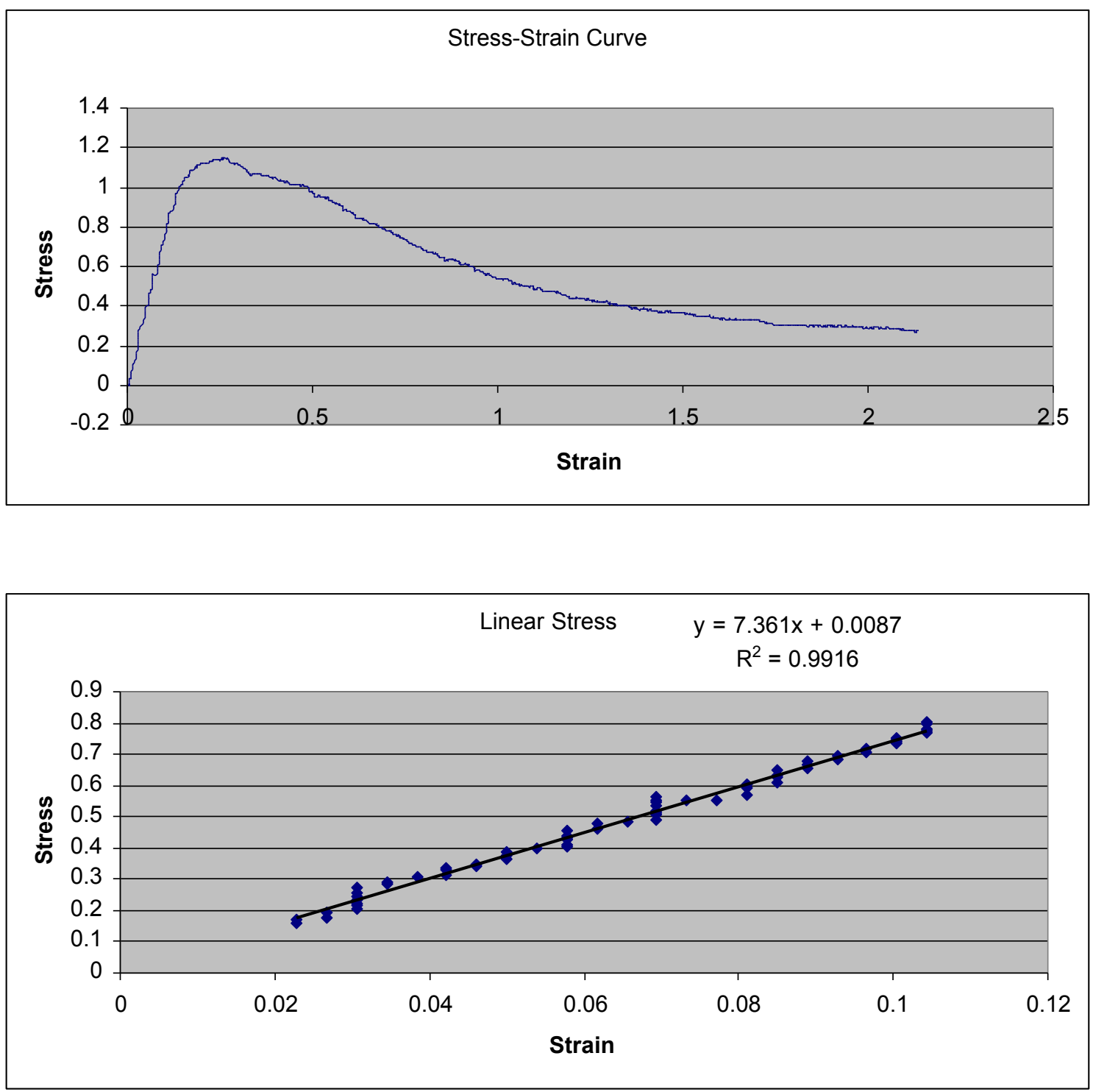


\section{Spin 4}

Section CD: Longitudinal Test
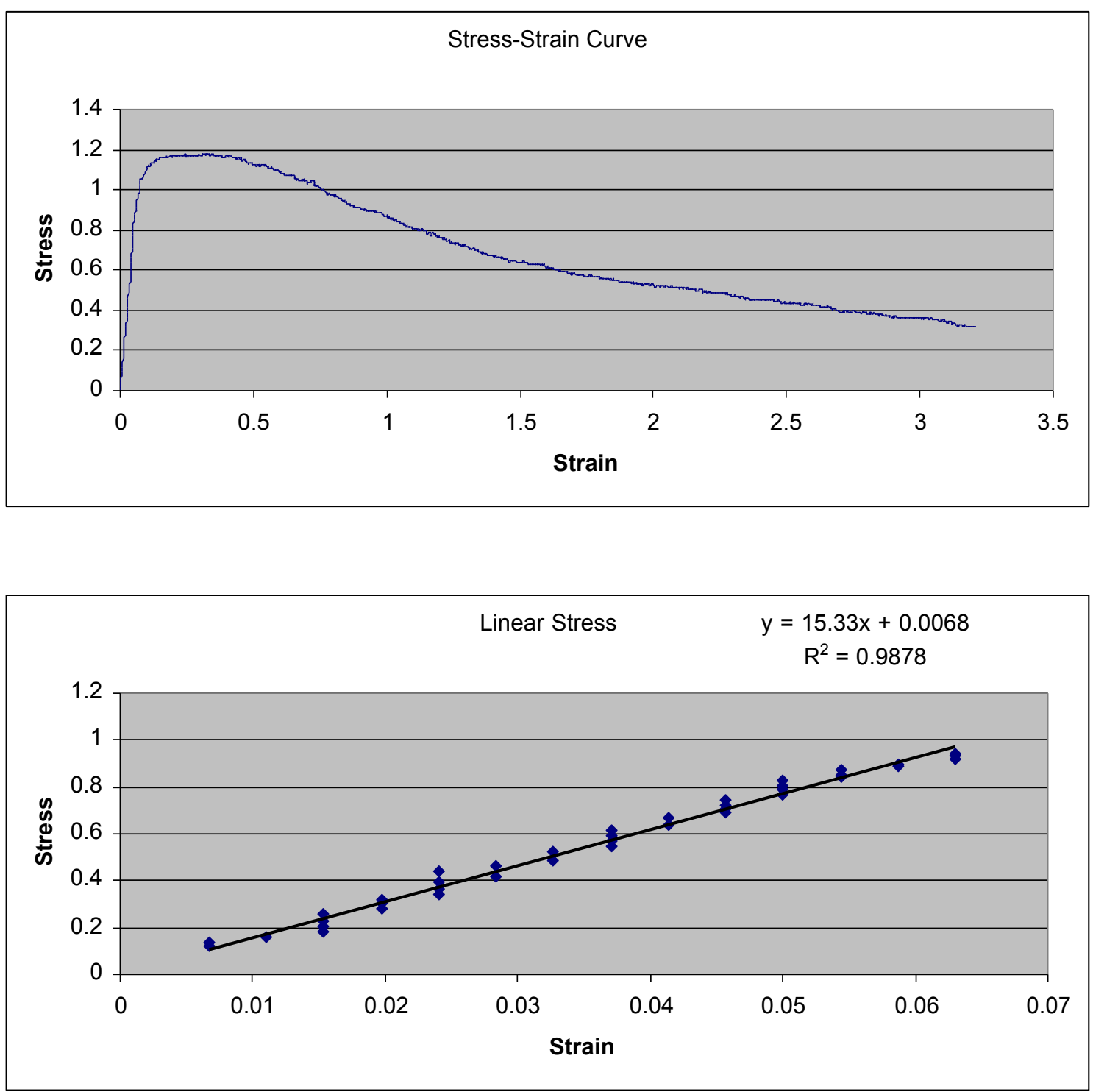


\section{Spin 4}

Section CD: Radial Test
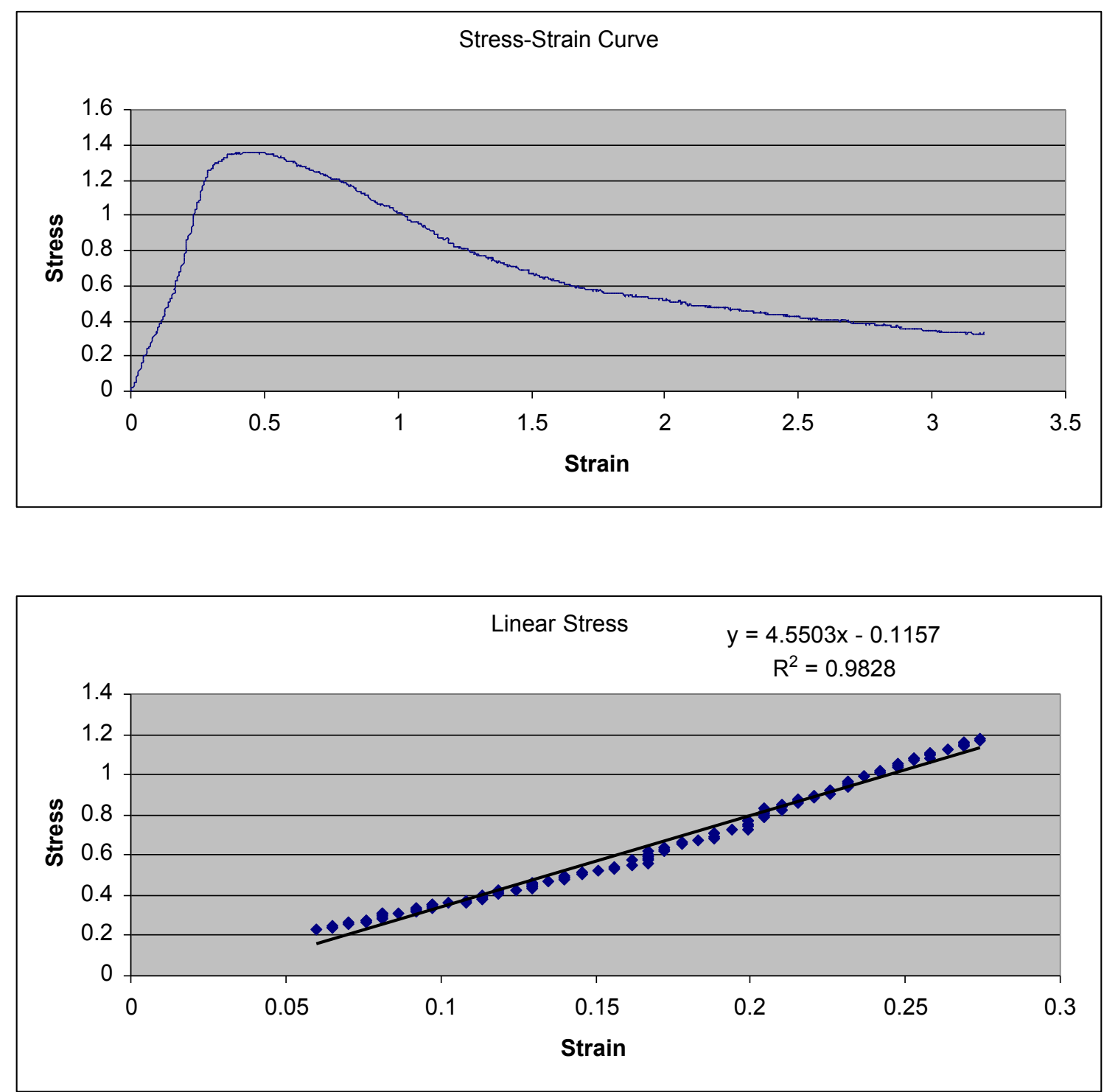


\section{Spin 5}

Section AB: Longitudinal Test
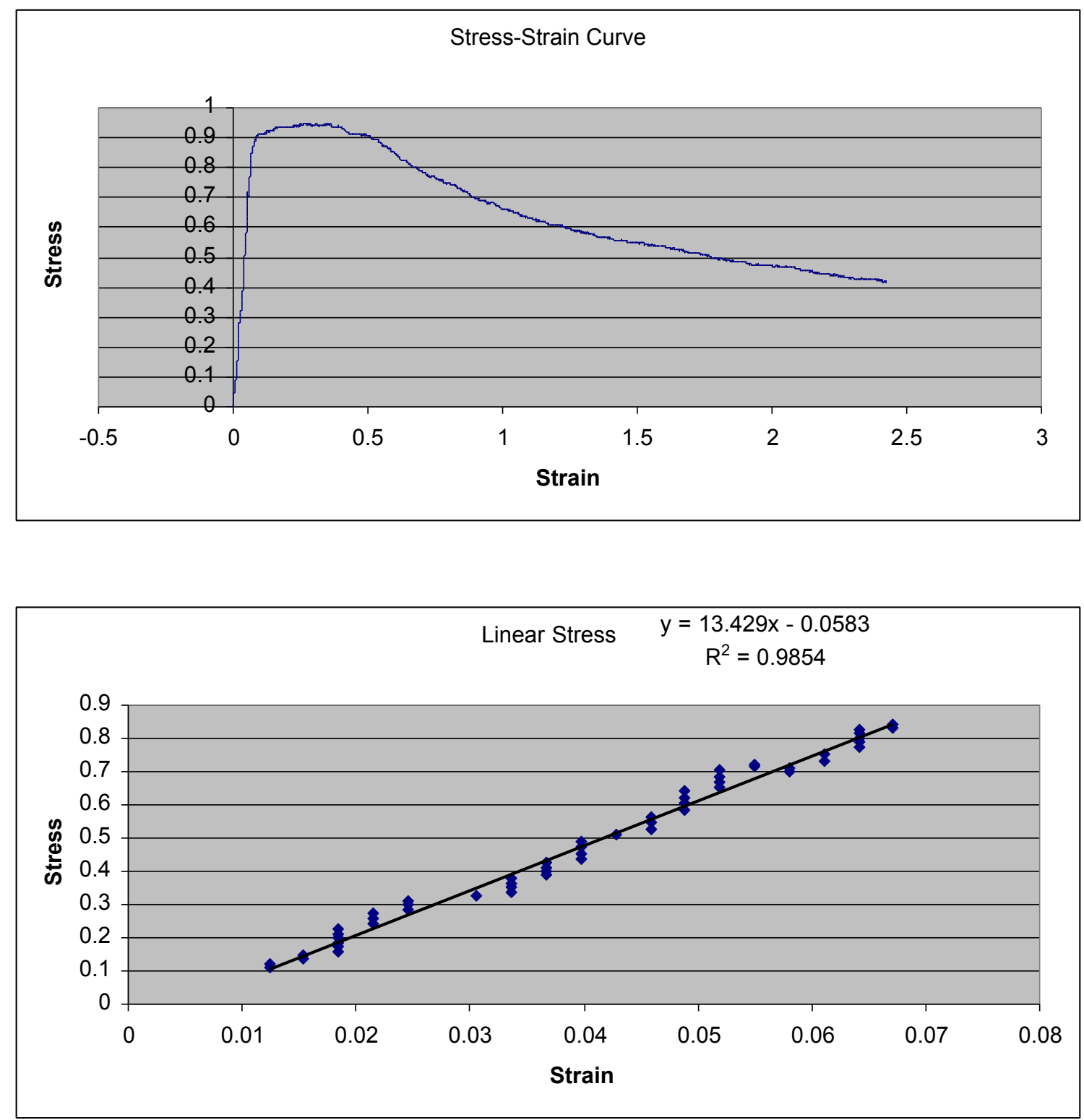


\section{Spin 5}

Section AB: Radial Test
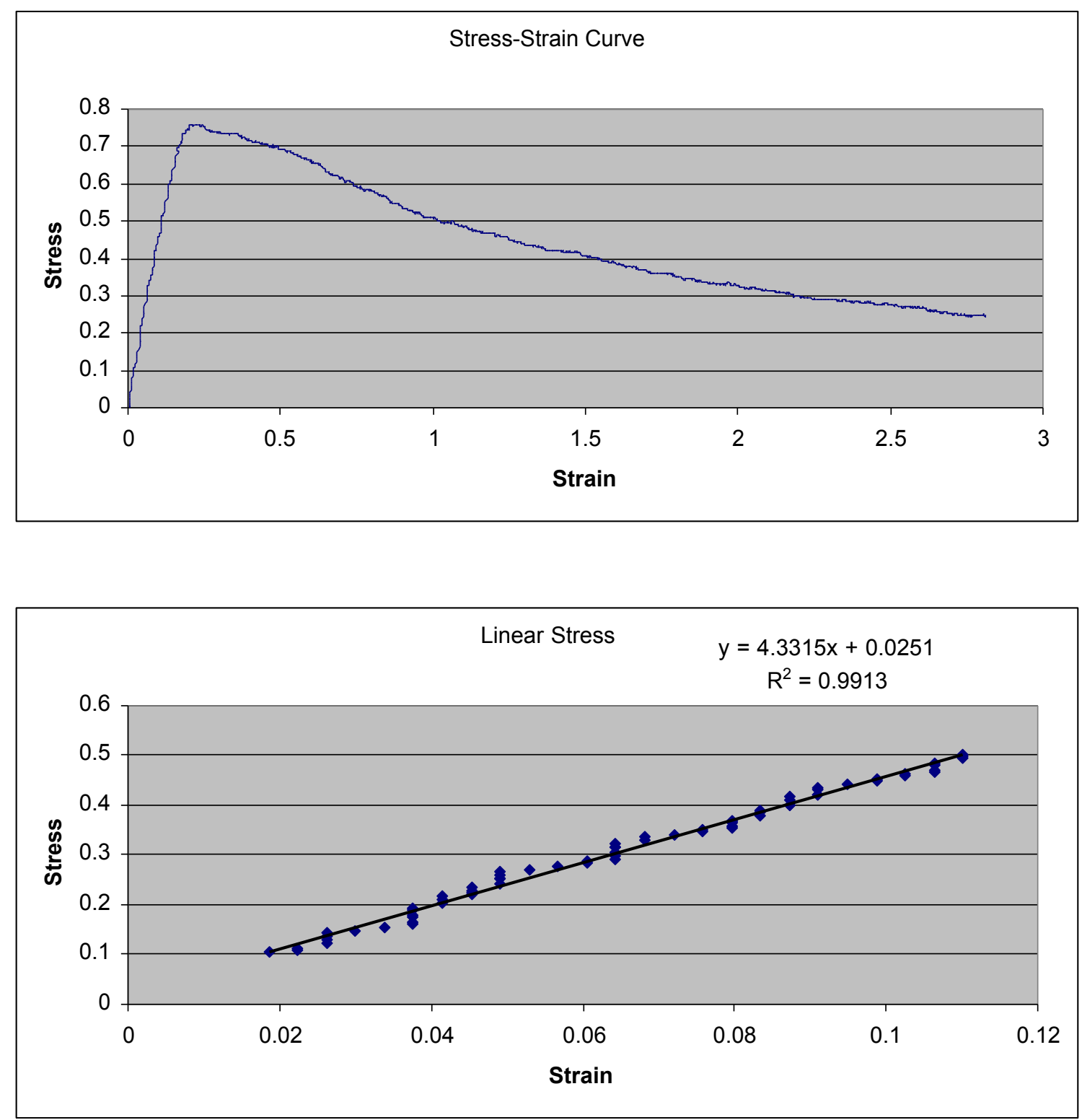


\section{Spin 5}

Section BC: Longitudinal Test
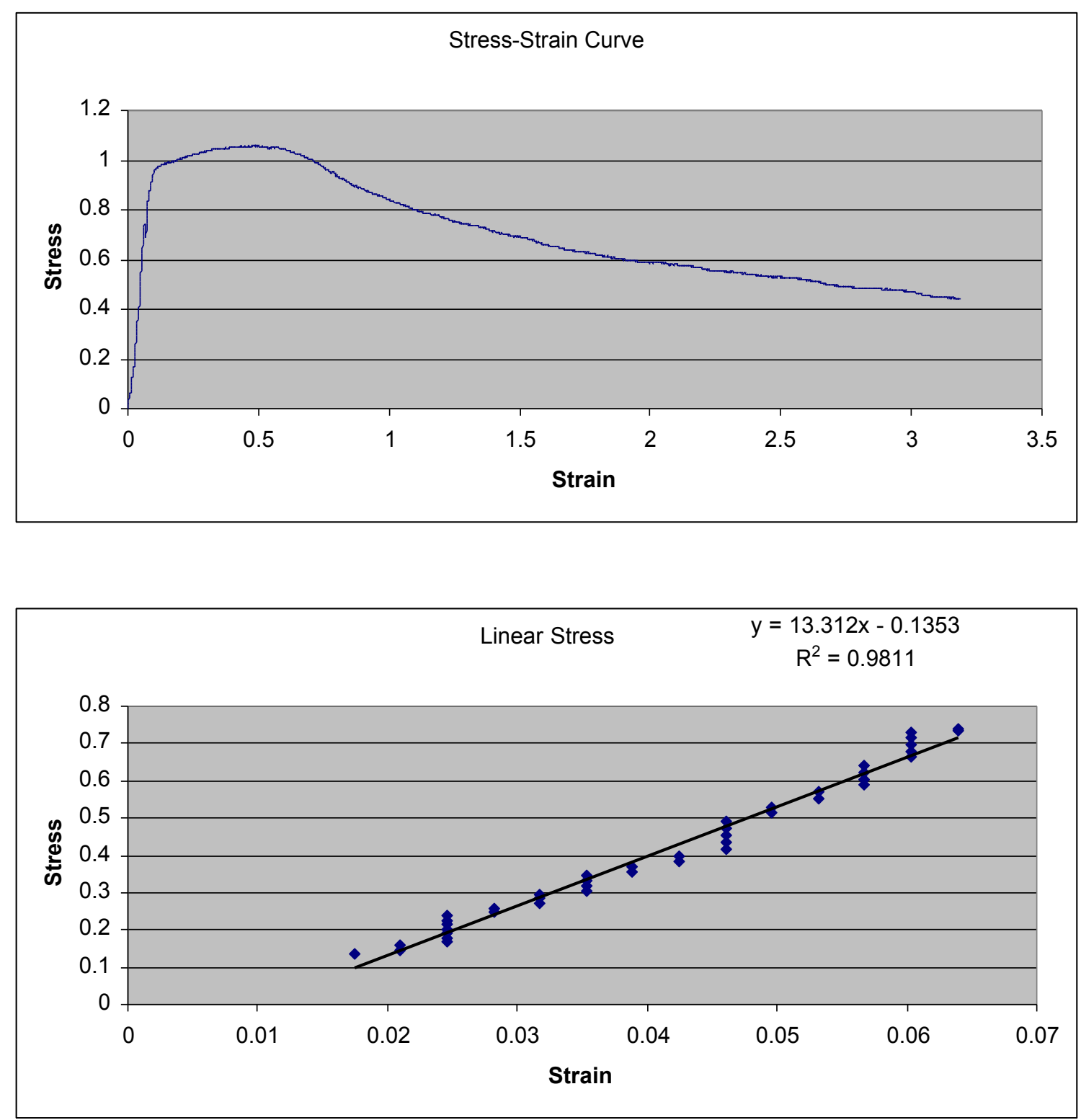


\section{Spin 5}

Section BC: Radial Test
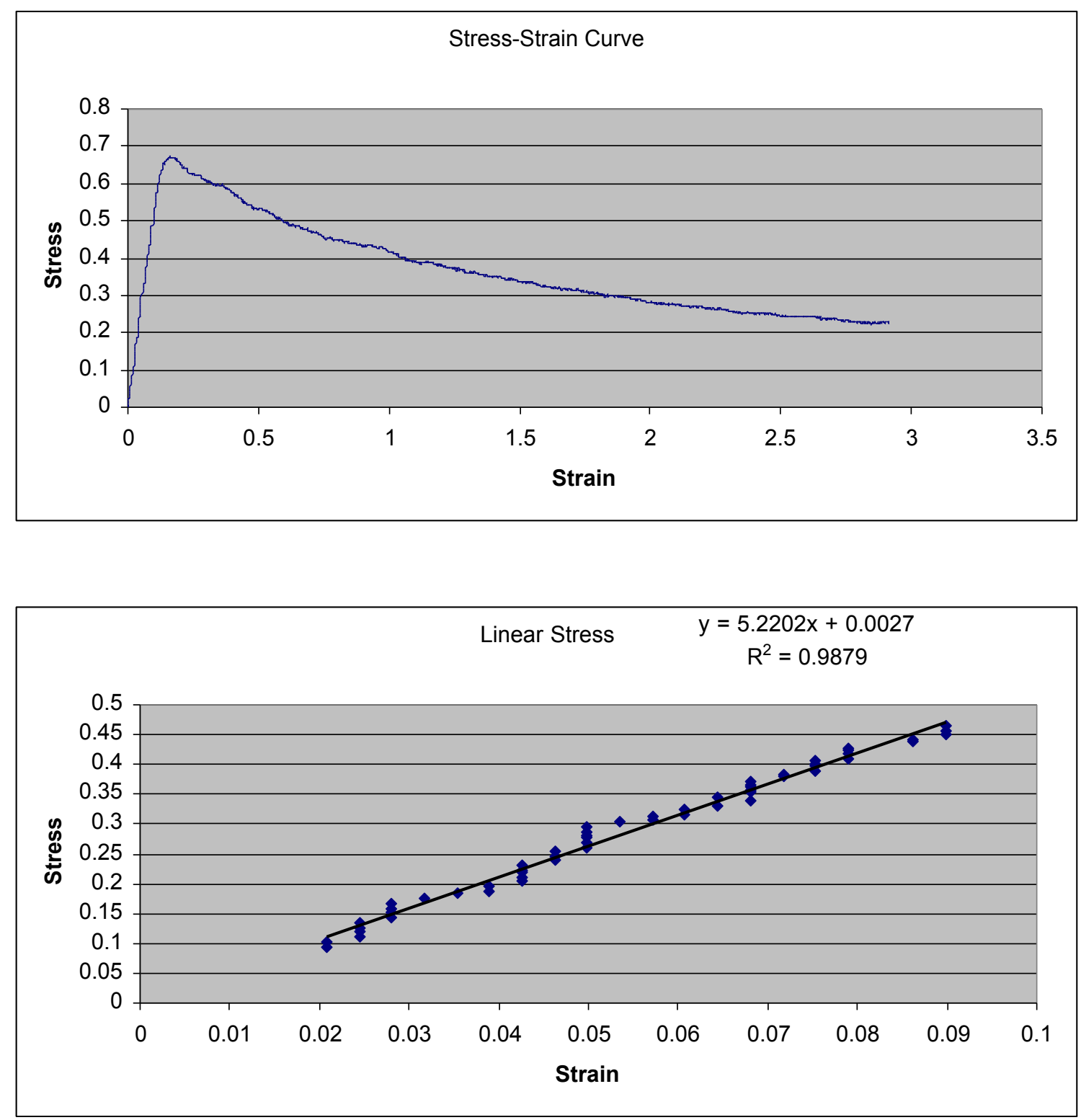


\section{Spin 5}

Section CD: Longitudinal Test
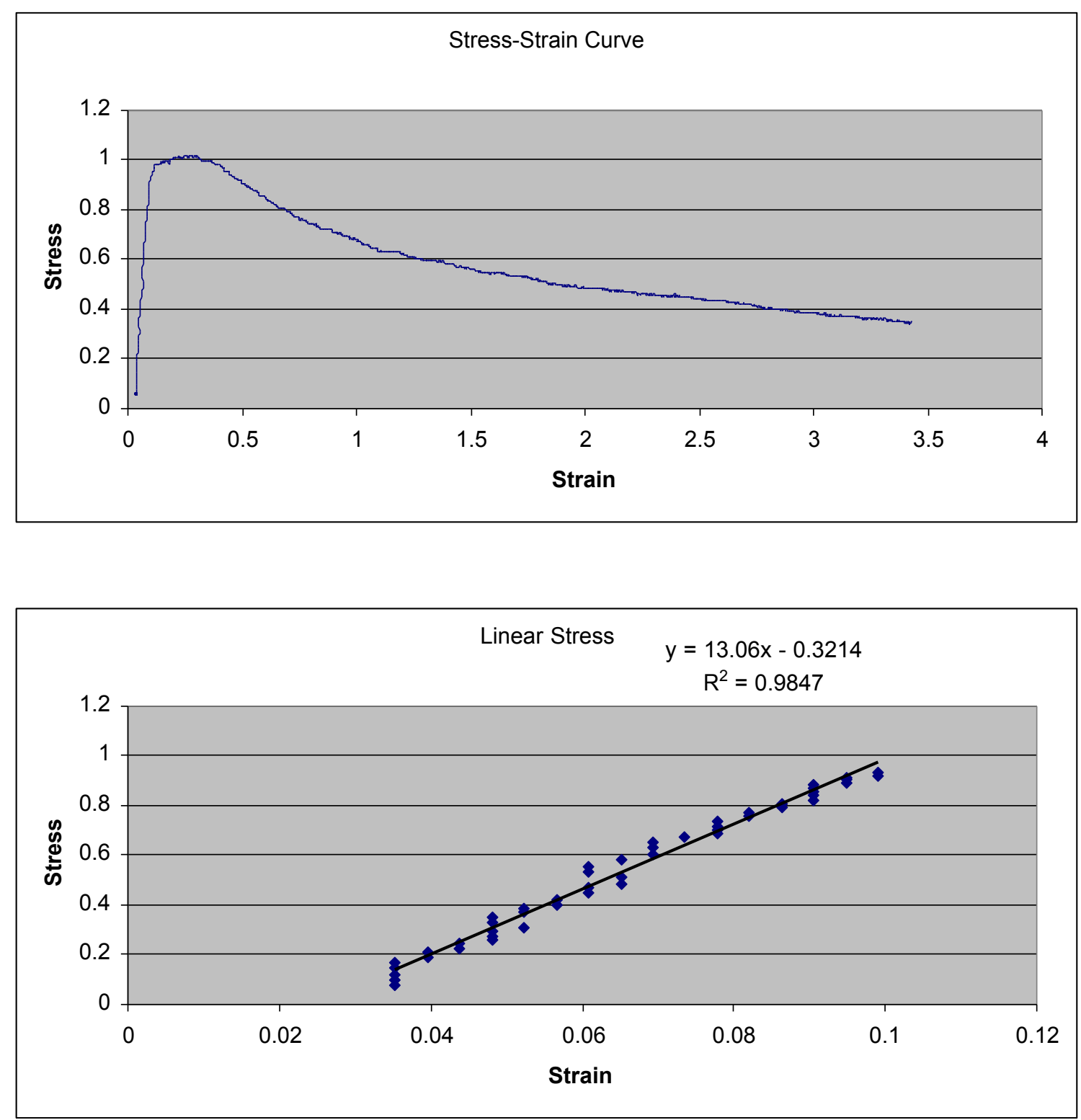


\section{Spin 5}

Section CD: Radial Test
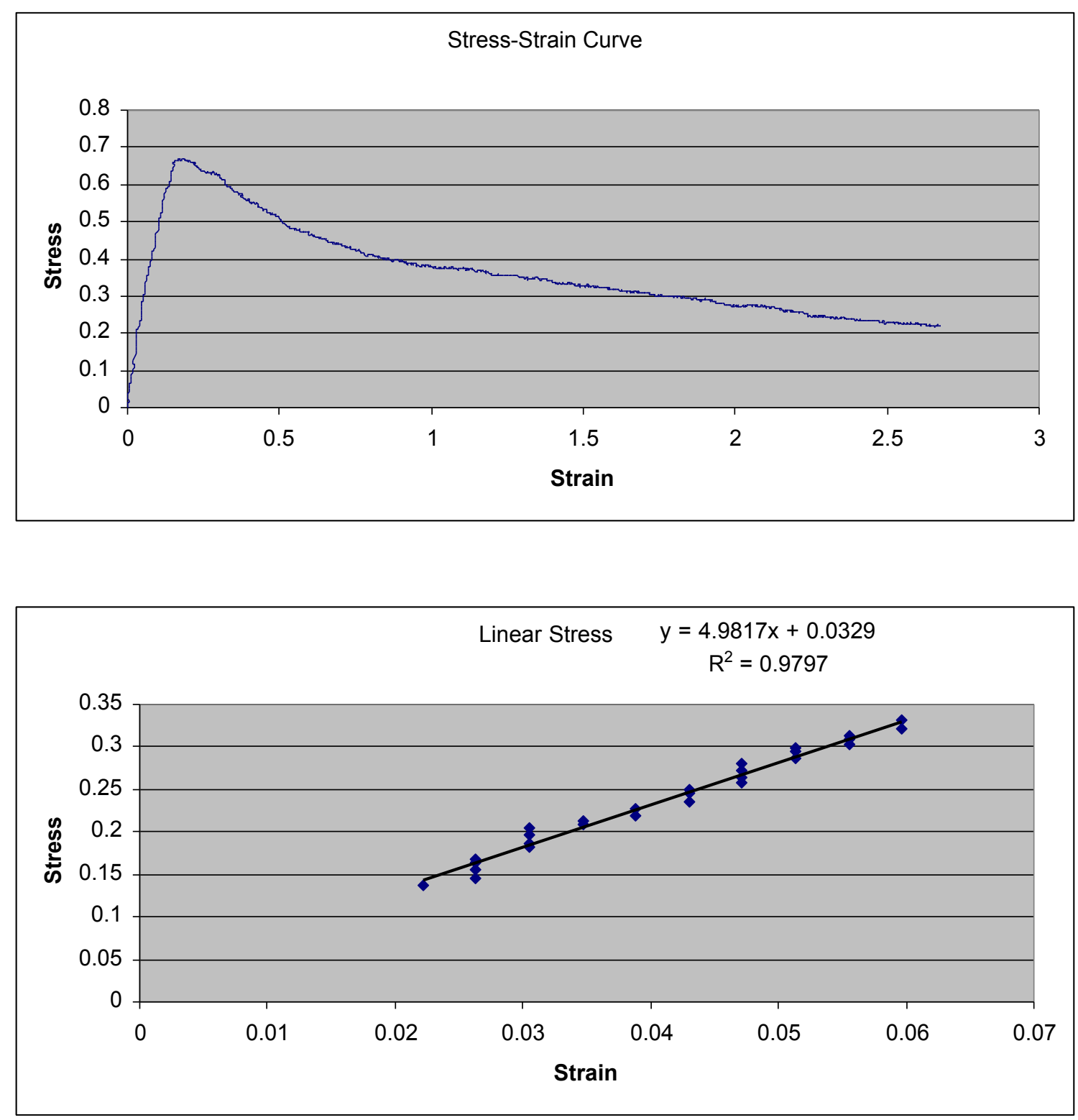


\section{Spin 6}

Section AB: Longitudinal Test
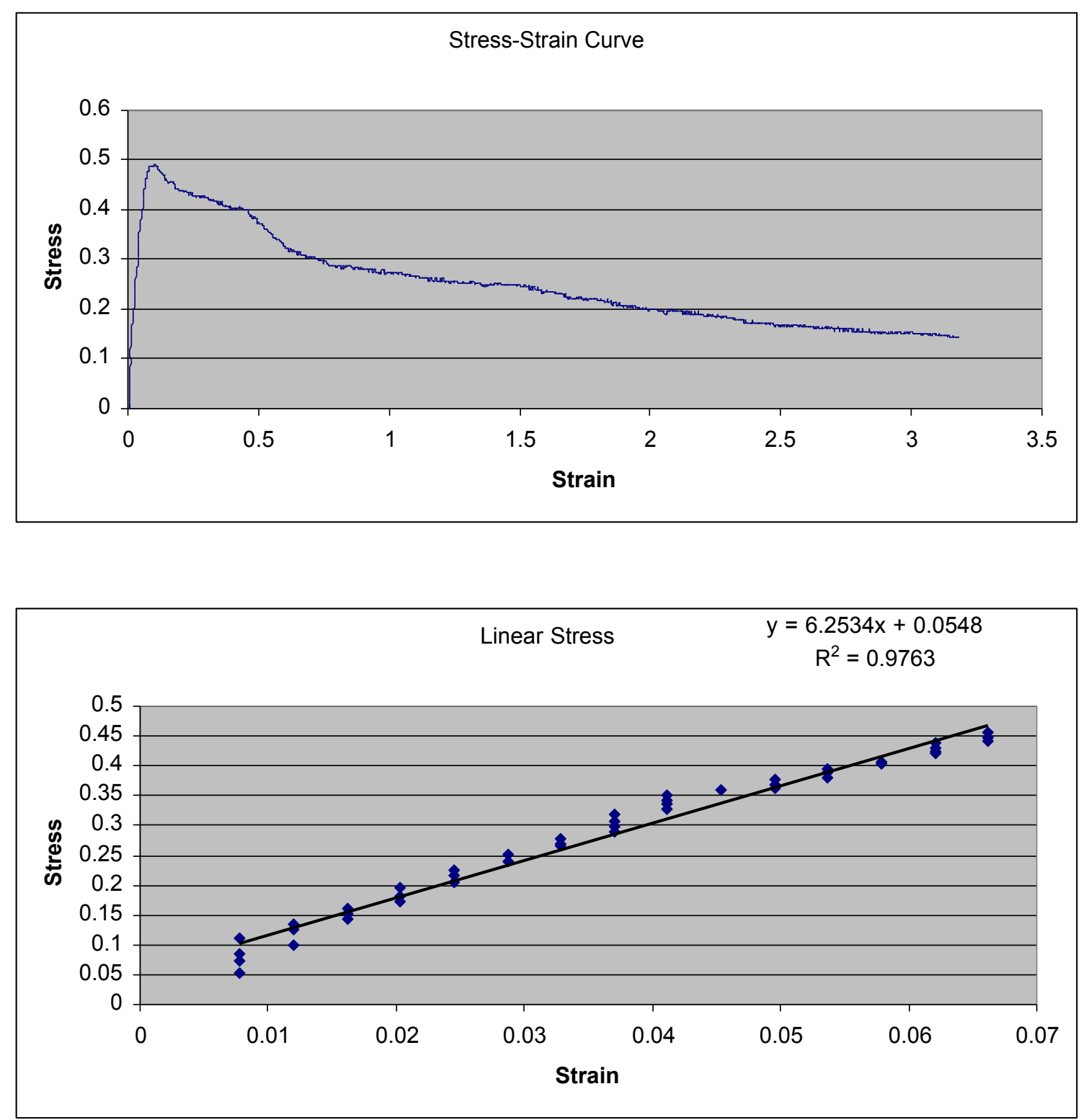


\section{Spin 6}

Section AB: Radial Test
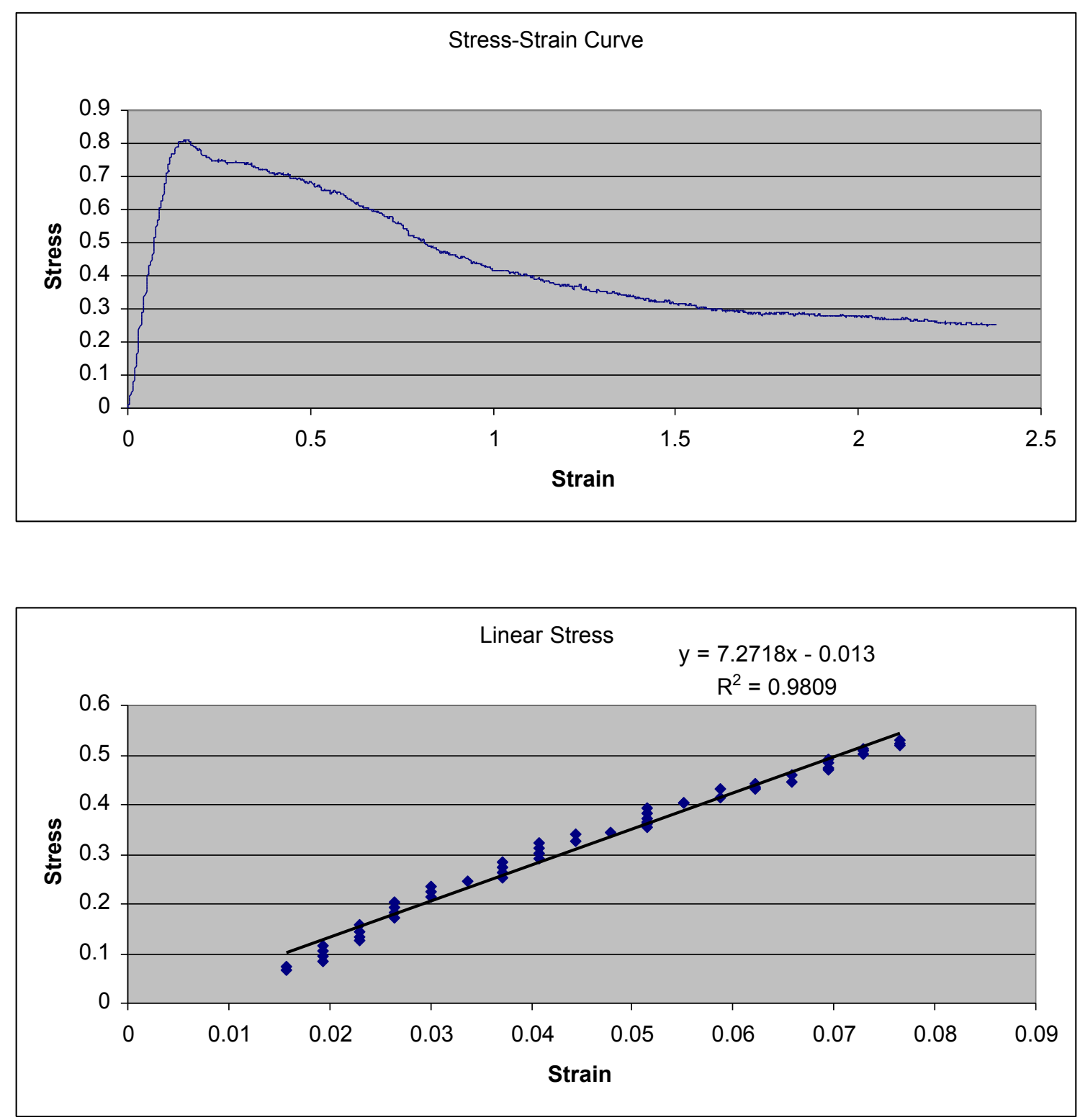


\section{Spin 6}

Section BC: Longitudinal Test
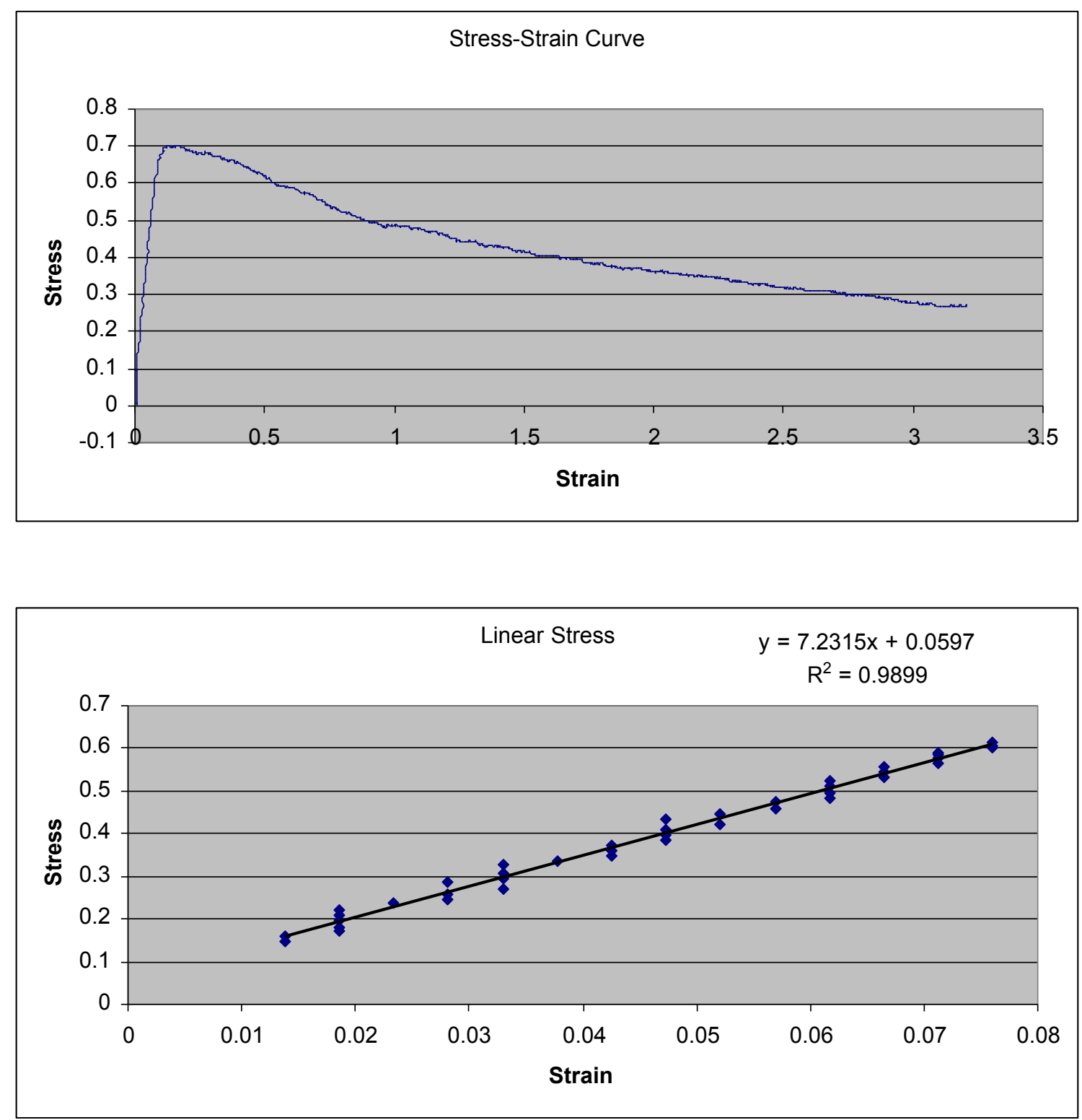


\section{Spin 6}

Section BC: Radial Test
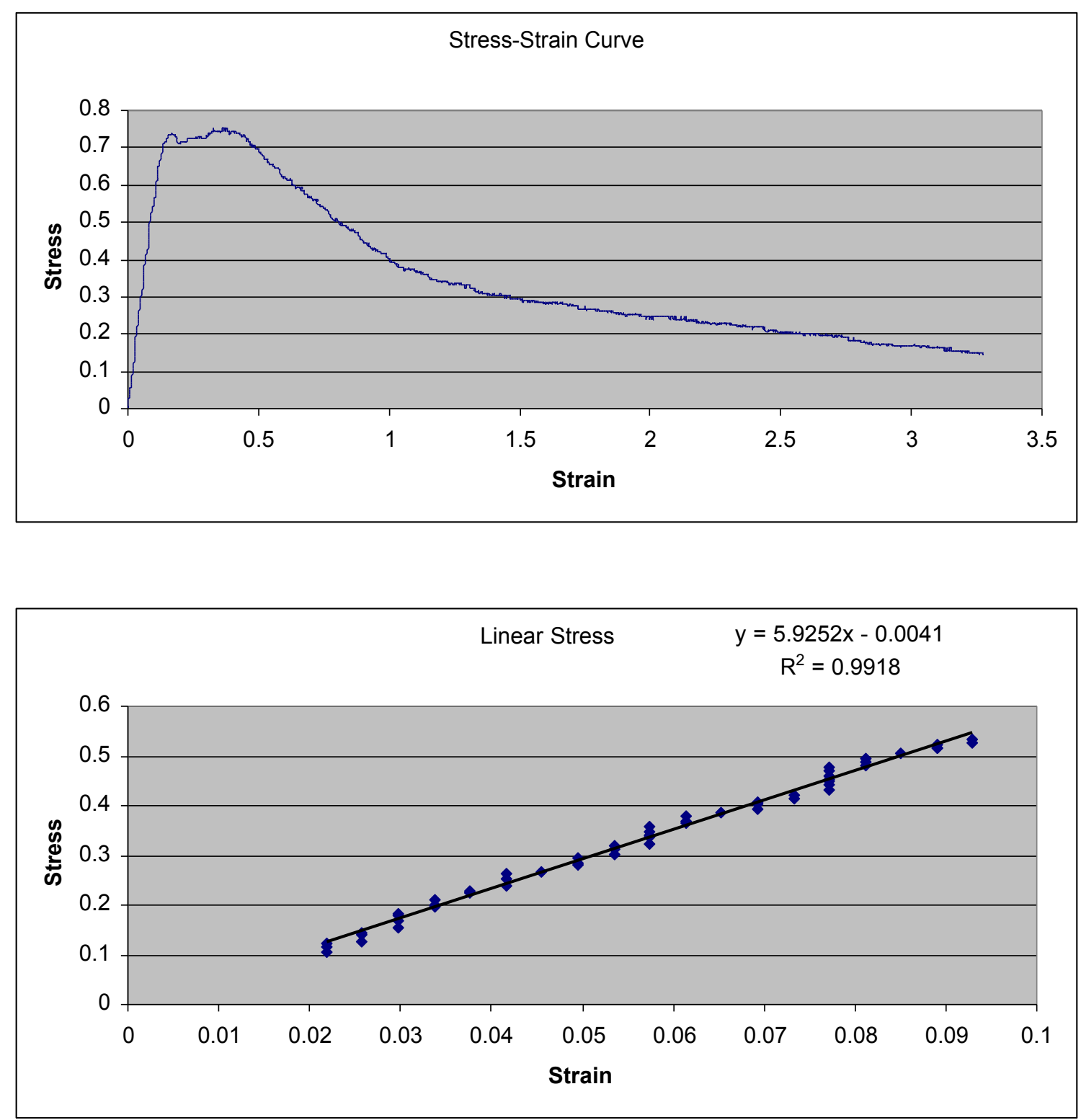


\section{Spin 6}

Section CD: Longitudinal Test
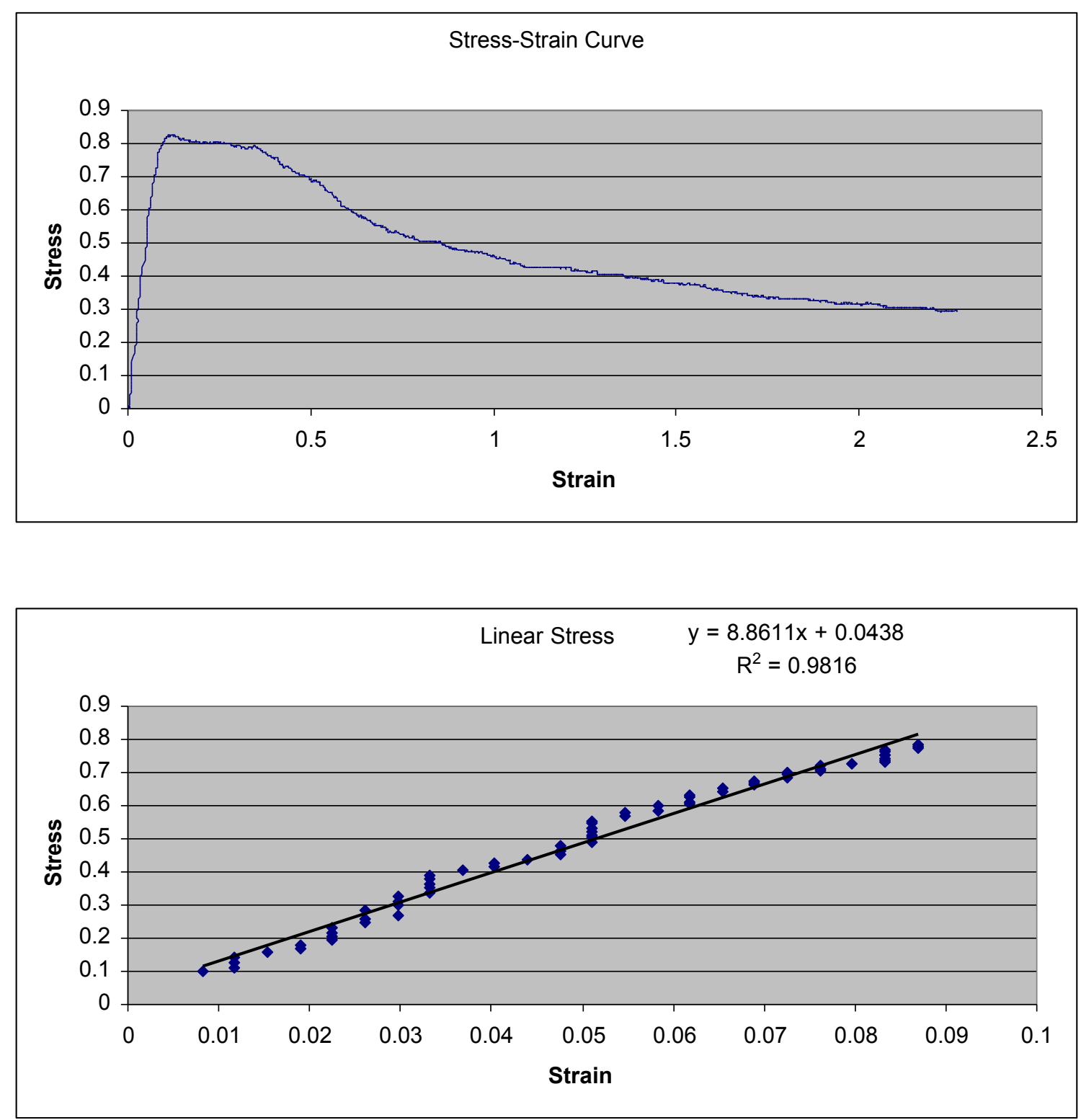


\section{Spin 6}

Section CD: Radial Test
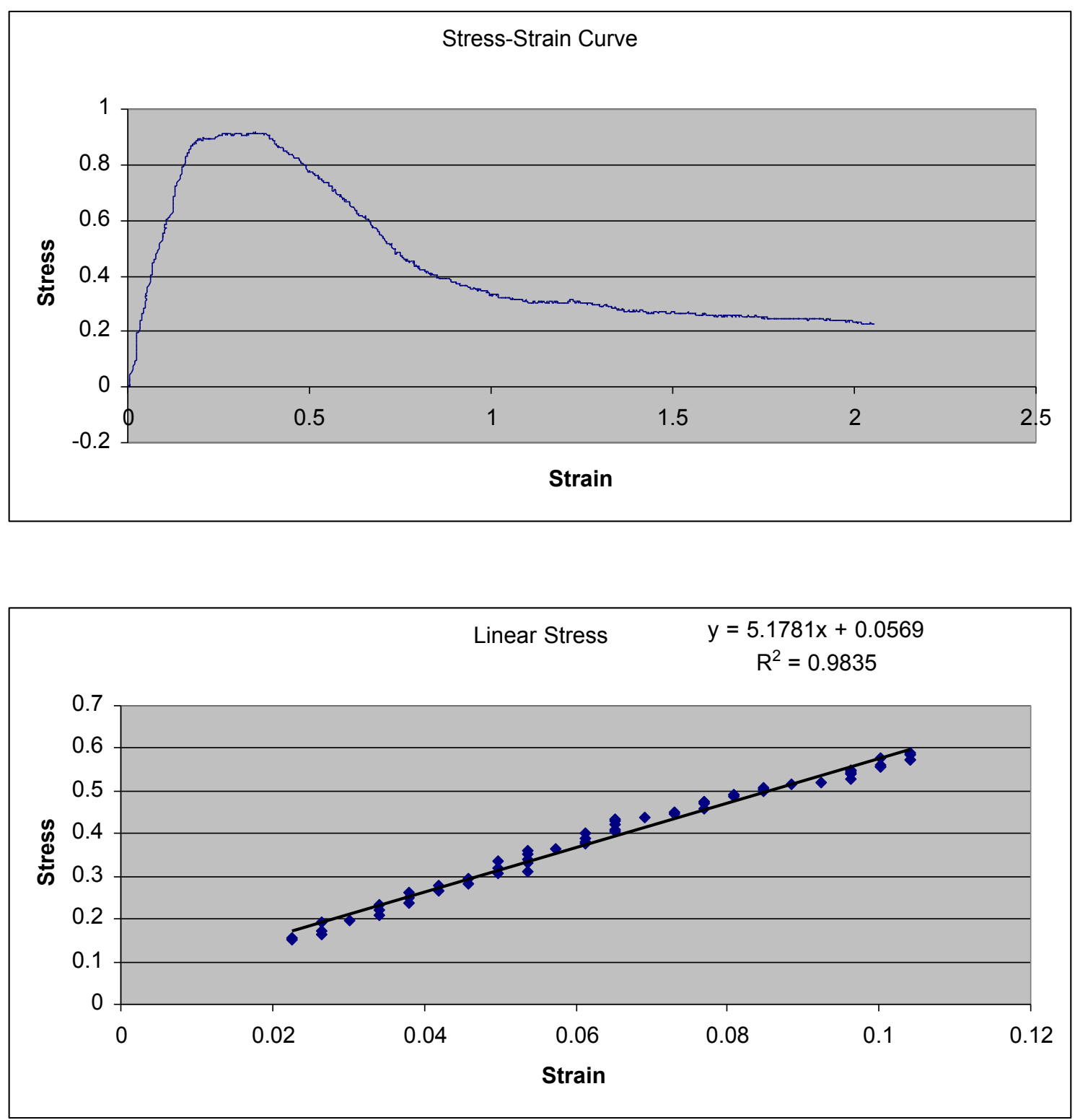


\section{Spin 7}

Section AB: Longitudinal Test
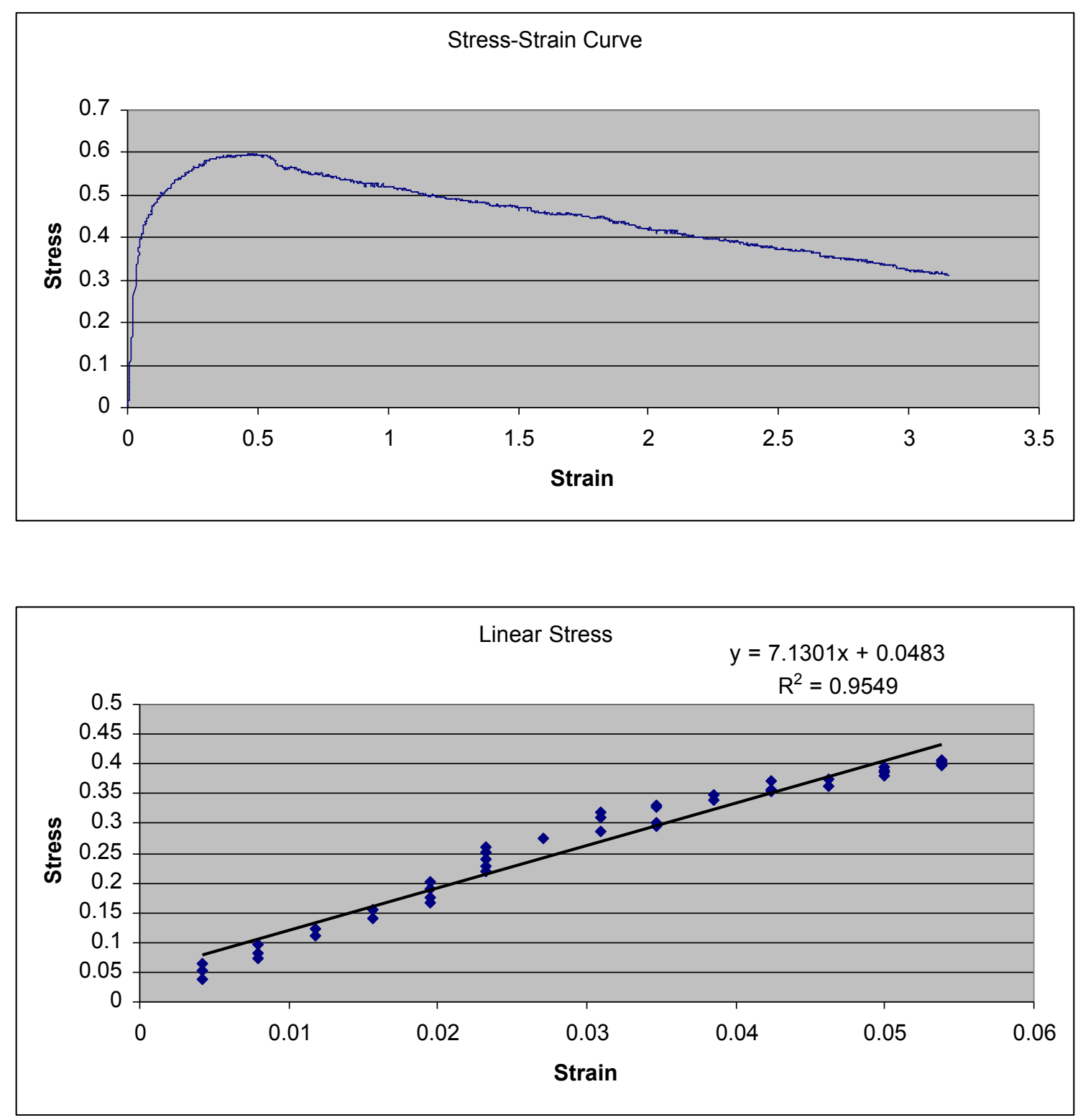


\section{Spin 7}

Section AB: Radial Test
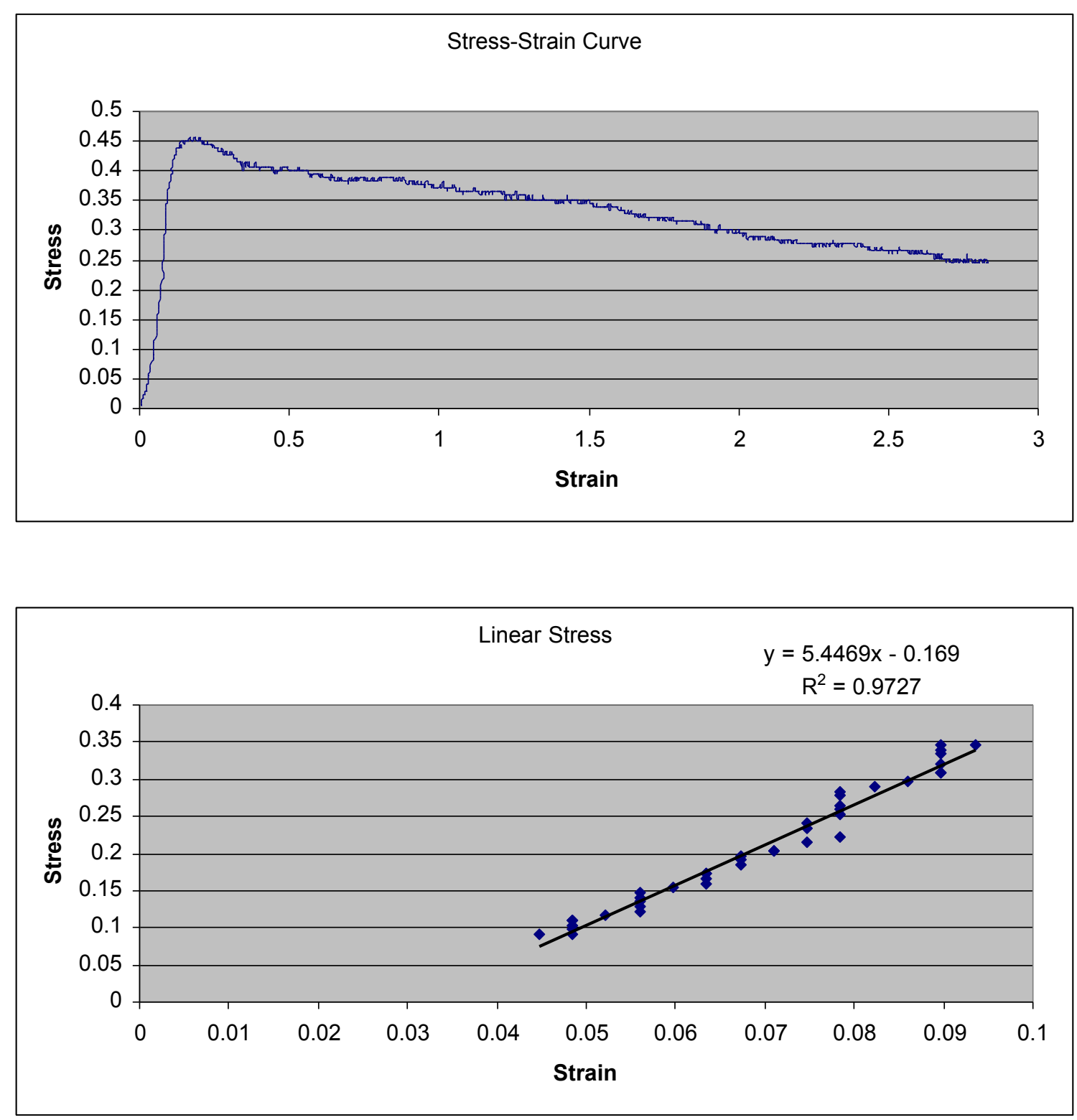


\section{Spin 7}

Section BC: Longitudinal Test
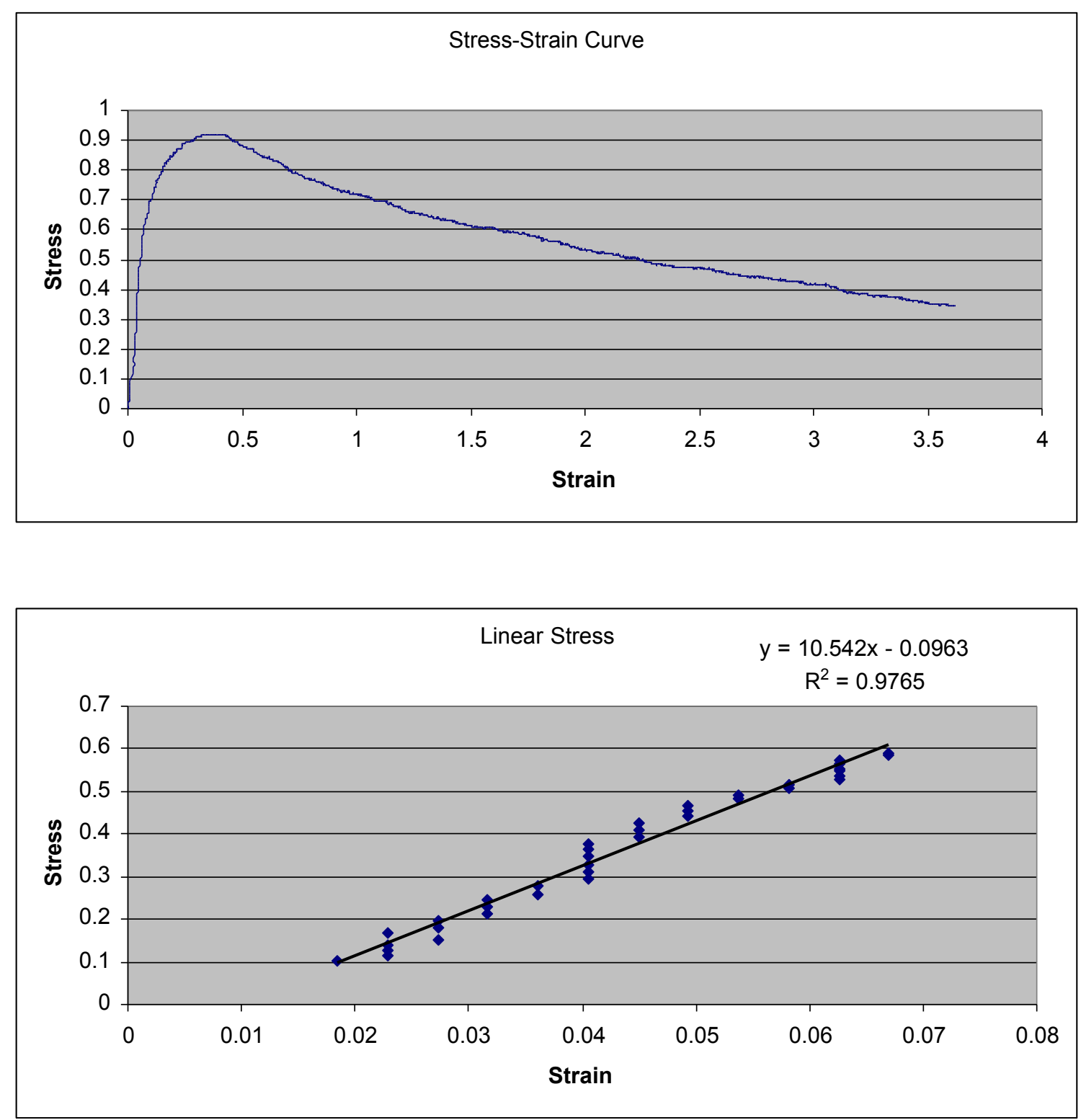


\section{Spin 7}

Section BC: Radial Test
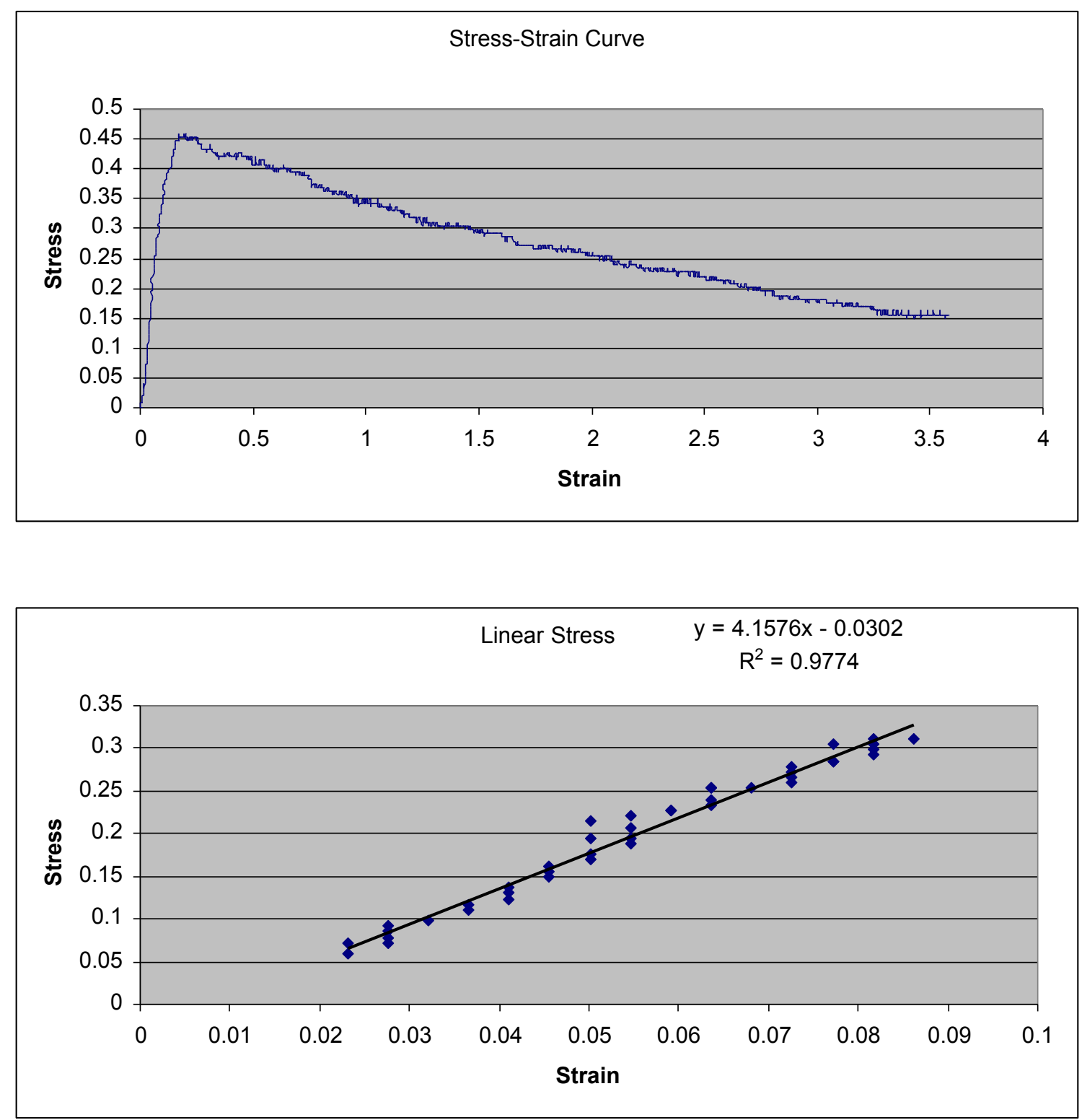


\section{Spin 7}

Section CD: Longitudinal Test
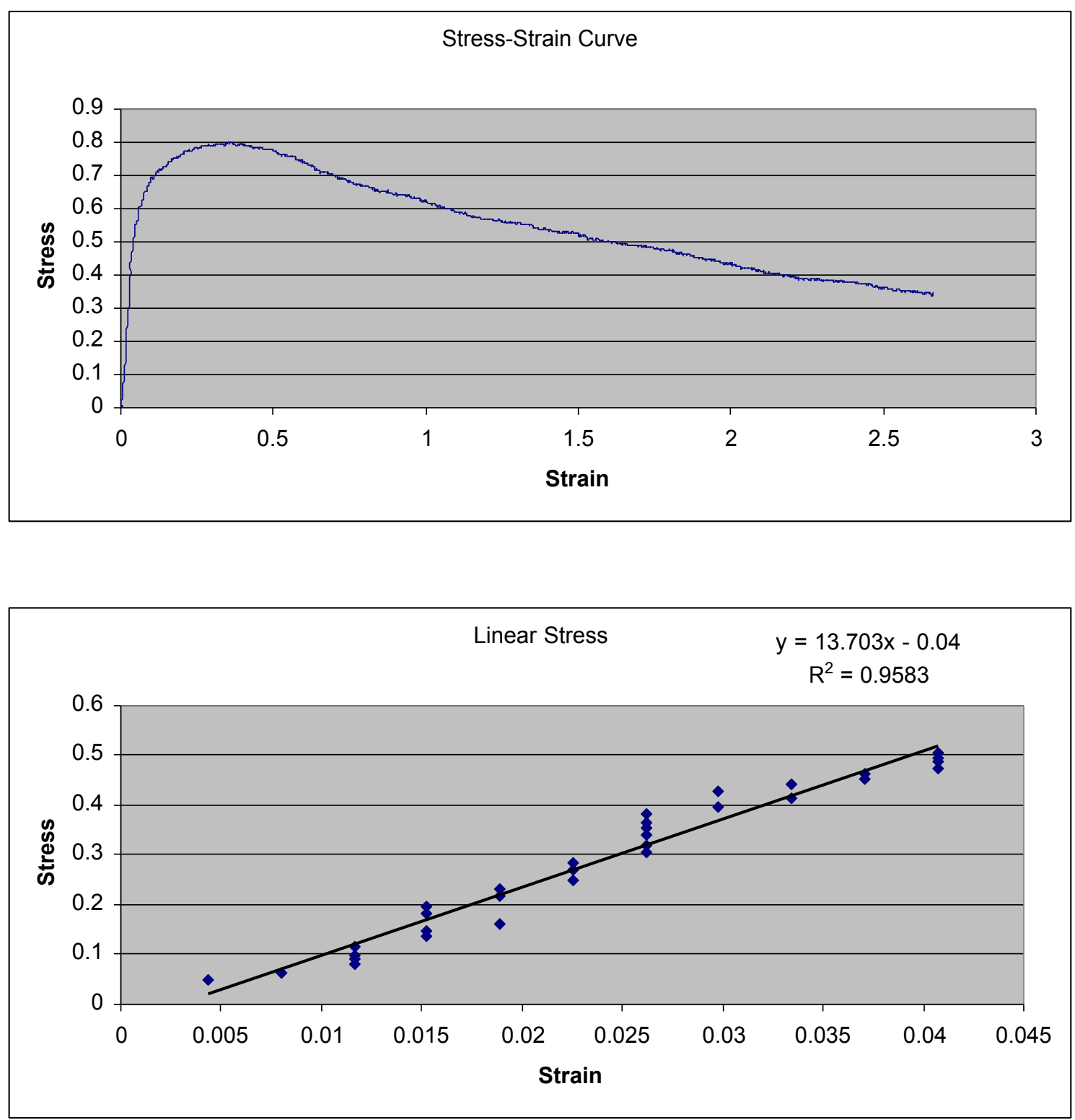


\section{Spin 7}

Section CD: Radial Test
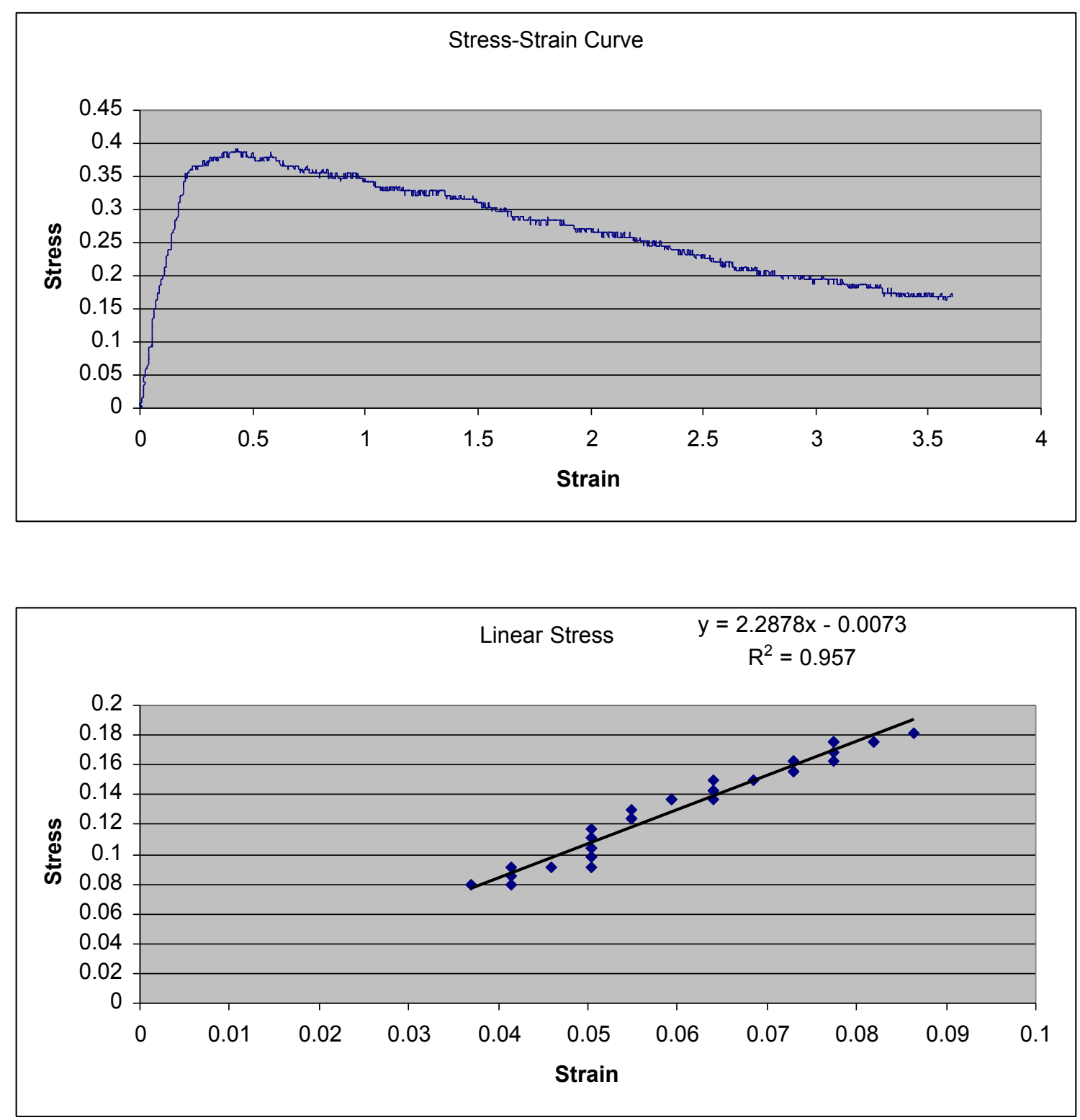


\section{Spin 8}

Section AB: Longitudinal Test
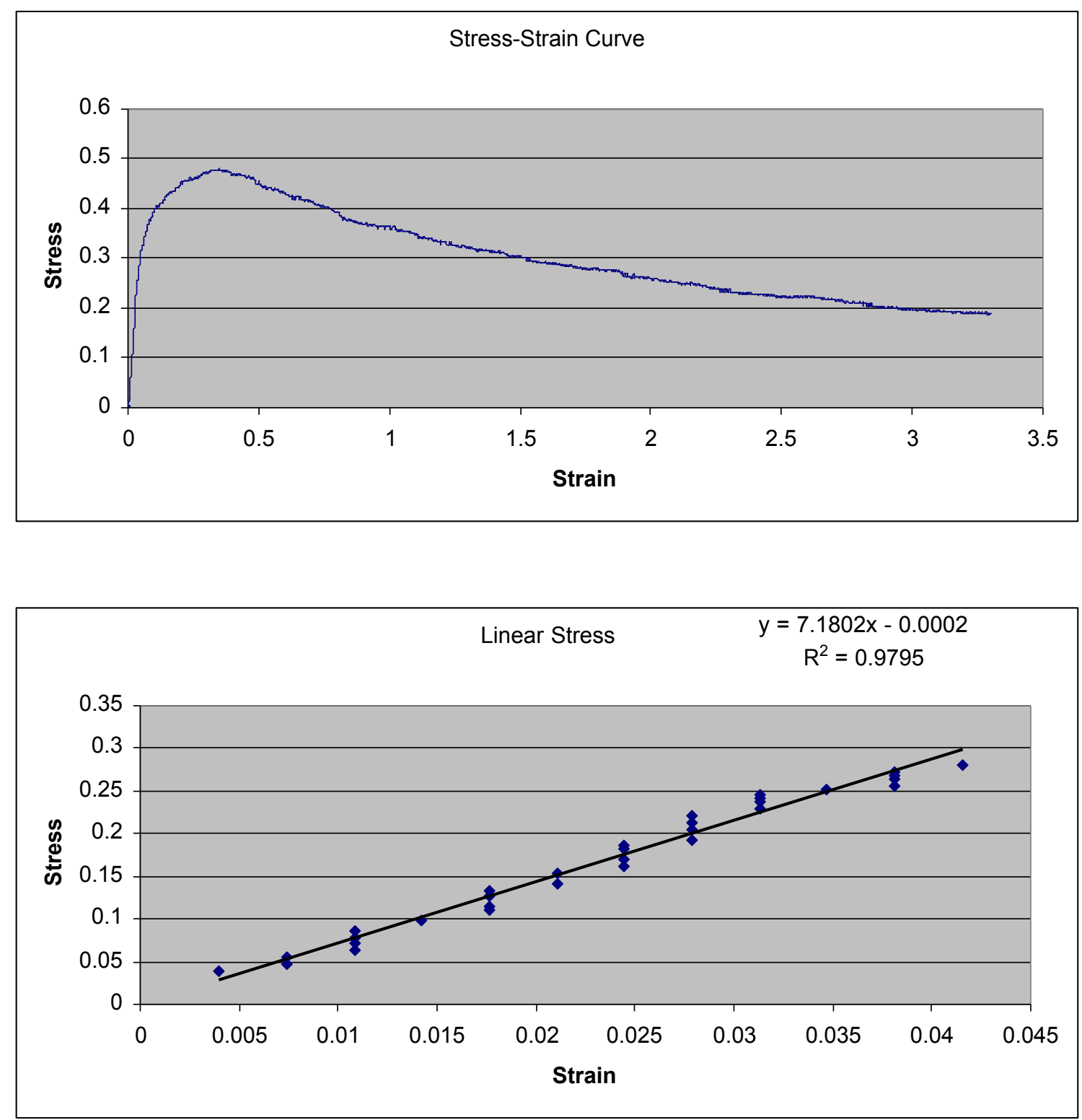


\section{Spin 8}

Section AB: Radial Test
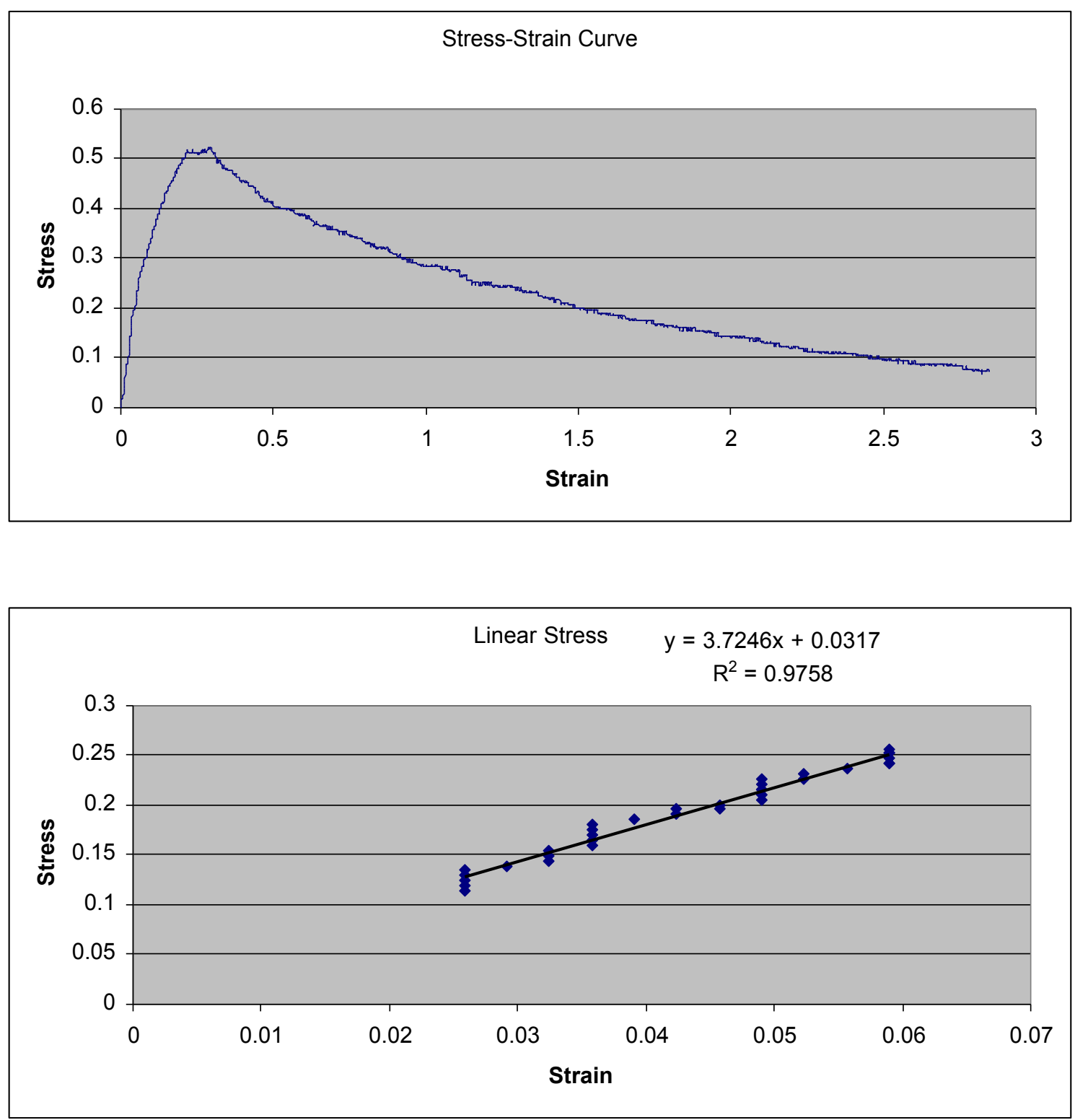


\section{Spin 8}

Section BC: Longitudinal Test
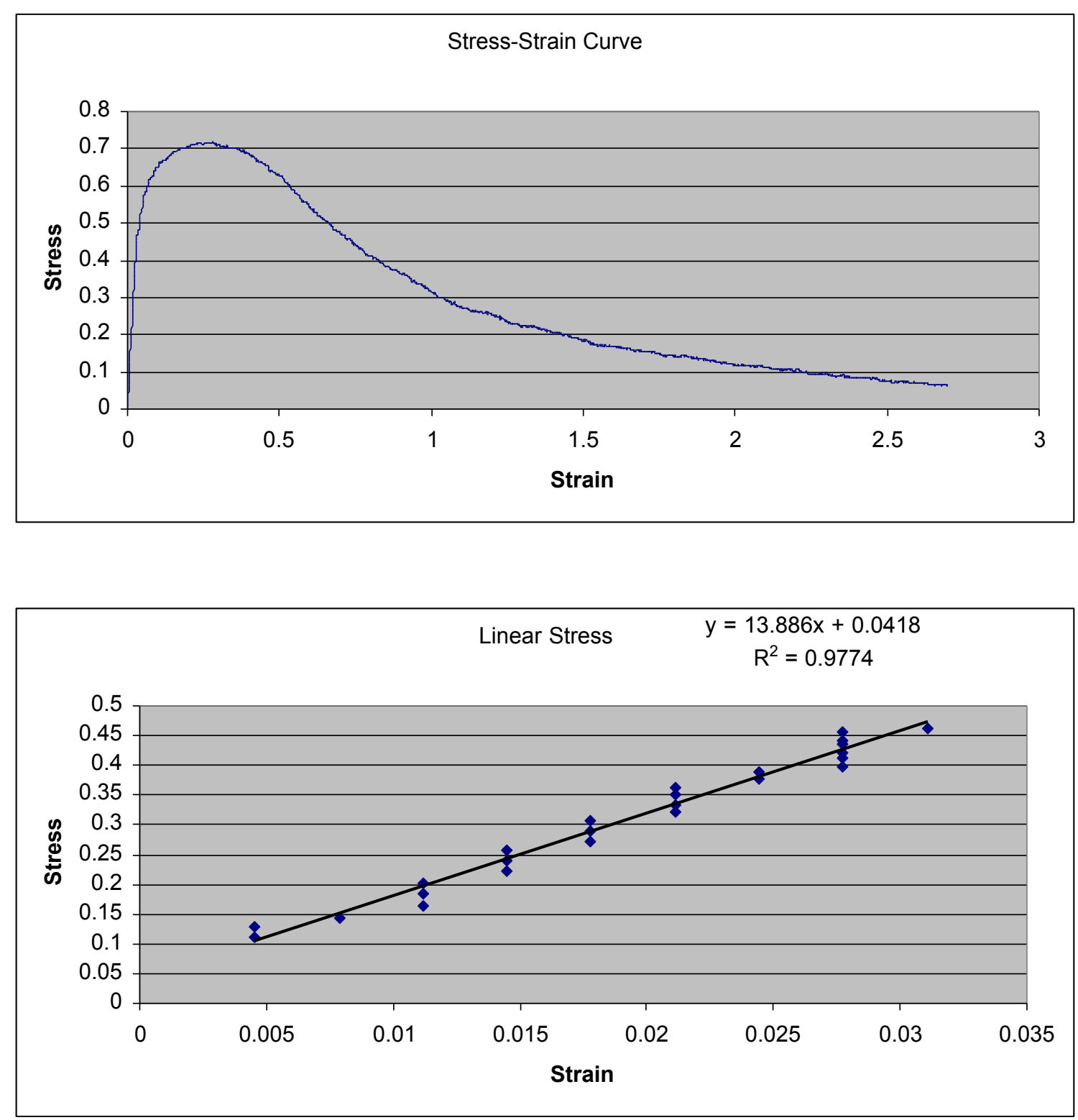


\section{Spin 8}

Section BC: Radial Test
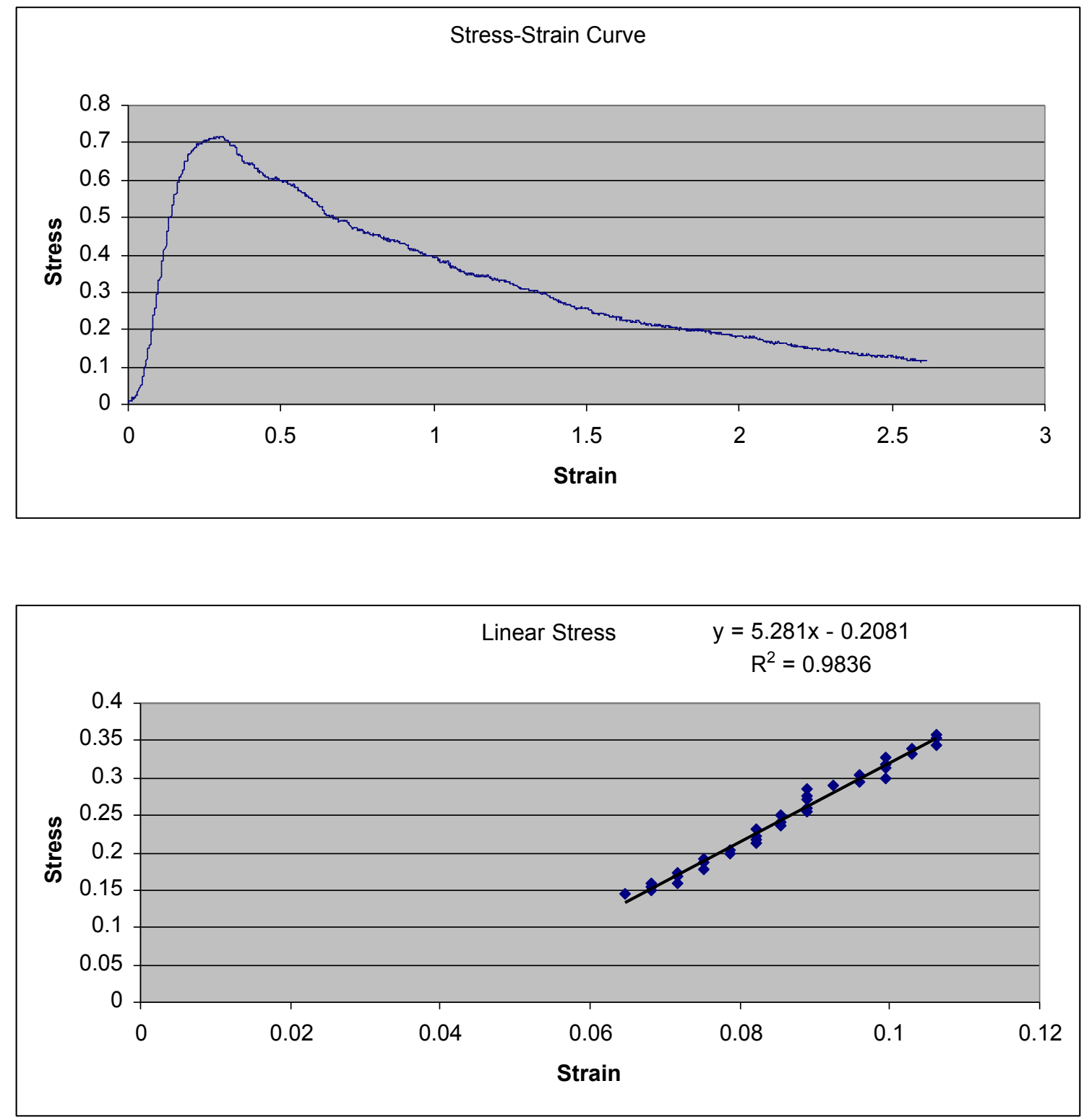


\section{Spin 8}

Section CD: Longitudinal Test
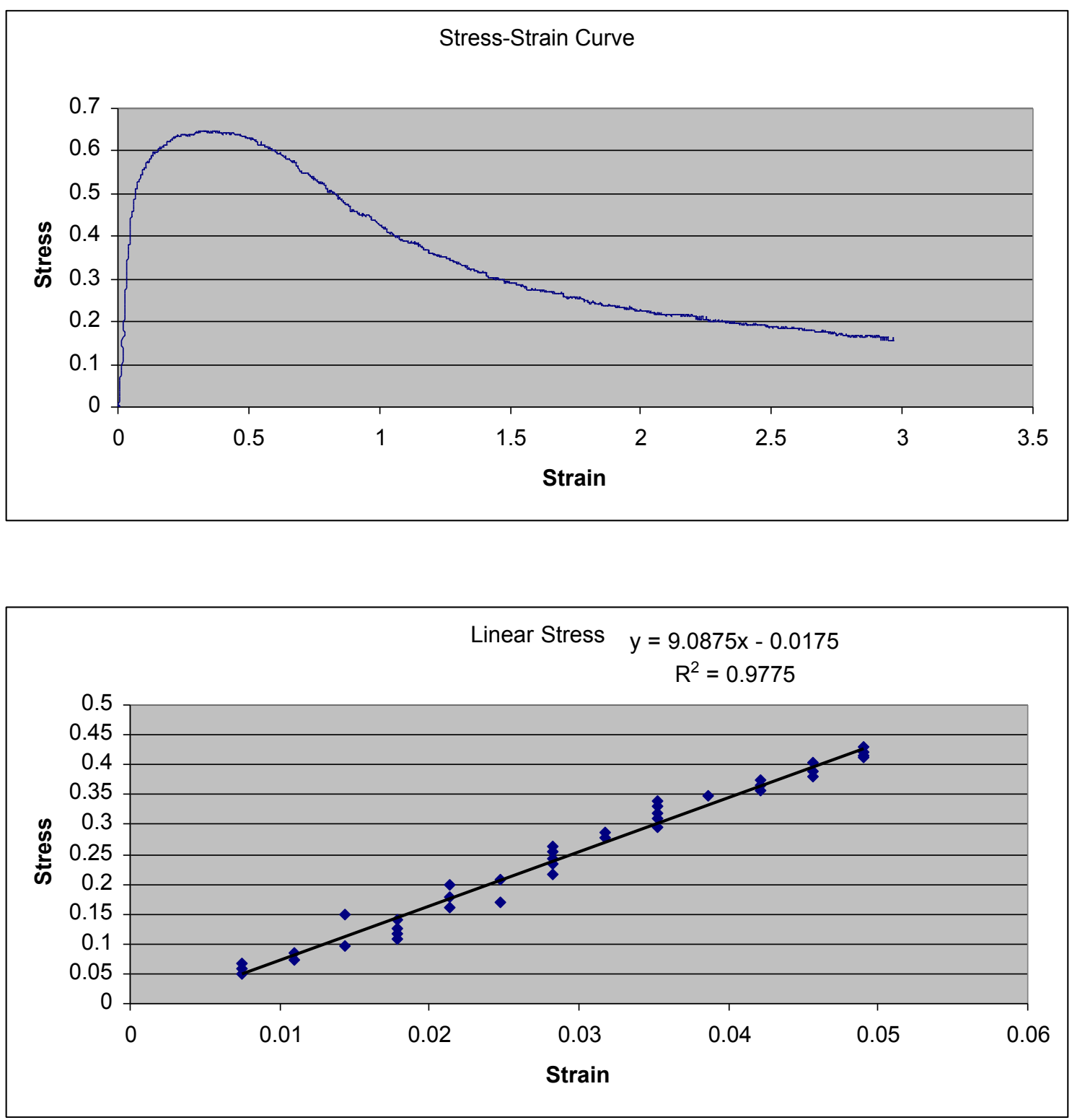


\section{Spin 8}

Section CD: Radial Test
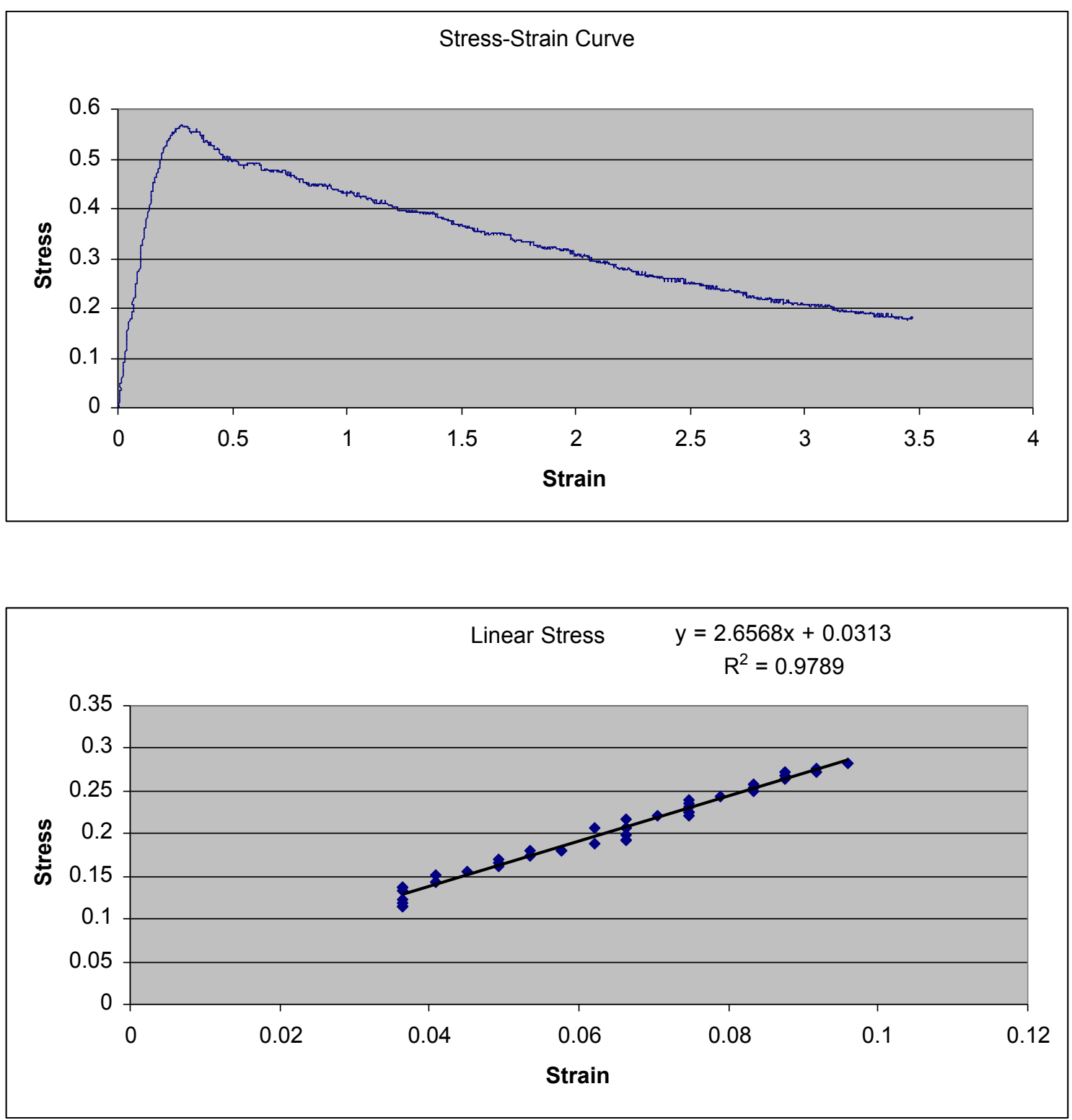


\section{Spin 9}

Section AB: Longitudinal Test
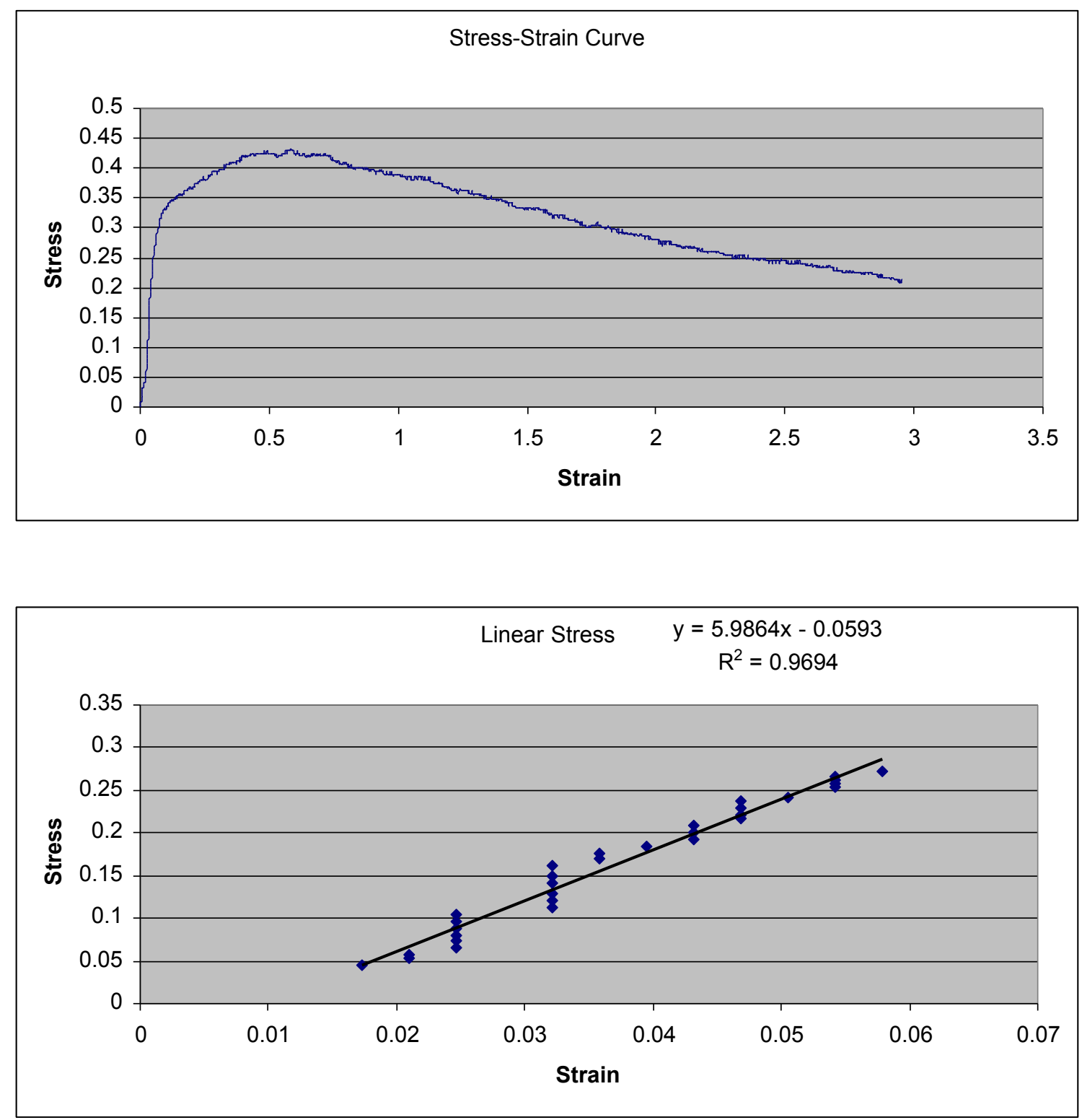


\section{Spin 9}

Section AB: Radial Test
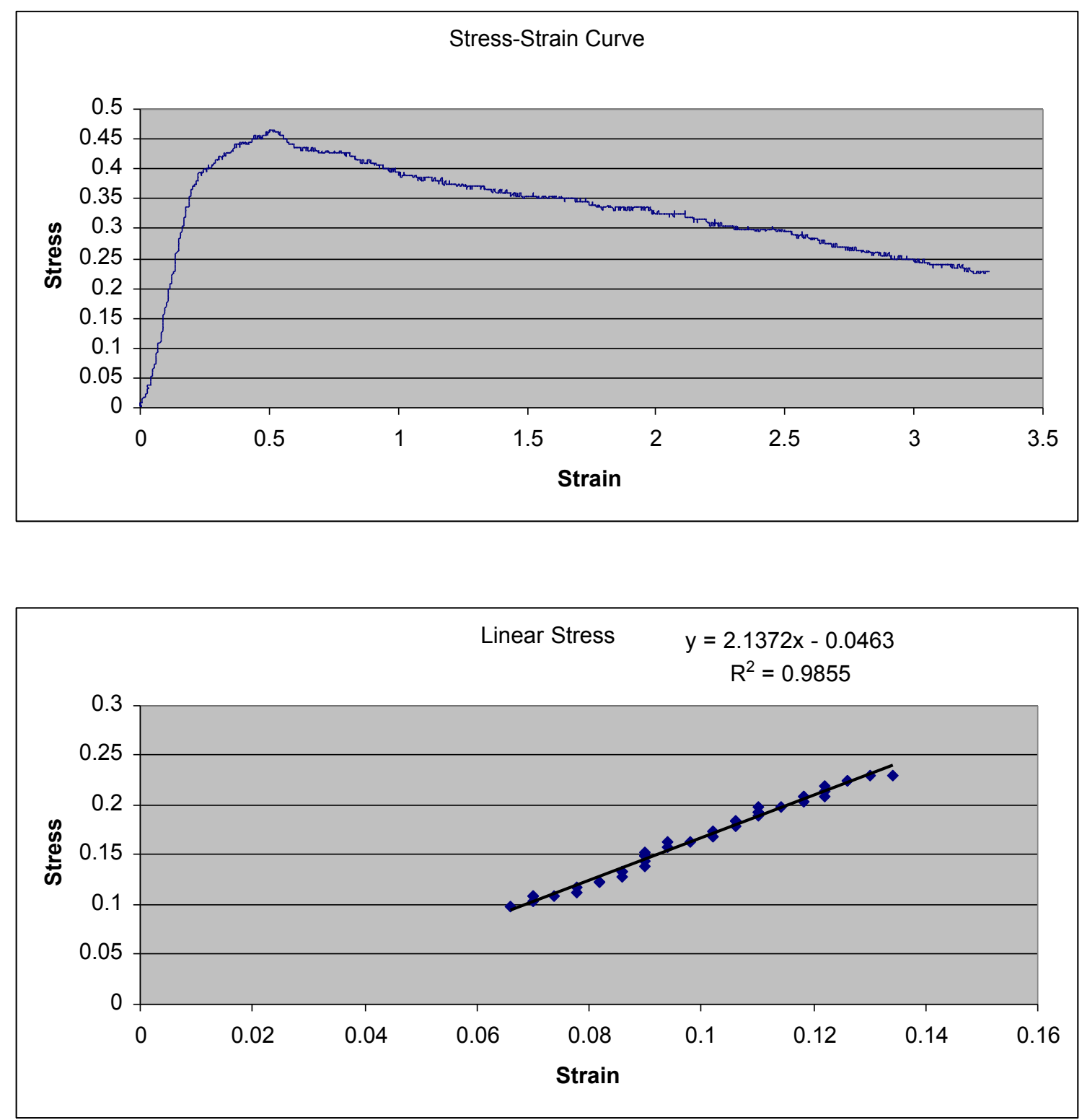


\section{Spin 9}

Section BC: Longitudinal Test
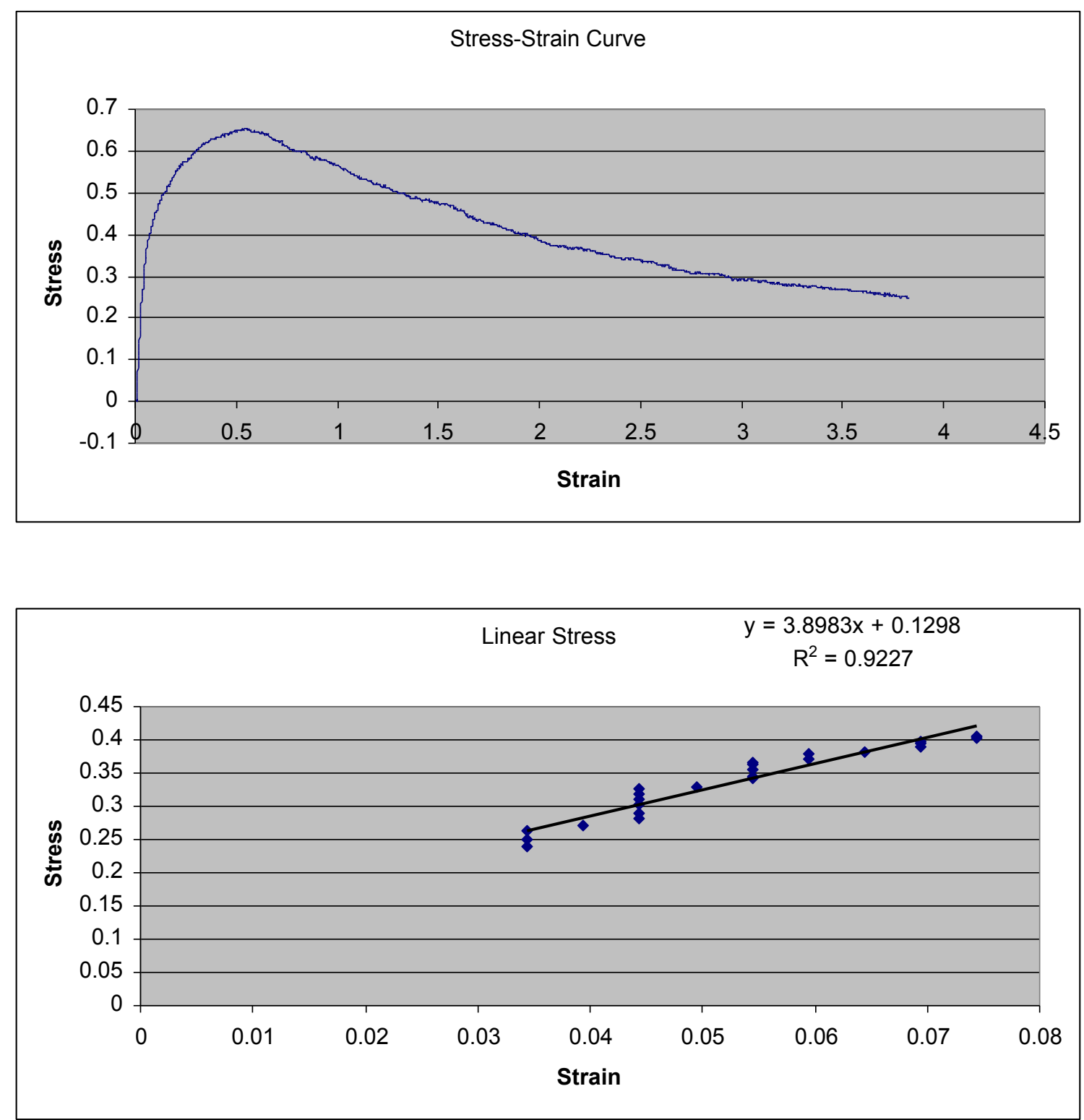


\section{Spin 9}

Section BC: Radial Test
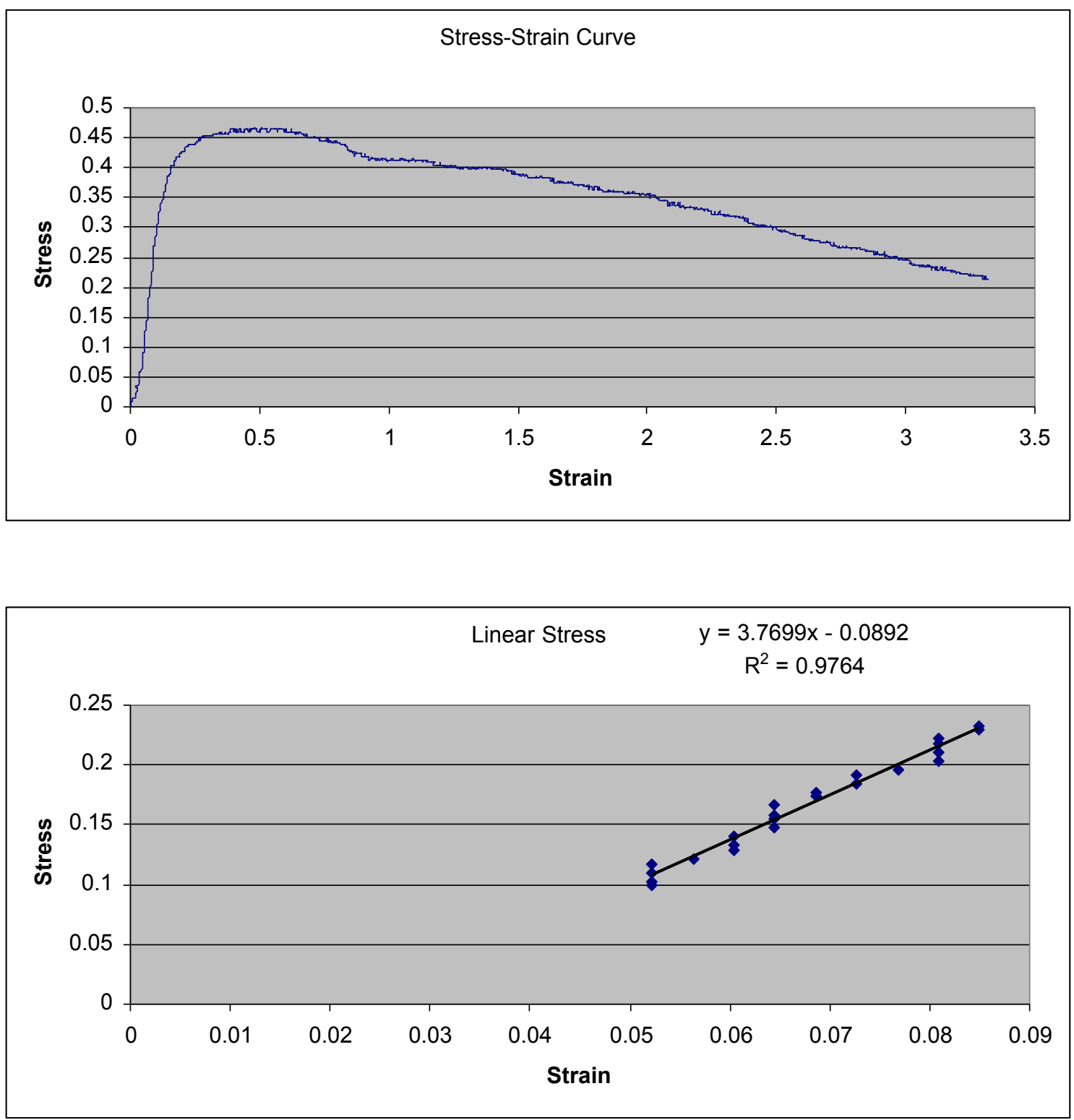


\section{Spin 9}

Section CD: Longitudinal Test
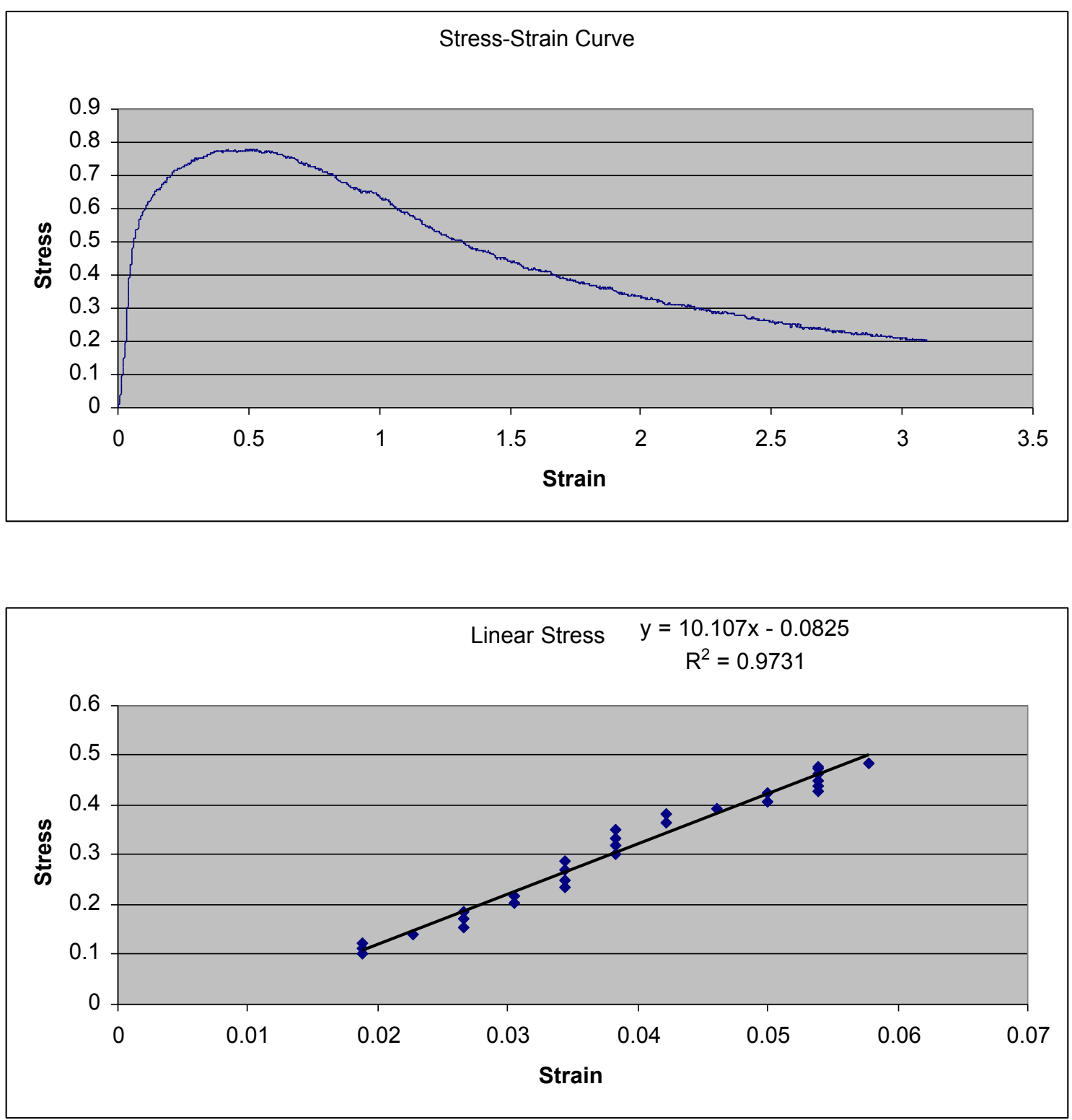


\section{Spin 9}

Section CD: Radial Test
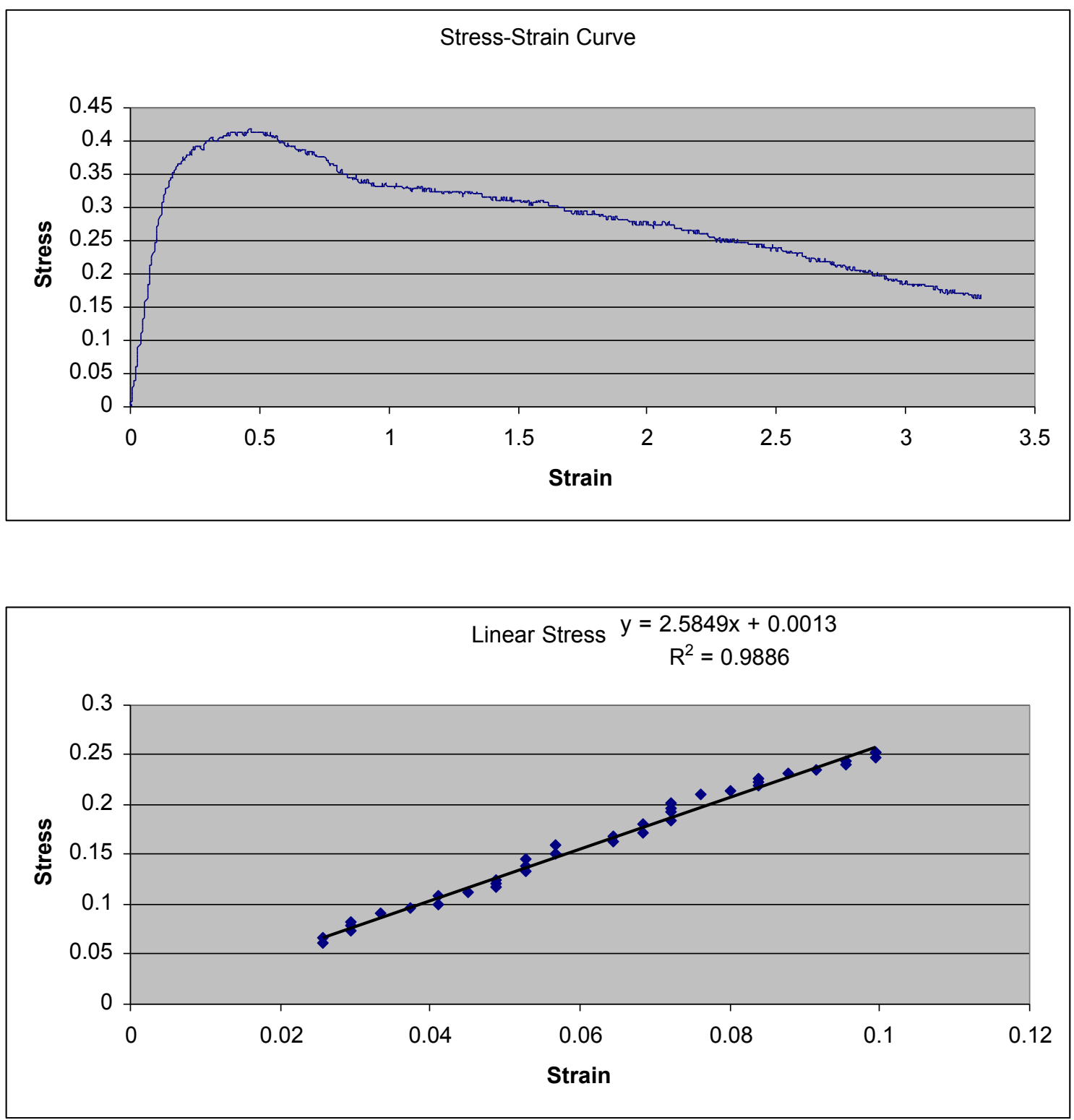


\section{Spin 10}

Section AB: Longitudinal Test
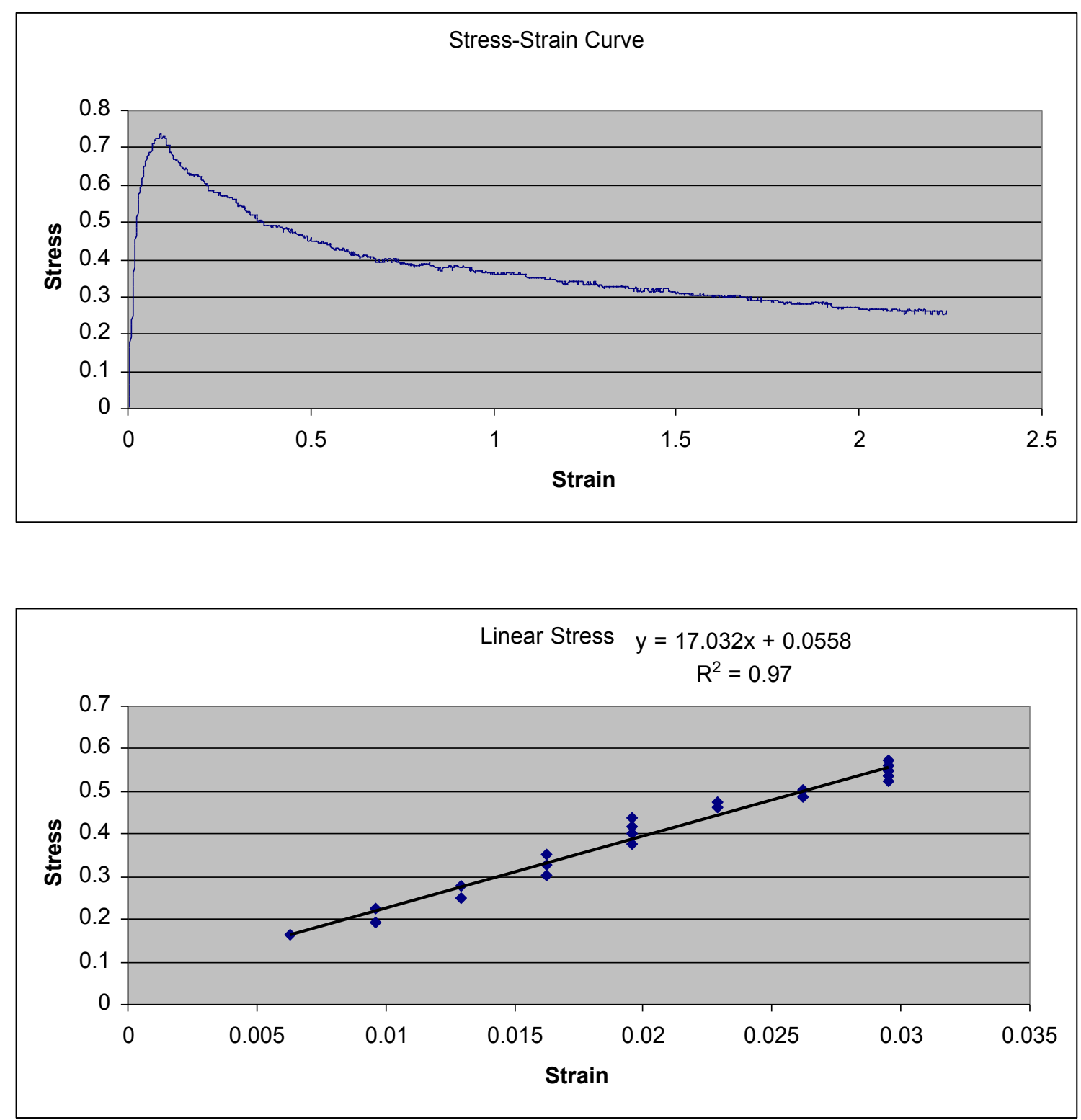


\section{Spin 10}

Section AB: Radial Test
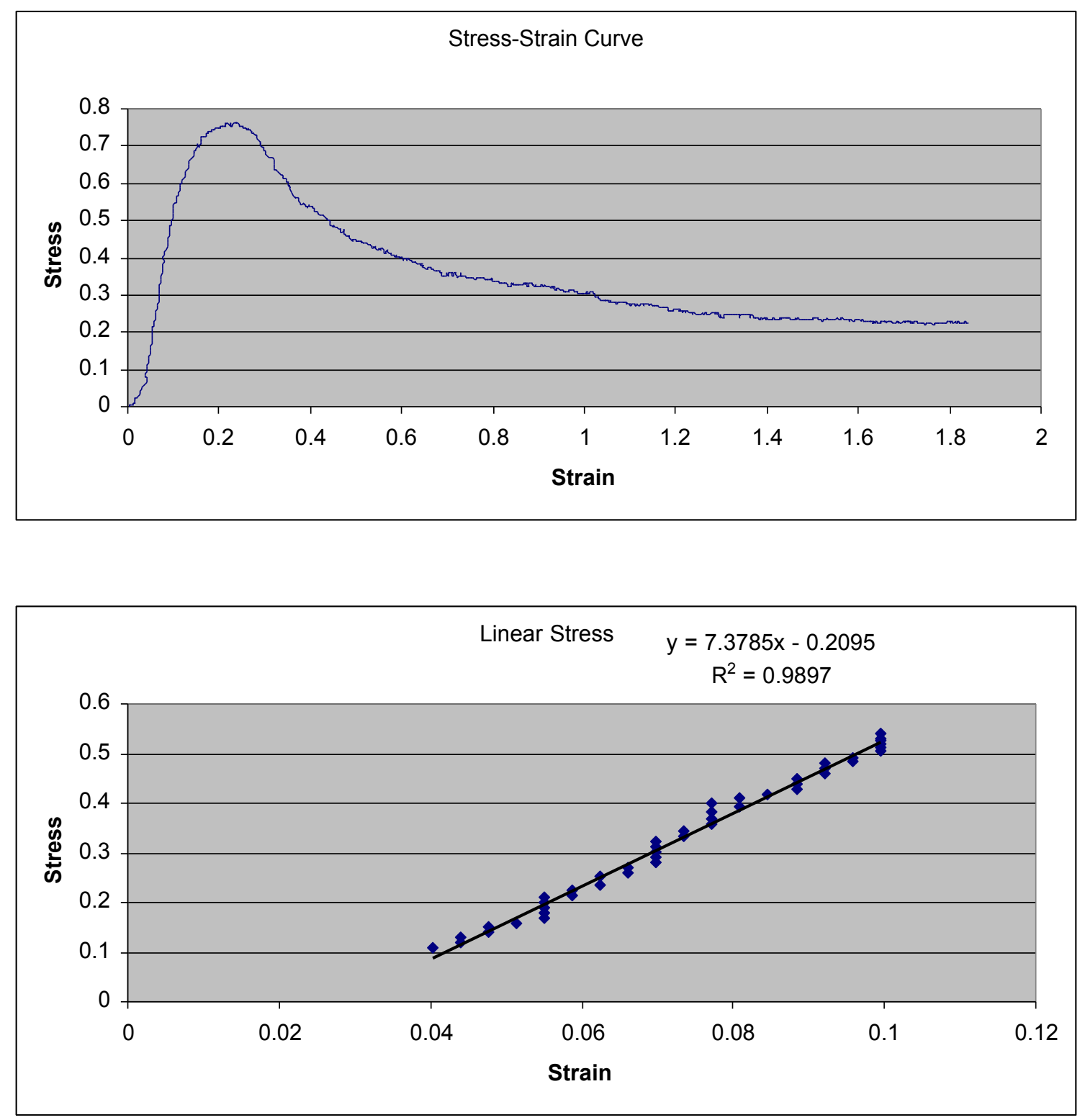


\section{Spin 10}

Section BC: Longitudinal Test
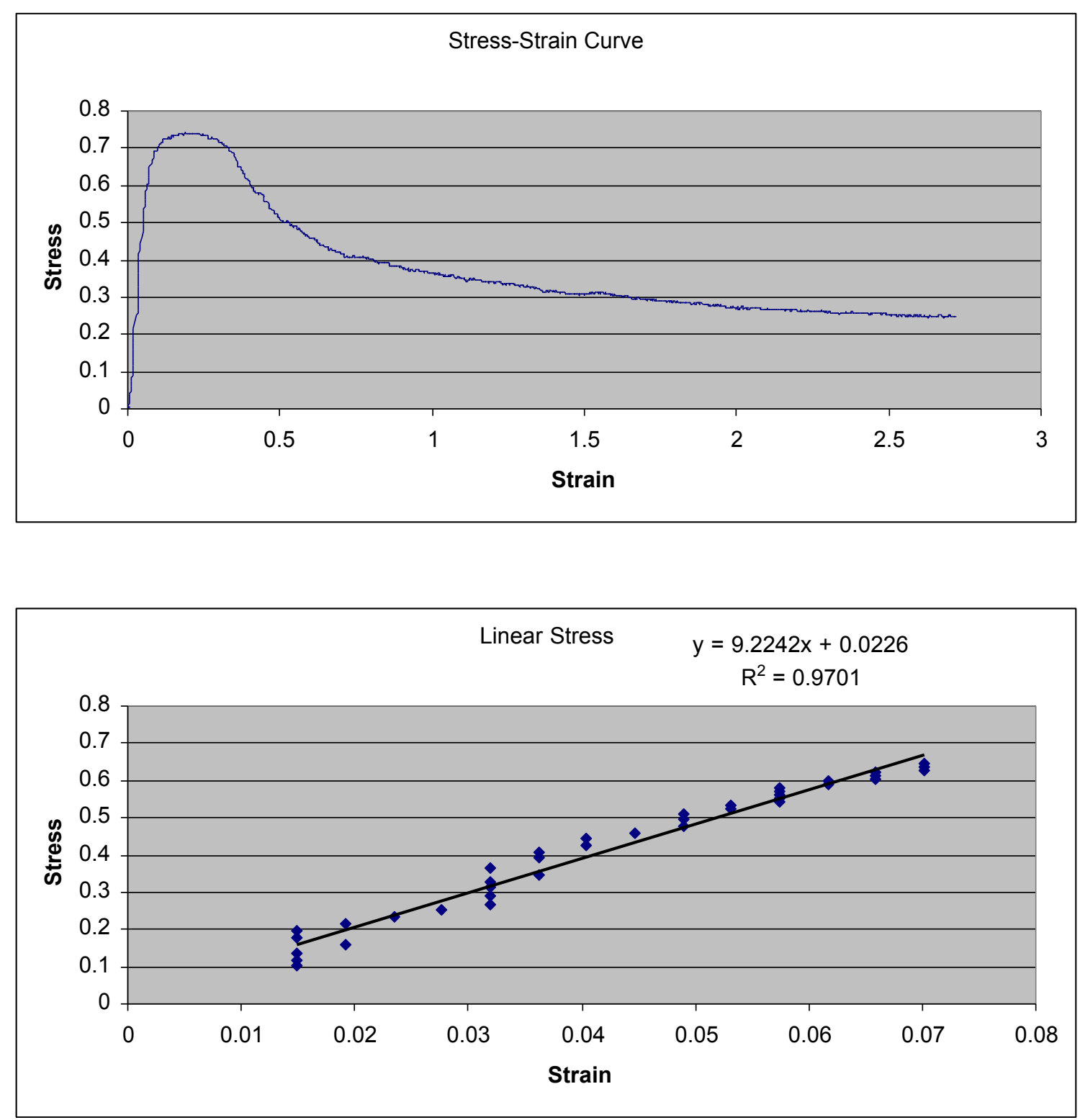


\section{Spin 10}

Section BC: Radial Test
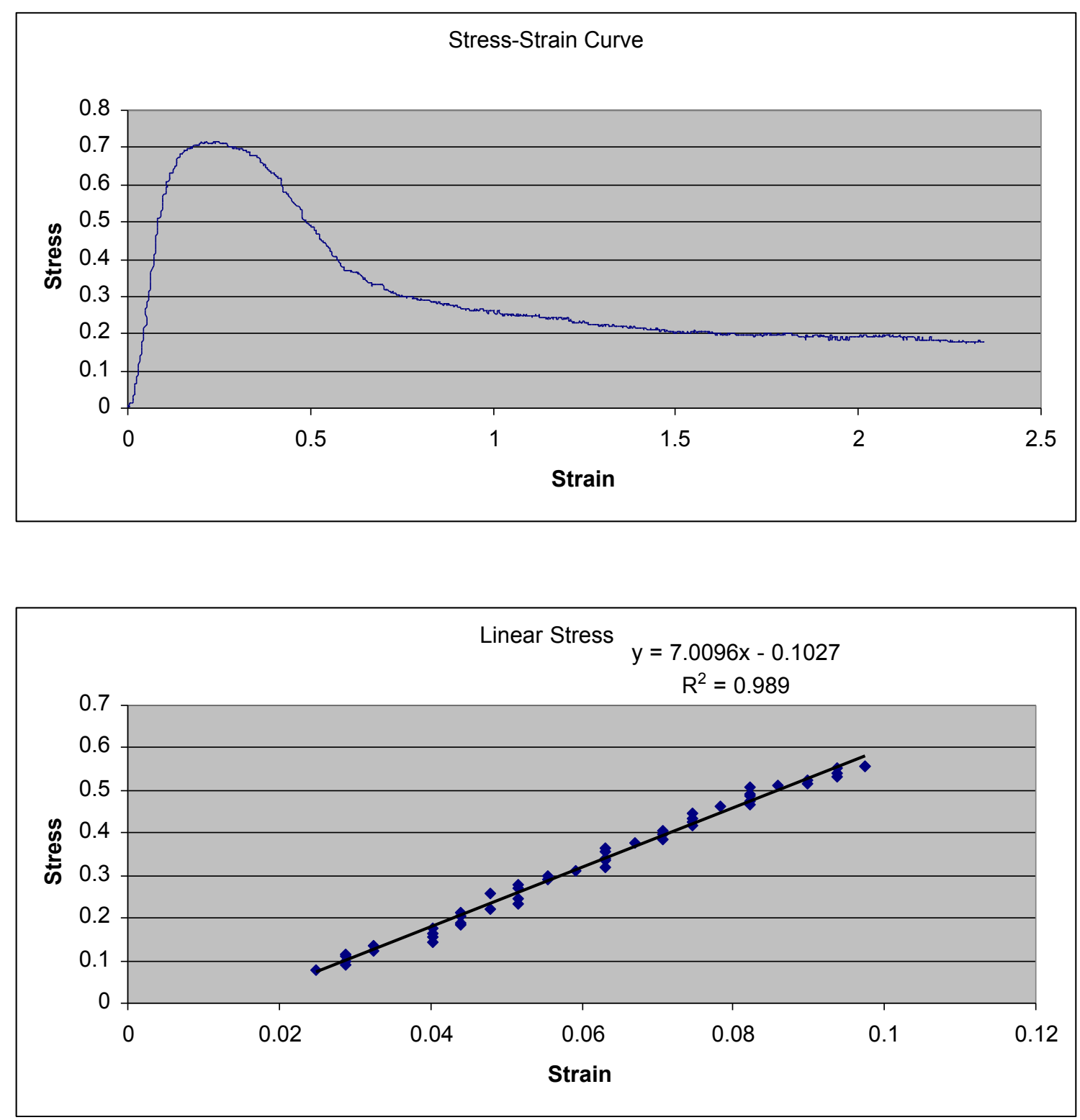


\section{Spin 10}

Section CD: Longitudinal Test
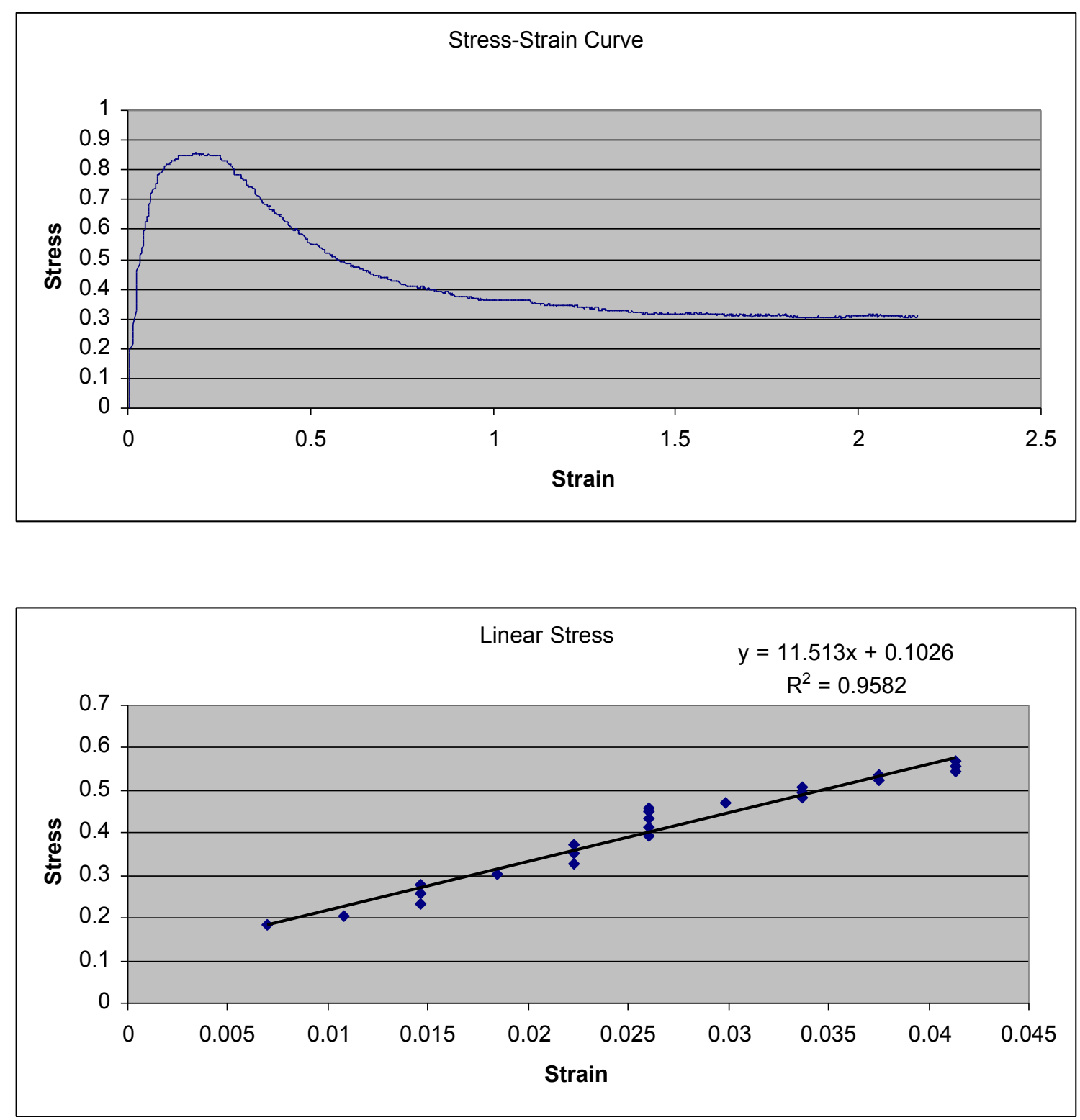


\section{Spin 10}

Section CD: Radial Test
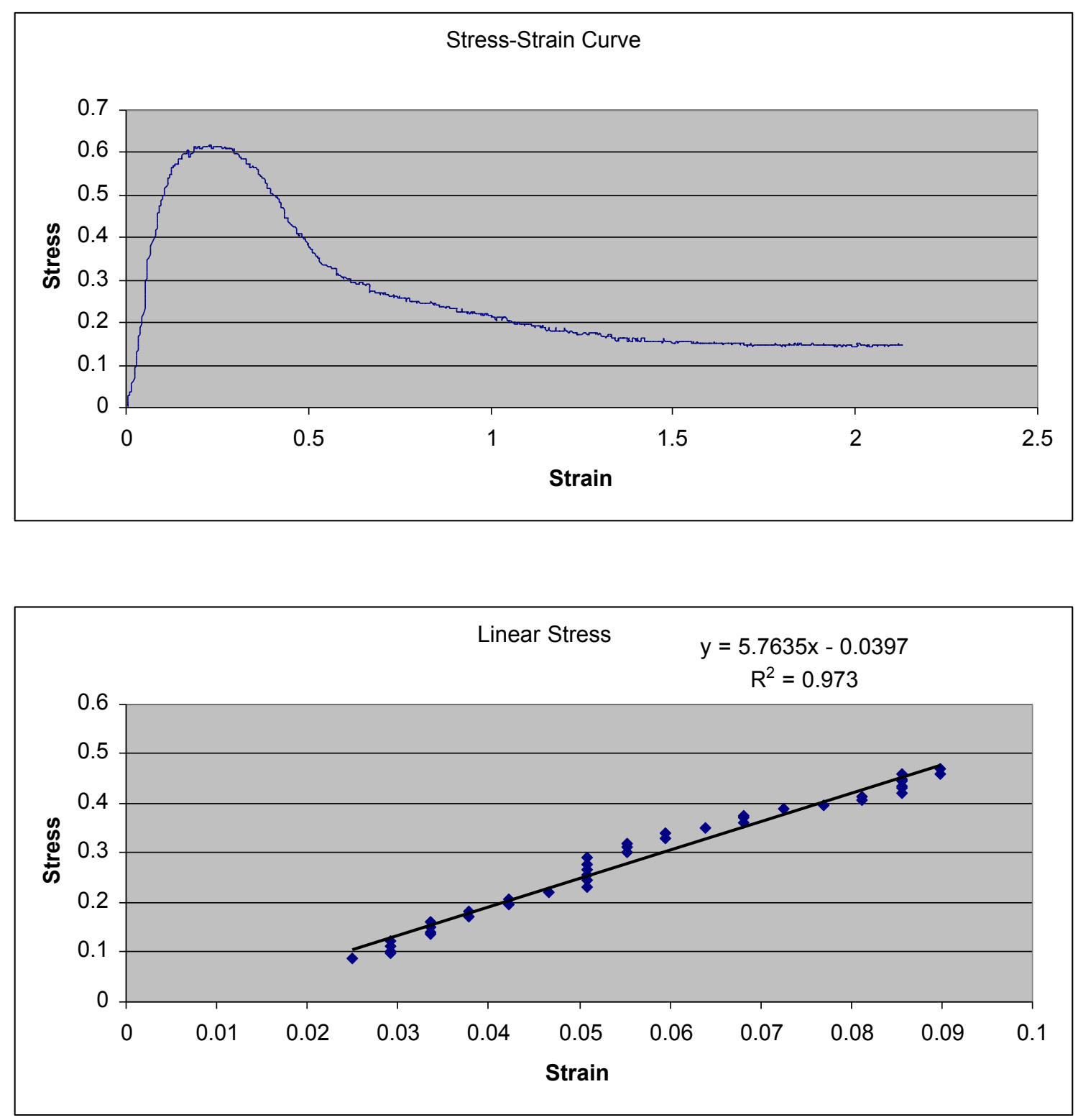


\section{Spin 11}

Section AB: Longitudinal Test
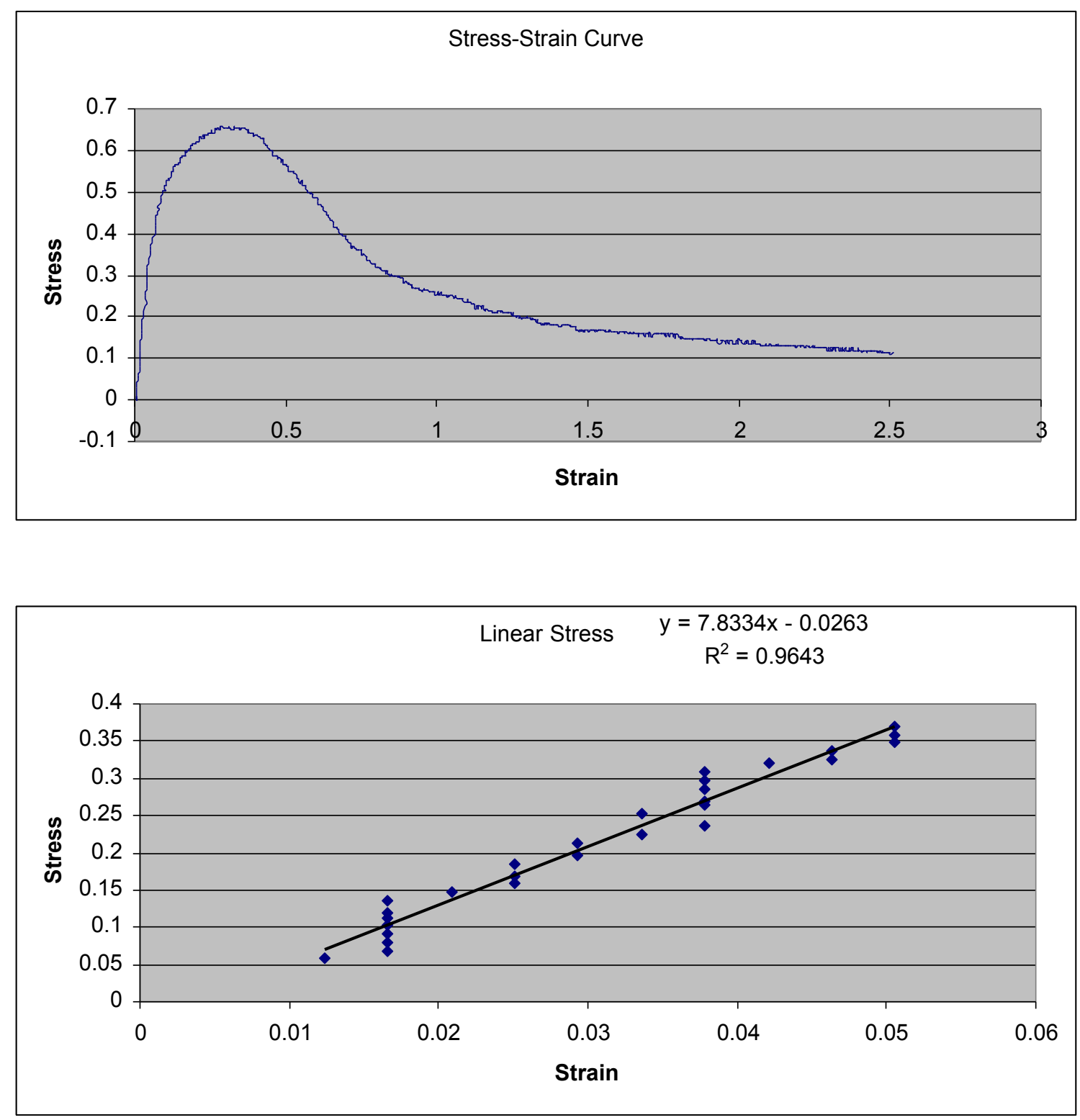


\section{Spin 11}

Section AB: Radial Test
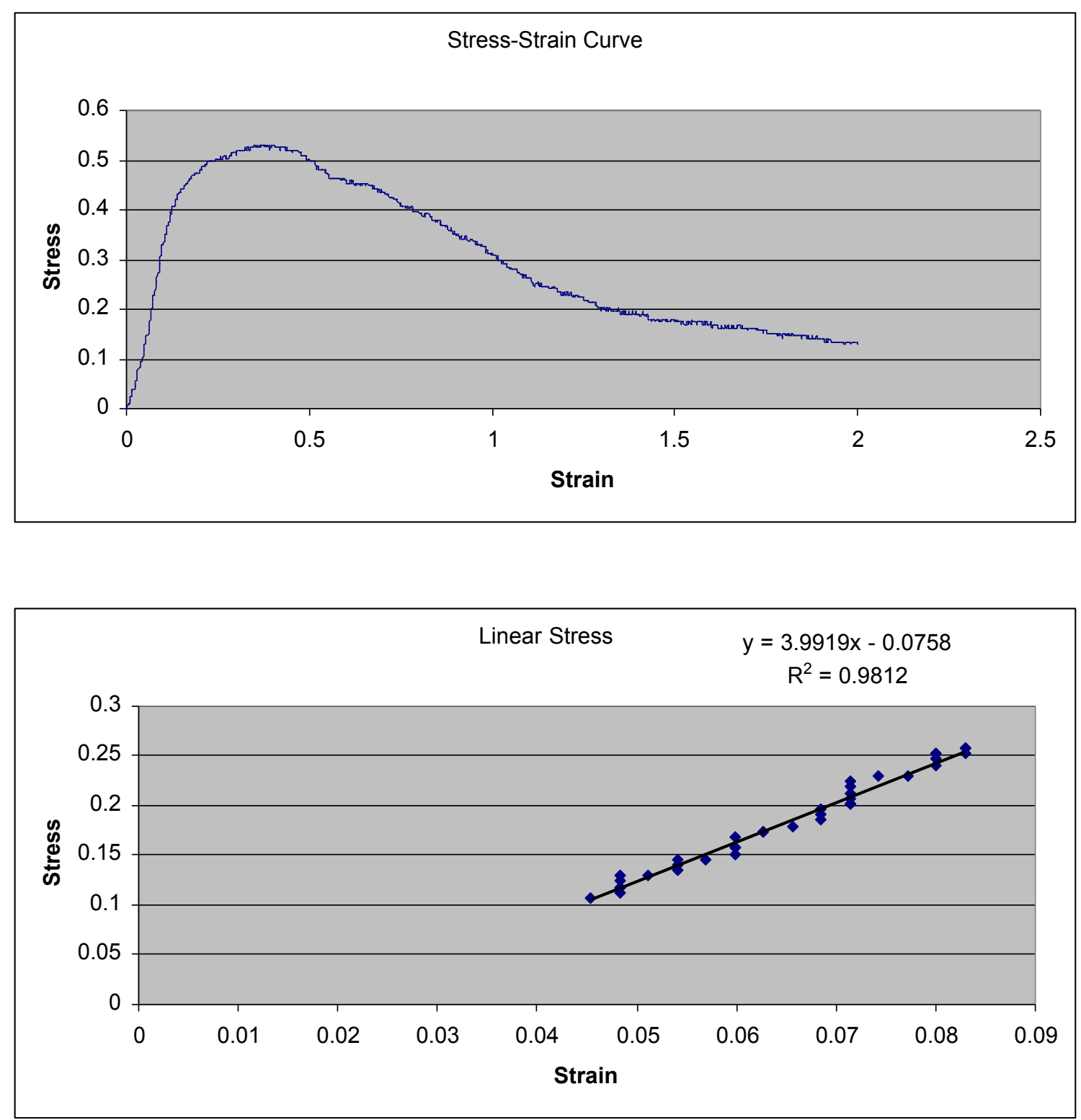


\section{Spin 11}

Section BC: Longitudinal Test
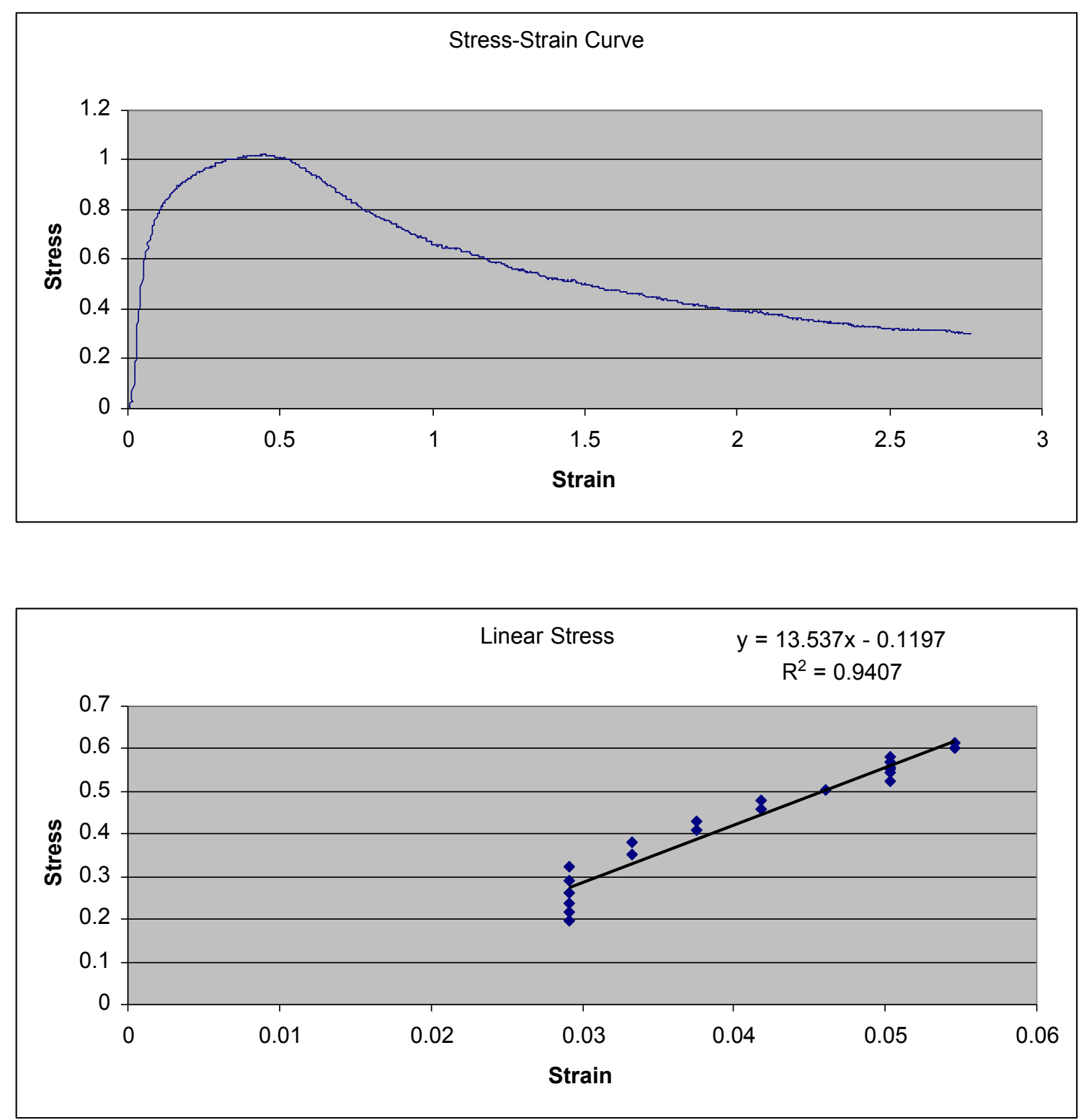


\section{Spin 11}

Section BC: Radial Test
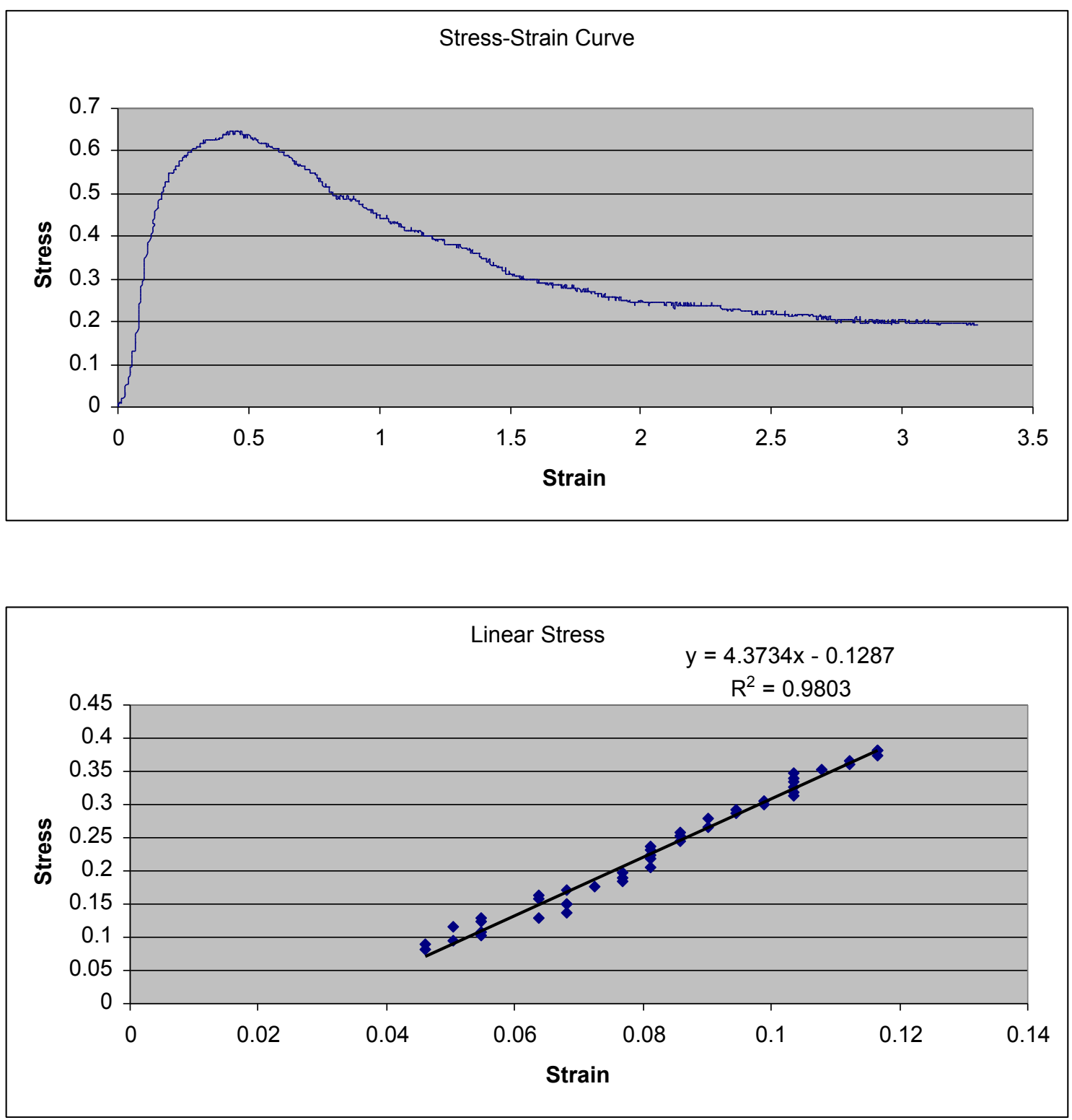


\section{Spin 11}

Section CD: Longitudinal Test
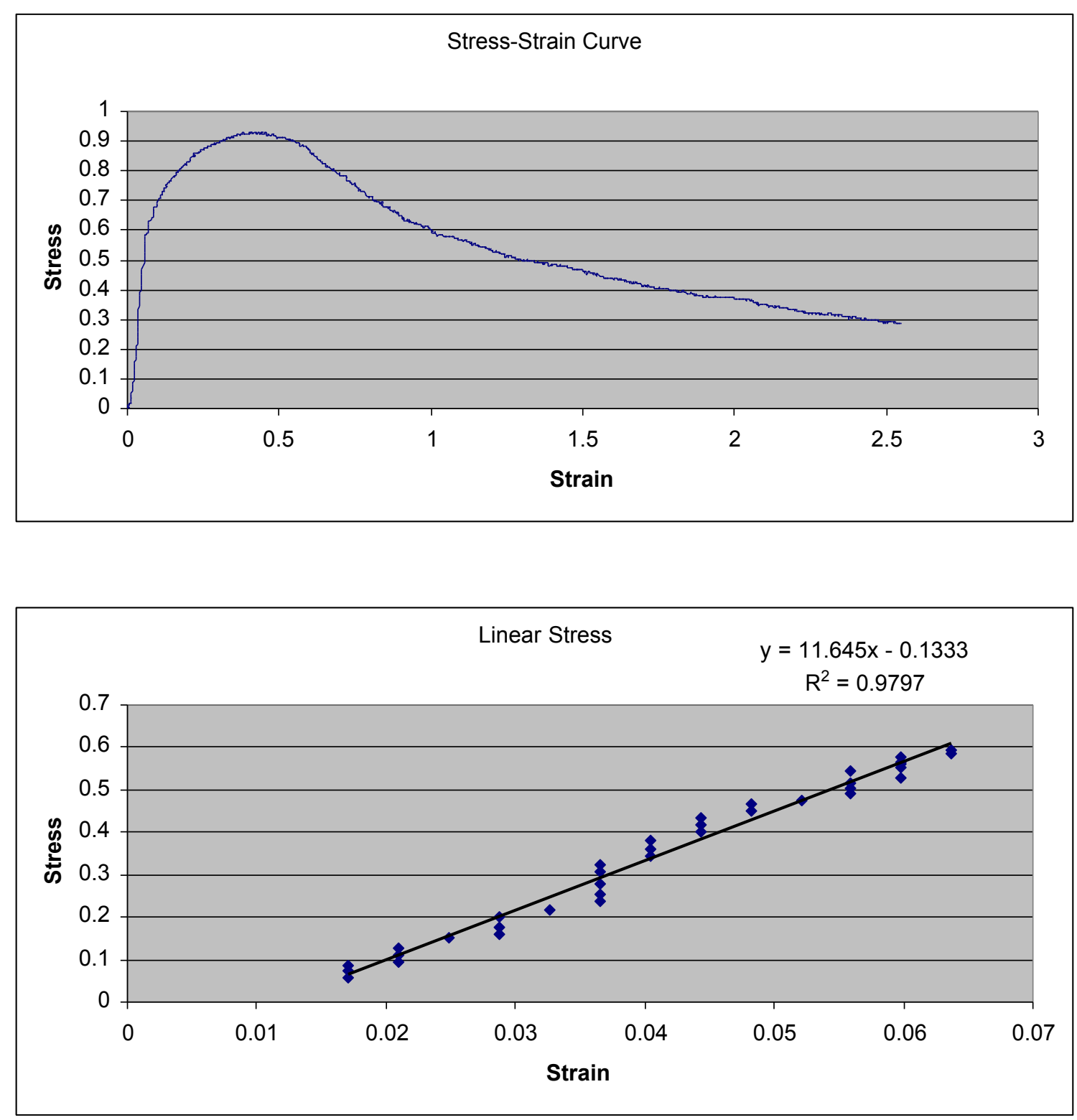


\section{Spin 11}

Section CD: Radial Test
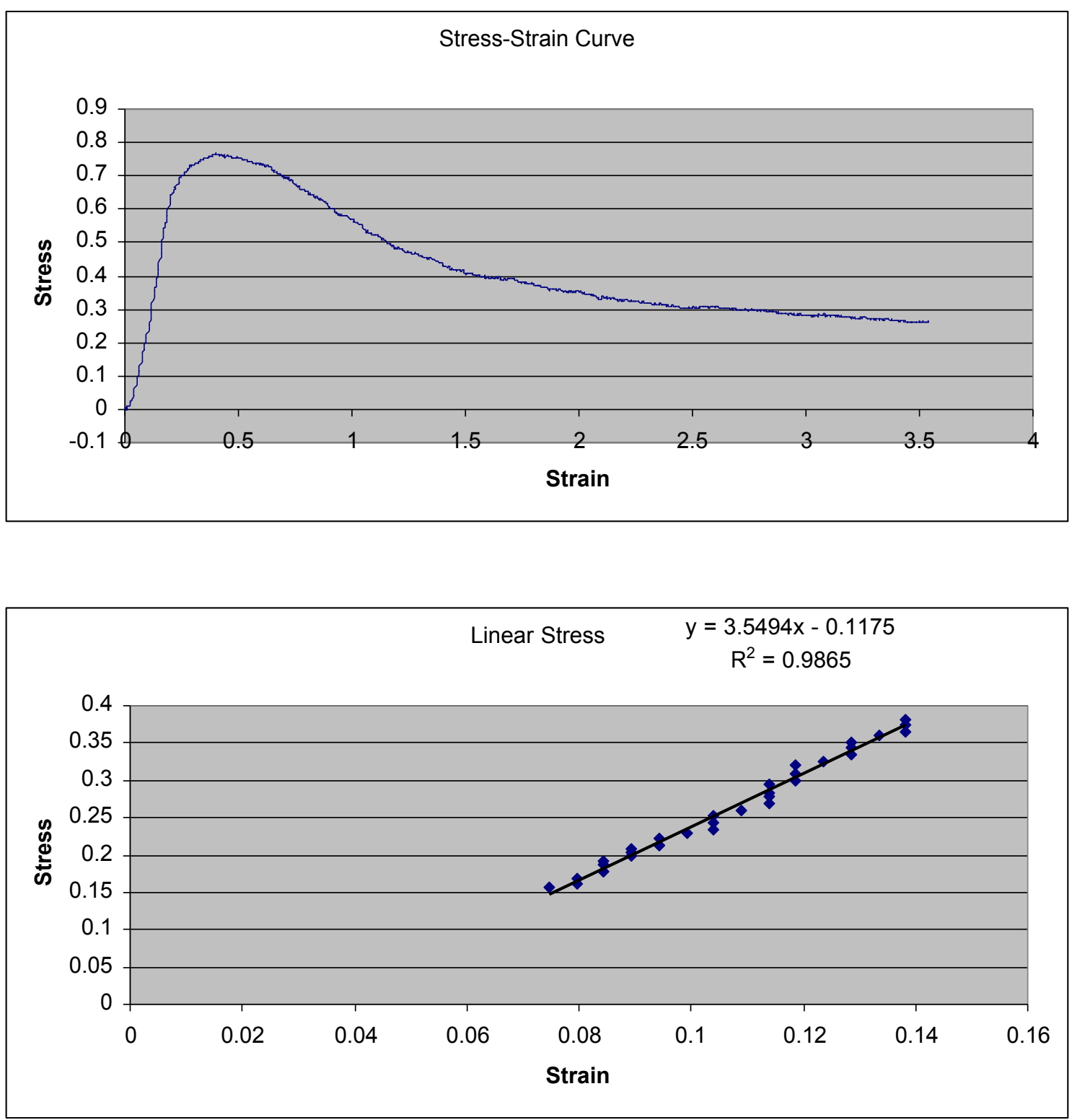


\section{APPENDIX H: INTRASCAFFOLD FIBER DIAMETER COMPARISON}

\section{Introduction}

This appendix contains ANOVA test results comparing the statistical similarity of fiber diameter data between sections of the same scaffold. Each test has four levels corresponding to the four sections imaged for fiber diameter from each scaffold (A, B, C, and D). Tukey's method of comparison was used to generate $95 \%$ confidence intervals for each ANOVA test and the intervals are presented below the ANOVA results. The confidence intervals show how the data from each section compares to the others based on individual means and a pooled standard deviation.

The key to understanding the intrascaffold fiber diameter ANOVA tests is the Pvalue. The P-value tells whether the null hypothesis (fiber diameter of all sections are equal) should be rejected or not. A small P-value indicates that there is a small chance of committing a type I error, which means rejecting the null hypothesis and being incorrect. 


\section{One-way ANOVA: Spin 1}

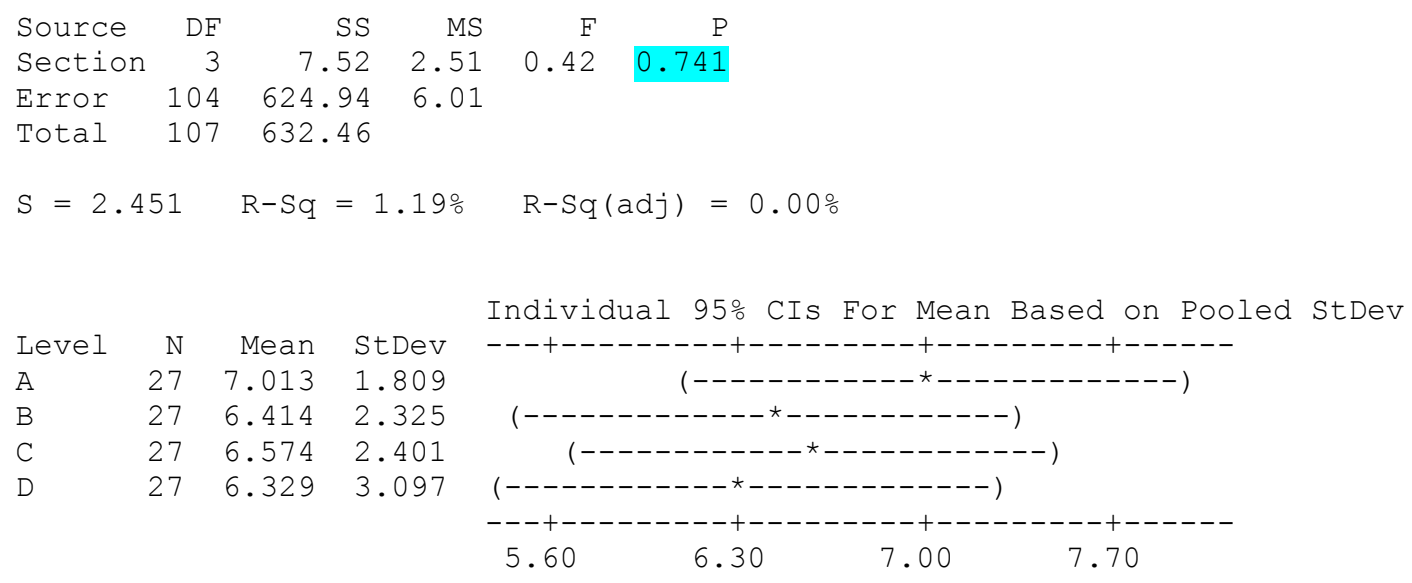

\section{One-way ANOVA: Spin 2}

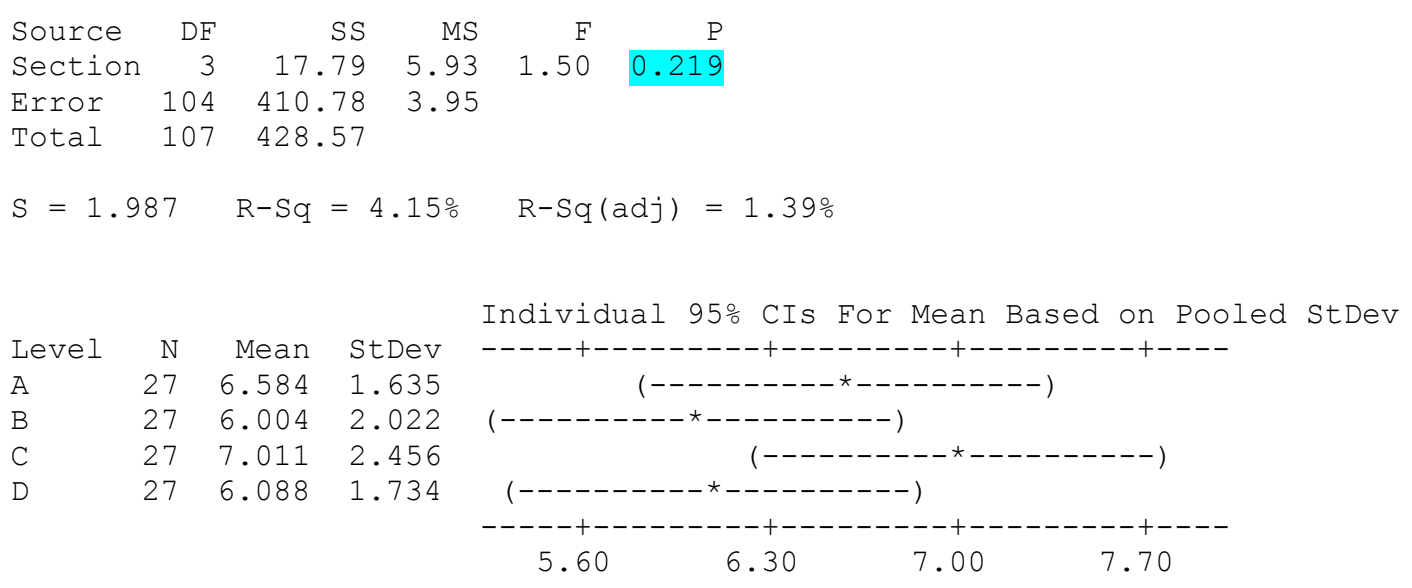

\section{One-way ANOVA: Spin 3}

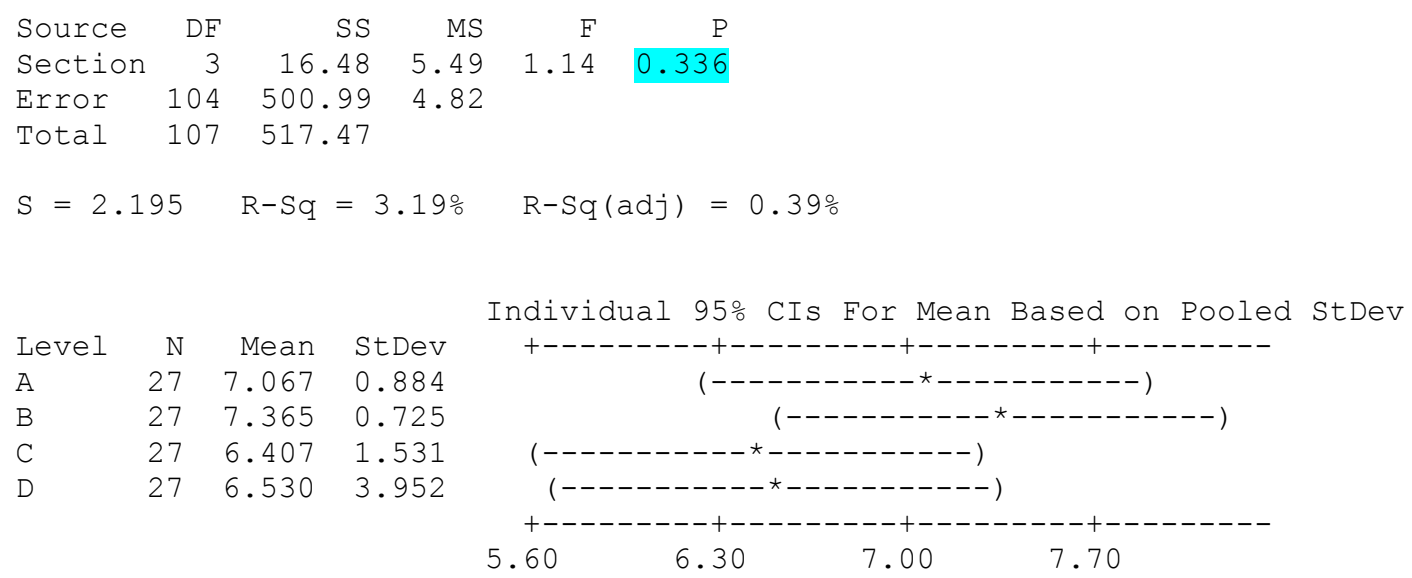


One-way ANOVA: Spin 4

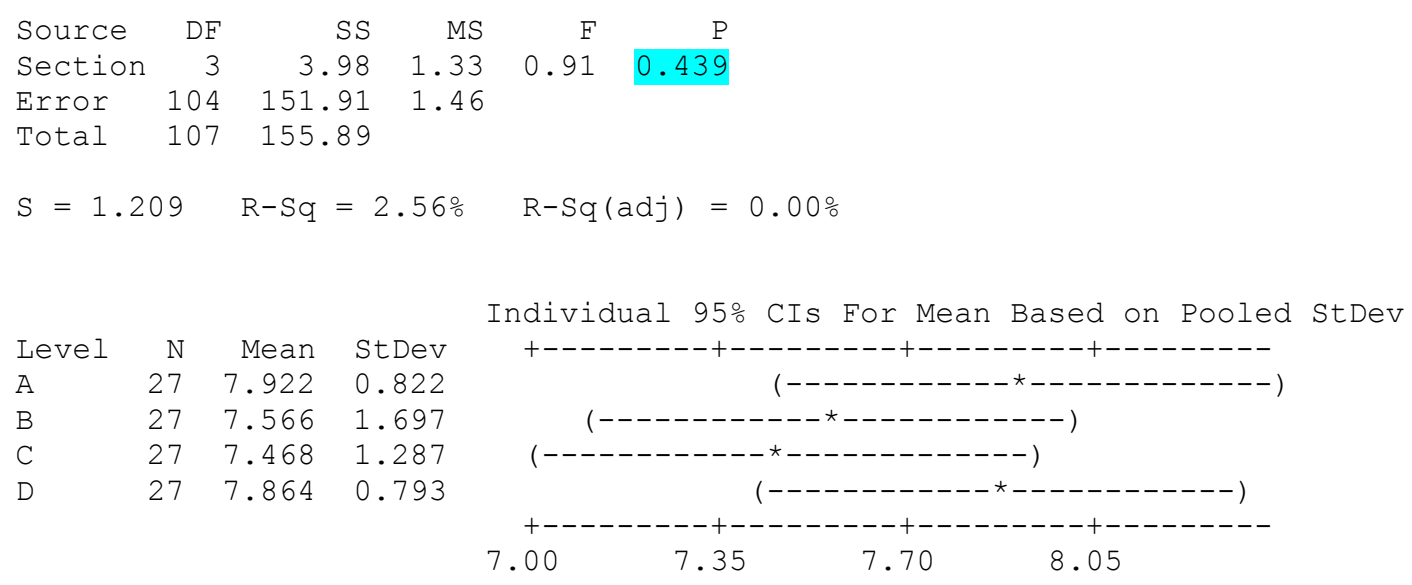

\section{One-way ANOVA: Spin 5}

\begin{tabular}{|c|c|c|c|c|}
\hline Source & $\mathrm{DF}$ & & SS & MS \\
\hline Sectio & 3 & 27. & 66 & 9.22 \\
\hline Error & 104 & 350. & 36 & 3.3 \\
\hline Total & $10^{\circ}$ & 378. & 02 & \\
\hline$S=1$. & 835 & $\mathrm{R}-\mathrm{Sq}$ & $=7$. & $32 \%$ \\
\hline Level & $\mathrm{N}$ & Mean & StD & Dev \\
\hline A & 27 & 6.339 & 1.8 & 398 \\
\hline B & 27 & 5.605 & 1.7 & 769 \\
\hline $\mathrm{C}$ & 27 & 5.647 & 1.7 & 735 \\
\hline D & 27 & 6.819 & 1.9 & 933 \\
\hline
\end{tabular}

$\begin{array}{rr}F & P \\ 2.74 & 0.047\end{array}$

$\mathrm{R}-\mathrm{Sq}(\mathrm{adj})=4.64 \%$

Individual 95\% CIs For Mean Based on Pooled StDev
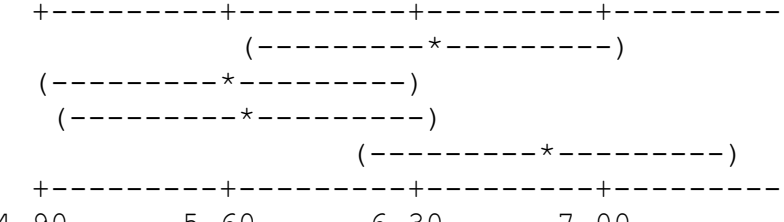

$\begin{array}{llll}4.90 & 5.60 & 6.30 & 7.00\end{array}$

One-way ANOVA: Spin 6

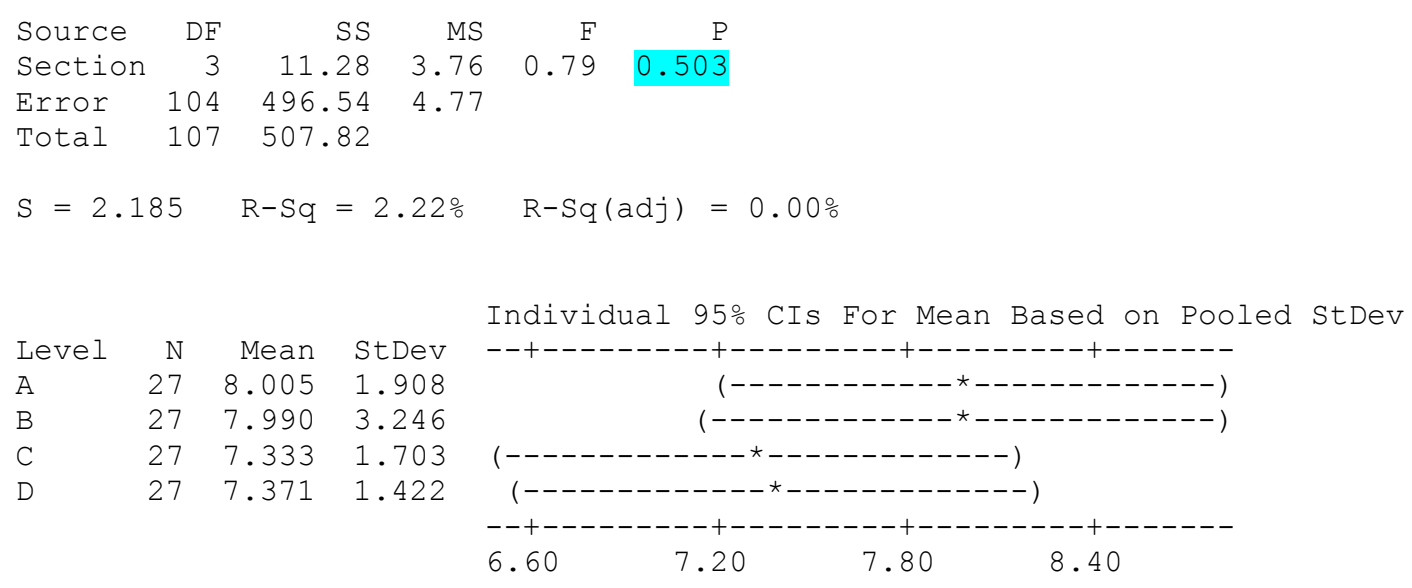


One-way ANOVA: Spin 7

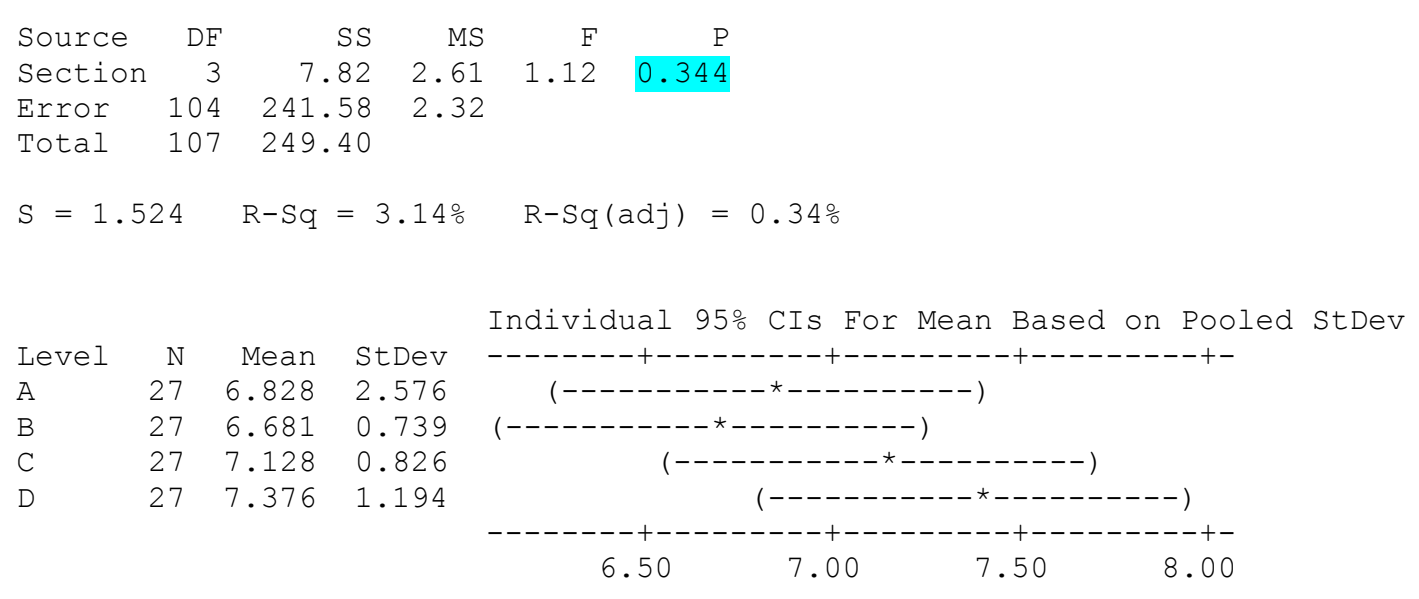

\section{One-way ANOVA: Spin 8}

\begin{tabular}{|c|c|c|c|c|}
\hline Source & $\mathrm{DF}$ & & SS & $\mathrm{MS}$ \\
\hline Sectio & 3 & & 73 & 3.24 \\
\hline Error & 104 & 264. & 81 & \\
\hline Total & 107 & 274 . & 54 & \\
\hline$S=1$. & 596 & $\mathrm{R}-\mathrm{Sq}$ & $=3.5$ & $54 \%$ \\
\hline Level & $\mathrm{N}$ & Mean & StDe & Dev \\
\hline A & 27 & 6.860 & 1.9 & 905 \\
\hline B & 27 & 7.609 & 1.5 & 597 \\
\hline C & 27 & 7.222 & 1.42 & 24 \\
\hline D & 27 & 7.556 & 1.4 & 406 \\
\hline
\end{tabular}

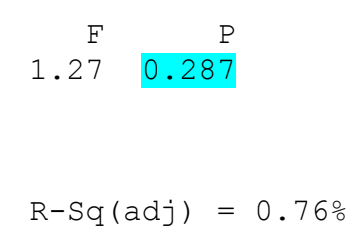

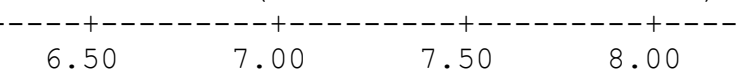

\section{One-way ANOVA: Spin 9}

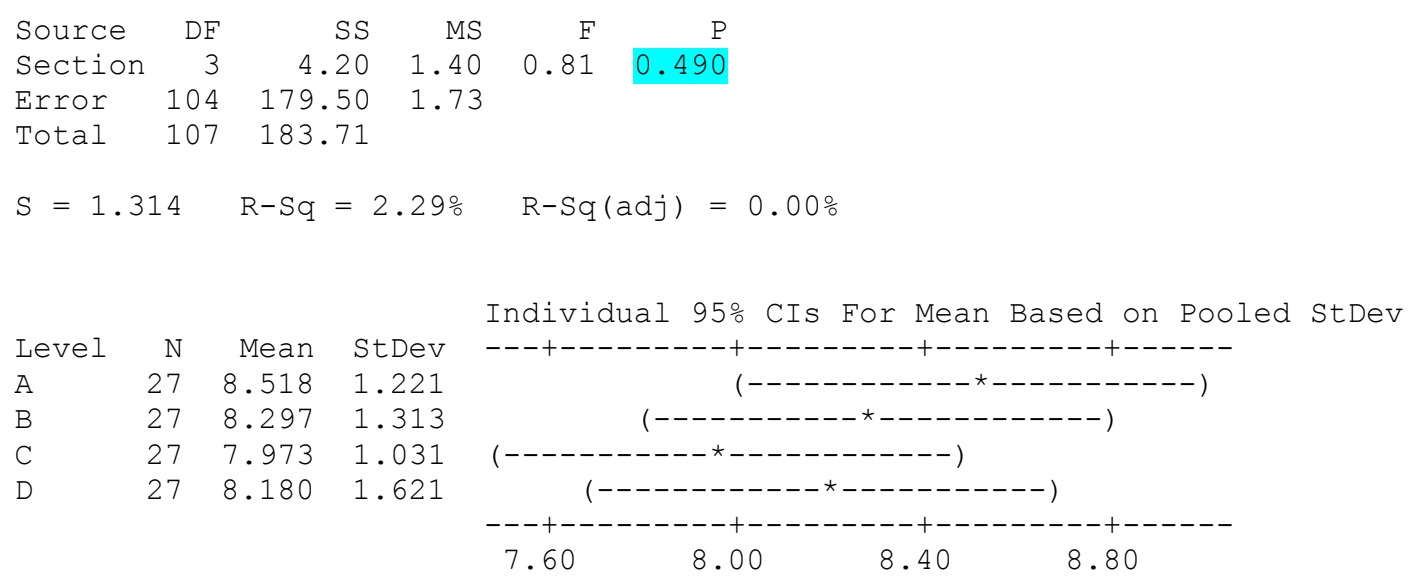


One-way ANOVA: Spin 10

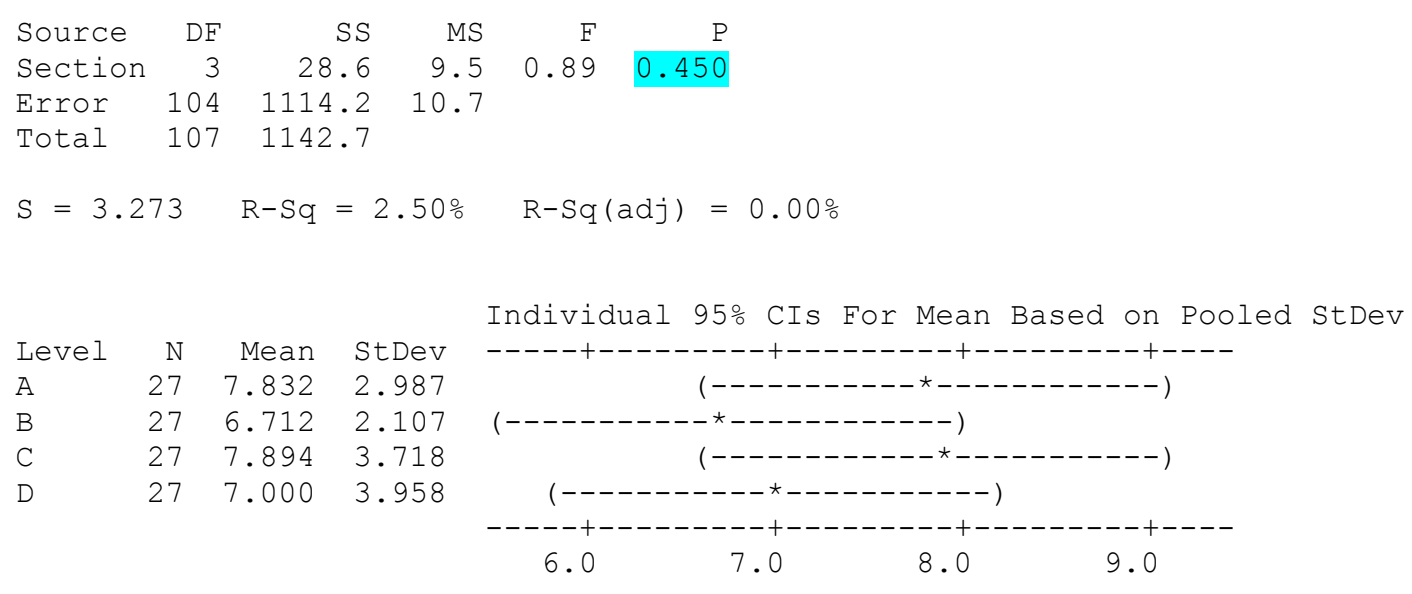

One-way ANOVA: Spin 11

\begin{tabular}{|c|c|c|c|c|}
\hline \multirow{2}{*}{$\begin{array}{l}\text { Source } \\
\text { Sectior }\end{array}$} & $\mathrm{DF}$ & SS & MS & $\mathrm{F} \quad \mathrm{P}$ \\
\hline & 3 & 53. & 17.8 & 3.150 .028 \\
\hline Error & 104 & 587. & 5.6 & \\
\hline Total & 107 & 641 & 18 & \\
\hline \multicolumn{2}{|c|}{$S=2.377$} & $\mathrm{R}-\mathrm{Sq}=$ & $=8.34 \%$ & $\mathrm{R}-\mathrm{Sq}(\mathrm{adj})=5.69 \%$ \\
\hline Level & $\mathrm{N}$ & Mean & StDev & Individual 95\% CIs For Mean Based on Pooled StDev \\
\hline A & 27 & 6.112 & 4.052 & $(--------\star--------)$ \\
\hline $\mathrm{B}$ & 27 & 8.083 & 0.734 & $(--------\star--------)$ \\
\hline C & 27 & 6.920 & 1.755 & $(--------\star--------)$ \\
\hline $\mathrm{D}$ & 27 & 7.184 & 1.601 & $(--------\star \star---------)$ \\
\hline & & & & $\begin{array}{cccc}6.0 & 7.0 & 8.0 & 9.0\end{array}$ \\
\hline
\end{tabular}




\section{APPENDIX I: INTRASCAFFOLD THICKNESS COMPARISON}

\section{Introduction}

This appendix contains ANOVA test results comparing the statistical similarity of wall thickness data between sections of the same scaffold. Each test has six levels corresponding to the six sections imaged for scaffold thickness from each scaffold (A1, B1, B2, C1, C2, and D1). Tukey's method of comparison was used to generate $95 \%$ confidence intervals for each ANOVA test and the intervals are presented below the ANOVA results. The confidence intervals show how the data from each section compares to the others based on individual means and a pooled standard deviation.

The key to understanding the intrascaffold wall thickness ANOVA tests is the Pvalue. The P-value tells whether the null hypothesis (wall thickness of all sections are equal) should be rejected or not. A small P-value indicates that there is a small chance of committing a type I error, which means rejecting the null hypothesis and being incorrect. 
One-way ANOVA: Spin 3

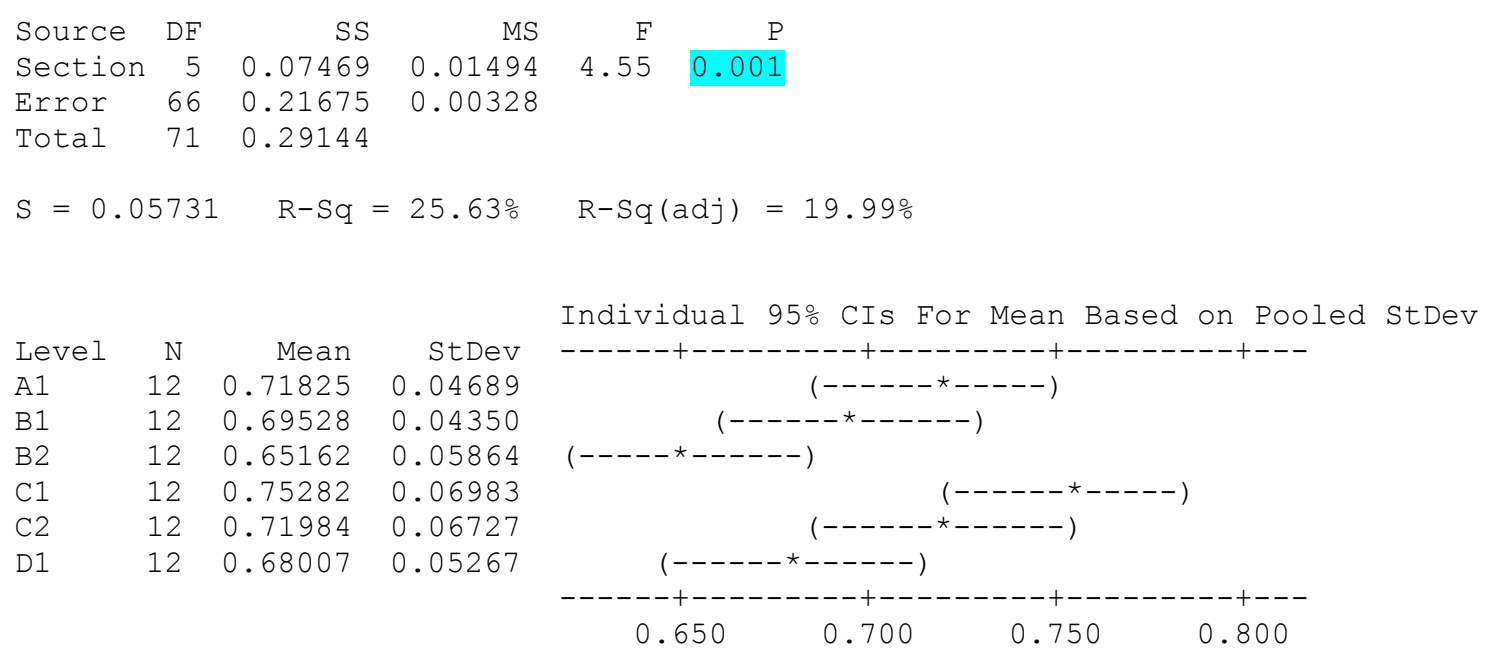

One-way ANOVA: Spin 4

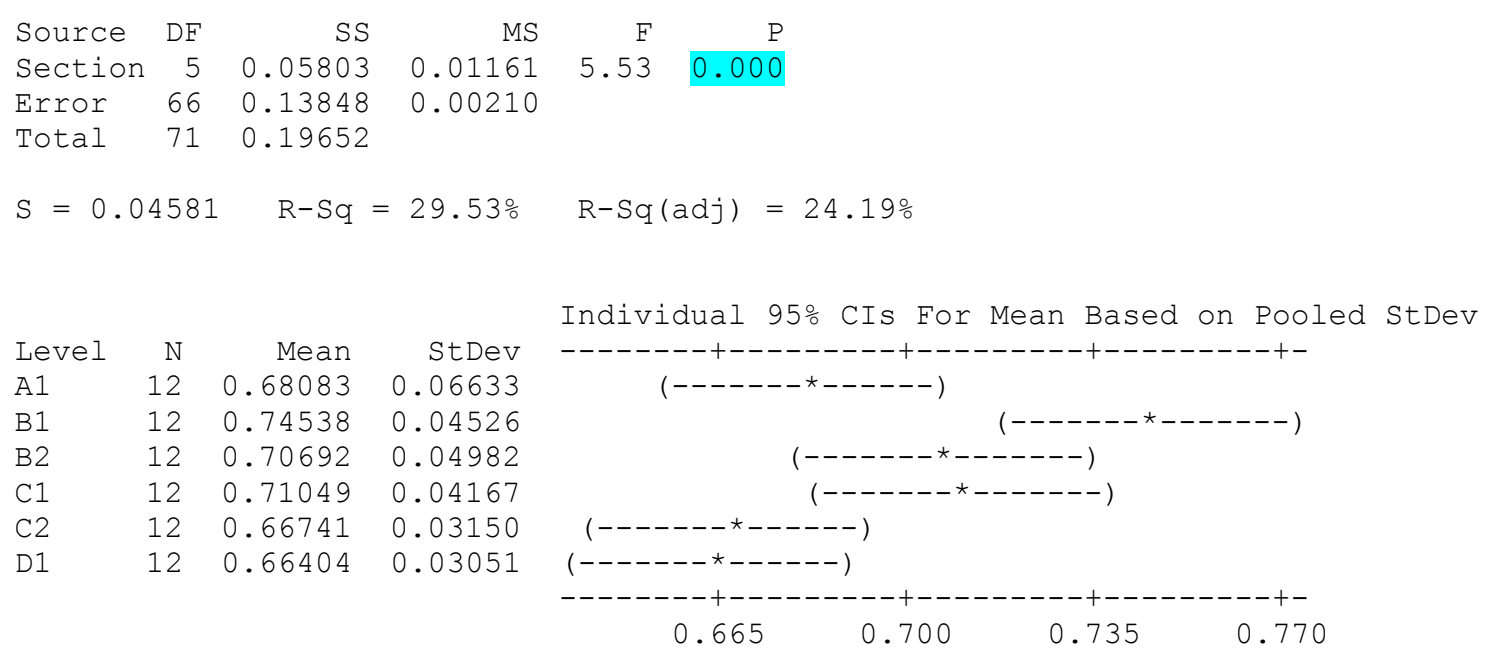


One-way ANOVA: Spin 5

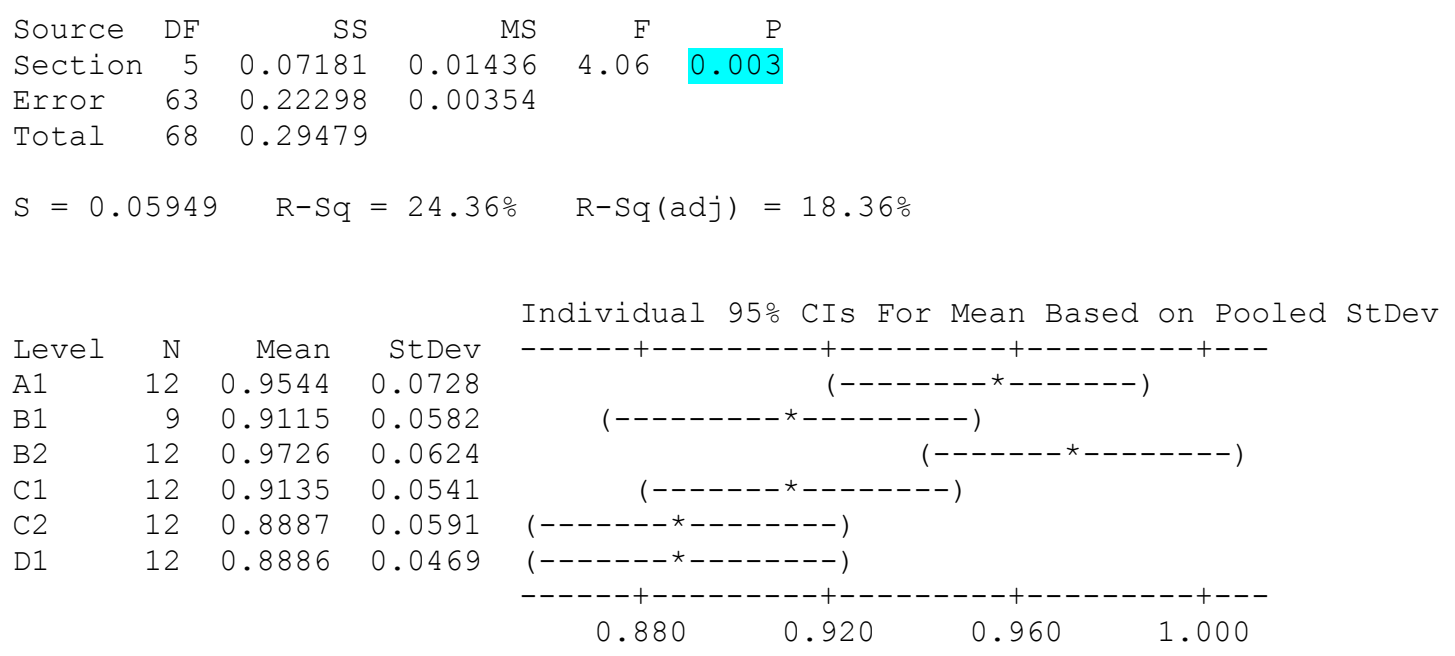

\section{One-way ANOVA: Spin 6}

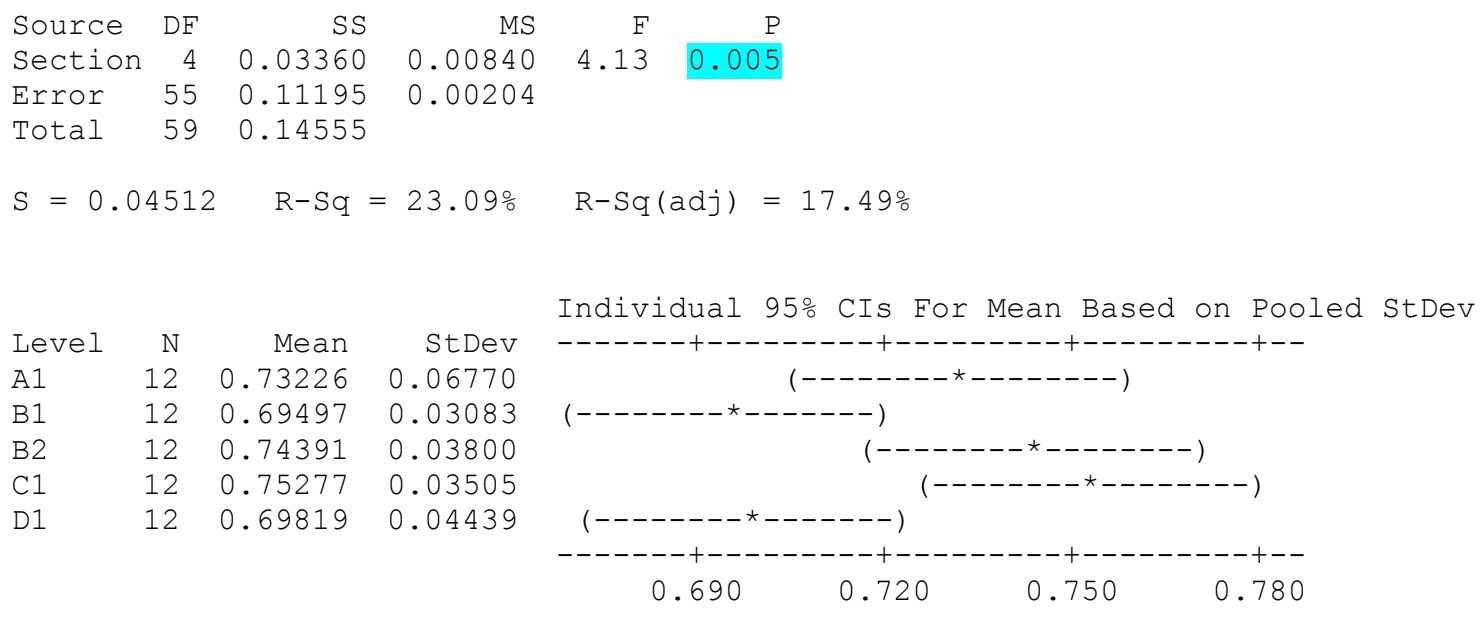


One-way ANOVA: Spin 7

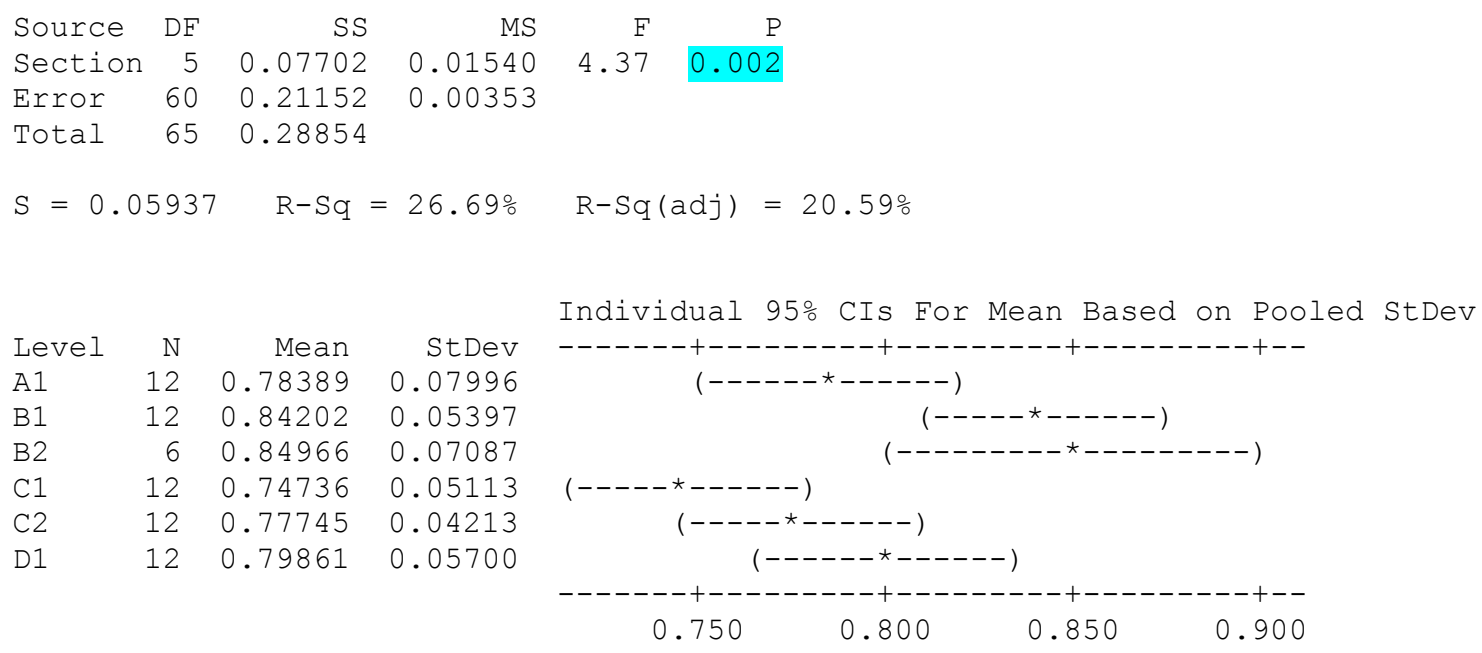

\section{One-way ANOVA: Spin 8}

$\begin{array}{lrrrrr}\text { Source } & \text { DF } & \text { SS } & \text { MS } & \text { F } & \text { P } \\ \text { Section } & 5 & 0.47638 & 0.09528 & 27.07 & 0.000 \\ \text { Error } & 60 & 0.21115 & 0.00352 & & \\ \text { Total } & 65 & 0.68752 & & & \\ \text { S }=0.05932 & \text { R-Sq }=69.29 \% & \text { R-Sq }(\text { adj })=66.73 \%\end{array}$

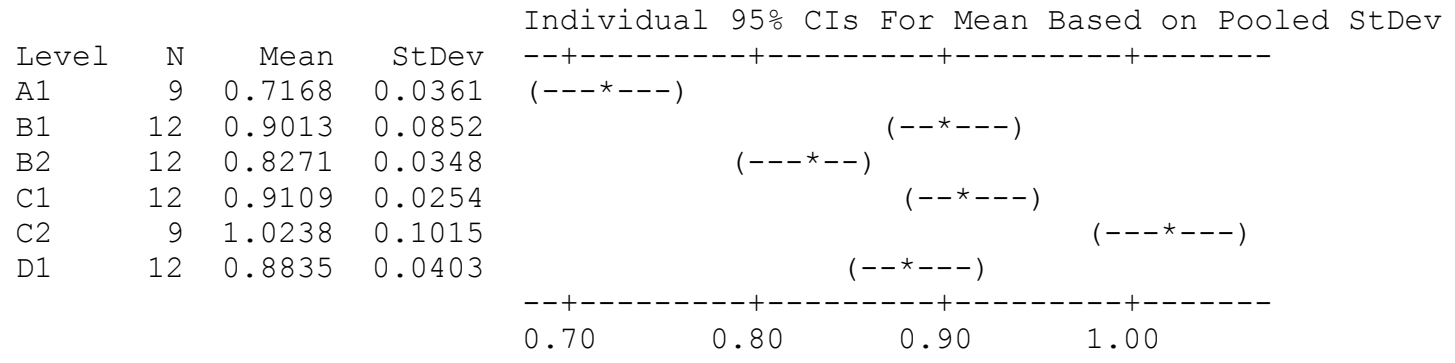




\section{One-way ANOVA: Spin 9}

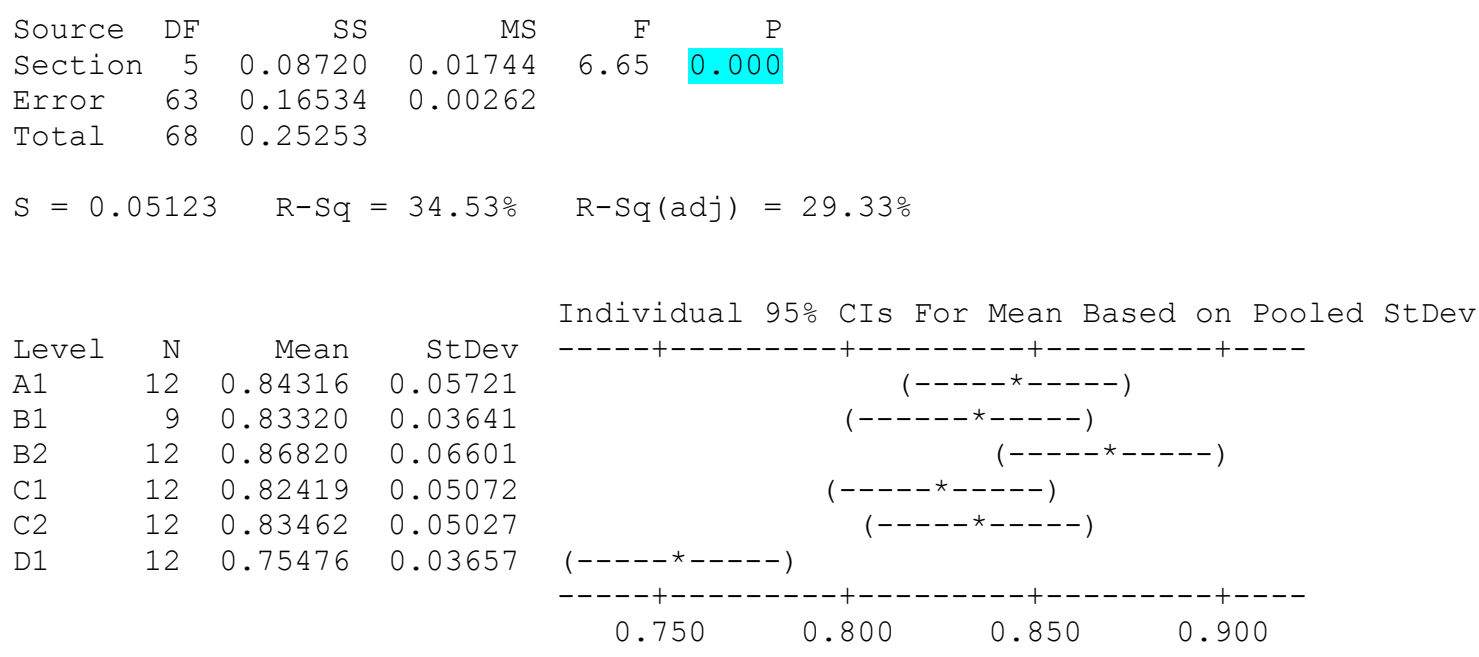
0.800
0.850
0.900

0.750

\section{One-way ANOVA: Spin 10}

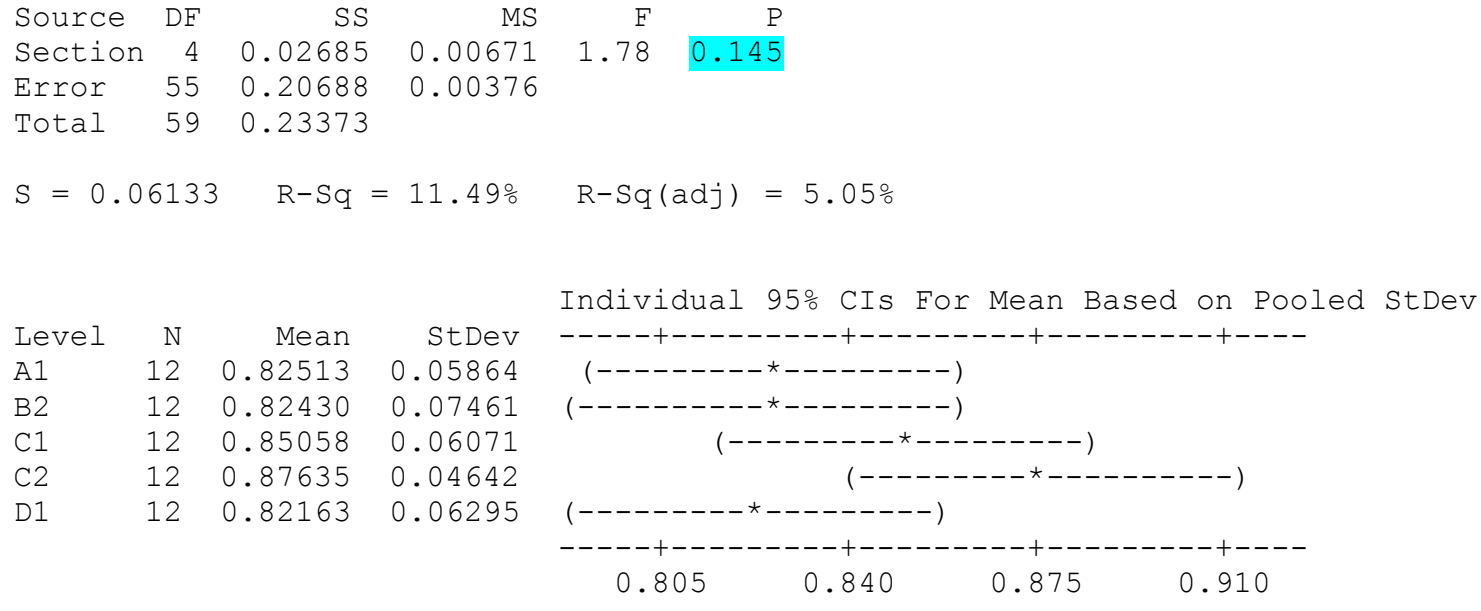
0.805
0.840

0.875

0.910 


\section{One-way ANOVA: Spin 11}

\begin{tabular}{|c|c|c|c|c|}
\hline Source & $\mathrm{DF}$ & SS & MS & $\mathrm{F}$ \\
\hline Section & 4 & 0.11431 & 0.02858 & 0.001 \\
\hline Error & 52 & 0.28742 & 0.00553 & \\
\hline Total & 56 & 0.40174 & & \\
\hline \multirow{2}{*}{\multicolumn{2}{|c|}{$S=0.07435$}} & $\mathrm{R}-\mathrm{Sq}$ & $=28.45 \%$ & $R-S q(\operatorname{adj})=22.95 \%$ \\
\hline & & & & $\begin{array}{l}\text { Individual 95\% CIs For Mean Based on } \\
\text { Pooled StDev }\end{array}$ \\
\hline Level & $\mathrm{N}$ & Mean & StDev & ---+---------+---------+---------+------ \\
\hline A1 & 12 & 0.76086 & 0.06483 & $(------*------)$ \\
\hline B1 & 12 & 0.74420 & 0.03681 & $(------\star------)$ \\
\hline B2 & 9 & 0.77799 & 0.13009 & $(--------\star-------)$ \\
\hline $\mathrm{C} 1$ & 12 & 0.86154 & 0.06589 & $(-------\star------)$ \\
\hline \multirow[t]{2}{*}{ D1 } & 12 & 0.82872 & 0.06262 & $(------\star------)$ \\
\hline & & & & $\begin{array}{c}---+--- \\
0.720\end{array}$ \\
\hline
\end{tabular}




\section{APPENDIX J: PRELIMINARY CELLULAR RESPONSE TO ELECTROSPUN P(LLA-CL) SCAFFOLDING}

\section{Introduction}

For electrospun scaffolding to be effective in the BVM, fiber diameter should be decreased to sub-micron levels. The process used in this thesis failed to produce fibers with diameters this small. Despite their large fiber diameter, the electropsun P(LLA-CL) constructs were implemented in the BVM as a preliminary cell viability test. Full confluency was not the anticipated outcome; rather, this experiment tested the hypothesis that the electrospun P(LLA-CL) would not have a cytotoxic effect in the BVM system.

\section{Methods and Materials}

Scaffold Fabrication and Preparation

All electrospinning parameters were the same as those used in the consistency study. Upon completion of the electrospinning process, the construct was allowed to dry in a dessicator for 24 hours. Two $2.5 \mathrm{~cm}$ long scaffolds were cut from the center of the construct, as well as four $0.2 \mathrm{~cm}$ long samples used to measure average fiber diameter (). Fiber diameter was measured using SEM micrographs in the same manner as the consistency study (Image J), but the fiber selection mask was not used. Instead, 15 fibers were randomly selected and measured. The two scaffolds were sterilized using ethylene oxide gas $(\mathrm{EtOH})$ prior to implementation in the BVM. 
Cell Sodding and Implentation Into the BVM

The sterilized scaffolds were sutured into the BVM system in the same manner as ePTFE scaffolding. Nutrient supplemented media was supplied to the system and the scaffolds were allowed to soak prior to cell sodding. The scaffolds were sodded at a density of $1 \times 10^{6}$ cells $/ \mathrm{cm}^{2}$ with $\mathrm{p} 7$ HUVECs and allowed to sit for 1 hour, then the 8roller peristaltic pump was turned on at a speed of $15 \mathrm{rpm}$, corresponding to a flow rate of approximately $1 \mathrm{ml} / \mathrm{min}$. After two hours, one scaffold was removed from a BVM system and placed in histochoice to halt cellular activity and fix cells to the scaffold. The second scaffold was allowed to stay in the BVM for 24 hours before it was removed and placed in histochoice.

Imaging of Scaffolds

Scaffolds were placed in a bis benzene sulphonylimide (BBI) staining solution overnight for histological examination. Scaffolds were cut in half lengthwise, and then imaged using a fluorescent light microscope. Following histological examination, scaffolds were placed in a desiccator to dry overnight. Once dry, the scaffolds were prepared for SEM imaging in the same manner as the samples were prepared in the consistency study. SEM images were then taken for high resolution topographical examination of the scaffolds. 


\section{Results}

Qualitative analysis of fluorescent microscope images revealed cell presence on the lumen of electrospun P(LLA-CL) scaffolds at both 2 (Figure J1) and 24 hours (Figure Figure J2). The fluorescent microscope images suggest that discrete sections of the scaffold lumens were confluent with ECs, while other sections were void of cells.

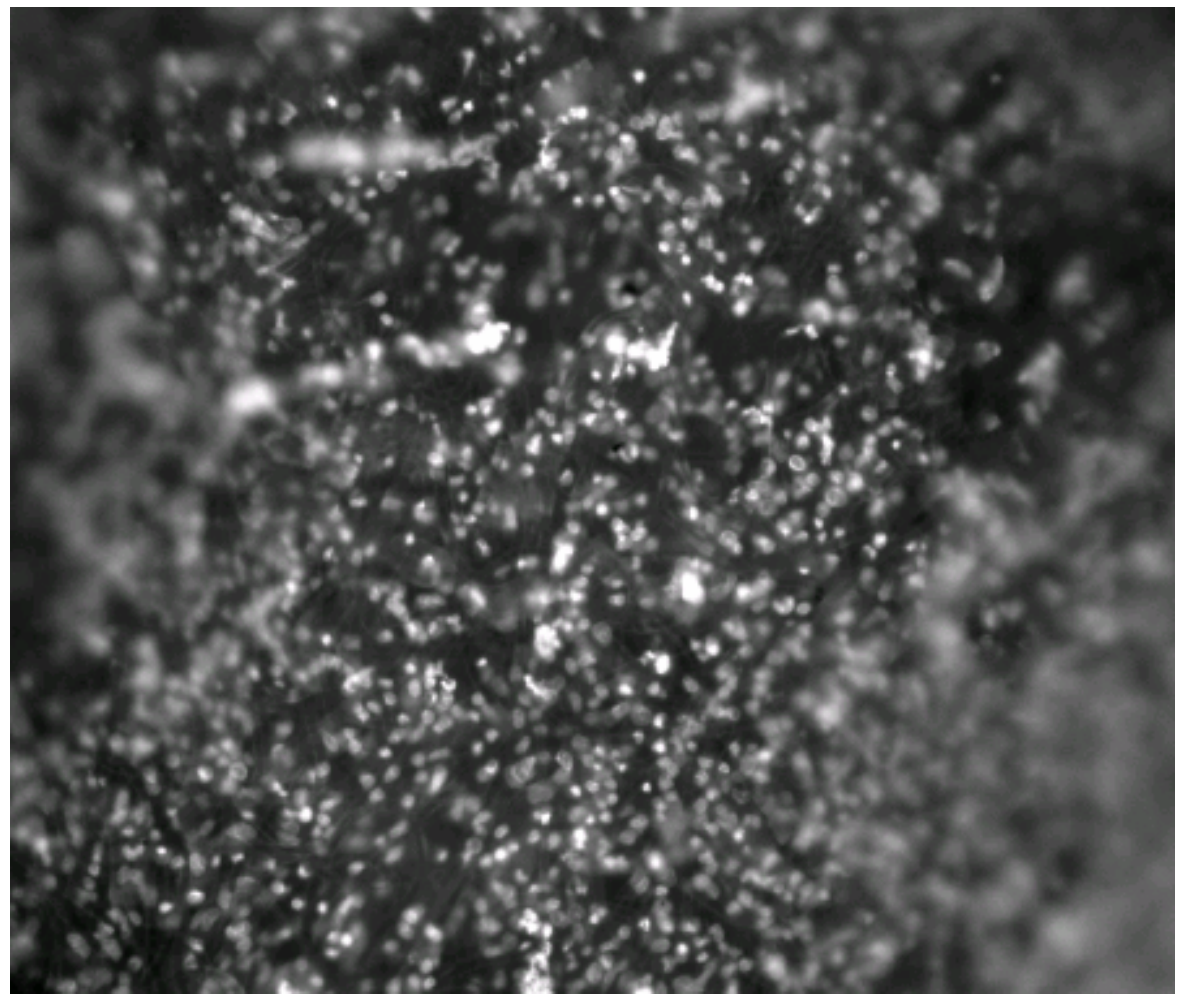

Figure J1 - Histological examination of electrospun P(LLA-CL) scaffolding sodded with HMVECs indicated that the in-house fabricated scaffolds did not have a cytotoxic effect after 2 hours in the BVM. (100x) 


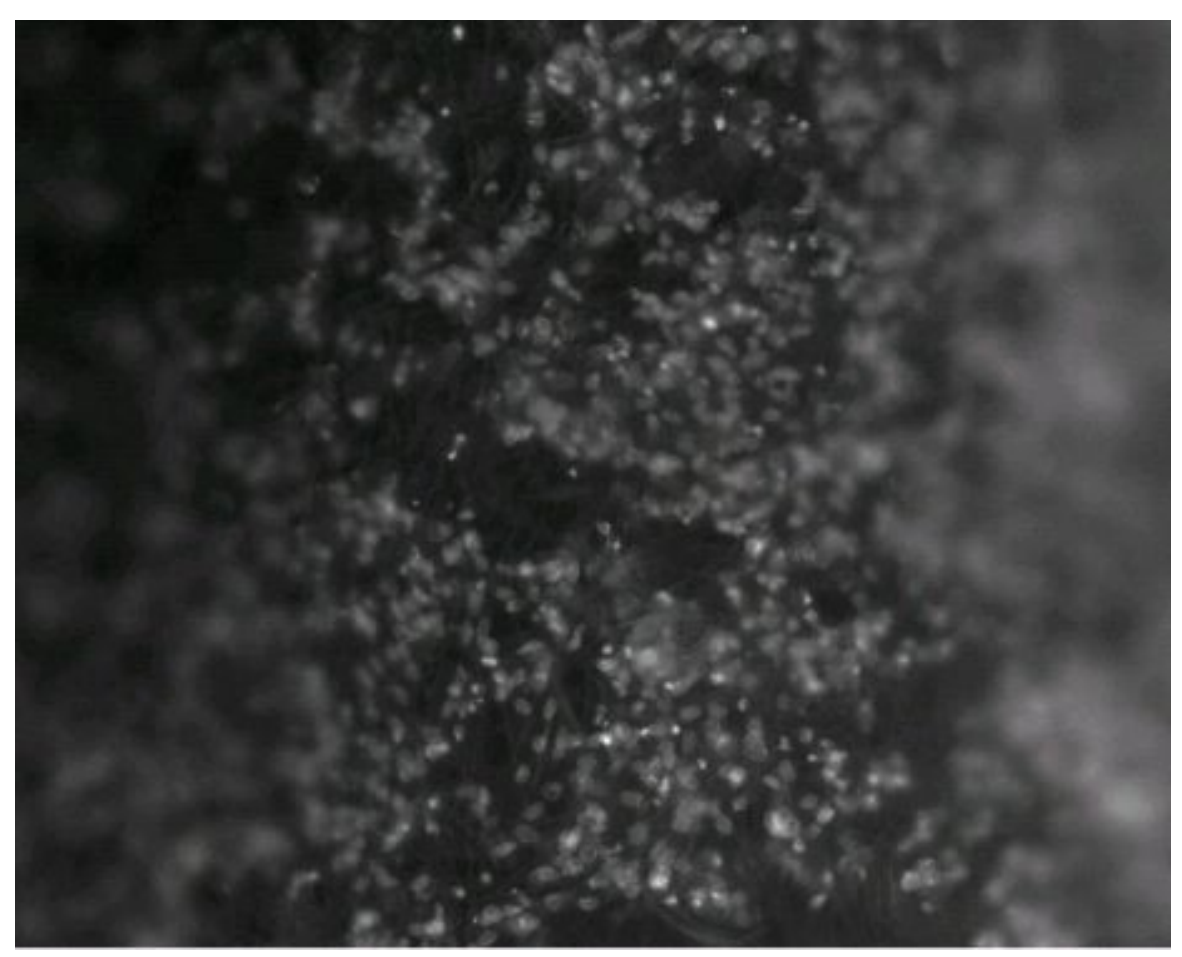

Figure J2 - Histological examination of an electrospun P(LLA-CL) scaffold sodded with HMVECs after 24 hours of flow conditions in the BVM. (100x)

Analysis of SEM images showing the lumen of the sodded electrospun scaffolds after 2 hours (Figure J3) and 24 hours (Figure J4) of flow conditions confirmed that discrete areas of the scaffolds were void of cells. In areas with ECs, cells stretched from one fiber to adjacent fibers. 


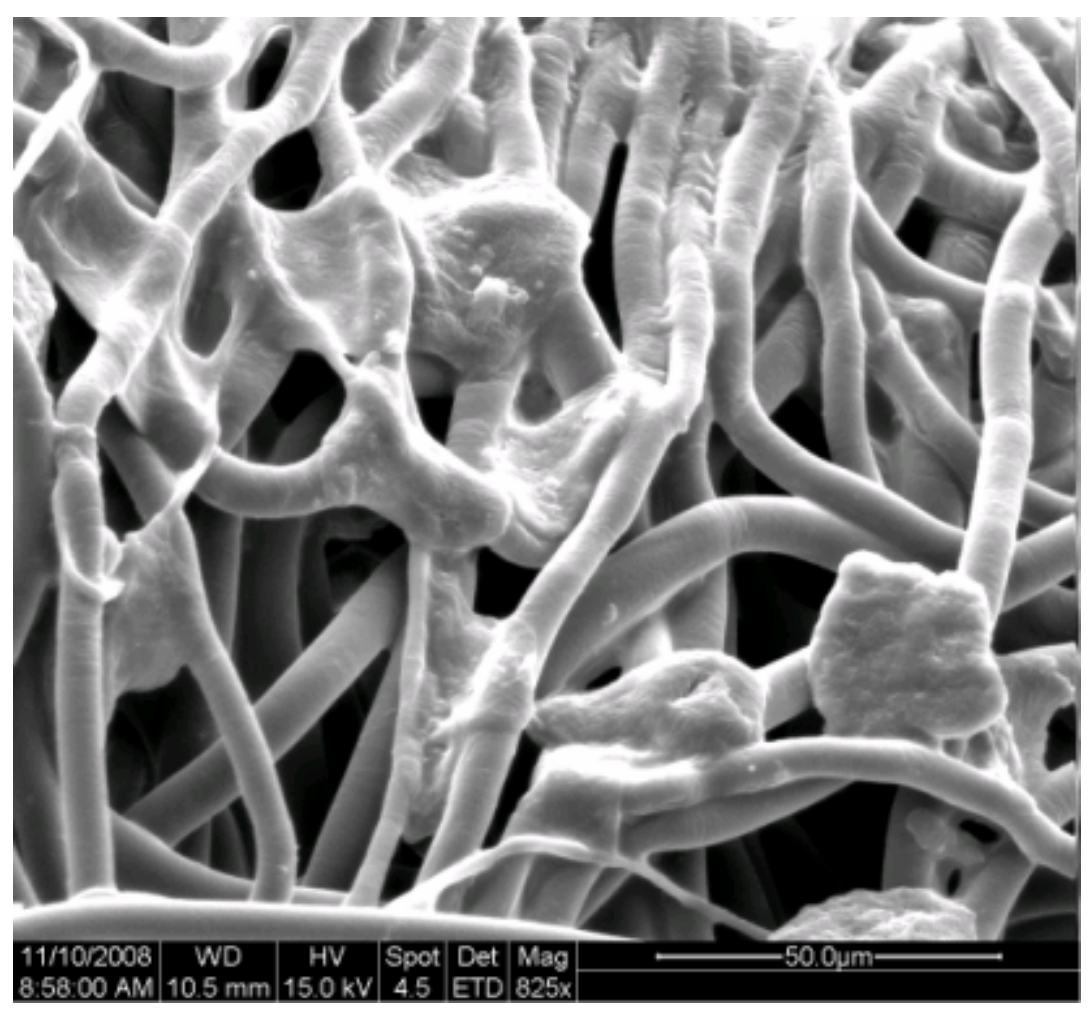

Figure J3 - SEM image of the lumen of an electrospun P(LLA-CL) scaffold sodded with ECs and cultured under flow conditions for 2 hours. (825x) 


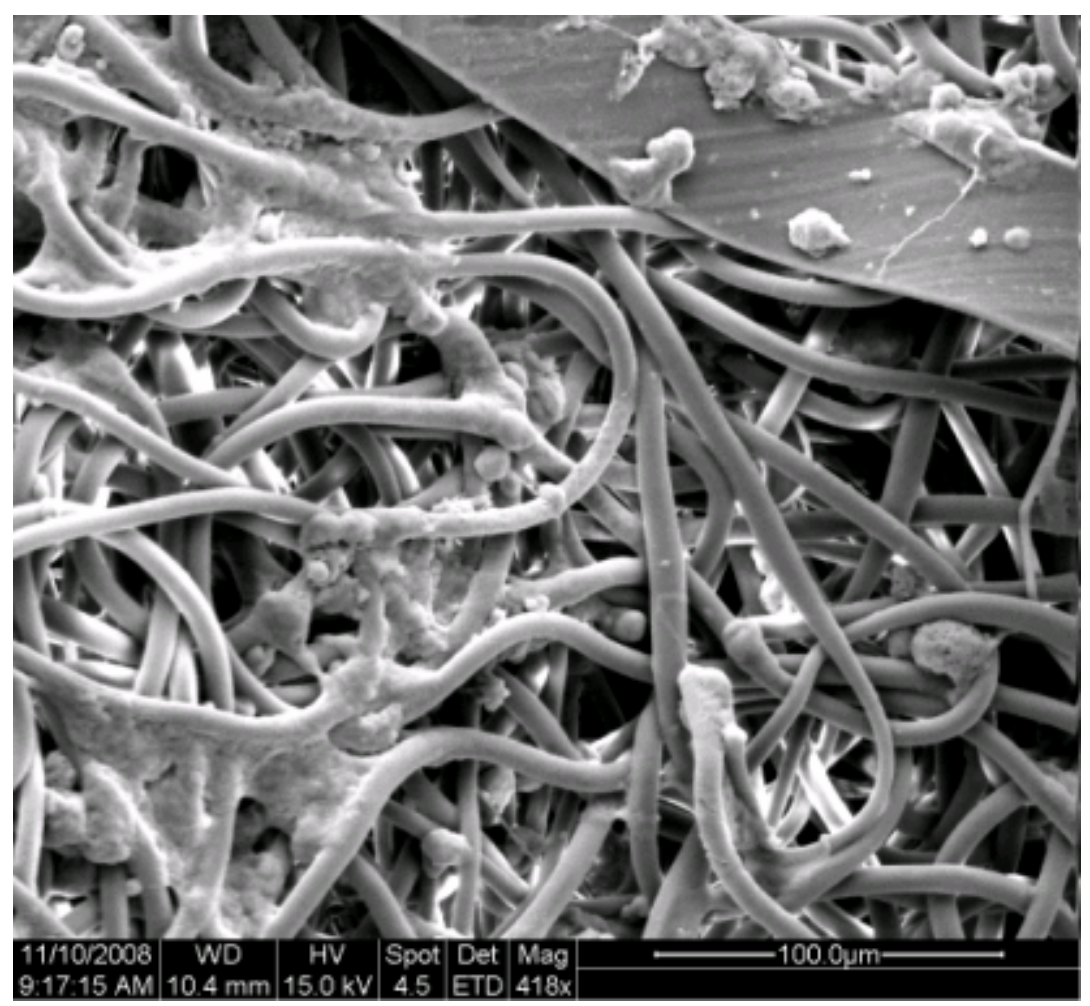

Figure J4 - SEM image after 24 hours of flow condition culturing for the sodded electrospun P(LLA-CL) scaffold. (418x)

\section{Discussion}

These preliminary results suggest that electrospun P(LLA-CL) scaffolds do not have a cytotoxic effect. A long term study is necessary to ascertain the true potential of these scaffolds to support ECs in the BVM. This may include looking over time at the migration, proliferation, and \% confluency of ECs, as well as the effect of local pH fluctuation due to scaffold degradation.

In addition, a confluent layer of ECs did not form on the lumen of the scaffolds. This outcome was anticipated, as some of the pore sizes were large enough to permit cells 
to pass through into the interior of the scaffold. For cells that did attach, stretching from fiber to fiber was characteristic.

Another possible use for these scaffolds is support for a dual-sodded BVM developed within our lab group. The dual-sodding procedure incorporates a base layer of SMCs in addition to a top layer of ECs. The large pores in the electrospun P(LLA-CL) may allow for infiltration of SMCs that would later provide support for a confluent luminal layer of ECs. 